\title{
Three-Dimensional Mixed-Mode Fracture of the Four-Point Shear Specimen
}

\author{
By
}

\author{
Mark Cohen
}

A thesis submitted to the Faculty of Graduate and Postdoctoral
Affairs in partial fulfillment of the requirements for the degree of

Master of Applied Science
in

Mechanical Engineering

Carleton University

Ottawa, Ontario

(C) 2015, Mark Cohen 


\begin{abstract}
Traditionally, fracture testing has focused on the fracture toughness of specimens undergoing pure mode I loading, often simplified by 2D assumptions of plane strain or plane stress. In reality, components have finite-thickness, and often see complex loading configurations. To study this complex behaviour, fracture specimens for mixed-mode loading have been developed. For these mixed-mode specimens, available literature solutions are primarily only for an idealized 2D case.

In this thesis, extensive three-dimensional finite element analysis is conducted to study two specimens: the (antisymmetric) four-point shear (FPS) specimen- a mode II test specimen, and the asymmetric four-point shear (AFPS) specimen - a mixed mode I/II specimen. Complete solutions of fracture mechanics parameters $K_{I}, K_{I I}, K_{I I I}, T_{11}$, and $T_{33}$ have been obtained for a wide range of $a / W$ and $t / W$ geometry combinations. The plastic zone sizes of these specimens have also been obtained and analyzed.

It is demonstrated that the thickness of the specimen has a significant effect on the variation of both fracture parameter values and plastic zone sizes. The results presented here will be very useful for the toughness testing of materials under mixed-mode loading conditions.
\end{abstract}




\section{Acknowledgements}

Firstly, I would like to express my sincere gratitude to my supervisor, Dr. Xin Wang, for giving me the opportunity to take part in this research project. I am very thankful for all the guidance and support Dr. Wang has provided me throughout the course of my studies.

I would like to thank my parents for their love and for always supporting and encouraging me in my studies. I am deeply grateful to my mother Cathy for her help proofreading and editing this thesis, and always being there for me with advice and a positive outlook throughout this process.

Finally, I would like to thank my wife Jennifer, for her love and understanding. Without her support, none of this would be possible.

Financial support for this research project was made possible through research grants from Natural Sciences and Engineering Research Council of Canada (NSERC). 


\section{Table of Contents}

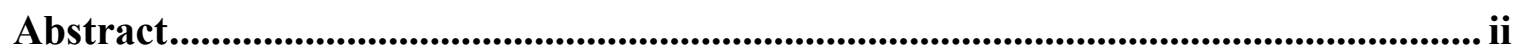

Acknowledgements .................................................................................................................. iii

List of Tables ............................................................................................................................ viii

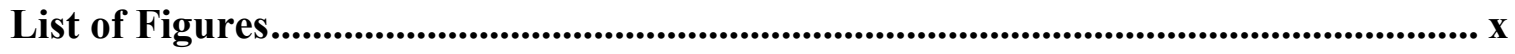

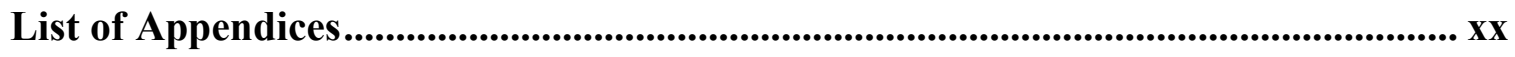

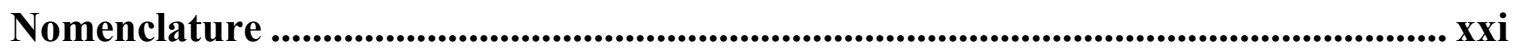

Chapter 1: $\quad$ Introduction ........................................................................................................... 1

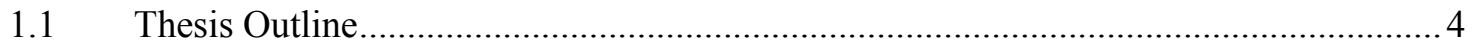

Chapter 2: $\quad$ Background and Literature Review ...................................................... 6

2.1 Linear Elastic Fracture Mechanics (LEFM) …..................................................... 6

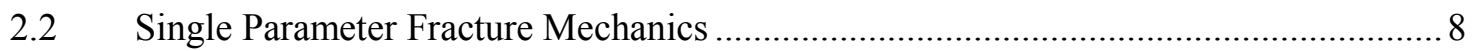

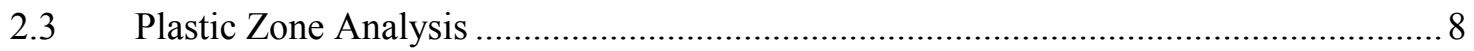

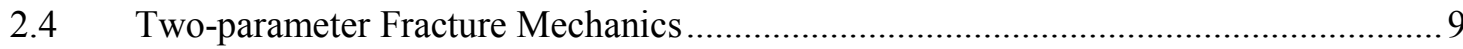

2.5 Literature Review: Three-dimensional (3D) Mixed Mode and T-stress ..................... 11

2.6 Plastic Zone Size from Three-dimensional Elastic FEM ....................................... 15

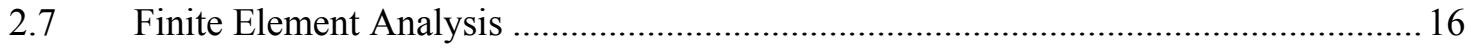

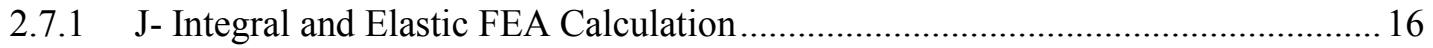

2.7.2 Contour Integral Evaluation in Abaqus/CAE ...................................................... 17

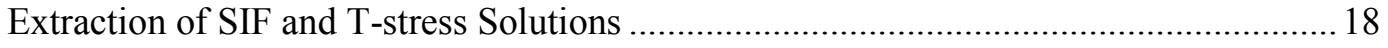


2.8 Mixed Mode: The Asymmetric Four-point Shear Specimen

2.9 Motivation for Current Work

Chapter 3: Three-Dimensional Fracture Analysis of FPS Test Specimens .......... 24

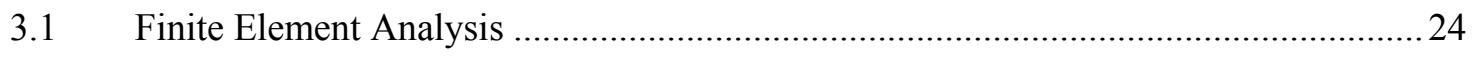

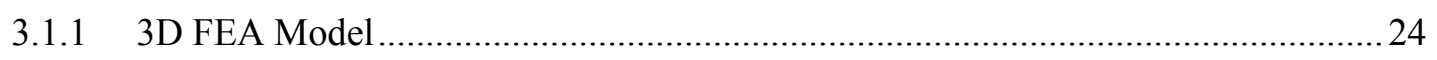

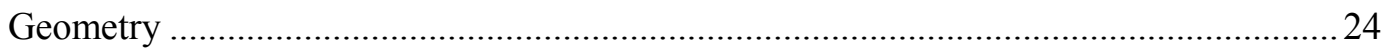

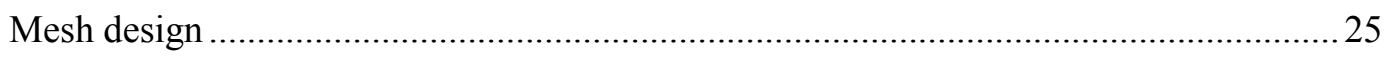

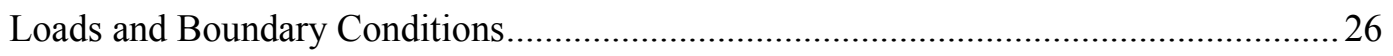

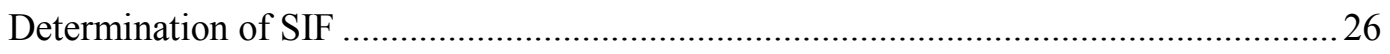

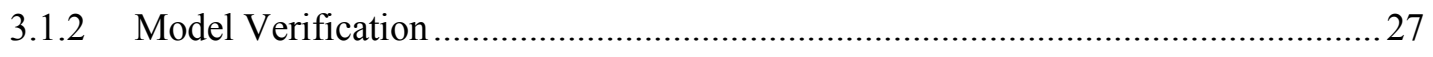

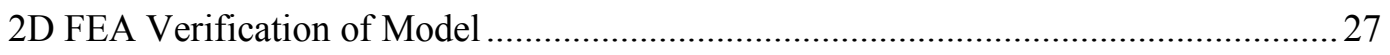

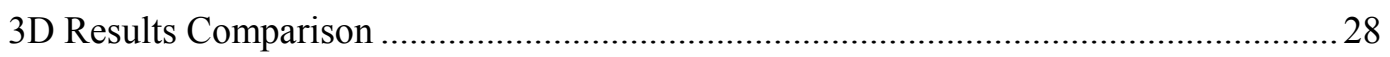

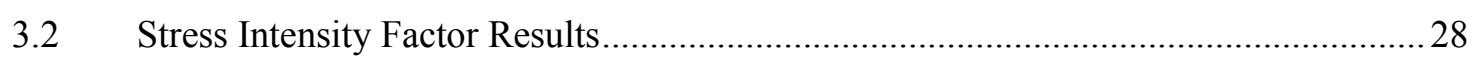

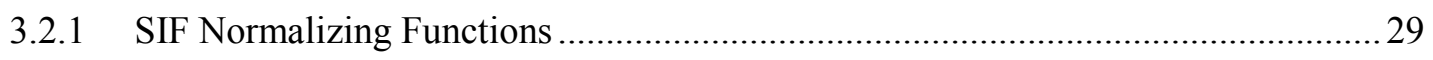

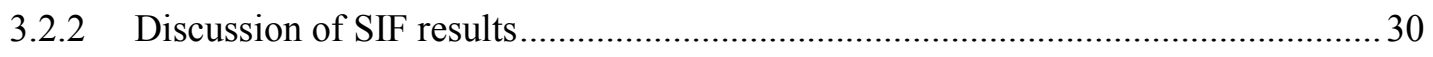

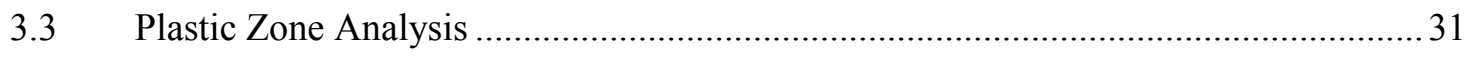

3.3.1 Calculating and Plotting the Plastic Zone from LEFM Parameters ......................... 31

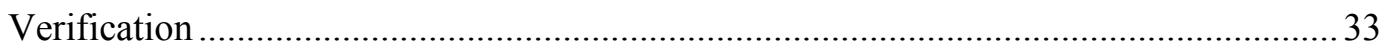

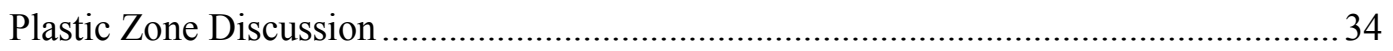

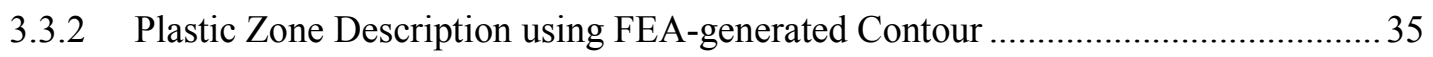

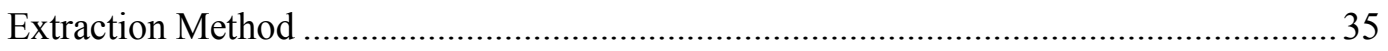




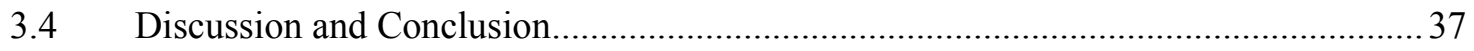

\section{Chapter 4: Three-Dimensional Fracture Analysis of Mixed-mode I/II Test Specimens $\quad 81$}

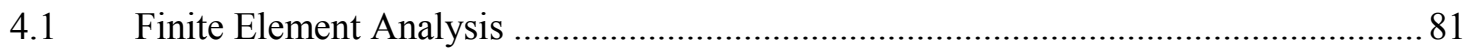

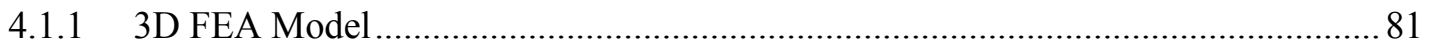

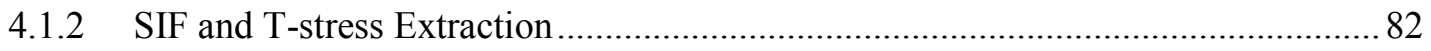

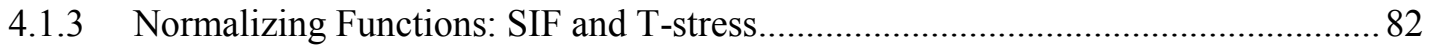

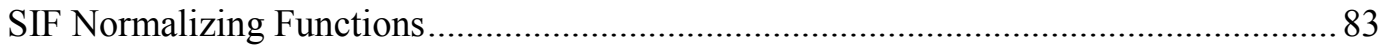

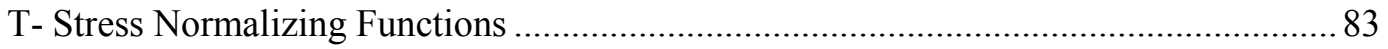

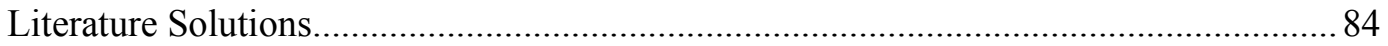

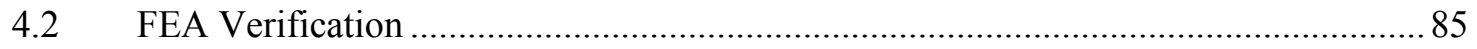

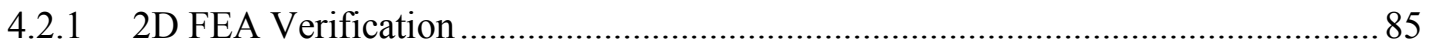

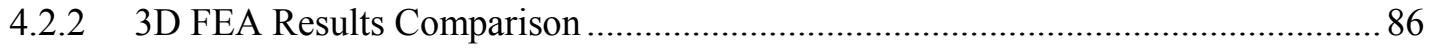

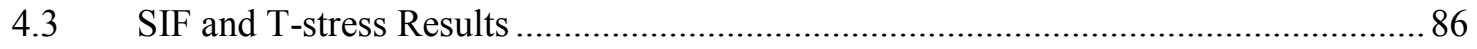

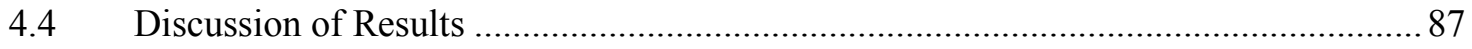

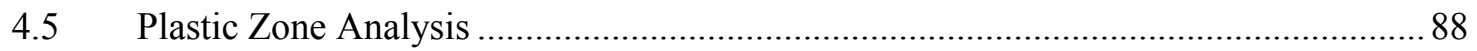

4.5.1 Calculating and Plotting the Plastic Zone from LEFM Parameters ......................... 88

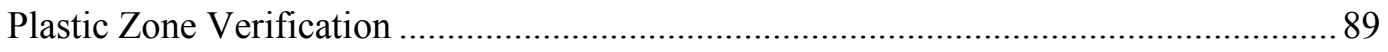

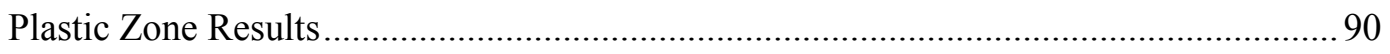

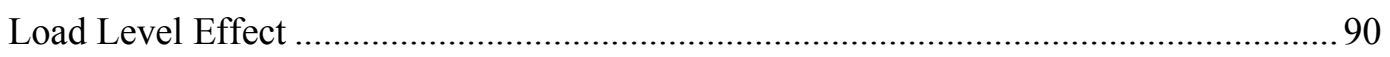


4.5.2 Plastic Zone Description using FEA-generated Contour .......................................92

Discussion on FEA Extracted Comparisons ……....................................................... 92

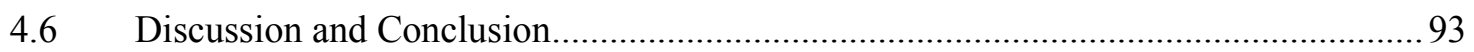

Chapter 5: Discussion and Conclusion ......................................................................... 180

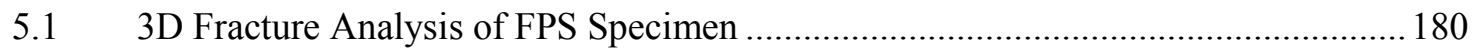

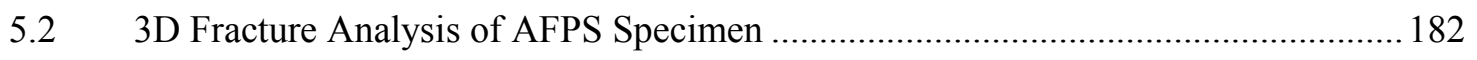

Appendices...................................................................................................................................... 185

Appendix A : Plastic Zone Calculation Method (Nazarali and Wang 2011) .......................... 185

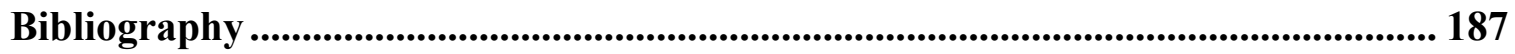




\section{List of Tables}

Table 3.1: Comparison of current $K_{I I}$ values and literature solution ............................... 39

Table 3.2: Normalized $K_{I I}$ values at the mid plane, for plates of different thickness ....... 39

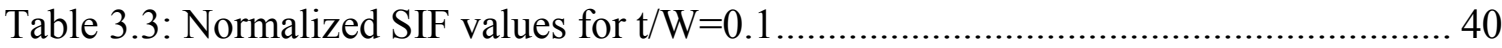

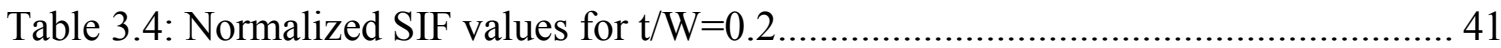

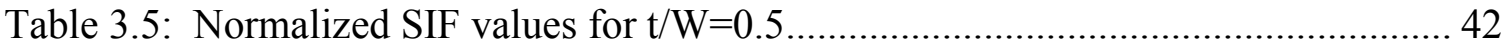

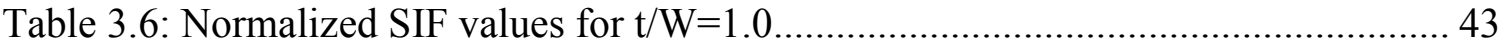

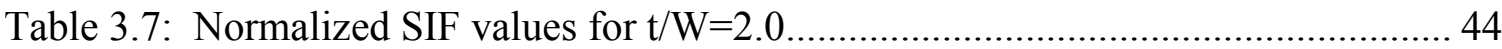

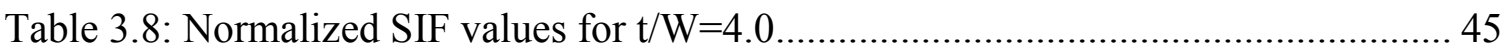

Table 4.1 : 2D FEA results comparison values............................................................... 95

Table 4.2: 3D FEA Mid plane results comparison values ................................................ 95

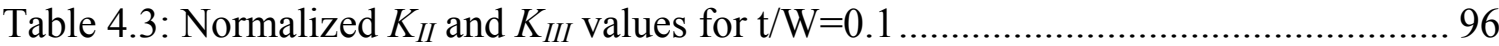

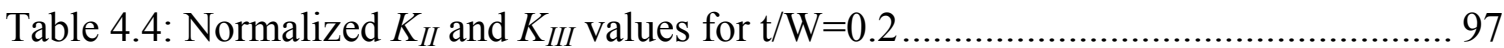

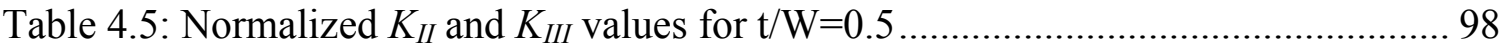

Table 4.6: Normalized $K_{I I}$ and $K_{I I I}$ values for t/W=1.0

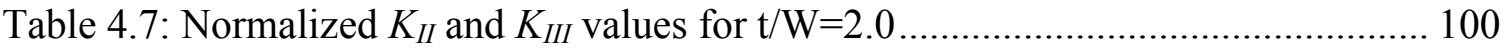

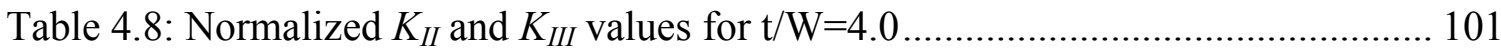

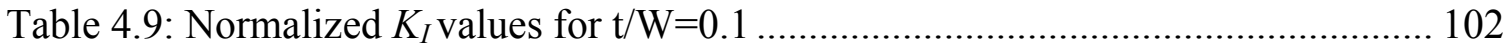

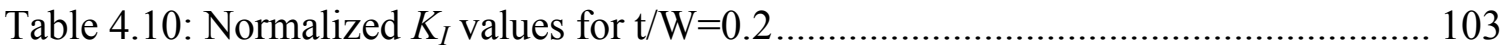


Table 4.11: Normalized $K_{I}$ values for $\mathrm{t} / \mathrm{W}=0.5$

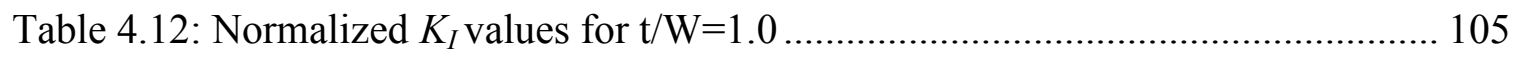

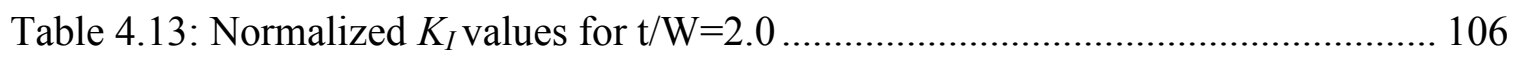

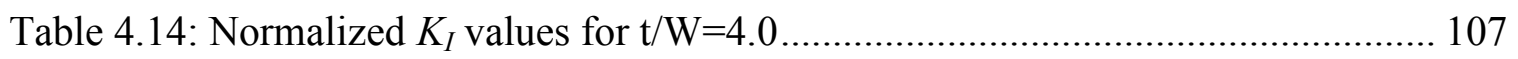

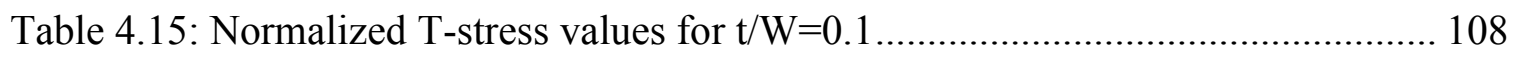

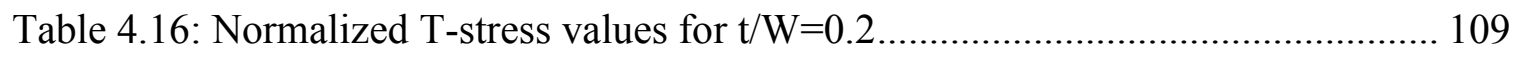

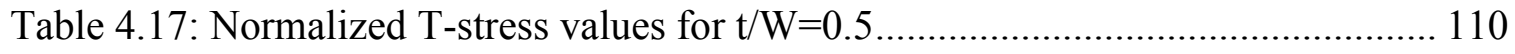

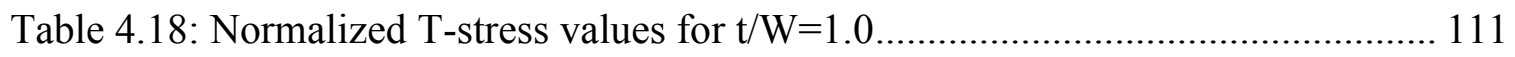

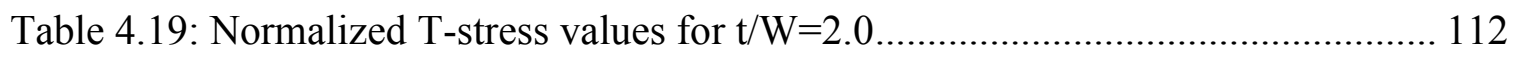

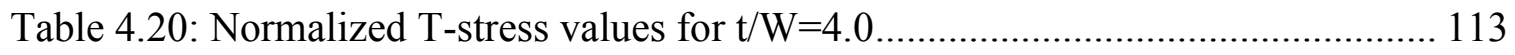




\section{List of Figures}

Figure 1.1: The asymmetric four-point shear specimen (He, Cao, and Evans 1990) ........ 5

Figure 1.2: The four-point shear (FPS) specimen............................................. 5

Figure 1.3: The asymmetric four-point shear (AFPS) specimen ................................ 5

Figure 2.1: Polar coordinate system of stress around a crack tip.................................. 21

Figure 2.2: Modes of loading in fracture mechanics ................................................ 21

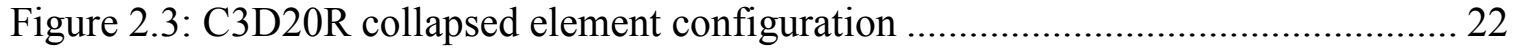

Figure 2.4: The asymmetric four-point-shear specimen ........................................ 23

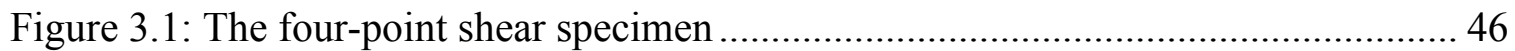

Figure 3.2: Typical finite element mesh for four-point shear specimen $(\mathrm{t} / \mathrm{W}=1.0$,

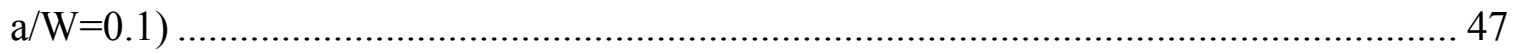

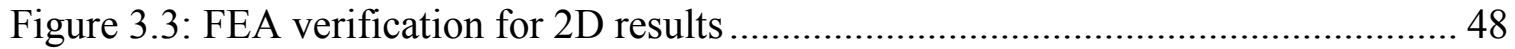

Figure 3.4: Comparison of normalized $K_{I I}$ values at the mid plane for plates of different

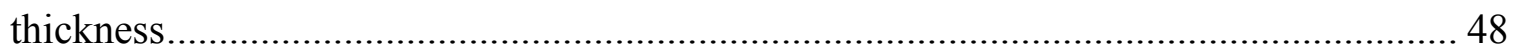

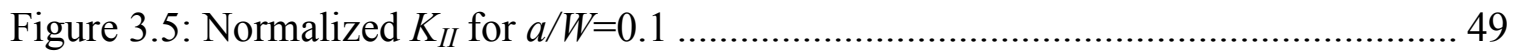

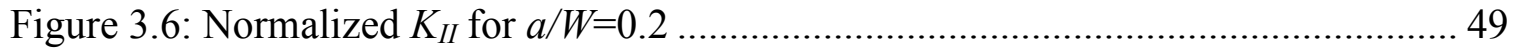

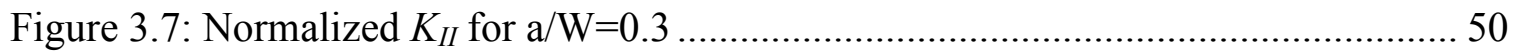

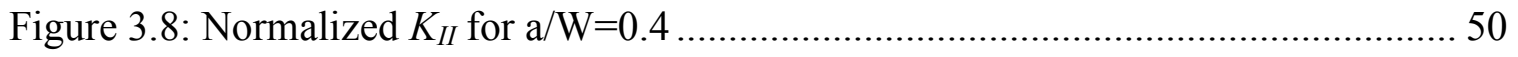

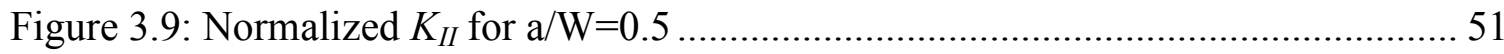

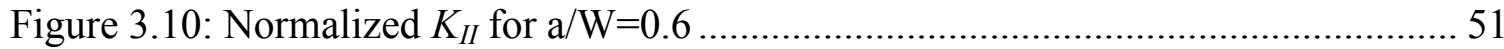

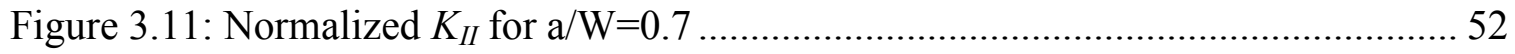


Figure 3.12: Normalized $K_{I I I}$ for a/W=0.1 53

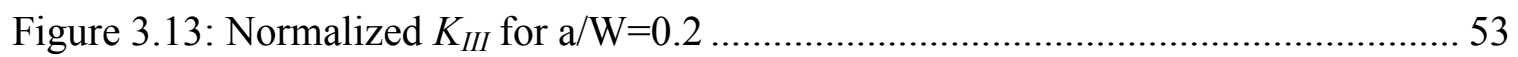

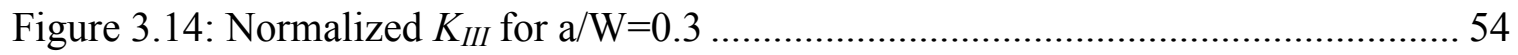

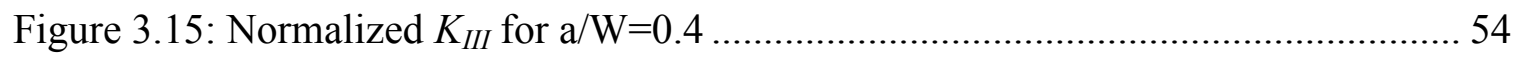

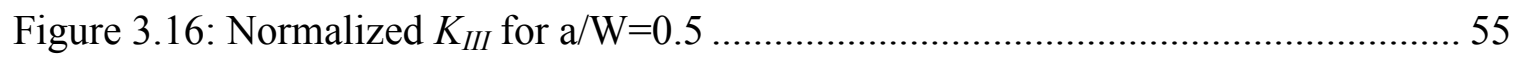

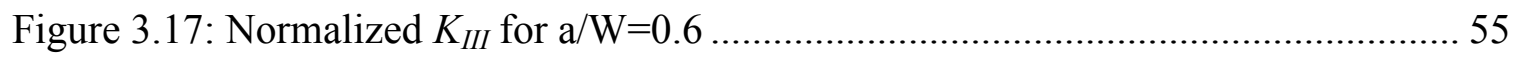

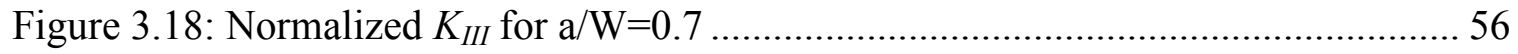

Figure 3.19: Plastic zone verification of pure mode II case ........................................ 57

Figure 3.20: Plastic zone verification of pure mode III case ..................................... 57

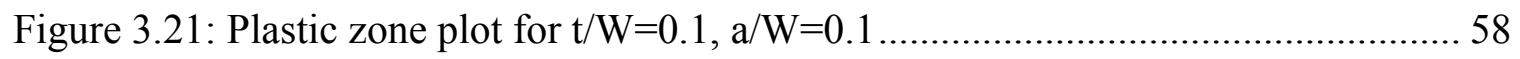

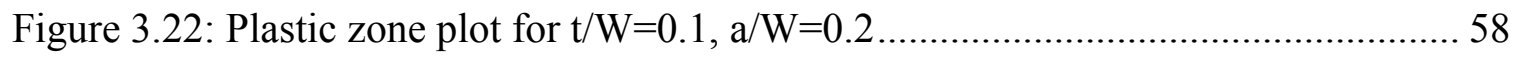

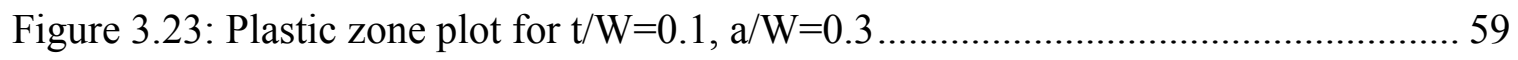

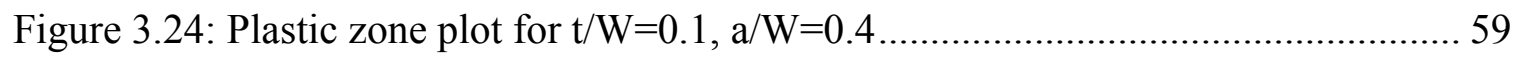

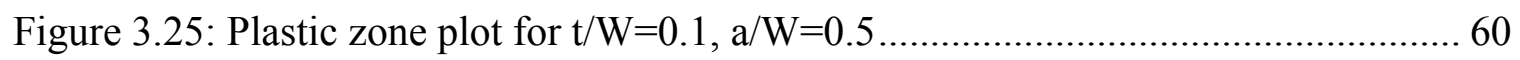

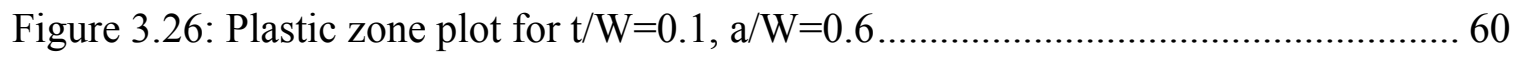

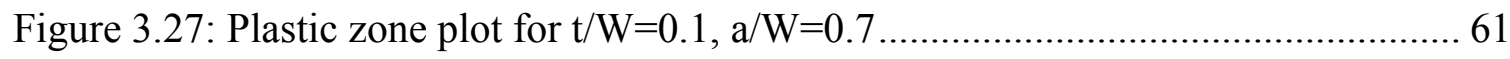

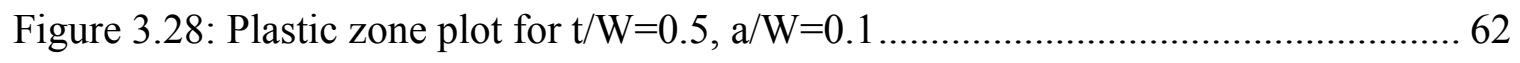

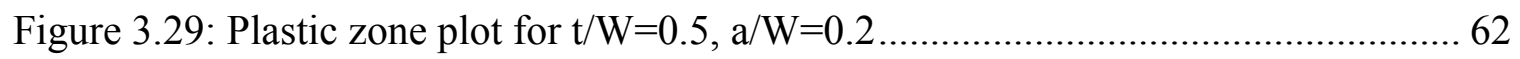

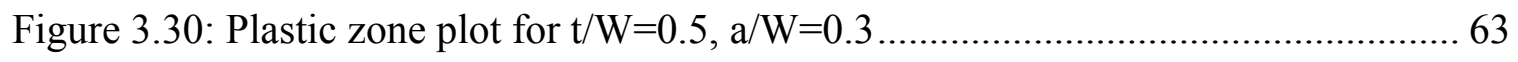

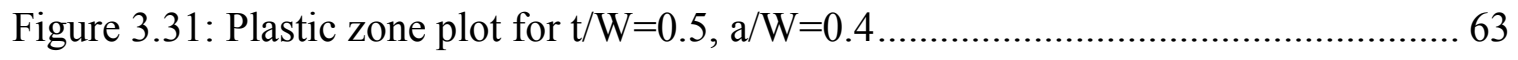


Figure 3.32: Plastic zone plot for $\mathrm{t} / \mathrm{W}=0.5$, $\mathrm{a} / \mathrm{W}=0.5$. 64

Figure 3.33: Plastic zone plot for $\mathrm{t} / \mathrm{W}=0.5, \mathrm{a} / \mathrm{W}=0.6$ 64

Figure 3.34: Plastic zone plot for $\mathrm{t} / \mathrm{W}=0.5, \mathrm{a} / \mathrm{W}=0.7$ 65

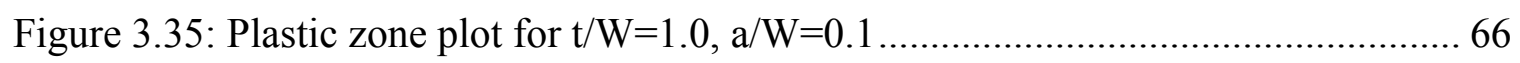

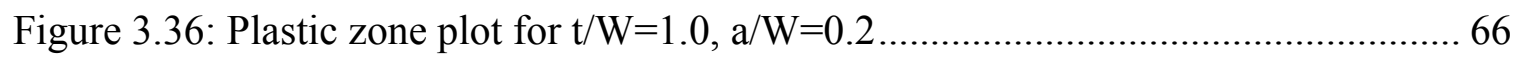

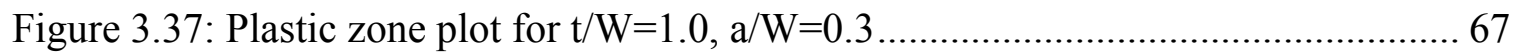

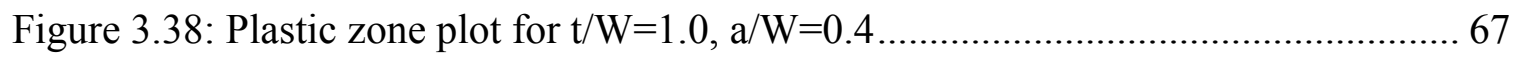

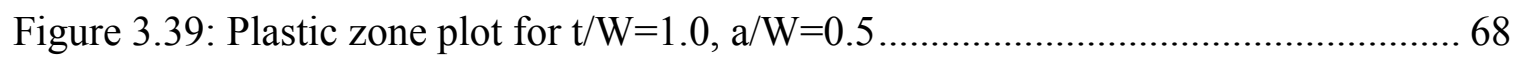

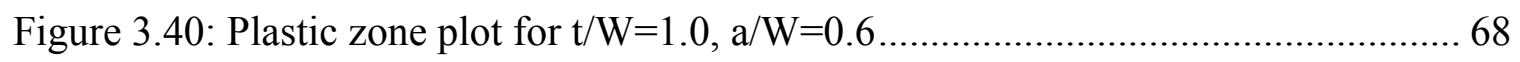

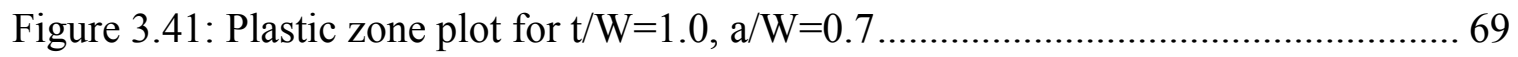

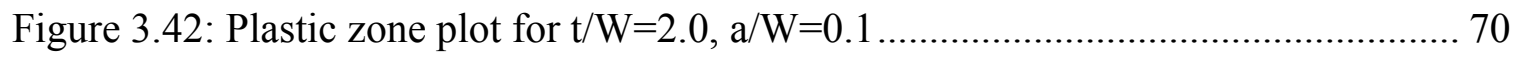

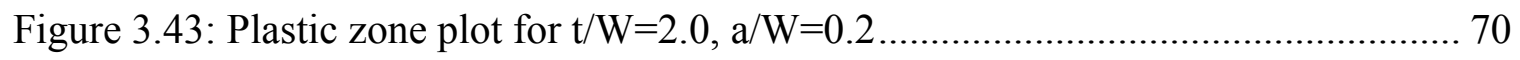

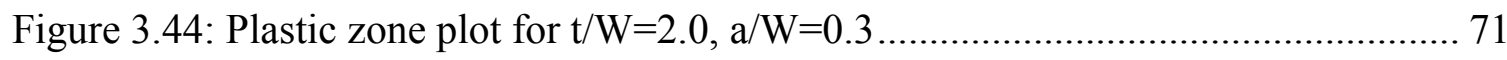

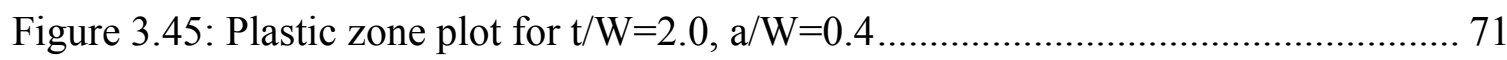

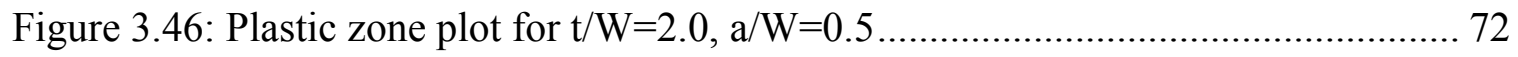

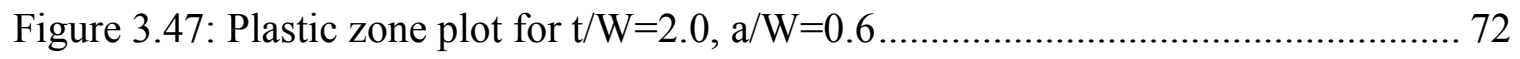

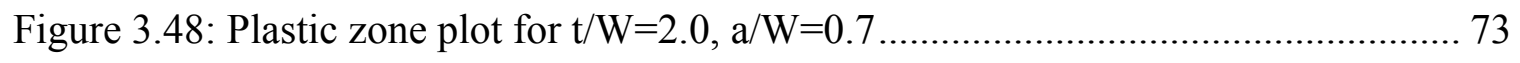

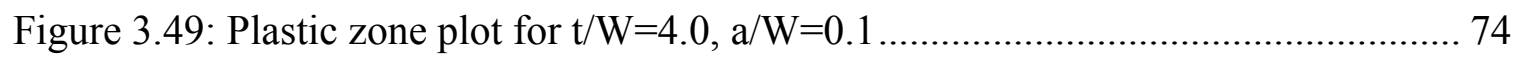

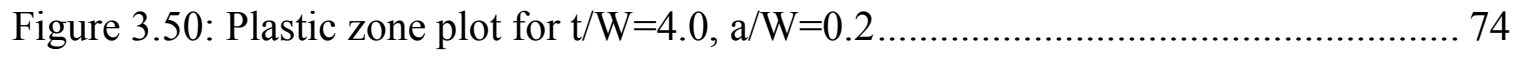

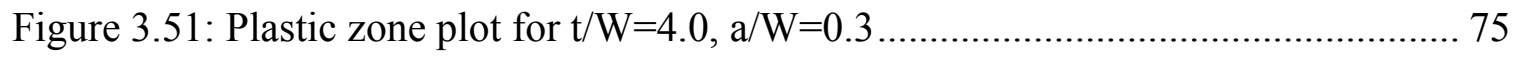




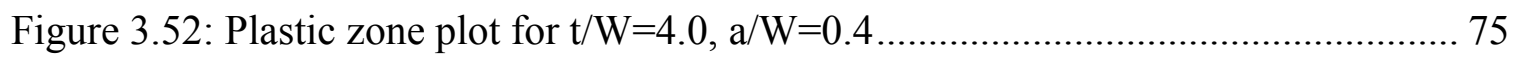

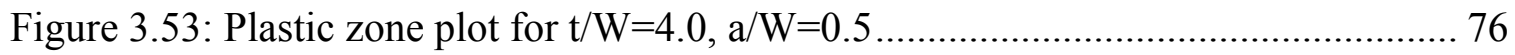

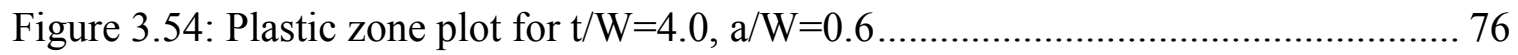

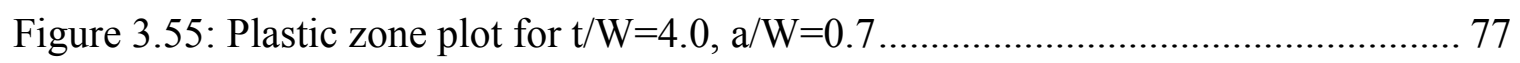

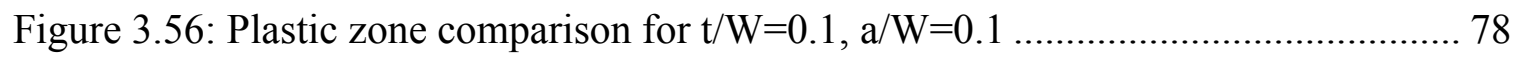

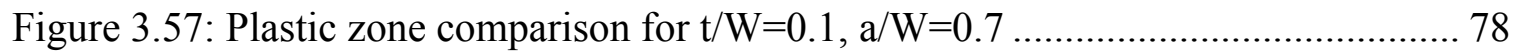

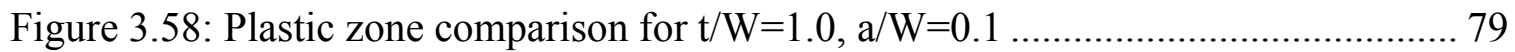

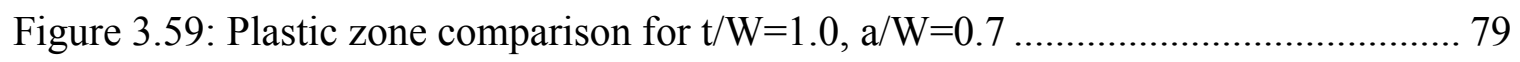

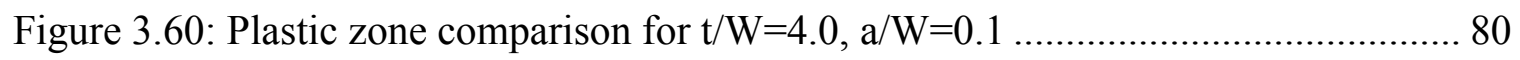

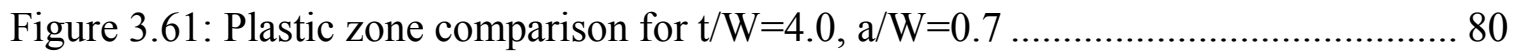

Figure 4.1: The asymmetric four-point shear specimen ....................................... 114

Figure 4.2: Typical mesh for AFPS specimen with $\mathrm{a} / \mathrm{W}=0.7, \mathrm{c} / \mathrm{W}=0.1$, and $\mathrm{t} / \mathrm{W}=1.0 \ldots 114$

Figure 4.3: 2D FEA Comparison with Literature Results ........................................ 115

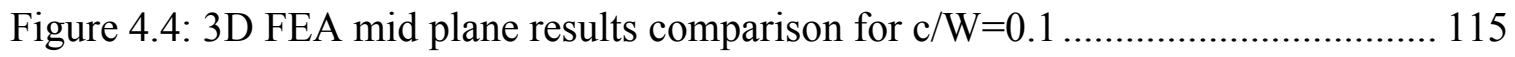

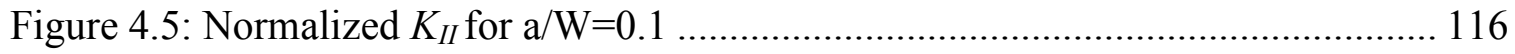

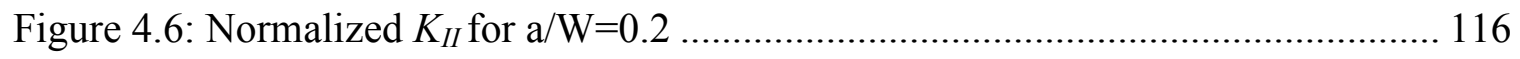

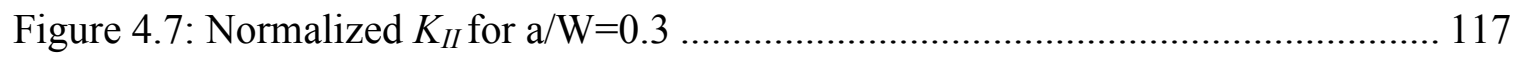

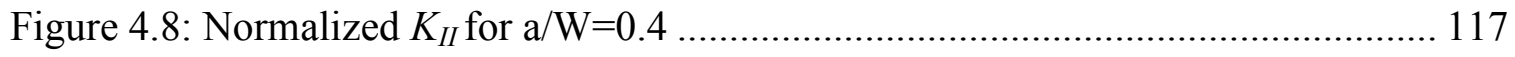

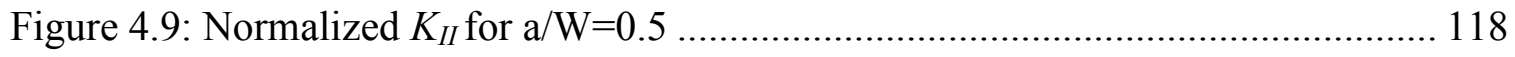

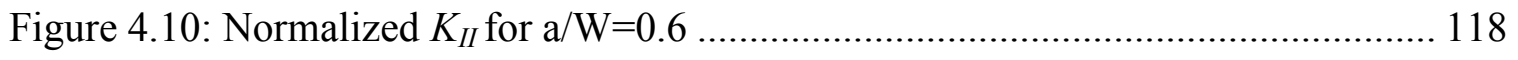


Figure 4.11: Normalized $K_{I I}$ for a/W=0.7 119

Figure 4.12: Normalized $K_{I I I}$ for a/W=0.1 120

Figure 4.13: Normalized $K_{I I I}$ for a/W=0.2 120

Figure 4.14: Normalized $K_{I I I}$ for a/W=0.3 121

Figure 4.15: Normalized $K_{I I I}$ for a/W=0.4 121

Figure 4.16: Normalized $K_{I I I}$ for a/W=0.5 122

Figure 4.17: Normalized $K_{I I I}$ for a/W=0.6 122

Figure 4.18: Normalized $K_{I I I}$ for a/W=0.7 123

Figure 4.19: Normalized $K_{I}$ for a/W=0.1 124

Figure 4.20: Normalized $K_{I}$ for a/W=0.2 124

Figure 4.21: Normalized $K_{I}$ for a/W=0.3 125

Figure 4.22: Normalized $K_{I}$ for a/W=0.4 125

Figure 4.23: Normalized $K_{I}$ for a/W=0.5 126

Figure 4.24: Normalized $K_{I}$ for a/W=0.6 126

Figure 4.25: Normalized $K_{I}$ for a/W=0.7 127

Figure 4.26: Normalized $T_{11}$ for a/W=0.1 128

Figure 4.27: Normalized $T_{11}$ for a/W=0.2 128

Figure 4.28: Normalized $T_{11}$ for $\mathrm{a} / \mathrm{W}=0.3$ 129

Figure 4.29: Normalized $T_{11}$ for $\mathrm{a} / \mathrm{W}=0.4$ 129

Figure 4.30: Normalized $T_{11}$ for $\mathrm{a} / \mathrm{W}=0.5$ 130 
Figure 4.31: Normalized $T_{11}$ for a/W=0.6 130

Figure 4.32: Normalized $T_{11}$ for $\mathrm{a} / \mathrm{W}=0.7$ 131

Figure 4.33: Normalized $T_{33}$ for $\mathrm{a} / \mathrm{W}=0.1$ 132

Figure 4.34: Normalized $T_{33}$ for $\mathrm{a} / \mathrm{W}=0.2$ 132

Figure 4.35: Normalized $T_{33}$ for $\mathrm{a} / \mathrm{W}=0.3$ 133

Figure 4.36: Normalized $T_{33}$ for $\mathrm{a} / \mathrm{W}=0.4$ 133

Figure 4.37: Normalized $T_{33}$ for $\mathrm{a} / \mathrm{W}=0.5$ 134

Figure 4.38: Normalized $T_{33}$ for $\mathrm{a} / \mathrm{W}=0.6$. 134

Figure 4.39: Normalized $T_{33}$ for $\mathrm{a} / \mathrm{W}=0.7$ 135

Figure 4.40: Plastic zone verification for $K_{I I} / K_{I}=1.0$ 136

Figure 4.41: Plastic zone map for $\mathrm{t} / \mathrm{W}=0.1, \mathrm{a} / \mathrm{W}=0.1$ 137

Figure 4.42: Plastic zone map for $\mathrm{t} / \mathrm{W}=0.1, \mathrm{a} / \mathrm{W}=0.2$ 137

Figure 4.43: Plastic zone map for $\mathrm{t} / \mathrm{W}=0.1, \mathrm{a} / \mathrm{W}=0.3$ 138

Figure 4.44: Plastic zone map for $\mathrm{t} / \mathrm{W}=0.1, \mathrm{a} / \mathrm{W}=0.4$ 138

Figure 4.45: Plastic zone map for $\mathrm{t} / \mathrm{W}=0.1, \mathrm{a} / \mathrm{W}=0.5$ 139

Figure 4.46: Plastic zone map for $\mathrm{t} / \mathrm{W}=0.1, \mathrm{a} / \mathrm{W}=0.6$ 139

Figure 4.47: Plastic zone map for $\mathrm{t} / \mathrm{W}=0.1, \mathrm{a} / \mathrm{W}=0.7$ 140

Figure 4.48: Plastic zone map for $\mathrm{t} / \mathrm{W}=0.5, \mathrm{a} / \mathrm{W}=0.1$ 141

Figure 4.49: Plastic zone map for $\mathrm{t} / \mathrm{W}=0.5, \mathrm{a} / \mathrm{W}=0.2$ 141

Figure 4.50: Plastic zone map for $\mathrm{t} / \mathrm{W}=0.5, \mathrm{a} / \mathrm{W}=0.3$ 142 
Figure 4.51: Plastic zone map for $\mathrm{t} / \mathrm{W}=0.5, \mathrm{a} / \mathrm{W}=0.4$ 142

Figure 4.52: Plastic zone map for $\mathrm{t} / \mathrm{W}=0.5, \mathrm{a} / \mathrm{W}=0.5$ 143

Figure 4.53: Plastic zone map for $\mathrm{t} / \mathrm{W}=0.5, \mathrm{a} / \mathrm{W}=0.6$ 143

Figure 4.54: Plastic zone map for $\mathrm{t} / \mathrm{W}=0.5, \mathrm{a} / \mathrm{W}=0.7$ 144

Figure 4.55: Plastic zone map for $\mathrm{t} / \mathrm{W}=1.0, \mathrm{a} / \mathrm{W}=0.1$ 145

Figure 4.56: Plastic zone map for $\mathrm{t} / \mathrm{W}=1.0, \mathrm{a} / \mathrm{W}=0.2$ 145

Figure 4.57: Plastic zone map for $\mathrm{t} / \mathrm{W}=1.0, \mathrm{a} / \mathrm{W}=0.3$ 146

Figure 4.58: Plastic zone map for $\mathrm{t} / \mathrm{W}=1.0, \mathrm{a} / \mathrm{W}=0.4$ 146

Figure 4.59: Plastic zone map for $\mathrm{t} / \mathrm{W}=1.0, \mathrm{a} / \mathrm{W}=0.5$ 147

Figure 4.60: Plastic zone map for $\mathrm{t} / \mathrm{W}=1.0, \mathrm{a} / \mathrm{W}=0.6$ 147

Figure 4.61: Plastic zone map for $\mathrm{t} / \mathrm{W}=1.0, \mathrm{a} / \mathrm{W}=0.7$ 148

Figure 4.62: Plastic zone map for $\mathrm{t} / \mathrm{W}=2.0, \mathrm{a} / \mathrm{W}=0.1$ 149

Figure 4.63: Plastic zone map for $\mathrm{t} / \mathrm{W}=2.0, \mathrm{a} / \mathrm{W}=0.2$ 149

Figure 4.64: Plastic zone map for $\mathrm{t} / \mathrm{W}=2.0, \mathrm{a} / \mathrm{W}=0.3$ 150

Figure 4.65: Plastic zone map for $\mathrm{t} / \mathrm{W}=2.0, \mathrm{a} / \mathrm{W}=0.4$ 150

Figure 4.66: Plastic zone map for $\mathrm{t} / \mathrm{W}=2.0, \mathrm{a} / \mathrm{W}=0.5$ 151

Figure 4.67: Plastic zone map for $\mathrm{t} / \mathrm{W}=2.0, \mathrm{a} / \mathrm{W}=0.6$ 151

Figure 4.68: Plastic zone map for $\mathrm{t} / \mathrm{W}=2.0, \mathrm{a} / \mathrm{W}=0.7$ 152

Figure 4.69: Plastic zone map for $\mathrm{t} / \mathrm{W}=4.0, \mathrm{a} / \mathrm{W}=0.1$ 153

Figure 4.70: Plastic zone map for $\mathrm{t} / \mathrm{W}=4.0, \mathrm{a} / \mathrm{W}=0.2$ 153 
Figure 4.71: Plastic zone map for $\mathrm{t} / \mathrm{W}=4.0, \mathrm{a} / \mathrm{W}=0.3$ 154

Figure 4.72: Plastic zone map for $\mathrm{t} / \mathrm{W}=4.0, \mathrm{a} / \mathrm{W}=0.4$ 154

Figure 4.73: Plastic zone map for $\mathrm{t} / \mathrm{W}=4.0, \mathrm{a} / \mathrm{W}=0.5$ 155

Figure 4.74: Plastic zone map for $\mathrm{t} / \mathrm{W}=4.0, \mathrm{a} / \mathrm{W}=0.6$ 155

Figure 4.75: Plastic zone map for $\mathrm{t} / \mathrm{W}=4.0, \mathrm{a} / \mathrm{W}=0.7$ 156

Figure 4.76: Plastic zone map including T-stress for $\mathrm{t} / \mathrm{W}=0.1, \mathrm{a} / \mathrm{W}=0.1$ 157

Figure 4.77: Plastic zone map including T-stress for $\mathrm{t} / \mathrm{W}=0.1, \mathrm{a} / \mathrm{W}=0.2$ 157

Figure 4.78: Plastic zone map including T-stress for $\mathrm{t} / \mathrm{W}=0.1, \mathrm{a} / \mathrm{W}=0.3$ 158

Figure 4.79: Plastic zone map including $T$-stress for $\mathrm{t} / \mathrm{W}=0.1, \mathrm{a} / \mathrm{W}=0.4$ 158

Figure 4.80: Plastic zone map including T-stress for $\mathrm{t} / \mathrm{W}=0.1, \mathrm{a} / \mathrm{W}=0.5$ 159

Figure 4.81: Plastic zone map including T-stress for $\mathrm{t} / \mathrm{W}=0.1$, $\mathrm{a} / \mathrm{W}=0.6$ 159

Figure 4.82: Plastic zone map including T-stress for $\mathrm{t} / \mathrm{W}=0.1, \mathrm{a} / \mathrm{W}=0.7$ 160

Figure 4.83: Plastic zone map including T-stress for $\mathrm{t} / \mathrm{W}=0.5$, $\mathrm{a} / \mathrm{W}=0.1$ 161

Figure 4.84: Plastic zone map including T-stress for $\mathrm{t} / \mathrm{W}=0.5$, $\mathrm{a} / \mathrm{W}=0.2$ 161

Figure 4.85: Plastic zone map including T-stress for $\mathrm{t} / \mathrm{W}=0.5, \mathrm{a} / \mathrm{W}=0.3$ 162

Figure 4.86: Plastic zone map including T-stress for $\mathrm{t} / \mathrm{W}=0.5$, $\mathrm{a} / \mathrm{W}=0.4$ 162

Figure 4.87: Plastic zone map including T-stress for $\mathrm{t} / \mathrm{W}=0.5$, $\mathrm{a} / \mathrm{W}=0.5$ 163

Figure 4.88: Plastic zone map including T-stress for $\mathrm{t} / \mathrm{W}=0.5, \mathrm{a} / \mathrm{W}=0.6$ 163

Figure 4.89: Plastic zone map including T-stress for $\mathrm{t} / \mathrm{W}=0.5, \mathrm{a} / \mathrm{W}=0.7$ 164

Figure 4.90: Plastic zone map including T-stress for $\mathrm{t} / \mathrm{W}=1.0, \mathrm{a} / \mathrm{W}=0.1$ 165 
Figure 4.91: Plastic zone map including T-stress for $\mathrm{t} / \mathrm{W}=1.0, \mathrm{a} / \mathrm{W}=0.2$ 165

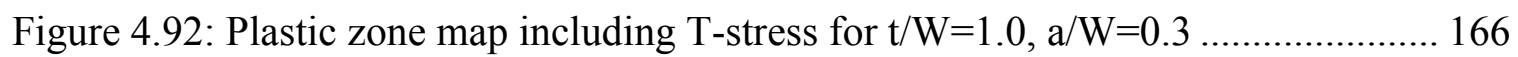

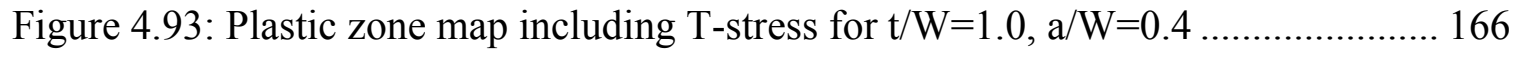

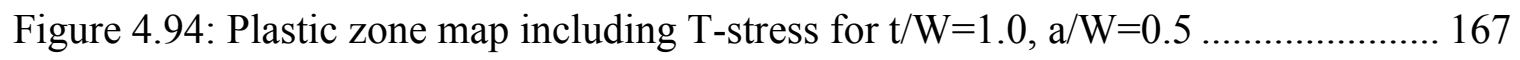

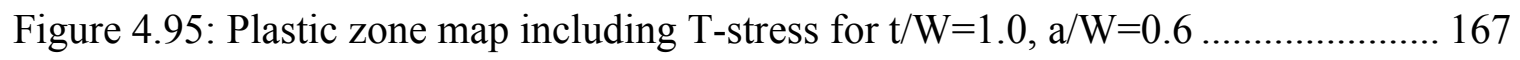

Figure 4.96: Plastic zone map including T-stress for $\mathrm{t} / \mathrm{W}=1.0, \mathrm{a} / \mathrm{W}=0.7 \ldots \ldots \ldots \ldots \ldots \ldots \ldots . . . . . . . .168$

Figure 4.97: Plastic zone map including T-stress for $\mathrm{t} / \mathrm{W}=2.0, \mathrm{a} / \mathrm{W}=0.1 \ldots \ldots \ldots \ldots \ldots \ldots . . . . . . . . .169$

Figure 4.98: Plastic zone map including T-stress for $\mathrm{t} / \mathrm{W}=2.0, \mathrm{a} / \mathrm{W}=0.2$.................... 169

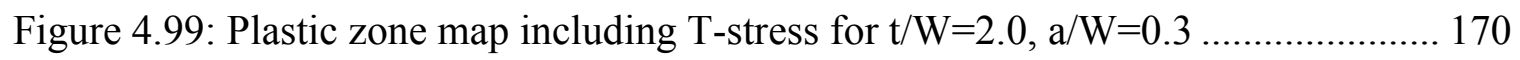

Figure 4.100: Plastic zone map including T-stress for $\mathrm{t} / \mathrm{W}=2.0, \mathrm{a} / \mathrm{W}=0.4 \ldots \ldots \ldots \ldots \ldots \ldots \ldots \ldots \ldots \ldots$

Figure 4.101: Plastic zone map including $\mathrm{T}$-stress for $\mathrm{t} / \mathrm{W}=2.0, \mathrm{a} / \mathrm{W}=0.5 \ldots \ldots \ldots \ldots \ldots \ldots . . .171$

Figure 4.102: Plastic zone map including T-stress for $\mathrm{t} / \mathrm{W}=2.0, \mathrm{a} / \mathrm{W}=0.6 \ldots \ldots \ldots \ldots \ldots \ldots \ldots \ldots \ldots \ldots$

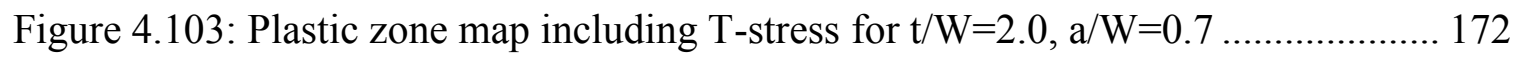

Figure 4.104: Plastic zone map including T-stress for $\mathrm{t} / \mathrm{W}=4.0, \mathrm{a} / \mathrm{W}=0.1 \ldots \ldots \ldots \ldots \ldots \ldots . . . \ldots \ldots$

Figure 4.105: Plastic zone map including T-stress for $\mathrm{t} / \mathrm{W}=4.0, \mathrm{a} / \mathrm{W}=0.2 \ldots \ldots \ldots \ldots \ldots \ldots \ldots \ldots$

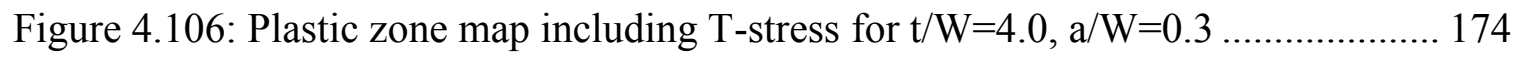

Figure 4.107: Plastic zone map including T-stress for $\mathrm{t} / \mathrm{W}=4.0, \mathrm{a} / \mathrm{W}=0.4 \ldots \ldots \ldots \ldots \ldots \ldots \ldots \ldots \ldots \ldots$

Figure 4.108: Plastic zone map including T-stress for $\mathrm{t} / \mathrm{W}=4.0, \mathrm{a} / \mathrm{W}=0.5 \ldots \ldots \ldots \ldots \ldots \ldots . . .175$

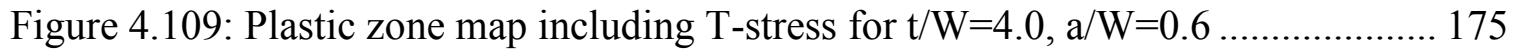

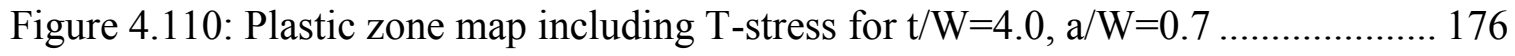


Figure 4.111: Plastic zone comparison for $\mathrm{t} / \mathrm{W}=0.1$, $\mathrm{a} / \mathrm{W}=0.1, \mathrm{~S} / \mathrm{Sy}=0.12$

Figure 4.112: Plastic zone comparison for $\mathrm{t} / \mathrm{W}=0.1, \mathrm{a} / \mathrm{W}=0.7, \mathrm{~S} / \mathrm{Sy}=0.06$ 177

Figure 4.113: Plastic zone comparison for $\mathrm{t} / \mathrm{W}=0.1, \mathrm{a} / \mathrm{W}=0.1, \mathrm{~S} / \mathrm{Sy}=0.09$ 178

Figure 4.114: Plastic zone comparison for $\mathrm{t} / \mathrm{W}=1.0, \mathrm{a} / \mathrm{W}=0.7, \mathrm{~S} / \mathrm{Sy}=0.06$ 178

Figure 4.115: Plastic zone comparison for $\mathrm{t} / \mathrm{W}=4.0$, $\mathrm{a} / \mathrm{W}=0.1, \mathrm{~S} / \mathrm{Sy}=0.12$ 179

Figure 4.116: Plastic zone comparison for $\mathrm{t} / \mathrm{W}=4.0$, $\mathrm{a} / \mathrm{W}=0.7, \mathrm{~S} / \mathrm{Sy}=0.06$ 179 


\section{List of Appendices}

Appendix A : Plastic Zone Calculation Method (Nazarali and Wang 2011) ........ 185 


\section{Nomenclature}

$2 \mathrm{D}$

$3 \mathrm{D}$

AFPS

$a / W$

$c / W$

E

FEA/FEM

FPS

LEFM

G

$J$

$K_{I}, K_{I I}, K_{I I I}$

$\mathrm{R}_{\mathrm{p}}$

$\mathrm{T}$

$T_{11}, T_{33}$

$t / W$

$v$

$\sigma_{\mathrm{ij}}$
Two-Dimensional

Three-Dimensional

Asymmetric Four Point Shear

Crack depth-to-width ratio

Crack offset-to-width ratio

Young's modulus

Finite Element Analysis/Method

Four Point Shear

Linear Elastic Fracture Mechanics

Energy Release Rate

J-integral

Mode I, II, and III stress intensity factors (SIF), respectively

Plastic zone radius

T-stress

T-stress in normal $\left(\mathrm{T}_{11}\right)$ and tangential $\left(\mathrm{T}_{33}\right)$ directions to the $3 \mathrm{D}$ crack plane

Plate thickness-to-width ratio

Poisson's ratio

Stress components surrounding crack tip 


\section{Chapter 1: Introduction}

Over the course of engineering history, countless failures of metallic components can be attributed to defects and flaws in material. While these imperfections have always existed in materials, only within the past century has their effect on structural behaviour really been considered.

Large scale failures have shown an ignorance of fracture behaviour, for example, the early Liberty Ships built between 1941 and 1945 for World War II (USCG 1957). These $10,000+$ ton ships were constructed rapidly, using new welding techniques, and they suffered widespread structural failures. The ships had a tendency to break in half; the failures were later determined to be caused by fast-propagating brittle cracks. These cracks were caused by a combination of the poor quality of steel, which became brittle at the low in-service temperatures, and the welded (as opposed to riveted) hull design, which allowed unhindered crack propagation.

The early Liberty Ships increased engineering awareness of fracture behaviour, but failures have still been common throughout modern history. Growth in the aviation, nuclear, and gas pipeline industries has led to advances in the field of fracture mechanics. In these areas, the presence of flaws in material is of special importance because of the impact of material failures on public safety.

Inglis (1913) published some of the earliest work on stresses in flawed plates. This early paper covered cases of circular and elliptical holes in plates, typical of riveted assemblies of the time. Soon after, Griffith (1921) published his work on the fracture of brittle materials, relating fracture to the energy released in creating new surfaces.

The early work on fracture behaviour focused on brittle materials, such as glass, but was later applied to a broader scope of materials. The work of Irwin (1957) and Williams (1957) forms the basis of the approach still in use today- linear elastic fracture mechanics (LEFM) - by relating the energy release rate to the stress around the crack tip. Williams published the description of the stress state around a crack tip, using empirical factors known as stress intensity factors (SIF) to take crack geometry effects 
into account. In the Williams expansion, stress contributions come from the loading stress, of which three modes describe the loading: mode I-opening mode, mode IIshearing mode, and mode III-tearing mode. The three modes of loading are described in more detail in Chapter 2.

With the introduction of the SIF, the notion of singularity at the crack tip developed, where infinite stress is predicted at the crack tip. Early researchers conceded that stress is not truly infinite at the crack tip, as elastic theory would predict, but instead, in a small area surrounding the crack, the material must exhibit plastic behaviour. It was determined that the theory of elasticity could still be used in the stress analysis of these cracked bodies, provided that the area of the material which was undergoing plastic deformation, known as the plastic zone, was small relative to other geometric features. This assumption forms the basis of small scale yielding, which is an approach still in use today.

The critical value of SIF, $K_{I C}$, is used as a material property to describe the resistance of a crack to propagate through a particular material. The value of $K_{I C}$, known as fracture toughness, is determined through experimental tests of material specimens with preexisting cracks. The most common test specimens to determine $K_{I C}$ are specimens loaded in pure mode I configuration, such as the single edge notch beam and compact tension specimens. These specimens are often used to represent idealized cases of plane stress and plane strain fracture under pure mode I loading. A cracked body can, however, be loaded in any number of combinations of the three primary loading directions, or modes. In order to determine the onset of fracture for the other modes of loading, and combinations thereof, fracture specimens for mixed-mode loading cases have been developed.

The asymmetric four-point shear (AFPS) is one such specimen designed to test a material's fracture behaviour under mixed-mode loading conditions. He, Cao, and Evans (1990) provide 2D plane strain solutions for the values of $K_{I}$ and $K_{I I}$, for the specimen shown in Figure 1.1. There are two configurations of this specimen: a pure mode II configuration, known as the (antisymmetric) four-point shear (FPS) specimen, shown in 
Figure 1.2, and a mixed-mode I/II configuration known as the AFPS specimen, shown in Figure 1.3. In the AFPS specimen, mixed mode loading is achieved by offsetting the crack with respect to the loading points, introducing asymmetry.

In recent studies, 3D finite element analysis has been used to study the effects of finitethickness on fracture behaviour. Researchers such as Jin and Wang (2014) have looked at the finite-thickness effect for mode I fracture specimens, but there is currently no comprehensive study focussing specifically on finite-thickness mixed-mode test specimens. In order to account for specimen thickness in the interpretation of fracture testing results, comprehensive SIF solutions are needed.

When considering specimens such as FPS and AFPS in 3D, forces due to Poisson's ratio, lead to a coupling of SIFs $K_{I I}$ and $K_{I I I}$. Currently, the effect of $K_{I I I}$ is neglected in the literature, which considers an idealized 2D case.

To help better understand the stress field surrounding the crack tip, the plastic zone can be calculated for given $K$ values. This type of plastic zone analysis determines the plastic zone shape and size for different mixed-mode cases, and is a useful indicator of crack tip stress. Currently, research is lacking into the effect of including the mode III contribution and $3 \mathrm{D}$ effects in the calculation of plastic zone. By considering specimens in $3 \mathrm{D}$, any variation of the plastic zone along the crack front can be seen.

Traditional fracture testing of mode I specimens yields a value of $K_{I C}$ that is applicable for situations of high crack tip constraint, which may be overly conservative. A number of researchers have shown that it is necessary to include an additional non-singular term in the Williams expansion to adequately describe the stress state surrounding the crack. This non-singular term is known as the T-stress, and forms the basis of the two-parameter fracture mechanics approach.

Recently, researchers have investigated the effects of T-stress magnitude on the plastic zone, for various mixed-mode cases. The T-stress values for mode I test specimens have been studied, but calculated values for the mixed-mode AFPS specimen are not readily available. 
The combination of applying two-parameter fracture mechanics to finite-thickness specimens undergoing mixed-modes I/II loading has been attempted for select cases, but no comprehensive analysis has been undertaken for the FPS or AFPS specimens.

In this thesis, the FPS and AFPS specimens are studied for different combinations of crack depth $(a / W)$ and specimen thickness $(t / W)$ to width ratios. Extensive 3D finite element analysis is carried out, and comprehensive solutions of fracture parameters are produced. From the results of the finite analysis, the size of the plastic zone is studied at various points through-thickness. Through these analyses, the $3 \mathrm{D}$ effects are illustrated.

\subsection{Thesis Outline}

The current thesis contains five chapters:

- Chapter 1 introduces the topics in the current research.

- Chapter 2 provides background information and a literature review of LEFM. One- and two-parameter fracture mechanics methods are discussed and the current literature in mixed-mode fracture and plastic zone analysis is reviewed.

- Chapter 3 presents results from the current analysis for pure mode II loading of the FPS specimen. Comprehensive solutions for through-thickness values of $K_{I I}$ and $K_{I I I}$ are given. The plastic zone is calculated using $K_{I I}$ and $K_{I I I}$ for number of through-thickness positions.

- Chapter 4 presents results from the current analysis for mixed-mode I/II loading of the AFPS specimen. Comprehensive solutions for through-thickness values of $K_{I}, K_{I I}, K_{I I I}, T_{11}$, and $T_{33}$ are given. Plastic zones are calculated first including only $K$ terms, and then including all $K$ and T-stress terms.

- Chapter 5 provides discussion and concluding remarks on the significance of the results. 


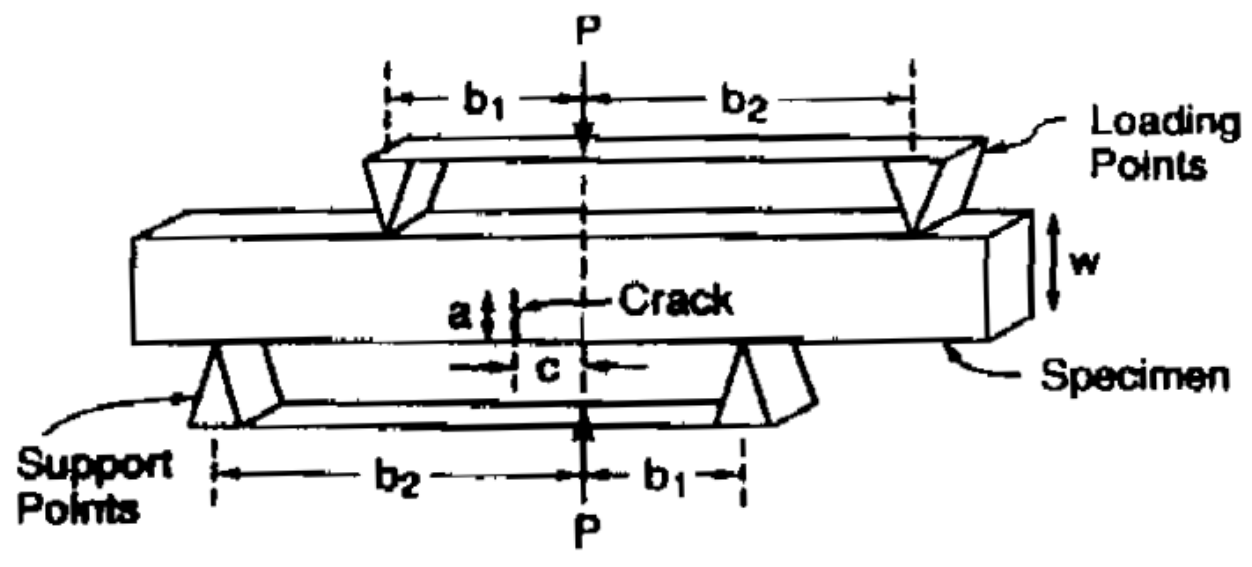

Figure 1.1: The asymmetric four-point shear specimen (He, Cao, and Evans 1990)

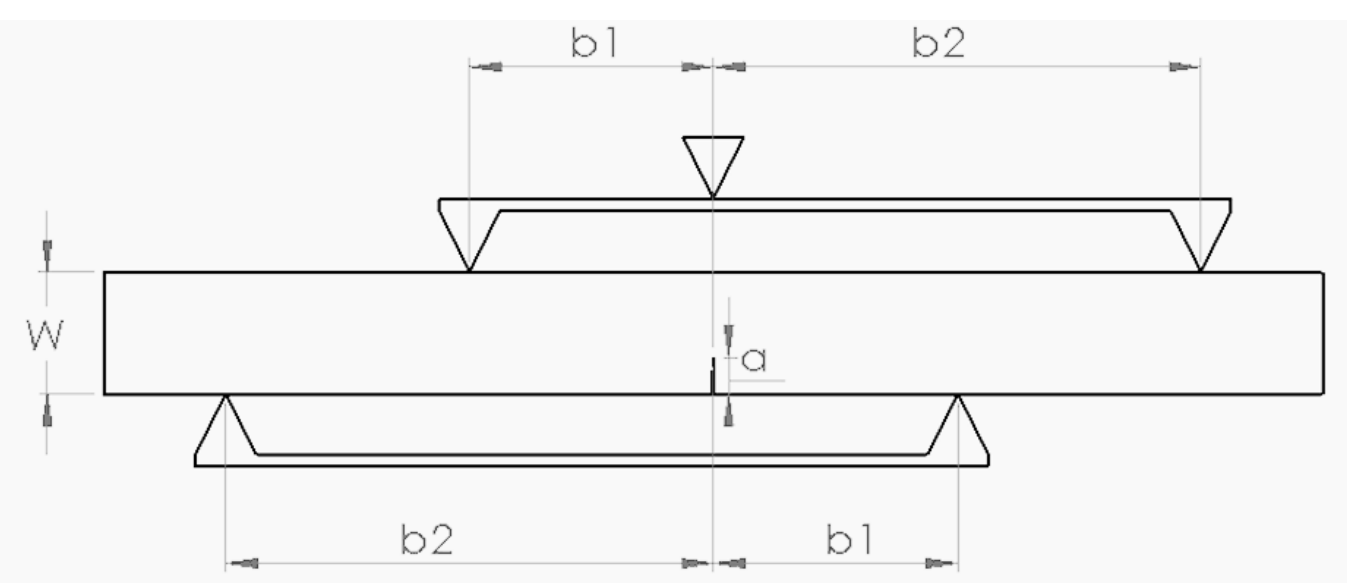

Figure 1.2: The four-point shear (FPS) specimen

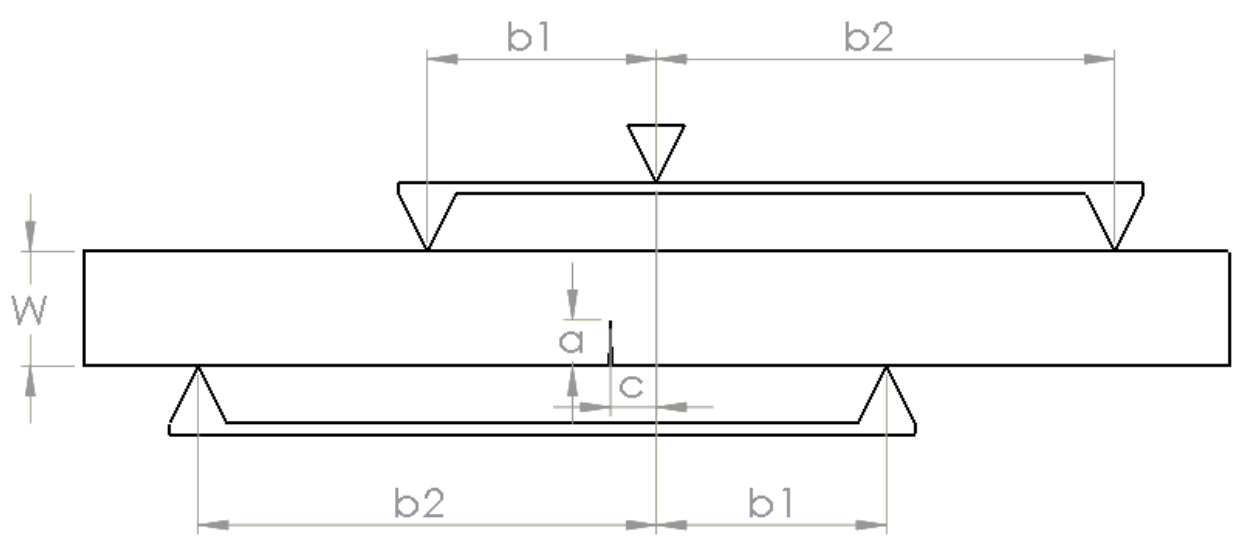

Figure 1.3: The asymmetric four-point shear (AFPS) specimen 


\section{Chapter 2: Background and Literature Review}

Engineering structures must be designed so that the effects of defects and flaws, which are inherent in all materials, can be accounted for. Linear Elastic Fracture Mechanics (LEFM) provides a basis to calculate the stress around a crack tip, based on the theory of elasticity. The textbook Fracture Mechanics: Fundamentals and Applications, by T.L. Anderson, provides an excellent overview to the basic theory of LEFM (Anderson 2006). Most of the topics within the background section are discussed more fully in Chapter 2: LEFM of the 2005 edition of this book.

\subsection{Linear Elastic Fracture Mechanics (LEFM)}

The methods used in the current research come from the well-developed concepts of Linear Elastic Fracture Mechanics (LEFM). Some of the most significant early work on this topic was done by Inglis and Griffith, where the former described the increase in stress in plates due to the presence of flaws and holes (Inglis 1913), and the latter described the stresses due to flaws in plates of plane stress and plane strain simplifications (Griffith, 1921). Griffith worked on an energy-based approach to fracture, a concept further developed by Irwin, using the energy release rate $G$, also known as crack driving force, as a measure of fracture toughness. Irwin (1957) added to Griffith's model for fracture by defining $G$ as the energy release rate:

$$
G=\frac{-d \prod}{d \mathrm{~A}}
$$

The energy release rate, $G$, is expressed in terms of strain energy with respect to crack area (not time). Irwin and Williams (1957) studied the effect of cracks on the stress field in a body comprised of a linear elastic material, and were among the first to relate $G$ to the local stress field around a crack tip. The most commonly used expression for the stress field comes from an expression known as the Williams series expansion, which describes the stress in terms of the angle (around crack tip polar coordinates), radial 
distance, and a term known as the stress intensity factor (SIF). Williams (1957) showed that the stress field in the cracked body is given by the expansion as follows:

$$
\sigma_{i j}=\left(\frac{k}{\sqrt{r}}\right) f_{i j}(\theta)+\sum_{m=0}^{\infty} \mathrm{A}_{m} r^{\frac{m}{2}} g_{i j}^{m}(\theta)
$$

where $k$ is a constant, known as the stress intensity factor (SIF); $r$ is the radial distance from the crack tip; and $\theta$ is the angle from the crack plane. $f_{i j}(\theta)$ in the first, singular, term is a function of crack geometry, while the second term is an infinite series which reduces to zero approaching the crack tip. Figure 2.1 shows the coordinates used in the Williams expansion. This expression yields an asymptotic relationship where stress approaches infinity very near the crack tip. The $\frac{1}{\sqrt{r}}$ singularity describes the stress state, while displacement is related to $\sqrt{r}$.

The deformation of a cracked body under load is classified into three modes, shown in Figure 2.2. Each mode has traditionally been studied separately, yielding many examples of closed-form solutions for pure modes of loading and specific specimen geometries. Each mode of loading contributes to the overall stress around the crack, and the stresses are additive. The three modes are as follows:

- Mode I, or opening mode, is where the stress is perpendicular to the crack plane, and it tends to cause an opening of the crack.

- Mode II, or in-plane shear mode, is where shear stress in the same plane as the crack drives crack extension.

- Mode III, or out-of plane shear mode, is a type of shear loading that is often referred to as tearing mode, due to the action of loading.

Mode I has historically been the best studied mode of crack loading, with common fracture toughness methods using $K_{l}$ as the critical crack driving force. Examples of common specimens include the three point bend and compact tension specimens, both used to determine fracture toughness, $K_{I C}$. Mode II loading has also been used for fracture specimens, such as the four-point shear specimen. 


\subsection{Single Parameter Fracture Mechanics}

The term single parameter fracture mechanics refers to the use of a single parameter, describing the crack driving force, to describe the stress state. Treating the first term in

the Williams expansion, containing the $\frac{1}{\sqrt{r}}$ stress singularity at the crack tip, as the dominant term, means that all terms after it are dropped. This allows the use of the single parameter, the critical stress intensity factor $\left(K_{I C}\right)$, to describe the stress state at the crack tip, and to predict the onset of fracture. Each individual mode of loading (I, II, and III) is additive and can first be calculated individually, and then superimposed to calculate total stress, as shown in Equation 2.3:

$$
\sigma_{i j}^{(\text {total })}=\sigma_{i j}^{(I)}+\sigma_{i j}^{(I I)}+\sigma_{i j}^{(I I I)}
$$

Real loading scenarios often include combinations of loading types, known as mixed mode loading. Solutions for SIF, required for interpretation of fracture tests, are available in handbooks and other literature, and are usually provided assuming 2D plane strain or plane stress conditions.

\subsection{Plastic Zone Analysis}

Because the stress field surrounding a crack is asymptotic approaching the crack tip, the stress predicted using LEFM methods is infinite at the tip of a sharp crack. No material can withstand infinite stress, so a small amount of plasticity around the crack tip must occur for metal. This region defined by the boundary between elastic and plastic behaviour is referred to as the plastic zone (PZ).

The PZ size and shape are of particular interest in analyzing the stress fields around the crack tip. At the PZ boundary, the stress is equal to the material yield stress. For the LEFM approach, the PZ size must be suitably small to apply the small scale yielding (SSY) assumption, allowing the application of elasticity. 
Irwin determined an estimate of the plastic zone size by algebraically solving the Williams expansion for the stress field at a crack tip, solving for $r_{y}$, which is an effective plastic zone radius size (Irwin, 1957).

The PZ boundary marks the transition from elastic behaviour, in the bulk of the material, to plastic behaviour, in a small zone surrounding the crack tip. A first order estimate from Irwin for the case of plane stress and pure mode I loading resolve to a plastic radius as calculated in Equation 2.4:

$$
r_{y}=\frac{1}{2 \pi}\left(\frac{K_{I}}{\sigma_{Y S}}\right)^{2}
$$

where $K_{I}$ is the mode I stress intensity factor and $\sigma_{Y S}$ is the material yield stress.

Other estimates have been made, such as a second order approximation, which can be proven using a force balance technique, resulting in a plastic radius twice the size of the first order estimate. Irwin attempted to provide an estimate for the plane strain case by accounting for the high stress triaxiality at the crack tip, reducing the PZ size to one-third that of the first-order estimate calculated using Equation 2.4.

While Irwin's early work on the size of the plastic zone provides several estimates of the plastic zone radius, Irwin assumed that the PZ shape would be circular. Anderson provides a summary of PZ maps, which show the shape of the plastic zone (how the radius changes with respect to angle $\theta$ around the crack tip) for each of the three modes of loading. The plots shown in Anderson (2005), for the cases of plane strain and plane stress, were created by equating the stress tensor, given by Williams's elastic solution, to the Von Mises yield criterion.

\subsection{Two-parameter Fracture Mechanics}

The Williams expansion, shown in Equation 2.2, is comprised of singular and nonsingular terms when expressed in polar coordinates about the crack tip. The first non- 
singular term is constant, known as the T-stress $\left(T \delta_{1 i} \delta_{1 j}\right)$. Rice (1974) concluded that the inclusion of this term had significant effect on the PZ size and shape for mode I loading. This approach is known as two-parameter fracture mechanics, or two-parameter LEFM, where SIF and T-stress are deemed sufficient to describe the stress state surrounding the crack-tip. The general stress state for mode I loading can be described as:

$$
\sigma_{i j}(r, \theta)=\frac{K_{I}}{\sqrt{2 \pi r}} f_{i j}(\theta)+T \delta_{1 i} \delta_{1 j}+O\left(r^{1 / 2}\right)
$$

Where the second term, $T \delta_{1 i} \delta_{1 j}$, represents constant stress components known as Tstress.

The inclusion of the T-stress term has been studied by a number of researchers after Rice. Nakamura and Parks (1992) looked at the calculation of elastic T-stress solutions for a single edge cracked plate (SECP) in bending and tension. Of interest is the throughthickness analysis given for plates with materials of varying $v$ (Poisson's ratio). The authors show that the amount of T-stress variation through-thickness is influenced by $v$.

The significance of the non-singular T-stress terms extends to the PZ size and shape. Nazarali and Wang (2011) determined the effect of the varying levels of T-stress on mixed and pure mode I and II cracks. It was found that both positive and negative values of T-stress have significant effect on the PZ size and shape. The PZ size was determined by algebraic rearrangement of the well-known Williams expansion, using the Von Mises yield criterion. The resulting expression was found to be discontinuous for certain combinations of $K_{I} / K_{I I}$ and T-stress.

Schutte and Molla-Abassi (2007) studied internal circular cracks, and produced PZ diagrams showing the influence of including various LEFM parameters in the calculation of PZ maps. Various angular positions were compared in the quarter-elliptical crack specimen, but no distinguishable differences were seen. This constant plastic zone around the crack front is due to symmetric boundary conditions, where no free edge effects are present (fully embedded crack). Mixed-mode loading was considered with various ratios 
of $K_{I} / K_{I I}$ and a variety of T-stress values. A discontinuous plastic zone is observed for cracks described with negative non-zero T-stress terms.

\subsection{Literature Review: Three-dimensional (3D) Mixed Mode and T-stress}

A review by G.C. Sih provided a detailed overview of three-dimensional cracked plate problems, and gave examples of variations in fracture parameters with position along the crack front, and varying specimen thickness (Sih 1971). The review highlighted the deficiency in applying two-dimensional (2D) plane strain and plane stress simplifications to certain cracked plate problems. At the free surface, the theory of elasticity cannot be satisfied by the assumptions required for plane strain/ plane stress simplification, so these cases cannot be truly expected to be limiting cases for 3D problems. The author conceded that due to the complexity of the three-dimensional equations of elasticity, it is not reasonable to derive an analytical solution to problems of cracks within finite-thickness plates.

Larsson and Carlsson (1973) concluded that a modified boundary layer method could adequately represent real cracked bodies for materials with low strain-hardening. Through the inclusion of additional non-singular terms - representing stress parallel to the crack face (T-stress) - in the analysis, results were found to agree with the reference solutions for the geometry. Four specimens were considered, all of them loaded in modeI loading: bending and tension.

Rice (Rice 1974) found agreement between results generated using his model, and the work previously done by Larsson and Carlsson (1973). Rice provided results that showed the relationship between $\mathrm{T}$-stress value and yield zone size, with a significant impact when large negative $\mathrm{T}$-stress values are considered.

Cotterell and Rice (1980) investigated the fracture of kinked and curved cracks, showing the influence of the sign of the T-stress on the stability of cracks, for small crack growth. 
Maccagno and Knott (1985) studied brittle fracture of specimens undergoing a wide range of mixed-mode I/II loading ratios through experimental asymmetric four-point shear specimen testing. Theoretical predictions of crack propagation angle, made using the maximum tensile stress criterion, were found to adequately match the observed trends. Photo-elastic analysis techniques were used to visualize stress contours around the crack tip, which appears in some cases discontinuous.

Nakamura and Parks (1989) investigated mixed mode loading in thin plates, looking at 3D variations of SIF for all modes. The authors described a corner singularity, where the free-edge and crack meet, and it was found that the $\frac{1}{\sqrt{r}}$ field is larger approaching the free edge. The influence of Poisson's ratio on $K_{I I}$ and $K_{I I I}$ variation through-thickness was shown for several values of $v$.

In the same year, Shih and Asaro (1989) used FEA to calculate the J-integral for the case of a crack in a bi-material interface. Stress contours were developed for varying load levels. The J-integral was found to be a satisfactory description of the state of stress in the near-tip field.

A later paper by Nakamura and Parks (1990) provided calculated plastic zone boundary plots for thin, ductile plates. The 3D analysis showed the plastic boundary at the mid plane and edge-plane (free-edge) for a range of applied-stress levels.

$\mathrm{He}$, Cao, and Evans (1990) studied the mixed-mode fracture of the four-point shear specimen, both experimentally, with PMMA and soda-lime glass specimens, and theoretically, by calculating SIF solutions for the considered geometries. The authors used a path-independent interaction integral to calculate the stress intensity factors $K_{I}$ and $K_{I I}$.

Maccagno and Knott (1991) investigated the effect of the proportion of mode I and mode II loading on low temperature brittle fracture behaviour for steel. The results showed a shift in fracture angle from a straight crack to an increasingly kinked crack with increasing mode II component. 
Tohgo and Ishii (1991) studied the mixed-mode fracture behaviour of aluminum alloy material under mode I, mode II, and mixed I/II loading. The authors found that under mode I dominated loading, the crack initiated in a brittle, tensile manner, while a predominantly mode II load produced a crack with shearing, ductile crack initiation.

Nakamura and Parks (1992) introduced a general method to calculate non-singular Tstress terms for three-dimensional crack fronts. Using FEA, an interaction-integral was calculated at the crack front, and LEFM parameters were extracted. From this analysis, it was determined that T-stress varies through-thickness on $3 \mathrm{D}$ plates, as well as with changing plate thickness. The effect of Poisson's ratio $(v)$ on the value of T-stress was examined, and found to be significant.

Hallbäck and Nilsson (1994) compared Abaqus finite element calculations of $J$ and $K$ with experimental results of an aluminum alloy. Interestingly, the authors highlighted the influence of T-stress as the key descriptor, between specimens of varying thickness, for crack-kinking angle.

Kwon and Sun (2000) examined the three-dimensional stress field around a crack in a single edge cracked plate. The authors identified a vertex-singularity at the intersection of the crack-line and the free-edge. They provided a simple ratio for estimating the threedimensional SIF solution from a given two-dimensional literature solution, at the mid plane:

$$
K_{3 D} / K_{2 D}=\sqrt{1 /\left(1-v^{2}\right)}
$$

,where $K_{3 D}$ is the $3 \mathrm{D} \mathrm{SIF}$, and $K_{2 D}$ is the $2 \mathrm{D} \mathrm{SIF}$. Kwon and Sun also provided some interesting observations as to the effect of thickness on the fracture process zone (plastic zone), and the suitable assumption of plane strain and plane stress solutions.

Fett (2001) studied mixed-mode loading of circular specimens, with geometry representative of compact tension and Brazilian disc specimens. Under mixed and pure modes I/II loading, fracture parameters were provided for a variety of specimen and loading geometries. T-stress values were computed for the considered geometries. The 
author also calculated T-stress values for loading cases that were considered pure mode II cases.

Dhondt, Chergui, and Bucholz (2001) used computational fracture mechanics to study several fracture specimens, comparing the $3 \mathrm{D}$ solutions with those from $2 \mathrm{D}$ reference. The authors noted apparent mode-coupling effects in the 3D results, something not covered in the literature solutions at the time. The authors used both a strain-energy release rate method and a virtual crack-closure method to analyze the cracked specimens.

Wang and Bell (2004) calculated T-stress values for semi-elliptical surface-cracked plates, using an interaction-integral method. The results provided an efficient method for calculating T-stress values in finite-thickness specimens.

Kacianauskas, Rimantas, et al. (2005) determined correction factors that reduced the uncertainty of applying Kwon and Sun's earlier 3D approximation method. Throughthickness variation of different plate thicknesses were investigated.

Ayatollahi and Aliha (2005) modified the maximum tangential stress (MTS) criterion originally suggested by Sih, to include the non-singular T-stress term, for better prediction of stress in mode II loading cracked Brazilian disc specimens. The authors highlighted the existence of T-stress in mode II loaded specimens, and concluded that mode II fracture toughness was a factor not just of material property, but also of loading and specimen geometry.

Zehnder and Viz (2005) provides a review of the fracture of thin plates and shells undergoing mixed loading, comparing the energy release rates calculated using different fracture theories. 3D analysis was performed for thicker plates to compare plate theory to three dimensional elasticity theory results.

Qu and Wang (2006) calculated T-stress values for the case of a quarter-elliptical corner crack in finite-thickness plates for the cases of bending and tension loading.

Ayatollahi and Aliha (2007) suggested a modified three-parameter model using mode I and II SIF values and T-stress values to describe the crack tip stress for plates subject to 
mixed-mode loading. The authors studied the brittle fracture of rocks, and the application of LEFM principles for rock failure analysis.

Shahani and Tabatabaei (2008) computed the mixed-mode stress intensity factors for a four-point bend specimen, and antisymmetric four-point shear specimen. The authors covered many different loading geometries in their analysis.

Aliha and Saghafi (2013) performed elastic FEA to examine the effect of $v$ on mixedmode specimens. The authors concluded that the SIF and T-stress values were dependent on specimen thickness and Poisson's ratio.

\subsection{Plastic Zone Size from Three-dimensional Elastic FEM}

Real materials and specimens need to be considered in 3D, and as a result, there has been a focus on 3D and thickness effects on the PZ size and shape. Nakamura and Parks (1990) presented results showing plastic zone maps at mid-plane and edges for various load levels. The results show that the loading level had a direct effect on the shape of the plastic zone. At high load levels, the authors attribute plastic zone variations to the competition between stress singularity amplitude and stress triaxiality.

McClung (1991) used elastic-plastic FEA to simulate fatigue crack growth, looking at plastic zone size as estimated by different prediction methods, and comparing these with finite element results. The results were extracted by locating integration points with stresses higher than yield stress.

Roy and Narasimhan (1998) studied J-dominance in mixed-mode loading of ductile specimens. The effect of the value of the T-stress term on the plastic zone was determined by applying a modified boundary-layer method.

Ayatollahi and Zakeri (2007) investigated the effect of T-stress on the plastic zone for mode II specimens. The authors compared calculated plastic zone values with the experimental results obtained from previous literature using the methods of photoelasticity. 
Kudari and Kodancha (2010) studied a Single Edge Notch Beam (SENB) specimen - a case of pure mode I opening loading - to analyze the size and shape of the plastic zone. The authors used a model similar to that used in the work of Qu and Wang (2006), which provided SIF and T-stress solutions for the SENB specimen. Kudari and Kodancha used elastic FEA to calculate $J$ and subsequently $K$ for 3D SENB specimens, using a postprocessor to generate the plots for the plastic zone size and shape. The reported PZ shapes did not correspond to what was expected from the 2D simplifications, which predicted a plastic zone bounded by plane strain and plane stress, and the authors further described the stress state at the free surface and throughout the crack front using the calculated FEA results. These results were again counter to the expectation of plane stress/ plane strain at free/mid planes. (Kudari and Kodancha, 2010). The method used by these authors was an iso-surface representation of the yield stress boundary region. Twodimensional comparisons of elastic-plastic and elastic FEA were also completed.

Matvienko and Pochinkov (2013) looked at a pure mode I opening case, and showed agreement between the FEA results and the theoretically calculated PZ size and shape. The results of this study covered a small variety of specimen thicknesses, and showed some comparisons of the $\mathrm{PZ}$ of the different thicknesses.

\subsection{Finite Element Analysis}

Due to the complex nature of elasticity theory, it is necessary to take a numerical, rather than analytical, approach to fracture problems. With ever-increasing computational power, analysis techniques previously deemed too computationally expensive are now available for widespread use. Of interest to the current research is the use of linear-elastic FEA to analyze problems involving fracture mechanics.

\subsubsection{J- Integral and Elastic FEA Calculation}

For a linear elastic material undergoing general loading, $G$ and $K$ are related by the expression: 


$$
G=\frac{1-v^{2}}{E}\left(K_{I}^{2}+K_{I I}^{2}\right)+\frac{1}{2 G} K_{I I I}^{2}
$$

In Equation 2.7, the stress intensity factor $(K)$ can be seen to be directly related to the energy release rate per unit crack front length $(G)$ (Dassault Systems/ SIMULIA, 2013). By calculating the value of $G$, the values of the SIFs can also be calculated.

The method used to calculate the value of $G$ is known as the J-integral $(J)$, which provides a convenient basis to calculate the SIF. Taking an arbitrary path around the crack tip, within the $K$ dominated zone, $J$ is shown to be path-independent, and is equal to $G$ for linear elastic materials.

In two-parameter fracture mechanics, $K$ and T-stress describe the stress surrounding a crack. It has been shown by Rice (1974) that the constant T-stress has no effect on Jintegral. This supports the conclusions made by many researchers that the inclusion of Tstress terms in the analysis of stress at the crack tip is necessary to adequately describe the stress field surrounding the crack tip. Equation 2.8 shows that $K$ and T-stress are independent in the expression for stress in a 2D cracked body.

$$
\begin{aligned}
{\left[\begin{array}{ll}
\sigma_{x x} & \sigma_{x y} \\
\sigma_{y x} & \sigma_{y y}
\end{array}\right]=} & \frac{K}{\sqrt{r}}\left[\begin{array}{ll}
f_{x x}(\theta) & f_{x y}(\theta) \\
f_{y x}(\theta) & f_{y y}(\theta)
\end{array}\right]+\left[\begin{array}{ll}
T & 0 \\
0 & 0
\end{array}\right] \\
& + \text { terms which vanish at crack tip }
\end{aligned}
$$

(Rice 1974)

\subsubsection{Contour Integral Evaluation in Abaqus/CAE}

Abaqus/CAE has built-in features to evaluate stresses in cracked bodies, using methods such as contour integral evaluation, for which SIF and T-stress extraction is provided. For a 3D model, Abaqus can calculate the J-integral around a number of paths, or contours, about the crack tip. The calculation of $J$ is performed at every node along the crack front (through-thickness direction), and each contour refers to each concentric ring of nodes extending radially outwards from the crack tip. The value of the J-integral is, for adequate 
mesh refinement, independent of mesh geometry. To ensure the path-independence of the J-integral, the finite element model must be created in such a way that the contour integral area must completely enclose the fracture process zone. Quadratic elements can reduce computation time and simplify the model. A report by Matos and Dodds (2011) showed that the 20-noded quadratic elements, shown in Figure 2.4, with reduced integration (C3D20R) yield excellent results for fracture analysis problems.

\section{Extraction of SIF and T-stress Solutions}

From the calculation of the J-integral, the interaction integral method is used to extract the numerical values of the three individual SIFs. The same interaction integral is used, along with an auxiliary stress solution of a line load applied along the crack line, to extract the value of T-stress. Detailed explanations for the exact methods used by Abaqus are available in the Abaqus Theory Manual (SIMULIA, 2014).

\subsection{Mixed Mode: The Asymmetric Four-point Shear Specimen}

Of particular interest to this thesis is the mixed-mode I/II case found in a specimen known as the asymmetric four-point shear (AFPS) specimen. Many researchers, such as Maccagno and Knott (1985), and He, Cao, and Evans (1990), have used this specimen to study mixed-mode fracture behaviour. More recently, He and Hutchinson (2000) provided more complete 2D solutions for the mixed-mode SIFs. Figure 2.4 depicts the AFPS specimen considered by $\mathrm{He}$ and Hutchinson (He and Hutchinson 2000), where crack depth or length is $a$, crack offset is $c$, plate width is $W$ or $w$, and plate thickness is $t$ (not shown). The following four ratios described the specimen geometry described in He and Hutchinson: $a / W, b_{1} / W, b_{2} / W$, and $c / W$. Note that thickness $(t / W)$ was not considered in the analysis. 
In a pure mode II case, $K_{I I}$ is defined as:

$$
K_{I I}^{R}=\frac{Q}{W^{\frac{1}{2}}} \frac{(a / W)^{\frac{3}{2}}}{(1-a / W)^{\frac{1}{2}}} F_{I I}(a / W)
$$

where $F_{I I}$ is the normalized SIF and $Q$ is shear force, calculated as follows:

$$
Q=P\left(b_{2}-b_{1}\right) /\left(b_{2}+b_{1}\right)
$$

$\mathrm{He}$, Cao, and Evans considered experimental data to empirically determine expressions for the SIF of this specimen for varying crack geometry (He, Cao, and Evans 1990) . Values for stress intensity factors $K_{I}$ and $K_{I I}$ were determined using a finite element approach and the energy release rate, G. A later paper by He and Hutchinson (2000) provided empirical formulae for the 2D stress intensity factors $K_{I}$ and $K_{I I}$. Figure 2.4 shows the four-point shear specimen used by $\mathrm{He}$ and Hutchinson. In this paper, corrections are made to account for finite-thickness specimen geometry. These small corrections are provided for a number of cases that have relatively small distance between loading points and crack tip.

A more recent paper by Shahani and Tabatabaei (2008) presented results for mode I and mode II stress intensity factors, generated using linear FEA . The correction factors from He and Hutchinson were further refined and presented.

He and Hutchinson (2000) provided solutions for the mixed-mode case of the asymmetric four-point shear specimen. The solution for $F_{I I}$ is given in terms of the $a / W$ ratio: 


$$
\begin{gathered}
F_{I I}\left(\frac{a}{W}\right)=7.264-9.37\left(\frac{a}{W}\right)+2.74\left(\frac{a}{W}\right)^{2}+1.87\left(\frac{a}{W}\right)^{3}-1.04\left(\frac{a}{W}\right)^{4} \\
\text { for } 0 \leq \frac{a}{W} \leq 1
\end{gathered}
$$

In mixed-mode loading, the mode I component solution is $F_{I}$ :

$$
\begin{gathered}
F_{I}\left(\frac{a}{W}\right)=1.122-1.121\left(\frac{a}{W}\right)+3.740\left(\frac{a}{W}\right)^{2}+3.873\left(\frac{a}{W}\right)^{3}-19.05\left(\frac{a}{W}\right)^{4} \\
+22.55\left(\frac{a}{W}\right)^{5} \\
\text { for } 0 \leq \frac{a}{W} \leq 0.7
\end{gathered}
$$

There are currently no literature solutions that provide values for $K_{I I I}, T_{11}$, or $T_{33}$. Fig. 2.5 shows the coordinate directions of the two T-stress terms relevant to the current work: $T_{11}$ and $T_{33}$.

\subsection{Motivation for Current Work}

The correct interpretation of fracture testing results requires accurate solutions of fracture mechanics parameters for the specimen geometry. The methods of fracture mechanics provide a good means to approximate the stress around a cracked body; however the solutions for fracture parameter values must be numerically calculated for each geometric case. Mixed-mode specimens have been developed to determine fracture behaviour under complex loading configurations.

Although the FPS and AFPS specimens have been used for fracture testing, the available solutions are based on 2D plane strain analysis. Clearly, 3D analysis is necessary to properly capture the complete fracture behaviour. In real test specimens, the variations caused by different specimen thicknesses have not been studied until now. In this thesis, the values of fracture parameters are determined through extensive 3D finite-element analysis on FPS and AFPS specimens of varying thickness. The 3D effects are illustrated through plastic zone analysis, taking into account all fracture mechanics parameters. 


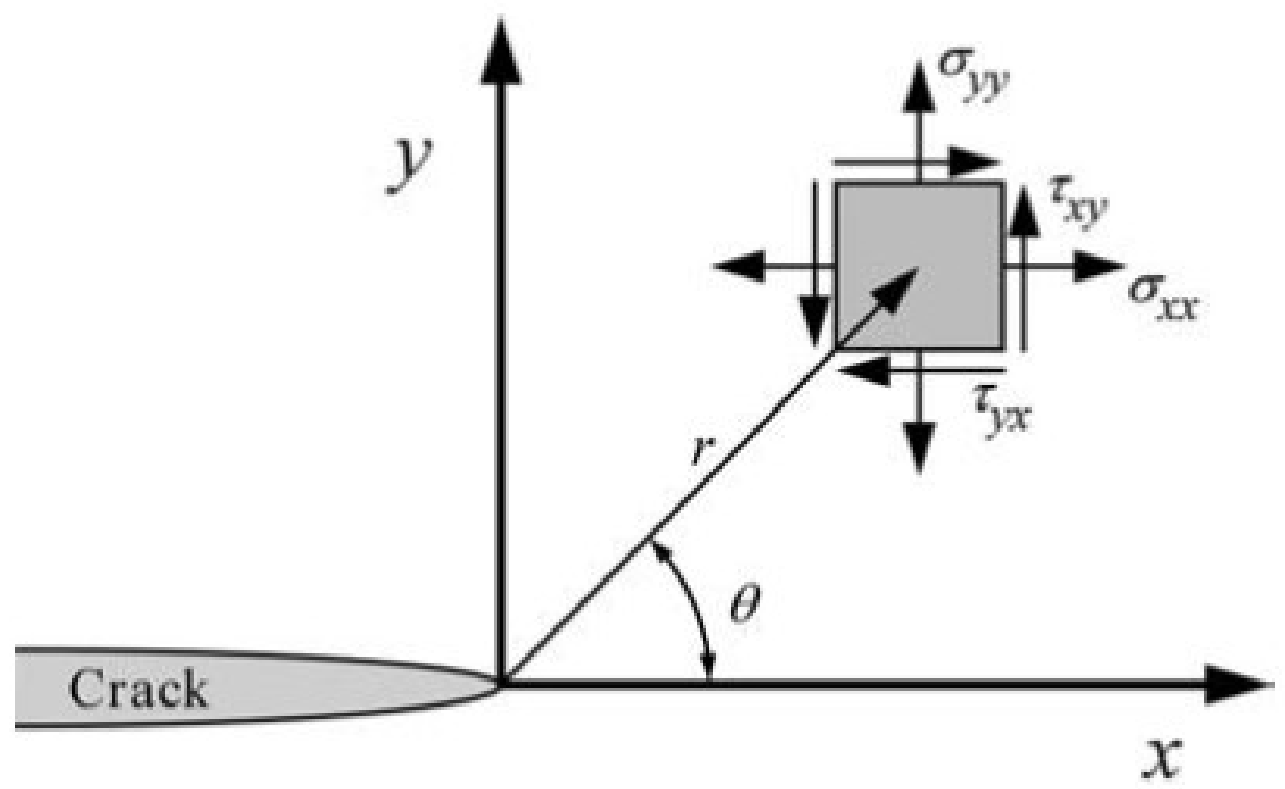

Figure 2.1: Polar coordinate system of stress around a crack tip

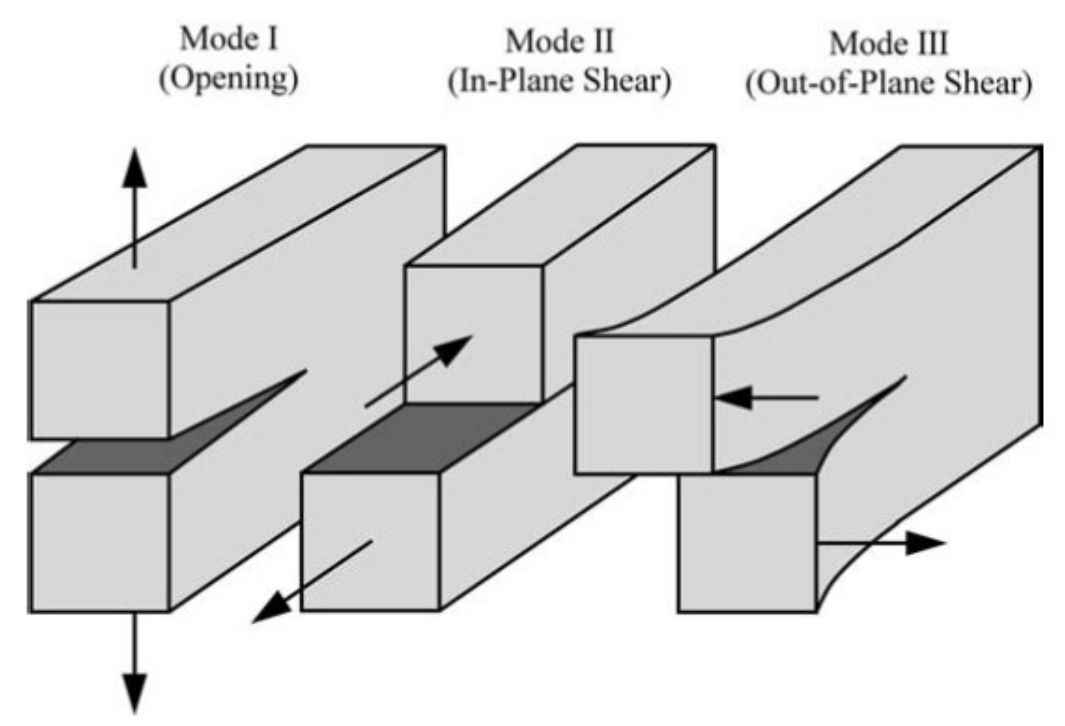

Figure 2.2: Modes of loading in fracture mechanics 


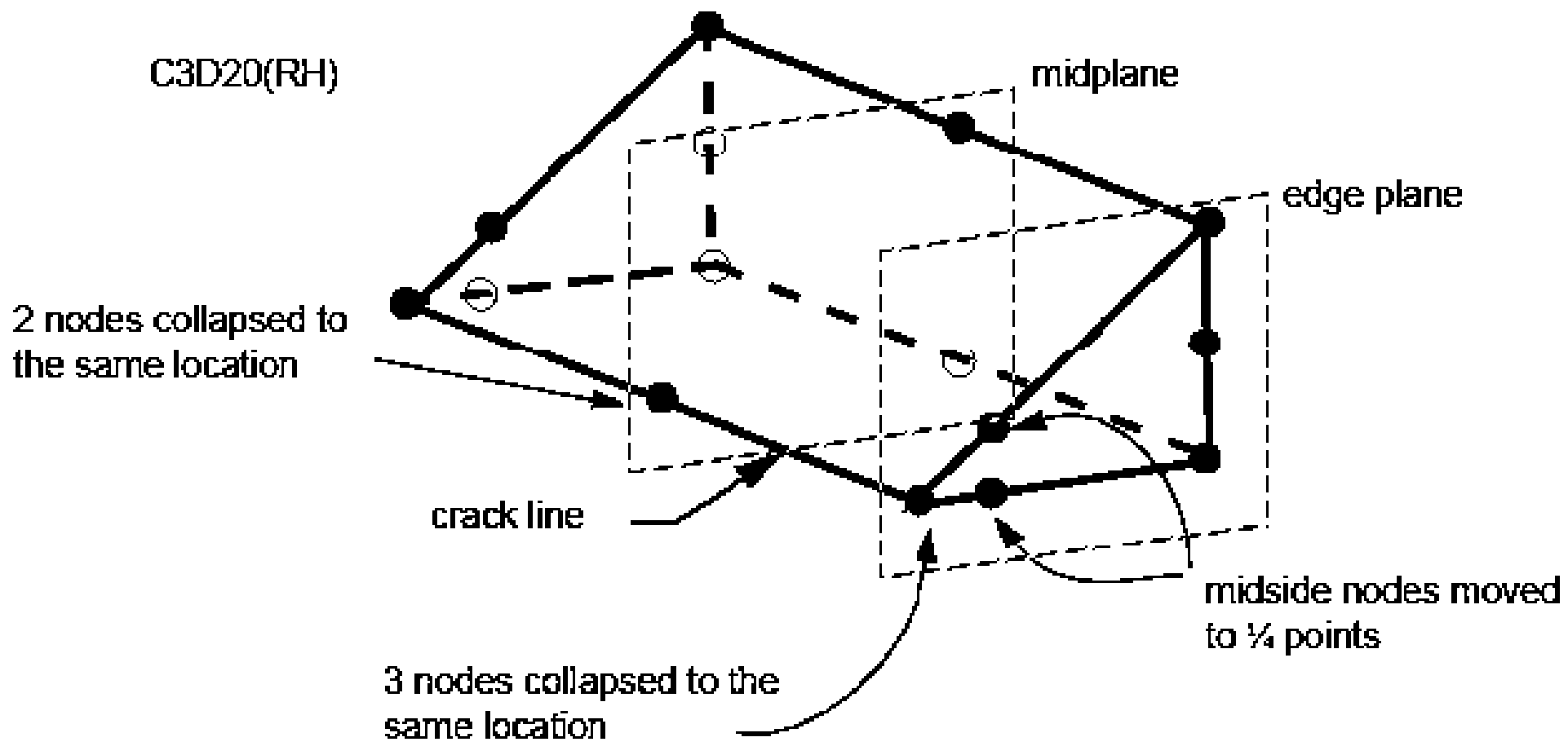

Figure 2.3: C3D20R collapsed element configuration 


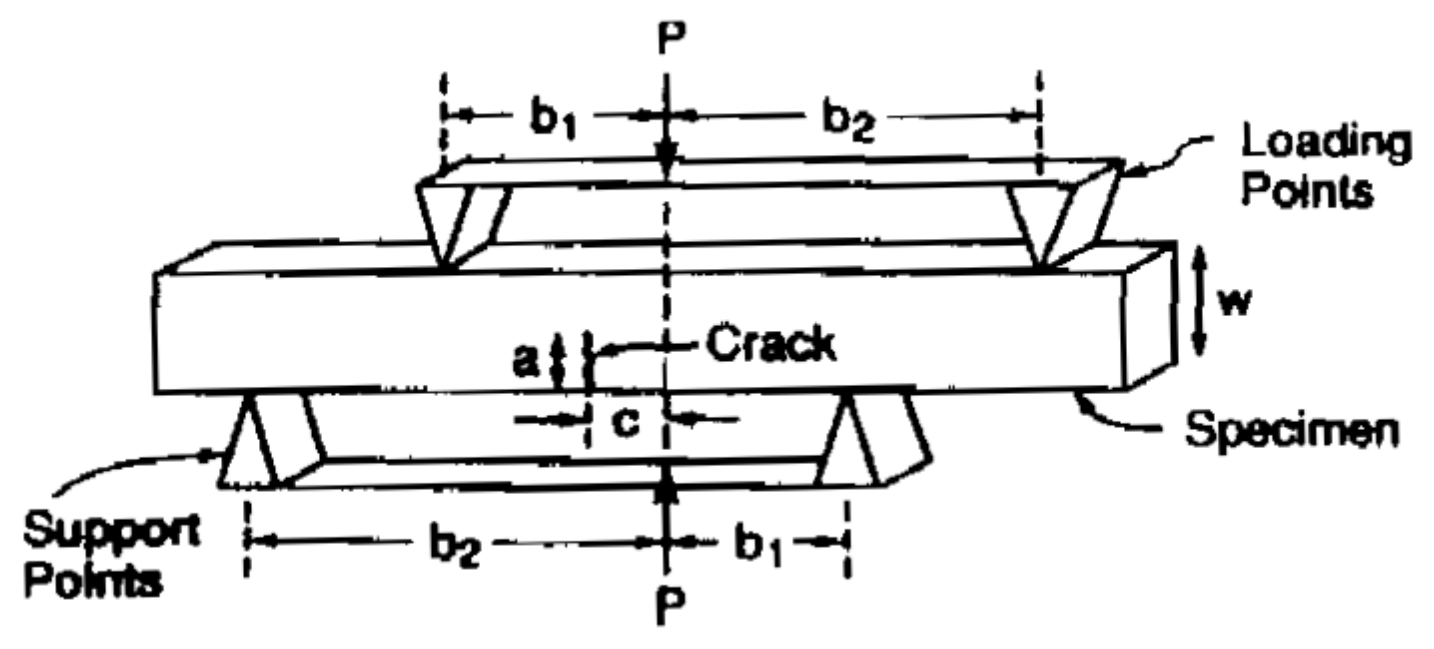

Figure 2.4: The asymmetric four-point-shear specimen

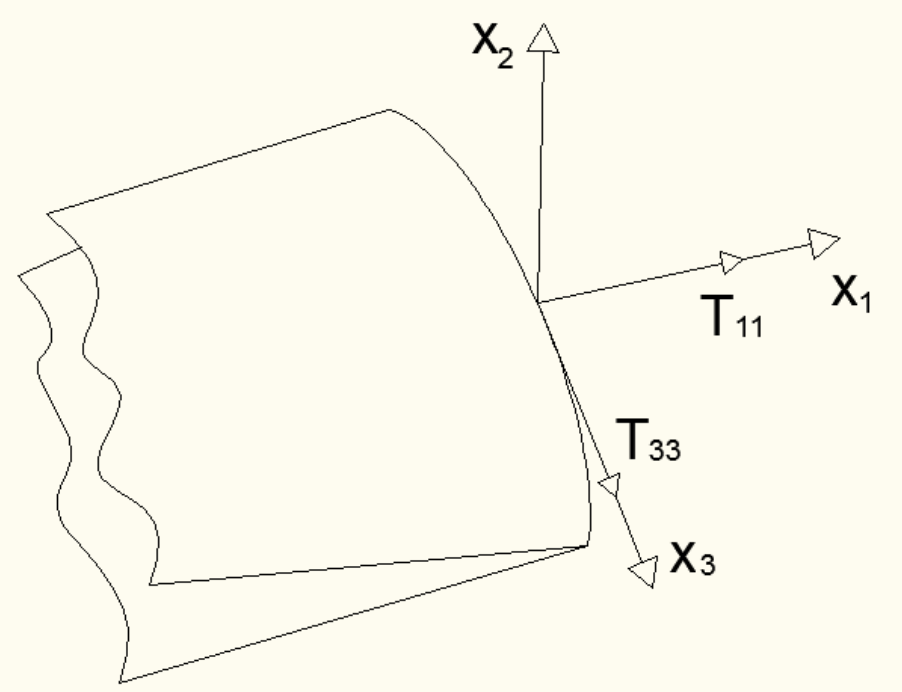

Figure 2.5: T-stress coordinate directions 


\section{Chapter 3: Three-Dimensional Fracture Analysis of FPS Test Specimens}

In this chapter, the fracture behaviour of the mode II loaded FPS specimen was examined. Comprehensive 3D FEA was conducted, and complete solutions of $K_{I I}$ and $K_{I I I}$ were obtained for a wide range of $a / W$ and $t / W$ ratios. The SIF values are presented for a number of points through-thickness for each specimen thickness considered.

A method for tracing the boundary of the plastic zone was created and verified against the previous methods of Nazarali and Wang (2011). Current FEA results were used to plot the plastic zone size and shape at various locations through-thickness, and some typical cases were compared with estimates from the stress contours of Abaqus FEA.

\subsection{Finite Element Analysis}

Elastic FEA was used to calculate the mode II and III SIF for various geometries of FPS specimens. Because the scope of the work was to cover a wide range of geometric variations, a parametric study was undertaken. Python scripts were used to generate Abaqus models for each different specimen geometry, which keep meshing constraints consistent between models. Figure 3.1 shows the four-point shear specimen, where the top of the specimen was loaded by load $P$ and the lower side of the specimen was reacted by the shown supports. The specifications of the finite element model are described in the following section.

\subsubsection{D FEA Model}

\section{Geometry}

Two parameters were varied in the models created for the pure mode II case: crack length-to-width ratios of $a / W=0.1,0.2,0.3,0.4,0.5,0.6$, and 0.7 ; and thickness-to-width ratios of $t / W=0.1,0.2,0.5,1.0,2.0$, and 4.0. In Figure 3.1, the following ratios were true: $b_{2} / b_{1}=2, b_{1} / W=2$. These values were selected based on the findings of He and Hutchinson (2000), who found good convergence with their solutions for $b_{1} / W>1.4 W$. By maintaining a (relatively) large distance from the loading and support points to the crack, 
the reference solutions can be compared with less error, and without needing to consider any correction factors.

The material was considered to be perfectly elastic, with an elastic modulus of $207 \mathrm{GPa}$, and a Poisson's ratio $(v)$ of 0.3 , common values for typical engineering materials. An individual case was performed for a value of $v=0$, and a thickness ratio $t / W=4.0$, to be used as a representative $2 \mathrm{D}$ case.

\section{Mesh Design}

The mesh design was a constant 2D mesh across the face of the plate, and was swept along the crack front. The mesh was refined around the loading and boundary condition locations, as well as at the crack tip. The element type throughout the model was a collapsed 20-noded quadratic element with reduced integration (C3D20R). At the crack tip, one edge of the element was collapsed to form a wedge, with the point at the crack. The mesh refinement around the crack tip was done to the recommended levels in the documentation, using 30 elements angularly around the crack tip (Dassault Systems/ SIMULIA, 2013). Midside nodes on the element sides directly adjacent to the crack tip were moved to the quarter point, to better capture the singularity at the crack tip. A typical specimen mesh for a plate with $t / W=1.0$ and $a / W=0.1$ is shown in Figure 3.2, and the C3D20R schematic is shown in Figure 2.3. Through-thickness, between 20 and 50 elements were used to calculate fracture parameters.

In order to maintain a consistent mesh for the many different geometric variations of the model, a script which creates models, parametrically varying crack length and offset, was used to generate the many models used. In this way, similar meshes were created for each geometric case considered. In total, 210 different models were created, with a varying number of elements from 10,000 to close to 500,000, depending on thickness and crack depth.

The mesh was refined in two ways: along the thickness direction, the mesh was refined towards the free edge; and around the crack tip, to meet sizing recommended by Abaqus training documentation (Dassault Systems/ SIMULIA, 2013). Additional mesh 
refinement was also done around the loading and support points, as a precaution to minimize any potential impact on the crack tip elements.

\section{Loads and Boundary Conditions}

The loading used in this model corresponded to the loading shown in Figure 3.1. The value of $P$ was set, and the force was distributed two-thirds and one-third to the points as shown in the same figure. The load used for each was a function of the plate thickness, where force $P$ was applied as force/unit length. The value of $P$ selected was $525 \mathrm{~N} / \mathrm{mm}$, corresponding to $175 \mathrm{~N} / \mathrm{mm}$ and $350 \mathrm{~N} / \mathrm{mm}$ applied to each of the two loading points (on top), respectively.

The lower support points were modeled as simple supports, and a single node at the bottom corner of the specimen was restrained from displacing along the length direction, preventing the specimen from having excessive displacement during the analysis.

Both the loading and support points were modeled by applying a pressure or restraining a small strip. The additional mesh refinement helped distribute the stress and deformation across many elements, relieving some of the stress spikes that can occur due to local boundary conditions and loads.

Symmetry was used to simplify the calculation analysis. The mid plane $(z / t=0)$ was chosen as the plane of symmetry, which had an insignificant effect on the results. All results were symmetric about the mid plane, with the exception of $K_{I I I}$, which was antisymmetric.

\section{Determination of SIF}

The FEA models were analyzed using Abaqus built-in contour integral calculation, which modified the crack tip mesh with collapsed element edges, forming degenerate cube (wedge) elements. These degenerate cube elements had four midside nodes which were moved to the quarter point, to better capture the singularity that occurs at the crack tip. A diagram of the element design around the crack tip is shown in Figure 2.3. Abaqus 
calculated the requested SIF values and outputted the results for each node along the crack tip. Five contours were used for the calculation, and the average of the outer three is presented as the result. For all geometries, convergence of the contour integral indicated that adequate meshing refinement was met, as values were stable after the first two contours.

These results were then extracted from the output files (.dat and .odb) and processed through the use of MATLAB scripts. Using scripted calculations, the results were organized, normalized, and plotted. The expressions used to normalize the results are described in Section 3.2.1.

Some previous researchers had found cases where specimens loaded in pure mode II had values of T-stress that were non-zero, and significant. In the current work, the values of $K_{I}, T_{11}$, and $T_{33}$ were all found to be near-zero, and were disregarded.

\subsubsection{Model Verification}

In this section, FEA models were created in Abaqus to reproduce the 2D results presented by He and Hutchinson (2000) for the four-point shear specimen. Both 2D and 3D FEA models were created to compare with the literature results. Verification of this kind served to confirm the modelling and calculation techniques that were used to produce the results.

\section{D FEA Verification of Model}

In order to verify the numerical modelling techniques and contour integral features, the results of $\mathrm{He}$ and Hutchinson (2000) were recreated using Abaqus/CAE 13.1. A 2D analysis was undertaken for a pure mode II case. The output from the current FEA gave the SIF solutions $K_{I I}$ and $K_{I I I}$, for the single node at the crack tip, using plane-stress elements. The values for $K_{I I}$ were normalized using the equations used by both early papers by He, and are given in Section 3.2.1. The results are presented in Figure 3.3 and Table 3.1, and show very good agreement between the current FEA results and the 
empirical solutions from the literature. Errors were calculated to be much less than $1 \%$ for the full range of $a / W$ values considered.

\section{D Results Comparison}

Before examining the through-thickness variation of each of the SIFs, the values of $K_{I I}$ at the mid plane were compared with the literature solutions of He and Hutchinson (2000). The normalized $K_{I I}$ value extracted at the mid plane was plotted, for each considered thickness, over the range of $a / W$ values. Figure 3.4 and Table 3.2 show the results of this comparison. For the purposes of verifying the 3D modelling techniques, the results from case of $t / W=4.0$, where $v=0$, were compared with the $2 \mathrm{D}$ results. The results from this case are representative of a $2 \mathrm{D}$ case because the effects of finite thickness can be attributed to the influence of $v$ on the free edges of the plate. As can be seen in Figure 3.4, the current $3 \mathrm{D}$ results matched the literature results almost exactly, varying at most $0.3 \%$ over the range of considered crack depths. The other thickness cases, where $v=0.3$, showed variations from the literature solutions of up to $20 \%$ in magnitude. The significance of these results is discussed in Section 3.2.2.

\subsection{Stress Intensity Factor Results}

In the case of a finite-thickness plate, the effects of Poisson's ratio and the free edge create stresses through the thickness that can be described by LEFM parameters. From this analysis, the calculated values of $K_{I I}$ and $K_{I I I}$ were provided. Each parameter is presented independently in tables and plots at the end of the chapter, comparing the normalized values from specimens of different thicknesses. The magnitude of the SIF values at the mid plane, and the through-thickness variation of SIF values are of particular interest. 


\subsubsection{SIF Normalizing Functions}

The SIFs for the FPS specimen are described below, using functions of the geometric parameters of the specimen, so that solutions can be presented in a more general manner. The values that are provided in the output databases (ODB) by Abaqus are given in units of $\mathrm{Pa} \sqrt{m}$. The non-dimensional functions $F_{I I}$ and $F_{I I I}$, known as the normalized SIFs, are functions of crack geometry. The normalizing factors are functions of crack length and loading force. He and Hutchinson (2000) provided these equations to normalize the mode I and II stress intensity factors. In a pure mode II case, $K_{I}$ is zero and $K_{I I}$ is defined as

$$
K_{I I}^{R}=\frac{Q}{W^{\frac{1}{2}}} \frac{(a / W)^{\frac{3}{2}}}{(1-a / W)^{\frac{1}{2}}} F_{I I}(a / W)
$$

where $F_{I I}$ is the normalized SIF and $Q$ is shear force, calculated as follows:

$$
Q=P\left(b_{2}-b_{1}\right) /\left(b_{2}+b_{1}\right)
$$

In this definition, $P$ is loading force, and both $\mathrm{Q}$ and $\mathrm{P}$ are defined per unit-thickness.

Because the reference solutions are applicable to the $2 \mathrm{D}$ case, there is currently no expression for normalizing $K_{I I I}$. The $K_{I I I}$ solutions are normalized using the same factor as the mode II component:

$$
K_{I I I}^{R}=\frac{Q}{W^{\frac{1}{2}}} \frac{(a / W)^{\frac{3}{2}}}{(1-a / W)^{\frac{1}{2}}} F_{I I I}(a / W)
$$

where $F_{I I I}$ is the normalized stress intensity factor. The values of $F_{I I}$ and $F_{I I I}$ solutions calculated in the current study are presented in the summary tables as $K_{I I}$ and $K_{I I I}$. A summary of the results can be found at the end of this chapter, in Table 3.3 through Table 3.8, and also plotted through-thickness from Figure 3.5 through Figure 3.18. 


\subsubsection{Discussion of SIF results}

Of the six thickness cases considered, the results can be split into two groups: thick plates and thin plates. The cases of $t / W=0.1,0.2$, and 0.5 , can be considered as thin plates, and show only minor variation in $K_{I I}$ value through-thickness, corresponding well to the $2 \mathrm{D}$ literature solutions. From Table 4.2 it can be seen that the thin plate cases are typically only a few percent from the literature solution, usually larger, at the mid plane. The $K_{I I}$ values of these thin plates are fairly constant, and positive in value, through the thickness, with a steep increase very close to the free edge $(z / t=0.5)$. The $K_{I I I}$ values have a different trend, with all cases having a value of 0 at the mid plane, and steadily decreasing (increasing magnitude in negative direction) towards the free edge, where, very close to the free edge, the values appear asymptotic to the free edge. The trends of throughthickness SIF variation of $K_{I I}$ and $K_{I I I}$ do not appear to change appreciably with varying $a / W$. The value of $K_{I I}$ decreases significantly with increasing $a / W$, while the value of $K_{I I I}$ only decreases slightly.

The plates which can be considered to represent thick cases, $t / W=1.0,2.0$, and 4.0, show significantly more variation through-thickness of both $K_{I I}$ and $K_{I I I}$. The value of $K_{I I}$ tends to be significantly lower (10-20\%) for the two thickest cases across all values of $a / W$. The case of the square plate $(t / W=1.0)$ shows intermediate behaviour between the extreme thickness cases, and the thin-plate behaviour. The mid plane results for the square case show a large deviation (10\%) from the literature solutions for shallow cracks, but show only minor deviation (2\%) for deep cracks. A defining feature of the $K_{I I}$ variation for these so-called thick plates is a noticeable increase in magnitude, from a position around $z / t=0.3$ to the free edge. For $t / W=1.0$, this increase is minor, but for the two thicker cases of $t / W=2.0$ and 4.0, the values increase significantly for the area near the free surface. Looking at $K_{I I I}$, the amount of the variation of through-thickness values between the thin plates, and even the square case, is dwarfed at shallow crack depths by the extreme variation of $K_{I I I}$ for the two thickest cases. For the shallow cracks of $a / W<0.4$, the thick cases show a sharp increase in magnitude $K_{I I I}$, which peaks at around $z / t=0.3$ for $t / W=2.0$, and $z / t=0.375$ for $t / W=4.0$. The values of $K_{I I I}$ are all large enough in magnitude that neglecting to take these into account would be a significant oversight. 


\subsection{Plastic Zone Analysis}

Of interest in fracture problems are the size and shape of the plastic zone, which is defined for our purpose as the boundary between elastic and plastic behaviour. The plastic zone is found by isolating the region bounded by stress equal to an effective yield stress. PZ size can be used to confirm SSY assumptions and to better understand the stress state around the crack tip.

In this section, the SIF results from the current FEA were used to predict PZ size and shape. The impact of including the $K_{I I I}$ term on the plastic zone was studied, as well as the plastic zone variation through-thickness for a variety of plate thickness.

\subsubsection{Calculating and Plotting the Plastic Zone from LEFM Parameters}

The Williams (1957) expansion for the stress around a crack tip calculates the stress components, in polar coordinates, at a distance $r$ from the crack tip and at angle $\theta$. For mode II loading, the stress state can be described in polar coordinates by the following (Anderson 2006):

$$
\begin{gathered}
\sigma_{11}(r, \theta)=-\frac{K_{I I}}{\sqrt{2 \pi r}} \sin \left(\frac{\theta}{2}\right)\left[2+\cos \left(\frac{\theta}{2}\right) \cos \left(\frac{3 \theta}{2}\right)\right] \\
\sigma_{22}(r, \theta)=\frac{K_{I I}}{\sqrt{2 \pi r}} \sin \left(\frac{\theta}{2}\right)\left[\cos \left(\frac{\theta}{2}\right) \cos \left(\frac{3 \theta}{2}\right)\right] \\
\sigma_{12}(r, \theta)=\frac{K_{I I}}{\sqrt{2 \pi r}} \cos \left(\frac{\theta}{2}\right)\left[1-\sin \left(\frac{\theta}{2}\right) \sin \left(\frac{3 \theta}{2}\right)\right] \\
\sigma_{33}(r, \theta)=v\left(\sigma_{11}+\sigma_{22}\right)
\end{gathered}
$$

where $v$ is Poisson's ratio. 
For pure mode III, the stress state can be described as follows:

$$
\begin{gathered}
\sigma_{13}(r, \theta)=-\frac{K_{I I I}}{\sqrt{2 \pi r}} \sin \left(\frac{\theta}{2}\right) \\
\sigma_{23}(r, \theta)=\frac{K_{I I I}}{\sqrt{2 \pi r}} \cos \left(\frac{\theta}{2}\right)
\end{gathered}
$$

Applying the principle of superposition for both modes, the total stress can be calculated:

$$
\sigma_{i j}^{(t o t a l)}=\sigma_{i j}^{(I I)}+\sigma_{i j}^{(I I I)}
$$

By using the components defined by the Williams' expansion, a yield criterion can be applied - in this case, Von Mises yield criterion. The effective Von-Mises stress is given by:

$$
\sigma_{\mathrm{e}}=\sqrt{\frac{\left(\sigma_{11}-\sigma_{22}\right)^{2}+\left(\sigma_{22}-\sigma_{33}\right)^{2}+\left(\sigma_{33}-\sigma_{11}\right)^{2}+6\left(\sigma_{12}^{2}+\sigma_{23}^{2}+\sigma_{31}^{2}\right)}{2}}
$$

Assuming an arbitrary value of yield stress, the Williams expansion terms in Equation 3.4 and 3.5 can be inserted into Equation 3.6 and subsequently equated to the Von-Mises yield criterion (Equation 3.7). This expression can be used to calculate $r$, in terms of $\theta$, around the crack tip, for an arbitrary yield stress value.

In order to determine $r$, the radius that defines the boundary between elastic and plastic behaviour, a symbolic solver was implemented in MATLAB to calculate the plastic zone for the FEA results previously presented. The function used in MATLAB was vpasolve, and the variable $r$, the radial distance from the crack tip in Equations 3.4 and 3.5, was assigned as a symbolic variable (MathWorks, 2015). Non-normalized SIF values were substituted into Equations 3.7, and $r$ was found for the full range of $\theta$ at several locations through the thickness. For pure mode II loading, the plastic zone was normalized as a function of $K$ and yield stress: 


$$
\text { Normalized plastic zone radius } R_{p}=\frac{r}{\frac{1}{\pi}\left[\frac{K_{I I}}{\sigma_{Y S}}\right]^{2}}
$$

To show the relative size of the plastic zone at various locations through-thickness, the value of $K_{I I}$ at the mid plane was used to normalize all values of $R_{p}$ for each of the different through-thickness cases. The value of $K_{I I I}$ was found to be zero at the mid plane, for all values of $t / W$, so the impact of its inclusion in calculating the PZ can be clearly seen. On the first plastic zone plot, the location of the crack line is shown for illustration purposes.

\section{Verification}

In order to confirm the current calculation method, some baseline cases were compared with values obtained from using the equations provided by Nazarali and Wang (2011). The full set of equations given by Nazarali and Wang is included in Appendix A, and the equations are valid for mode I and II loading cases, which may include T-stress.

A pure mode II loading case was used, with a non-zero $K_{I I}$ value, and a zero value of $K_{I I I}$. This is plotted in Figure 3.19, which shows that the current calculated plastic zone matches the results from Nazarali and Wang (2011) exactly. This figure is also in agreement with the shape and magnitude of the plastic zones given in Anderson (2005), who used a similar method, also equating the Von-Mises yield stress to the Williams expansion, and solving for $r$.

It is worth noting that the only difference between the calculation method being employed in the current work, and the calculation method given by the expressions in Nazarali and Wang, is the inclusion of the $K_{I I I}$ term. Both methods use the Von-Mises yield criterion, so the comparison between methods is valid.

The work of Nazarali and Wang (2011) can only be used to verify the calculation of plastic zones without the inclusion of $K_{I I I}$, the mode III contribution. A pure mode III case was considered using the current calculation method, and was compared with the 
results presented in Anderson (2005). It can be seen that for the case of pure mode III loading, the plastic zone was perfectly circular, with a normalized radius value of 1.5 , exactly matching the results of Anderson.

\section{Plastic Zone Discussion}

From the produced plots of the normalized plastic radius, it is initially noticeable that the behaviour can again be grouped into two types of specimen: thick and thin. The considered specimens for the plastic zone analysis have $t / W$ ratios of $0.1,0.5,1.0,2.0$, and 4.0. The case of $t / W=0.2$ has been excluded for space considerations, and it is expected to be almost identical to the case of $t / W=0.1$.

When comparing the plastic zones, for all cases, the smallest plastic zone (throughthickness) was located at the mid plane, and the plastic zone radius increased at all positions towards the free surface. The value of $K_{I I I}$ always reached zero at the mid plane, so the plastic zone in the middle of the plate can be expected to resemble that of pure mode II loading. The relative increase in plastic zone size was small for the thin $(t / W=$ $0.1,0.5)$ plates considered, but was significant for the thicker cases. The thin plates showed a plastic zone which stayed at a fairly constant size, in the shape expected of pure mode II loading. The case of $t / W=1.0$ showed an increased plastic zone at locations approaching the free surface, still in the shape of pure mode II loading. The size increase (from the mid plane) of the plastic zone at outer positions appeared to decrease with increasing $a / W$, where shallower cracks had larger variations in plastic zone size than deep cracks.

As might be expected from the extreme $K_{I I I}$ variations in Figure 3.12 and Figure 3.13, the thickest cases had the largest plastic zone variations through-thickness. In the case of $t / W=2.0$, for a shallow crack of $a / W=0.1$ (Figure 3.42), the plastic zone was exaggerated, and no longer resembled a pure-mode II shape, being an oblong circle. This change in shape and increase in size corresponded with the high value of $K_{I I I}$ at that location through-thickness. This effect was even more noticeable in the shallow-crack case of the thickest plate considered, $t / W=4.0$, where the plastic zone at the mid plane appeared to be just a fraction of that at the near-surface point $(z / t=0.375)$. At this near-surface point, the 
plastic zone appeared almost perfectly round, and represented a high amount of mode III contribution.

The current analysis found that for the FPS specimen, the values calculated for $T$ and $K_{I}$ were zero, and did not have any influence on the size of shape of the plastic zone. The large distance from the loading points to the crack ensured a zero value of $K_{I}$ and $T$. It could be concluded from this result that the T-stress contribution from pure mode II loading was negligible, and it was not included in this plastic zone analysis.

\subsubsection{Plastic Zone Description using FEA-generated Contour}

The calculated plastic zone sizes described using the Williams expansion and the VonMises yield criterion provided results showing large variations of plastic zone size and shape through-thickness. In order to verify that these predicted values were consistent with the stress results from elastic FEA, the PZ shape and size were estimated from the stress contour and compared with the prediction, for some typical cases.

Abaqus/CAE has built-in features to calculate and display stress contour regions as part of its post-processing analysis. This feature interpolates between the integration points of individual elements, providing a scaled, accurate representation of the stress field surrounding engineering features. By analyzing image captures of the stress contours, the region bounded by a certain stress level can be determined through simple scripting functions. The contour provides better estimates than would be extracted from the integration points alone, due to the higher-order interpolation between points.

\section{Extraction Method}

An image from the output database stress contour was imported into MATLAB and saved as an RGB image variable. The image was converted into a binary image and the boundary of the contour was traced and saved. A reference element edge was used to scale the boundary trace, and the calculated data was converted from Cartesian to polar coordinates after the center (crack tip) was found. The FEA-extracted plastic zone data was normalized using the same method and values as used for the predicted plastic zones. 
Some typical thickness cases were considered, $t / W=0.1,1.0$, and 4.0 , for the cases of crack depth extremes, $a / W=0.1$ and 0.7. In these figures, the number of data points shown for the FEA-extracted results was reduced for clarity.

\section{Discussion on FEA Extracted Comparisons}

This verification analysis proved to be a less efficient method than the prediction method for calculating the plastic zone, as the level of mesh refinement that would be necessary for low load levels was unreasonably high. In the current analysis, specimens with relatively small plastic zones for example, mid plane at shallow cracks, the plastic zone could be confined to be within a single element. This was not an ideal situation, as the built-in stress contour within Abaqus must be used to approximate the boundary. As seen in the comparison case, the plastic zone estimated by the SIF solutions showed good agreement with the FEA images, for all typical cases. The thin case, $t / W=0.1$, had little variation through-thickness for both thick and thin cracks. The resulting plots, from Figure 3.21 and Figure 3.56 through Figure 3.61, show good correlation with size and shape of the extracted and calculated plastic zones. There was some overlap of the FEAextracted plastic zones for the two positions in each of the two thin cases, and this was attributed to inaccuracies in the extraction method, and coarseness of the contour refinement.

The square $(t / W=1.0)$ specimen showed very good agreement between the calculated and extracted values for both crack depth ratios considered. Similar agreement was seen with the thick case of $t / W=4.0$; however, it appeared that the FEA-extracted values were slightly larger than the calculated values for the shallow crack $(a / W=0.1)$ case. This apparent difference could be attributed to contour coarseness, or even mesh coarseness in the area surrounding the crack. The deep-crack case showed better agreement with the calculated values, and supported the predicted values. Overall, the comparison cases showed that the plastic zone size and shape, as predicted by the current calculation method, was consistent with results from the FEA stress contours. 


\subsection{Discussion and Conclusion}

From the plots of the results, some key observations can be made about the inclusion of $K_{I I I}$ in the fracture analysis of the FPS specimen. Primarily, it can be seen that the $K_{I I I}$ influence on pure mode II loading cannot be ignored, and can influence PZ size and shape greatly. The coupling of $K_{I I}$ and $K_{I I I}$ is caused by Poisson's ratio effects from the free surface, which can be significant for thick specimens. This is seen most easily in the exaggerated case shown in Figure $3.49(t / W=4.0, a / W=0.1)$, where a large magnitude of $K_{I I I}$ is seen to greatly affect the plastic zone size and shape, resembling a circular shape, similar to that expected of pure mode III loading scenario. The trends seen in the results indicate that thick plates containing shallow cracks are most affected by the influence of $K_{I I I}$. The through-thickness comparison of SIF variation also showed an interesting trend, with some extreme cases showing significant variation in plastic zone size and shape at different location through-thickness.

Another key observation is that the amount of through-thickness variation of $K_{I I}$ appeared to be a function of the thickness of the plate. The trends identified show an increasing $K_{I I}$ variation for increased plate thickness. For the thicker cases $(t / W>1.0)$ it was observed that the value of $K_{I I}$ at the mid plane was lower than the literature solution, but increased to higher values closer to the free surface.

To see the total effect of finite-thickness considerations on the pure mode II loading case, the PZ size and shape were used as an indicator of crack tip stress state. It was observed that the through-thickness variation in the SIFs (increasing magnitude in positive $K_{I I}$ and negative $K_{I I I}$ ) had a tendency to increase PZ size. Inspection of the Von-Mises yield criterion showed that, in the absence of $K_{I}$, the $K_{I I I}$ stress contributions served to increase the effective stress, which in turn increased the radius of the predicted plastic zone. The produced PZ plots show that, for relatively thick plates $(t / W>=1.0)$, the plastic zone is not constant through-thickness, and enlarges at locations approaching the free surface.

The main conclusion that can be drawn from the pure mode II analysis of the FPS specimen is that the inclusion of $3 \mathrm{D}$ effects can have a very large effect on the plastic zone and stress field surrounding a crack, and must be considered. Of the cases 
considered in this analysis, the relatively thick $(t / W>1.0)$ plate specimens were affected most when shallow cracks were present, with the largest values of $K_{I I I}$ and biggest amount of through-thickness variation. What this could mean, in practical terms, is that the critical area to consider in these shallow-crack thick-plate bodies is not necessarily located at either mid or edge plane, but rather at some location between. This conclusion is counter to results for $K_{I}$ in pure mode I specimens (Jin and Wang 2014), which showed thick plates in good agreement with a plane strain 2D reference solution. The current results suggest that applying a similar assumption to mode II specimens, when considering mode III influence, would be incorrect.

The current analysis also suggests that for thin plates $(t / W<1.0)$, the variation throughthickness appears to be less significant than for thick plates, and the inclusion of the $K_{I I I}$ term has a less pronounced effect. This conclusion is arrived at after inspection of the plastic zone plots, which can be used qualitatively to estimate the influence of each mode's SIF. Thin FPS fracture specimens appear to be consistent with the 2D reference solutions, and test results could be interpreted using these solutions with reasonable accuracy.

The fracture parameter solutions obtained in this chapter will be very useful for fracture toughness testing of FPS specimens. The results of the plastic zone analysis performed in this chapter provide a clear illustration of the 3D effects on fracture specimens of varying thicknesses undergoing pure mode II loading. 
Table 3.1: Comparison of current $K_{I I}$ values and literature solution

\begin{tabular}{|c|r|r|r|}
\hline \multirow{2}{*}{ a/w } & \multicolumn{2}{|c|}{ Normalized $\boldsymbol{K}_{\boldsymbol{\prime}}$} & \multirow{2}{*}{ \% Difference } \\
\cline { 2 - 3 } & Reference & Current FEA & \\
\hline 0.1 & 6.3562 & 6.3603 & $0.06 \%$ \\
\hline 0.2 & 5.5129 & 5.5140 & $0.02 \%$ \\
\hline 0.3 & 4.7417 & 4.7392 & $-0.05 \%$ \\
0.4 & 4.0475 & 4.0460 & $-0.04 \%$ \\
0.5 & 3.4328 & 3.4334 & $0.02 \%$ \\
0.6 & 2.8975 & 2.8974 & $0.00 \%$ \\
0.7 & 2.4393 & 2.4341 & $-0.22 \%$ \\
\hline
\end{tabular}

Table 3.2: Normalized $K_{I I}$ values at the mid plane, for plates of different thickness

\begin{tabular}{|c|c|c|c|c|c|c|c|c|c|c|c|c|c|c|}
\hline \multirow{3}{*}{$t / w$} & \multicolumn{14}{|c|}{$a / w$} \\
\hline & \multicolumn{2}{|c|}{0.1} & \multicolumn{2}{|c|}{0.2} & \multicolumn{2}{|c|}{0.3} & \multicolumn{2}{|c|}{0.4} & \multicolumn{2}{|c|}{0.5} & \multicolumn{2}{|c|}{0.6} & \multicolumn{2}{|c|}{0.7} \\
\hline & $\boldsymbol{K}_{I I}$ & $\%$ & $K_{I I}$ & $\%$ & $K_{I I}$ & $\%$ & $K_{I I}$ & $\%$ & $K_{I I}$ & $\%$ & $K_{l I}$ & $\%$ & $K_{I I}$ & $\%$ \\
\hline 0.1 & 6.4867 & $2.1 \%$ & 5.6219 & $2.0 \%$ & 4.8260 & $1.8 \%$ & 4.1150 & $1.7 \%$ & 3.4882 & $1.6 \%$ & 2.9408 & $1.5 \%$ & 2.4669 & $1.1 \%$ \\
\hline 0.2 & 6.4465 & $1.4 \%$ & 5.6241 & $2.0 \%$ & 4.8308 & $1.9 \%$ & 4.1187 & $1.8 \%$ & 3.4898 & $1.7 \%$ & 2.9401 & $1.5 \%$ & 2.4634 & $1.0 \%$ \\
\hline 0.5 & 6.1036 & $-4.0 \%$ & 5.4811 & $-0.6 \%$ & 4.7776 & $0.8 \%$ & 4.0927 & $1.1 \%$ & 3.4716 & $1.1 \%$ & 2.9224 & $0.9 \%$ & 2.4422 & $0.1 \%$ \\
\hline 1.0 & 5.6646 & $-11 \%$ & 5.0403 & $-8.6 \%$ & 4.4534 & $-6.1 \%$ & 3.8827 & $-4.1 \%$ & 3.3369 & $-2.8 \%$ & 2.8351 & $-2.2 \%$ & 2.3885 & $-2.1 \%$ \\
\hline 2.0 & 5.0623 & $-20 \%$ & 4.4541 & $-19 \%$ & 3.8784 & $-18 \%$ & 3.3684 & $-17 \%$ & 2.9196 & $-15 \%$ & 2.5224 & $-13 \%$ & 2.1706 & $-11 \%$ \\
\hline 4.0 & 5.4983 & $-13 \%$ & 4.7524 & $-14 \%$ & 4.0643 & $-14 \%$ & 3.4496 & $-15 \%$ & 2.9130 & $-15 \%$ & 2.4518 & $-15 \%$ & 2.0612 & $-15 \%$ \\
\hline $4.0, v=0$ & 6.3488 & $-0.1 \%$ & 5.5093 & $-0.1 \%$ & 4.7391 & $-0.1 \%$ & 4.0456 & $0.0 \%$ & 3.4328 & $0.0 \%$ & 2.8968 & $0.0 \%$ & 2.4322 & $-0.3 \%$ \\
\hline Reference & 6.35 & & 5.51 & & 4.74 & & 4.04 & & 3.43 & & & & & \\
\hline
\end{tabular}


Table 3.3: Normalized SIF values for $t / W=0.1$

\begin{tabular}{|c|c|c|c|c|c|c|c|c|c|c|c|c|c|c|}
\hline & \multicolumn{2}{|c|}{$\mathrm{a} / \mathbf{W}=\mathbf{0 . 1}$} & \multicolumn{2}{|c|}{$\mathrm{a} / \mathrm{W}=0.2$} & \multicolumn{2}{|c|}{$\mathrm{a} / \mathrm{W}=\mathbf{0 . 3}$} & \multicolumn{2}{|c|}{$\mathrm{a} / \mathrm{W}=0.4$} & \multicolumn{2}{|c|}{$\mathrm{a} / \mathrm{W}=0.5$} & \multicolumn{2}{|c|}{$\mathrm{a} / \mathrm{W}=\mathbf{0 . 6}$} & \multicolumn{2}{|c|}{$\mathrm{a} / \mathrm{W}=\mathbf{0 . 7}$} \\
\hline & $K_{I I}$ & $K_{I I I}$ & $K_{I I}$ & $K_{I I I}$ & $K_{I I}$ & $K_{I I I}$ & $K_{I I}$ & $K_{I I I}$ & $K_{I I}$ & $K_{I I I}$ & $K_{I I}$ & $K_{I I I}$ & $K_{I I}$ & $K_{I I I}$ \\
\hline 0.0000 & 6.4867 & 0.0000 & 5.6219 & 0.0000 & 8260 & 0.0000 & 4.1150 & 0.0000 & 3.4882 & 0.0000 & 2.9408 & 0.0000 & 2.4669 & 0.0000 \\
\hline 0.0325 & 6.4857 & -0.0603 & 5.6212 & -0.0656 & 4.8255 & -0.0608 & 4.1146 & -0.0539 & 3.4879 & -0.0469 & 2.9407 & -0.0403 & 2.4667 & -0.0343 \\
\hline 0.0650 & 6.4846 & -0.1207 & 5.6203 & -0.1312 & 4.8249 & -0.1216 & & & 3.4875 & -0.0938 & 2.9404 & -0.0805 & 2.4665 & -0.0685 \\
\hline 0.0975 & 6.4814 & -0.1846 & 5.6180 & -0.2000 & 4.8232 & -0.1850 & 4.1129 & -0.1641 & 3.4866 & -0.1426 & 2.9397 & -0.1224 & 2.4661 & -0.1041 \\
\hline 0.1300 & 6.4781 & -0.2487 & 5.6155 & -0.2687 & 4.8213 & -0.2485 & 4.1115 & -0.2202 & 3.4856 & -0.1913 & 2.9390 & -0.1642 & 2.4655 & -0.1396 \\
\hline 0.1625 & 6.4724 & -0.3206 & 5.6114 & -0.3443 & 4.8185 & -0.3178 & 4.1095 & -0.2814 & 3.4842 & -0.2443 & 2.9379 & -0.2096 & 2.4648 & -0.1782 \\
\hline 0.1950 & 6.4663 & -0.3928 & 5.6071 & -0.4200 & 4.8153 & -0.3870 & 4.1072 & -0.3424 & 3.4825 & -0.2972 & 2.9367 & -0.2549 & 2.4639 & -0.2166 \\
\hline 0.2275 & 6.4575 & -0.4786 & 5.6013 & -0.5075 & 4.8114 & -0.4664 & 4.1045 & -0.4122 & 3.4806 & -0.3575 & 2.9354 & -0.3064 & 2.4631 & -0.2603 \\
\hline 0.2600 & 6.4479 & -0.5662 & 5.5947 & -0.5961 & 4.8068 & -0.5466 & 4.1013 & -0.4824 & 3.4783 & -0.4181 & 2.9337 & -0.3582 & 2.4619 & -0.3041 \\
\hline 0.2925 & 6.4354 & -0.6757 & 5.5878 & -0.7038 & 4.8028 & -0.6432 & 4.0989 & -0.5667 & 3.4770 & -0.4906 & 2.9331 & -0.4200 & 2.4616 & -0.3564 \\
\hline 0.3250 & 6.4212 & -0.7923 & 5.5794 & -0.8166 & 4.7976 & -0.7437 & 4.0956 & -0.6541 & 3.4749 & -0.5656 & 2.9318 & -0.4839 & 2.4609 & -0.4104 \\
\hline 0.3338 & 6.4174 & -0.8303 & 5.5782 & -0.8534 & 4.7975 & -0.7765 & 4.0960 & -0.6827 & 3.4755 & -0.5902 & 2.9325 & -0.5048 & 2.4616 & -0.4281 \\
\hline 0.3425 & 6.4121 & -0.8727 & 5.5753 & -0.8922 & 4.7957 & -0.8105 & 4.0949 & -0.7120 & 3.4748 & -0.6152 & 2.9321 & -0.5259 & 2.4614 & -0.4459 \\
\hline 0.3512 & 6.4087 & -0.9077 & 5.5745 & -0.9267 & 4.7959 & -0.8413 & 4.0956 & -0.7388 & 3.4757 & -0.6383 & 2.9330 & -0.5456 & 2.4623 & -0.4625 \\
\hline 0.3600 & 6.4029 & -0.9583 & 5.5715 & -0.9723 & 4.7942 & -0.8811 & 4.0946 & -0.7730 & 3.4752 & -0.6674 & 2.9328 & -0.5703 & 2.4623 & -0.4833 \\
\hline 0.3688 & 6.3999 & -0.9939 & 5.5715 & -1.0073 & 4.7953 & -0.9124 & 4.0961 & -0.8003 & 3.4768 & -0.6909 & 2.9343 & -0.5903 & 2.4637 & -0.5002 \\
\hline 0.3775 & 6.3935 & -1.0547 & 5.5686 & -1.0612 & 4.7939 & -0.9591 & 4.0955 & -0.8403 & 3.4766 & -0.7249 & 2.9344 & -0.6191 & 2.4640 & -0.5244 \\
\hline 0.3950 & 6.3849 & -1.1659 & 5.5678 & -1.1625 & 4.7958 & -1.0475 & 4.0985 & -0.9165 & 3.4800 & -0.7899 & 2.9378 & -0.6741 & 2.4671 & -0.5708 \\
\hline 0.4125 & 6.3786 & -1.2972 & 5.5710 & -1.2805 & 4.8019 & -1.1503 & 4.1054 & -1.0047 & 3.4868 & -0.8651 & 2.9442 & -0.7378 & 2.4729 & -0.6243 \\
\hline 0.4300 & 6.3791 & -1.4588 & 5.5830 & -1.4242 & 4.8166 & -1.2748 & 4.1201 & -1.1115 & 3.5005 & -0.9560 & 2.9565 & -0.8146 & 2.4838 & -0.6889 \\
\hline 0.4475 & 6.3981 & -1.6738 & 5.6161 & -1.6140 & 4.8509 & -1.4389 & 4.1522 & -1.2521 & 3.5293 & -1.0755 & 2.9819 & -0.9156 & 2.5058 & -0.7739 \\
\hline 0.4650 & 6.4856 & -2.0141 & 5.7188 & -1.9166 & 4.9482 & -1.7015 & 4.2396 & -1.4774 & 3.6060 & -1.2673 & 3.0481 & -1.0778 & 2.5624 & -0.9103 \\
\hline 0.4825 & 6.7726 & -2.4097 & 6.0191 & -2.2682 & 5.2231 & -2.0064 & 4.4821 & -1.7390 & 3.8161 & -1.4900 & 3.2281 & -1.2662 & 2.7153 & -1.0688 \\
\hline 0.5000 & 9.5642 & -7.8703 & 8.6743 & -7.1629 & 7.5800 & -6.2639 & 6.5286 & -5.3964 & 5.5717 & -4.6060 & 4.7213 & -3.9032 & 3.9765 & -3.2877 \\
\hline
\end{tabular}


Table 3.4: Normalized SIF values for $t / \mathrm{W}=0.2$

\begin{tabular}{|c|c|c|c|c|c|c|c|c|c|c|c|c|c|c|}
\hline \multirow{2}{*}{$z / t$} & \multicolumn{2}{|c|}{$\mathrm{a} / \mathrm{W}=\mathbf{0 . 1}$} & \multicolumn{2}{|c|}{$\mathrm{a} / \mathrm{W}=0.2$} & \multicolumn{2}{|c|}{$\mathrm{a} / \mathrm{W}=0.3$} & \multicolumn{2}{|c|}{$\mathrm{a} / \mathrm{W}=0.4$} & \multicolumn{2}{|c|}{$\mathrm{a} / \mathrm{W}=0.5$} & \multicolumn{2}{|c|}{$a / W=0.6$} & \multicolumn{2}{|c|}{$\mathrm{a} / \mathrm{W}=\mathbf{0 . 7}$} \\
\hline & \begin{tabular}{|l|}
$K_{I I}$ \\
\end{tabular} & $K_{\text {III }}$ & \begin{tabular}{l|l}
$K_{I I}$ \\
\end{tabular} & $K_{I I I}$ & \begin{tabular}{l|l}
$K_{I I}$ \\
\end{tabular} & $K_{I I I}$ & $\begin{array}{ll}K_{I I} \\
\end{array}$ & $K_{I I I}$ & $K_{I I}$ & $K_{I I I}$ & $K_{I I}$ & $K_{I I I}$ & $K_{I I}$ & $K_{I I I}$ \\
\hline 0.0000 & 6.4465 & 0000 & 5.6241 & 0.0000 & 4.8308 & 0.0000 & 4.1187 & 0.0000 & 3.4898 & 0.0000 & 2.9401 & 0.0000 & 2.4634 & 0.0000 \\
\hline 0.0295 & 466 & & & & & & & & & & & & & \\
\hline 0.0589 & 4467 & & & & & & & & & & & & & \\
\hline & 467 & & & & & & & & & & & & & \\
\hline 0.1179 & 4468 & -0.1428 & 6192 & & 4.8267 & & & & 3.4873 & -0.1643 & 2.9383 & -0.1436 & & 0.1236 \\
\hline 0.1473 & 4464 & & & & & & & & & & & & & \\
\hline 0.1768 & 6.4459 & -0.2282 & 5.6125 & & 4.8212 & -0.3098 & & 2853 & 3.4842 & -0.2539 & 2.9361 & -0.2216 & 0 & -0.1907 \\
\hline 0.2062 & 4440 & -0.2810 & 6073 & -0.3779 & 4.8171 & -0.3726 & & & 3.4820 & -0.3041 & 2.9347 & -0.2651 & & -0.2279 \\
\hline & & & & & & & & & & & & & & \\
\hline 0.2652 & 6.4372 & -0.4028 & 5.5946 & -0.5220 & 4.8076 & -0.5098 & & 4659 & 3.4772 & -0.4127 & 2.9315 & -0.3590 & 1584 & -0.3082 \\
\hline 0.2946 & 4322 & -0.4709 & 5869 & -0.6002 & 4.8019 & -0.5837 & 4.0972 & .5320 & 3.4745 & -0.4707 & .9298 & .4090 & & -0.3509 \\
\hline 0.3241 & 6.4220 & -0.5695 & & & & & & & & & & & & \\
\hline 0.3536 & 6.4106 & -0.6660 & 5.5656 & -0.8064 & 4.7879 & -0.7731 & 4.0884 & -0.6994 & 3.4692 & -0.6159 & 2.9271 & -0.5335 & .4568 & $\begin{array}{l}-0.4566 \\
\end{array}$ \\
\hline 0.3830 & 6.3927 & -0.8218 & & -0.9578 & 4.7853 & -0.9084 & & & $3.4 / 25$ & & 2.9312 & & & -0.5295 \\
\hline 0.4169 & 6.3728 & -1.0401 & 5.5512 & -1.1662 & 4.7889 & -1.0936 & & & 3.4819 & & 2.9414 & & & -0.6287 \\
\hline 0.4256 & 6.3598 & -1.1008 & 5.5449 & -1.2183 & 4.7862 & -1.1383 & 4.0965 & -1.0161 & 3.4823 & -0.8873 & 2.9424 & -0.7642 & 2.4729 & -0.6514 \\
\hline 0.4344 & 6.3544 & -1.1786 & 5.5477 & -1.2894 & 4.7923 & -1.2005 & 4.1037 & -1.0698 & 3.4896 & -0.9331 & 2.9494 & -0.8031 & 2.4794 & -0.6841 \\
\hline 0.4431 & 6.3490 & -1.2676 & 5.5523 & -1.3686 & 4.8004 & -1.2692 & 4.1130 & -1.1287 & 3.4989 & -0.9833 & 2.9582 & -0.8455 & 2.4874 & -0.7198 \\
\hline 0.4519 & 6.3455 & -1.3675 & 5.5612 & -1.4566 & 4.8132 & -1.3453 & 4.1266 & -1.1939 & 3.5121 & -1.0386 & 2.9704 & -0.8923 & 2.4984 & -0.7592 \\
\hline 0.4606 & 6.3461 & -1.4797 & 5.5772 & -1.5540 & 4.8334 & -1.4291 & 4.1471 & -1.2654 & 3.5315 & -1.0993 & 2.9881 & -0.9435 & 2.5141 & -0.8022 \\
\hline 0.4781 & & & & & & & & & & & & & & -0.8870 \\
\hline 0.4825 & 6.4851 & -2.1433 & 5.7747 & -2.1283 & 5.0320 & -1.9239 & 4.3313 & -1.6885 & 3.6963 & -1.4588 & 3.1324 & -1.2472 & 2.6389 & -1.0575 \\
\hline 0.4913 & 6.8052 & -2.5292 & 6.1304 & -2.4699 & 5.3655 & -2.2207 & 4.6295 & -1.9436 & 3.9570 & -1.6763 & 3.3573 & -1.4314 & 2.8308 & -1.2126 \\
\hline 0.5000 & 9.7396 & -8.0383 & 9.0291 & -7.4890 & 7.9819 & -6.6285 & 6.9240 & -5.7531 & 5.9386 & -4.9358 & 5.0510 & -4.1989 & 4.2666 & -3.5474 \\
\hline
\end{tabular}


Table 3.5: Normalized SIF values for $t / W=0.5$

\begin{tabular}{|c|c|c|c|c|c|c|c|c|c|c|c|c|c|c|}
\hline \multirow{2}{*}{$z / t$} & \multicolumn{2}{|c|}{$\mathrm{a} / \mathrm{W}=\mathbf{0 . 1}$} & \multicolumn{2}{|c|}{$\mathrm{a} / \mathbf{W}=0.2$} & \multicolumn{2}{|c|}{$\mathbf{a} / \mathbf{W}=\mathbf{0 . 3}$} & \multicolumn{2}{|c|}{$\mathrm{a} / \mathrm{W}=0.4$} & \multicolumn{2}{|c|}{$\mathrm{a} / \mathrm{W}=0.5$} & \multicolumn{2}{|c|}{$a / W=0.6$} & \multicolumn{2}{|c|}{$\mathrm{a} / \mathbf{W}=\mathbf{0 . 7}$} \\
\hline & $K_{I I}$ & $K_{I I I}$ & $K_{I I}$ & $K_{I I I}$ & $K_{I I}$ & $K_{I I I}$ & $K_{I I}$ & $K_{I I I}$ & $K_{I I}$ & $\overline{K_{I I I}}$ & $K_{I I}$ & $K_{I I I}$ & $K_{I I}$ & $K_{I I I}$ \\
\hline 0.0000 & 6.1036 & 0.0000 & 5.4811 & 0.0000 & 4.7776 & 0.0000 & 4.0927 & 0.0000 & 3.4716 & 0.0000 & 2.9224 & 0.0000 & 2.4422 & 0.0000 \\
\hline 0.0500 & 6.1118 & -0.0572 & 5.4848 & -0.0427 & 4.7787 & -0.0546 & 4.0931 & -0.0580 & 3.4717 & -0.0561 & 2.9226 & -0.0518 & 2.4424 & -0.0459 \\
\hline 0.1000 & 6.1368 & -0.1134 & 5.4958 & -0.0880 & 4.7817 & -0.1118 & 4.0939 & -0.1181 & 3.4720 & -0.1141 & 2.9229 & -0.1052 & 2.4429 & -0.0931 \\
\hline 0.1500 & 6.1792 & -0.1684 & 5.5132 & -0.1393 & 4.7863 & -0.1743 & 4.0952 & -0.1829 & 3.4724 & -0.1760 & 2.9233 & -0.1619 & 2.4438 & -0.1432 \\
\hline 0.2000 & 6.2398 & -0.2234 & 5.5359 & -0.2002 & 4.7919 & -0.2457 & 4.0963 & -0.2552 & 3.4726 & -0.2443 & 2.9237 & -0.2239 & 2.4449 & -0.1978 \\
\hline 0.2250 & 6.2793 & -0.2528 & 5.5489 & -0.2384 & 4.7945 & -0.2883 & 4.0965 & -0.2973 & 3.4724 & -0.2834 & 2.9238 & -0.2591 & 2.4457 & -0.2287 \\
\hline 0.2500 & 6.3189 & -0.2819 & 5.5619 & -0.2764 & 4.7973 & -0.3306 & 4.0967 & -0.3392 & 3.4723 & -0.3224 & 2.9238 & -0.2942 & 2.4464 & -0.2594 \\
\hline 0.2750 & 6.3671 & -0.3175 & 5.5751 & -0.3266 & 4.7991 & -0.3837 & 4.0962 & -0.3901 & 3.4716 & -0.3689 & 2.9238 & -0.3356 & 2.4473 & -0.2955 \\
\hline 0.3000 & 6.4154 & -0.3525 & 5.5884 & -0.3763 & 4.8010 & -0.4364 & 4.0957 & -0.4406 & 3.4709 & -0.4152 & 2.9237 & -0.3767 & 2.4482 & -0.3313 \\
\hline 0.3250 & 6.4698 & -0.4034 & 5.5996 & -0.4462 & 4.8011 & -0.5064 & 4.0941 & -0.5056 & 3.4699 & -0.4734 & 2.9237 & -0.4278 & 2.4497 & -0.3755 \\
\hline 0.3500 & 6.5247 & -0.4525 & 5.6110 & -0.5148 & 4.8011 & -0.5752 & 4.0923 & -0.5696 & 3.4686 & -0.5308 & 2.9236 & -0.4782 & 2.4510 & -0.4191 \\
\hline 0.3750 & 6.5798 & -0.5391 & 5.6174 & -0.6205 & 4.7987 & -0.6747 & 4.0902 & -0.6590 & 3.4683 & -0.6092 & 2.9252 & -0.5460 & 2.4545 & -0.4772 \\
\hline 0.4000 & 6.6357 & -0.6185 & 5.6230 & -0.7209 & 4.7948 & -0.7700 & 4.0865 & -0.7449 & 3.4664 & -0.6847 & 2.9253 & -0.6113 & 2.4567 & -0.5331 \\
\hline 0.4250 & 6.6801 & -0.7991 & 5.6227 & -0.9062 & 4.7942 & -0.9339 & 4.0909 & -0.8870 & 3.4747 & -0.8066 & 2.9362 & -0.7151 & 2.4696 & -0.6211 \\
\hline 0.4500 & 6.7220 & -0.9925 & 5.6221 & -1.1005 & 4.7946 & -1.1046 & 4.0967 & -1.0345 & 3.4846 & -0.9328 & 2.9488 & -0.8224 & 2.4840 & -0.7119 \\
\hline 0.4525 & 6.7227 & -1.0530 & 5.6233 & -1.1529 & 4.7992 & -1.1486 & 4.1033 & -1.0716 & 3.4920 & -0.9641 & 2.9562 & -0.8487 & 2.4912 & -0.7340 \\
\hline 0.4600 & 6.7312 & -1.1582 & 5.6255 & -1.2473 & 4.8057 & -1.2284 & 4.1130 & -1.1391 & 3.5032 & -1.0211 & 2.9679 & -0.8967 & 2.5028 & -0.7743 \\
\hline 0.4700 & 6.7426 & -1.3428 & 5.6353 & -1.4070 & 4.8242 & -1.3622 & 4.1365 & -1.2519 & 3.5284 & -1.1160 & 2.9929 & -0.9765 & 2.5266 & -0.8413 \\
\hline 0.4750 & 6.7504 & -1.4630 & 5.6465 & -1.5080 & 4.8413 & -1.4463 & 4.1563 & -1.3225 & 3.5486 & -1.1754 & 3.0123 & -1.0263 & 2.5447 & -0.8832 \\
\hline 0.4800 & 6.7631 & -1.6130 & 5.6667 & -1.6317 & 4.8686 & -1.5488 & 4.1862 & -1.4085 & 3.5782 & -1.2477 & 3.0401 & -1.0870 & 2.5701 & -0.9341 \\
\hline 0.4850 & 6.7874 & -1.8087 & 5.7041 & -1.7907 & 4.9144 & -1.6803 & 4.2339 & -1.5188 & 3.6241 & -1.3404 & 3.0825 & -1.1647 & 2.6081 & -0.9993 \\
\hline 0.4900 & 6.8574 & -2.1184 & 5.7937 & -2.0448 & 5.0119 & -1.8938 & 4.3295 & -1.6998 & 3.7129 & -1.4937 & 3.1625 & -1.2941 & 2.6787 & -1.1083 \\
\hline 0.4950 & 7.2166 & -2.6395 & 6.1703 & -2.4844 & 5.3774 & -2.2715 & 4.6666 & -2.0246 & 4.0143 & -1.7714 & 3.4270 & -1.5302 & 2.9074 & -1.3081 \\
\hline 0.5000 & 9.7033 & -7.0776 & 8.5478 & -6.3119 & 7.5683 & -5.6198 & 6.6284 & -4.9367 & 5.7359 & -4.2799 & 4.9175 & -3.6739 & 4.1836 & -3.1281 \\
\hline
\end{tabular}


Table 3.6: Normalized SIF values for $t / W=1.0$

\begin{tabular}{|c|c|c|c|c|c|c|c|c|c|c|c|c|c|c|}
\hline \multirow{2}{*}{$z / t$} & \multicolumn{2}{|c|}{$\mathrm{a} / \mathrm{W}=\mathbf{0 . 1}$} & \multicolumn{2}{|c|}{$\mathrm{a} / \mathrm{W}=0.2$} & \multicolumn{2}{|c|}{$\mathbf{a} / \mathbf{W}=\mathbf{0 . 3}$} & \multicolumn{2}{|c|}{$\mathrm{a} / \mathrm{W}=0.4$} & \multicolumn{2}{|c|}{$\mathrm{a} / \mathrm{W}=0.5$} & \multicolumn{2}{|c|}{$\mathrm{a} / \mathrm{W}=0.6$} & \multicolumn{2}{|c|}{$\mathrm{a} / \mathbf{W}=\mathbf{0 . 7}$} \\
\hline & $K_{I I}$ & $K_{I I I}$ & $K_{I I}$ & $K_{I I I}$ & $K_{I I}$ & $K_{I I I}$ & $K_{I I}$ & $K_{I I I}$ & $K_{I I}$ & $\overline{K_{I I I}}$ & $K_{I I}$ & $K_{I I I}$ & $K_{I I}$ & $K_{I I I}$ \\
\hline 0.0000 & 5.6646 & 0.0000 & 5.0403 & 0.0000 & 4.4534 & 0.0000 & 3.8827 & 0.0000 & 3.3369 & 0.0000 & 2.8351 & 0.0000 & 2.3885 & 0.0000 \\
\hline 0.0578 & 5.6857 & -0.3803 & 5.0609 & -0.1076 & 4.4691 & -0.0507 & 3.8929 & -0.0407 & 3.3434 & -0.0396 & 2.8391 & -0.0379 & 2.3909 & -0.0333 \\
\hline 0.0964 & 5.7202 & -0.6225 & 5.0942 & -0.1768 & 4.4942 & -0.0852 & 3.9091 & -0.0694 & 3.3535 & -0.0677 & 2.8455 & -0.0647 & 2.3946 & -0.0569 \\
\hline 0.1542 & 5.8044 & -0.9549 & 5.1748 & -0.2747 & 4.5534 & -0.1398 & 3.9469 & -0.1171 & 3.3773 & -0.1144 & 2.8605 & -0.1092 & 2.4035 & -0.0962 \\
\hline 0.1734 & 5.8466 & -1.0484 & 5.2140 & -0.3046 & 4.5810 & -0.1599 & 3.9643 & -0.1359 & 3.3882 & -0.1328 & 2.8674 & -0.1267 & 2.4076 & -0.1117 \\
\hline 0.2120 & 5.9437 & -1.2182 & 5.3023 & -0.3627 & 4.6417 & -0.2026 & 4.0022 & -0.1766 & 3.4119 & -0.1727 & 2.8824 & -0.1644 & 2.4169 & -0.1452 \\
\hline 0.2698 & 6.1399 & -1.4056 & 5.4694 & -0.4464 & 4.7507 & -0.2788 & 4.0692 & -0.2521 & 3.4536 & -0.2461 & 2.9092 & -0.2330 & 2.4338 & -0.2063 \\
\hline 0.3083 & 6.3220 & -1.4710 & 5.6072 & -0.5062 & 4.8346 & -0.3455 & 4.1194 & -0.3190 & 3.4846 & -0.3100 & 2.9294 & -0.2920 & 2.4473 & -0.2586 \\
\hline 0.3276 & 6.4401 & -1.4817 & 5.6849 & -0.5434 & 4.8792 & -0.3896 & 4.1454 & -0.3627 & 3.5006 & -0.3509 & 2.9400 & -0.3290 & 2.4549 & -0.2914 \\
\hline 0.3661 & 6.7108 & -1.4872 & 5.8470 & -0.6335 & 4.9690 & -0.4953 & 4.1969 & -0.4657 & 3.5323 & -0.4458 & 2.9616 & -0.4141 & 2.4712 & -0.3662 \\
\hline 0.4047 & 7.0573 & -1.4864 & 6.0184 & -0.7752 & 5.0572 & -0.6516 & 4.2459 & -0.6121 & 3.5630 & -0.5767 & 2.9843 & -0.5291 & 2.4907 & -0.4662 \\
\hline 0.4240 & 7.2510 & -1.4830 & 6.1048 & -0.8582 & 5.0993 & -0.7419 & 4.2683 & -0.6957 & 3.5768 & -0.6508 & 2.9948 & -0.5936 & 2.5004 & -0.5221 \\
\hline 0.4432 & 7.4839 & -1.5741 & 6.1894 & -1.0384 & 5.1392 & -0.9126 & 4.2928 & -0.8444 & 3.5966 & -0.7774 & 3.0140 & -0.7009 & 2.5202 & -0.6132 \\
\hline 0.4625 & 7.7169 & -1.6801 & 6.2736 & -1.2298 & 5.1795 & -1.0921 & 4.3183 & -0.9999 & 3.6178 & -0.9094 & 3.0347 & -0.8125 & 2.5417 & -0.7078 \\
\hline 0.4644 & 7.7482 & -1.7345 & 6.2827 & -1.2861 & 5.1853 & -1.1398 & 4.3243 & -1.0393 & 3.6246 & -0.9419 & 3.0420 & -0.8393 & 2.5491 & -0.7302 \\
\hline 0.4662 & 7.7737 & -1.7581 & 6.2920 & -1.3161 & 5.1906 & -1.1665 & 4.3286 & -1.0618 & 3.6287 & -0.9607 & 3.0461 & -0.8551 & 2.5533 & -0.7435 \\
\hline 0.4681 & 7.7991 & -1.7864 & 6.3006 & -1.3497 & 5.1956 & -1.1959 & 4.3329 & -1.0864 & 3.6330 & -0.9811 & 3.0506 & -0.8721 & 2.5577 & -0.7577 \\
\hline 0.4700 & 7.8245 & -1.8153 & 6.3095 & -1.3835 & 5.2007 & -1.2253 & 4.3372 & -1.1110 & 3.6374 & -1.0015 & 3.0551 & -0.8890 & 2.5623 & -0.7719 \\
\hline 0.4719 & 7.8516 & -1.8507 & 6.3197 & -1.4227 & 5.2073 & -1.2592 & 4.3431 & -1.1394 & 3.6432 & -1.0251 & 3.0610 & -0.9087 & 2.5681 & -0.7884 \\
\hline 0.4794 & 7.9661 & -2.0254 & 6.3657 & -1.6046 & 5.2399 & -1.4141 & 4.3735 & -1.2679 & 3.6738 & -1.1314 & 3.0914 & -0.9969 & 2.5976 & -0.8621 \\
\hline 0.4831 & 8.0324 & -2.1472 & 6.3971 & -1.7219 & 5.2650 & -1.5123 & 4.3977 & -1.3487 & 3.6979 & -1.1980 & 3.1149 & -1.0521 & 2.6198 & -0.9081 \\
\hline 0.4869 & 8.1125 & -2.3046 & 6.4412 & -1.8662 & 5.3028 & -1.6318 & 4.4344 & -1.4468 & 3.7337 & -1.2786 & 3.1491 & -1.1187 & 2.6515 & -0.9636 \\
\hline 0.4963 & 8.7498 & -3.3798 & 6.9262 & -2.7578 & 5.7327 & -2.3638 & 4.8271 & -2.0525 & 4.0915 & -1.7832 & 3.4706 & -1.5408 & 2.9347 & -1.3177 \\
\hline 0.5000 & 11.5054 & -7.3678 & 9.1860 & -5.9259 & 7.6821 & -4.9860 & 6.5300 & -4.2595 & 5.5787 & -3.6532 & 4.7611 & -3.1267 & 4.0420 & -2.6590 \\
\hline
\end{tabular}


Table 3.7: Normalized SIF values for $t / W=2.0$

\begin{tabular}{|c|c|c|c|c|c|c|c|c|c|c|c|c|c|c|}
\hline \multirow{2}{*}{$z / t$} & \multicolumn{2}{|c|}{$\mathrm{a} / \mathrm{W}=0.1$} & \multicolumn{2}{|c|}{$\mathrm{a} / \mathrm{W}=0.2$} & \multicolumn{2}{|c|}{$\mathrm{a} / \mathrm{W}=\mathbf{0 . 3}$} & \multicolumn{2}{|c|}{$\mathrm{a} / \mathrm{W}=0.4$} & \multicolumn{2}{|c|}{$\mathrm{a} / \mathrm{W}=0.5$} & \multicolumn{2}{|c|}{$a / W=0.6$} & \multicolumn{2}{|c|}{$\mathrm{a} / \mathrm{W}=\mathbf{0 . 7}$} \\
\hline & \begin{tabular}{|l|}
$K_{I I}$ \\
\end{tabular} & $K_{I I I}$ & \begin{tabular}{l|l}
$K_{I I}$ \\
\end{tabular} & $K_{I I I}$ & \begin{tabular}{l|l}
$K_{I I}$ \\
\end{tabular} & $K_{I I I}$ & $\begin{array}{ll}K_{I I} \\
\end{array}$ & $K_{I I I}$ & $K_{I I}$ & $K_{I I I}$ & $K_{I I}$ & $K_{I I I}$ & $K_{I I}$ & $K_{I I I}$ \\
\hline 0.0000 & 5.062 & & & & 3.8784 & & 3.3684 & & 2.9196 & 00000 & 2.5224 & 0.0000 & 2.1706 & 0.0000 \\
\hline & & & & & & & & & & & & & & \\
\hline & & & & & & & & & & & & & & \\
\hline & 5 & & & & 65 & & & & & & & & & \\
\hline 0.2026 & 4288 & & 7865 & & 4.1958 & & 3.6546 & & & & & & 2962 & 0.0268 \\
\hline & 4863 & & & & & & & & & & & & & \\
\hline 0.2280 & 5.5438 & -5.5738 & 8916 & -1.9869 & 4.2947 & & 3.7393 & & 210 & -0.216 & 7492 & & מ & .0384 \\
\hline 0.2406 & 5.6129 & -5.7678 & 4.9554 & & 4.3537 & 310 & 3.7886 & & 2591 & & & & & \\
\hline & 5. & & & & & & & & & & & & & \\
\hline 0.2913 & 5.9471 & -6.3670 & 2668 & -2.2596 & 4.6344 & -1.0381 & 4.0161 & 326 & & -0.2923 & .9021 & 0.1646 & 347 & -0.0872 \\
\hline 0.3039 & 6.0464 & -6.4689 & 3603 & -2.2952 & 4.7164 & -1.0602 & 4.0808 & .5518 & 4802 & -0.3 & 9367 & & & \\
\hline 0.3419 & 6.4330 & & & & & & & & & & & & & \\
\hline 0.3799 & 6.9468 & -6.3175 & 6.1979 & -2.3084 & 5.3764 & -1.1699 & 4.5671 & -0.7024 & 3.8317 & -0.4695 & 3.1859 & \begin{tabular}{|c|}
-0.3329 \\
\end{tabular} & & -0.2359 \\
\hline 0.3926 & 7.1876 & -6.1374 & 6.3993 & & 5.5162 & & 4.6637 & & 3.8994 & & & & & -0.2739 \\
\hline 0.4053 & 7.4269 & -5.9556 & 6.6007 & -2.2674 & 5.6561 & -1.2241 & 4.7603 & & & -0.5575 & & & & \\
\hline 0.4306 & 8.0891 & -5.4288 & 7.0784 & -2.2406 & 5.9643 & -1.3158 & 4.9662 & -0.9062 & 4.1093 & -0.6804 & 3.3816 & -0.5317 & .7647 & -0.4143 \\
\hline 0.4559 & 9.0401 & -4.8000 & 7.6333 & -2.2855 & 6.2982 & -1.4865 & 5.1813 & -1.0984 & 4.2555 & -0.8640 & & & & -0.5391 \\
\hline 0.4686 & 9.7438 & -4.5997 & 7.9760 & -2.4492 & 6.4958 & -1.6932 & 5.3077 & -1.2950 & 4.3432 & -1.0378 & 3.5511 & -0.8467 & 8934 & -0.6868 \\
\hline 0.4841 & 10.7277 & -4.5014 & 8.4489 & -2.7696 & 6.7716 & -2.0441 & 5.4883 & -1.6147 & 4.4725 & -1.3142 & 3.6506 & -1.0809 & .9743 & -0.8839 \\
\hline 0.4878 & 11.0504 & -4.5789 & 8.6074 & -2.9297 & 6.8688 & -2.1958 & 5.5560 & -1.7458 & 4.5240 & -1.4243 & 3.6920 & -1.1724 & .0088 & -0.9598 \\
\hline 0.4916 & & & & & & & & & & & & & & \\
\hline 0.4953 & 12.0661 & -5.0778 & & -3.5238 & 7.2428 & -2.7177 & 5.8366 & & 4.7479 & -1.7857 & 3.8765 & -1.4695 & 3.1624 & -1.2040 \\
\hline 0.4991 & 13.4638 & -4.9740 & 10.0465 & -3.5977 & 7.8973 & -2.8091 & 6.3557 & -2.2633 & 5.1732 & -1.8498 & 4.2295 & -1.5193 & 3.4545 & -1.2440 \\
\hline 0.5000 & 18.5921 & -12.2656 & 13.7543 & -9.0274 & 10.7908 & -7.0753 & 8.6872 & -5.6980 & 7.0794 & -4.6474 & 5.7959 & -3.8083 & 4.7379 & -3.1145 \\
\hline
\end{tabular}


Table 3.8: Normalized SIF values for $t / W=4.0$

\begin{tabular}{|c|c|c|c|c|c|c|c|c|c|c|c|c|c|c|}
\hline \multirow{2}{*}{$t$} & \multicolumn{2}{|c|}{$\mathrm{a} / \mathbf{W}=\mathbf{0 . 1}$} & \multicolumn{2}{|c|}{$\mathrm{a} / \mathbf{W}=\mathbf{0 . 2}$} & \multicolumn{2}{|c|}{$\mathrm{a} / \mathbf{W}=\mathbf{0 . 3}$} & \multicolumn{2}{|c|}{$\mathrm{a} / \mathbf{W}=0.4$} & \multicolumn{2}{|c|}{$\mathrm{a} / \mathbf{W}=\mathbf{0 . 5}$} & \multicolumn{2}{|c|}{$\mathrm{a} / \mathbf{W}=\mathbf{0 . 6}$} & \multicolumn{2}{|c|}{$\mathrm{a} / \mathbf{W}=\mathbf{0 . 7}$} \\
\hline & $K_{I I}$ & $K_{I I I}$ & $K_{I I}$ & $K_{I I I}$ & $K_{I I}$ & $K_{I I I}$ & $K_{I I}$ & $K_{\text {III }}$ & $K_{I I}$ & $K_{I I I}$ & $K_{I I}$ & $K_{I I I}$ & $K_{I I}$ & $K_{\text {III }}$ \\
\hline 0.0000 & 5.4983 & 0.0000 & 4.7524 & 0.0000 & 4.0643 & 0.0000 & 3.4496 & 0.0000 & 2.9130 & 0.0000 & 2.4518 & 0.0000 & 2.0612 & 0.0000 \\
\hline 0.1188 & 5.4575 & -4.1466 & 4.7269 & -1.7136 & 4.0507 & -0.9095 & 3.4463 & -0.5148 & 2.9198 & -0.2858 & 2.4676 & -0.1423 & 2.0836 & -0.0509 \\
\hline 0.2078 & 5.4263 & -7.5690 & 4.7218 & -3.0787 & 4.0655 & -1.6107 & 3.4803 & -0.8974 & 2.9718 & -0.4885 & 2.5324 & -0.2371 & 2.1539 & -0.0802 \\
\hline 0.2375 & 5.4425 & -8.7497 & 4.7463 & -3.5340 & 4.0966 & -1.8375 & 3.5190 & -1.0169 & 3.0160 & -0.5498 & 2.5781 & -0.2650 & 2.1975 & -0.0885 \\
\hline 0.2923 & 5.5638 & -10.8328 & 4.8742 & -4.3116 & 4.2343 & -2.2139 & 3.6679 & -1.2106 & 3.1659 & -0.6496 & 2.7176 & -0.3134 & 2.3193 & -0.1068 \\
\hline 0.3106 & 5.6469 & -11.4458 & 4.9548 & -4.5323 & 4.3172 & -2.3174 & 3.7515 & -1.2637 & 3.2438 & -0.6789 & 2.7852 & -0.3302 & 2.3749 & -0.1158 \\
\hline 0.3197 & 5.7045 & -11.7140 & 5.0093 & -4.6265 & 4.3725 & -2.3609 & 3.8054 & -1.2866 & 3.2922 & -0.6928 & 2.8258 & -0.3395 & 2.4072 & -0.1218 \\
\hline 0.3380 & 5.8401 & -12.1974 & 5.1368 & -4.7937 & 4.5009 & -2.4375 & 3.9280 & -1.3285 & 3.3999 & -0.7203 & 2.9146 & -0.3595 & 2.4771 & -0.1359 \\
\hline 0.3471 & 5.9181 & -12.4126 & 5.2097 & -4.8667 & 4.5739 & -2.4707 & 3.9966 & -1.3475 & 3.4592 & -0.7340 & 2.9629 & -0.3702 & 2.5145 & -0.1441 \\
\hline 0.3563 & 6.0223 & -12.5555 & 5.3070 & -4.9123 & 4.6696 & -2.4919 & 4.0830 & -1.3627 & 3.5313 & -0.7482 & 3.0199 & -0.3836 & 2.5577 & -0.1555 \\
\hline 0.3654 & 6.1262 & -12.6988 & 5.4040 & -4.9580 & 4.7652 & -2.5130 & 4.1694 & -1.3779 & 3.6034 & -0.7624 & 3.0770 & -0.3969 & 2.6009 & -0.1668 \\
\hline 0.3745 & 6.2639 & -12.7471 & 5.5335 & -4.9695 & 4.8894 & -2.5209 & 4.2768 & -1.3903 & 3.6900 & -0.7785 & 3.1438 & -0.4143 & 2.6503 & -0.1828 \\
\hline 0.3837 & 6.4012 & -12.7957 & 5.6627 & -4.9809 & 5.0135 & -2.5286 & 4.3842 & -1.4026 & 3.7767 & -0.7947 & 3.2106 & -0.4316 & 2.6998 & -0.1987 \\
\hline 0.3928 & 6.5826 & -12.7219 & 5.8361 & -4.9513 & 5.1730 & -2.5233 & 4.5158 & -1.4140 & 3.8794 & -0.8150 & 3.2883 & -0.4551 & 2.7563 & -0.2212 \\
\hline 0.4019 & 6.7632 & -12.6484 & 6.0091 & -4.9214 & 5.3325 & -2.5178 & 4.6474 & -1.4253 & 3.9823 & -0.8352 & 3.3660 & -0.4785 & 2.8128 & -0.2436 \\
\hline 0.4385 & 7.9024 & -11.3535 & 7.1112 & -4.5268 & 6.2406 & -2.4498 & 5.3409 & -1.5021 & 4.5016 & -0.9735 & 3.7497 & -0.6368 & 3.0882 & -0.3978 \\
\hline 0.4476 & 8.3965 & -10.7102 & 7.5392 & -4.3815 & 6.5482 & -2.4485 & 5.5603 & -1.5510 & 4.6606 & -1.0399 & 3.8658 & -0.7070 & 3.1721 & -0.4648 \\
\hline 0.4567 & 8.8793 & -10.0550 & 7.9662 & -4.2303 & 6.8546 & -2.4432 & 5.7788 & -1.5968 & 4.8188 & -1.1036 & 3.9814 & -0.7751 & 3.2554 & -0.5303 \\
\hline 0.4659 & 9.7071 & -9.2058 & 8.5347 & -4.1206 & 7.2268 & -2.5010 & 6.0342 & -1.7028 & 5.0007 & -1.2210 & 4.1139 & -0.8898 & 3.3529 & -0.6363 \\
\hline 0.4750 & 10.5655 & -8.3638 & 9.1099 & -4.0215 & 7.6023 & -2.5669 & 6.2917 & -1.8155 & 5.1840 & -1.3438 & 4.2476 & -1.0089 & 3.4514 & -0.7461 \\
\hline 0.4850 & 12.0852 & -7.4741 & 9.9385 & -4.0913 & 8.1173 & -2.8029 & 6.6383 & -2.0797 & 5.4290 & -1.5968 & 4.4273 & -1.2384 & 3.5870 & -0.9495 \\
\hline 0.4938 & 14.0149 & -7.1374 & 10.9720 & -4.5018 & 8.7740 & -3.2997 & 7.0919 & -2.5504 & 5.7587 & -2.0146 & 4.6748 & -1.5999 & 3.7766 & -1.2591 \\
\hline 0.4988 & 16.9274 & -7.5542 & 12.7243 & -5.2815 & 10.0012 & -4.0414 & 8.0109 & -3.1964 & 6.4701 & -2.5623 & 5.2329 & -2.0594 & 4.2135 & -1.6437 \\
\hline 0.5000 & 21.4002 & -11.2669 & 15.8079 & -8.1837 & 12.3401 & -6.3514 & 9.8515 & -5.0577 & 7.9411 & -4.0711 & 6.4130 & -3.2834 & 5.1540 & -2.6329 \\
\hline
\end{tabular}



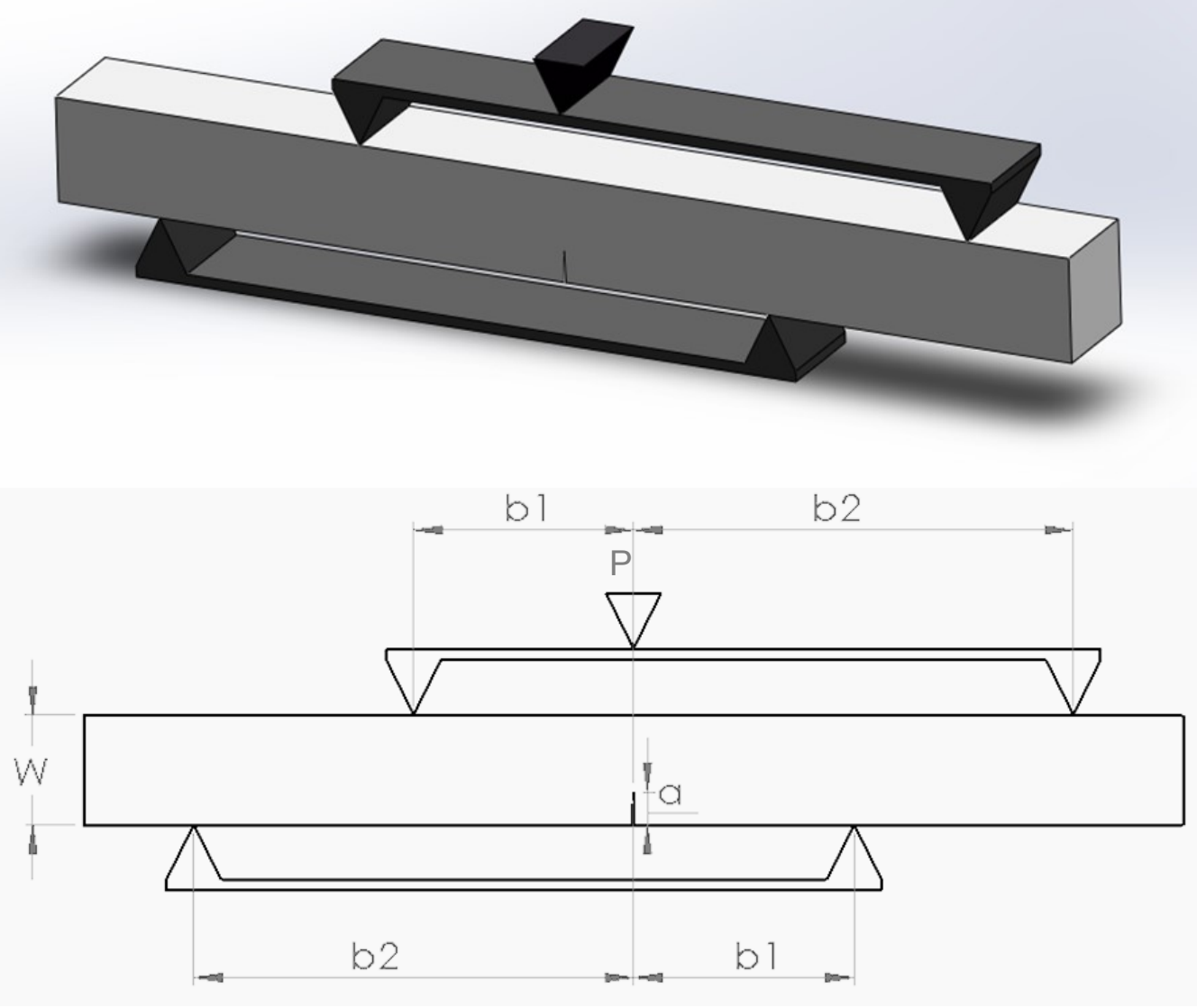

Figure 3.1: The four-point shear specimen 


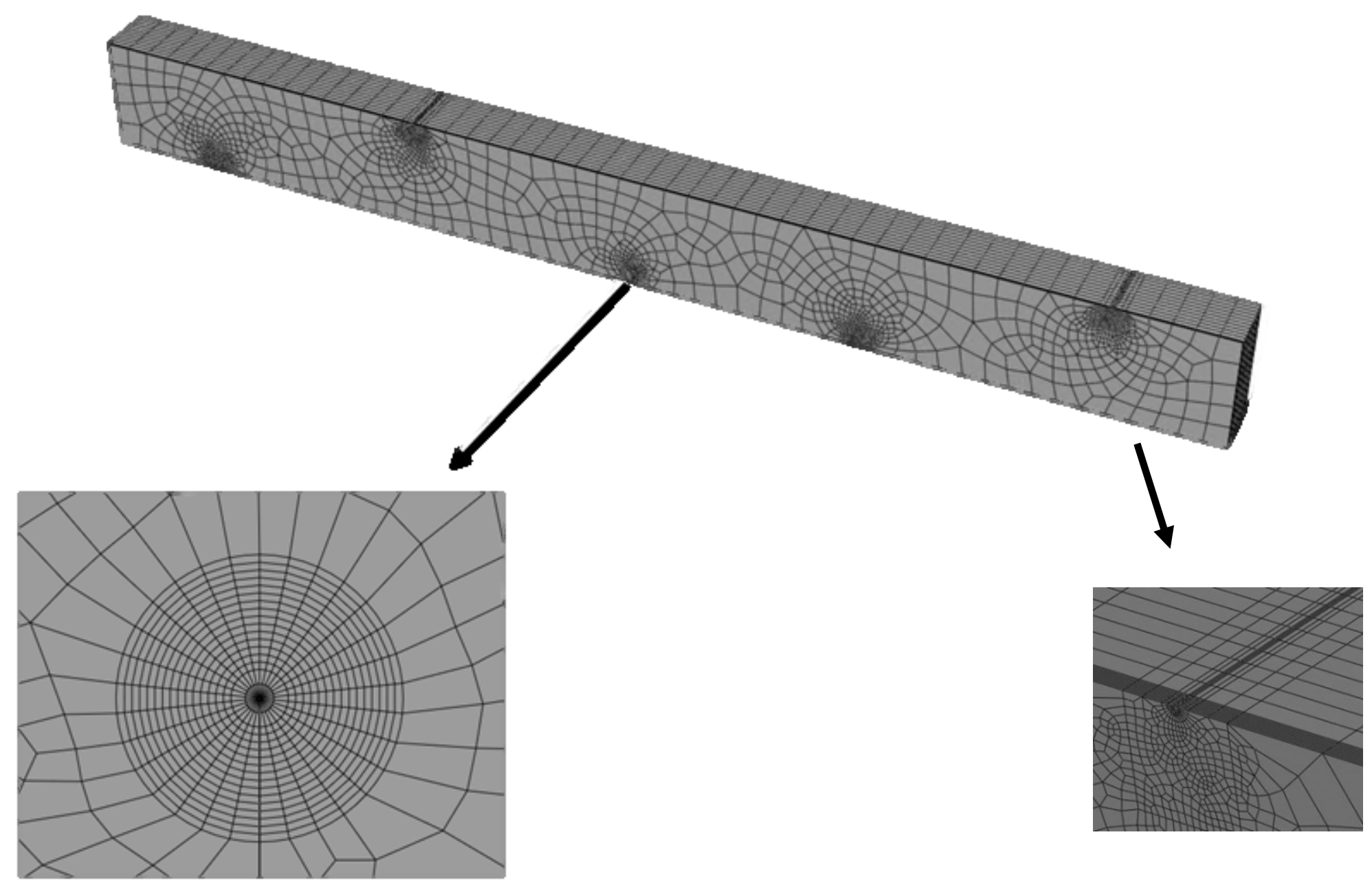

Figure 3.2: Typical finite element mesh for four-point shear specimen $(t / W=1.0, a / W=0.1)$ 


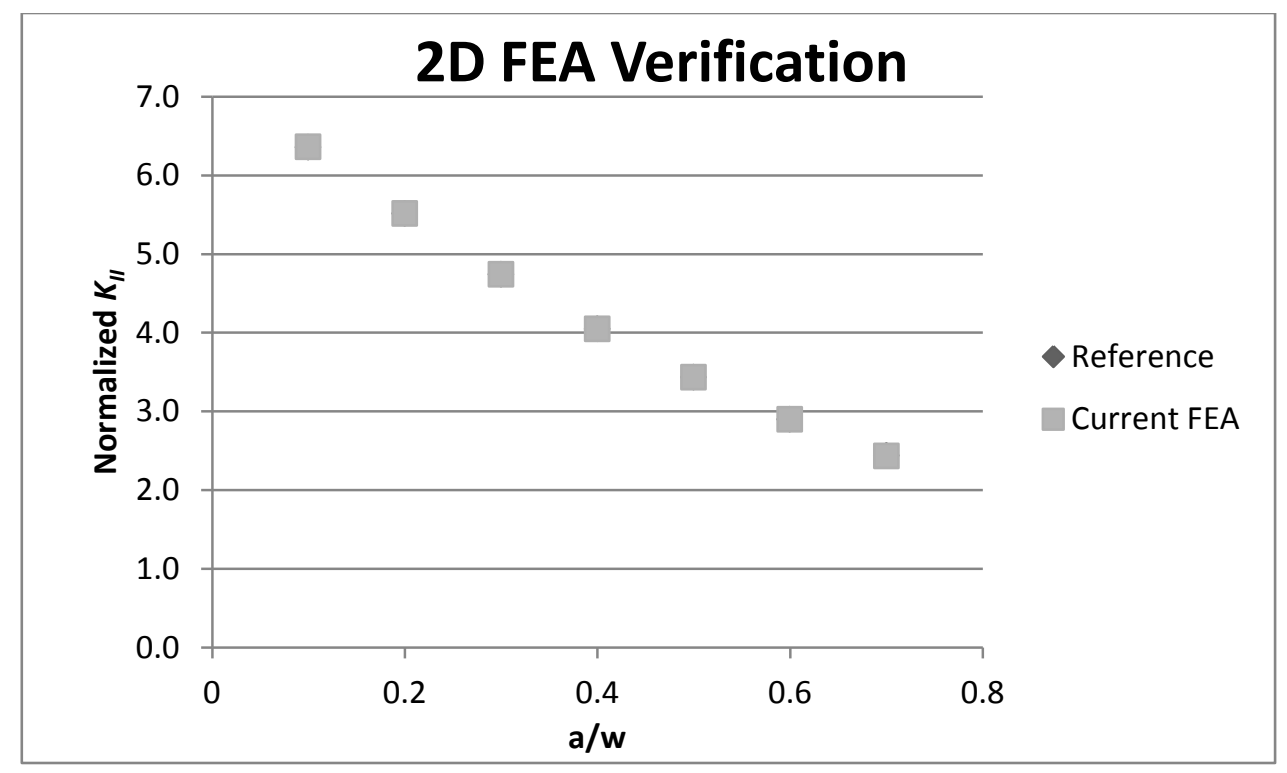

Figure 3.3: FEA verification for 2D results

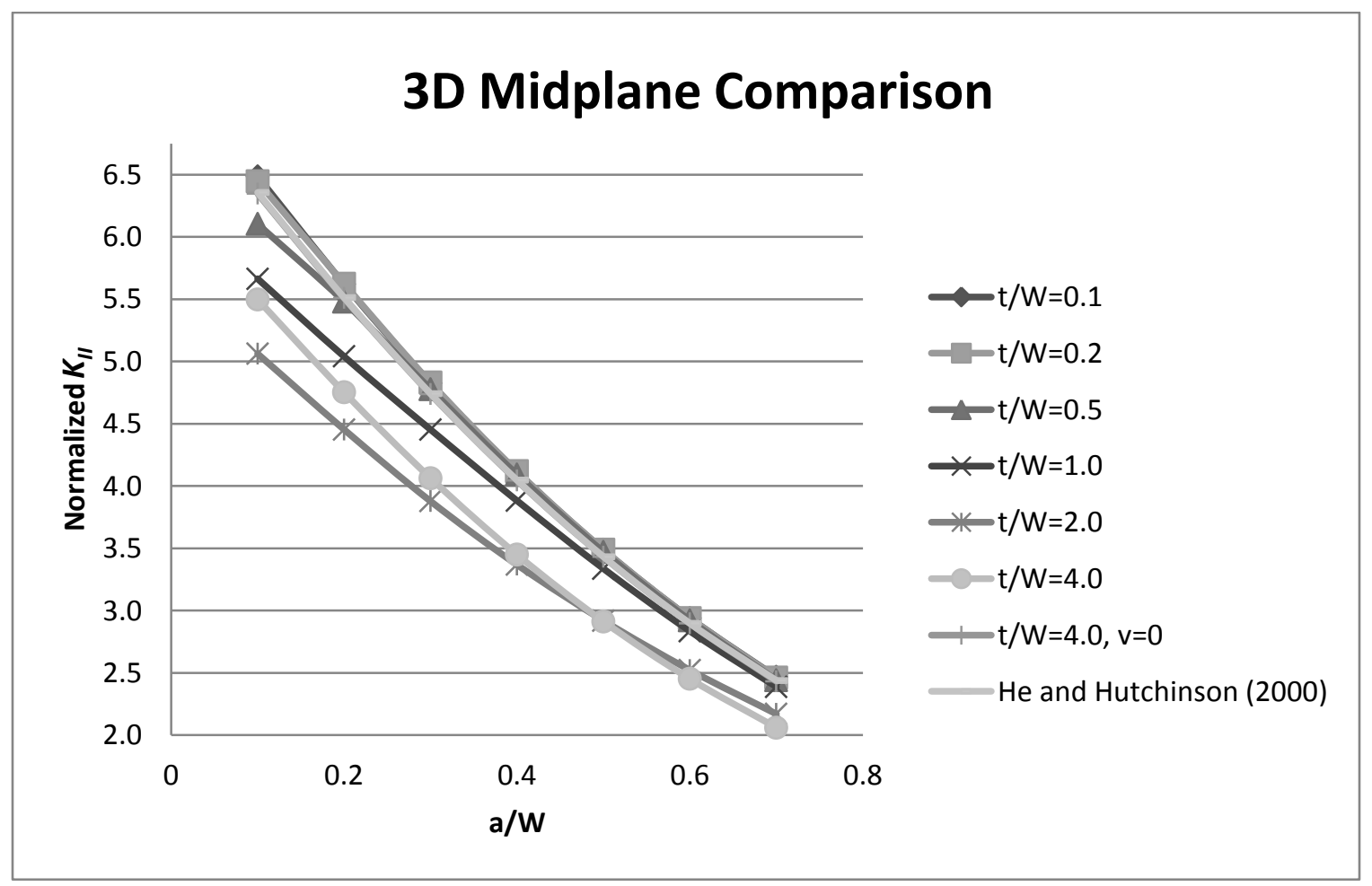

Figure 3.4: Comparison of normalized $K_{I I}$ values at the mid plane for plates of different thickness 


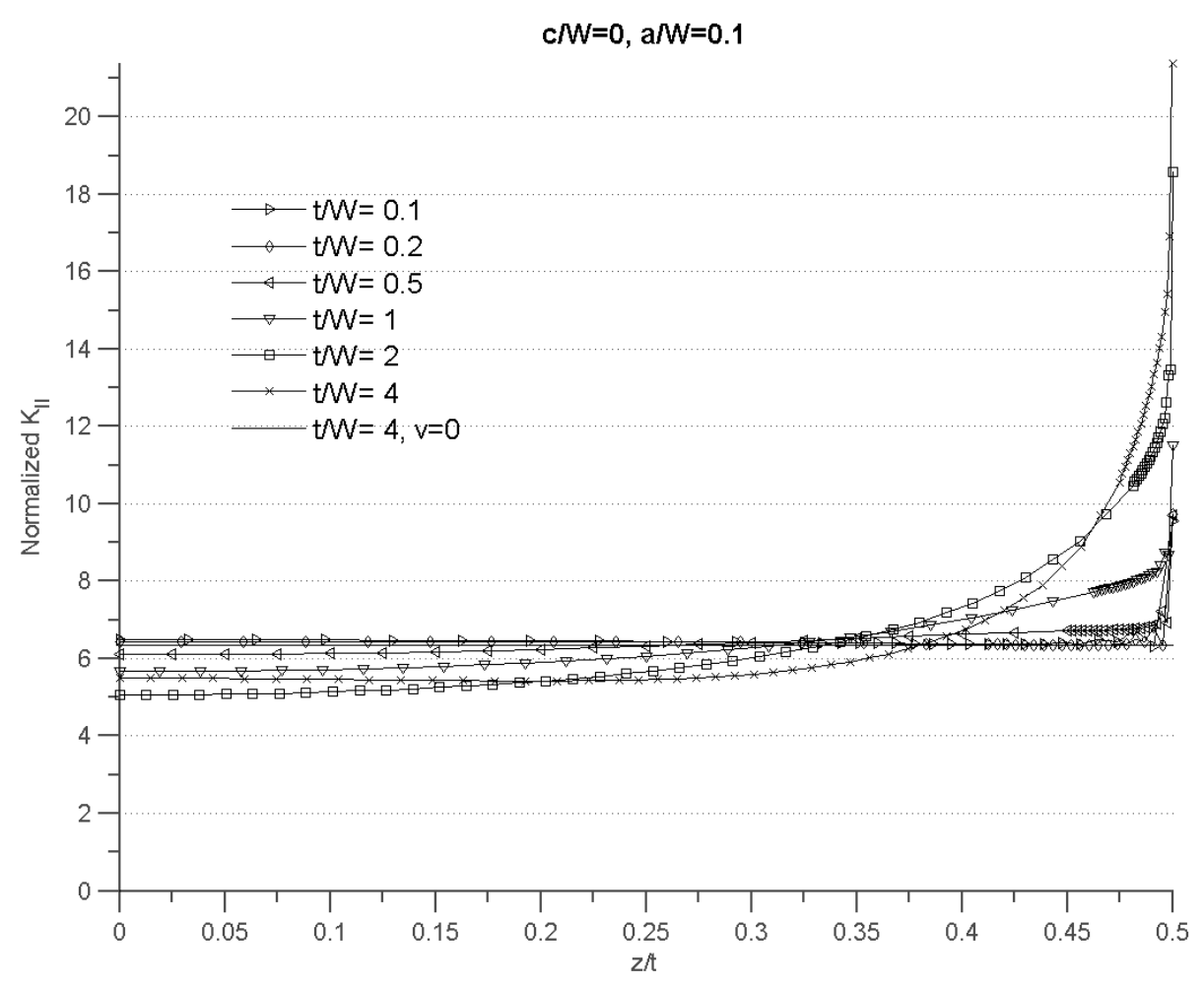

Figure 3.5: Normalized $K_{I I}$ for $a / W=0.1$

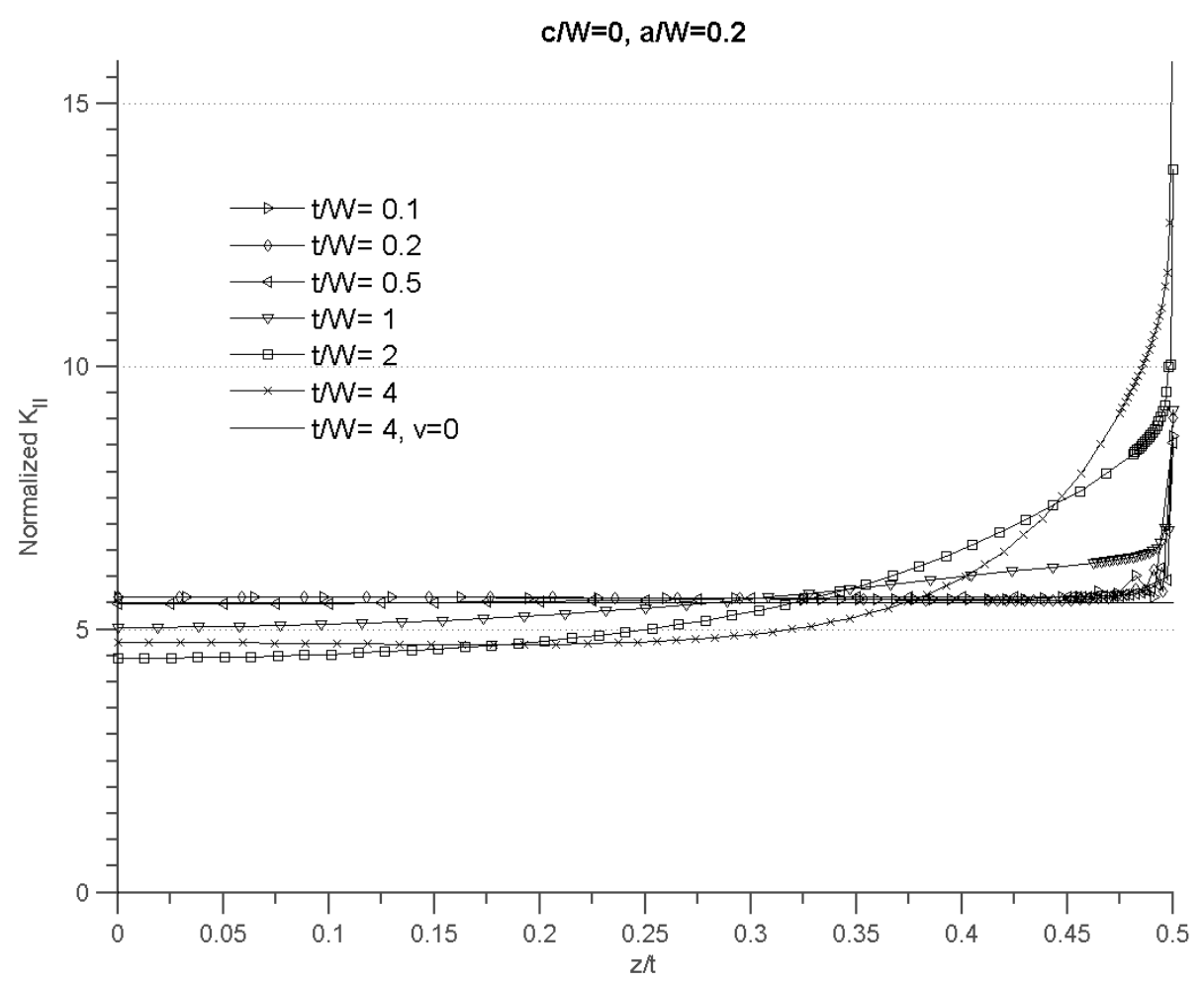

Figure 3.6: Normalized $K_{I I}$ for $a / W=0.2$ 


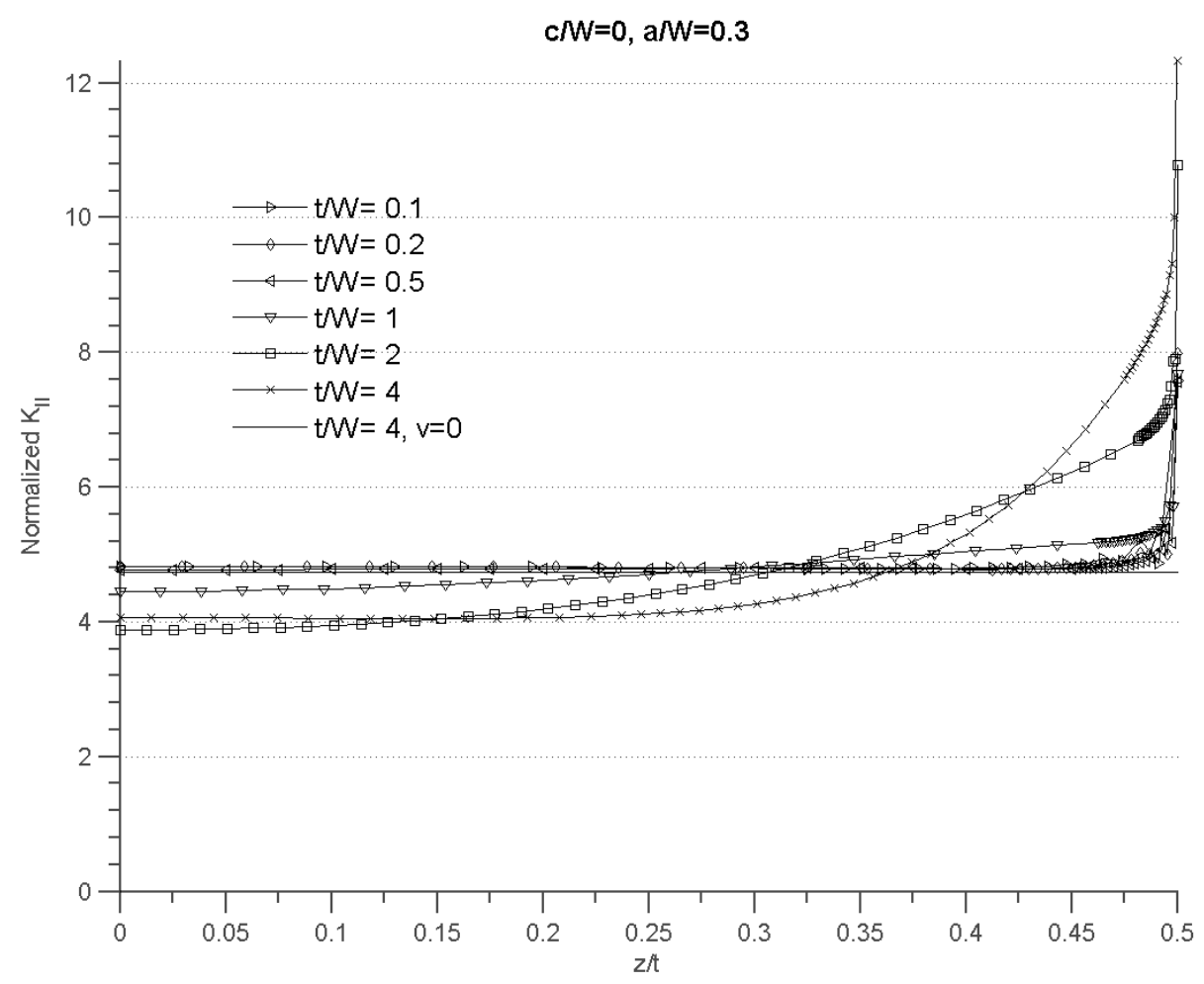

Figure 3.7: Normalized $K_{I I}$ for $\mathrm{a} / \mathrm{W}=\mathbf{0 . 3}$

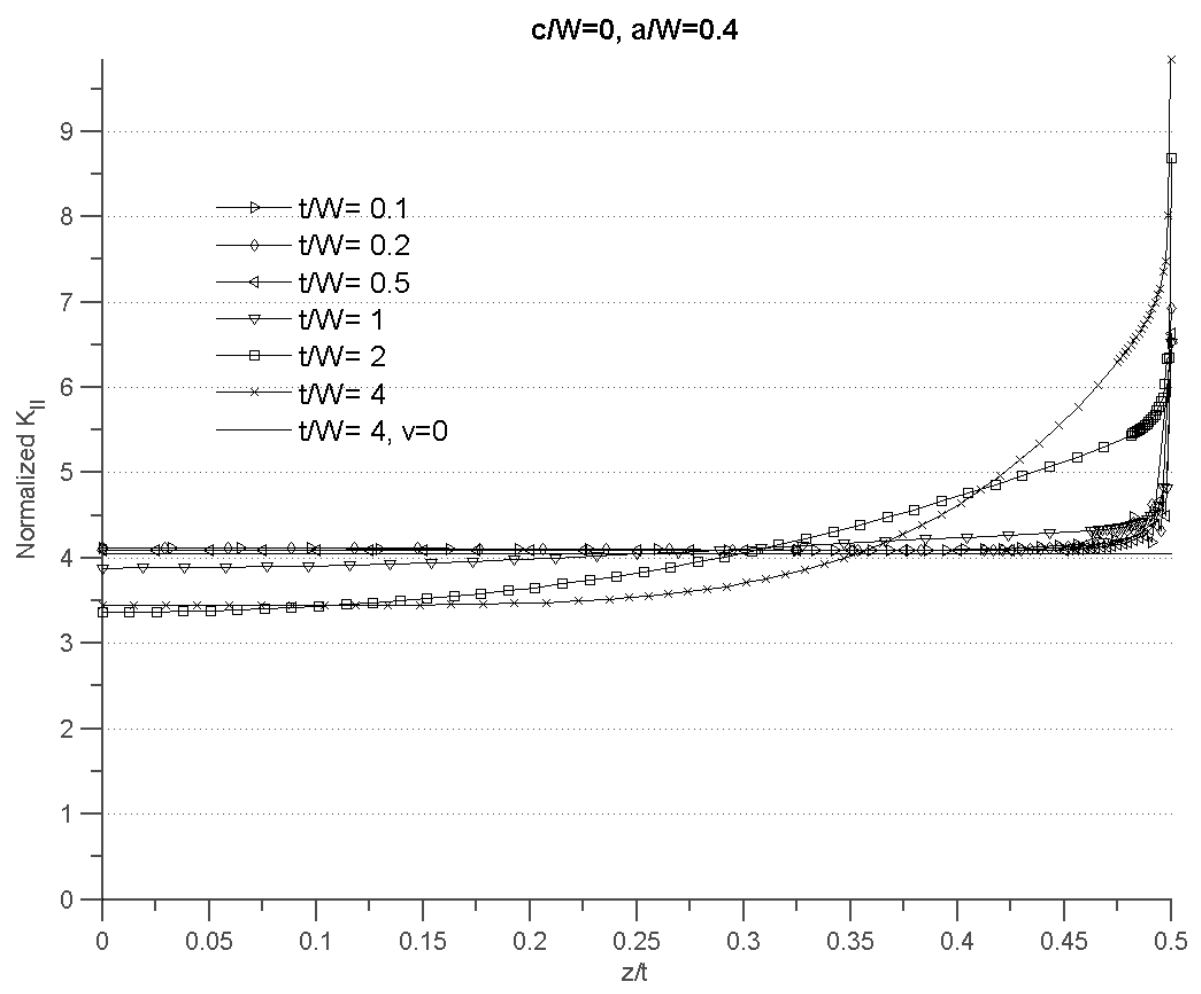

Figure 3.8: Normalized $K_{I I}$ for $\mathrm{a} / \mathrm{W}=0.4$ 


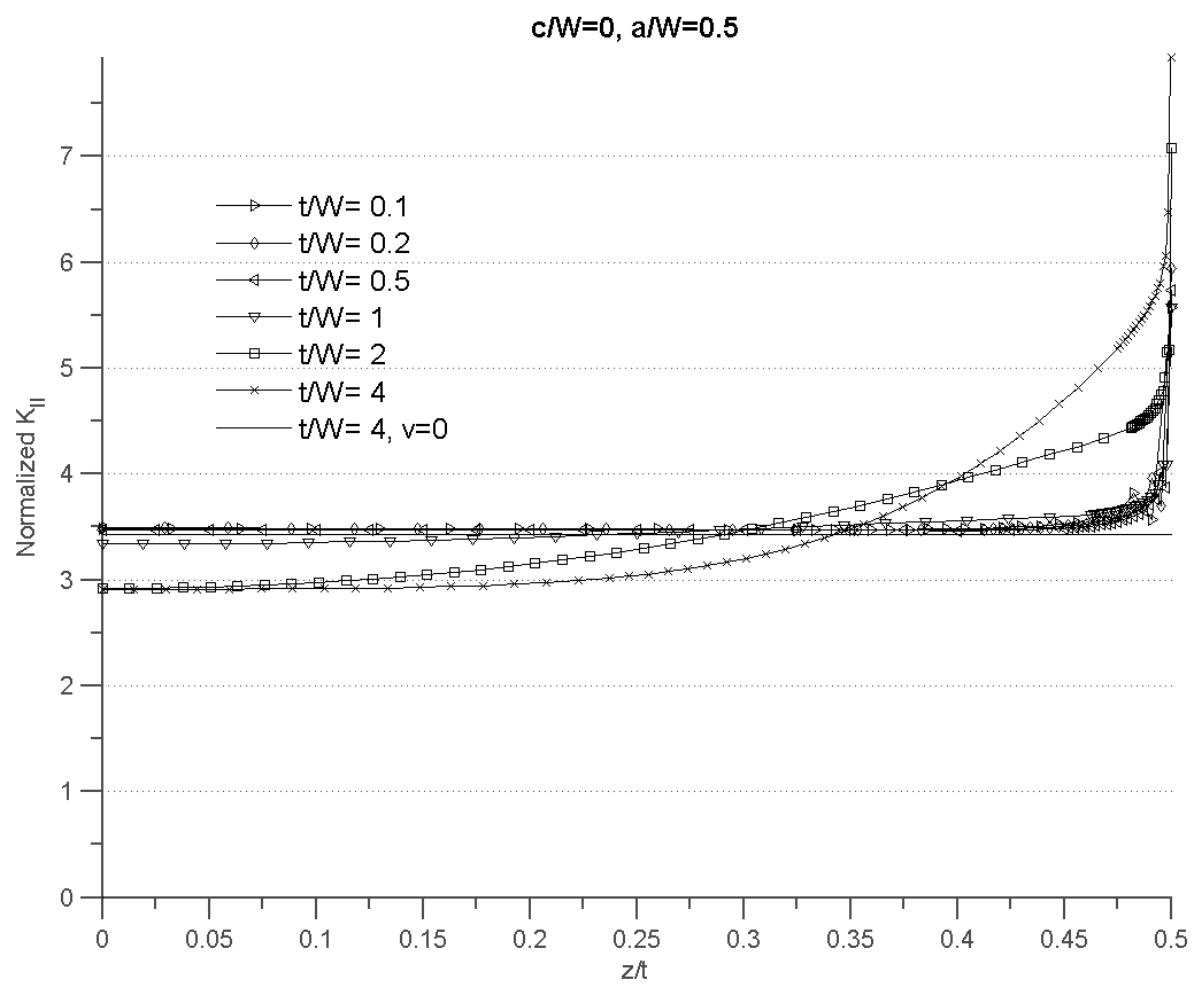

Figure 3.9: Normalized $K_{I I}$ for $\mathrm{a} / \mathrm{W}=0.5$

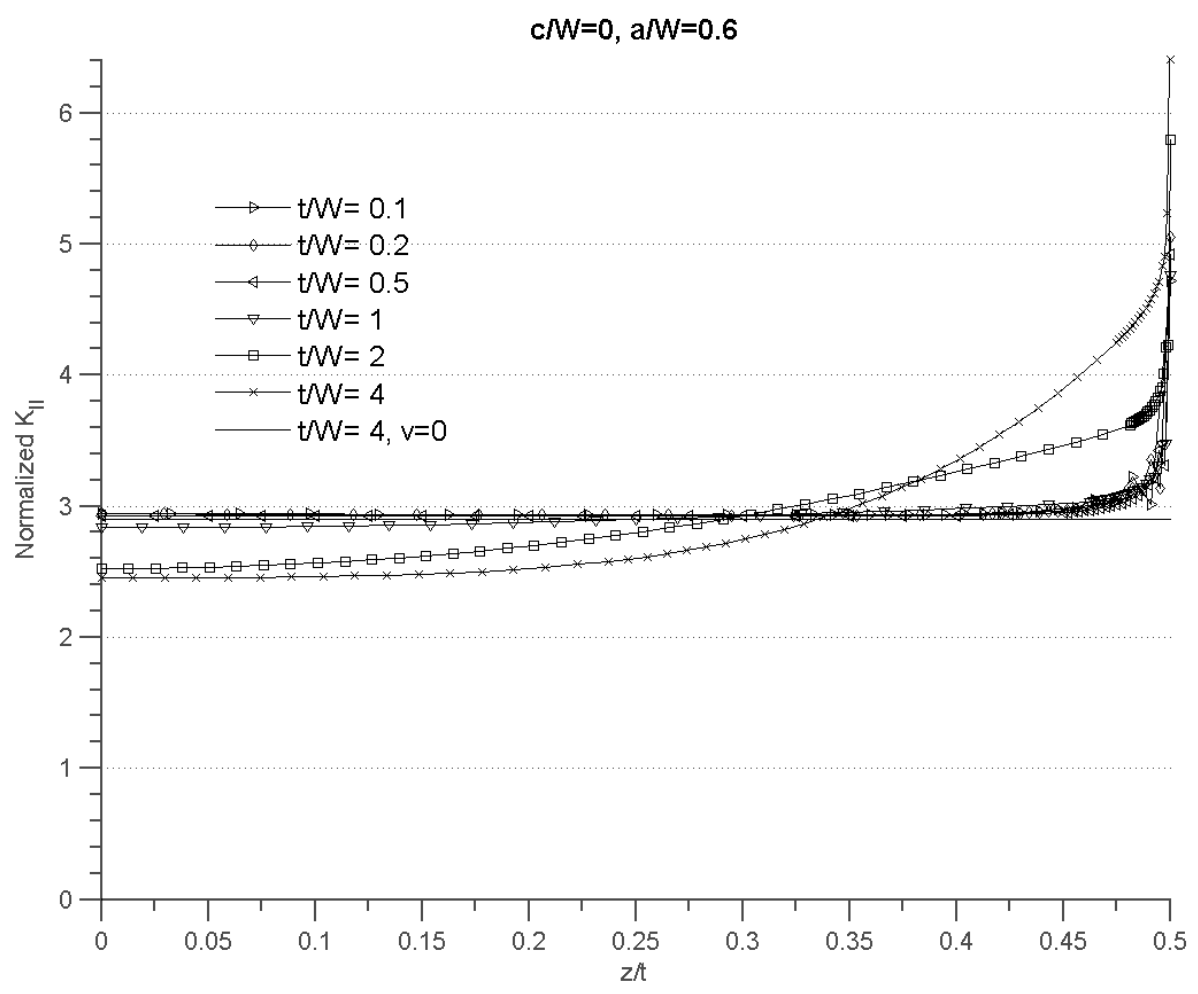

Figure 3.10: Normalized $K_{I I}$ for $\mathrm{a} / \mathrm{W}=\mathbf{0 . 6}$ 


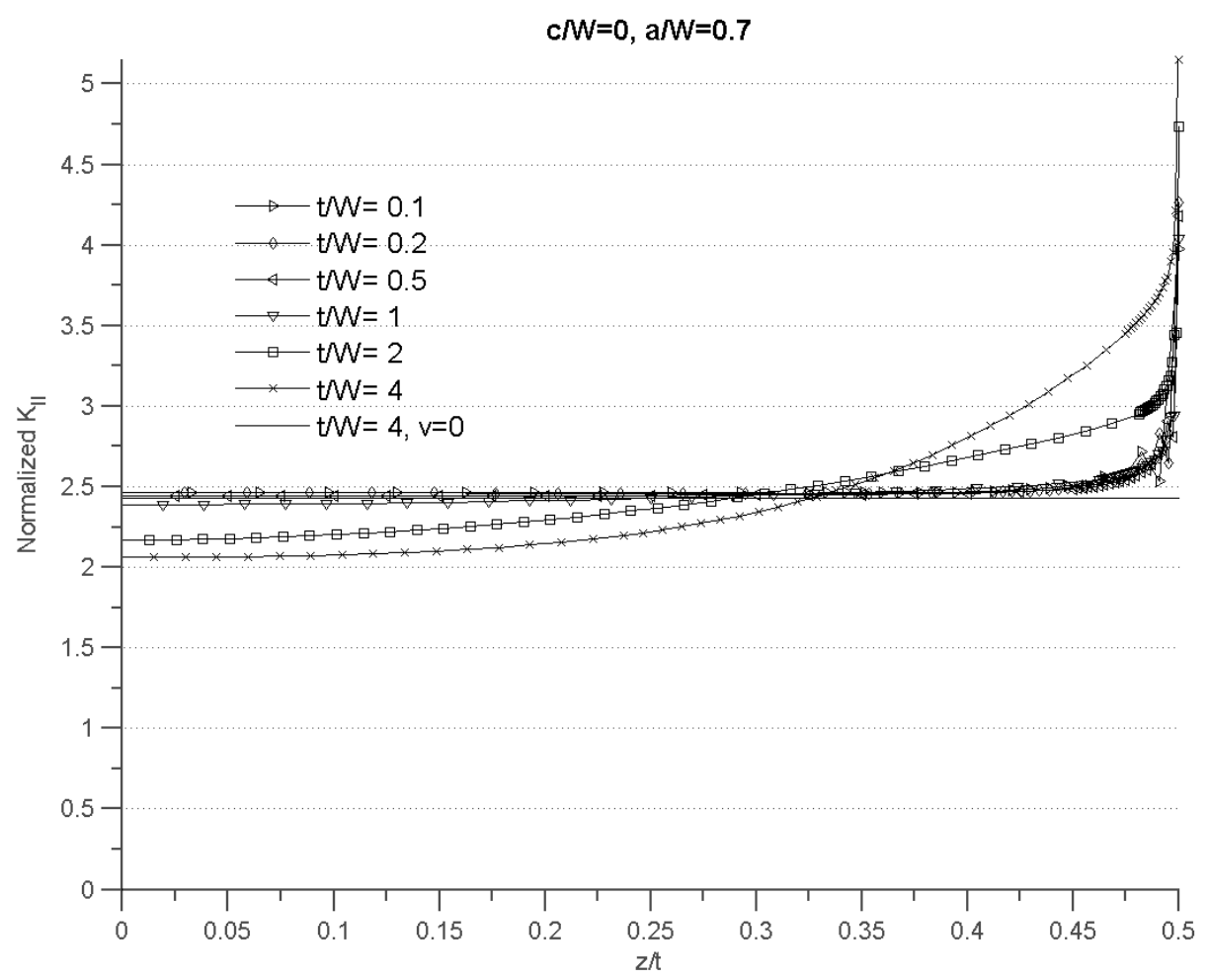

Figure 3.11: Normalized $K_{I I}$ for $\mathrm{a} / \mathrm{W}=\mathbf{0 . 7}$ 


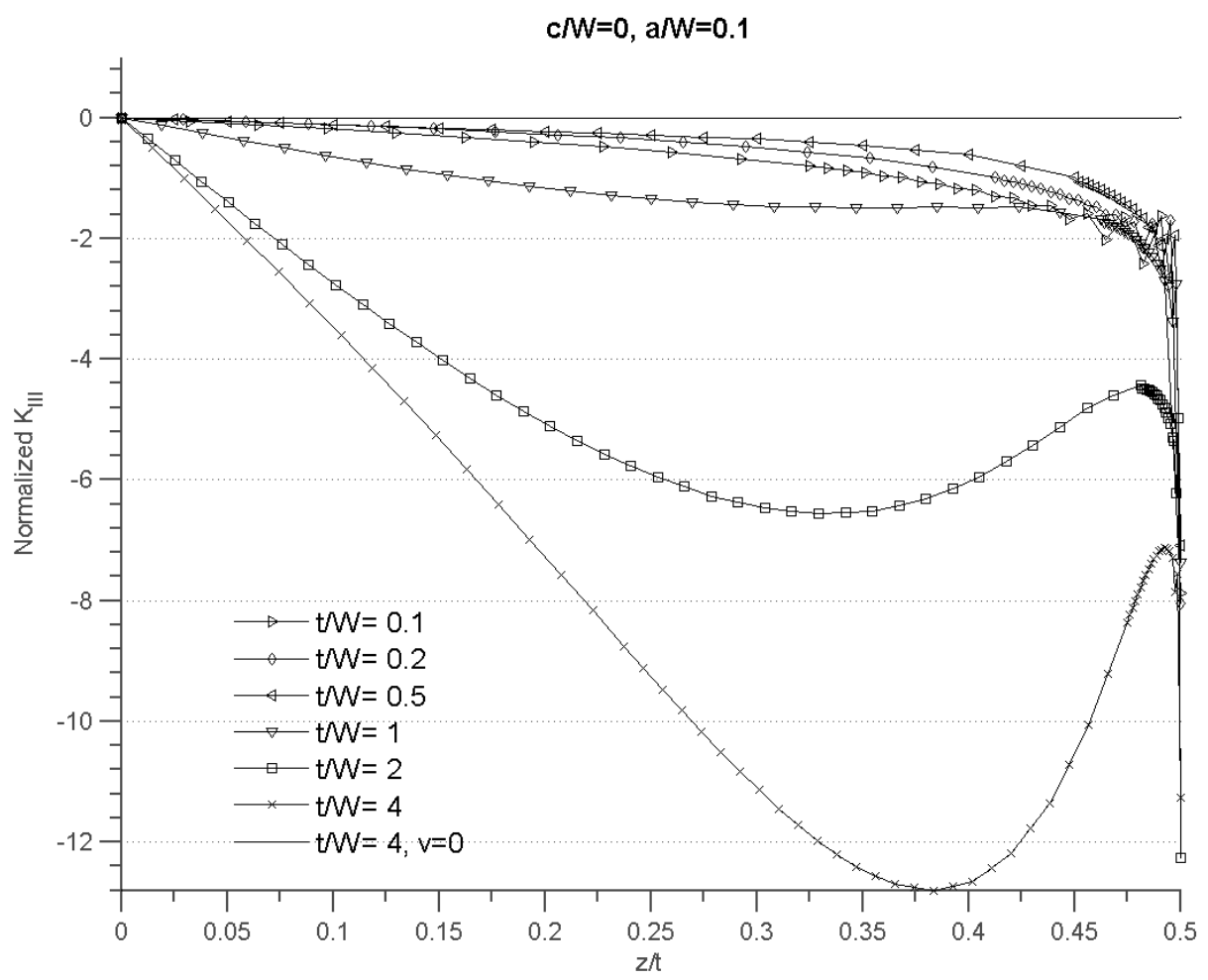

Figure 3.12: Normalized $K_{I I I}$ for $\mathrm{a} / \mathrm{W}=\mathbf{0 . 1}$

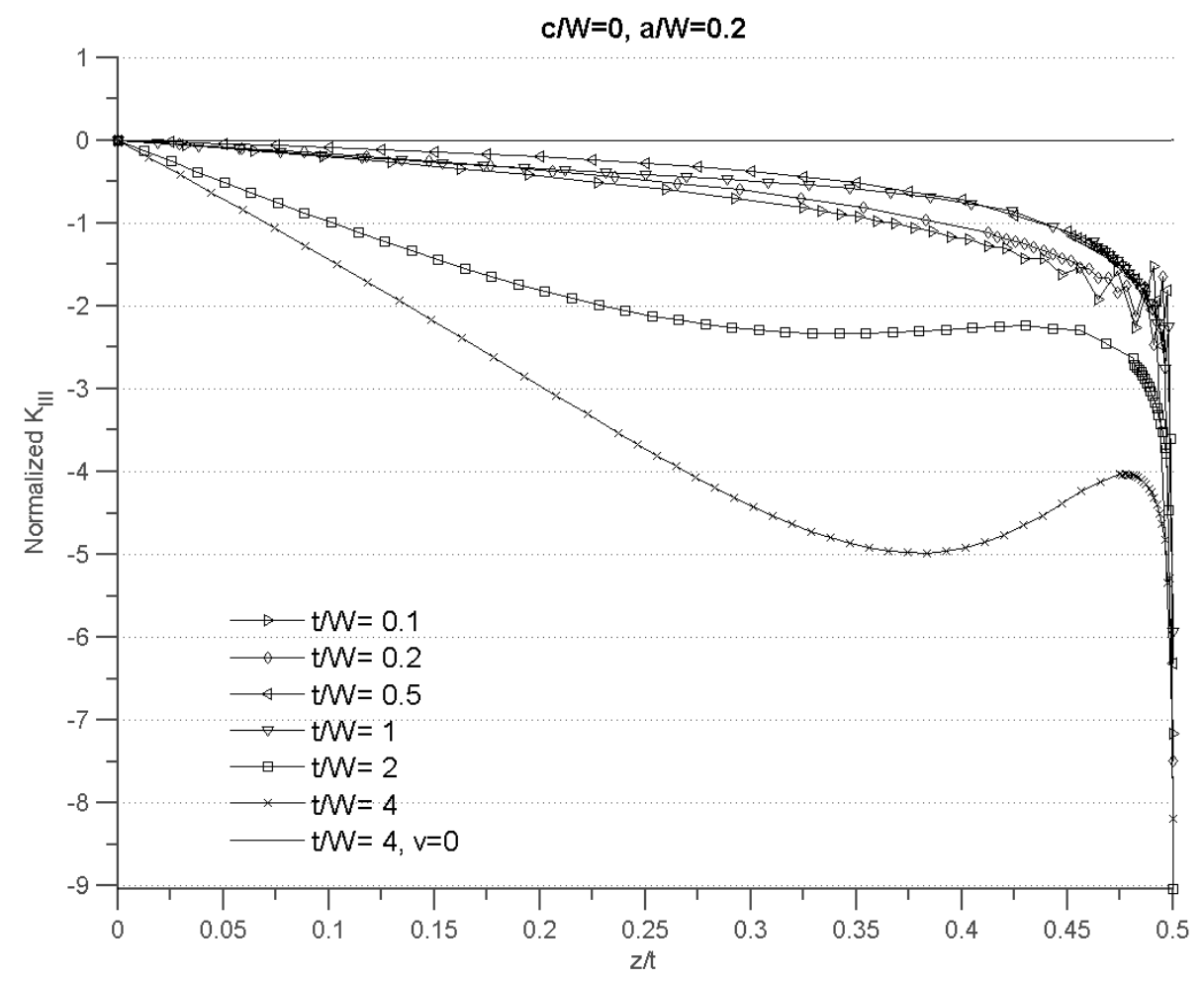

Figure 3.13: Normalized $K_{I I I}$ for $\mathrm{a} / \mathrm{W}=\mathbf{0 . 2}$ 


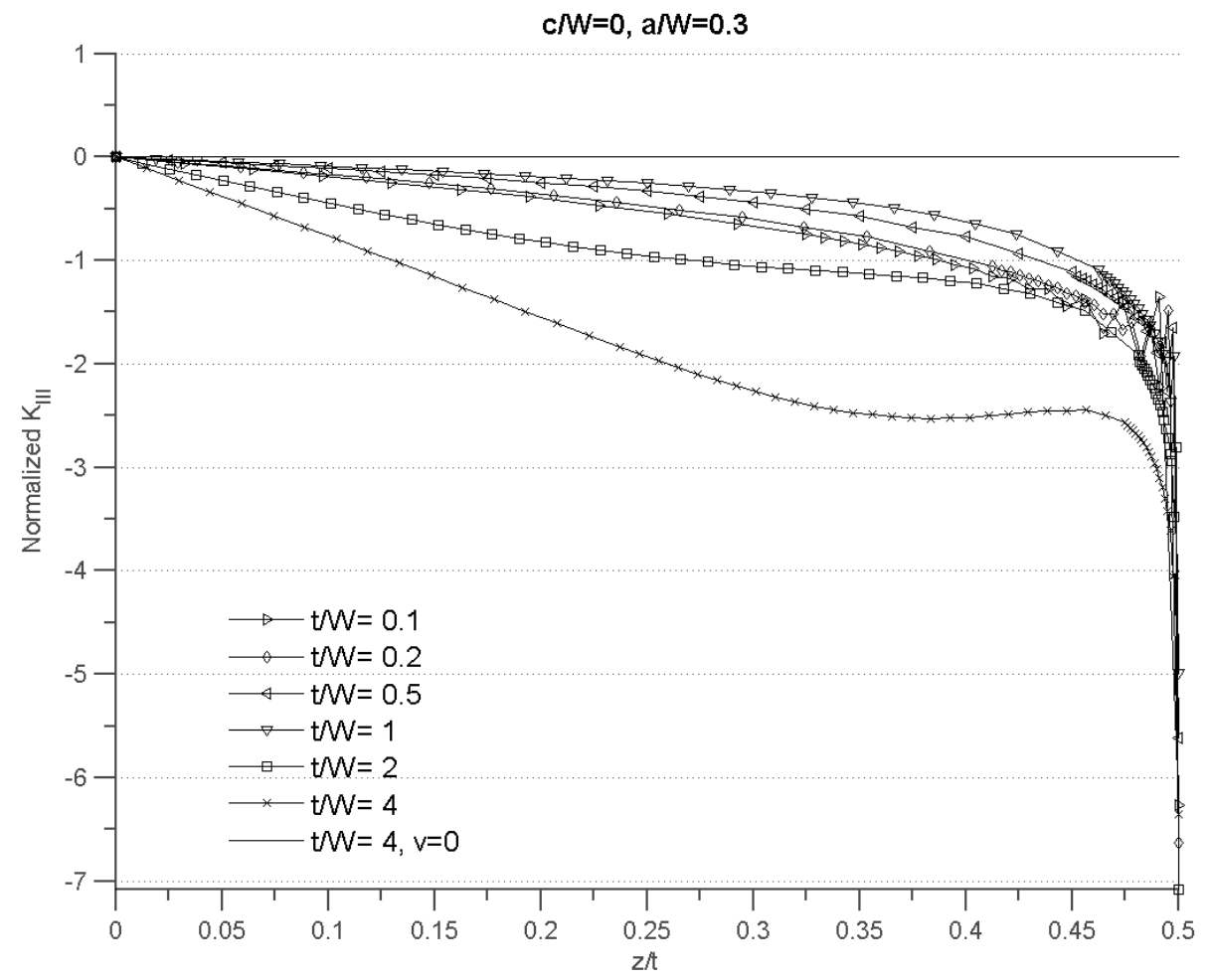

Figure 3.14: Normalized $K_{I I I}$ for $\mathrm{a} / \mathrm{W}=\mathbf{0 . 3}$

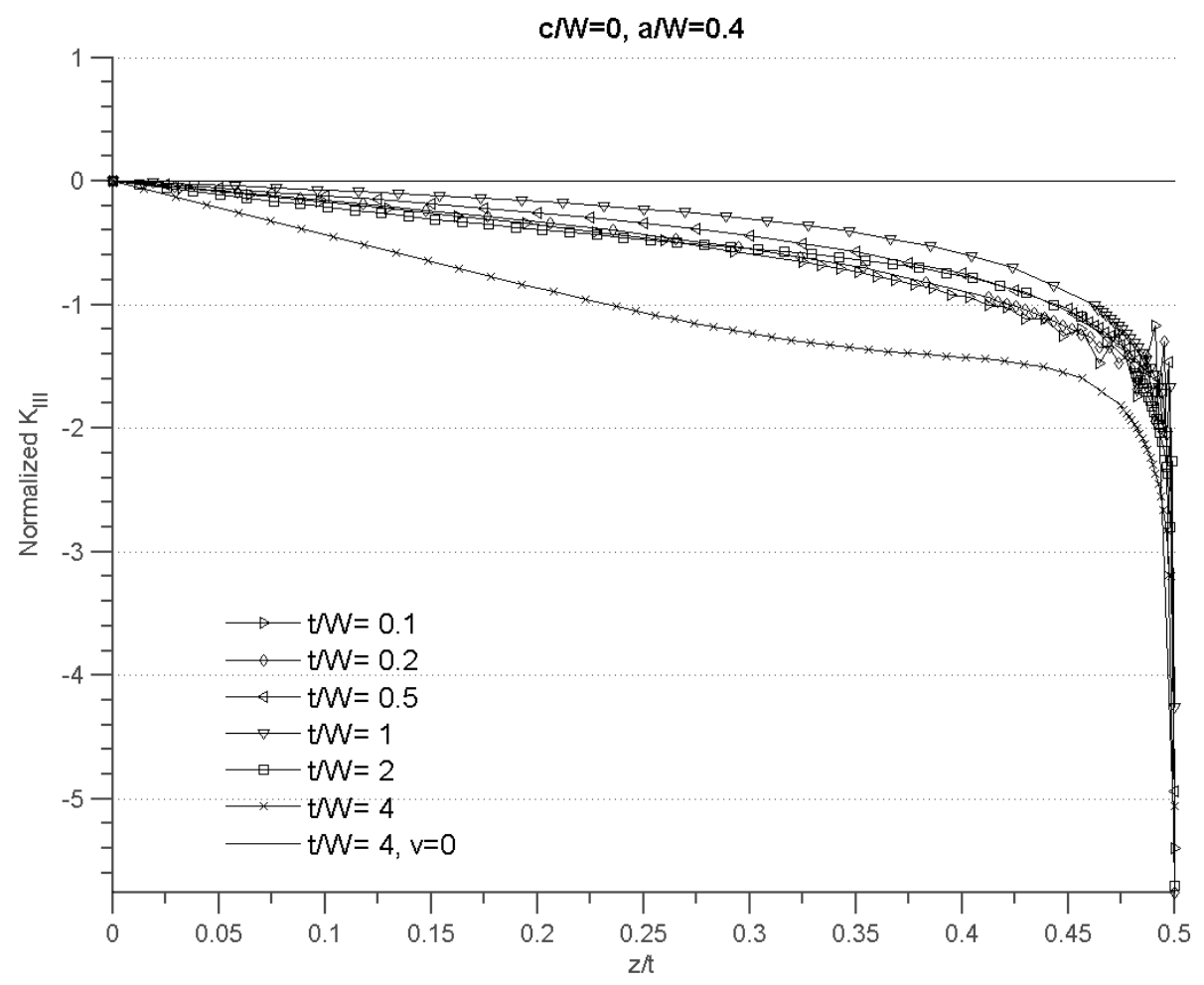

Figure 3.15: Normalized $K_{I I I}$ for $\mathrm{a} / \mathrm{W}=\mathbf{0 . 4}$ 


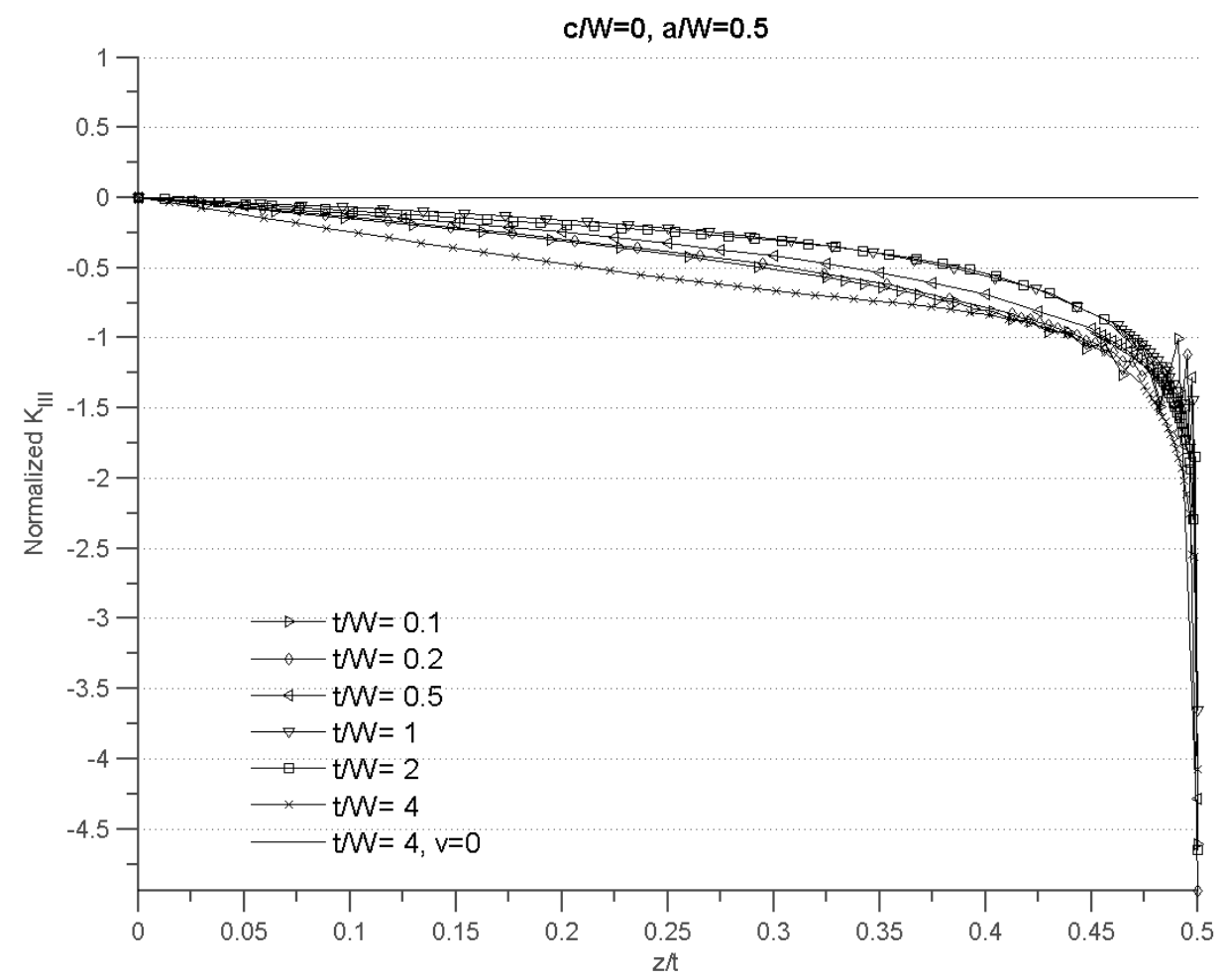

Figure 3.16: Normalized $K_{I I I}$ for $\mathrm{a} / \mathrm{W}=\mathbf{0 . 5}$

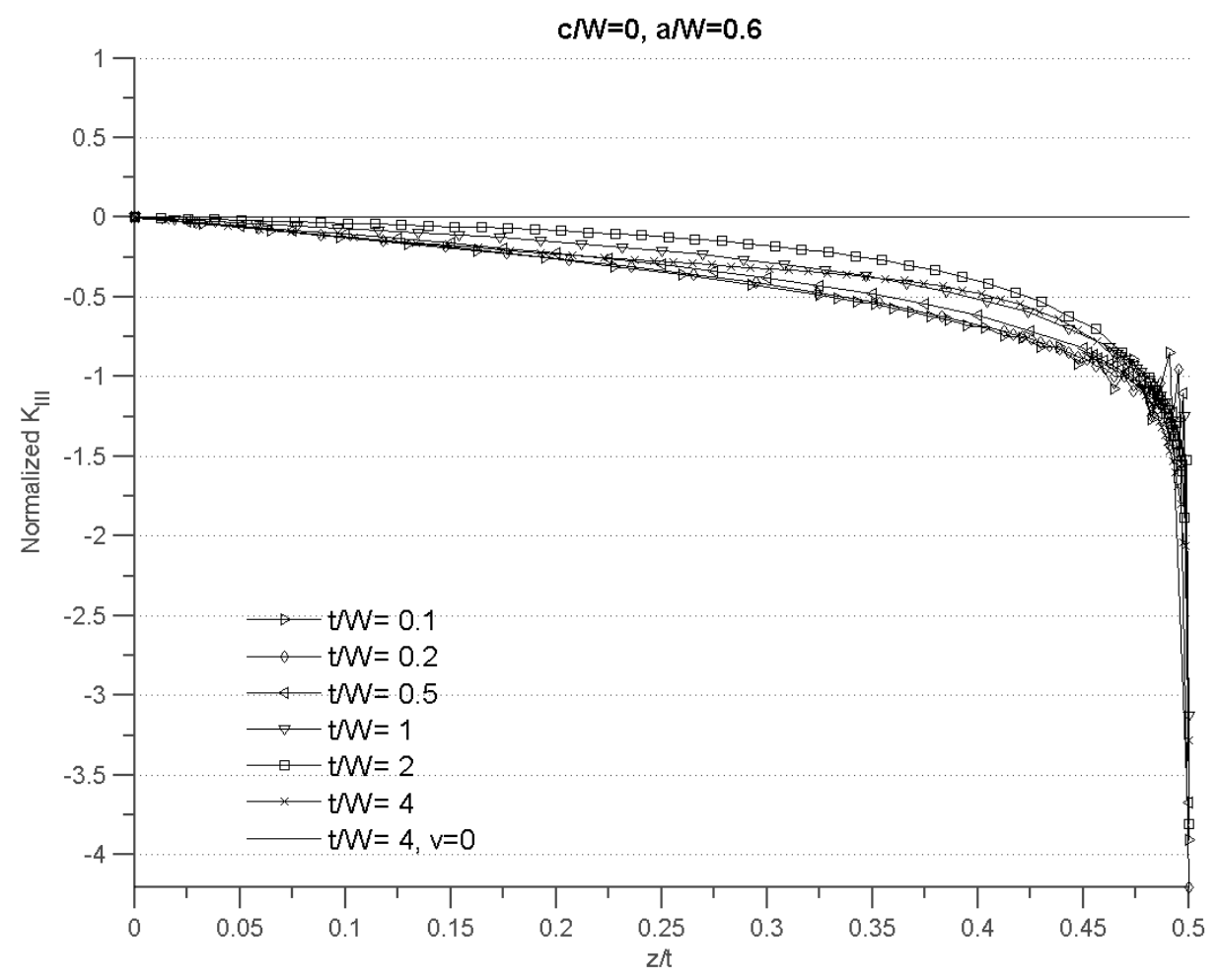

Figure 3.17: Normalized $K_{I I I}$ for $\mathrm{a} / \mathrm{W}=\mathbf{0 . 6}$ 


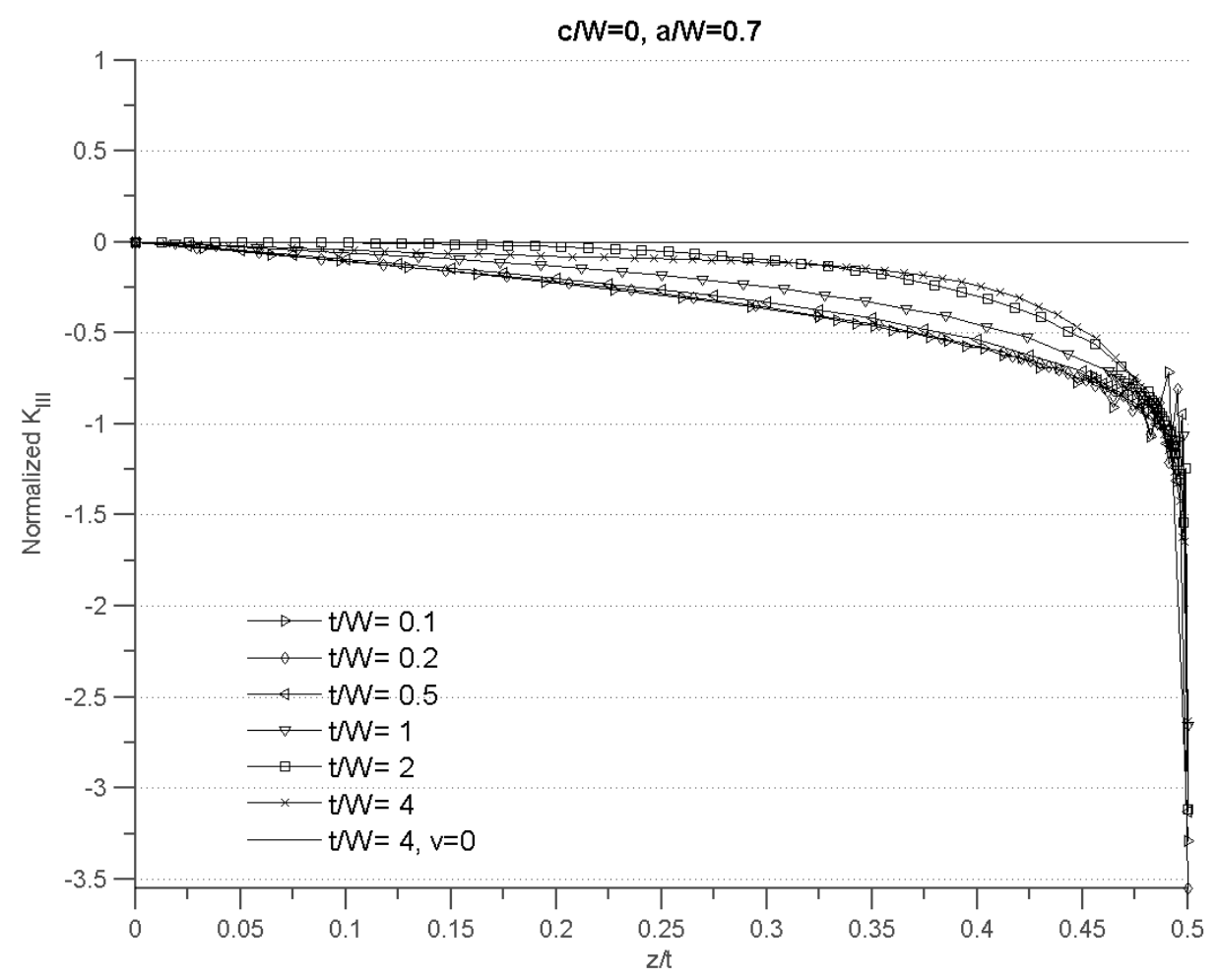

Figure 3.18: Normalized $K_{I I}$ for $\mathrm{a} / \mathrm{W}=0.7$ 


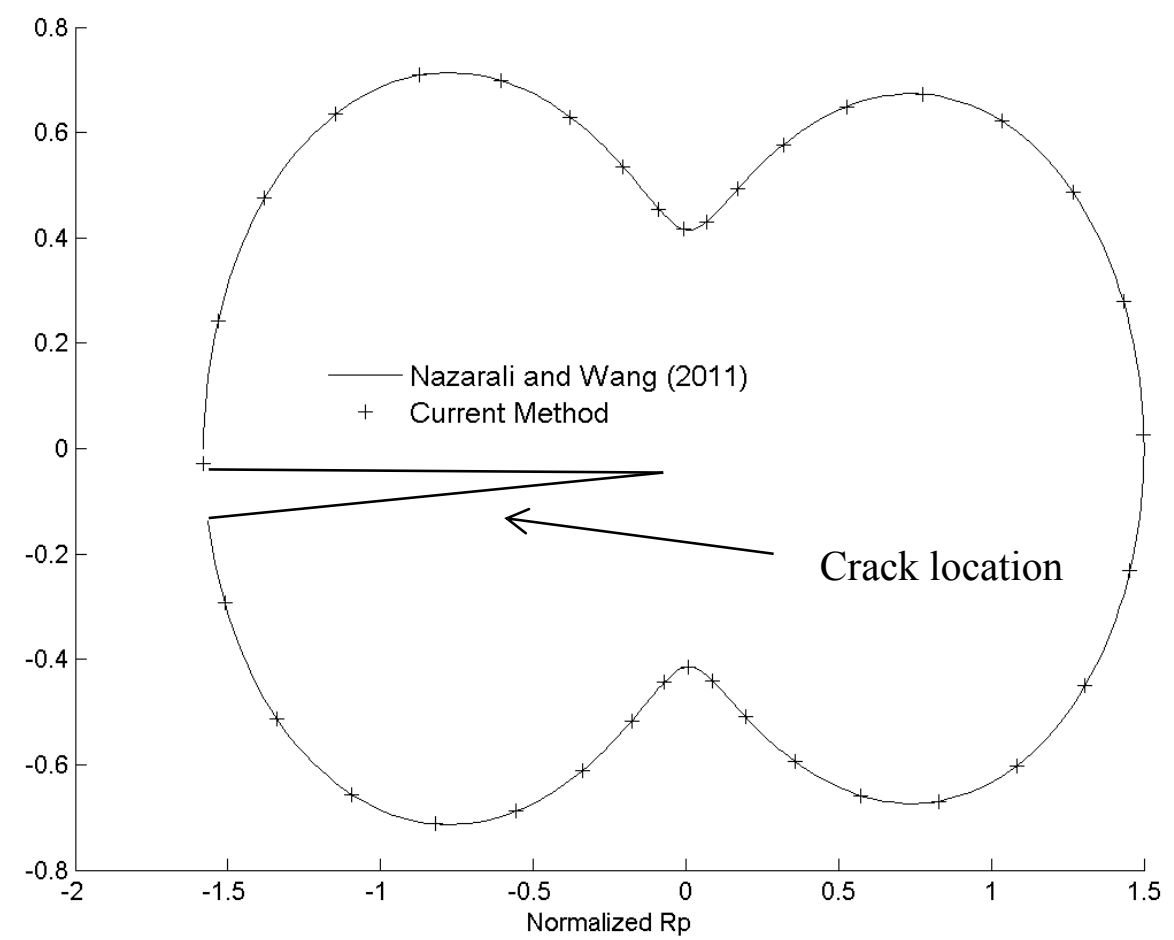

Figure 3.19: Plastic zone verification of pure mode II case

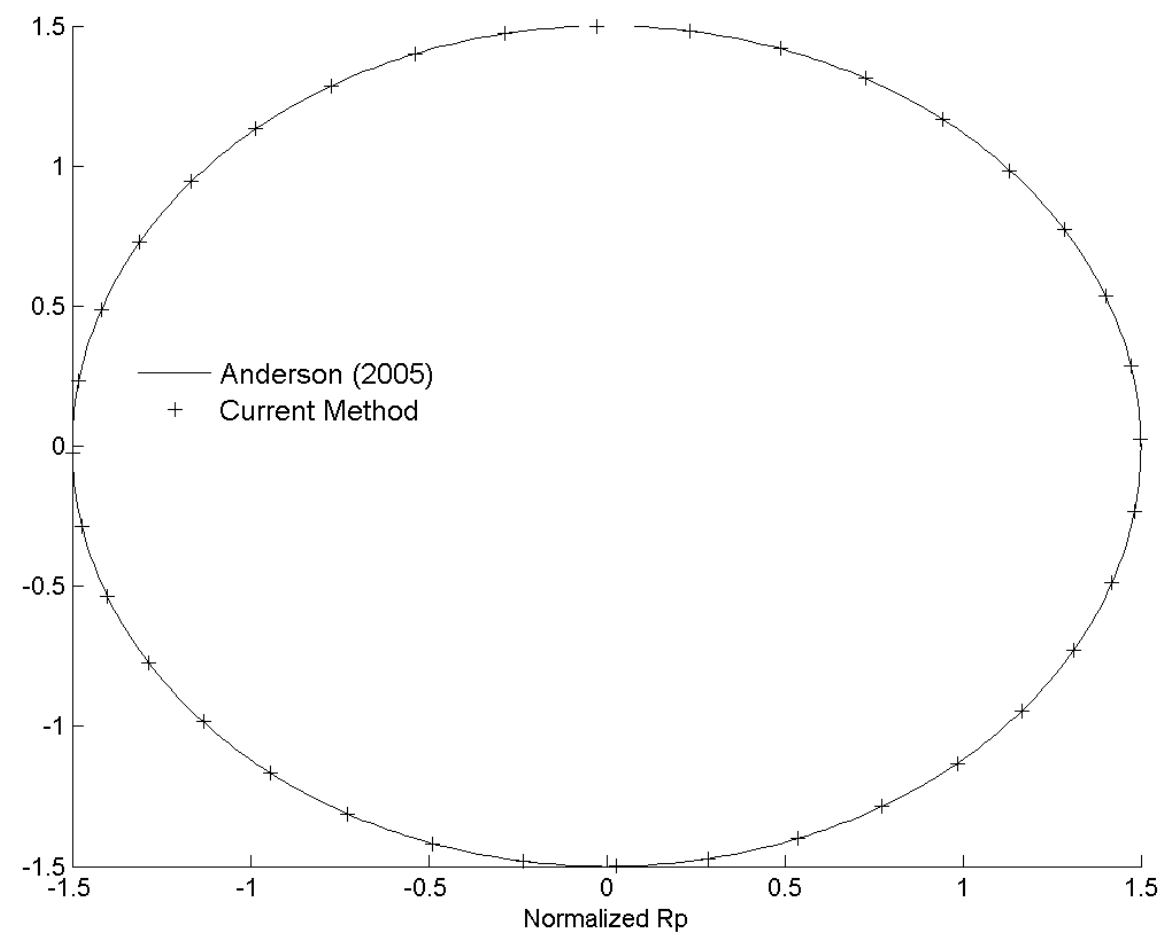

Figure 3.20: Plastic zone verification of pure mode III case 


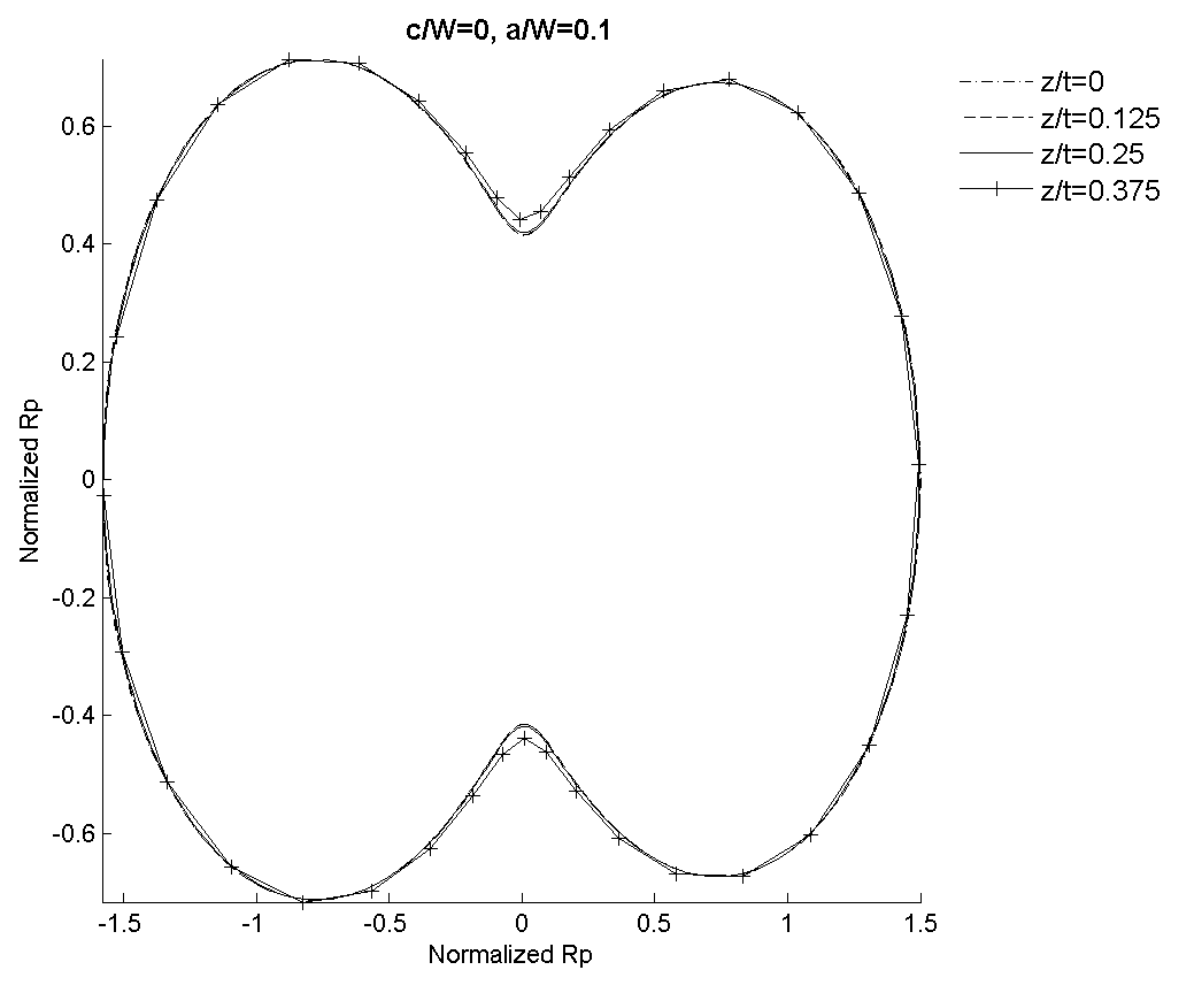

Figure 3.21: Plastic zone plot for $t / W=0.1, a / W=0.1$

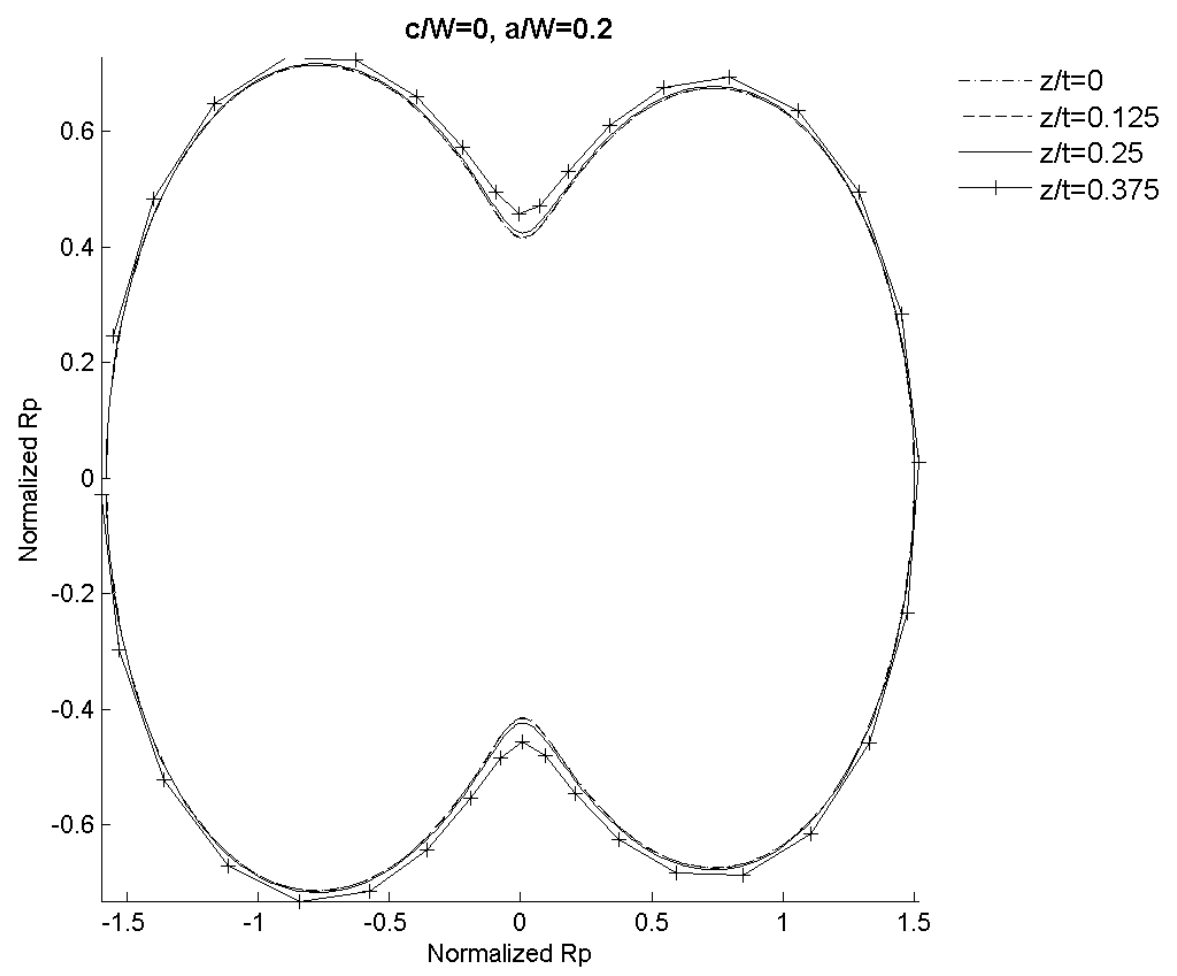

Figure 3.22: Plastic zone plot for $\mathrm{t} / \mathrm{W}=0.1, \mathrm{a} / \mathrm{W}=\mathbf{0 . 2}$ 


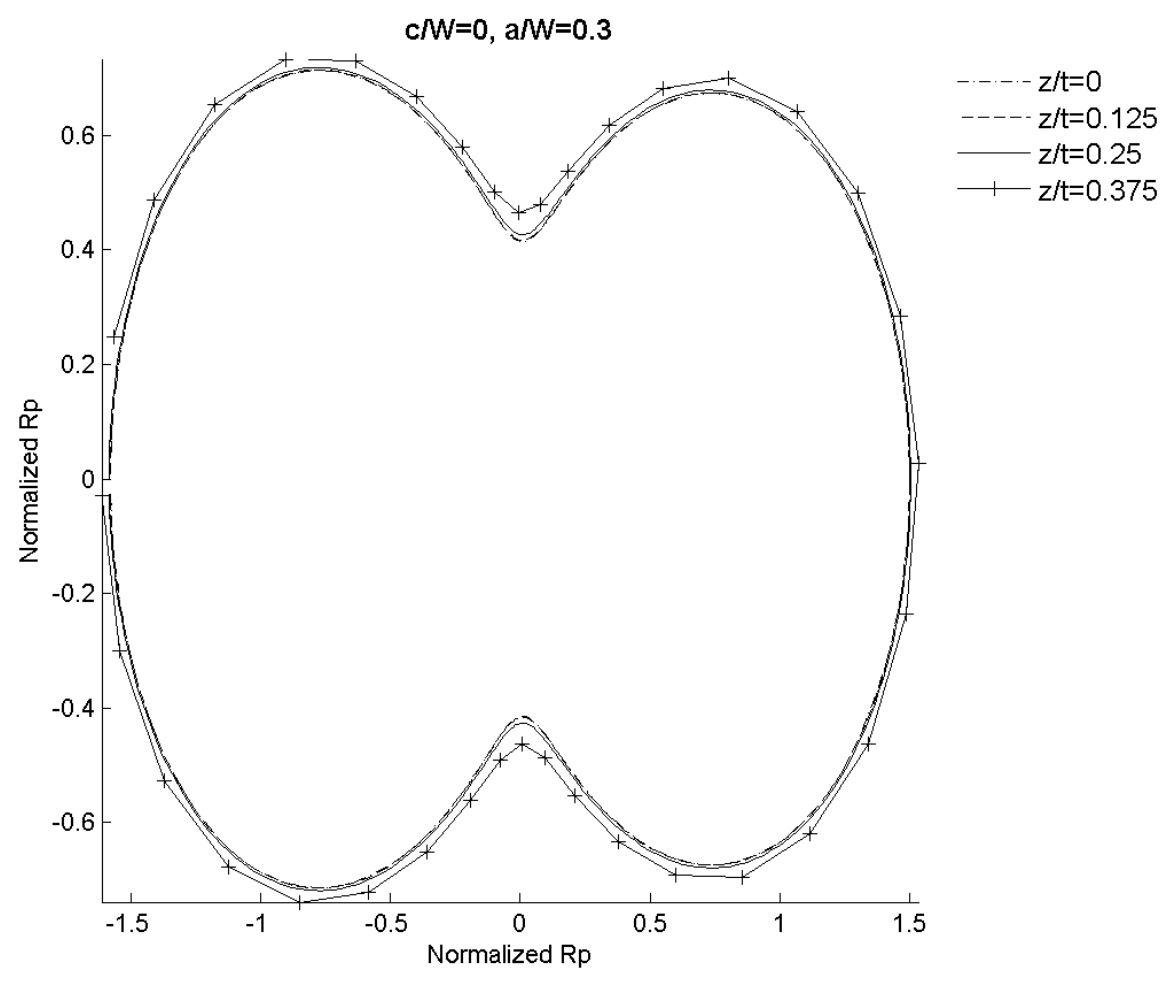

Figure 3.23: Plastic zone plot for $\mathrm{t} / \mathrm{W}=\mathbf{0 . 1}, \mathrm{a} / \mathrm{W}=\mathbf{0 . 3}$

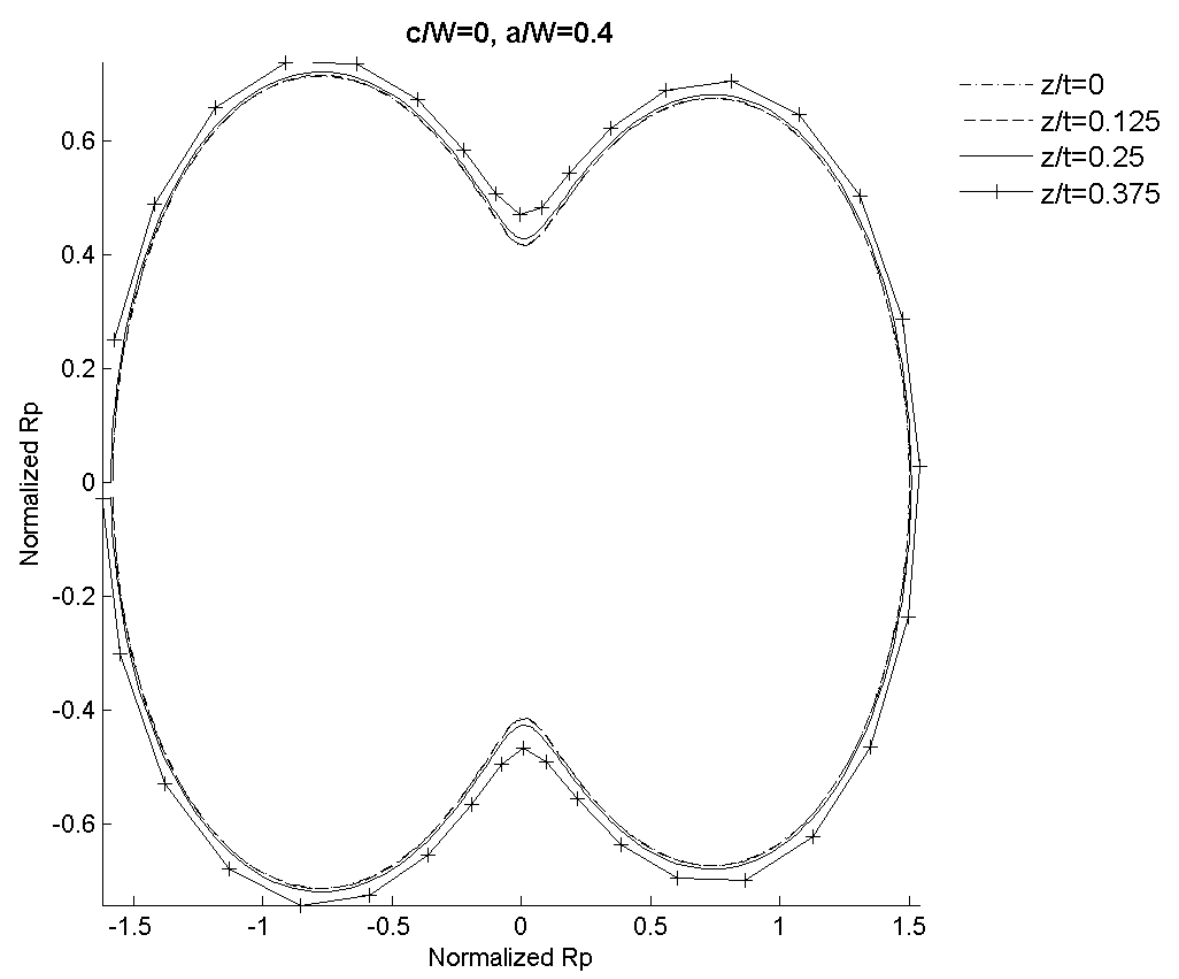

Figure 3.24: Plastic zone plot for $\mathrm{t} / \mathrm{W}=0.1, \mathrm{a} / \mathrm{W}=\mathbf{0 . 4}$ 


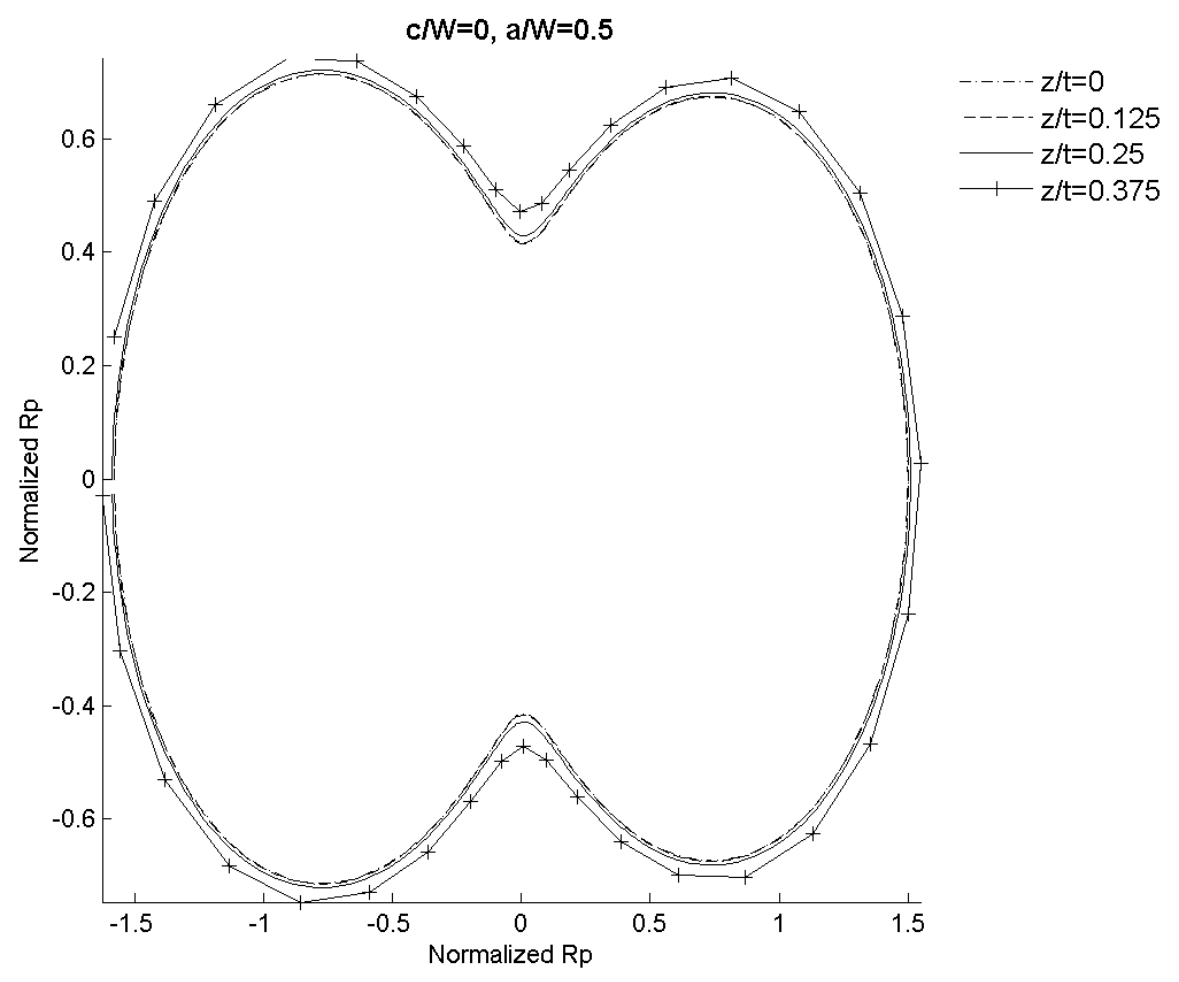

Figure 3.25: Plastic zone plot for $\mathrm{t} / \mathrm{W}=\mathbf{0 . 1}, \mathrm{a} / \mathrm{W}=\mathbf{0 . 5}$

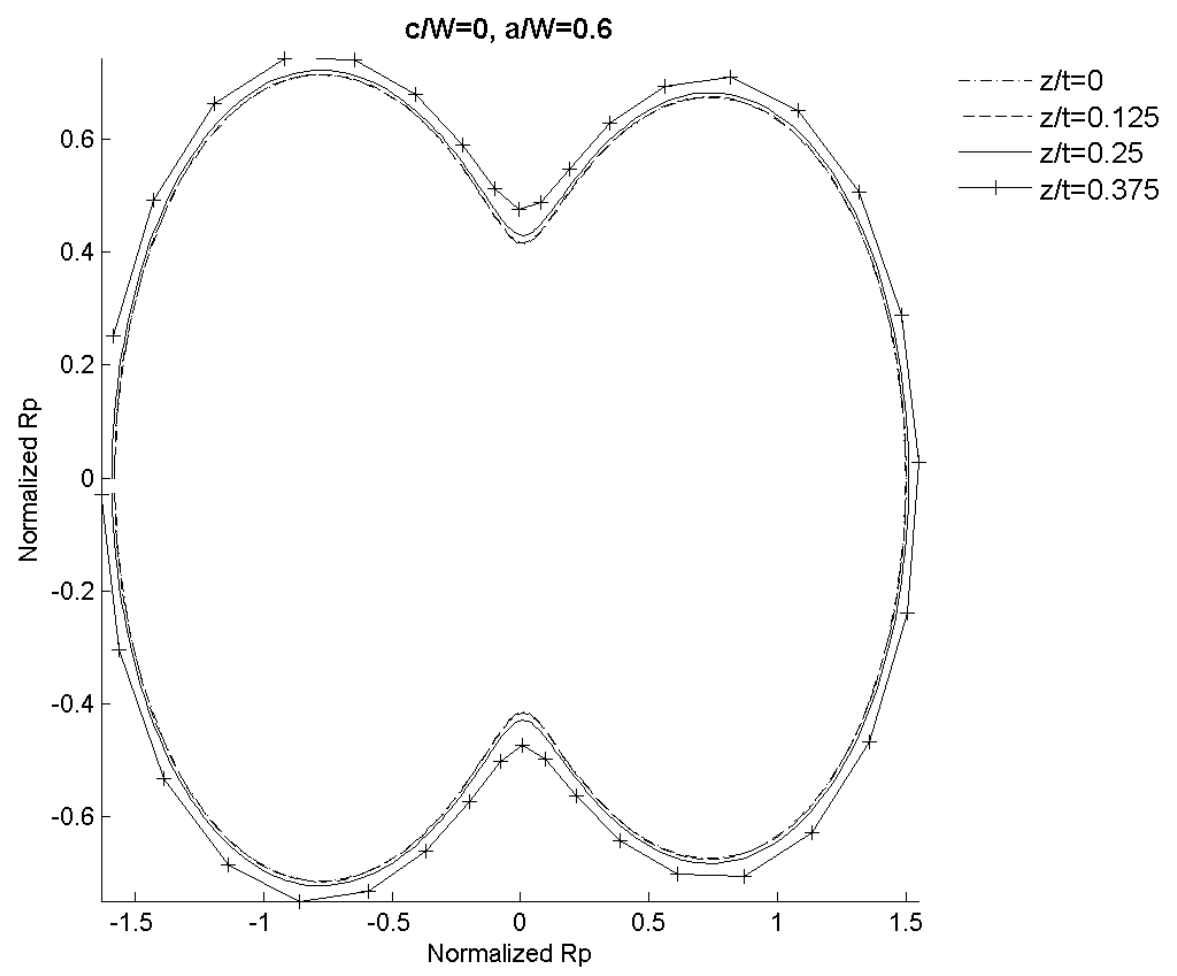

Figure 3.26: Plastic zone plot for $\mathrm{t} / \mathrm{W}=\mathbf{0 . 1}, \mathrm{a} / \mathrm{W}=\mathbf{0 . 6}$ 


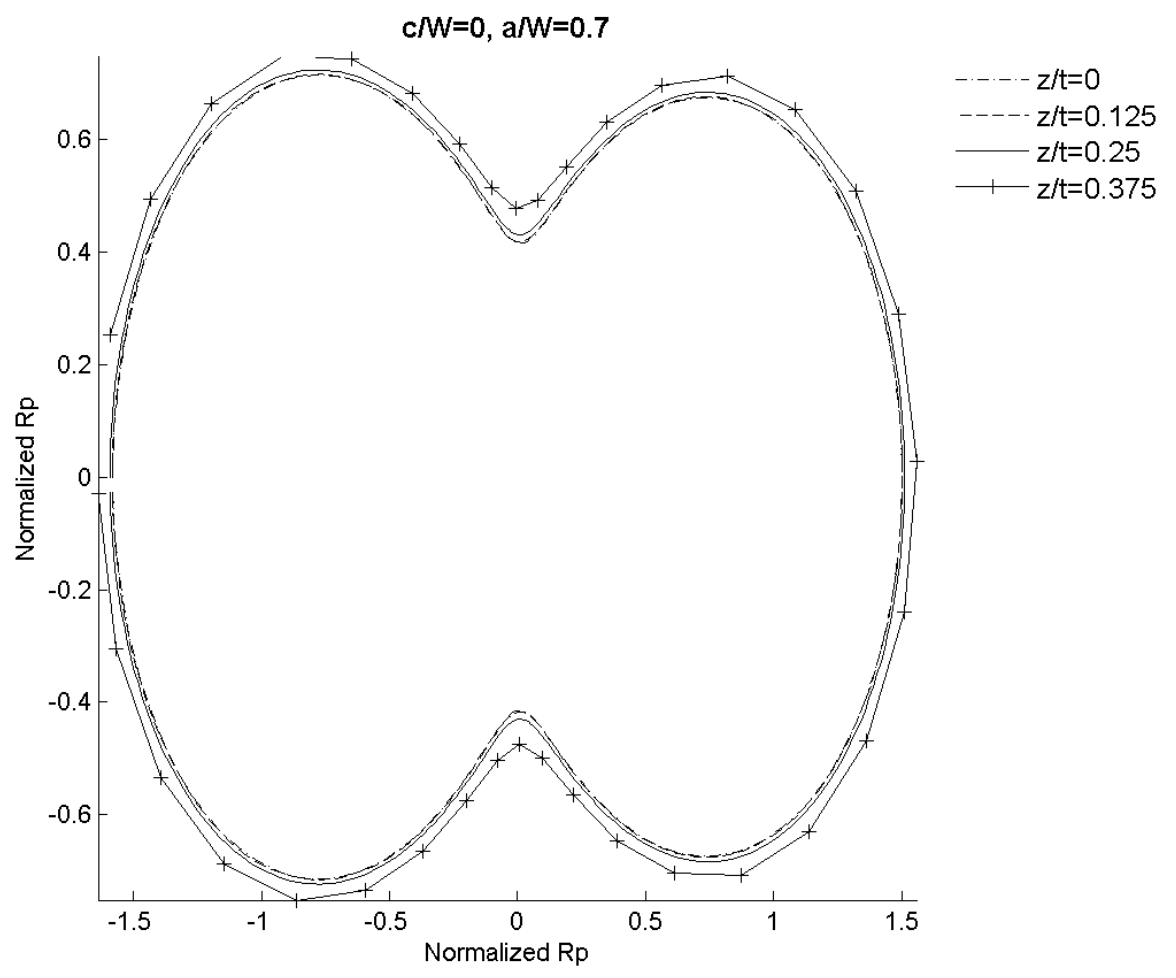

Figure 3.27: Plastic zone plot for $t / W=0.1, a / W=0.7$ 


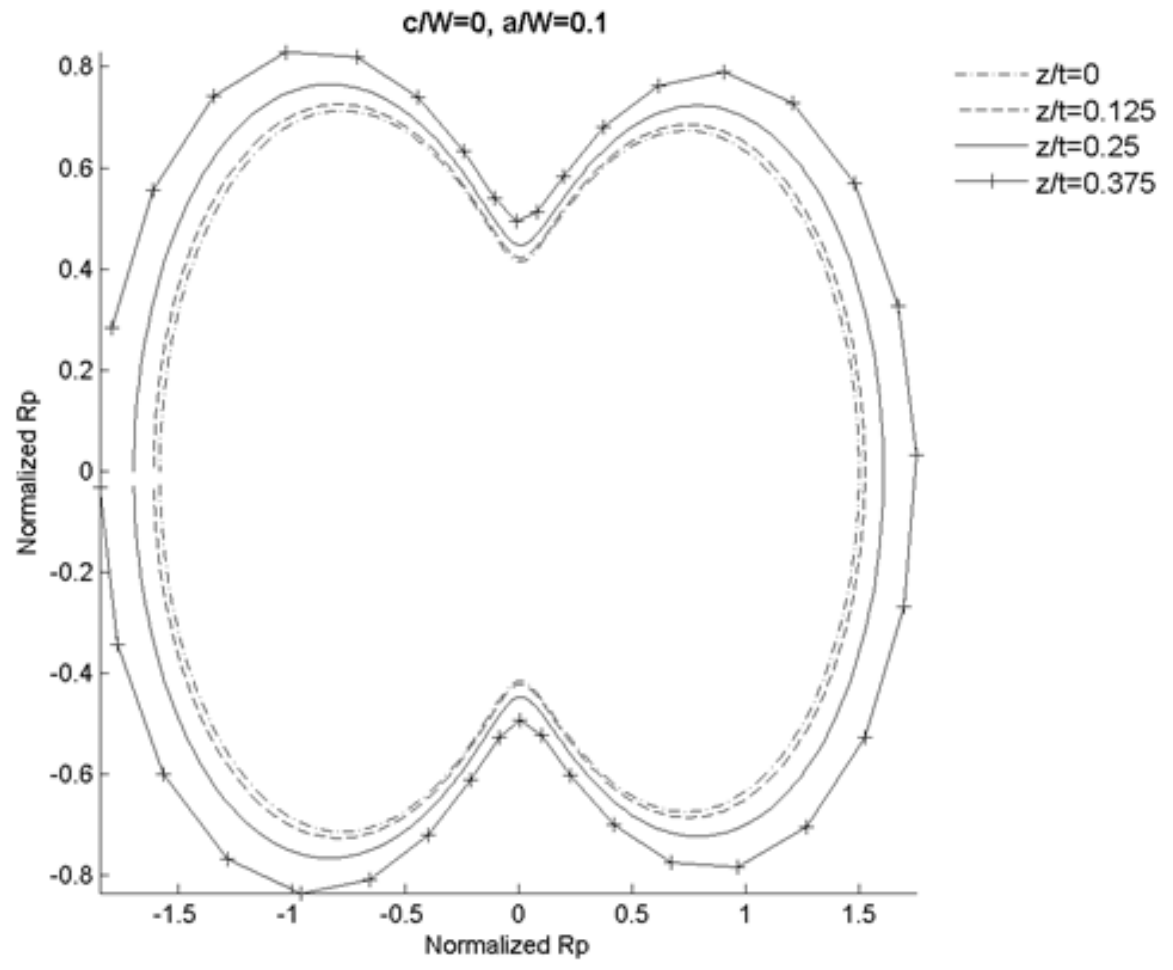

Figure 3.28: Plastic zone plot for $\mathrm{t} / \mathrm{W}=0.5, \mathrm{a} / \mathrm{W}=\mathbf{0 . 1}$

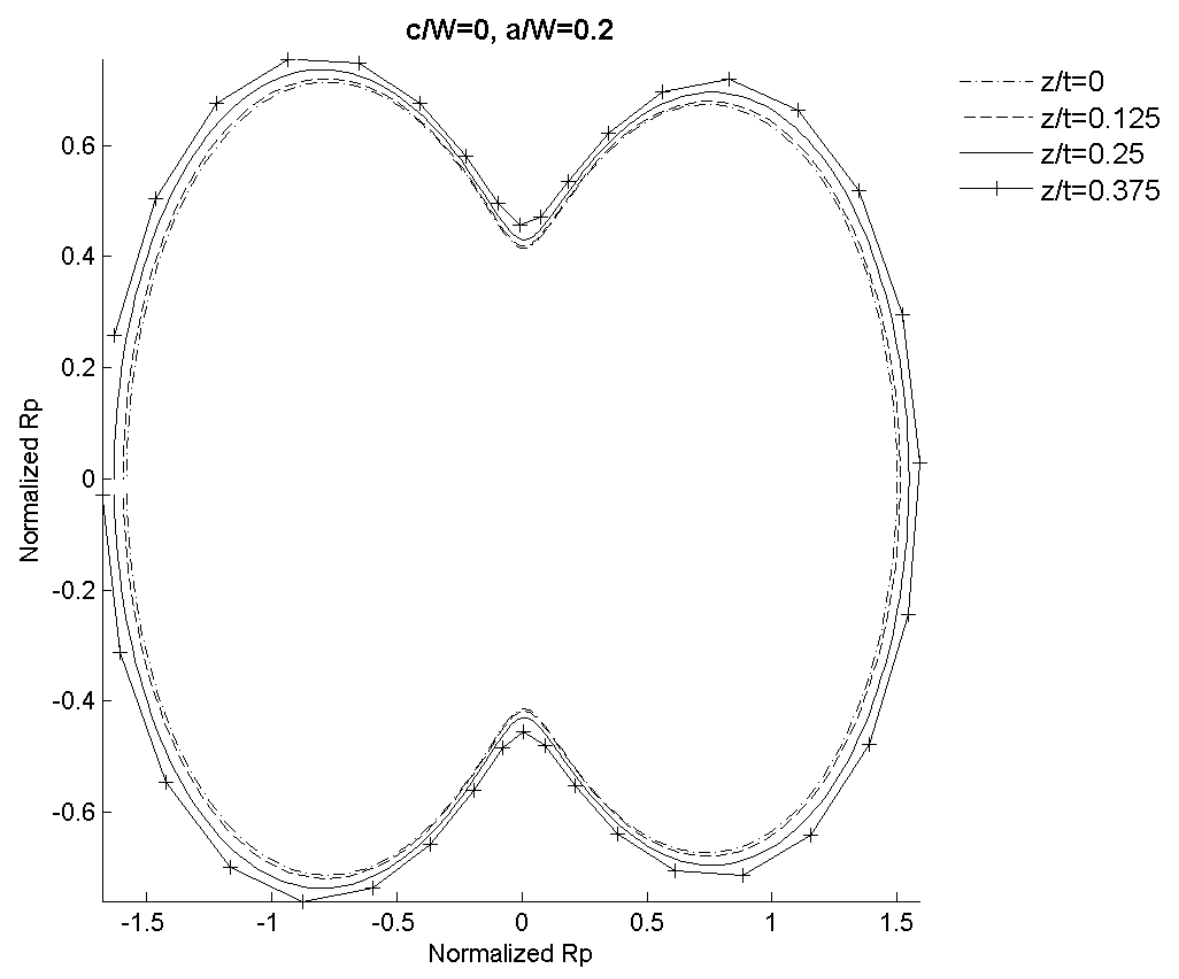

Figure 3.29: Plastic zone plot for $\mathrm{t} / \mathrm{W}=0.5, \mathrm{a} / \mathrm{W}=\mathbf{0 . 2}$ 


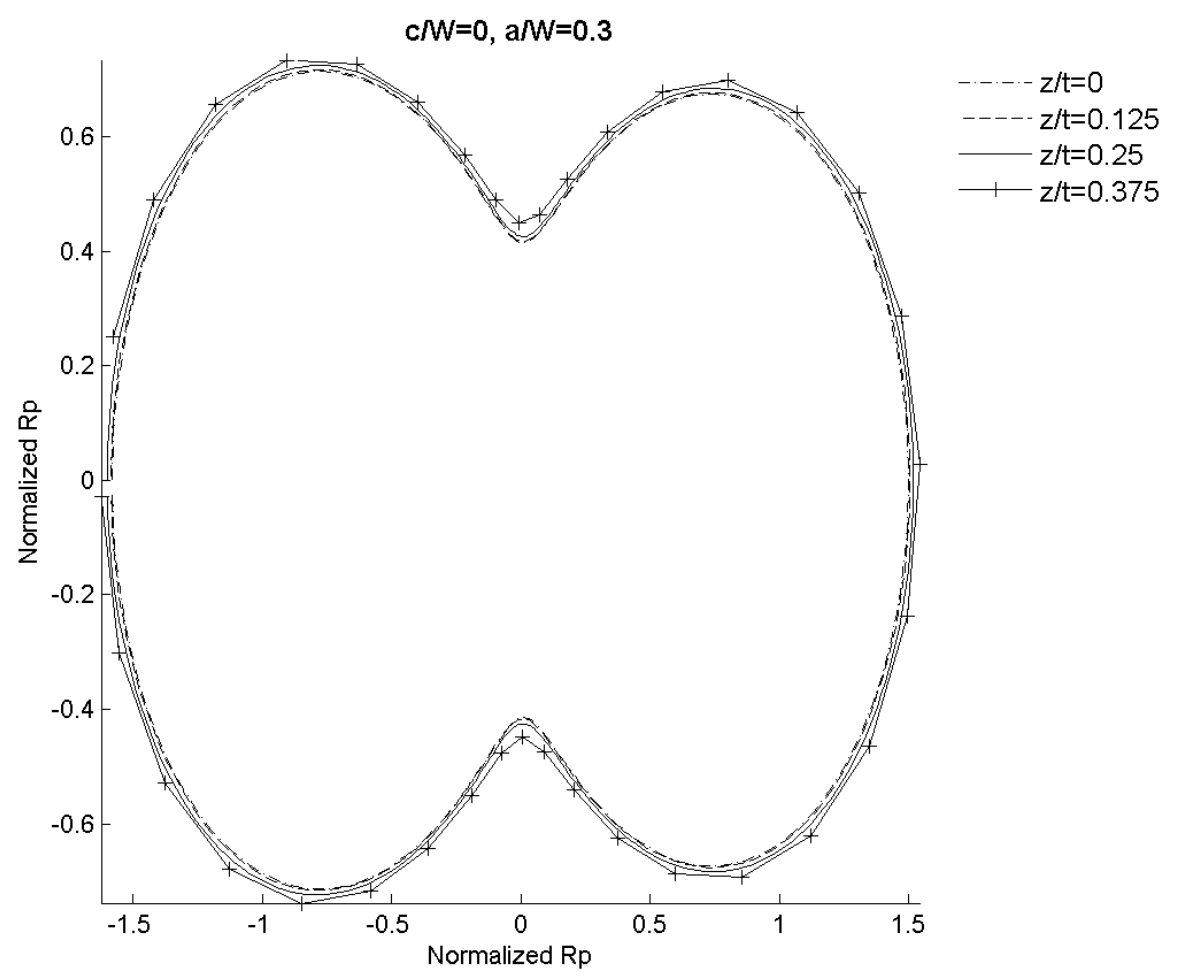

Figure 3.30: Plastic zone plot for $\mathrm{t} / \mathrm{W}=\mathbf{0 . 5}, \mathrm{a} / \mathrm{W}=\mathbf{0 . 3}$

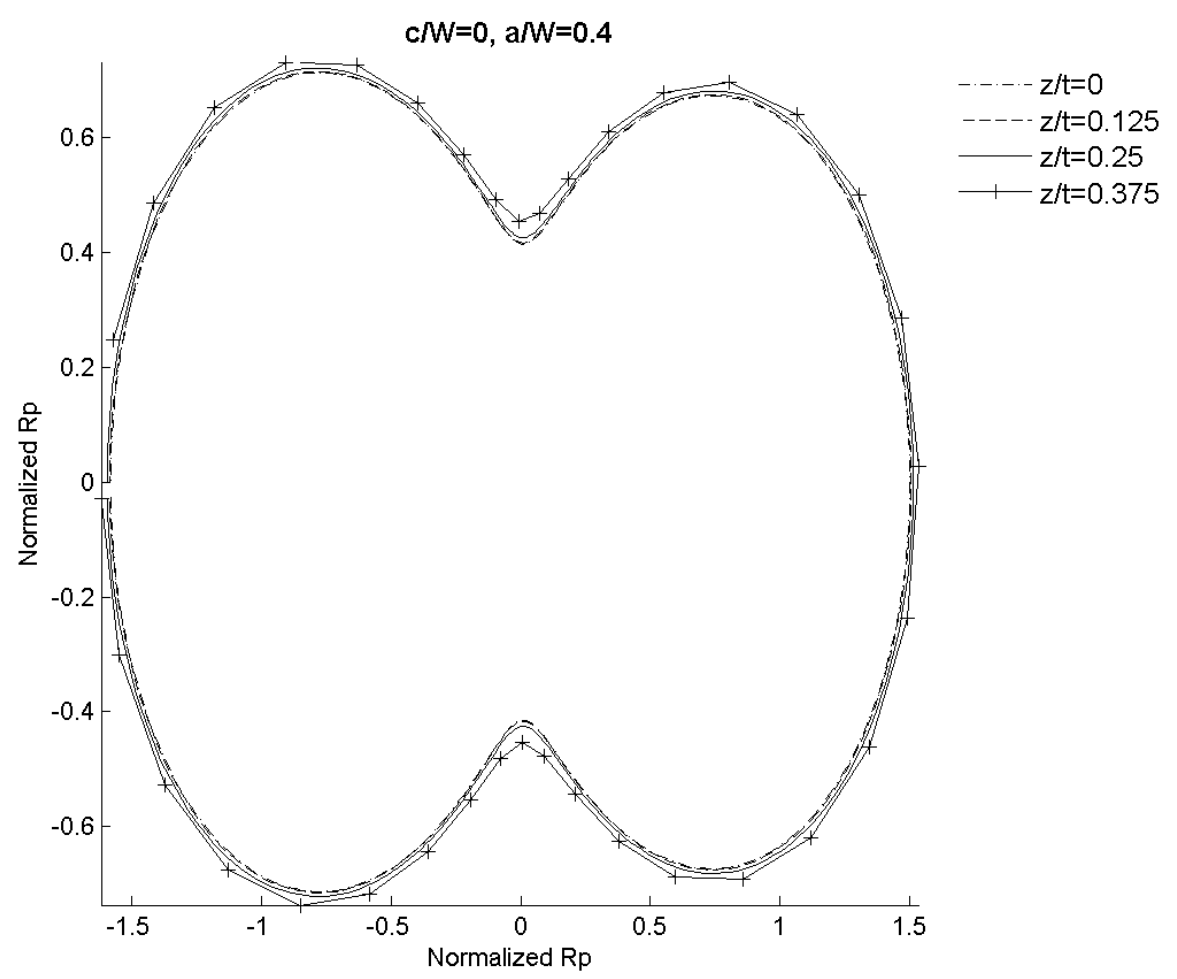

Figure 3.31: Plastic zone plot for $\mathrm{t} / \mathrm{W}=0.5, \mathrm{a} / \mathrm{W}=\mathbf{0 . 4}$ 


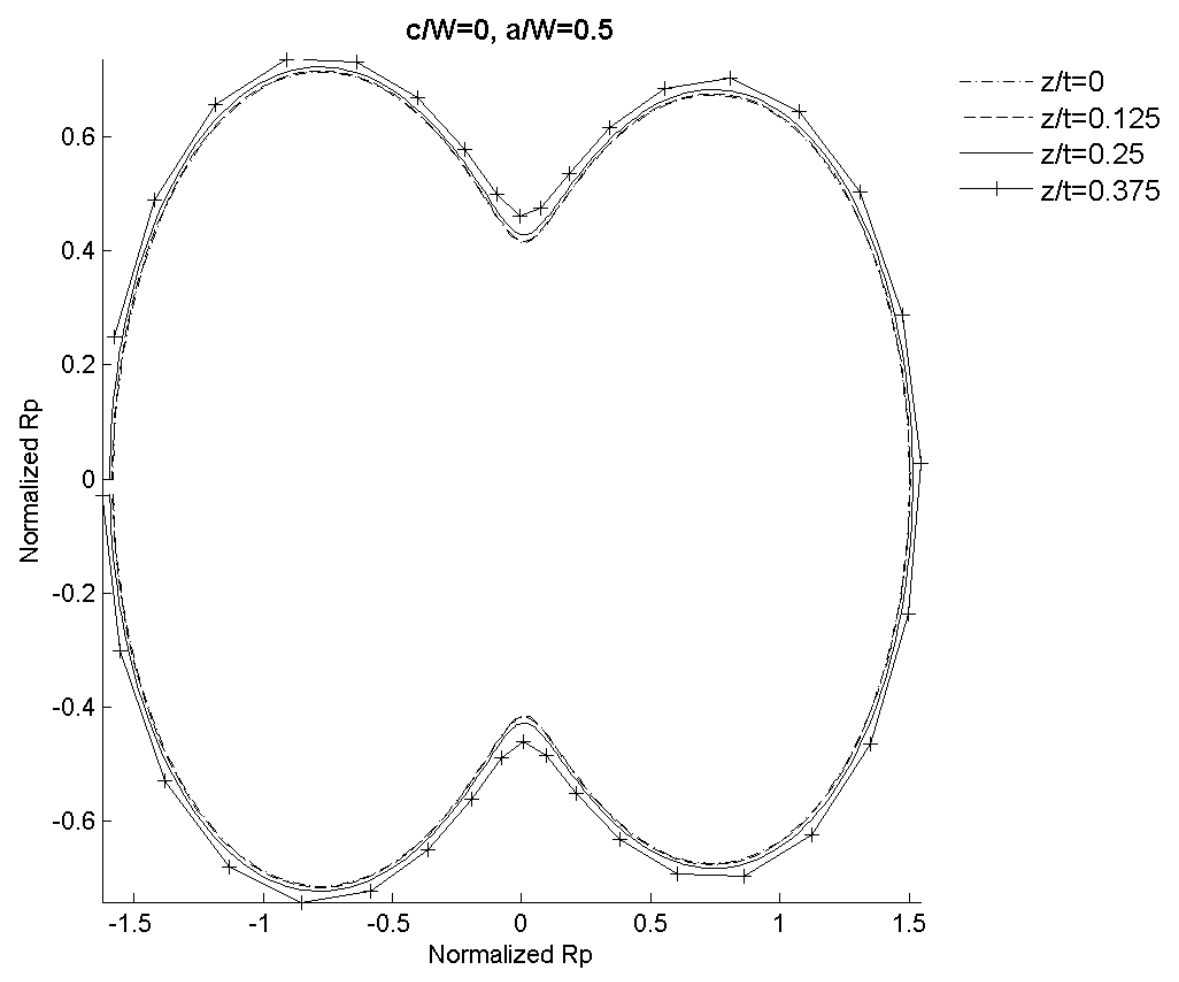

Figure 3.32: Plastic zone plot for $\mathrm{t} / \mathrm{W}=\mathbf{0 . 5}, \mathrm{a} / \mathrm{W}=\mathbf{0 . 5}$

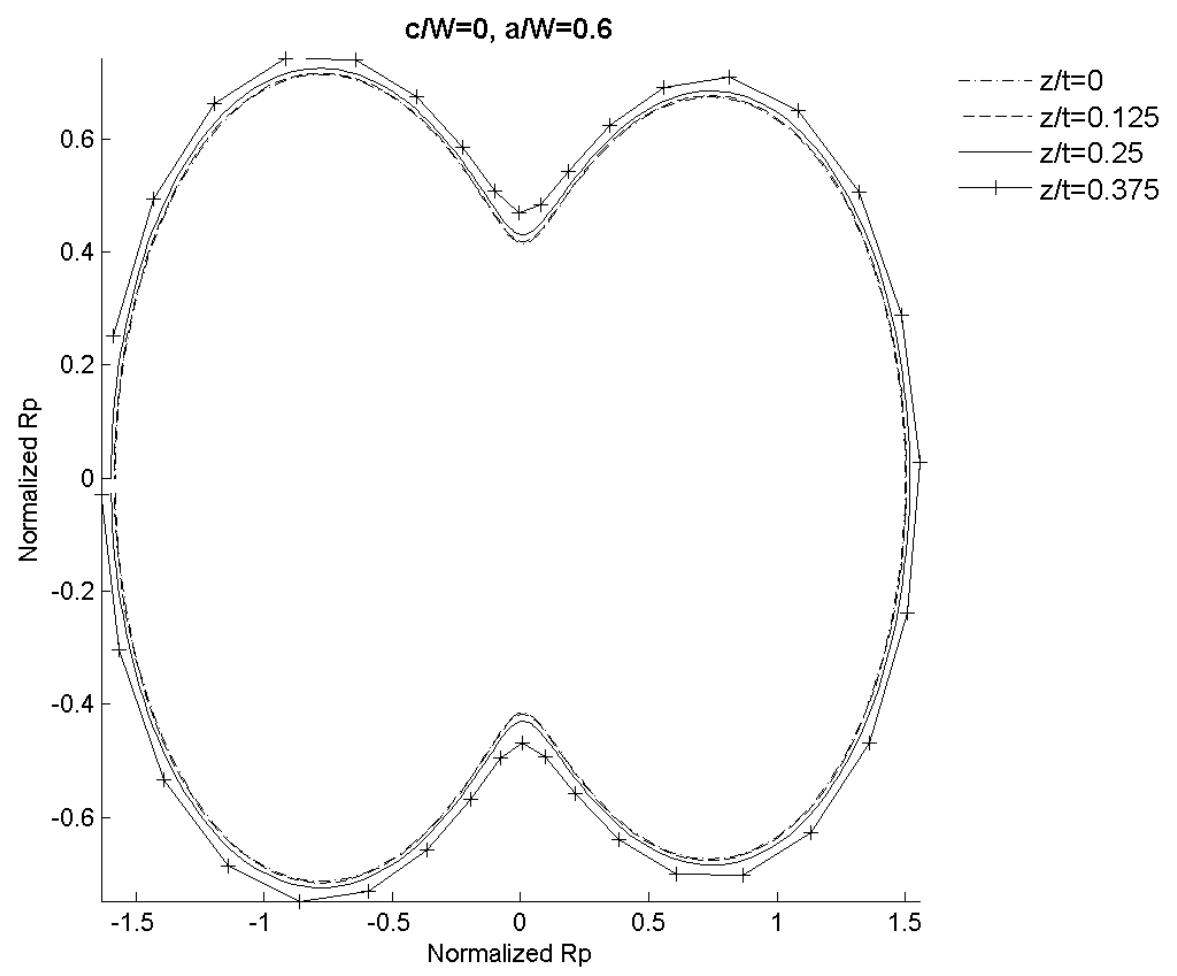

Figure 3.33: Plastic zone plot for $\mathrm{t} / \mathrm{W}=\mathbf{0 . 5}, \mathrm{a} / \mathrm{W}=\mathbf{0 . 6}$ 


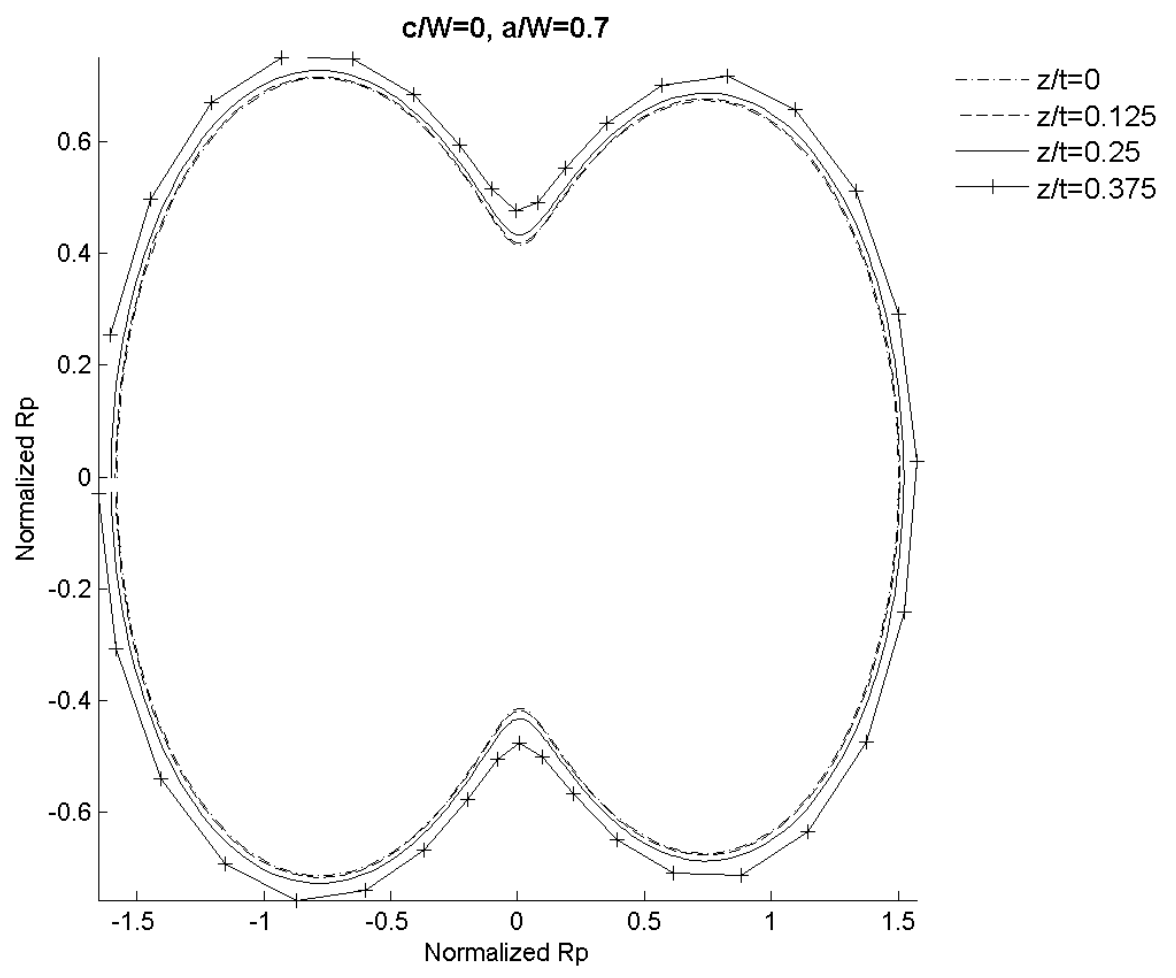

Figure 3.34: Plastic zone plot for $t / W=0.5, a / W=0.7$ 


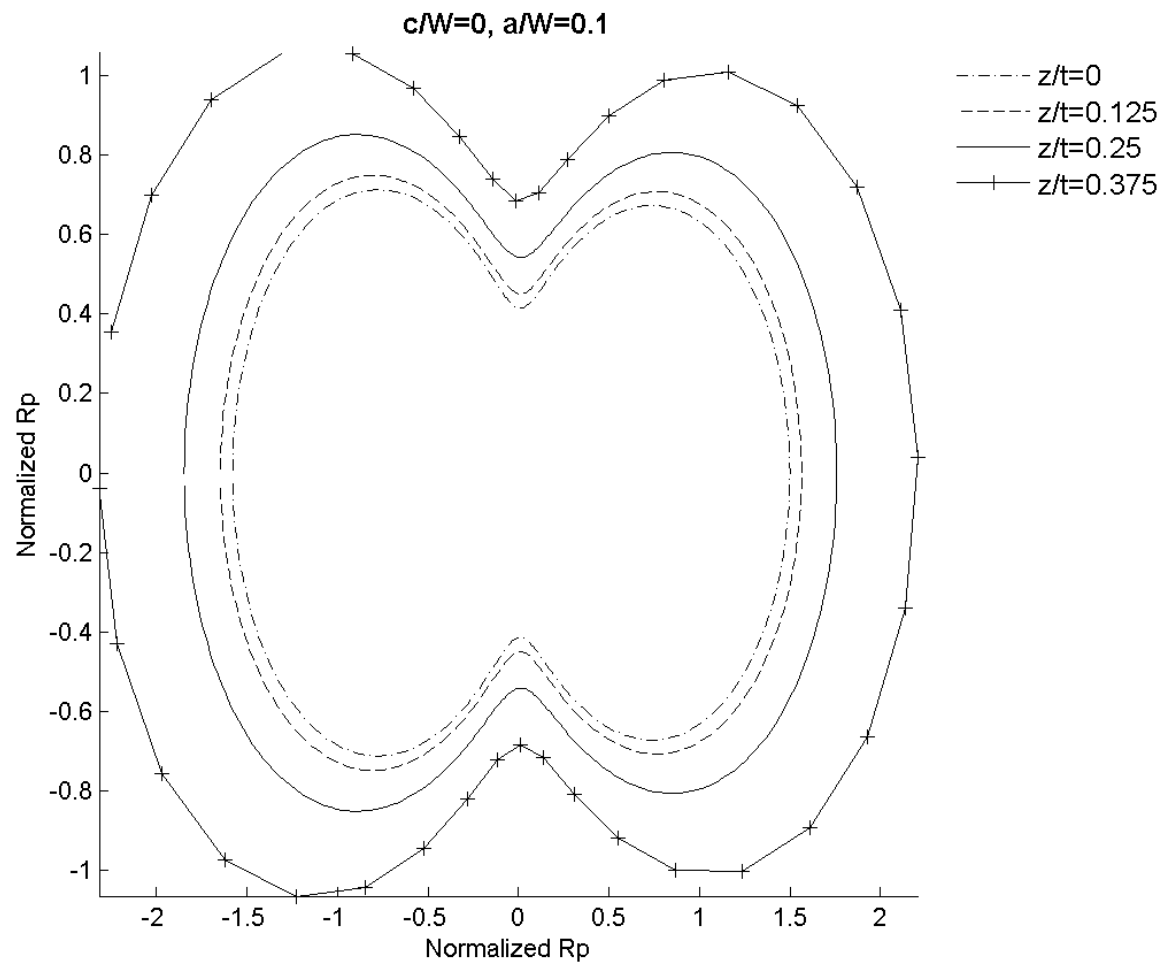

Figure 3.35: Plastic zone plot for $\mathrm{t} / \mathrm{W}=1.0, \mathrm{a} / \mathrm{W}=\mathbf{0 . 1}$

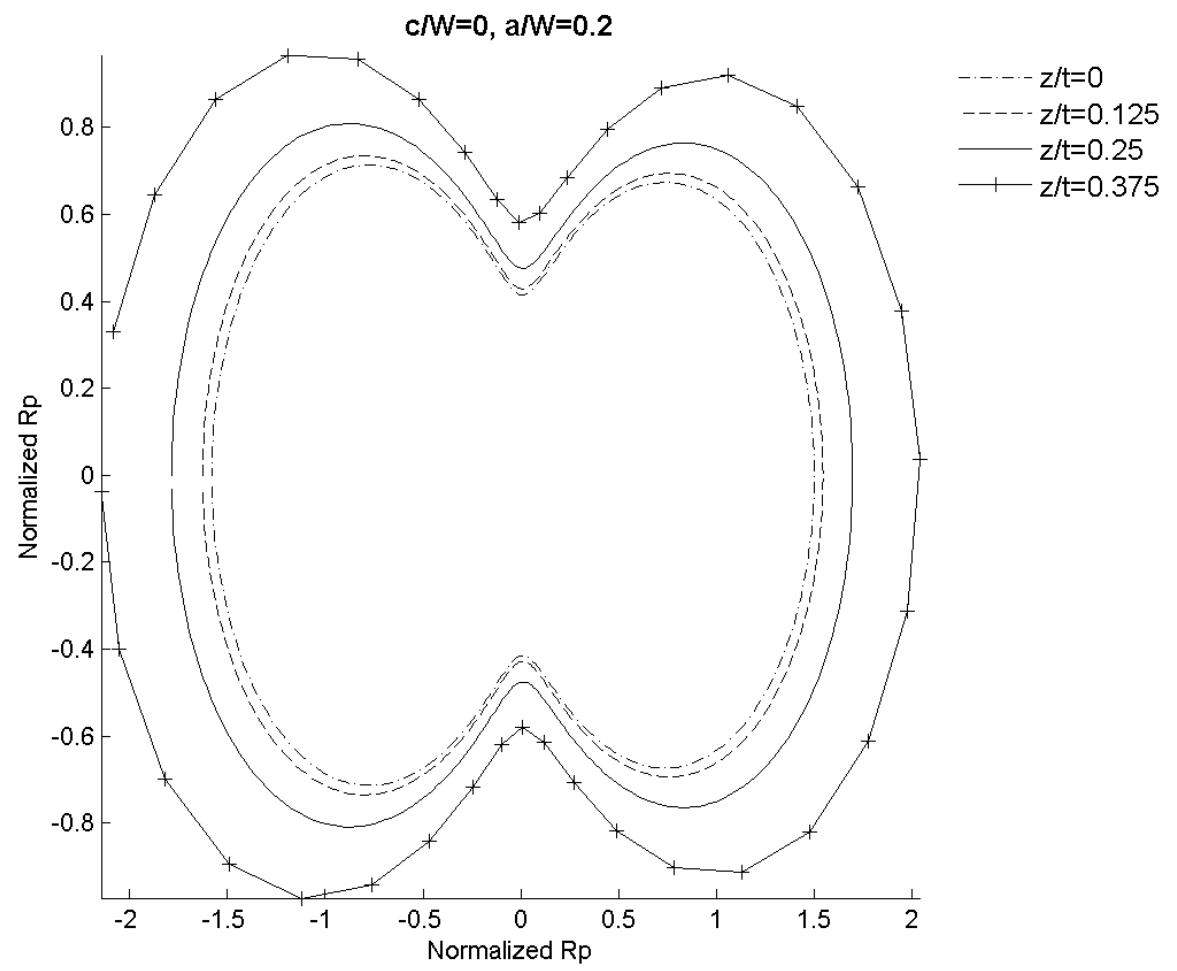

Figure 3.36: Plastic zone plot for $t / W=1.0, a / W=0.2$ 


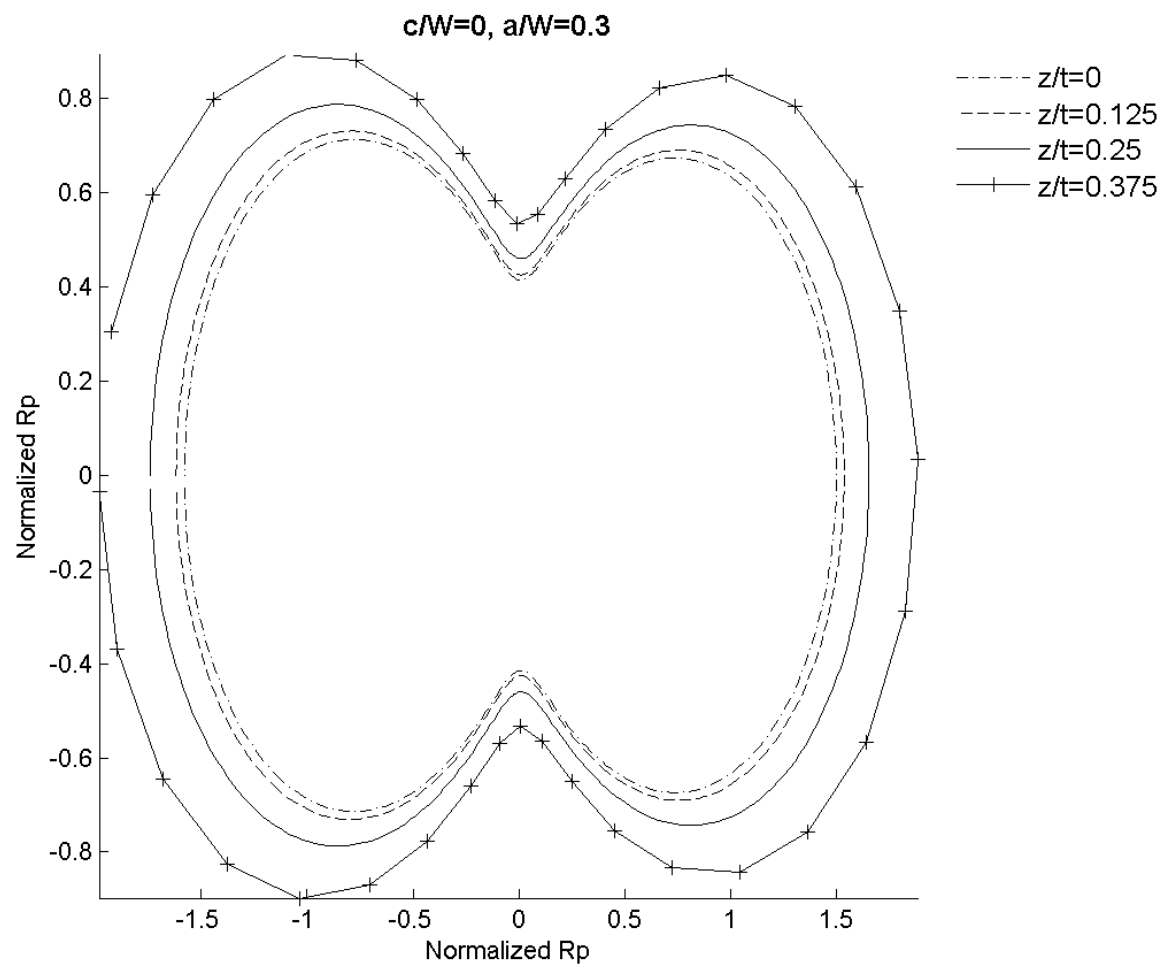

Figure 3.37: Plastic zone plot for $t / W=1.0, a / W=0.3$

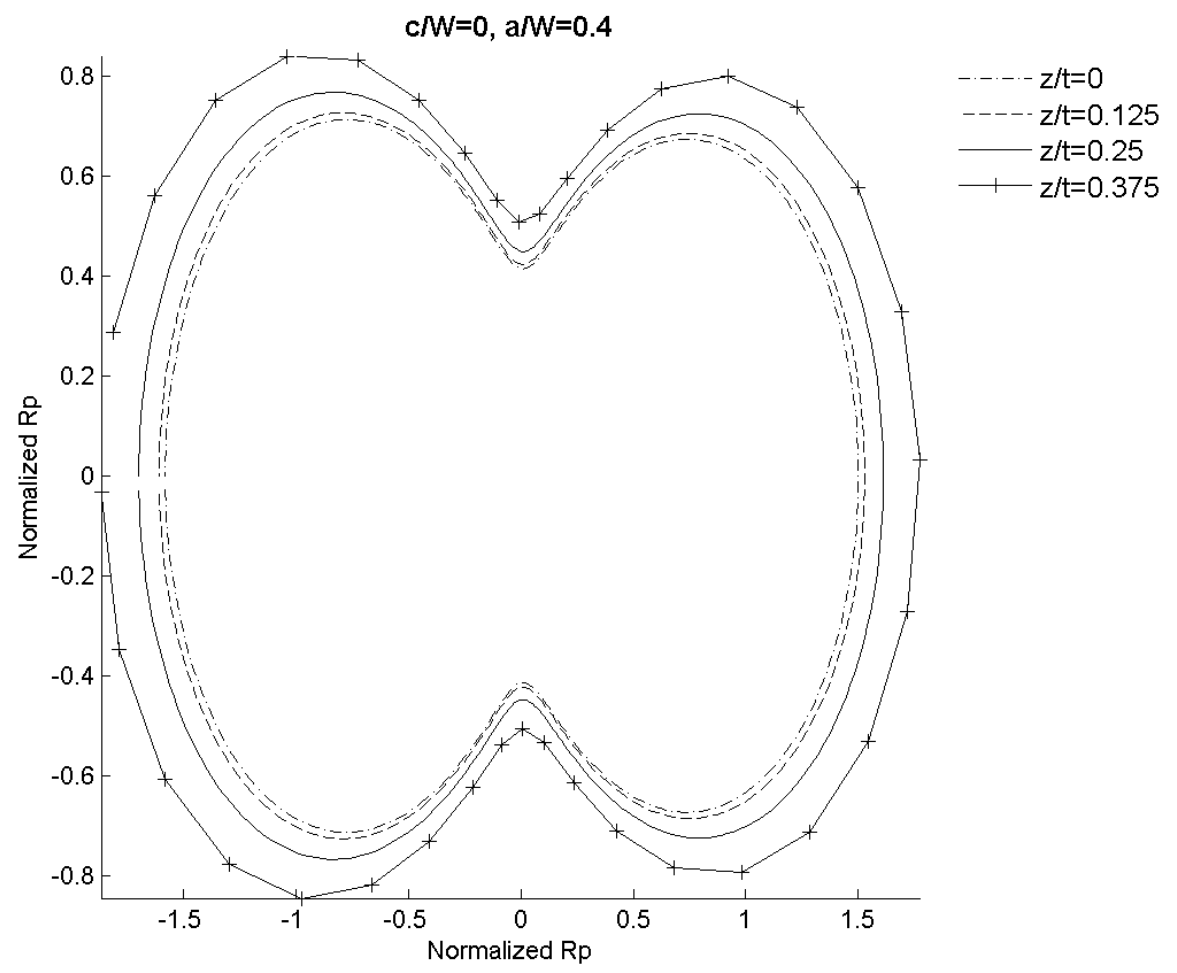

Figure 3.38: Plastic zone plot for $\mathrm{t} / \mathrm{W}=1.0, \mathrm{a} / \mathrm{W}=\mathbf{0 . 4}$ 


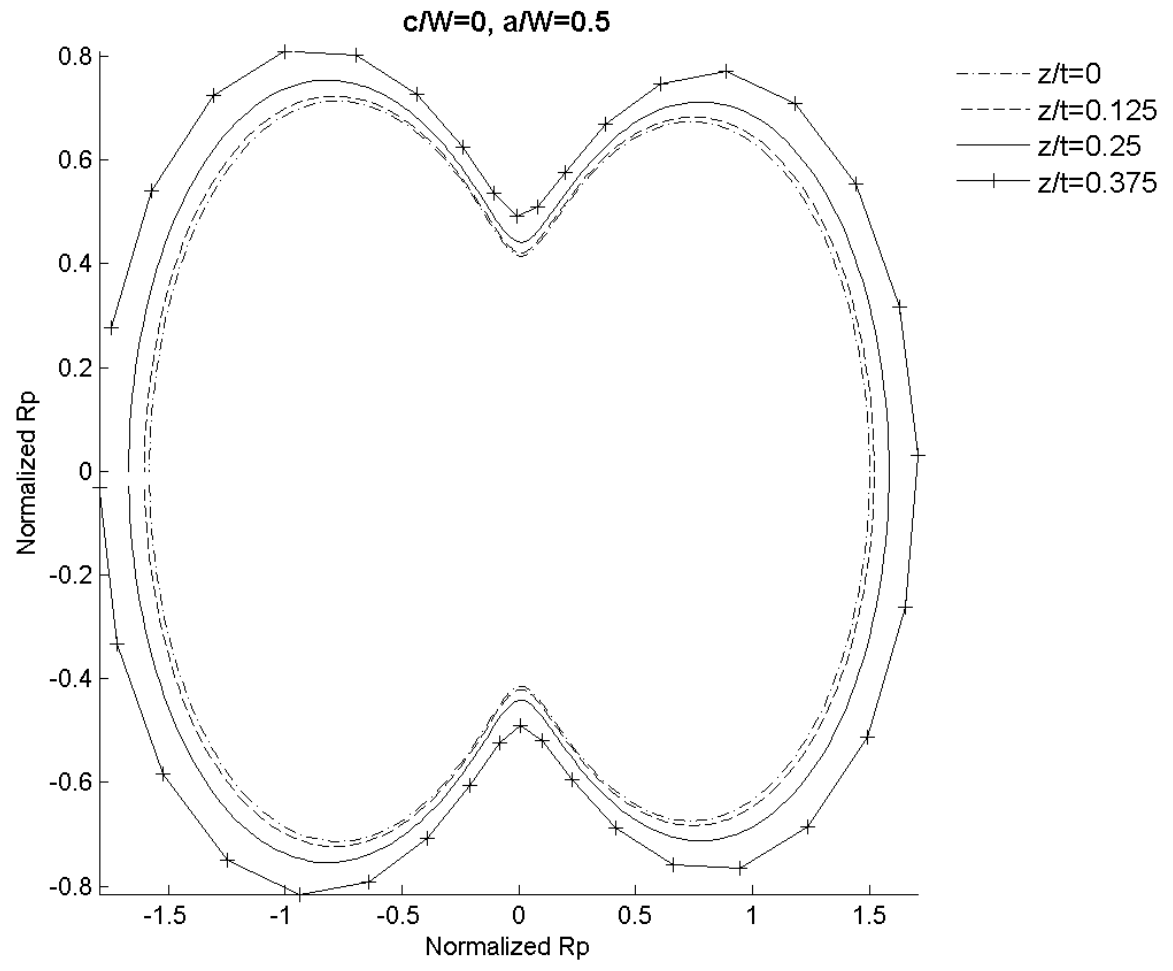

Figure 3.39: Plastic zone plot for $\mathrm{t} / \mathrm{W}=1.0, \mathrm{a} / \mathrm{W}=\mathbf{0 . 5}$

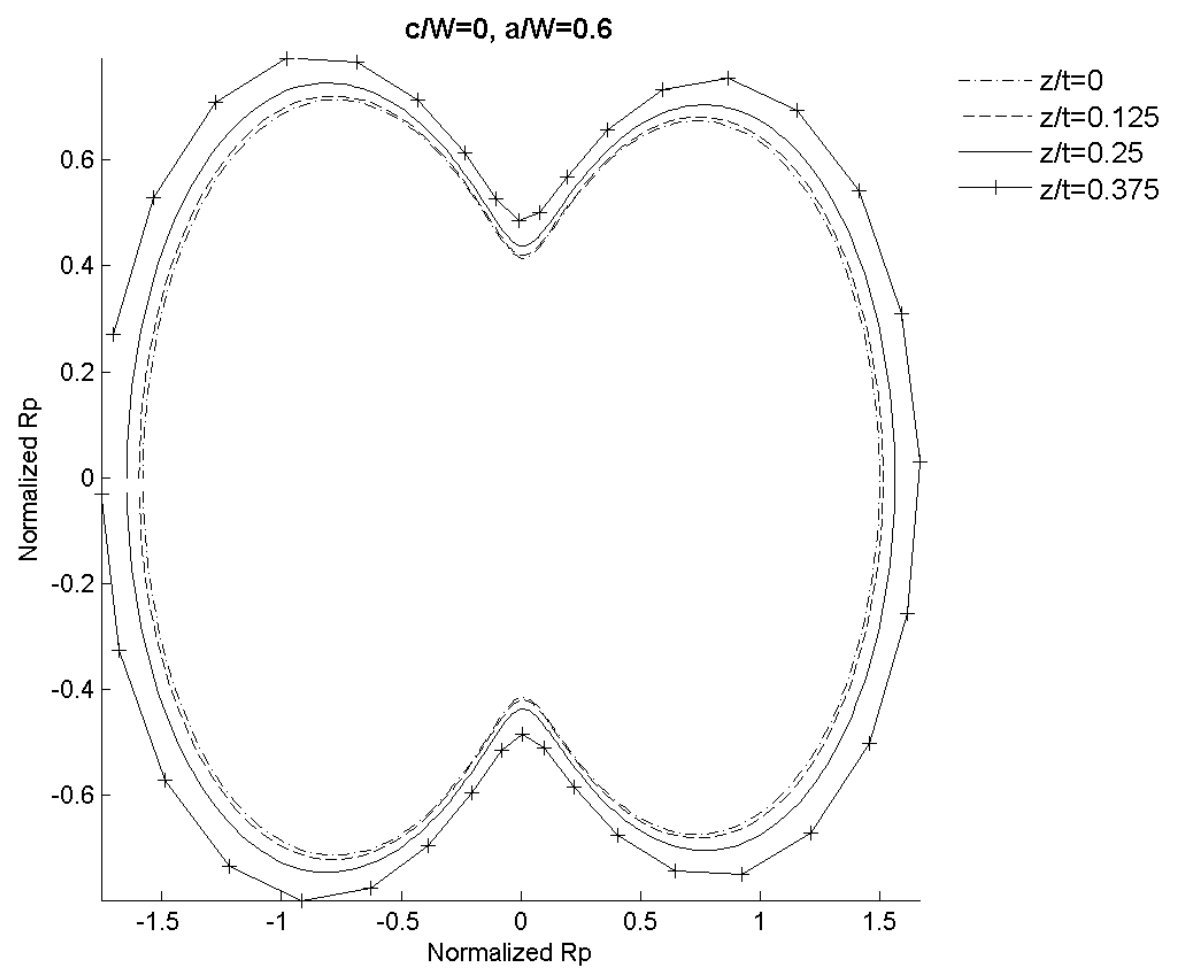

Figure 3.40: Plastic zone plot for $t / W=1.0, a / W=0.6$ 


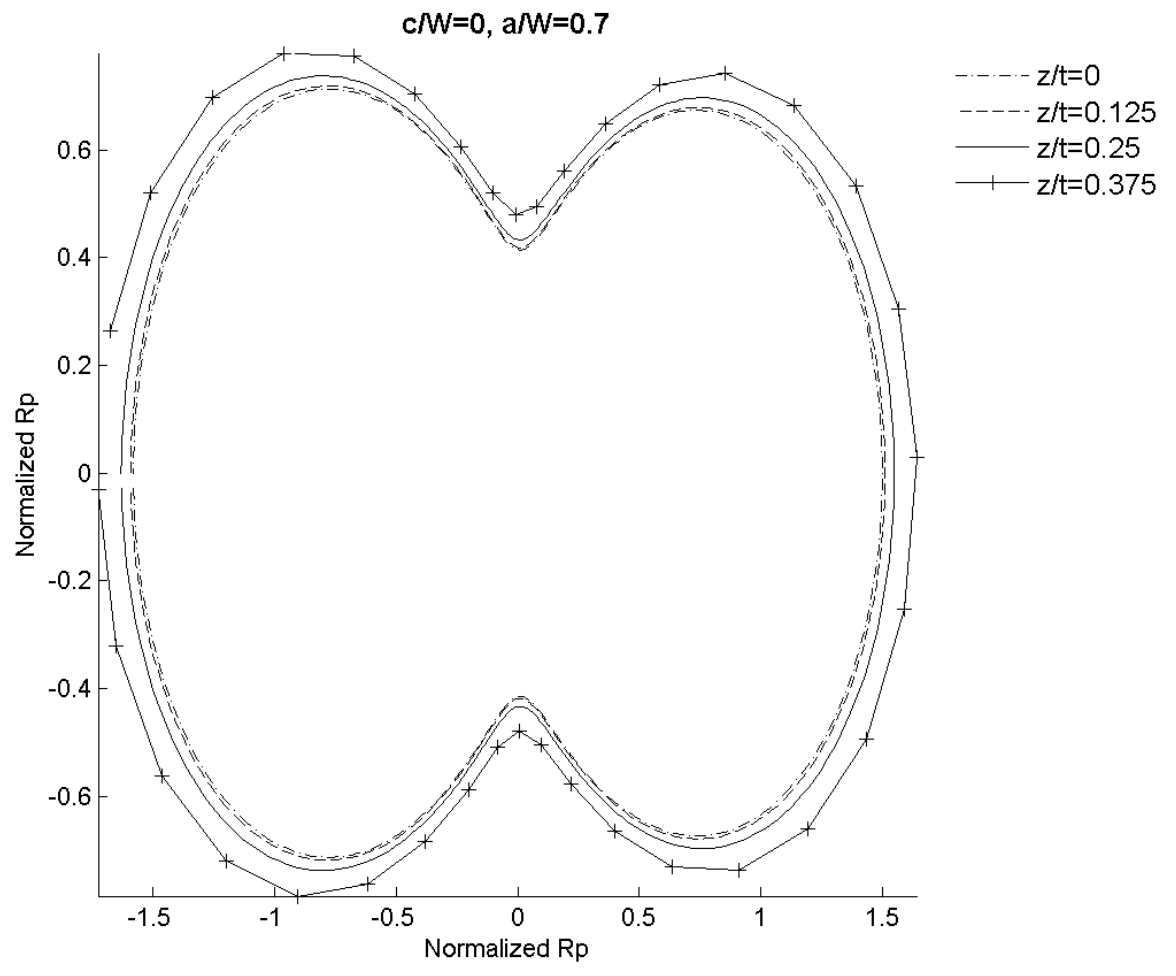

Figure 3.41: Plastic zone plot for $\mathrm{t} / \mathrm{W}=1.0, \mathrm{a} / \mathrm{W}=0.7$ 


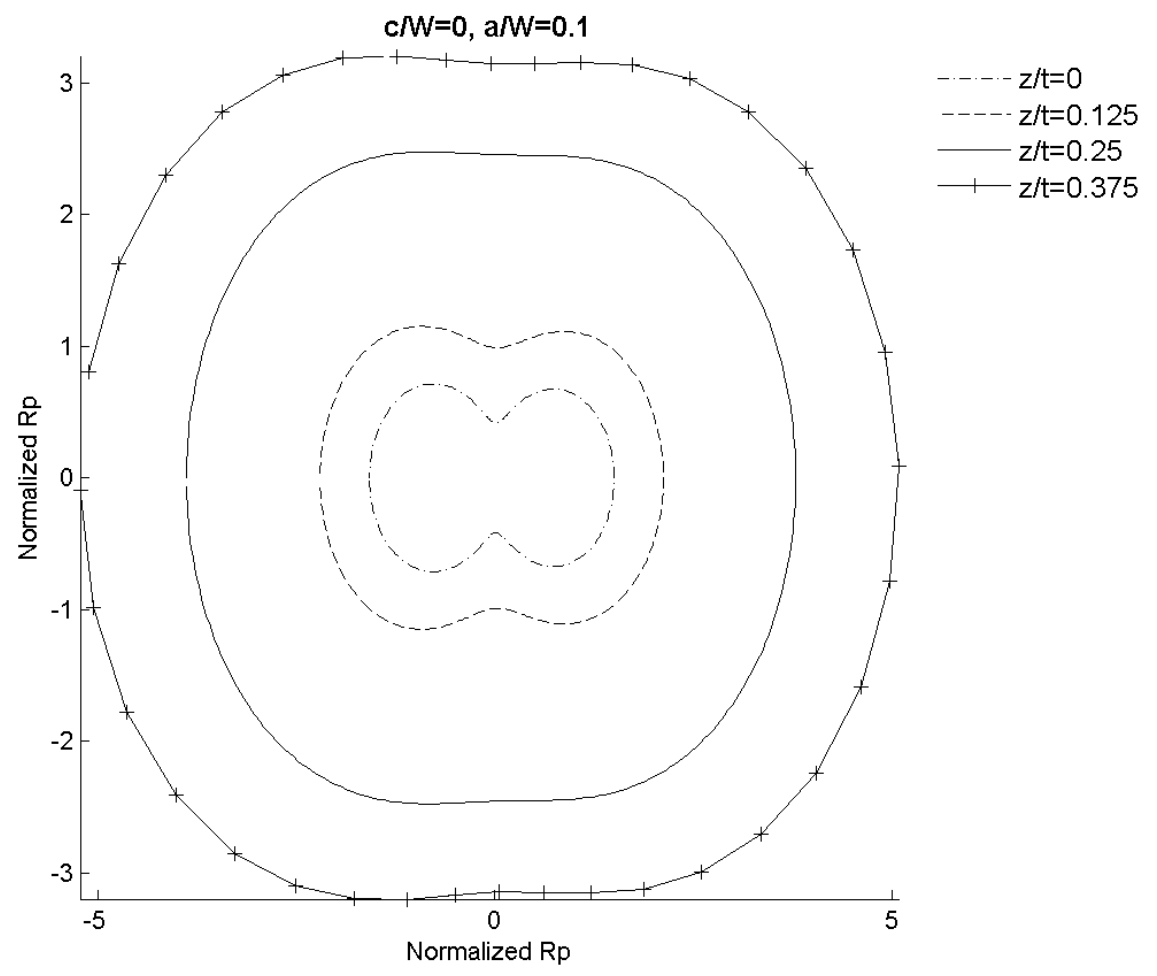

Figure 3.42: Plastic zone plot for $t / W=2.0, a / W=0.1$

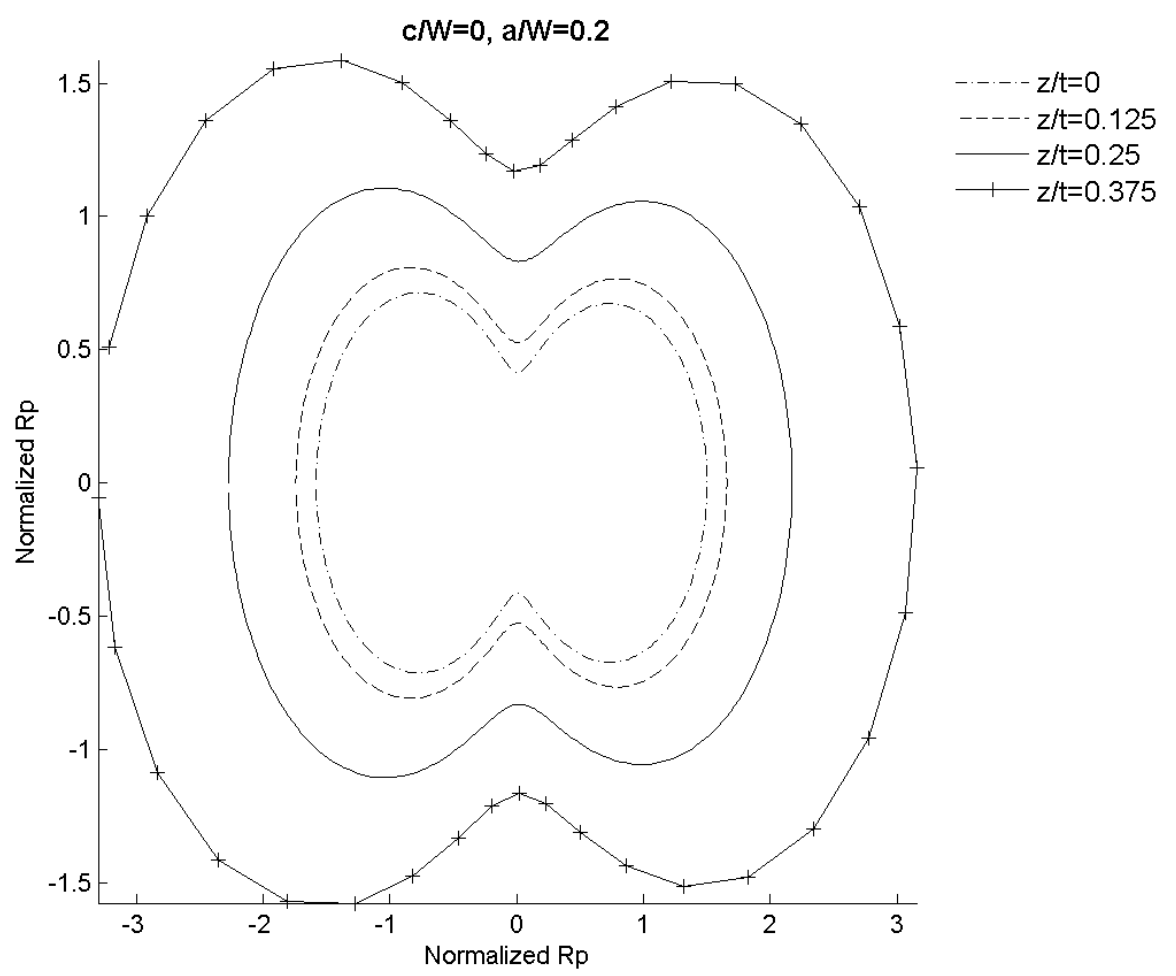

Figure 3.43: Plastic zone plot for $\mathrm{t} / \mathrm{W}=\mathbf{2 . 0}, \mathrm{a} / \mathrm{W}=\mathbf{0 . 2}$ 


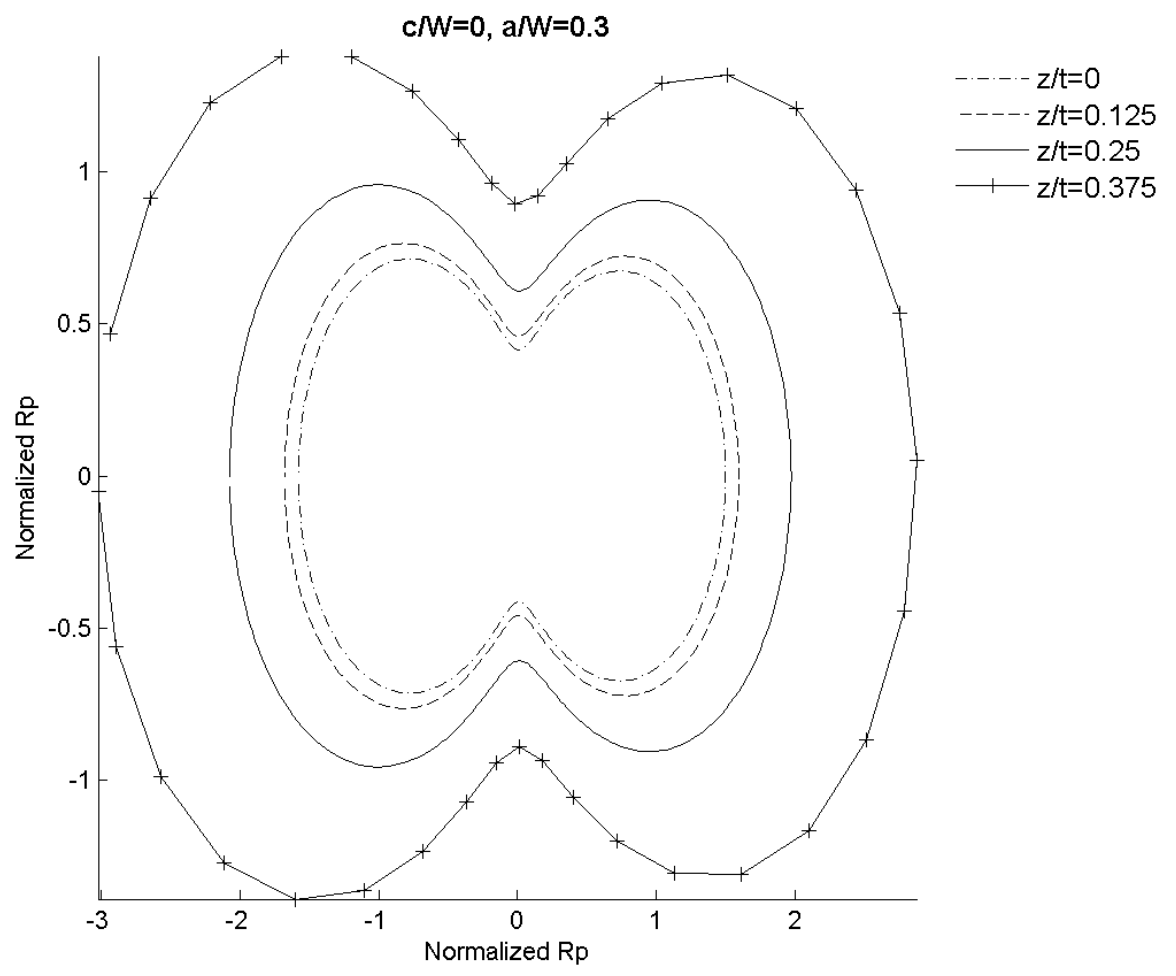

Figure 3.44: Plastic zone plot for $\mathrm{t} / \mathrm{W}=\mathbf{2 . 0}, \mathrm{a} / \mathrm{W}=\mathbf{0 . 3}$

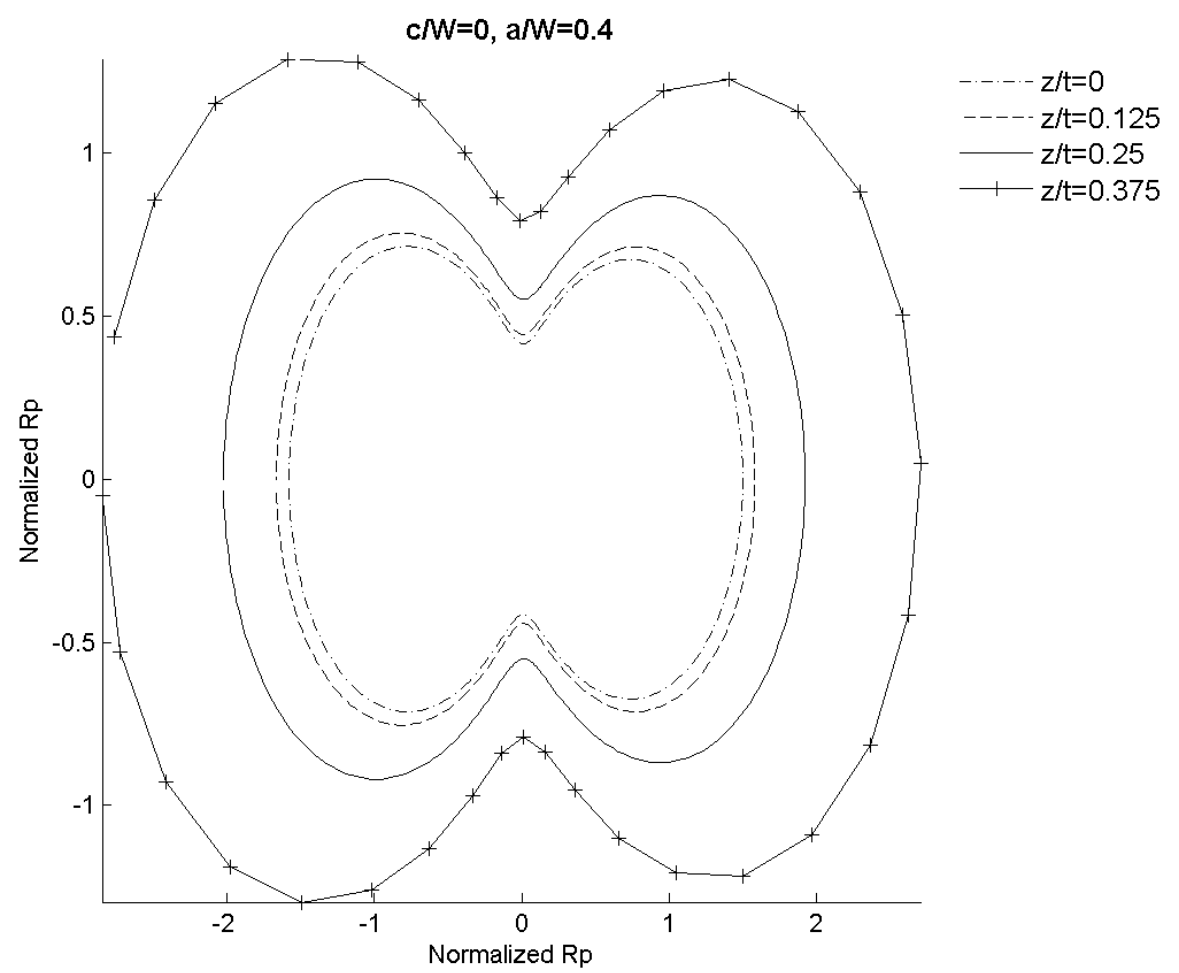

Figure 3.45: Plastic zone plot for $\mathrm{t} / \mathrm{W}=\mathbf{2 . 0}, \mathrm{a} / \mathrm{W}=\mathbf{0 . 4}$ 


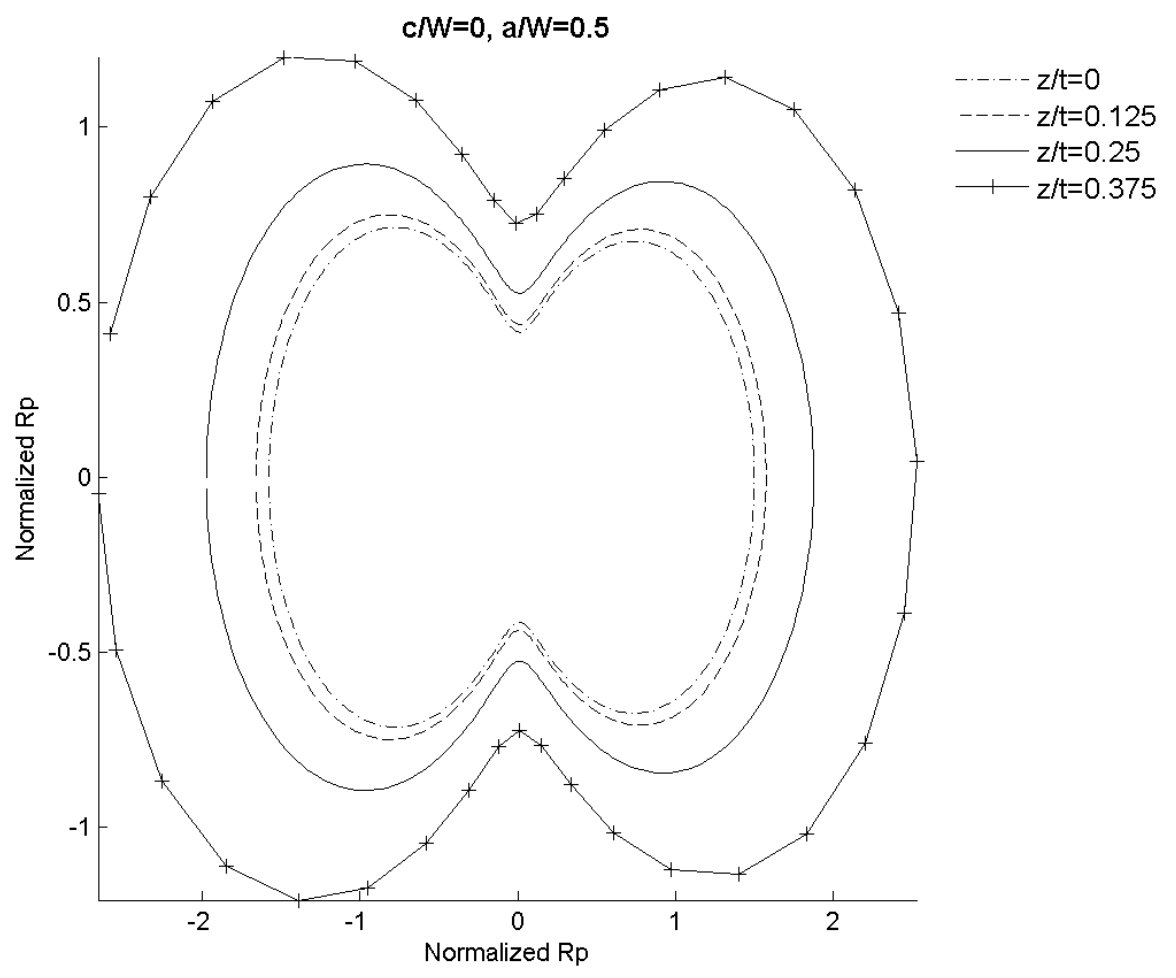

Figure 3.46: Plastic zone plot for $\mathrm{t} / \mathrm{W}=\mathbf{2 . 0}, \mathrm{a} / \mathrm{W}=\mathbf{0 . 5}$

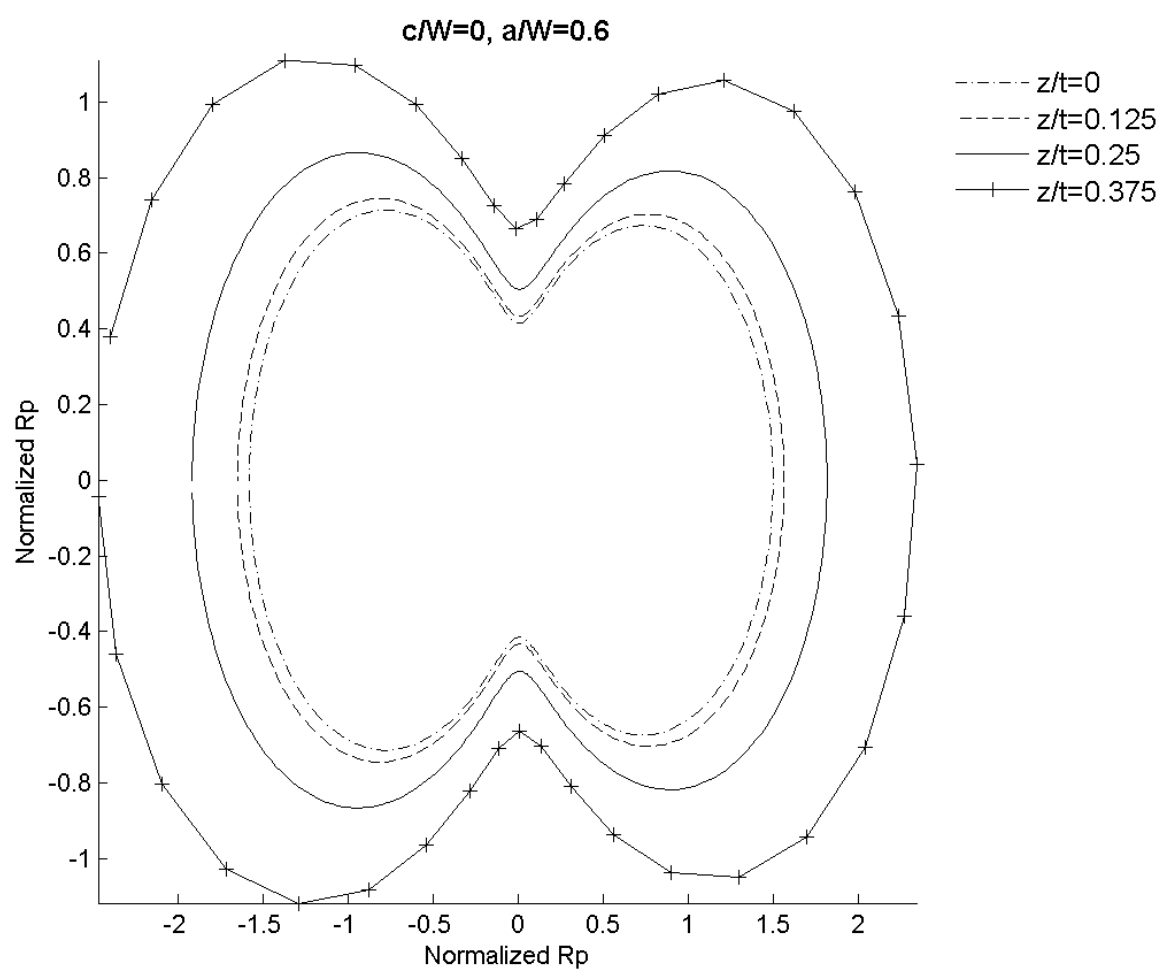

Figure 3.47: Plastic zone plot for $t / W=2.0, a / W=0.6$ 


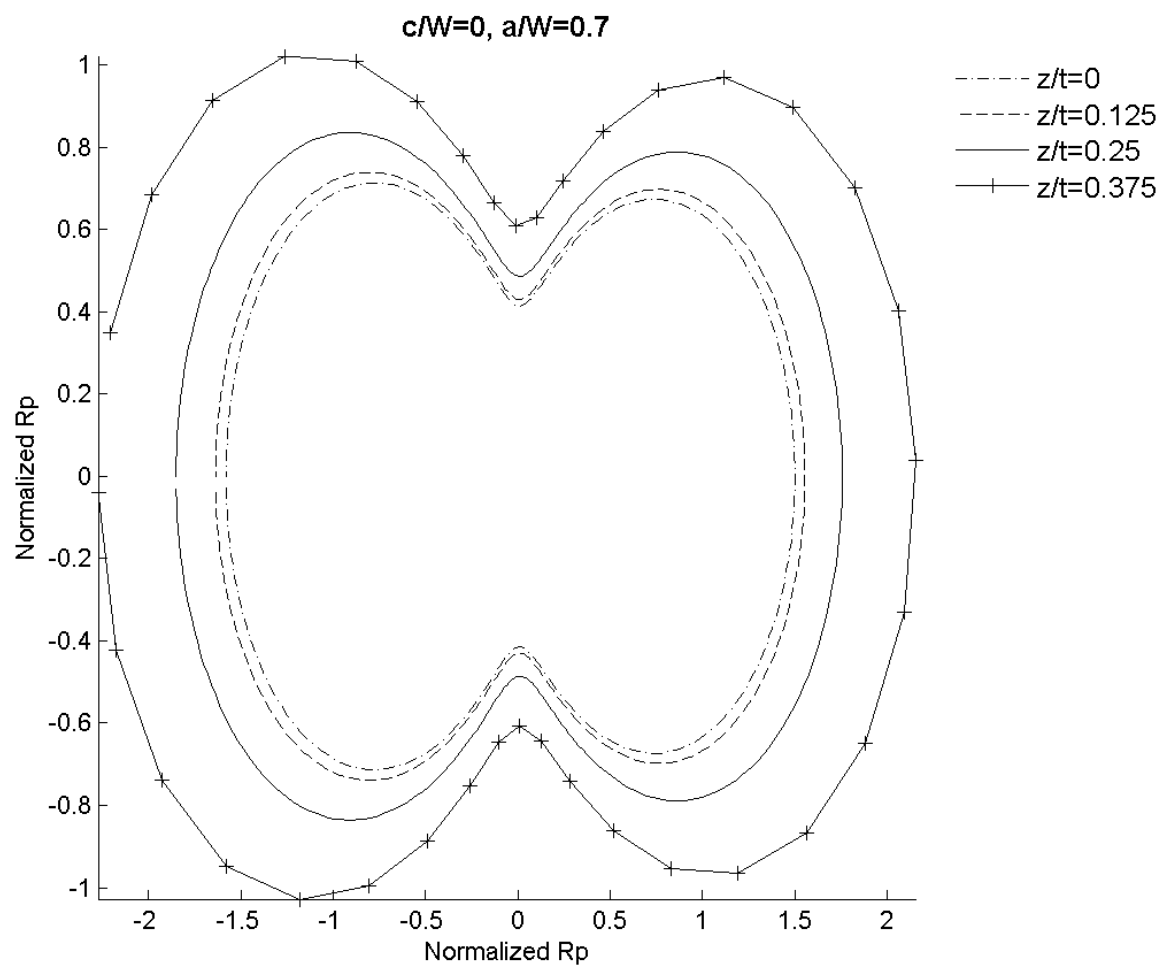

Figure 3.48: Plastic zone plot for $t / W=2.0, a / W=0.7$ 


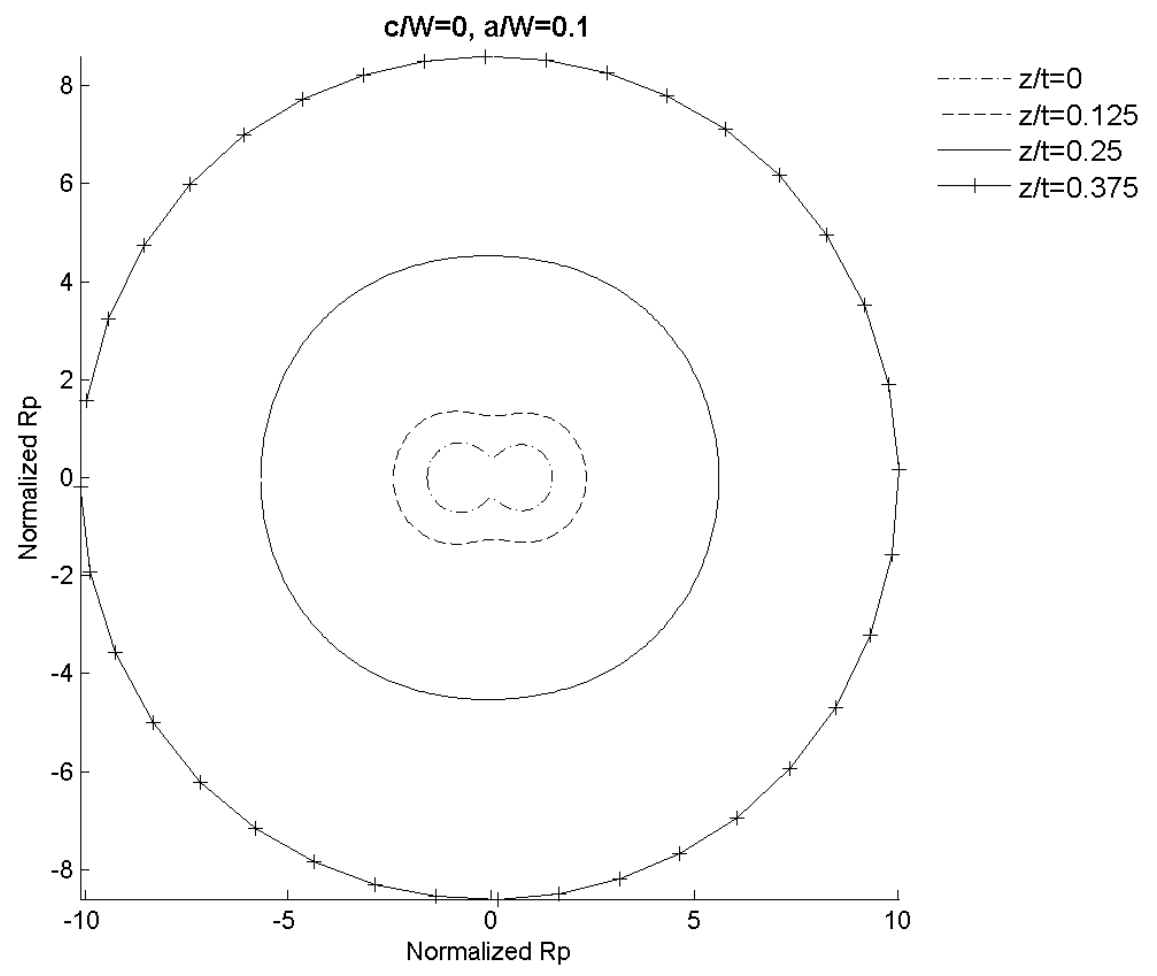

Figure 3.49: Plastic zone plot for $t / W=4.0, a / W=0.1$

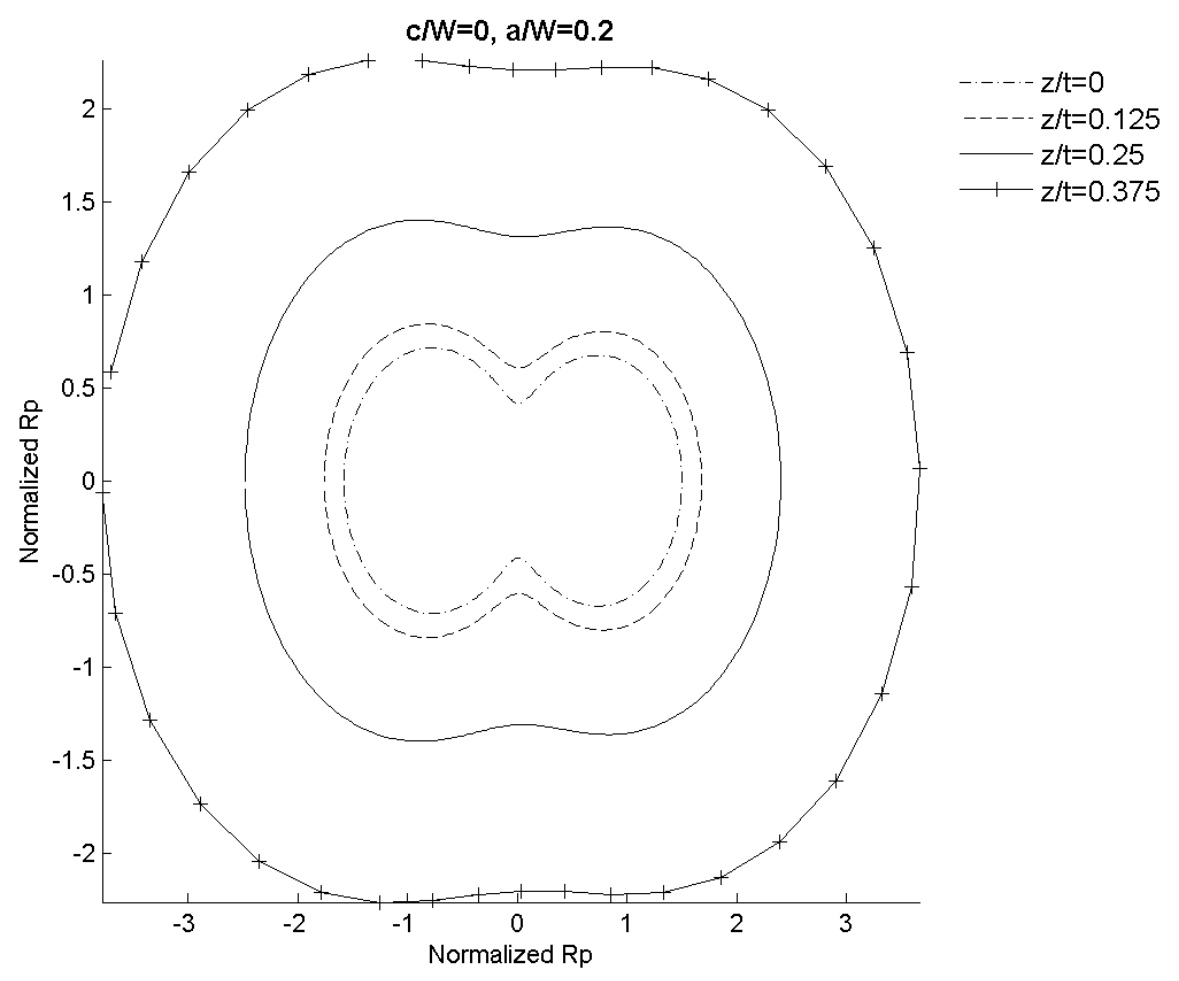

Figure 3.50: Plastic zone plot for $\mathrm{t} / \mathrm{W}=4.0, \mathrm{a} / \mathrm{W}=\mathbf{0 . 2}$ 


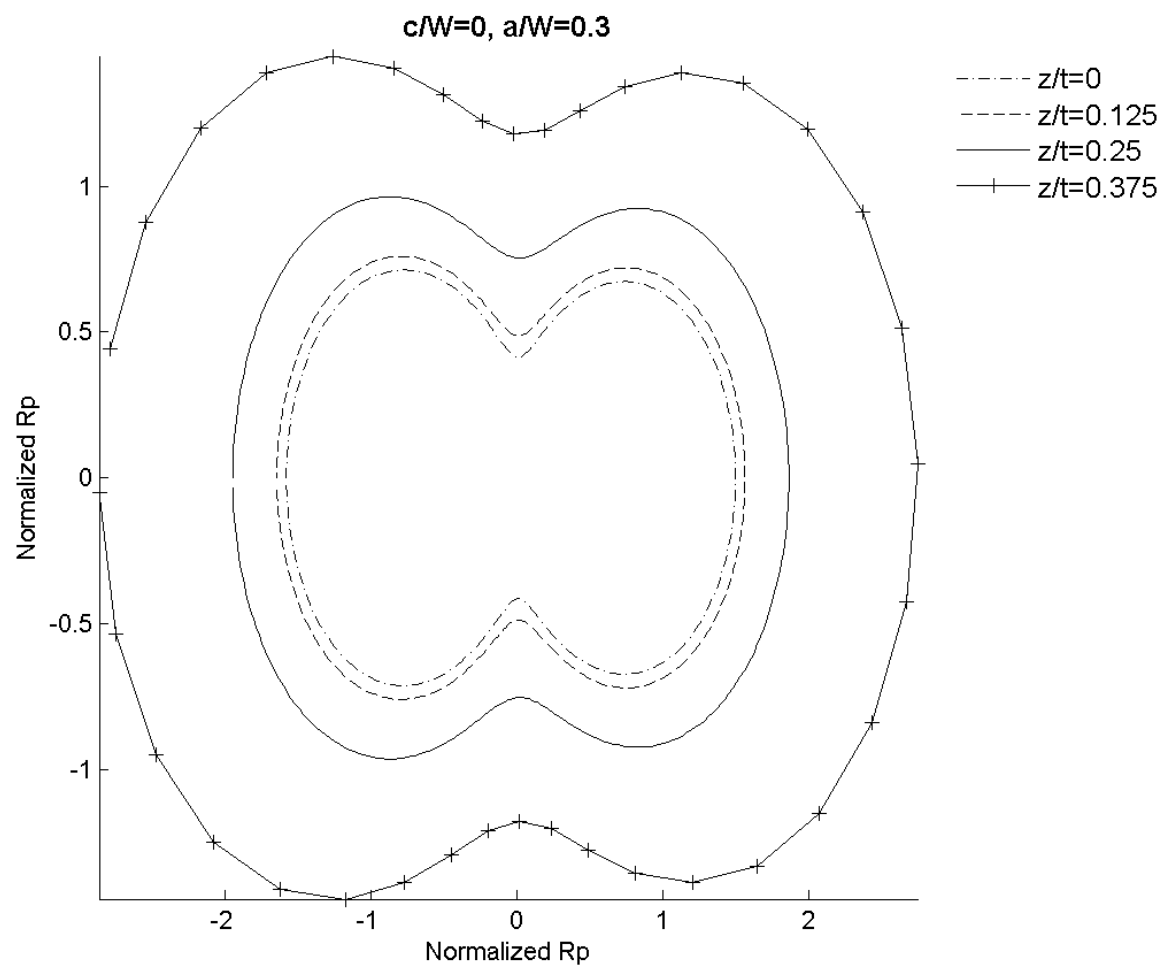

Figure 3.51: Plastic zone plot for $\mathrm{t} / \mathrm{W}=\mathbf{4 . 0}, \mathrm{a} / \mathrm{W}=\mathbf{0 . 3}$

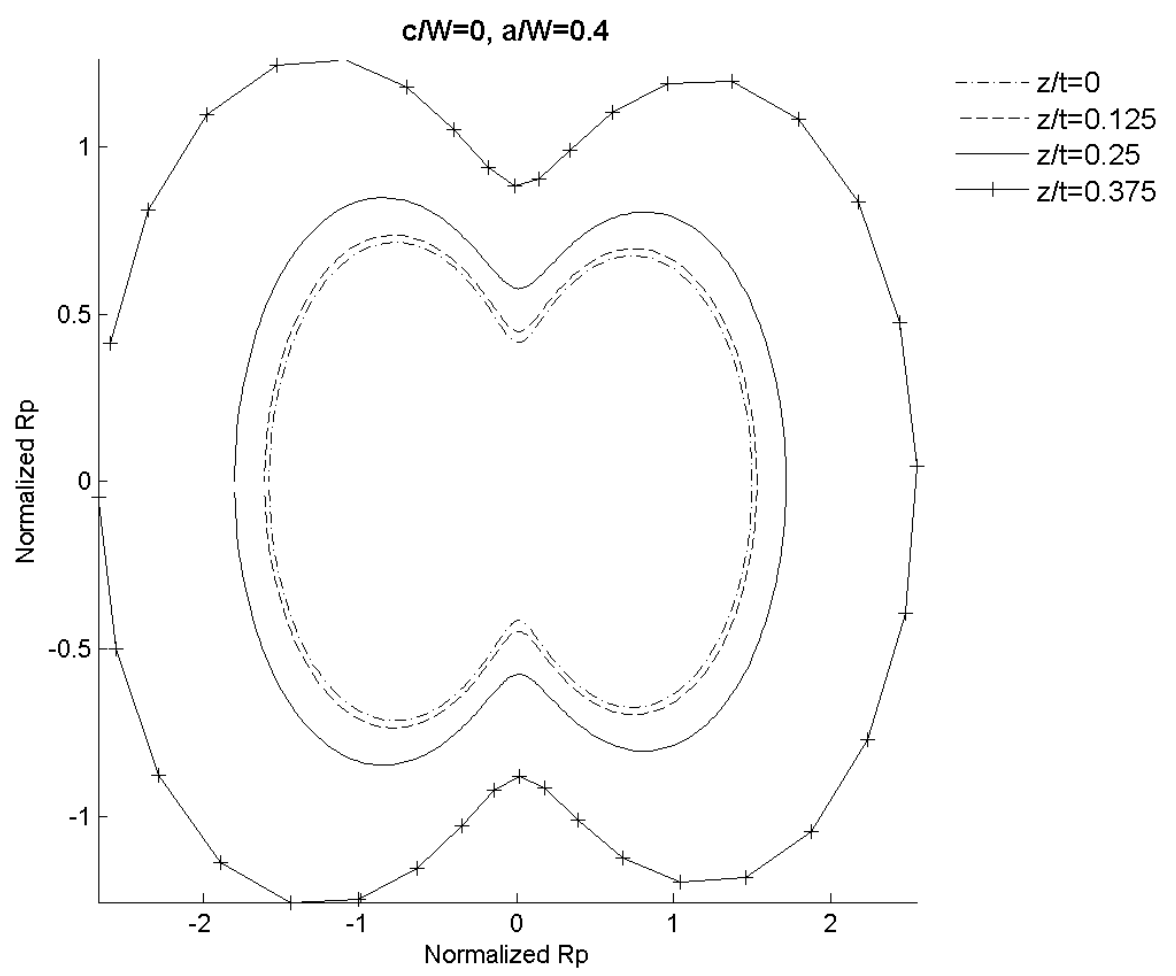

Figure 3.52: Plastic zone plot for $\mathrm{t} / \mathrm{W}=4.0, \mathrm{a} / \mathrm{W}=\mathbf{0 . 4}$ 


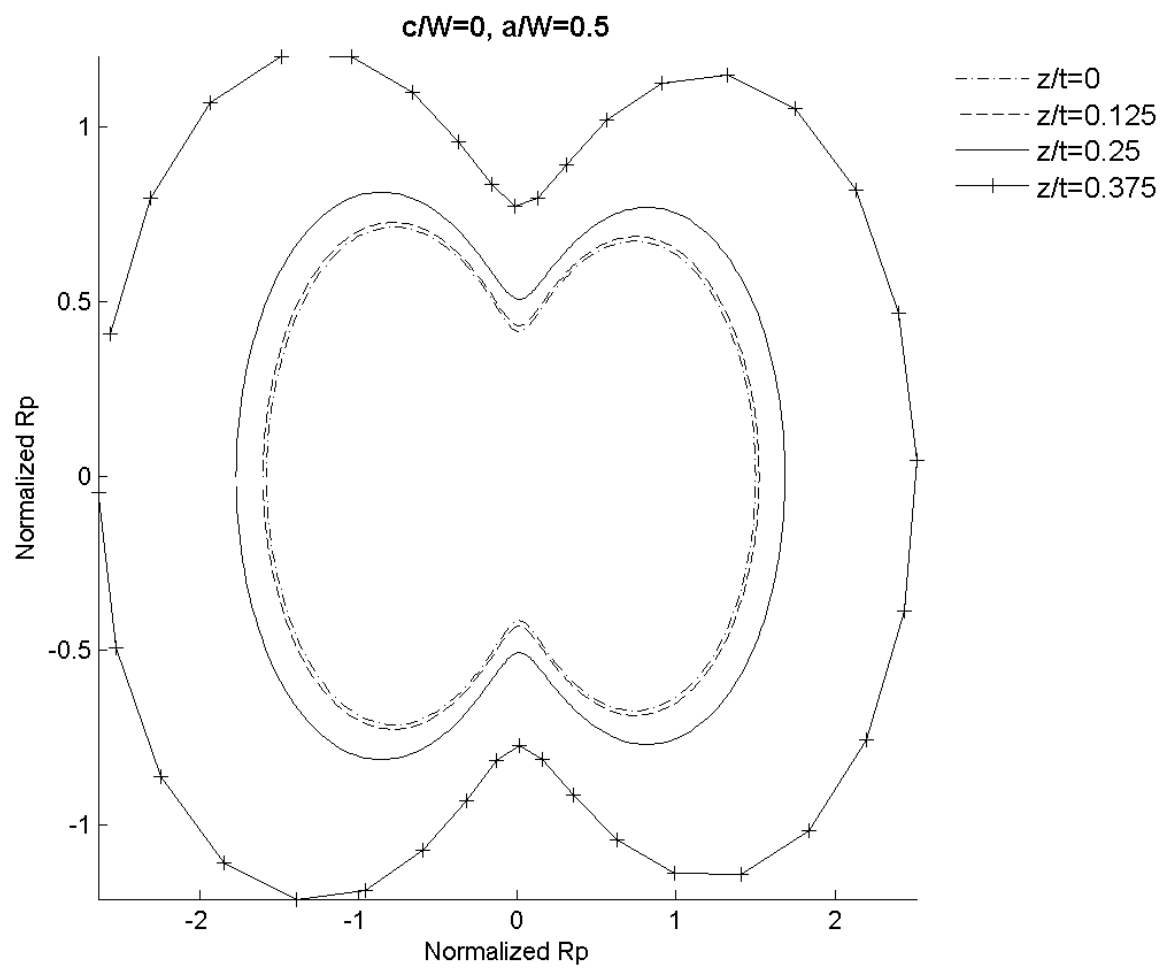

Figure 3.53: Plastic zone plot for $\mathrm{t} / \mathrm{W}=4.0, \mathrm{a} / \mathrm{W}=\mathbf{0 . 5}$

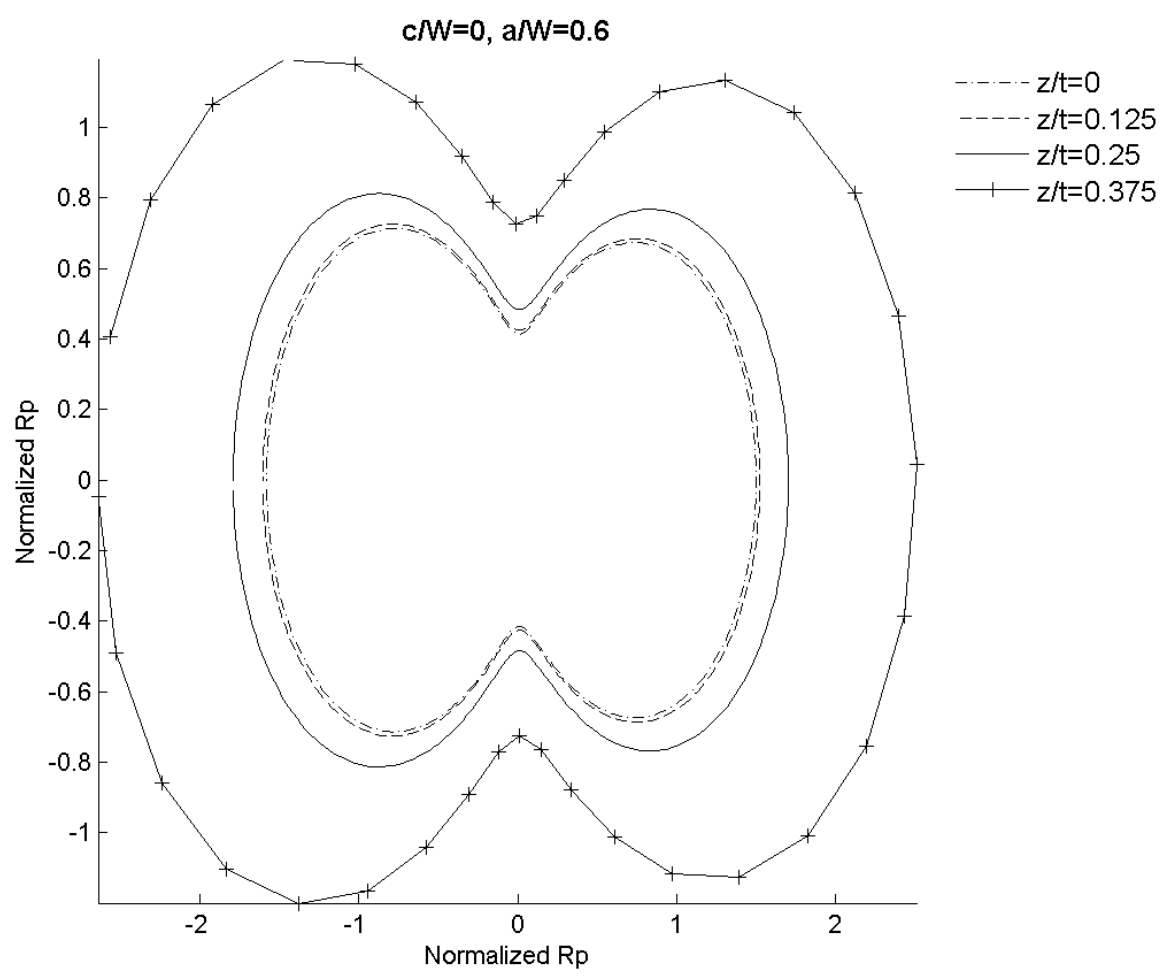

Figure 3.54: Plastic zone plot for $t / W=4.0, a / W=0.6$ 


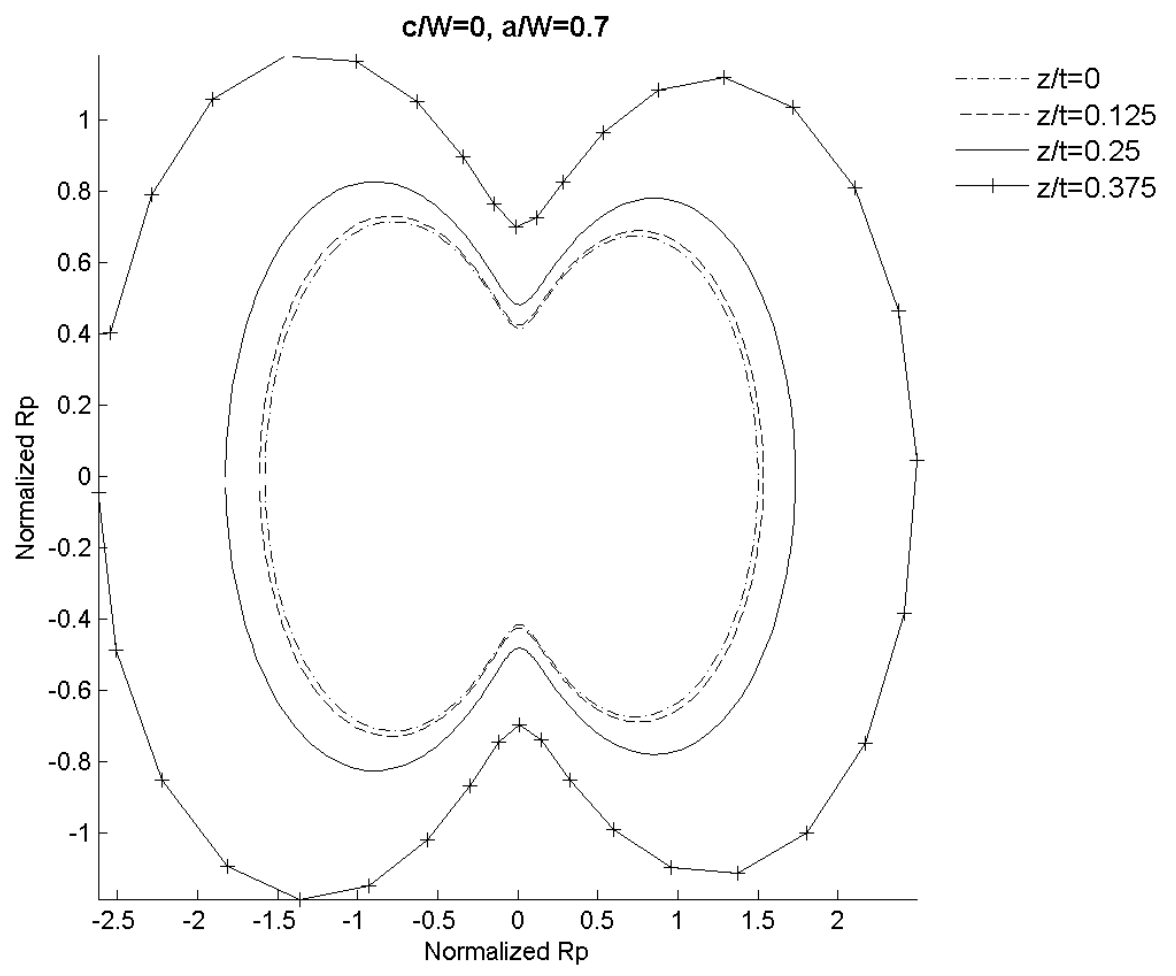

Figure 3.55: Plastic zone plot for $t / W=4.0, a / W=0.7$ 


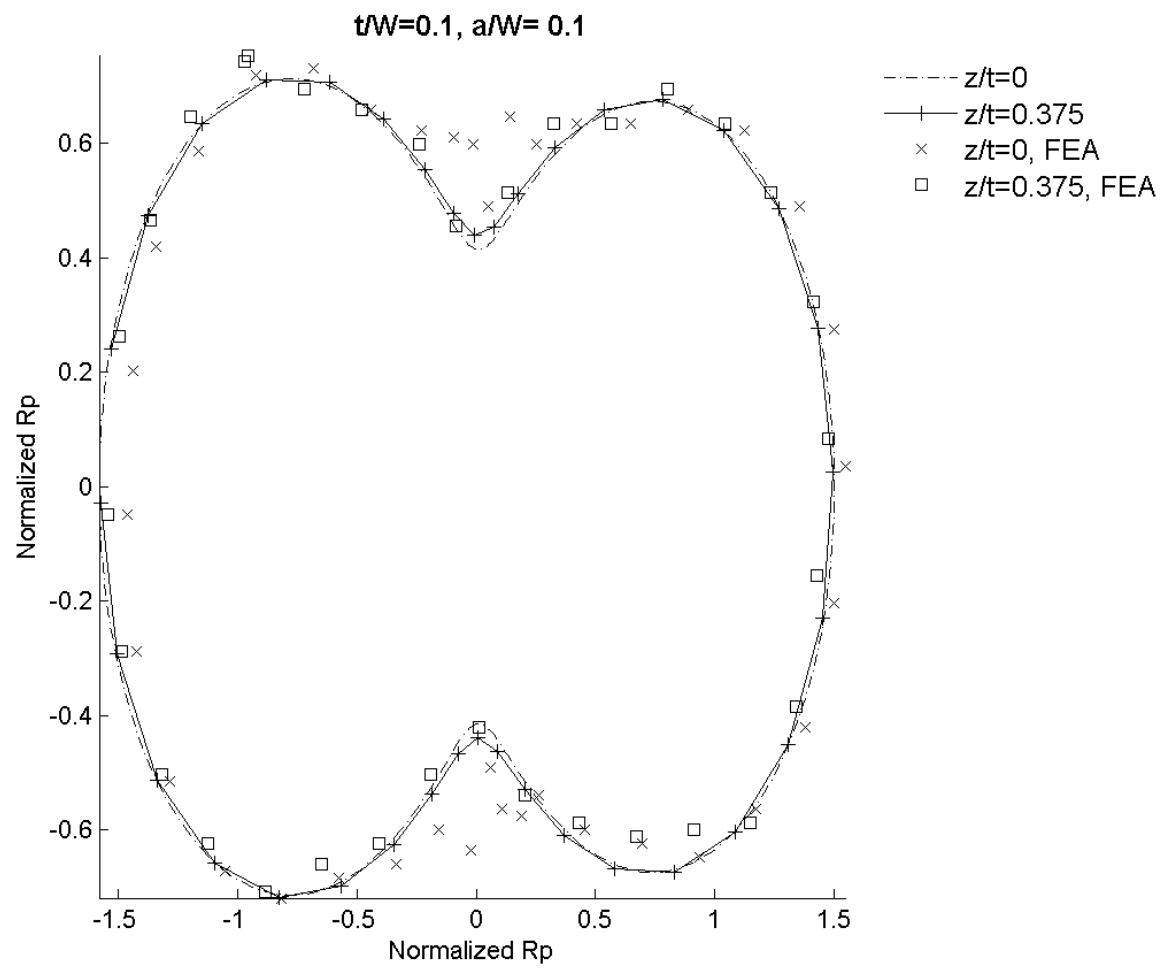

Figure 3.56: Plastic zone comparison for $\mathrm{t} / \mathrm{W}=\mathbf{0 . 1}, \mathrm{a} / \mathrm{W}=\mathbf{0 . 1}$

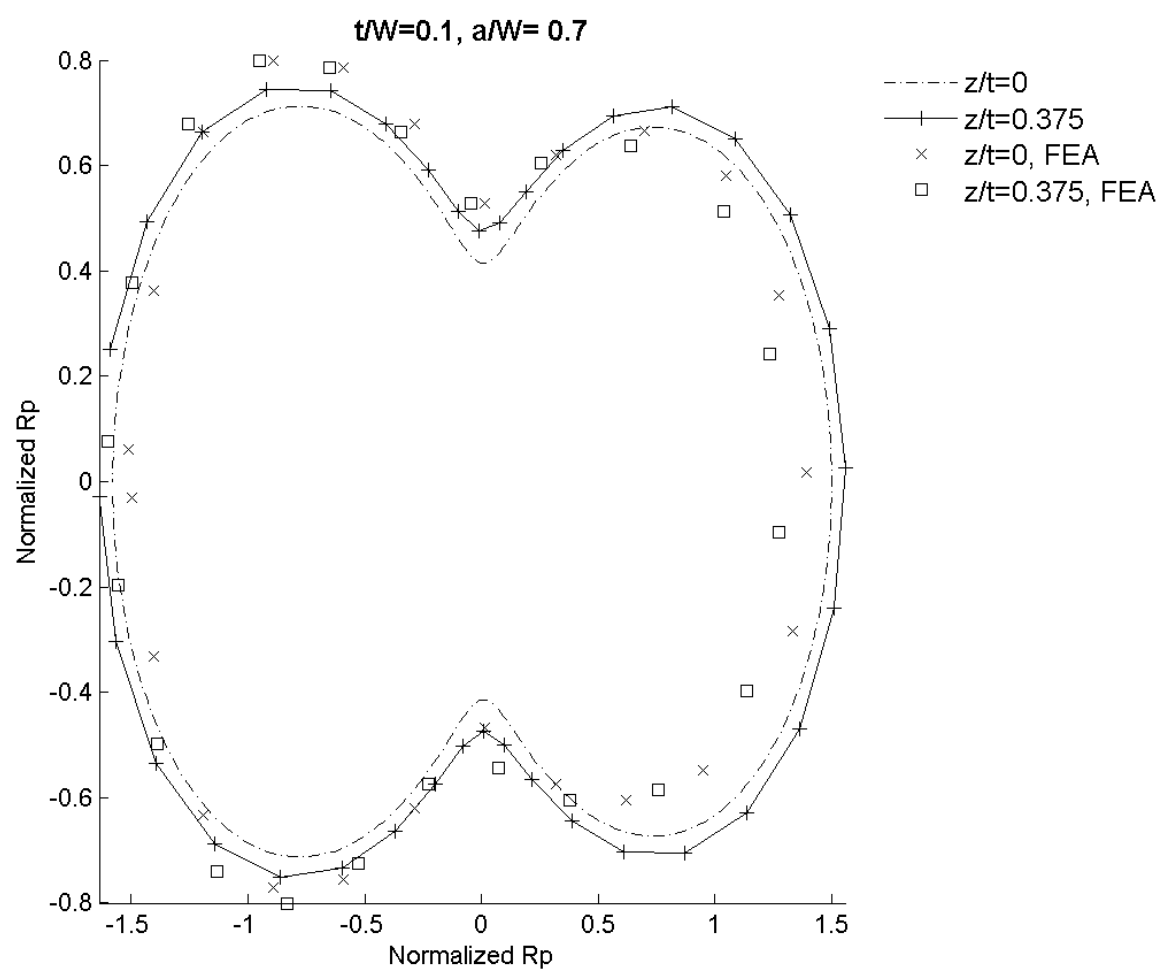

Figure 3.57: Plastic zone comparison for $\mathrm{t} / \mathrm{W}=\mathbf{0 . 1}, \mathrm{a} / \mathrm{W}=\mathbf{0 . 7}$ 


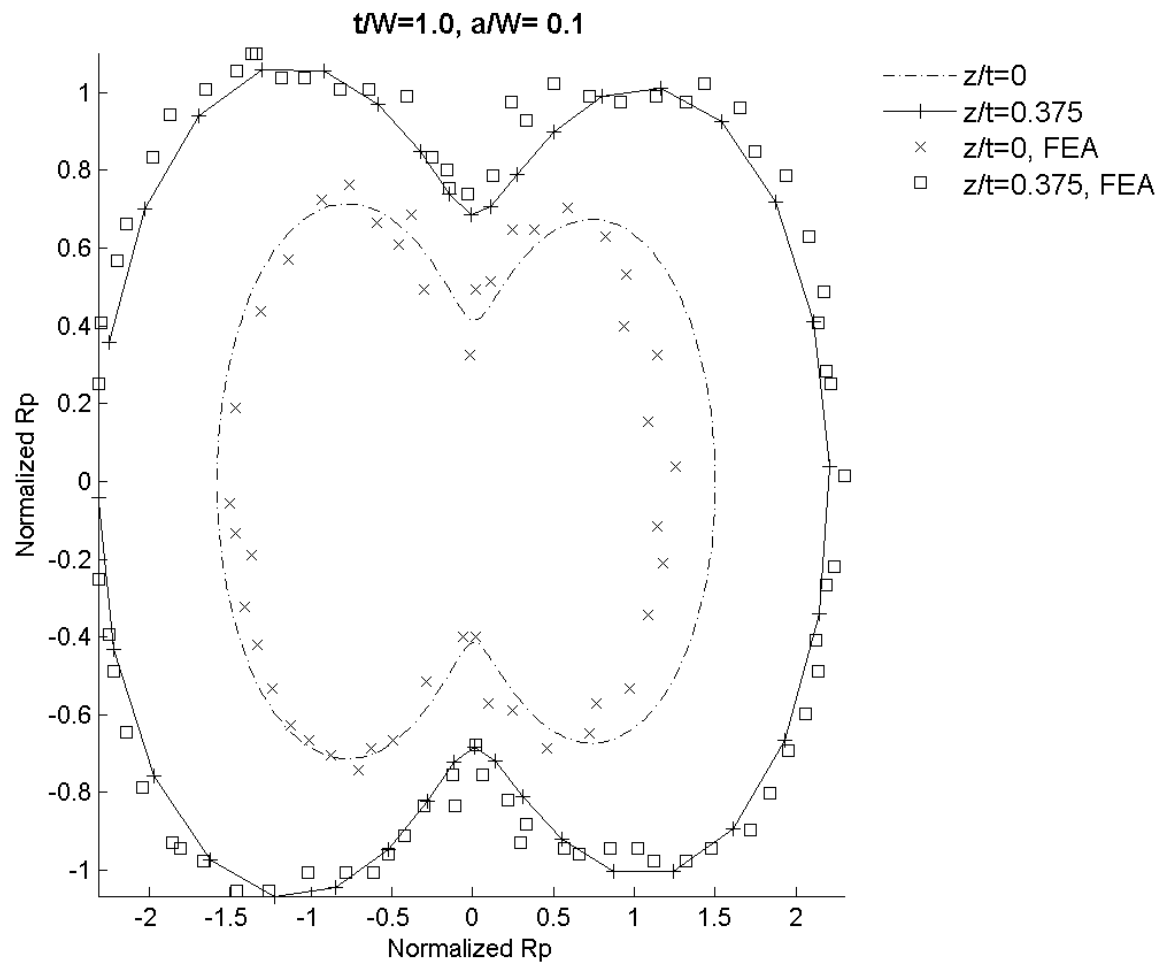

Figure 3.58: Plastic zone comparison for $t / W=1.0, a / W=0.1$

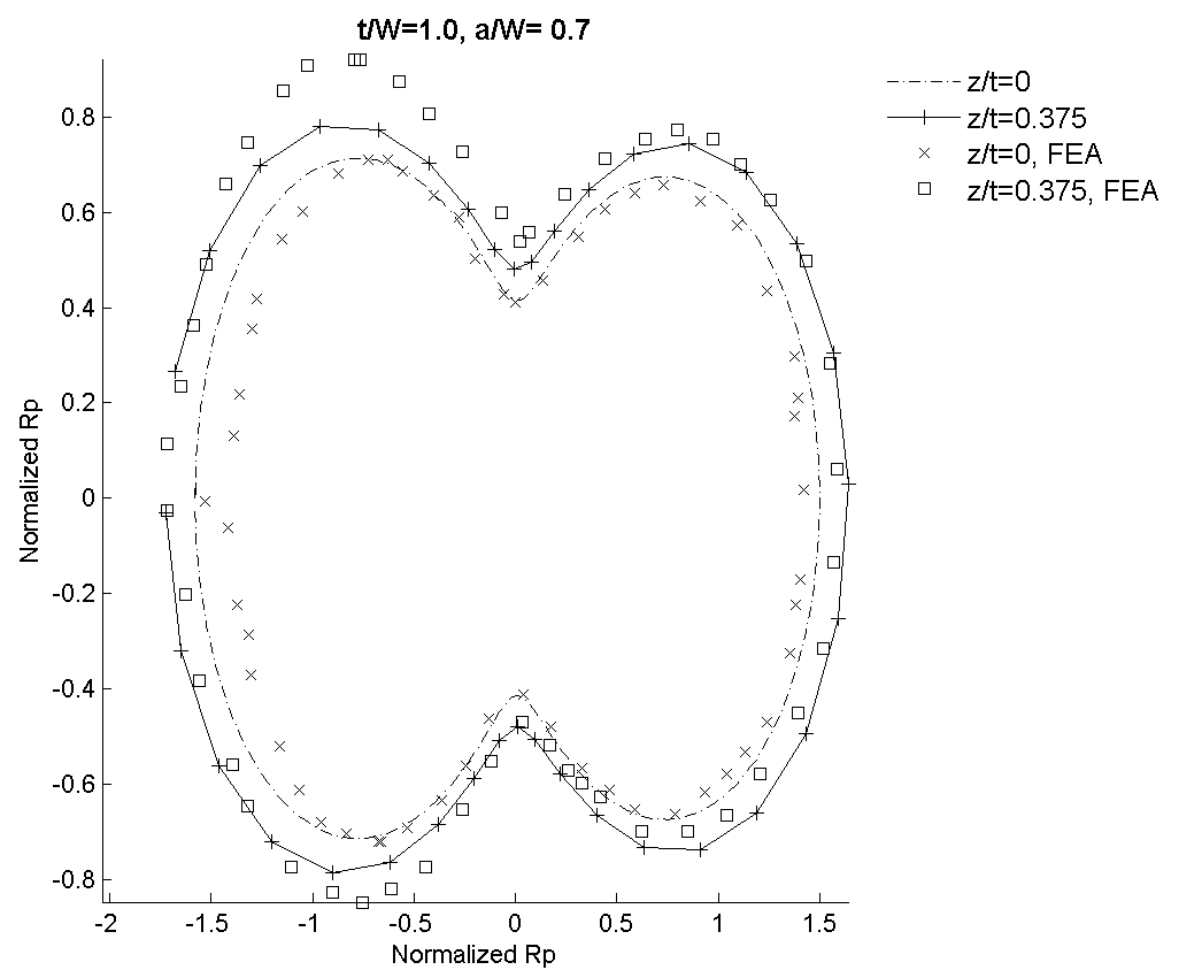

Figure 3.59: Plastic zone comparison for $t / W=1.0, a / W=0.7$ 


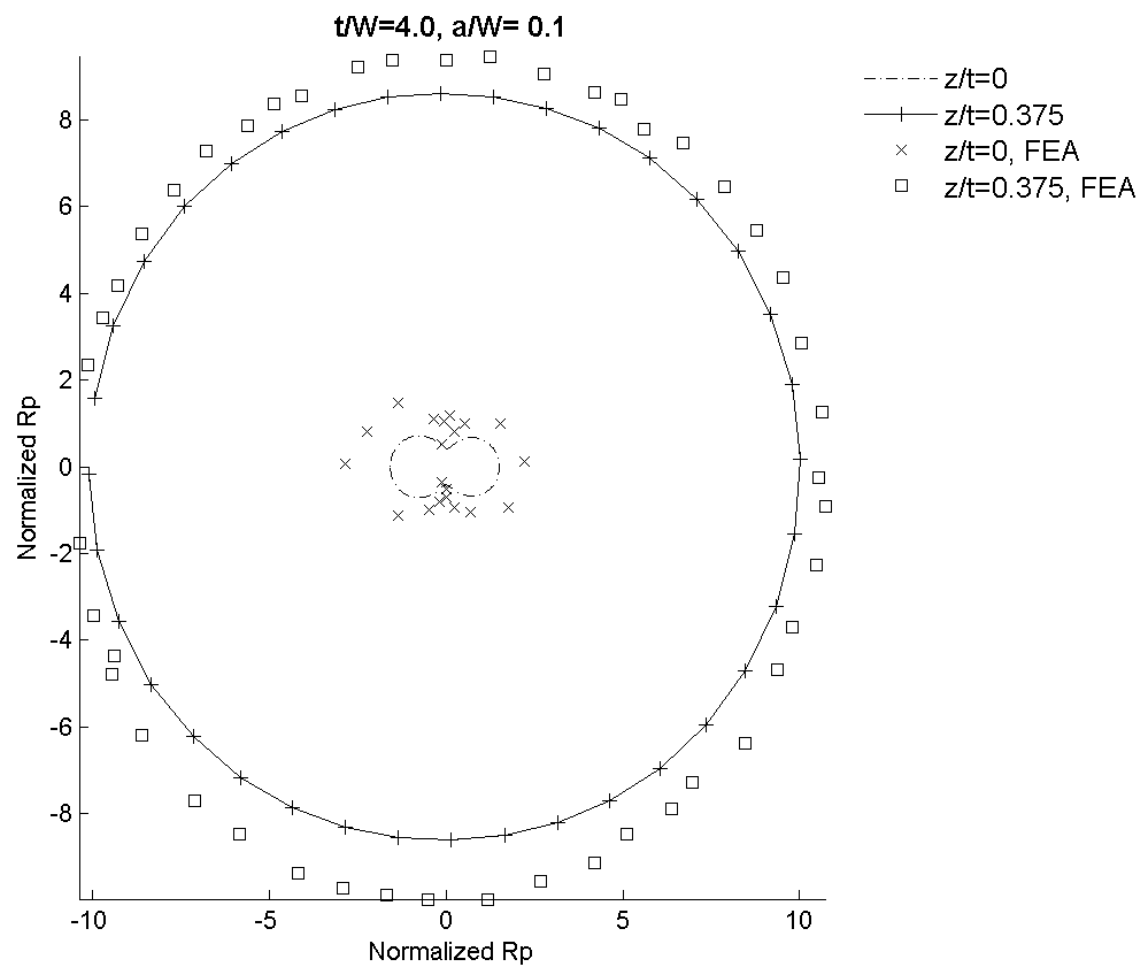

Figure 3.60: Plastic zone comparison for $\mathrm{t} / \mathrm{W}=4.0, \mathrm{a} / \mathrm{W}=\mathbf{0 . 1}$

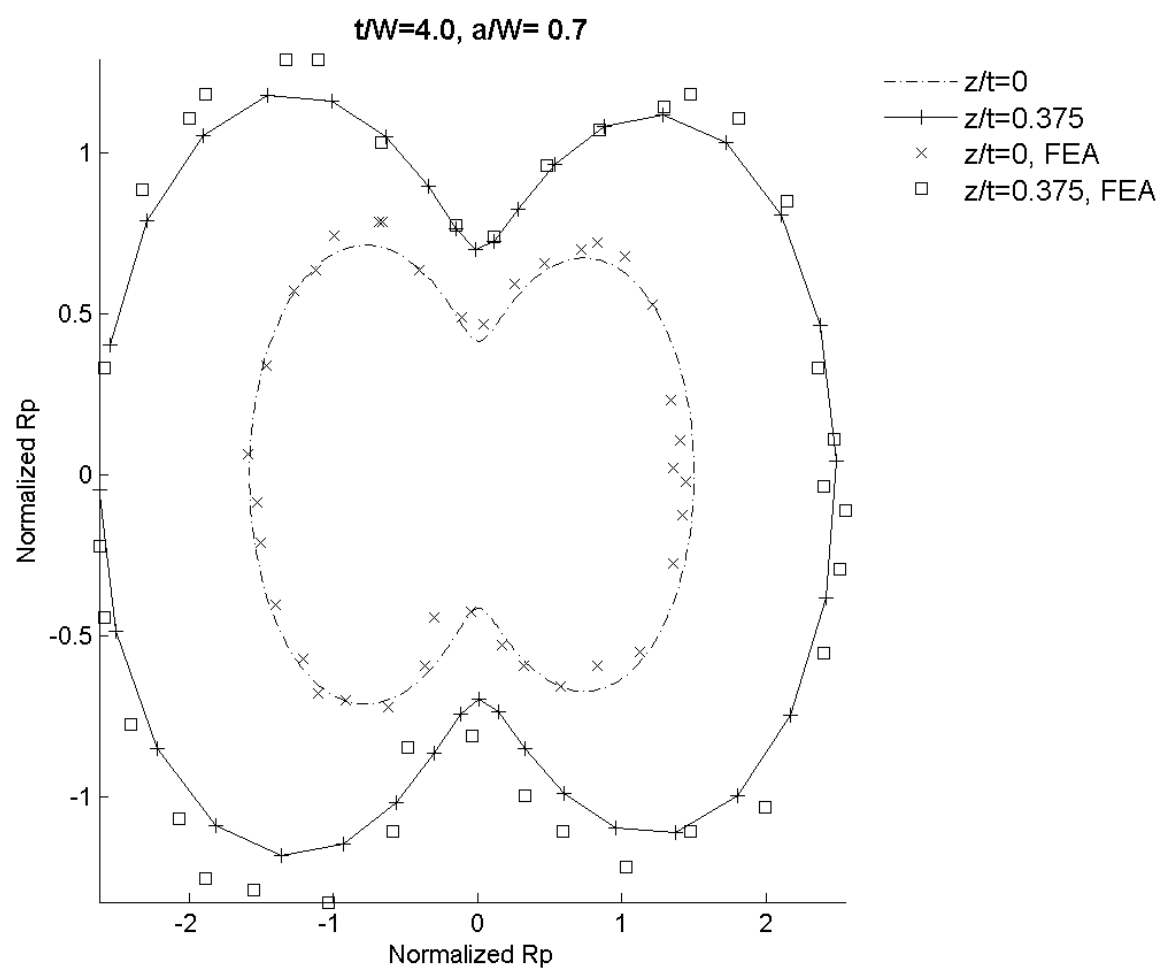

Figure 3.61: Plastic zone comparison for $\mathrm{t} / \mathrm{W}=4.0, \mathrm{a} / \mathrm{W}=\mathbf{0 . 7}$ 


\section{Chapter 4: Three-Dimensional Fracture Analysis of Mixed-mode I/II Test Specimens}

In this chapter, the fracture behaviour of the mixed-mode I/II AFPS specimen was studied in a similar manner as the FPS specimen was studied in the previous chapter. Through extensive 3D finite element analysis, the values of the fracture parameters $K_{I}$, $K_{I I}, K_{I I I}, T_{11}$, and $T_{33}$ were obtained and presented for various specimen geometries. Specimens with a wide range of combinations of $a / W$ and $t / W$ ratios were studied.

Of particular interest to the current work is the effect of the inclusion of $K_{I I I}$ and $T_{33}$ terms on the stress field surrounding the crack tip. Plastic zone analysis was used to study the 3D effects on finite-thickness specimens. The influence of T-stress terms on the plastic zone size was illustrated by comparing the plastic zone calculated using only SIF terms, with the plastic zone calculated including SIF and T-stress terms. For the plastic zones calculated using all SIF and T-stress terms, several different loading levels were considered, demonstrating different amounts of T-stress influence.

\subsection{Finite Element Analysis}

Similarly to the pure mode II case presented in Chapter 3, 3D elastic FEA provides the means to calculate the SIF and T-stress values for the considered mixed-mode loading cases. Abaqus/CAE is again used in the analysis, and the following sections provide details on the creation of the models used, and the extraction of the parameters of interest.

\subsubsection{D FEA Model}

The mixed-mode specimen, the AFPS, combines mode II in-plane shear loading with mode I opening (or bending) loading by offsetting the crack location, setting up asymmetric loading. The amount of mode I contribution is controlled by the value of $c / W$, or the rack offset ratio. Figure 4.1 depicts the AFPS specimen, which is identical to the 
FPS specimen used in Chapter 3; however the value of $c$ is non-zero. Throughout the course of the current research, it was also planned to study the influence of $c / W$. It was determined from the results that the variation of fracture parameters with respect to $c / W$ was not significant, and the value of $c / W$ only served to provide cases with different ratios of mixed mode loading. Therefore, the results discussed in this chapter are for the case of $c / W=0.1$, which provides a good range of mixed-mode loading from primarilymode I to primarily-mode II loading.

Because the FEA model used in this chapter is created in an identical manner as that in Chapter 3, the model specifics that are not changed are not presented again in this chapter. For further details on the mesh design, loading and boundary conditions, and determination of SIF results, refer to Section 3.1.1 in Chapter 3. A typical mesh design for a plate with $t / W=1.0, a / W=0.7$, and $c / W=0.1$, is shown in Figure 4.2.

\subsubsection{SIF and T-stress Extraction}

In an identical manner as performed in Chapter 3, the SIF values are extracted from the ODB produced in Abaqus. Because T-stress is non-zero in the case of mixed-mode loading, these values are calculated and extracted, as are values of $e_{33}$, which are required in the calculation of $T_{33}$. These results are then processed through MATLAB to first normalize (as described in the following section), then sort and plot the values.

\subsubsection{Normalizing Functions: SIF and T-stress}

To provide a more generalized form of the SIF and T-stress solutions, the values of these parameters are normalized as functions of geometry and loading. 


\section{SIF Normalizing Functions}

As shown in the previous chapter, He and Hutchinson provide the mode I and II stress intensity factors as functions of crack and loading geometry. In a mixed-mode I/II case, $K_{I}$ can be described as a function of crack and loading geometry:

$$
K_{I}^{R}=\frac{6 \mathrm{c} Q}{W^{2}} \sqrt{\pi a} F_{I}(a / W)
$$

where $F_{I}$ is the normalized mode I SIF, a function of crack depth and other geometry. $K_{I I}$ and $K_{I I I}$ are defined in the same way as they were in Chapter 3:

$$
\begin{gathered}
K_{I I}^{R}=\frac{Q}{W^{\frac{1}{2}}} \frac{(a / W)^{\frac{3}{2}}}{(1-a / W)^{\frac{1}{2}}} F_{I I}(a / W) \\
K_{I I I}^{R}=\frac{Q}{W^{\frac{1}{2}}} \frac{(a / W)^{\frac{3}{2}}}{(1-a / W)^{\frac{1}{2}}} F_{I I I}(a / W)
\end{gathered}
$$

where $F_{I I}$ and $F_{I I I}$ are the normalized mode II and III SIFs, and Q is shear force, calculated as follows:

$$
Q=P\left(b_{2}-b_{1}\right) /\left(b_{2}+b_{1}\right)
$$

There is no literature expression currently in use to normalize the value of $K_{I I I}$, however since it is coupled with $K_{I I}$, the two SIFs associated with mode II loading have the same normalizing factors.

\section{T- Stress Normalizing Functions}

The first non-singular term in the Williams expansion is known as T-stress, and the nonzero components can be described as: 


$$
\begin{gathered}
T_{11}=T \\
T_{33}=E e_{33}+v T
\end{gathered}
$$

It is typical, in 2D and 3D cases, for T-stress to be normalized with respect to the loading, or far-field stress. In the case of the AFPS specimen, the loading stress associated with Tstress comes from the mode I bending stress. The normalizing factor for the T-Stress is introduced as $\sigma^{\text {nom }}$, the nominal bending stress, and is calculated as follows:

$$
\sigma^{n o m}=\frac{6 c Q}{W^{2}}
$$

The normalized T-stress values are presented as

$$
\begin{aligned}
& T 11=\frac{T_{11}}{\sigma^{\text {nom }}} \\
& T 33=\frac{T_{33}}{\sigma^{\text {nom }}}
\end{aligned}
$$

The values of the five non-dimensional fracture parameters are summarized in Table 4.3 through Table 4.20, at the end of this chapter.

\section{Literature Solutions}

From the literature, there are existing solutions for the values of $F_{I}$ and $F_{I I}$, determined through FEA. These results are available from He and Hutchinson (2000), and are given as 


$$
\begin{gathered}
F_{I}\left(\frac{a}{W}\right)=1.122-1.121\left(\frac{a}{W}\right)+3.740\left(\frac{a}{W}\right)^{2}+3.873\left(\frac{a}{W}\right)^{3} \\
-19.05\left(\frac{a}{W}\right)^{4}+22.55\left(\frac{a}{W}\right)^{5} \\
\text { for } 0 \leq \frac{a}{W} \leq 0.7 \\
F_{I I}\left(\frac{a}{W}\right)=7.264-9.37\left(\frac{a}{W}\right)+2.74\left(\frac{a}{W}\right)^{2}+1.87\left(\frac{a}{W}\right)^{3} \\
-1.04\left(\frac{a}{W}\right)^{4} \\
\text { for } 0 \leq \frac{a}{W} \leq 1
\end{gathered}
$$

As identified in the literature review (Chapter 2), there are currently no literature solutions available for $K_{I I I}, T_{11}$, or $T_{33}$ parameters.

\subsection{FEA Verification}

In order to verify the modelling techniques used to calculate the mixed-mode fracture parameters, results from the current work are compared with literature solutions. Twodimensional FEA is first performed to verify the expected values of normalized SIF, after which results from 3D FEA (at the mid plane) are compared with the literature solutions.

\subsubsection{D FEA Verification}

He and Hutchinson (2000) provide solutions for the AFPS specimen, which have been used for comparison with a two-dimensional model in Abaqus FEA. The current analysis shows good agreement with the literature solutions over the full range of crack depths considered. Figure 4.3 shows the current FEA results for various crack depths and crack

offset ratios $(c / W)$. The percent differences are seen to be small for all cases of $a / W$ and $c / W$, as shown in Table 4.1. It can be expected that the effect of increasing $c / W$ ratio is 
linear, with respect to SIF and T-stress. The normalizing method accounts for $c / W$, and therefore, results can be presented for a single $c / W$ value.

\subsubsection{D FEA Results Comparison}

In Chapter 3, the mode II SIF results at the mid plane, for each $t / W$ ratio considered, were compared with the 2D literature solutions as a means of verifying the FEA modelling methods. An example case of a thick plate $(t / W=4.0)$, with $v=0$, is used as a representative case of $2 \mathrm{D}$ results. The effects of finite thickness are attributed to the effect of Poisson's ratio on the free surface of the plate, which can be verified by comparing the values of the $2 \mathrm{D}$ results to the thick plate, with $v=0$. In a similar manner as in Chapter 3 , the mode I SIF results are compared with the literature solutions, as shown in Figure 4.4.

The results from this comparison show much less variation of $K_{I}$ at the mid plane, with typical results showing deviation from the $2 \mathrm{D}$ solutions of $1-6 \%$. The increase in SIF value for the $3 \mathrm{D}$ case appears to be in line with the simple method given by Kwon and Sun (2000), which (for $v=0.3$ ) estimates an increase in $K_{I}$ value of approximately $5 \%$ at the mid plane when compared with the $2 \mathrm{D}$ value. The values calculated for the example case of a thick plate $(t / W=4.0)$, with $v=0$, show excellent agreement $(<0.5 \%$ difference $)$ with the literature solutions, validating the modelling methods used.

\subsection{SIF and T-stress Results}

The results from linear elastic FEA are given in tabular and graphical form at the end of this chapter. The results are summarized in Table 4.3 through Table 4.20, and SIF and Tstress values are plotted for different thickness in Figure 4.5 through 4.39. 


\subsection{Discussion of Results}

With the introduction of mode I loading in the AFPS specimen, the mode I SIF and Tstress values can be calculated, in addition to the mode II and III SIFs calculated in the pure mode II loading case. Each parameter is examined individually; the variation of the value through-thickness being or particular interest.

The SIFs associated with the mode II loading, $K_{I I}$ and $K_{I I I}$, are examined and compared with the values calculated in the pure mode II result. The trends identified in the pure mode II case of Chapter 3 are applicable to the mixed-mode case, and are expected to be consistent for all values of $c / W$. This observation supports a conclusion of independence of $K_{I I}$ and $K_{I I I}$ from mode I loading.

The trends in the parameters associated with the mode I loading, $K_{I}, T_{11}$, and $T_{33}$, are observed to have significant through-thickness variation, and each parameter has its own trend with respect to crack depth and thickness ratios.

The mode I SIF values show a general trend of decreasing value of $K_{I}$ as the position through-thickness approaches the free surface. This trend of $K_{I}$ value dropping towards the free surface appears for all thickness cases, and for all considered crack depths. For the case of thin plates $(t / W<1.0)$, the values of $K_{I}$ are all very close and show similar through-thickness variation. Increasing thickness appears to decrease the value of $K_{I}$, an effect that is most pronounced for the shallowest crack $(a / W=0.1)$ case. The two thickest cases $(t / W=2.0,4.0)$ show significantly reduced values of $K_{I}$ located at a position of $z / t=0.25$ up to the free surface, when compared with the values of the thinner cases. The effect of increasing thickness appears to be most significant at low values of $a / W$.

Until now, all the results presented have been for the values used in a single-parameter fracture mechanics approach. With the introduction of the two T-stress components, $T_{11}$ and $T_{33}$, the analysis becomes a two-parameter approach.

The trends of T-stress variation through-thickness, and with respect to $t / W$ ratio, are seen to be consistent with the trends identified in Jin and Wang (2014). The value of $T_{11}$ is negative for $a / W<=0.3$, and positive for the deeper cracks where $a / W>0.3$. The trends of 
variation through-thickness appear mostly independent of $a / W$, and the value of $T_{11}$ is, for the most part, constant across the thickness. The values of $T_{11}$ are observed to increase as thickness decreases, as observed at the mid plane, and most of the through-thickness locations. The cases of thicker plates $(t / W>1.0)$ show increasing values of $T_{11}$ for the locations from $z / t=0.3$ up to the free surface.

The plots of $T_{33}$ show trends that can be described more easily. Thickness appears to inversely correlate to $T_{33}$ value, where the thinner plates have larger magnitude of $T_{33} . T_{33}$ increases with increasing $a / W$ ratio, which could be attributed to the fact that $a / W$ ratio is not considered in the normalizing functions. The value of $T_{33}$ is also observed to decrease as the position approaches the free surface, with the thicker plates showing a decrease beginning at higher values of $z / t$, while thinner plates show a decrease which starts to become evident at lower values of $z / t$.

\subsection{Plastic Zone Analysis}

Many researchers have used the plastic zone as a means to describe the effect that the value of fracture parameters have on the stress around a crack tip. Using single- and twoparameter fracture mechanics methods, the size and shape of the plastic zone can be estimated either with or without T-stress effect, under mixed mode loading.

In this section, the SIF results extracted from the current FEA are used to predict the size and shape of the plastic zone. The impact of including the $K_{I I I}$ term is studied, as is the plastic zone variation through-thickness for a variety of plate thickness.

\subsubsection{Calculating and Plotting the Plastic Zone from LEFM Parameters}

In the case of pure mode II loading, as seen in Chapter 3, there exist only mode II and mode III SIF contributions to the stress described by the Williams expansion. In mixedmode loading the mode II and mode III stress contributions, seen in Equations 3.4 and 3.5, are combined with the contributions of mode I: 


$$
\begin{gathered}
\sigma_{11}(r, \theta)=\frac{K_{I}}{\sqrt{2 \pi r}} \cos \left(\frac{\theta}{2}\right)\left[1-\sin \left(\frac{\theta}{2}\right) \sin \left(\frac{3 \theta}{2}\right)\right]+T_{11} \\
\sigma_{22}(r, \theta)=\frac{K_{I}}{\sqrt{2 \pi r}} \cos \left(\frac{\theta}{2}\right)\left[1+\sin \left(\frac{\theta}{2}\right) \cos \left(\frac{3 \theta}{2}\right)\right] \\
\sigma_{12}(r, \theta)=\frac{K_{I}}{\sqrt{2 \pi r}} \cos \left(\frac{\theta}{2}\right) \sin \left(\frac{\theta}{2}\right) \cos \left(\frac{3 \theta}{2}\right) \\
\sigma_{33}(r, \theta)=v\left(\frac{K_{I}}{\sqrt{2 \pi r}} \cos \left(\frac{\theta}{2}\right)\left[1-\sin \left(\frac{\theta}{2}\right) \sin \left(\frac{3 \theta}{2}\right)\right]+\sigma_{22}\right)+T_{33}
\end{gathered}
$$

Applying the principle of superposition, including all three modes, the total stress can be calculated:

$$
\sigma_{i j}{ }^{(t o t a l)}=\sigma_{i j}{ }^{(I)}+\sigma_{i j}{ }^{(I I)}+\sigma_{i j}{ }^{(I I I)}
$$

The stress components from Equations 4.12, 3.4, and 3.5 are substituted into the VonMises yield criterion in Equation 3.7. The plastic zone radius is extracted using the same process as in Chapter 3; however an effective SIF, $K_{\text {eff, }}$ is used to normalize the plastic zone, and includes the mode I component, as seen in Equation 4.14:

$$
K_{e f f}=\sqrt{K_{I}^{2}+K_{I I}^{2}}
$$

The plastic zone is normalized using the values only at the mid plane; in this way, the through-thickness variation of the plastic zone can be clearly seen.

\section{Plastic Zone Verification}

Nazarali and Wang (2011) provide an expression describing the plastic zone radius for cracked bodies undergoing mixed-mode I/II loading. This expression is used to verify the calculation method used in the current analysis. The expression given by Nazarali and Wang takes $K_{I}, K_{I I}$, and T-stress into account, but has no provision for $K_{I I I}$ or individual $T_{33}$ component. The equations taken from Nazarali and Wang can be found in Appendix 
A. The current calculated values of T-stress range from relatively low negative values, to high positive values. Figure 4.40 shows the effect of various T-stress values on the plastic zone size, for the mixed-mode loading case of $K_{I I} / K_{I}=1.0$. The current analysis exactly matches the expression from Nazarali and Wang.

\section{Plastic Zone Results}

In the current analysis, two plastic zone size predictions are made, the first calculated including the effects of only the three SIFs, and the second including all SIF and T-stress values. The plastic zones calculated including only SIF are presented at the end of the chapter from Figure 4.41 through Figure 4.75, while the plastic zones calculated using all SIF and T-stress terms are in Figure 4.76 through Figure 4.110.

From the analysis, the influence of T-stress can be seen in the shape of the plastic zone, which is similar to, albeit less exaggerated than, that observed by Nazarali and Wang (2011). It should be noted that, in Nazarali and Wang, the T-stress magnitude is in the same order of magnitude as the yield stress (YS) value, and is normalized with respect to the YS. In the current work, the T-stress is normalized with respect to loading stress, which varies with the value of $c / W$. The plastic zone calculations are performed using the non-normalized SIF and T-stress values, and the value of the plastic radius is then normalized itself. In this way, the plastic zone will vary with $c / W$ value, because there is a different mixed-mode loading ratio for each value of $c / W$. In light of this, the plastic zone plots are only shown for the case of $c / W=0.1$.

\section{Load Level Effect}

The influence of T-stress can be subtle or extreme, as seen in Nazarali and Wang (2011), depending on the relative T-stress and yield stress values. In this way, the effect of the relative T-stress can be studied, as previous researchers have done; but since the values of T-stress calculated in the current analysis vary through-thickness, this ratio is not constant. To provide some means to evaluate the increased effect that T-stress has on the plastic zone size and shape, a loading level ratio is introduced as 


$$
\text { Load level }=\frac{\sigma^{\text {nom }}}{\sigma_{Y S}}
$$

In the current analysis the values of load level considered are 0.006 (a low-loading scenario), 0.06, 0.09, and 0.12 (a high-loading case); providing a good range of T-stress effects that can be observed. The ratio $S / S y$ used in the plastic zone plots in Figure 4.111 through Figure 4.116 refers to the load level ratio $\sigma^{\text {nom }} / \sigma_{Y S}$.

\section{Discussion of Mixed-mode Plastic Zone Results}

The plastic zone plots calculated using just the three SIFs show results consistent with those found in Chapter 3, namely, an increase in plastic zone size from including a nonzero $K_{I I I}$ term in the analysis. In these plots, the mixed-mode effect is seen to influence the shape of the plastic zone, depending on the mixed-mode ratio. The proportion of $K_{I I}$ to $K_{I}$, the mixed-mode ratio, determines the shape of the plastic zone at the mid plane (where $K_{I I I}=0$ ). The variation of all three SIFs through-thickness influences the plastic zone by apparently increasing the size of the plastic zone, at locations approaching the free surface. The effect of considering just the SIF parameters is shown for only one load level $\left(\sigma^{\mathrm{nom}} / \sigma_{\mathrm{YS}}=0.02\right)$ because the shape and normalized size of the plastic zone does not change at different load levels.

The plots which include the effects of T-stress show four different cases, each with a different load level. As seen in Figure 4.40 and the work of Nazarali and Wang (2011), the sign and magnitude of T-stress directly, and drastically, affect the size and shape of the plastic zone. In the current analysis, the values of T-stress calculated from elastic FEA solutions are used to predict the plastic zone size. By comparing the plastic zone predictions calculated with and without T-stress, the influence of T-stress can be shown. At low load levels, the value of T-stress, with respect to yield stress, is low, and the plastic zone contribution is small. The plastic zone plots for relatively low load levels $\left(\sigma^{\mathrm{nom}} / \sigma_{\mathrm{YS}}=0.006,0.06\right)$ show plastic zones consistent with those predicted without Tstress effects. 


\subsubsection{Plastic Zone Description using FEA-generated Contour}

Using the extraction method described in Chapter 3, Section 3.3.2, the plastic zone plots for select cases are produced from the stress contours in the FEA, and compared with the predicted values. Considering mixed-mode loading does not appear to have any effect on the extraction method used.

The resulting plots are found at the end of the chapter, for the cases considered; $t / W=0.1$, $a / W=0.1, \sigma^{\mathrm{nom}} / \sigma_{Y S}=0.12$ (Figure 4.11), $t / W=0.1, a / W=0.7, \sigma^{\mathrm{nom}} / \sigma_{Y S}=0.06$ (Figure 4.112) $, \quad t / W=0.1, \quad a / W=0.1, \quad \sigma^{\text {nom }} / \sigma_{Y S}=0.09 \quad$ (Figure 4.113$), \quad t / W=1.0, \quad a / W=0.7$, $\sigma^{\mathrm{nom}} / \sigma_{\mathrm{YS}}=0.06$ (Figure 4.114 ), $t / W=4.0, a / W=0.1, \sigma^{\mathrm{nom}} / \sigma_{\mathrm{YS}}=0.12$ (Figure 4.115), and $t / W=4.0, a / W=0.7, \sigma^{\text {nom }} / \sigma_{Y S}=0.06$ (Figure 4.116).

\section{Discussion on FEA Extracted Comparisons}

In general, the extracted values are consistent with the predicted plastic zone. For the cases considered with significant T-stress influence, such as Figure 4.112, a case where $t / W=0.1, a / W=0.7$, the discontinuous plastic zone is seen in the FEA-extracted plastic zone. This result is consistent with the predicted plastic zones, which roughly match the shape and magnitude of the extracted plastic zone.

The same conclusion can be drawn from the mixed-mode FEA plastic zone comparison as from the pure mode II comparisons; the methods used can be used to determine the plastic zone size, but they are not as convenient as calculating the plastic zone.

The use of large T-stress values can lead to the calculation of extreme plastic zone size variations through-thickness. These large variations cannot be modelled sufficiently by elastic FEA that does not take plasticity into account (purely elastic). The load levels chosen in the FEA comparison cases have a moderate amount of T-stress induced discontinuity across the crack line, in the plastic zone plot. 


\subsection{Discussion and Conclusion}

The results of the current work again support the grouping of the results into thick and thin plate behaviour. However, with the introduction of mode I loading, the finite thickness effects are no longer only significant for thick specimens, as thin-plates undergoing mode I loading have high through-thickness T-stress effects.

The thin plates considered in this analysis $(\mathrm{t} / \mathrm{W}<1.0)$ can be grouped together and discussed as a whole due to the similar nature of fracture parameter values and variations. As was seen in the case of pure mode II, the KII values of thin plates have little variation through-thickness, and thin plate results are almost indistinguishable from one another. This is clearly shown in Figure 4.5 through Figure 4.11 , which shows very close $K_{I I}$ values between all thin plate cases. A similar observation can be made of the $K_{I}$ values, which also exhibit similar behaviour for $t / W<1.0$. $K_{I I I}$ values were seen to be significant, and slightly different for each of the thickness cases considered.

The $K_{I I}$ and $K_{I I I}$ results are consistent with what was observed in Chapter 3, and from the plastic zone analysis, it could be expected that the plastic zone size would not have much variation through-thickness. When considering only SIF values in the calculation of plastic zone, the size and shape of the plastic zone are consistent through-thickness, for the thin cases.

Including T-stress in the calculation of the plastic zone shows some cases with discontinuous plastic zones. The maximum T-stress values were located in the thin specimens with deep cracks, and the impact on the plastic zone can be seen to be extreme. An example of the extreme effect can be seen in Figure 4.82 which shows the plastic zone for different load levels with $t / W=0.1$, and $a / W=0.7$. The T-stress effect is most noticeable at deep cracks, where T-stress is largest, in proportion with $K$, but still has a noticeable effect over a wide range of crack geometries.

Because T-stress is relative to material yield stress, the different load levels used in the calculation of the plastic zone show different amounts of T-stress influence. The results presented here cover a wide range of crack geometries and loading scenarios. 
From the analysis of the AFPS specimen, the same conclusion can be drawn as was with the mode II FPS specimen: finite thickness effects have significant effect. For mixedmode I/II loading, the finite thickness effect is present at all thicknesses. For thin specimens, T-stress magnitudes are large approaching the free surface, enlarging the plastic zone greatly. At the other end of the spectrum, thick plates do not see the same amount of T-stress effect, but large $K_{I I}$ and $K_{I I I}$ values cause enlarged plastic zones at locations between the mid plane and free edge.

The findings from the current analysis show that using 2D solutions to interpret fracture specimens undergoing mixed-mode loading will not adequately describe the stress at the crack. It is necessary to consider the finite thickness effects on the plate, as well as the crack tip constraint effect from the T-stress. Currently, the results indicate that, unlike the case of pure mode I loading, very large specimen thicknesses do not approach the reference plane strain solution for $K_{I I}$.

The fracture parameter solutions obtained in this analysis will be very useful for fracture testing of mixed-mode I/II AFPS specimens. The plastic zone analysis performed in this chapter serves to illustrate the importance of considering specimen thickness in the interpretation of fracture testing results. 
Table 4.1 : 2D FEA results comparison values

\begin{tabular}{|c|r|r|r|}
\hline \multirow{2}{*}{ a/W } & \multicolumn{2}{|c|}{ Normalized $\boldsymbol{K}_{\boldsymbol{I}}$} & \multirow{2}{*}{ \% Difference } \\
\cline { 2 - 3 } & Reference & Current $\boldsymbol{F E A}$ & \\
\hline 0.1 & 1.0495 & 1.0447 & $-0.46 \%$ \\
\hline 0.2 & 1.0551 & 1.0537 & $-0.14 \%$ \\
\hline 0.3 & 1.1274 & 1.1231 & $-0.38 \%$ \\
\hline 0.4 & 1.2631 & 1.2599 & $-0.26 \%$ \\
\hline 0.5 & 1.4947 & 1.4978 & $0.21 \%$ \\
\hline 0.6 & 1.9170 & 1.9135 & $-0.18 \%$ \\
\hline 0.7 & 2.7144 & 2.7250 & $0.39 \%$ \\
\hline
\end{tabular}

Table 4.2: 3D FEA Mid plane results comparison values

\begin{tabular}{|c|c|c|c|c|c|c|c|c|c|c|c|c|c|c|}
\hline \multirow{3}{*}{$t / N$} & \multicolumn{14}{|c|}{$\mathrm{a} / \mathrm{W}$} \\
\hline & \multicolumn{2}{|c|}{0.1} & \multicolumn{2}{|c|}{0.2} & \multicolumn{2}{|c|}{0.3} & \multicolumn{2}{|c|}{0.4} & \multicolumn{2}{|c|}{0.5} & \multicolumn{2}{|c|}{0.6} & \multicolumn{2}{|c|}{0.7} \\
\hline & $K_{I}$ & $\%$ & $K_{I}$ & $\%$ & $K_{I}$ & $\%$ & $\boldsymbol{K}_{I}$ & $\%$ & $\boldsymbol{K}_{I}$ & $\%$ & $\boldsymbol{K}_{I}$ & $\%$ & $\boldsymbol{K}_{\boldsymbol{I}}$ & $\%$ \\
\hline 0.1 & 11068 & $5.5 \%$ & 1.1216 & $6.3 \%$ & 1.1968 & $6.2 \%$ & 1.3438 & $6.4 \%$ & 15968 & $6.8 \%$ & 20421 & $6.5 \%$ & 29053 & $7.0 \%$ \\
\hline 0.2 & 11033 & $5.1 \%$ & 1.1203 & $6.2 \%$ & 1.1967 & $6.1 \%$ & 1.3447 & $6.5 \%$ & 15986 & $6.9 \%$ & 20449 & $6.7 \%$ & 29085 & $7.2 \%$ \\
\hline 0.5 & 10834 & $3.2 \%$ & 1.1119 & $5.4 \%$ & 1.1924 & $5.8 \%$ & 1.3413 & $6.2 \%$ & 15952 & $6.7 \%$ & 20374 & $6.3 \%$ & 28845 & $6.3 \%$ \\
\hline 1.0 & 10568 & $0.7 \%$ & 1.0856 & $29 \%$ & 1.1711 & $3.9 \%$ & 1.3211 & $4.6 \%$ & 15716 & $5.1 \%$ & 20043 & $4.6 \%$ & 28319 & $4.3 \%$ \\
\hline 2.0 & 10743 & $24 \%$ & 1.0856 & $29 \%$ & 1.1616 & $3.0 \%$ & 1.3056 & $3.4 \%$ & 15500 & $3.7 \%$ & 19744 & $3.0 \%$ & 27894 & $28 \%$ \\
\hline 4.0 & 11048 & $5.3 \%$ & 1.1100 & $5.2 \%$ & 1.1793 & $4.6 \%$ & 1.3170 & $4.3 \%$ & 15552 & $4.0 \%$ & 19730 & $2.9 \%$ & 27814 & $25 \%$ \\
\hline $4.0 v=0$ & 10451 & $-0.4 \%$ & 1.0520 & $-0.3 \%$ & 1.1213 & $-0.5 \%$ & 1.2578 & $-0.4 \%$ & 14938 & $-0.1 \%$ & 19087 & $-0.4 \%$ & 27127 & -0.18 \\
\hline Reference & 1.04 & & 1.05 & & 1.12 & & 126 & & 1494 & & 1917 & & 2.71 & \\
\hline
\end{tabular}


Table 4.3: Normalized $K_{I I}$ and $K_{I I I}$ values for $\mathrm{t} / \mathrm{W}=0.1$

\begin{tabular}{|c|c|c|c|c|c|c|c|c|c|c|c|c|c|c|}
\hline \multirow{2}{*}{$z / t$} & \multicolumn{2}{|c|}{$\mathrm{a} / \mathrm{w}=\mathbf{0 . 1}$} & \multicolumn{2}{|c|}{$\mathrm{a} / \mathrm{w}=0.2$} & \multicolumn{2}{|c|}{$\mathrm{a} / \mathrm{w}=\mathbf{0 . 3}$} & \multicolumn{2}{|c|}{$\mathrm{a} / \mathrm{w}=0.4$} & \multicolumn{2}{|c|}{$\mathrm{a} / \mathrm{w}=0.5$} & \multicolumn{2}{|c|}{$\mathrm{a} / \mathrm{w}=0.6$} & \multicolumn{2}{|c|}{$\mathrm{a} / \mathrm{w}=0.7$} \\
\hline & $K_{\text {II }}$ & $K_{\text {III }}$ & $K_{I I}$ & $K_{I I I}$ & $K_{I I}$ & $K_{\text {III }}$ & $K_{I I}$ & $K_{I I I}$ & $K_{I I}$ & $K_{I I I}$ & $K_{I I}$ & $K_{I I I}$ & $K_{I I}$ & $K_{\text {III }}$ \\
\hline 0.0000 & 6.4793 & 0.0000 & 5.6199 & 0.0000 & 4.8256 & 0.0000 & 4.1154 & 0.0000 & 3.4885 & 0.0000 & 2.9404 & 0.0000 & 2.4660 & 0.0000 \\
\hline 0.0650 & 6.4782 & -0.0606 & 5.6191 & -0.0656 & 4.8251 & -0.0608 & 4.1150 & -0.0539 & 3.4883 & -0.0469 & 2.9399 & -0.0805 & 2.4656 & -0.0685 \\
\hline 0.0975 & 6.4771 & -0.1213 & 5.6183 & -0.1311 & 4.8244 & -0.1216 & 4.1146 & -0.1079 & 3.4879 & -0.0938 & 2.9393 & -0.1224 & 2.4652 & -0.1041 \\
\hline 0.1300 & 6.4738 & -0.1854 & 5.6160 & -0.1998 & 4.8227 & -0.1850 & 4.1133 & -0.1641 & 3.4870 & -0.1426 & 2.9385 & -0.1642 & 2.4646 & -0.1396 \\
\hline 0.1625 & 6.4704 & -0.2498 & 5.6134 & -0.2685 & 4.8209 & -0.2484 & 4.1120 & -0.2202 & 3.4860 & -0.1914 & 2.9375 & -0.2096 & 2.4639 & -0.1781 \\
\hline 0.1950 & 6.4645 & -0.3218 & 5.6094 & -0.3441 & 4.8180 & -0.3177 & 4.1099 & -0.2814 & 3.4845 & -0.2444 & 2.9362 & -0.2549 & 2.4630 & -0.2166 \\
\hline 0.2275 & 6.4584 & -0.3943 & 5.6050 & -0.4197 & 4.8149 & -0.3870 & 4.1077 & -0.3425 & 3.4828 & -0.2973 & 2.9350 & -0.3063 & 2.4621 & -0.2602 \\
\hline 0.2600 & 6.4494 & -0.4802 & 5.5992 & -0.5072 & 4.8110 & -0.4664 & 4.1050 & -0.4122 & 3.4810 & -0.3575 & 2.9333 & -0.3581 & 2.4609 & -0.3040 \\
\hline 0.2925 & 6.4397 & -0.5679 & 5.5927 & -0.5957 & 4.8064 & -0.5465 & 4.1017 & -0.4825 & 3.4786 & -0.4181 & 2.9326 & -0.4199 & 2.4607 & -0.3563 \\
\hline 0.3250 & 6.4271 & -0.6774 & 5.5857 & -0.7034 & 4.8024 & -0.6431 & 4.0994 & -0.5667 & 3.4773 & -0.4906 & 2.9313 & -0.4838 & 2.4600 & -0.4103 \\
\hline 0.3338 & 6.4129 & -0.7940 & 5.5774 & -0.8161 & 4.7972 & -0.7436 & 4.0961 & -0.6542 & 3.4752 & -0.5657 & 2.9320 & -0.5047 & 2.4607 & -0.4280 \\
\hline 0.3425 & 6.4091 & -0.8321 & 5.5761 & -0.8529 & 4.7971 & -0.7764 & 4.0965 & -0.6827 & 3.4758 & -0.5903 & 2.9316 & -0.5259 & 2.4605 & -0.4458 \\
\hline 0.3512 & 6.4038 & -0.8744 & 5.5732 & -0.8917 & 4.7953 & -0.8104 & 4.0953 & -0.7120 & 3.4751 & -0.6153 & 2.9325 & -0.5455 & 2.4614 & -0.4624 \\
\hline 0.3600 & 6.4005 & -0.9094 & 5.5724 & -0.9261 & 4.7955 & -0.8412 & 4.0960 & -0.7389 & 3.4760 & -0.6383 & 2.9323 & -0.5702 & 2.4613 & -0.4832 \\
\hline 0.3688 & 6.3947 & -0.9600 & 5.5694 & -0.9717 & 4.7939 & -0.8810 & 4.0951 & -0.7731 & 3.4755 & -0.6675 & 2.9339 & -0.5902 & 2.4628 & -0.5001 \\
\hline 0.3775 & 6.3916 & -0.9956 & 5.5694 & -1.0067 & 4.7949 & -0.9123 & 4.0966 & -0.8004 & 3.4771 & -0.6910 & 2.9340 & -0.6190 & 2.4630 & -0.5243 \\
\hline 0.3863 & 6.3854 & -1.0562 & 5.5665 & -1.0606 & 4.7935 & -0.9590 & 4.0960 & -0.8404 & 3.4769 & -0.7250 & 2.9365 & -0.6392 & 2.4653 & -0.5414 \\
\hline 0.3950 & 6.3830 & -1.0919 & 5.5676 & -1.0959 & 4.7959 & -0.9906 & 4.0986 & -0.8680 & 3.4796 & -0.7488 & 2.9373 & -0.6740 & 2.4662 & -0.5706 \\
\hline 0.4125 & 6.3753 & -1.2002 & 5.5684 & -1.1949 & 4.7995 & -1.0772 & 4.1032 & -0.9426 & 3.4844 & -0.8125 & 2.9437 & -0.7376 & 2.4720 & -0.6241 \\
\hline 0.4300 & 6.3702 & -1.3216 & 5.5737 & -1.3045 & 4.8078 & -1.1727 & 4.1122 & -1.0247 & 3.4930 & -0.8824 & 2.9560 & -0.8145 & 2.4829 & -0.6887 \\
\hline 0.4475 & 6.3709 & -1.4538 & 5.5872 & -1.4222 & 4.8242 & -1.2747 & 4.1286 & -1.1122 & 3.5083 & -0.9568 & 2.9814 & -0.9155 & 2.5049 & -0.7736 \\
\hline 0.4650 & 6.3818 & -1.5861 & 5.6145 & -1.5369 & 4.8541 & -1.3732 & 4.1572 & -1.1962 & 3.5344 & -1.0280 & 3.0476 & -1.0777 & 2.5614 & -0.9100 \\
\hline 0.4825 & 6.4178 & -1.6455 & 5.6721 & -1.5786 & 4.9127 & -1.4058 & 4.2116 & -1.2226 & 3.5830 & -1.0496 & 3.2276 & -1.2660 & 2.7143 & -1.0684 \\
\hline 0.5000 & 6.3291 & -1.6318 & 5.6244 & -1.5277 & 4.8817 & -1.3496 & 4.1898 & -1.1689 & 3.5671 & -1.0009 & 4.7206 & -3.9026 & 3.9751 & -3.2864 \\
\hline
\end{tabular}


Table 4.4: Normalized $K_{I I}$ and $K_{I I}$ values for $\mathrm{t} / \mathrm{W}=\mathbf{0 . 2}$

\begin{tabular}{|c|c|c|c|c|c|c|c|c|c|c|c|c|c|c|}
\hline \multirow{2}{*}{$z / t$} & \multicolumn{2}{|c|}{$\mathrm{a} / \mathrm{w}=\mathbf{0 . 1}$} & \multicolumn{2}{|c|}{$\mathrm{a} / \mathrm{w}=0.2$} & \multicolumn{2}{|c|}{$\mathrm{a} / \mathrm{w}=\mathbf{0 . 3}$} & \multicolumn{2}{|c|}{$\mathrm{a} / \mathrm{w}=0.4$} & \multicolumn{2}{|c|}{$\mathrm{a} / \mathrm{w}=0.5$} & \multicolumn{2}{|c|}{$a / w=0.6$} & \multicolumn{2}{|c|}{$\mathrm{a} / \mathrm{w}=\mathbf{0 . 7}$} \\
\hline & \begin{tabular}{|l|}
$K_{I I}$ \\
\end{tabular} & $K_{I I I}$ & $K_{I I}$ & $K_{I I I}$ & \begin{tabular}{|l|}
$K_{I I}$ \\
\end{tabular} & $K_{I I I}$ & \begin{tabular}{|l|}
$K_{I I}$ \\
\end{tabular} & $K_{I I I}$ & $K_{I I}$ & $K_{I I I}$ & \begin{tabular}{|l|}
$K_{I I}$ \\
\end{tabular} & $K_{I I I}$ & $\boldsymbol{K}_{\boldsymbol{I I}}$ & $K_{I I I}$ \\
\hline 0.0000 & 6.4399 & 0.0000 & 5.6219 & 0.0000 & 4.8306 & 0.0000 & 4.1193 & 0.0000 & 3.4901 & 0.0000 & 2.9398 & 0.0000 & 2.4625 & 0.0000 \\
\hline 0.0589 & 6.4400 & -0.0689 & 5.6207 & -0.0977 & 4.8296 & -0.0977 & 4.1185 & -0.0904 & 3.4895 & -0.0807 & 2.9393 & -0.0706 & 2.4622 & -0.0608 \\
\hline 0.0884 & 6.4400 & -0.1061 & 5.6188 & -0.1490 & 4.8281 & -0.1486 & 4.1172 & -0.1374 & 3.4886 & -0.1226 & 2.9387 & -0.1071 & 2.4618 & -0.0922 \\
\hline 0.1179 & 6.4400 & -0.1431 & 5.6169 & -0.2001 & 4.8265 & -0.1995 & 4.1160 & -0.1843 & 3.4876 & -0.1643 & 2.9380 & -0.1435 & 2.4614 & -0.1236 \\
\hline 0.1473 & 6.4394 & -0.1861 & 5.6136 & -0.2565 & 4.8237 & -0.2547 & 4.1138 & -0.2349 & 3.4861 & -0.2092 & 2.9369 & -0.1826 & 2.4607 & -0.1572 \\
\hline 0.1768 & 6.4388 & -0.2288 & 5.6102 & -0.3126 & 4.8210 & -0.3098 & 4.1117 & -0.2853 & 3.4845 & -0.2540 & 2.9358 & -0.2216 & 2.4600 & -0.1906 \\
\hline 0.2062 & 6.4366 & -0.2818 & 5.6051 & -0.3777 & 4.8169 & -0.3726 & 4.1087 & -0.3423 & 3.4823 & -0.3041 & 2.9344 & -0.2651 & 2.4592 & -0.2279 \\
\hline 0.2357 & 6.4345 & -0.3343 & 5.5999 & -0.4423 & 4.8129 & -0.4349 & 4.1056 & -0.3988 & 3.4801 & -0.3540 & 2.9329 & -0.3083 & 2.4583 & -0.2649 \\
\hline 0.2652 & 6.4291 & -0.4040 & 5.5923 & -0.5217 & 4.8074 & -0.5098 & 4.1017 & -0.4660 & 3.4776 & -0.4128 & 2.9313 & -0.3590 & 2.4575 & -0.3082 \\
\hline 0.2946 & 6.4237 & -0.4724 & 5.5847 & -0.5999 & 4.8017 & -0.5836 & 4.0977 & -0.5322 & 3.4748 & -0.4707 & 2.9295 & -0.4090 & 2.4565 & -0.3508 \\
\hline 0.3241 & 6.4129 & -0.5712 & 5.5748 & -0.7038 & 4.7954 & -0.6792 & 4.0939 & -0.6166 & 3.4728 & -0.5440 & 2.9287 & -0.4718 & 2.4566 & -0.4041 \\
\hline 0.3536 & 6.4010 & -0.6679 & 5.5634 & -0.8059 & 4.7877 & -0.7730 & 4.0888 & -0.6996 & 3.4696 & -0.6159 & 2.9268 & -0.5334 & 2.4558 & -0.4565 \\
\hline 0.3830 & 6.3827 & -0.8237 & 5.5541 & -0.9572 & 4.7851 & -0.9083 & 4.0899 & -0.8174 & 3.4726 & -0.7172 & 2.9309 & -0.6197 & 2.4605 & -0.5294 \\
\hline 0.4125 & 6.3597 & -0.9912 & 5.5419 & -1.1168 & 4.7803 & -1.0501 & 4.0893 & -0.9404 & 3.4744 & -0.8227 & 2.9340 & -0.7094 & 2.4643 & -0.6051 \\
\hline 0.4212 & 6.3580 & -1.0709 & 5.5482 & -1.1901 & 4.7896 & -1.1144 & 4.0993 & -0.9959 & 3.4841 & -0.8701 & 2.9431 & -0.7495 & 2.4726 & -0.6390 \\
\hline 0.4300 & 6.3458 & -1.1466 & 5.5420 & -1.2554 & 4.7868 & -1.1706 & 4.0984 & -1.0439 & 3.4843 & -0.9108 & 2.9439 & -0.7839 & 2.4737 & -0.6678 \\
\hline 0.4387 & 6.3388 & -1.2341 & 5.5452 & -1.3338 & 4.7938 & -1.2387 & 4.1066 & -1.1023 & 3.4927 & -0.9606 & 2.9518 & -0.8260 & 2.4810 & -0.7032 \\
\hline 0.4475 & 6.3341 & -1.3372 & 5.5515 & -1.4246 & 4.8039 & -1.3172 & 4.1178 & -1.1695 & 3.5037 & -1.0177 & 2.9621 & -0.8742 & 2.4904 & -0.7438 \\
\hline 0.4563 & 6.3330 & -1.4591 & 5.5641 & -1.5306 & 4.8205 & -1.4085 & 4.1350 & -1.2475 & 3.5201 & -1.0839 & 2.9772 & -0.9301 & 2.5038 & -0.7907 \\
\hline 0.4650 & 6.3407 & -1.6100 & 5.5889 & -1.6607 & 4.8492 & -1.5202 & 4.1633 & -1.3429 & 3.5463 & -1.1647 & 3.0007 & -0.9983 & 2.5246 & -0.8480 \\
\hline 0.4737 & 6.3703 & -1.8131 & 5.6402 & -1.8358 & 4.9032 & -1.6708 & 4.2144 & -1.4714 & 3.5926 & -1.2738 & 3.0416 & -1.0904 & 2.5601 & -0.9253 \\
\hline 0.4825 & 6.4753 & -2.1428 & 5.7722 & -2.1272 & 5.0318 & -1.9238 & 4.3318 & -1.6887 & 3.6966 & -1.4590 & 3.1321 & -1.2471 & 2.6379 & -1.0572 \\
\hline 0.4913 & 6.7958 & -2.5280 & 6.1277 & -2.4686 & 5.3652 & -2.2206 & 4.6301 & -1.9438 & 3.9574 & -1.6765 & 3.3570 & -1.4313 & 2.8298 & -1.2122 \\
\hline 0.5000 & 9.7291 & -8.0299 & 9.0249 & -7.4855 & 7.9815 & -6.6281 & 6.9249 & -5.7538 & 5.9392 & -4.9363 & 5.0505 & -4.1984 & 4.2652 & -3.5461 \\
\hline
\end{tabular}


Table 4.5: Normalized $K_{I I}$ and $K_{I I I}$ values for $\mathrm{t} / \mathrm{W}=0.5$

\begin{tabular}{|c|c|c|c|c|c|c|c|c|c|c|c|c|c|c|}
\hline \multirow{2}{*}{$z / t$} & \multicolumn{2}{|c|}{$\mathrm{a} / \mathrm{w}=\mathbf{0 . 1}$} & \multicolumn{2}{|c|}{$\mathrm{a} / \mathrm{w}=0.2$} & \multicolumn{2}{|c|}{$\mathrm{a} / \mathrm{w}=\mathbf{0 . 3}$} & \multicolumn{2}{|c|}{$\mathrm{a} / \mathrm{w}=0.4$} & \multicolumn{2}{|c|}{$\mathrm{a} / \mathrm{w}=0.5$} & \multicolumn{2}{|c|}{$a / w=0.6$} & \multicolumn{2}{|c|}{$\mathrm{a} / \mathrm{w}=\mathbf{0 . 7}$} \\
\hline & \begin{tabular}{|l|}
$K_{I I}$ \\
\end{tabular} & $K_{I I I}$ & $K_{I I}$ & $K_{I I I}$ & \begin{tabular}{|l|}
$K_{I I}$ \\
\end{tabular} & $K_{I I I}$ & $\boldsymbol{K}_{\boldsymbol{I I}}$ & $K_{I I I}$ & $K_{I I}$ & $K_{I I I}$ & \begin{tabular}{|l|}
$K_{I I}$ \\
\end{tabular} & $K_{I I I}$ & $\boldsymbol{K}_{\boldsymbol{I I}}$ & $K_{I I I}$ \\
\hline 0.0000 & 6.0983 & 0.0000 & 5.4798 & 0.0000 & 4.7787 & 0.0000 & 4.0929 & 0.0000 & 3.4717 & 0.0000 & 2.9222 & 0.0000 & 2.4418 & 0.0000 \\
\hline 0.0500 & 6.1066 & -0.0569 & 5.4835 & -0.0427 & 4.7797 & -0.0547 & 4.0932 & -0.0580 & 3.4718 & -0.0561 & 2.9223 & -0.0518 & 2.4420 & -0.0459 \\
\hline 0.0750 & 6.1191 & -0.0849 & 5.4890 & -0.0654 & 4.7812 & -0.0833 & 4.0937 & -0.0881 & 3.4720 & -0.0851 & 2.9224 & -0.0785 & 2.4423 & -0.0695 \\
\hline 0.1250 & 6.1527 & -0.1403 & 5.5032 & -0.1137 & 4.7850 & -0.1431 & 4.0947 & -0.1506 & 3.4724 & -0.1451 & 2.9228 & -0.1335 & 2.4430 & -0.1182 \\
\hline 0.1500 & 6.1739 & -0.1676 & 5.5119 & -0.1392 & 4.7874 & -0.1744 & 4.0954 & -0.1829 & 3.4726 & -0.1760 & 2.9230 & -0.1618 & 2.4434 & -0.1432 \\
\hline 0.2000 & 6.2345 & -0.2224 & 5.5346 & -0.2002 & 4.7929 & -0.2457 & 4.0965 & -0.2553 & 3.4728 & -0.2443 & 2.9234 & -0.2239 & 2.4446 & -0.1978 \\
\hline 0.2250 & 6.2740 & -0.2517 & 5.5476 & -0.2383 & 4.7956 & -0.2883 & 4.0967 & -0.2973 & 3.4726 & -0.2834 & 2.9235 & -0.2591 & 2.4453 & -0.2287 \\
\hline 0.2500 & 6.3136 & -0.2807 & 5.5606 & -0.2763 & 4.7983 & -0.3307 & 4.0969 & -0.3392 & 3.4724 & -0.3224 & 2.9236 & -0.2942 & 2.4460 & -0.2594 \\
\hline 0.2750 & 6.3617 & -0.3163 & 5.5738 & -0.3265 & 4.8002 & -0.3838 & 4.0964 & -0.3901 & 3.4717 & -0.3689 & 2.9235 & -0.3355 & 2.4469 & -0.2954 \\
\hline 0.3000 & 6.4101 & -0.3512 & 5.5870 & -0.3763 & 4.8021 & -0.4365 & 4.0959 & -0.4407 & 3.4711 & -0.4152 & 2.9234 & -0.3767 & 2.4478 & -0.3313 \\
\hline 0.3250 & 6.4646 & -0.4020 & 5.5983 & -0.4462 & 4.8022 & -0.5065 & 4.0943 & -0.5056 & 3.4700 & -0.4734 & 2.9235 & -0.4277 & 2.4493 & -0.3754 \\
\hline 0.3500 & 6.5195 & -0.4509 & 5.6097 & -0.5148 & 4.8022 & -0.5753 & 4.0925 & -0.5697 & 3.4688 & -0.5308 & 2.9233 & -0.4782 & 2.4506 & -0.4190 \\
\hline 0.3750 & 6.5749 & -0.5372 & 5.6160 & -0.6205 & 4.7997 & -0.6749 & 4.0904 & -0.6591 & 3.4685 & -0.6093 & 2.9249 & -0.5460 & 2.4542 & -0.4771 \\
\hline 0.4000 & 6.6310 & -0.6164 & 5.6216 & -0.7209 & 4.7959 & -0.7702 & 4.0868 & -0.7449 & 3.4666 & -0.6847 & 2.9250 & -0.6113 & 2.4564 & -0.5331 \\
\hline 0.4500 & 6.7185 & -0.9894 & 5.6207 & -1.1004 & 4.7956 & -1.1049 & 4.0969 & -1.0346 & 3.4848 & -0.9329 & 2.9484 & -0.8223 & 2.4837 & -0.7118 \\
\hline 0.4600 & 6.7280 & -1.1548 & 5.6240 & -1.2472 & 4.8067 & -1.2287 & 4.1132 & -1.1393 & 3.5033 & -1.0212 & 2.9676 & -0.8966 & 2.5025 & -0.7742 \\
\hline 0.4650 & 6.7334 & -1.2394 & 5.6273 & -1.3211 & 4.8140 & -1.2908 & 4.1228 & -1.1917 & 3.5139 & -1.0653 & 2.9783 & -0.9337 & 2.5128 & -0.8054 \\
\hline 0.4700 & 6.7396 & -1.3393 & 5.6338 & -1.4068 & 4.8252 & -1.3625 & 4.1367 & -1.2520 & 3.5285 & -1.1162 & 2.9926 & -0.9764 & 2.5263 & -0.8412 \\
\hline 0.4750 & 6.7474 & -1.4595 & 5.6450 & -1.5078 & 4.8424 & -1.4466 & 4.1565 & -1.3227 & 3.5487 & -1.1756 & 3.0120 & -1.0262 & 2.5443 & -0.8831 \\
\hline 0.4800 & 6.7601 & -1.6094 & 5.6652 & -1.6314 & 4.8697 & -1.5492 & 4.1864 & -1.4087 & 3.5783 & -1.2478 & 3.0398 & -1.0868 & 2.5697 & -0.9339 \\
\hline 0.4850 & 6.7843 & -1.8052 & 5.7026 & -1.7903 & 4.9155 & -1.6807 & 4.2341 & -1.5190 & 3.6242 & -1.3405 & 3.0822 & -1.1646 & 2.6078 & -0.9992 \\
\hline 0.4900 & 6.8539 & -2.1148 & 5.7922 & -2.0443 & 5.0130 & -1.8942 & 4.3297 & -1.7000 & 3.7130 & -1.4938 & 3.1622 & -1.2940 & 2.6783 & -1.1081 \\
\hline 0.4950 & 7.2123 & -2.6358 & 6.1687 & -2.4838 & 5.3786 & -2.2720 & 4.6668 & -2.0248 & 4.0145 & -1.7716 & 3.4267 & -1.5300 & 2.9070 & -1.3079 \\
\hline 0.5000 & 9.6952 & -7.0711 & 8.5456 & -6.3102 & 7.5700 & -5.6211 & 6.6288 & -4.9369 & 5.7363 & -4.2801 & 4.9170 & -3.6734 & 4.1830 & -3.1276 \\
\hline
\end{tabular}


Table 4.6: Normalized $K_{I I}$ and $K_{I I I}$ values for $\mathrm{t} / \mathrm{W}=1.0$

\begin{tabular}{|c|c|c|c|c|c|c|c|c|c|c|c|c|c|c|}
\hline \multirow{2}{*}{$z / t$} & \multicolumn{2}{|c|}{$\mathrm{a} / \mathrm{w}=0.1$} & \multicolumn{2}{|c|}{$\mathrm{a} / \mathrm{w}=0.2$} & \multicolumn{2}{|c|}{$\mathrm{a} / \mathrm{w}=0.3$} & \multicolumn{2}{|c|}{$\mathrm{a} / \mathrm{w}=0.4$} & \multicolumn{2}{|c|}{$\mathrm{a} / \mathrm{w}=0.5$} & \multicolumn{2}{|c|}{$a / w=0.6$} & \multicolumn{2}{|c|}{$\mathrm{a} / \mathrm{w}=\mathbf{0 . 7}$} \\
\hline & $K_{I I}$ & $K_{I I I}$ & \begin{tabular}{|l|}
$K_{I I}$ \\
\end{tabular} & $K_{I I I}$ & \begin{tabular}{|l|}
$K_{I I}$ \\
\end{tabular} & $K_{I I I}$ & $\boldsymbol{K}_{\boldsymbol{I I}}$ & $K_{I I I}$ & $\boldsymbol{K}_{\boldsymbol{I I}}$ & $K_{I I I}$ & $\boldsymbol{K}_{I I}$ & $K_{I I I}$ & $\boldsymbol{K}_{\boldsymbol{I I}}$ & $K_{I I I}$ \\
\hline 0.0000 & 5.6598 & 0.0000 & 5.0388 & 0.0000 & 4.4542 & 0.0000 & 3.8828 & 0.0000 & 3.3370 & 0.0000 & 2.8349 & 0.0000 & 2.3884 & 0.0000 \\
\hline 0.0193 & 5.6640 & -0.1281 & 5.0429 & -0.0362 & 4.4574 & -0.0168 & 3.8848 & -0.0134 & 3.3383 & -0.0131 & 2.8357 & -0.0125 & 2.3888 & -0.0110 \\
\hline 0.0578 & 5.6810 & -0.3810 & 5.0594 & -0.1077 & 4.4700 & -0.0507 & 3.8930 & -0.0407 & 3.3434 & -0.0396 & 2.8389 & -0.0379 & 2.3907 & -0.0333 \\
\hline 0.0964 & 5.7155 & -0.6235 & 5.0927 & -0.1771 & 4.4951 & -0.0853 & 3.9092 & -0.0695 & 3.3536 & -0.0677 & 2.8453 & -0.0647 & 2.3944 & -0.0569 \\
\hline 0.1349 & 5.7686 & -0.8488 & 5.1435 & -0.2430 & 4.5325 & -0.1214 & 3.9331 & -0.1008 & 3.3686 & -0.0983 & 2.8547 & -0.0939 & 2.4000 & -0.0827 \\
\hline 0.1734 & 5.8423 & -1.0498 & 5.2126 & -0.3049 & 4.5819 & -0.1600 & 3.9644 & -0.1359 & 3.3882 & -0.1328 & 2.8671 & -0.1267 & 2.4075 & -0.1117 \\
\hline 0.2120 & 5.9397 & -1.2195 & 5.3009 & -0.3631 & 4.6426 & -0.2028 & 4.0024 & -0.1767 & 3.4120 & -0.1728 & 2.8822 & -0.1644 & 2.4167 & -0.1452 \\
\hline 0.2505 & 6.0657 & -1.3512 & 5.4090 & -0.4190 & 4.7137 & -0.2523 & 4.0461 & -0.2254 & 3.4393 & -0.2202 & 2.8996 & -0.2089 & 2.4277 & -0.1848 \\
\hline 0.2891 & 6.2278 & -1.4393 & 5.5370 & -0.4767 & 4.7937 & -0.3124 & 4.0945 & -0.2857 & 3.4692 & -0.2782 & 2.9190 & -0.2626 & 2.4404 & -0.2325 \\
\hline 0.3276 & 6.4372 & -1.4821 & 5.6837 & -0.5436 & 4.8803 & -0.3898 & 4.1456 & -0.3627 & 3.5007 & -0.3509 & 2.9397 & -0.3290 & 2.4547 & -0.2914 \\
\hline 0.3661 & 6.7083 & -1.4872 & 5.8460 & -0.6337 & 4.9702 & -0.4955 & 4.1972 & -0.4657 & 3.5324 & -0.4458 & 2.9613 & -0.4141 & 2.4710 & -0.3662 \\
\hline 0.4047 & 7.0552 & -1.4861 & 6.0174 & -0.7754 & 5.0585 & -0.6518 & 4.2463 & -0.6122 & 3.5632 & -0.5768 & 2.9840 & -0.5291 & 2.4906 & -0.4662 \\
\hline 0.4240 & 7.2492 & -1.4826 & 6.1039 & -0.8584 & 5.1006 & -0.7421 & 4.2687 & -0.6958 & 3.5770 & -0.6508 & 2.9946 & -0.5936 & 2.5003 & -0.5221 \\
\hline 0.4625 & 7.7169 & -1.6785 & 6.2728 & -1.2299 & 5.1809 & -1.0924 & 4.3187 & -1.0000 & 3.6180 & -0.9095 & 3.0344 & -0.8124 & 2.5416 & -0.7078 \\
\hline 0.4662 & 7.7742 & -1.7563 & 6.2911 & -1.3162 & 5.1920 & -1.1668 & 4.3290 & -1.0620 & 3.6289 & -0.9608 & 3.0458 & -0.8551 & 2.5532 & -0.7434 \\
\hline 0.4700 & 7.8252 & -1.8133 & 6.3086 & -1.3837 & 5.2021 & -1.2256 & 4.3376 & -1.1112 & 3.6376 & -1.0016 & 3.0547 & -0.8890 & 2.5622 & -0.7718 \\
\hline 0.4738 & 7.8790 & -1.8831 & 6.3283 & -1.4610 & 5.2148 & -1.2923 & 4.3489 & -1.1667 & 3.6489 & -1.0477 & 3.0662 & -0.9272 & 2.5736 & -0.8038 \\
\hline 0.4775 & 7.9358 & -1.9693 & 6.3507 & -1.5511 & 5.2304 & -1.3691 & 4.3635 & -1.2303 & 3.6636 & -1.1002 & 3.0809 & -0.9708 & 2.5879 & -0.8402 \\
\hline 0.4813 & 7.9974 & -2.0776 & 6.3775 & -1.6580 & 5.2509 & -1.4590 & 4.3832 & -1.3044 & 3.6834 & -1.1613 & 3.1004 & -1.0213 & 2.6065 & -0.8824 \\
\hline 0.4850 & 8.0675 & -2.2184 & 6.4123 & -1.7895 & 5.2798 & -1.5683 & 4.4113 & -1.3942 & 3.7112 & -1.2351 & 3.1274 & -1.0823 & 2.6318 & -0.9332 \\
\hline 0.4887 & 8.1548 & -2.4096 & 6.4633 & -1.9592 & 5.3247 & -1.7078 & 4.4549 & -1.5082 & 3.7537 & -1.3287 & 3.1677 & -1.1596 & 2.6689 & -0.9976 \\
\hline 0.4925 & 8.2628 & -2.6980 & 6.5350 & -2.2029 & 5.3907 & -1.9066 & 4.5192 & -1.6707 & 3.8158 & -1.4624 & 3.2261 & -1.2704 & 2.7220 & -1.0899 \\
\hline 0.4963 & 8.7512 & -3.3780 & 6.9254 & -2.7576 & 5.7343 & -2.3645 & 4.8275 & -2.0528 & 4.0917 & -1.7834 & 3.4702 & -1.5407 & 2.9346 & -1.3177 \\
\hline 0.5000 & 11.5051 & -7.3668 & 9.1850 & -5.9253 & 7.6842 & -4.9874 & 6.5307 & -4.2600 & 5.5791 & -3.6535 & 4.7606 & -3.1264 & 4.0418 & -2.6588 \\
\hline
\end{tabular}


Table 4.7: Normalized $K_{I I}$ and $K_{I I}$ values for $\mathrm{t} / \mathrm{W}=\mathbf{2 . 0}$

\begin{tabular}{|c|c|c|c|c|c|c|c|c|c|c|c|c|c|c|}
\hline \multirow{2}{*}{$z / t$} & \multicolumn{2}{|c|}{$\mathrm{a} / \mathrm{w}=\mathbf{0 . 1}$} & \multicolumn{2}{|c|}{$\mathrm{a} / \mathrm{w}=0.2$} & \multicolumn{2}{|c|}{$\mathrm{a} / \mathrm{w}=\mathbf{0 . 3}$} & \multicolumn{2}{|c|}{$\mathrm{a} / \mathrm{w}=0.4$} & \multicolumn{2}{|c|}{$\mathrm{a} / \mathrm{w}=0.5$} & \multicolumn{2}{|c|}{$a / w=0.6$} & \multicolumn{2}{|c|}{$\mathrm{a} / \mathrm{w}=\mathbf{0 . 7}$} \\
\hline & $K_{I I}$ & $K_{I I I}$ & $K_{I I}$ & $K_{I I I}$ & \begin{tabular}{|l|}
$K_{I I}$ \\
\end{tabular} & $K_{I I I}$ & \begin{tabular}{|l|}
$K_{I I}$ \\
\end{tabular} & $K_{I I I}$ & $\boldsymbol{K}_{I I}$ & $K_{I I I}$ & $\boldsymbol{K}_{I I}$ & $K_{I I I}$ & $\boldsymbol{K}_{\boldsymbol{I I}}$ & $\overline{K_{I I I}}$ \\
\hline 0.0000 & 5.0538 & 0.0000 & 4.4503 & 0.0000 & 3.8772 & 0.0000 & 3.3669 & 0.0000 & 2.9184 & 0.0000 & 2.5212 & 0.0000 & 2.1697 & 0.0000 \\
\hline 0.0253 & 5.0588 & -0.7120 & 4.4548 & -0.2574 & 3.8815 & -0.1171 & 3.3709 & -0.0549 & 2.9219 & -0.0243 & 2.5239 & -0.0087 & 2.1717 & -0.0007 \\
\hline 0.0507 & 5.0739 & -1.4181 & 4.4685 & -0.5123 & 3.8947 & -0.2330 & 3.3832 & -0.1094 & 2.9323 & -0.0486 & 2.5320 & -0.0177 & 2.1775 & -0.0016 \\
\hline 0.0760 & 5.0997 & -2.1119 & 4.4918 & -0.7622 & 3.9169 & -0.3465 & 3.4040 & -0.1628 & 2.9499 & -0.0727 & 2.5456 & -0.0269 & 2.1872 & -0.0029 \\
\hline 0.1013 & 5.1366 & -2.7871 & 4.5252 & -1.0046 & 3.9489 & -0.4562 & 3.4336 & -0.2147 & 2.9747 & -0.0967 & 2.5647 & -0.0366 & 2.2009 & -0.0051 \\
\hline 0.1266 & 5.1858 & -3.4367 & 4.5697 & -1.2367 & 3.9915 & -0.5611 & 3.4726 & -0.2648 & 3.0072 & -0.1206 & 2.5894 & -0.0472 & 2.2186 & -0.0083 \\
\hline 0.1520 & 5.2486 & -4.0531 & 4.6264 & -1.4558 & 4.0458 & -0.6598 & 3.5218 & -0.3127 & 3.0476 & $\mid-0.1446$ & 2.6200 & -0.0588 & 2.2402 & -0.0128 \\
\hline 0.1773 & 5.3266 & -4.6281 & 4.6971 & -1.6587 & 4.1133 & -0.7511 & 3.5819 & -0.3582 & 3.0963 & -0.1687 & 2.6567 & -0.0718 & 2.2659 & -0.0192 \\
\hline 0.2026 & 5.4220 & -5.1523 & 4.7837 & -1.8422 & 4.1956 & -0.8341 & 3.6539 & -0.4012 & 3.1538 & -0.1935 & 2.6995 & -0.0869 & 2.2958 & -0.0280 \\
\hline 0.2280 & 5.5375 & -5.6155 & 4.8892 & -2.0028 & 4.2948 & -0.9077 & 3.7388 & -0.4419 & 3.2206 & -0.2194 & 2.7487 & -0.1045 & 2.3299 & -0.0397 \\
\hline 0.2533 & 5.6763 & -6.0060 & 5.0169 & -2.1371 & 4.4132 & -0.9714 & 3.8377 & -0.4808 & 3.2971 & -0.2472 & 2.8045 & -0.1255 & 2.3683 & -0.0552 \\
\hline 0.2786 & 5.8427 & -6.3107 & 5.1716 & -2.2415 & 4.5533 & -1.0251 & 3.9516 & -0.5187 & 3.3836 & -0.2780 & 2.8671 & -0.1511 & 2.4110 & -0.0755 \\
\hline 0.3039 & 6.0424 & -6.5151 & 5.3590 & -2.3129 & 4.7177 & -1.0696 & 4.0813 & -0.5573 & 3.4805 & -0.3133 & 2.9366 & -0.1825 & 2.4582 & -0.1018 \\
\hline 0.3293 & 6.2833 & -6.6030 & 5.5867 & -2.3496 & 4.9089 & -1.1069 & 4.2273 & -0.5989 & 3.5879 & -0.3552 & 3.0131 & -0.2217 & 2.5101 & -0.1358 \\
\hline 0.3546 & 6.5778 & -6.5569 & 5.8633 & -2.3519 & 5.1291 & -1.1410 & 4.3900 & -0.6471 & 3.7055 & -0.4069 & 3.0965 & -0.2713 & 2.5666 & -0.1800 \\
\hline 0.3799 & 6.9471 & -6.3584 & 6.1988 & -2.3251 & 5.3794 & -1.1790 & 4.5688 & -0.7077 & 3.8328 & -0.4730 & 3.1863 & -0.3352 & 2.6280 & -0.2374 \\
\hline 0.4053 & 7.4293 & -5.9923 & 6.6025 & -2.2831 & 5.6598 & -1.2328 & 4.7625 & -0.7902 & 3.9687 & -0.5608 & 3.2820 & -0.4192 & 2.6942 & -0.3132 \\
\hline 0.4306 & 8.0938 & -5.4607 & 7.0814 & -2.2550 & 5.9689 & -1.3240 & 4.9689 & -0.9112 & 4.1111 & -0.6837 & 3.3825 & -0.5338 & 2.7654 & -0.4157 \\
\hline 0.4813 & 10.4778 & -4.4463 & 8.3335 & -2.6437 & 6.7057 & -1.9225 & 5.4426 & -1.5084 & 4.4375 & -1.2244 & 3.6212 & -1.0058 & 2.9494 & -0.8216 \\
\hline 0.4850 & 10.8192 & -4.5306 & 8.4926 & -2.8140 & 6.8007 & -2.0835 & 5.5076 & -1.6474 & 4.4866 & -1.3411 & 3.6608 & -1.1026 & 2.9828 & -0.9018 \\
\hline 0.4887 & 11.1577 & -4.6202 & 8.6600 & -2.9850 & 6.9042 & -2.2442 & 5.5802 & -1.7858 & 4.5422 & -1.4571 & 3.7057 & -1.1988 & 3.0203 & -0.9815 \\
\hline 0.4925 & 11.5912 & -4.8186 & 8.8870 & -3.2423 & 7.0523 & -2.4739 & 5.6889 & -1.9795 & 4.6278 & -1.6176 & 3.7760 & -1.3310 & 3.0789 & -1.0903 \\
\hline 0.4963 & 12.2538 & -5.3031 & 9.2651 & -3.7227 & 7.3154 & -2.8805 & 5.8906 & -2.3150 & 4.7908 & -1.8925 & 3.9110 & -1.5559 & 3.1911 & -1.2744 \\
\hline 0.5000 & 18.6379 & -12.2970 & 13.7790 & -9.0449 & 10.8096 & -7.0882 & 8.6989 & -5.7061 & 7.0872 & -4.6528 & 5.8003 & -3.8113 & 4.7412 & -3.1168 \\
\hline
\end{tabular}


Table 4.8: Normalized $K_{I I}$ and $K_{I I}$ values for $\mathrm{t} / \mathrm{W}=4.0$

\begin{tabular}{|c|c|c|c|c|c|c|c|c|c|c|c|c|c|c|}
\hline \multirow{2}{*}{$z / t$} & \multicolumn{2}{|c|}{$\mathrm{a} / \mathrm{w}=\mathbf{0 . 1}$} & \multicolumn{2}{|c|}{$\mathrm{a} / \mathrm{w}=0.2$} & \multicolumn{2}{|c|}{$\mathrm{a} / \mathrm{w}=0.3$} & \multicolumn{2}{|c|}{$\mathrm{a} / \mathrm{w}=0.4$} & \multicolumn{2}{|c|}{$\mathrm{a} / \mathrm{w}=0.5$} & \multicolumn{2}{|c|}{$a / w=0.6$} & \multicolumn{2}{|c|}{$\mathrm{a} / \mathrm{w}=\mathbf{0 . 7}$} \\
\hline & $K_{I I}$ & $K_{I I I}$ & $K_{I I}$ & $K_{I I I}$ & \begin{tabular}{|l|}
$K_{I I}$ \\
\end{tabular} & $K_{I I I}$ & $\boldsymbol{K}_{\boldsymbol{I I}}$ & $K_{I I I}$ & $\boldsymbol{K}_{\boldsymbol{I I}}$ & $K_{I I I}$ & $\boldsymbol{K}_{I I}$ & $K_{I I I}$ & $\boldsymbol{K}_{\boldsymbol{I I}}$ & $K_{I I I}$ \\
\hline 0.0000 & 5.4811 & 0.0000 & 4.7436 & 0.0000 & 4.0568 & 0.0000 & 3.4432 & 0.0000 & 2.9078 & 0.0000 & 2.4474 & 0.0000 & 2.0571 & 0.0000 \\
\hline 0.1039 & 5.4495 & -3.6161 & 4.7240 & -1.4943 & 4.0465 & -0.7932 & 3.4409 & -0.4488 & 2.9132 & -0.2488 & 2.4598 & -0.1235 & 2.0746 & -0.0435 \\
\hline 0.1336 & 5.4341 & -4.7092 & 4.7158 & -1.9385 & 4.0437 & -1.0253 & 3.4430 & -0.5779 & 2.9202 & -0.3188 & 2.4708 & -0.1570 & 2.0882 & -0.0545 \\
\hline 0.1633 & 5.4207 & -5.8382 & 4.7108 & -2.3918 & 4.0451 & -1.2595 & 3.4503 & -0.7065 & 2.9331 & -0.3874 & 2.4882 & -0.1893 & 2.1078 & -0.0644 \\
\hline 0.1930 & 5.4143 & -6.9999 & 4.7133 & -2.8515 & 4.0544 & -1.4939 & 3.4664 & -0.8333 & 2.9554 & -0.4539 & 2.5145 & -0.2199 & 2.1355 & -0.0735 \\
\hline 0.2227 & 5.4218 & -8.1821 & 4.7296 & -3.3115 & 4.0774 & -1.7250 & 3.4966 & -0.9562 & 2.9915 & -0.5173 & 2.5532 & -0.2488 & 2.1735 & -0.0819 \\
\hline 0.2466 & 5.4440 & -9.1365 & 4.7577 & -3.6767 & 4.1105 & -1.9056 & 3.5356 & -1.0507 & 3.0342 & -0.5656 & 2.5960 & -0.2709 & 2.2133 & -0.0888 \\
\hline 0.2649 & 5.4762 & -9.8497 & 4.7929 & -3.9450 & 4.1491 & -2.0365 & 3.5783 & -1.1184 & 3.0784 & -0.6002 & 2.6380 & -0.2872 & 2.2507 & -0.0944 \\
\hline 0.2832 & 5.5256 & -10.5368 & 4.8436 & -4.1996 & 4.2027 & -2.1589 & 3.6352 & -1.1812 & 3.1345 & -0.6328 & 2.6894 & -0.3035 & 2.2949 & -0.1010 \\
\hline 0.3014 & 5.5973 & -11.1810 & 4.9140 & -4.4340 & 4.2756 & -2.2699 & 3.7098 & -1.2381 & 3.2052 & -0.6634 & 2.7517 & -0.3201 & 2.3468 & -0.1092 \\
\hline 0.3197 & 5.6978 & -11.7608 & 5.0099 & -4.6405 & 4.3733 & -2.3662 & 3.8063 & -1.2881 & 3.2933 & -0.6923 & 2.8267 & -0.3380 & 2.4075 & -0.1198 \\
\hline 0.3380 & 5.8349 & -12.2488 & 5.1385 & -4.8095 & 4.5027 & -2.4438 & 3.9298 & -1.3306 & 3.4018 & -0.7203 & 2.9162 & -0.3582 & 2.4778 & -0.1341 \\
\hline 0.3563 & 6.0187 & -12.6110 & 5.3100 & -4.9298 & 4.6725 & -2.4991 & 4.0858 & -1.3654 & 3.5341 & -0.7485 & 3.0222 & -0.3827 & 2.5590 & -0.1539 \\
\hline 0.3745 & 6.2623 & -12.8057 & 5.5381 & -4.9883 & 4.8937 & -2.5289 & 4.2808 & -1.3935 & 3.6938 & -0.7793 & 3.1468 & -0.4137 & 2.6522 & -0.1815 \\
\hline 0.3928 & 6.5834 & -12.7823 & 5.8425 & -4.9711 & 5.1788 & -2.5320 & 4.5210 & -1.4178 & 3.8842 & -0.8162 & 3.2921 & -0.4549 & 2.7589 & -0.2202 \\
\hline 0.4111 & 7.0082 & -12.4808 & 6.2517 & -4.8660 & 5.5422 & -2.5114 & 4.8127 & -1.4439 & 4.1087 & -0.8649 & 3.4602 & -0.5113 & 2.8805 & -0.2748 \\
\hline 0.4385 & 7.9114 & -11.4101 & 7.1239 & -4.5466 & 6.2514 & -2.4592 & 5.3501 & -1.5068 & 4.5096 & -0.9757 & 3.7558 & -0.6375 & 3.0926 & -0.3976 \\
\hline 0.4659 & 9.7215 & -9.2546 & 8.5538 & -4.1385 & 7.2420 & -2.5103 & 6.0466 & -1.7080 & 5.0110 & -1.2239 & 4.1217 & -0.8912 & 3.3585 & -0.6368 \\
\hline 0.4775 & 10.9701 & -8.1574 & 9.3411 & -4.0489 & 7.7476 & -2.6311 & 6.3911 & -1.8841 & 5.2554 & -1.4082 & 4.3000 & -1.0665 & 3.4907 & -0.7966 \\
\hline 0.4813 & 11.5071 & -7.8187 & 9.6398 & -4.0635 & 7.9344 & -2.7092 & 6.5170 & -1.9742 & 5.3446 & -1.4956 & 4.3653 & -1.1464 & 3.5397 & -0.8677 \\
\hline 0.4850 & 12.1098 & -7.5103 & 9.9640 & -4.1077 & 8.1365 & -2.8124 & 6.6533 & -2.0856 & 5.4412 & -1.6006 & 4.4363 & -1.2407 & 3.5934 & -0.9507 \\
\hline 0.4887 & 12.8241 & -7.2771 & 10.3404 & -4.2151 & 8.3720 & -2.9686 & 6.8133 & -2.2407 & 5.5558 & -1.7416 & 4.5213 & -1.3645 & 3.6581 & -1.0579 \\
\hline 0.4925 & 13.6800 & -7.1516 & 10.7947 & -4.4099 & 8.6604 & -3.1976 & 7.0124 & -2.4557 & 5.7005 & -1.9316 & 4.6298 & -1.5284 & 3.7412 & -1.1979 \\
\hline 0.5000 & 21.4713 & -11.3008 & 15.8563 & -8.2095 & 12.3737 & -6.3693 & 9.8759 & -5.0704 & 7.9600 & -4.0808 & 6.4264 & -3.2901 & 5.1630 & -2.6373 \\
\hline
\end{tabular}


Table 4.9: Normalized $K_{I}$ values for $t / W=0.1$

\begin{tabular}{|c|c|c|c|c|c|c|c|}
\hline \multirow{2}{*}{$z \boldsymbol{t}$} & $\mathbf{a} / \mathbf{W}=\mathbf{0 . 1}$ & $\mathbf{a} / \mathbf{W}=\mathbf{0 . 2}$ & $\mathbf{a} / \mathbf{W}=\mathbf{0 . 3}$ & $\mathbf{a} / \mathbf{W}=\mathbf{0 . 4}$ & $\mathbf{a} / \mathbf{W}=\mathbf{0 . 5}$ & $\mathbf{a} / \mathbf{W}=\mathbf{0 . 6}$ & $\mathbf{a} / \mathbf{W}=\mathbf{0 . 7}$ \\
\cline { 2 - 8 } & $\boldsymbol{K}_{\boldsymbol{I}}$ & $\boldsymbol{K}_{\boldsymbol{I}}$ & $\boldsymbol{K}_{\boldsymbol{I}}$ & $\boldsymbol{K}_{\boldsymbol{I}}$ & $\boldsymbol{K}_{\boldsymbol{I}}$ & $\boldsymbol{K}_{\boldsymbol{I}}$ & $\boldsymbol{K}_{\boldsymbol{I}}$ \\
\hline 0.0000 & 1.1068 & 1.1216 & 1.1968 & 1.3438 & 1.5968 & 2.0421 & 2.9053 \\
\hline 0.0325 & 1.1067 & 1.1214 & 1.1966 & 1.3435 & 1.5964 & 2.0416 & 2.9045 \\
\hline 0.0650 & 1.1065 & 1.1211 & 1.1963 & 1.3432 & 1.5960 & 2.0410 & 2.9036 \\
\hline 0.0975 & 1.1059 & 1.1205 & 1.1955 & 1.3422 & 1.5948 & 2.0393 & 2.9009 \\
\hline 0.1300 & 1.1053 & 1.1198 & 1.1947 & 1.3412 & 1.5935 & 2.0375 & 2.8981 \\
\hline 0.1625 & 1.1042 & 1.1185 & 1.1932 & 1.3394 & 1.5912 & 2.0344 & 2.8932 \\
\hline 0.1950 & 1.1030 & 1.1172 & 1.1916 & 1.3375 & 1.5888 & 2.0311 & 2.8881 \\
\hline 0.2275 & 1.1011 & 1.1150 & 1.1891 & 1.3345 & 1.5850 & 2.0259 & 2.8800 \\
\hline 0.2600 & 1.0992 & 1.1128 & 1.1865 & 1.3314 & 1.5810 & 2.0205 & 2.8717 \\
\hline 0.2925 & 1.0960 & 1.1092 & 1.1823 & 1.3264 & 1.5748 & 2.0120 & 2.8586 \\
\hline 0.3250 & 1.0924 & 1.1052 & 1.1778 & 1.3210 & 1.5680 & 2.0029 & 2.8446 \\
\hline 0.3338 & 1.0910 & 1.1035 & 1.1759 & 1.3187 & 1.5652 & 1.9991 & 2.8389 \\
\hline 0.3425 & 1.0896 & 1.1020 & 1.1741 & 1.3166 & 1.5626 & 1.9956 & 2.8336 \\
\hline 0.3512 & 1.0881 & 1.1003 & 1.1722 & 1.3144 & 1.5599 & 1.9920 & 2.8280 \\
\hline 0.3600 & 1.0863 & 1.0983 & 1.1700 & 1.3118 & 1.5567 & 1.9876 & 2.8214 \\
\hline 0.3688 & 1.0847 & 1.0965 & 1.1679 & 1.3093 & 1.5536 & 1.9835 & 2.8151 \\
\hline 0.3775 & 1.0825 & 1.0941 & 1.1651 & 1.3061 & 1.5496 & 1.9782 & 2.8072 \\
\hline 0.3950 & 1.0777 & 1.0888 & 1.1592 & 1.2992 & 1.5411 & 1.9668 & 2.7901 \\
\hline 0.4125 & 1.0717 & 1.0822 & 1.1518 & 1.2906 & 1.5306 & 1.9529 & 2.7691 \\
\hline 0.4300 & 1.0641 & 1.0740 & 1.1427 & 1.2800 & 1.5175 & 1.9356 & 2.7434 \\
\hline 0.4475 & 1.0549 & 1.0639 & 1.1315 & 1.2670 & 1.5017 & 1.9146 & 2.7121 \\
\hline 0.4650 & 1.0427 & 1.0506 & 1.1168 & 1.2501 & 1.4810 & 1.8873 & 2.6716 \\
\hline 0.4825 & 1.0090 & 1.0154 & 1.0786 & 1.2067 & 1.4289 & 1.8198 & 2.5736 \\
\hline 0.5000 & 0.8087 & 0.8108 & 0.8597 & 0.9604 & 1.1357 & 1.4438 & 2.0365 \\
\hline
\end{tabular}


Table 4.10: Normalized $K_{I}$ values for $t / W=0.2$

\begin{tabular}{|c|c|c|c|c|c|c|c|}
\hline \multirow{2}{*}{$\boldsymbol{t} \boldsymbol{t}$} & $\mathbf{a} / \mathbf{W}=\mathbf{0 . 1}$ & $\mathbf{a} / \mathbf{W}=\mathbf{0 . 2}$ & $\mathbf{a} / \mathbf{W}=\mathbf{0 . 3}$ & $\mathbf{a} / \mathbf{W}=\mathbf{0 . 4}$ & $\mathbf{a} / \mathbf{W}=\mathbf{0 . 5}$ & $\mathbf{a} / \mathbf{W}=\mathbf{0 . 6}$ & $\mathbf{a} / \mathbf{W}=\mathbf{0 . 7}$ \\
\cline { 2 - 8 } & $\boldsymbol{K}_{\boldsymbol{I}}$ & $\boldsymbol{K}_{\boldsymbol{I}}$ & $\boldsymbol{K}_{\boldsymbol{I}}$ & $\boldsymbol{K}_{\boldsymbol{I}}$ & $\boldsymbol{K}_{\boldsymbol{I}}$ & $\boldsymbol{K}_{\boldsymbol{I}}$ & $\boldsymbol{K}_{\boldsymbol{I}}$ \\
\hline 0.0000 & 1.1033 & 1.1203 & 1.1967 & 1.3447 & 1.5986 & 2.0449 & 2.9085 \\
\hline 0.0295 & 1.1032 & 1.1201 & 1.1964 & 1.3444 & 1.5982 & 2.0443 & 2.9076 \\
\hline 0.0589 & 1.1031 & 1.1199 & 1.1962 & 1.3441 & 1.5978 & 2.0437 & 2.9067 \\
\hline 0.0884 & 1.1026 & 1.1193 & 1.1955 & 1.3431 & 1.5965 & 2.0420 & 2.9039 \\
\hline 0.1179 & 1.1022 & 1.1187 & 1.1947 & 1.3422 & 1.5953 & 2.0402 & 2.9011 \\
\hline 0.1473 & 1.1014 & 1.1176 & 1.1934 & 1.3405 & 1.5930 & 2.0370 & 2.8961 \\
\hline 0.1768 & 1.1005 & 1.1166 & 1.1920 & 1.3388 & 1.5908 & 2.0339 & 2.8911 \\
\hline 0.2062 & 1.0992 & 1.1148 & 1.1899 & 1.3361 & 1.5872 & 2.0288 & 2.8831 \\
\hline 0.2357 & 1.0978 & 1.1130 & 1.1877 & 1.3334 & 1.5837 & 2.0239 & 2.8752 \\
\hline 0.2652 & 1.0956 & 1.1102 & 1.1843 & 1.3292 & 1.5782 & 2.0162 & 2.8631 \\
\hline 0.2946 & 1.0933 & 1.1075 & 1.1810 & 1.3250 & 1.5728 & 2.0086 & 2.8512 \\
\hline 0.3241 & 1.0896 & 1.1030 & 1.1756 & 1.3184 & 1.5642 & 1.9967 & 2.8325 \\
\hline 0.3536 & 1.0858 & 1.0984 & 1.1702 & 1.3117 & 1.5557 & 1.9848 & 2.8139 \\
\hline 0.4125 & 1.0706 & 1.0808 & 1.1497 & 1.2869 & 1.5243 & 1.9419 & 2.7476 \\
\hline 0.4212 & 1.0669 & 1.0767 & 1.1448 & 1.2812 & 1.5170 & 1.9320 & 2.7323 \\
\hline 0.4300 & 1.0628 & 1.0719 & 1.1395 & 1.2748 & 1.5091 & 1.9212 & 2.7159 \\
\hline 0.4387 & 1.0575 & 1.0661 & 1.1328 & 1.2670 & 1.4994 & 1.9082 & 2.6962 \\
\hline 0.4475 & 1.0519 & 1.0599 & 1.1257 & 1.2586 & 1.4890 & 1.8943 & 2.6751 \\
\hline 0.4563 & 1.0450 & 1.0522 & 1.1171 & 1.2485 & 1.4764 & 1.8775 & 2.6499 \\
\hline 0.4650 & 1.0365 & 1.0428 & 1.1065 & 1.2362 & 1.4613 & 1.8573 & 2.6196 \\
\hline 0.4737 & 1.0262 & 1.0315 & 1.0939 & 1.2215 & 1.4432 & 1.8333 & 2.5836 \\
\hline 0.4825 & 1.0129 & 1.0169 & 1.0777 & 1.2027 & 1.4202 & 1.8029 & 2.5383 \\
\hline 0.4913 & 0.9785 & 0.9809 & 1.0386 & 1.1582 & 1.3667 & 1.7335 & 2.4375 \\
\hline 0.5000 & 0.7814 & 0.7798 & 0.8238 & 0.9170 & 1.0802 & 1.3670 & 1.9159 \\
\hline
\end{tabular}


Table 4.11: Normalized $K_{I}$ values for $t / W=0.5$

\begin{tabular}{|c|c|c|c|c|c|c|c|}
\hline \multirow{2}{*}{$\boldsymbol{t}$} & $\mathbf{a} / \mathbf{W}=\mathbf{0 . 1}$ & $\mathbf{a} / \mathbf{W}=\mathbf{0 . 2}$ & $\mathbf{a} / \mathbf{W}=\mathbf{0 . 3}$ & $\mathbf{a} / \mathbf{W}=\mathbf{0 . 4}$ & $\mathbf{a} / \mathbf{W}=\mathbf{0 . 5}$ & $\mathbf{a} / \mathbf{W}=\mathbf{0 . 6}$ & $\mathbf{a} / \mathbf{W}=\mathbf{0 . 7}$ \\
\cline { 2 - 8 } & $\boldsymbol{K}_{\boldsymbol{I}}$ & $\boldsymbol{K}_{\boldsymbol{I}}$ & $\boldsymbol{K}_{\boldsymbol{I}}$ & $\boldsymbol{K}_{\boldsymbol{I}}$ & $\boldsymbol{K}_{\boldsymbol{I}}$ & $\boldsymbol{K}_{\boldsymbol{I}}$ & $\boldsymbol{K}_{\boldsymbol{I}}$ \\
\hline 0.0000 & 1.0834 & 1.1119 & 1.1924 & 1.3413 & 1.5952 & 2.0374 & 2.8845 \\
\hline 0.0250 & 1.0834 & 1.1118 & 1.1922 & 1.3411 & 1.5949 & 2.0369 & 2.8838 \\
\hline 0.0500 & 1.0835 & 1.1117 & 1.1920 & 1.3408 & 1.5945 & 2.0364 & 2.8831 \\
\hline 0.0750 & 1.0837 & 1.1113 & 1.1915 & 1.3401 & 1.5934 & 2.0349 & 2.8809 \\
\hline 0.1000 & 1.0839 & 1.1110 & 1.1909 & 1.3393 & 1.5924 & 2.0334 & 2.8788 \\
\hline 0.1250 & 1.0842 & 1.1104 & 1.1899 & 1.3379 & 1.5905 & 2.0307 & 2.8749 \\
\hline 0.1500 & 1.0845 & 1.1098 & 1.1889 & 1.3365 & 1.5885 & 2.0280 & 2.8710 \\
\hline 0.1750 & 1.0847 & 1.1089 & 1.1874 & 1.3344 & 1.5856 & 2.0239 & 2.8650 \\
\hline 0.2000 & 1.0850 & 1.1079 & 1.1858 & 1.3322 & 1.5827 & 2.0198 & 2.8590 \\
\hline 0.2500 & 1.0853 & 1.1049 & 1.1812 & 1.3259 & 1.5741 & 2.0077 & 2.8412 \\
\hline 0.3000 & 1.0848 & 1.1003 & 1.1744 & 1.3168 & 1.5617 & 1.9901 & 2.8151 \\
\hline 0.3500 & 1.0826 & 1.0929 & 1.1640 & 1.3032 & 1.5433 & 1.9642 & 2.7760 \\
\hline 0.4000 & 1.0768 & 1.0805 & 1.1475 & 1.2819 & 1.5150 & 1.9243 & 2.7150 \\
\hline 0.4500 & 1.0601 & 1.0542 & 1.1147 & 1.2408 & 1.4615 & 1.8500 & 2.6008 \\
\hline 0.4550 & 1.0556 & 1.0482 & 1.1075 & 1.2321 & 1.4503 & 1.8347 & 2.5773 \\
\hline 0.4600 & 1.0517 & 1.0430 & 1.1013 & 1.2245 & 1.4407 & 1.8216 & 2.5573 \\
\hline 0.4650 & 1.0473 & 1.0373 & 1.0944 & 1.2162 & 1.4302 & 1.8072 & 2.5354 \\
\hline 0.4700 & 1.0420 & 1.0305 & 1.0864 & 1.2066 & 1.4180 & 1.7906 & 2.5103 \\
\hline 0.4750 & 1.0355 & 1.0223 & 1.0769 & 1.1952 & 1.4037 & 1.7713 & 2.4810 \\
\hline 0.4800 & 1.0270 & 1.0121 & 1.0651 & 1.1812 & 1.3863 & 1.7479 & 2.4459 \\
\hline 0.4850 & 1.0160 & 0.9991 & 1.0503 & 1.1638 & 1.3647 & 1.7191 & 2.4027 \\
\hline 0.4900 & 1.0026 & 0.9835 & 1.0325 & 1.1430 & 1.3390 & 1.6847 & 2.3514 \\
\hline 0.4950 & 0.9749 & 0.9533 & 0.9992 & 1.1047 & 1.2925 & 1.6240 & 2.2625 \\
\hline 0.5000 & 0.8124 & 0.7891 & 0.8244 & 0.9091 & 1.0611 & 1.3293 & 1.8447 \\
\hline
\end{tabular}


Table 4.12: Normalized $K_{I}$ values for $t / W=1.0$

\begin{tabular}{|c|c|c|c|c|c|c|c|}
\hline \multirow{2}{*}{$\boldsymbol{t}$} & $\mathbf{a} / \mathbf{W}=\mathbf{0 . 1}$ & $\mathbf{a} / \mathbf{W}=\mathbf{0 . 2}$ & $\mathbf{a} / \mathbf{W}=\mathbf{0 . 3}$ & $\mathbf{a} / \mathbf{W}=\mathbf{0 . 4}$ & $\mathbf{a} / \mathbf{W}=\mathbf{0 . 5}$ & $\mathbf{a} / \mathbf{W}=\mathbf{0 . 6}$ & $\mathbf{a} / \mathbf{W}=\mathbf{0 . 7}$ \\
\cline { 2 - 8 } & $\boldsymbol{K}_{\boldsymbol{I}}$ & $\boldsymbol{K}_{\boldsymbol{I}}$ & $\boldsymbol{K}_{\boldsymbol{I}}$ & $\boldsymbol{K}_{\boldsymbol{I}}$ & $\boldsymbol{K}_{\boldsymbol{I}}$ & $\boldsymbol{K}_{\boldsymbol{I}}$ & $\boldsymbol{K}_{\boldsymbol{I}}$ \\
\hline 0.0000 & 1.0568 & 1.0856 & 1.1711 & 1.3211 & 1.5716 & 2.0043 & 2.8319 \\
\hline 0.0193 & 1.0569 & 1.0856 & 1.1710 & 1.3210 & 1.5715 & 2.0041 & 2.8317 \\
\hline 0.0385 & 1.0570 & 1.0857 & 1.1710 & 1.3210 & 1.5714 & 2.0039 & 2.8315 \\
\hline 0.0771 & 1.0576 & 1.0860 & 1.1709 & 1.3204 & 1.5705 & 2.0028 & 2.8303 \\
\hline 0.1156 & 1.0587 & 1.0865 & 1.1705 & 1.3195 & 1.5691 & 2.0009 & 2.8281 \\
\hline 0.1542 & 1.0601 & 1.0870 & 1.1700 & 1.3181 & 1.5668 & 1.9979 & 2.8246 \\
\hline 0.1927 & 1.0620 & 1.0876 & 1.1690 & 1.3159 & 1.5636 & 1.9936 & 2.8194 \\
\hline 0.2313 & 1.0643 & 1.0880 & 1.1675 & 1.3128 & 1.5589 & 1.9874 & 2.8119 \\
\hline 0.2698 & 1.0671 & 1.0880 & 1.1651 & 1.3083 & 1.5523 & 1.9786 & 2.8009 \\
\hline 0.3083 & 1.0701 & 1.0872 & 1.1613 & 1.3018 & 1.5429 & 1.9659 & 2.7846 \\
\hline 0.3469 & 1.0729 & 1.0850 & 1.1553 & 1.2921 & 1.5293 & 1.9473 & 2.7600 \\
\hline 0.3854 & 1.0747 & 1.0803 & 1.1456 & 1.2776 & 1.5090 & 1.9195 & 2.7219 \\
\hline 0.4240 & 1.0737 & 1.0706 & 1.1294 & 1.2544 & 1.4772 & 1.8756 & 2.6596 \\
\hline 0.4625 & 1.0623 & 1.0468 & 1.0956 & 1.2093 & 1.4168 & 1.7923 & 2.5366 \\
\hline 0.4662 & 1.0584 & 1.0410 & 1.0881 & 1.1997 & 1.4043 & 1.7752 & 2.5108 \\
\hline 0.4700 & 1.0553 & 1.0362 & 1.0819 & 1.1918 & 1.3941 & 1.7611 & 2.4897 \\
\hline 0.4738 & 1.0517 & 1.0308 & 1.0749 & 1.1830 & 1.3827 & 1.7455 & 2.4664 \\
\hline 0.4775 & 1.0472 & 1.0244 & 1.0669 & 1.1730 & 1.3697 & 1.7278 & 2.4397 \\
\hline 0.4813 & 1.0415 & 1.0167 & 1.0573 & 1.1611 & 1.3546 & 1.7072 & 2.4089 \\
\hline 0.4850 & 1.0341 & 1.0071 & 1.0456 & 1.1468 & 1.3364 & 1.6827 & 2.3721 \\
\hline 0.4887 & 1.0237 & 0.9942 & 1.0304 & 1.1284 & 1.3134 & 1.6517 & 2.3258 \\
\hline 0.4925 & 1.0098 & 0.9773 & 1.0108 & 1.1051 & 1.2843 & 1.6128 & 2.2678 \\
\hline 0.4963 & 0.9871 & 0.9514 & 0.9813 & 1.0705 & 1.2418 & 1.5566 & 2.1845 \\
\hline 0.5000 & 0.8497 & 0.8123 & 0.8338 & 0.9062 & 1.0476 & 1.3086 & 1.8293 \\
\hline
\end{tabular}


Table 4.13: Normalized $K_{I}$ values for $t / W=2.0$

\begin{tabular}{|c|c|c|c|c|c|c|c|}
\hline \multirow{2}{*}{$\boldsymbol{t}$} & $\mathbf{a} / \mathbf{w}=\mathbf{0 . 1}$ & $\mathbf{a} / \mathbf{w}=\mathbf{0 . 2}$ & $\mathbf{a} / \mathbf{w}=\mathbf{0 . 3}$ & $\mathbf{a} / \mathbf{w}=\mathbf{0 . 4}$ & $\mathbf{a} / \mathbf{w}=\mathbf{0 . 5}$ & $\mathbf{a} / \mathbf{w}=\mathbf{0 . 6}$ & $\mathbf{a} / \mathbf{w}=\mathbf{0 . 7}$ \\
\cline { 2 - 8 } & $\boldsymbol{K}_{\boldsymbol{I}}$ & $\boldsymbol{K}_{\boldsymbol{I}}$ & $\boldsymbol{K}_{\boldsymbol{I}}$ & $\boldsymbol{K}_{\boldsymbol{I}}$ & $\boldsymbol{K}_{\boldsymbol{I}}$ & $\boldsymbol{K}_{\boldsymbol{I}}$ & $\boldsymbol{K}_{\boldsymbol{I}}$ \\
\hline 0.0000 & 1.0743 & 1.0856 & 1.1616 & 1.3056 & 1.5500 & 1.9744 & 2.7894 \\
\hline 0.0253 & 1.0741 & 1.0854 & 1.1614 & 1.3054 & 1.5498 & 1.9742 & 2.7892 \\
\hline 0.0507 & 1.0733 & 1.0849 & 1.1610 & 1.3050 & 1.5494 & 1.9738 & 2.7888 \\
\hline 0.0760 & 1.0720 & 1.0840 & 1.1603 & 1.3043 & 1.5487 & 1.9731 & 2.7882 \\
\hline 0.1013 & 1.0703 & 1.0828 & 1.1593 & 1.3033 & 1.5477 & 1.9721 & 2.7872 \\
\hline 0.1266 & 1.0682 & 1.0813 & 1.1580 & 1.3021 & 1.5464 & 1.9708 & 2.7860 \\
\hline 0.1773 & 1.0628 & 1.0776 & 1.1548 & 1.2987 & 1.5428 & 1.9671 & 2.7826 \\
\hline 0.2280 & 1.0566 & 1.0734 & 1.1507 & 1.2942 & 1.5378 & 1.9619 & 2.7775 \\
\hline 0.2786 & 1.0504 & 1.0690 & 1.1458 & 1.2882 & 1.5307 & 1.9541 & 2.7696 \\
\hline 0.3039 & 1.0477 & 1.0668 & 1.1428 & 1.2843 & 1.5260 & 1.9486 & 2.7639 \\
\hline 0.3293 & 1.0455 & 1.0646 & 1.1394 & 1.2796 & 1.5201 & 1.9416 & 2.7563 \\
\hline 0.3546 & 1.0440 & 1.0622 & 1.1353 & 1.2737 & 1.5123 & 1.9322 & 2.7456 \\
\hline 0.3673 & 1.0437 & 1.0608 & 1.1326 & 1.2697 & 1.5071 & 1.9256 & 2.7378 \\
\hline 0.3799 & 1.0433 & 1.0594 & 1.1299 & 1.2658 & 1.5019 & 1.9190 & 2.7301 \\
\hline 0.3926 & 1.0433 & 1.0575 & 1.1261 & 1.2602 & 1.4944 & 1.9094 & 2.7181 \\
\hline 0.4306 & 1.0435 & 1.0495 & 1.1113 & 1.2385 & 1.4648 & 1.8706 & 2.6682 \\
\hline 0.4686 & 1.0353 & 1.0244 & 1.0730 & 1.1856 & 1.3935 & 1.7736 & 2.5312 \\
\hline 0.4831 & 1.0232 & 1.0024 & 1.0429 & 1.1461 & 1.3412 & 1.7019 & 2.4257 \\
\hline 0.4859 & 1.0182 & 0.9949 & 1.0332 & 1.1338 & 1.3253 & 1.6801 & 2.3931 \\
\hline 0.4887 & 1.0118 & 0.9857 & 1.0216 & 1.1193 & 1.3066 & 1.6547 & 2.3549 \\
\hline 0.4916 & 1.0026 & 0.9734 & 1.0065 & 1.1006 & 1.2828 & 1.6224 & 2.3065 \\
\hline 0.4944 & 0.9885 & 0.9557 & 0.9855 & 1.0754 & 1.2511 & 1.5797 & 2.2425 \\
\hline 0.4972 & 0.9615 & 0.9244 & 0.9498 & 1.0334 & 1.1993 & 1.5109 & 2.1402 \\
\hline 0.5000 & 0.8196 & 0.7797 & 0.7960 & 0.8615 & 0.9954 & 1.2486 & 1.7603 \\
\hline
\end{tabular}


Table 4.14: Normalized $K_{I}$ values for $t / W=4.0$

\begin{tabular}{|c|c|c|c|c|c|c|c|}
\hline \multirow{2}{*}{$\boldsymbol{t}$} & $\mathbf{a} / \mathbf{w}=\mathbf{0 . 1}$ & $\mathbf{a} / \mathbf{w}=\mathbf{0 . 2}$ & $\mathbf{a} / \mathbf{w}=\mathbf{0 . 3}$ & $\mathbf{a} / \mathbf{w}=\mathbf{0 . 4}$ & $\mathbf{a} / \mathbf{w}=\mathbf{0 . 5}$ & $\mathbf{a} / \mathbf{w}=\mathbf{0 . 6}$ & $\mathbf{a} / \mathbf{w}=\mathbf{0 . 7}$ \\
\cline { 2 - 8 } & $\boldsymbol{K}_{\boldsymbol{I}}$ & $\boldsymbol{K}_{\boldsymbol{I}}$ & $\boldsymbol{K}_{\boldsymbol{I}}$ & $\boldsymbol{K}_{\boldsymbol{I}}$ & $\boldsymbol{K}_{\boldsymbol{I}}$ & $\boldsymbol{K}_{\boldsymbol{I}}$ & $\boldsymbol{K}_{\boldsymbol{I}}$ \\
\hline 0.0000 & 1.1048 & 1.1100 & 1.1793 & 1.3170 & 1.5552 & 1.9730 & 2.7814 \\
\hline 0.0445 & 1.1039 & 1.1091 & 1.1785 & 1.3162 & 1.5544 & 1.9723 & 2.7808 \\
\hline 0.1039 & 1.1002 & 1.1055 & 1.1750 & 1.3129 & 1.5513 & 1.9694 & 2.7782 \\
\hline 0.1633 & 1.0923 & 1.0980 & 1.1680 & 1.3064 & 1.5452 & 1.9637 & 2.7729 \\
\hline 0.2227 & 1.0787 & 1.0854 & 1.1564 & 1.2956 & 1.5352 & 1.9543 & 2.7641 \\
\hline 0.2558 & 1.0680 & 1.0758 & 1.1477 & 1.2876 & 1.5276 & 1.9472 & 2.7575 \\
\hline 0.2740 & 1.0609 & 1.0695 & 1.1421 & 1.2824 & 1.5227 & 1.9425 & 2.7530 \\
\hline 0.2923 & 1.0529 & 1.0626 & 1.1359 & 1.2766 & 1.5171 & 1.9372 & 2.7481 \\
\hline 0.3014 & 1.0486 & 1.0588 & 1.1325 & 1.2734 & 1.5141 & 1.9343 & 2.7453 \\
\hline 0.3106 & 1.0442 & 1.0551 & 1.1291 & 1.2702 & 1.5110 & 1.9313 & 2.7425 \\
\hline 0.3197 & 1.0394 & 1.0511 & 1.1255 & 1.2667 & 1.5076 & 1.9280 & 2.7393 \\
\hline 0.3288 & 1.0347 & 1.0470 & 1.1218 & 1.2632 & 1.5042 & 1.9247 & 2.7361 \\
\hline 0.3563 & 1.0193 & 1.0341 & 1.1098 & 1.2514 & 1.4923 & 1.9128 & 2.7247 \\
\hline 0.3837 & 1.0032 & 1.0206 & 1.0967 & 1.2378 & 1.4781 & 1.8982 & 2.7102 \\
\hline 0.4111 & 0.9878 & 1.0066 & 1.0815 & 1.2208 & 1.4592 & 1.8776 & 2.6886 \\
\hline 0.4385 & 0.9743 & 0.9916 & 1.0632 & 1.1986 & 1.4329 & 1.8470 & 2.6545 \\
\hline 0.4659 & 0.9627 & 0.9703 & 1.0328 & 1.1584 & 1.3812 & 1.7815 & 2.5721 \\
\hline 0.4775 & 0.9559 & 0.9558 & 1.0119 & 1.1303 & 1.3443 & 1.7327 & 2.5061 \\
\hline 0.4813 & 0.9525 & 0.9489 & 1.0021 & 1.1173 & 1.3270 & 1.7095 & 2.4734 \\
\hline 0.4850 & 0.9480 & 0.9405 & 0.9903 & 1.1017 & 1.3065 & 1.6817 & 2.4335 \\
\hline 0.4887 & 0.9413 & 0.9289 & 0.9747 & 1.0813 & 1.2797 & 1.6452 & 2.3802 \\
\hline 0.4925 & 0.9314 & 0.9130 & 0.9538 & 1.0546 & 1.2448 & 1.5976 & 2.3096 \\
\hline 0.4962 & 0.9077 & 0.8812 & 0.9149 & 1.0066 & 1.1837 & 1.5145 & 2.1851 \\
\hline 0.5000 & 0.7980 & 0.7605 & 0.7806 & 0.8510 & 0.9934 & 1.2629 & 1.8111 \\
\hline
\end{tabular}


Table 4.15: Normalized T-stress values for $t / W=0.1$

\begin{tabular}{|c|c|c|c|c|c|c|c|c|c|c|c|c|c|c|}
\hline \multirow{2}{*}{$z / t$} & \multicolumn{2}{|c|}{$\mathrm{a} / \mathbf{W}=\mathbf{0 . 1}$} & \multicolumn{2}{|c|}{$\mathrm{a} / \mathrm{W}=\mathbf{0 . 2}$} & \multicolumn{2}{|c|}{$\mathrm{a} / \mathrm{W}=\mathbf{0 . 3}$} & \multicolumn{2}{|c|}{$\mathrm{a} / \mathrm{W}=0.4$} & \multicolumn{2}{|c|}{$\mathrm{a} / \mathrm{W}=0.5$} & \multicolumn{2}{|c|}{$\mathrm{a} / \mathrm{W}=\mathbf{0 . 6}$} & \multicolumn{2}{|c|}{$\mathrm{a} / \mathrm{W}=\mathbf{0 . 7}$} \\
\hline & $T_{11}$ & $T_{33}$ & $T_{11}$ & $T_{33}$ & $T_{11}$ & $T_{33}$ & $T_{11}$ & $T_{33}$ & $T_{11}$ & $T_{33}$ & $T_{11}$ & $T_{33}$ & $T_{11}$ & $T_{33}$ \\
\hline 0.0000 & & & & -5.5087 & & -7.0326 & & & & -11.6905 & 6.7201 & -16.0032 & 12.7592 & -23.7133 \\
\hline 0.0325 & -1.9415 & -4.0845 & -0.9306 & & 0.2000 & -7.0732 & 1.6212 & -9.0122 & & -11.7618 & 6.7256 & -16.1044 & 12.7663 & -23.8718 \\
\hline 0.0650 & -1.9445 & -4.1024 & -0.9346 & -5.5664 & & -7.1091 & & & 3.5801 & -11.8248 & 6.7114 & -16.1939 & 12.7432 & -24.0121 \\
\hline 0.0975 & -1.9372 & -4.1625 & & & 0.2096 & & & & & & & & & -24.4779 \\
\hline 0.1300 & -1.9390 & -4.2200 & -0.9245 & -5.7431 & & -7.3433 & & -9.3656 & 3.5996 & -12.2356 & 6.7363 & -16.7767 & 12.7752 & -24.9244 \\
\hline & & & & & & & & & & & & & 12.8468 & -25.7798 \\
\hline 0.1950 & -1.9301 & -4.4362 & -0.9086 & -6.0668 & & -7.7718 & & & & -12.9861 & & -17.8402 & 12.8229 & -26.5872 \\
\hline & & & & & & & & & & & & & & -28.0143 \\
\hline 0.2600 & -1.9162 & -4.7902 & -0.8860 & -6.5935 & 0.2530 & -8.4673 & 1.6866 & -10.8339 & 3.6719 & -14.2012 & 6.8282 & -19.5594 & 12.8904 & -29.2691 \\
\hline 0.2925 & -1.8896 & & -0.8482 & & 0.3007 & & 1.7474 & -11.6140 & 3.7512 & & 6.9355 & & 13.0447 & -31.5547 \\
\hline 0.3250 & -1.8512 & -5.4201 & -0.7934 & -7.5231 & 0.3705 & -9.6920 & 1.8369 & -12.4297 & 3.8686 & -16.3314 & 7.0962 & -22.5625 & 13.2810 & -33.9309 \\
\hline 0.3338 & -1.8736 & -5.5390 & -0.8263 & -7.6985 & 0.3272 & -9.9233 & 1.7806 & -12.7308 & 3.7935 & -16.7320 & 6.9899 & -23.1242 & 13.1146 & -34.7959 \\
\hline 0.3425 & -1.8667 & -5.6541 & -0.8169 & -7.8677 & 0.3389 & -10.1460 & 1.7957 & -13.0206 & 3.8127 & -17.1188 & 7.0154 & -23.6690 & 13.1503 & -35.6405 \\
\hline 0.3512 & -1.8618 & -5.8015 & -0.8108 & -8.0833 & 0.3462 & -10.4291 & 1.8047 & -13.3887 & 3.8243 & -17.6093 & 7.0304 & -24.3588 & 13.1693 & -36.7079 \\
\hline 0.3600 & -1.8525 & -5.9317 & -0.7980 & -8.2741 & 0.3625 & -10.6800 & 1.8254 & -13.7152 & 3.8515 & -18.0444 & 7.0673 & -24.9707 & 13.2230 & -37.6542 \\
\hline 0.3688 & -1.8521 & -6.1057 & -0.7988 & -8.5280 & 0.3607 & -11.0133 & 1.8228 & -14.1484 & 3.8474 & -18.6213 & 7.0601 & -25.7811 & 13.2076 & -38.9056 \\
\hline 0.3775 & -1.8392 & & -0.7810 & & & & & & 3.8860 & & & & 13.2858 & -39.9835 \\
\hline 0.3950 & -1.8233 & -6.6384 & -0.7610 & -9.3054 & 0.4085 & -12.0342 & 1.8840 & -15.4753 & 3.9272 & -20.3875 & 7.1682 & -28.2608 & 13.3626 & -42.7312 \\
\hline 0.4125 & -1.8030 & & -0.7356 & & 0.4407 & -12.9270 & 1.9251 & -16.6348 & 3.9809 & & 7.2408 & -30.4228 & 13.4659 & -46.0596 \\
\hline 0.4300 & -1.7752 & -7.7150 & -0.7001 & -10.8691 & 0.4859 & -14.0846 & 1.9834 & -18.1373 & 4.0575 & -23.9259 & 7.3457 & -33.2185 & 13.6190 & -50.3565 \\
\hline 0.4475 & -1.7251 & -8.7169 & -0.6335 & & 0.5722 & -15.9795 & 2.0952 & -20.5946 & 4.2055 & -27.1876 & 7.5509 & -37.7793 & 13.9268 & -57.3490 \\
\hline 0.4650 & -1.6757 & -11.5911 & -0.5676 & -16.4371 & 0.6587 & -21.3572 & 2.2083 & -27.5553 & 4.3568 & -36.4168 & 7.7633 & -50.6741 & 14.2501 & -77.0943 \\
\hline 0.4825 & -1.9299 & -20.0289 & -0.9266 & -28.5054 & 0.1986 & -37.0951 & 1.6217 & -47.9140 & 3.5896 & -63.3889 & 6.7077 & -88.3192 & 12.6663 & -134.6366 \\
\hline 0.5000 & -5.7355 & -25.1754 & -6.2744 & -35.8060 & -6.7090 & -46.5777 & -7.2524 & -60.1402 & -8.0909 & -79.5288 & -9.4758 & -110.7393 & -11.8030 & -168.662 \\
\hline
\end{tabular}


Table 4.16: Normalized T-stress values for $t / W=0.2$

\begin{tabular}{|c|c|c|c|c|c|c|c|c|c|c|c|c|c|c|}
\hline \multirow{2}{*}{$z / t$} & \multicolumn{2}{|c|}{$\mathrm{a} / \mathbf{W}=\mathbf{0 . 1}$} & \multicolumn{2}{|c|}{$\mathrm{a} / \mathrm{W}=\mathbf{0 . 2}$} & \multicolumn{2}{|c|}{$\mathrm{a} / \mathbf{W}=\mathbf{0 . 3}$} & \multicolumn{2}{|c|}{$\mathrm{a} / \mathrm{W}=0.4$} & \multicolumn{2}{|c|}{$\mathrm{a} / \mathrm{W}=\mathbf{0 . 5}$} & \multicolumn{2}{|c|}{$\mathrm{a} / \mathrm{W}=\mathbf{0 . 6}$} & \multicolumn{2}{|c|}{$\mathrm{a} / \mathrm{W}=\mathbf{0 . 7}$} \\
\hline & $T_{11}$ & $T_{33}$ & $T_{11}$ & $T_{33}$ & $T_{11}$ & $T_{33}$ & $T_{11}$ & $T_{33}$ & $T_{11}$ & $T_{33}$ & $T_{11}$ & $T_{33}$ & $T_{11}$ & $T_{33}$ \\
\hline 0.0000 & & -2.9602 & & -3.8266 & & -4.7516 & 1.3910 & -5.9361 & & -7.5886 & 6.3573 & -10.1020 & 12.2933 & -14.2688 \\
\hline 0.0295 & -1.9769 & -2.9712 & -1.0668 & -3.8430 & 0.0202 & -4.7740 & 1.3923 & -5.9657 & & -7.6289 & 6.3586 & -10.1598 & & -14.3616 \\
\hline 0.0589 & -1.9800 & & & -3.8599 & & -4.7969 & & -5.9961 & 3.3000 & -7.6701 & & -10.2189 & 12.2758 & -14.4564 \\
\hline 0.0884 & & -3.0154 & & & & & & & & & & & & -14.7370 \\
\hline 0.1179 & -1.9881 & -3.0493 & -1.0653 & -3.9613 & 0.0235 & -4.9346 & & -6.1784 & & & & -10.5736 & 12.2690 & -15.0244 \\
\hline & & & & & & & & & & & & & & -15.5283 \\
\hline 0.1768 & -2.0013 & -3.1700 & -1.0588 & -4.1440 & & -5.1821 & & -6.5058 & & -8.3598 & 6.3653 & -11.2086 & 12.2563 & -16.0384 \\
\hline & & & & & & & & & & & & & & -16.8404 \\
\hline 0.2357 & -2.0191 & -3.3609 & -1.0498 & -4.4332 & 0.0472 & -5.5722 & 1.4216 & -7.0206 & & -9.0547 & 6.3766 & -12.2031 & 12.2351 & -17.6200 \\
\hline 0.2652 & -2.0218 & & -1.0325 & -4.6664 & 0.0703 & -5.8846 & & -7.4312 & & -9.6070 & 6.4180 & -12.9901 & 12.2741 & -18.8626 \\
\hline 0.2946 & -2.0448 & -3.6499 & -1.0450 & -4.8712 & 0.0543 & -6.1603 & 1.4272 & -7.7946 & 3.3360 & -10.0969 & 6.3649 & -13.6904 & 12.1674 & -19.9734 \\
\hline 0.3241 & -2.0362 & & -1.0106 & -5.3091 & 0.0984 & -6.7416 & 1.4822 & -8.5547 & 3.4052 & -11.1144 & 6.4528 & -15.1315 & 12.2715 & -22.2272 \\
\hline 0.3536 & -2.0728 & & -1.0410 & -5.6093 & 0.0582 & -7.1428 & 1.4282 & -9.0812 & 3.3301 & -11.8219 & 6.3395 & -16.1385 & 12.0679 & -23.8142 \\
\hline & -1.9545 & -5.2390 & -0.8329 & -7.2277 & 0.3263 & -9.2747 & 1.7721 & -11.8538 & 3.7793 & -15.5165 & 6.9490 & -21.3371 & 12.9369 & -31.8687 \\
\hline & -2.0675 & & -0.9991 & & 0.1044 & -9.0800 & 1.4822 & -11.6040 & 3.3881 & -15.1879 & 6.3914 & -20.8831 & 12.0529 & -31.1886 \\
\hline 0.4300 & -2.0379 & -5.5753 & -0.9511 & -7.7218 & 0.1669 & -9.9223 & 1.5614 & -12.6928 & 3.4946 & -16.6293 & 6.5377 & -22.8959 & 12.2680 & -34.2708 \\
\hline 0.4387 & -2.0415 & & -0.9533 & & & & & & & & & & 12.2496 & -37.2394 \\
\hline 0.4475 & -2.0366 & -6.3940 & -0.9452 & -8.9157 & 0.1731 & -11.4873 & 1.5696 & -14.7218 & 3.5046 & -19.3242 & 6.5473 & -26.6708 & 12.2630 & -40.0794 \\
\hline 0.4563 & -2.0294 & & -0.9348 & & 0.1855 & & 1.5855 & -15.8499 & & -20.8189 & 6.5729 & -28.7577 & 12.2900 & -43.2759 \\
\hline 0.4650 & -2.0155 & -7.4473 & -0.9157 & -10.4399 & 0.2094 & -13.4788 & 1.6164 & -17.2976 & & -22.7354 & 6.6275 & -31.4299 & 12.3622 & -47.3597 \\
\hline 0.4737 & -1.9808 & -8.4207 & -0.8678 & -11.8408 & 0.2713 & -15.3064 & 1.6971 & -19.6591 & 3.6730 & -25.8583 & 6.7762 & -35.7779 & 12.5799 & -53.9871 \\
\hline 0.4825 & -1.9471 & -11.2107 & -0.8210 & -15.8251 & 0.3331 & -20.4881 & 1.7794 & -26.3432 & 3.7846 & -34.6888 & 6.9345 & -48.0652 & 12.8192 & -72.6953 \\
\hline 0.4913 & -2.2032 & -19.3949 & -1.1769 & -27.4865 & -0.1193 & -35.6458 & 1.2078 & -45.8866 & 3.0441 & -60.4896 & 5.9266 & \begin{tabular}{|l|}
-83.9230 \\
\end{tabular} & 11.3250 & -127.1853 \\
\hline 0.5000 & -5.8486 & -24.3316 & -6.2630 & -34.4511 & -6.6529 & -44.6511 & -7.1420 & -57.4473 & -7.8849 & -75.6780 & -9.1161 & -104.9007 & -11.2160 & -158.768 \\
\hline
\end{tabular}


Table 4.17: Normalized T-stress values for $t / W=0.5$

\begin{tabular}{|c|c|c|c|c|c|c|c|c|c|c|c|c|c|c|}
\hline \multirow{2}{*}{$z / t$} & \multicolumn{2}{|c|}{$\mathrm{a} / \mathrm{W}=\mathbf{0 . 1}$} & \multicolumn{2}{|c|}{$\mathrm{a} / \mathrm{W}=\mathbf{0 . 2}$} & \multicolumn{2}{|c|}{$\mathrm{a} / \mathrm{W}=\mathbf{0 . 3}$} & \multicolumn{2}{|c|}{$\mathrm{a} / \mathrm{W}=\mathbf{0 . 4}$} & \multicolumn{2}{|c|}{$\mathrm{a} / \mathrm{W}=\mathbf{0 . 5}$} & \multicolumn{2}{|c|}{$\mathrm{a} / \mathrm{W}=\mathbf{0 . 6}$} & \multicolumn{2}{|c|}{$\mathrm{a} / \mathbf{W}=\mathbf{0 . 7}$} \\
\hline & $T_{11}$ & $\boldsymbol{T}_{33}$ & $T_{11}$ & $T_{33}$ & $T_{11}$ & $\boldsymbol{T}_{33}$ & $T_{11}$ & $T_{33}$ & $T_{11}$ & $T_{33}$ & $T_{11}$ & $T_{33}$ & $T_{11}$ & $T_{33}$ \\
\hline 0.0000 & -2.2107 & -2.1374 & & -2.2828 & & -2.6156 & 1.2860 & -3.0102 & & -3.5004 & 6.2636 & -4.0149 & 12.2498 & -4.0278 \\
\hline 0.0250 & -2.2093 & -2.1412 & -1.1238 & -2.2908 & -0.0565 & -2.6265 & 1.2849 & -3.0253 & 3.1791 & -3.5216 & 6.2596 & -4.0472 & 12.2419 & -4.0837 \\
\hline 0.0500 & -2.2087 & & & & & & 1.2814 & & 3.1738 & & 6.2505 & -4.0806 & 12.2253 & -4.1415 \\
\hline 0.0750 & -2.2039 & -2.1567 & -1.1282 & -2.3233 & -0.0611 & -2.6707 & 1.2807 & -3.0860 & 3.1720 & -3.6076 & 6.2440 & -4.1776 & 12.2106 & -4.3090 \\
\hline 0.1000 & -2.2008 & -2.1686 & & & -0.0666 & -2.7045 & 1.2747 & -3.1326 & & -3.6734 & 6.2264 & -4.2772 & 12.1772 & -4.4812 \\
\hline & -2.1939 & -2.1899 & & & & & & & & & & -4.4468 & & -4.7733 \\
\hline 0.1500 & -2.1889 & -2.2117 & & -2.4340 & & -2.8216 & & -3.2932 & & & 6.1853 & -4.6191 & 12.0931 & -5.0702 \\
\hline & & & & & & & & & & & & & & -5.5072 \\
\hline 0.2000 & -2.1756 & -2.2806 & -1.1619 & -2.5641 & -0.0941 & -2.9995 & 1.2485 & -3.5364 & & -4.2411 & 6.1260 & -5.1319 & 11.9669 & -5.9484 \\
\hline 0.2500 & -2.1655 & -2.3859 & -1.1851 & -2.7520 & -0.1140 & -3.2568 & 1.2288 & & & -4.7295 & 6.0460 & -5.8604 & 11.7889 & -7.1863 \\
\hline 0.3000 & -2.1658 & -2.5450 & -1.2146 & -3.0230 & -0.1379 & -3.6277 & 1.2036 & -4.3877 & 3.0358 & -5.4235 & 5.9406 & -6.8860 & 11.5450 & -8.9094 \\
\hline 0.3500 & -2.1860 & -2.7926 & -1.2516 & -3.4295 & -0.1691 & -4.1813 & 1.1677 & -5.1290 & 2.9707 & -6.4399 & 5.7972 & -8.3714 & 11.2062 & -11.3662 \\
\hline 0.4000 & -2.2378 & -3.2059 & -1.3067 & -4.0852 & -0.2246 & -5.0658 & 1.0972 & -6.3004 & 2.8556 & -8.0272 & 5.5693 & -10.6612 & 10.6929 & -15.0792 \\
\hline 0.4500 & -2.2342 & -4.2721 & -1.2827 & -5.6706 & -0.1858 & -7.1555 & 1.1418 & -9.0184 & 2.8871 & -11.6472 & 5.5314 & -15.7686 & 10.4081 & -23.0846 \\
\hline 0.4550 & -2.2544 & -4.4965 & -1.3152 & -5.9972 & -0.2297 & -7.5825 & 1.0835 & -9.5699 & 2.8061 & -12.3774 & 5.4085 & -16.7901 & 10.1849 & -24.6661 \\
\hline 0.4600 & -2.2479 & -4.7577 & -1.3070 & -6.3716 & -0.2191 & -8.0696 & 1.0959 & -10.1976 & 2.8195 & -13.2064 & 5.4182 & -17.9453 & 10.1729 & -26.4423 \\
\hline 0.4650 & -2.2516 & -5.0395 & -1.3147 & -6.7750 & -0.2294 & -8.5941 & 1.0819 & -10.8732 & 2.7981 & -14.0976 & 5.3802 & -19.1856 & 10.0867 & -28.3453 \\
\hline 0.4700 & -2.2540 & -5.3766 & -1.3213 & -7.2550 & -0.2381 & -9.2168 & 1.0701 & -11.6740 & 2.7800 & -15.1525 & 5.3469 & -20.6506 & 10.0080 & -30.5859 \\
\hline & -2.2565 & -5.7954 & -1.3292 & -7.8485 & -0.2485 & -9.9855 & 1.0563 & -12.6612 & & -16.4511 & 5.3110 & -22.4505 & 9.9252 & -33.3299 \\
\hline 0.4800 & -2.2590 & -6.3310 & -1.3380 & -8.6040 & -0.2599 & -10.9624 & 1.0418 & -13.9141 & 2.7390 & -18.0969 & 5.2755 & -24.7270 & 9.8435 & -36.7891 \\
\hline 0.4850 & -2.2552 & -6.9915 & -1.3388 & -9.5333 & -0.2605 & -12.1634 & 1.0421 & -15.4534 & 2.7390 & -20.1165 & 5.2700 & -27.5159 & 9.8091 & -41.0145 \\
\hline 0.4900 & -2.2257 & -8.3472 & -1.3041 & -11.4286 & -0.2141 & -14.6073 & 1.1038 & -18.5812 & 2.8216 & -24.2149 & 5.3810 & -33.1659 & 9.9541 & -49.5462 \\
\hline 0.4950 & -2.2401 & -15.4851 & -1.3173 & -21.3474 & -0.2195 & -27.3717 & 1.1101 & -34.8984 & 2.8448 & -45.5734 & 5.4307 & -62.5693 & 10.0460 & -93.8145 \\
\hline 0.5000 & -5.0603 & -21.9738 & -5.1137 & -30.2416 & -5.0199 & -38.7414 & -4.9444 & -49.3579 & -4.9789 & -64.4037 & -5.1923 & -88.3378 & -5.6665 & -132.298 \\
\hline
\end{tabular}


Table 4.18: Normalized T-stress values for $t / W=1.0$

\begin{tabular}{|c|c|c|c|c|c|c|c|c|c|c|c|c|c|c|}
\hline \multirow{2}{*}{$z / t$} & \multicolumn{2}{|c|}{$\mathrm{a} / \mathrm{W}=\mathbf{0 . 1}$} & \multicolumn{2}{|c|}{$\mathrm{a} / \mathrm{W}=\mathbf{0 . 2}$} & \multicolumn{2}{|c|}{$\mathrm{a} / \mathrm{W}=\mathbf{0 . 3}$} & \multicolumn{2}{|c|}{$\mathrm{a} / \mathrm{W}=0.4$} & \multicolumn{2}{|c|}{$\mathrm{a} / \mathrm{W}=\mathbf{0 . 5}$} & \multicolumn{2}{|c|}{$\mathrm{a} / \mathbf{W}=\mathbf{0 . 6}$} & \multicolumn{2}{|c|}{$\mathrm{a} / \mathbf{W}=\mathbf{0 . 7}$} \\
\hline & $T_{11}$ & $T_{33}$ & $T_{11}$ & $T_{33}$ & $T_{11}$ & $T_{33}$ & $T_{11}$ & $T_{33}$ & $T_{11}$ & $T_{33}$ & $T_{11}$ & $T_{33}$ & $T_{11}$ & $T_{33}$ \\
\hline 0.0000 & & & & & & -1.4190 & & -1.2300 & & -0.9056 & 5.9251 & -0.1594 & 11.1900 & 1.6729 \\
\hline 0.0193 & -2.3068 & -2.1144 & & -1.6533 & & -1.4242 & 1.2081 & & & -0.9170 & & -0.1765 & & 1.6481 \\
\hline 0.0385 & -2.3064 & & -1.3246 & -1.6560 & & -1.4297 & 1.2060 & -1.2459 & 3.0665 & -0.9290 & 5.9218 & -0.1943 & 11.1925 & 1.6223 \\
\hline 0.0771 & -2.3044 & -2.1129 & & & & & & & & & & & & 1.4714 \\
\hline 0.1156 & -2.3005 & -2.1113 & & -1.6995 & & -1.5161 & & -1.3740 & & & 5.9148 & -0.4754 & & 1.2104 \\
\hline & & & & & & & & & & & & & & 0.8241 \\
\hline 0.1927 & -2.2848 & -2.1120 & -1.2864 & -1.8025 & & -1.7046 & 1.1594 & -1.6511 & & -1.5220 & & -1.0852 & & 0.2893 \\
\hline & & & & & & & & & & & & & & -0.4278 \\
\hline 0.2698 & -2.2513 & -2.1401 & -1.2685 & -2.0025 & -0.2237 & -2.0374 & 1.0955 & -2.1331 & 2.9111 & -2.2229 & & -2.1450 & 11.2888 & -1.3766 \\
\hline 0.3083 & -2.2259 & & & & & -2.2874 & 1.0472 & & 2.8400 & -2.7362 & 5.6887 & -2.9213 & & -2.6321 \\
\hline 0.3469 & -2.1983 & -2.2712 & -1.2871 & -2.3882 & -0.2921 & -2.6270 & 0.9844 & -2.9664 & 2.7444 & -3.4158 & 5.5478 & -3.9458 & 11.0274 & -4.3153 \\
\hline 0.3854 & -2.1819 & -2.4352 & -1.3227 & -2.7229 & -0.3476 & -3.1130 & 0.9006 & -3.6360 & 2.6124 & -4.3563 & 5.3384 & -5.3516 & 10.7030 & -6.6463 \\
\hline 0.4240 & -2.2093 & -2.7410 & -1.3862 & -3.2623 & -0.4326 & -3.8685 & 0.7741 & -4.6535 & 2.4127 & 7595 & 5.0084 & -7.4193 & 1289 & .0718 \\
\hline 0.4625 & -2.2476 & -3.5919 & -1.4189 & -4.5806 & -0.4903 & -5.6192 & 0.6704 & -6.9308 & 2.2302 & -8.8033 & 4.6654 & -11.7727 & 9.4373 & -17.0732 \\
\hline 0.4662 & -2.2469 & -3.7746 & -1.4159 & -4.8501 & -0.4930 & -5.9712 & 0.6608 & -7.3836 & 2.2099 & -9.4026 & 4.6230 & -12.6184 & 9.3375 & .4123 \\
\hline 0.4700 & -2.2468 & -3.9799 & -1.4162 & -5.1484 & -0.4969 & -6.3563 & 0.6516 & -7.8757 & 2.1923 & -10.0496 & 4.5879 & -13.5248 & 9.2594 & -19.8325 \\
\hline & -2.2519 & & & & & & & & & & & -14.5214 & & -21.3921 \\
\hline 0.4775 & -2.2558 & -4.4888 & -1.4328 & -5.8802 & -0.5268 & -7.2987 & 0.6055 & -9.0776 & 2.1211 & -11.6276 & 4.4682 & -15.7287 & 9.0221 & -23.2715 \\
\hline 0.4813 & -2.2606 & -4.8471 & & & & & & & 2.0786 & -12.7085 & 4.3992 & -17.2308 & 8.8877 & -25.5986 \\
\hline 0.4850 & -2.2686 & -5.3207 & -1.4626 & -7.0537 & -0.5743 & -8.7983 & 0.5380 & -10.9801 & 2.0234 & -14.1127 & 4.3133 & -19.1755 & 8.7269 & -28.5960 \\
\hline 0.4887 & -2.2764 & -5.9914 & -1.4830 & -7.9882 & -0.6048 & -9.9867 & 0.4972 & & 1.9672 & -16.0691 & 4.2272 & -21.8764 & 8.5660 & -32.7378 \\
\hline 0.4925 & -2.2852 & -6.6994 & -1.5080 & -8.9754 & -0.6405 & -11.2428 & 0.4512 & -14.0707 & 1.9062 & -18.1334 & 4.1360 & -24.7207 & 8.3974 & -37.0889 \\
\hline 0.4963 & -2.2448 & -11.5656 & -1.4588 & -15.6532 & -0.5732 & -19.7022 & 0.5459 & -24.7411 & 2.0402 & -31.9763 & 4.3282 & -43.7279 & 8.6819 & -65.9187 \\
\hline 0.5000 & -4.2158 & -19.8794 & -4.0633 & -26.8964 & -3.7874 & -33.8281 & -3.4225 & -42.4517 & -3.0108 & -54.8366 & -2.4872 & -74.9656 & -1.4671 & -113.0271 \\
\hline
\end{tabular}


Table 4.19: Normalized T-stress values for $t / W=2.0$

\begin{tabular}{|c|c|c|c|c|c|c|c|c|c|c|c|c|c|c|}
\hline \multirow{2}{*}{$z / t$} & \multicolumn{2}{|c|}{$\mathrm{a} / \mathrm{w}=\mathbf{0 . 1}$} & \multicolumn{2}{|c|}{$\mathrm{a} / \mathrm{w}=0.2$} & \multicolumn{2}{|c|}{$\mathrm{a} / \mathrm{w}=\mathbf{0 . 3}$} & \multicolumn{2}{|c|}{$\mathrm{a} / \mathrm{w}=0.4$} & \multicolumn{2}{|c|}{$\mathrm{a} / \mathrm{w}=0.5$} & \multicolumn{2}{|c|}{$\mathrm{a} / \mathrm{w}=\mathbf{0 . 6}$} & \multicolumn{2}{|c|}{$\mathrm{a} / \mathrm{w}=\mathbf{0 . 7}$} \\
\hline & $T_{11}$ & $T_{33}$ & $T_{11}$ & $T_{33}$ & $T_{11}$ & $\boldsymbol{T}_{33}$ & $T_{11}$ & $T_{33}$ & $T_{11}$ & $T_{33}$ & $T_{11}$ & $T_{33}$ & $T_{11}$ & $T_{33}$ \\
\hline 0.0000 & & -2.3832 & -1.5248 & -1.7769 & & -1.1604 & 0.7068 & -0.4946 & 2.4009 & 0.3004 & 5.0438 & 1.4003 & 10.1319 & 3.2557 \\
\hline 0.0253 & -2.3917 & -2.3816 & -1.5235 & -1.7756 & -0.5370 & -1.1604 & 0.7094 & -0.4964 & 2.4041 & 0.2969 & 5.0475 & 1.3960 & 10.1355 & 3.2522 \\
\hline 0.0507 & -2.3893 & & -1.5200 & -1.7716 & -0.5311 & -1.1602 & & & 2.4148 & 0.2866 & 5.0599 & 1.3832 & 10.1476 & 3.2414 \\
\hline 0.0760 & -2.3853 & -2.3689 & -1.5140 & -1.7652 & -0.5212 & -1.1604 & 0.7319 & -0.5111 & 2.4326 & 0.2686 & 5.0807 & 1.3608 & 10.1681 & 3.2222 \\
\hline 0.1013 & -2.3797 & -2.3578 & -1.5056 & -1.7564 & & -1.1615 & 0.7515 & & 2.4572 & & 5.1099 & 1.3269 & 10.1976 & 3.1923 \\
\hline 0.1266 & -2.3726 & & -1.4946 & & & & & & 2.4884 & & 5.1475 & & 10.2368 & 3.1487 \\
\hline 0.1773 & -2.3541 & -2.3063 & -1.4640 & -1.7212 & & -1.1807 & 0.8400 & -0.6139 & 2.5683 & 0.0870 & 5.2472 & 1.1265 & 10.3482 & 2.9997 \\
\hline & -2.3300 & & & & & & & & 2.6641 & & 5.3751 & & & 2.7116 \\
\hline 0.2786 & -2.2998 & -2.1998 & -1.3617 & -1.6969 & -0.3061 & -1.3201 & 0.9955 & -0.9377 & 2.7598 & -0.4358 & 5.5155 & 0.4058 & 10.7268 & 2.1642 \\
\hline 0.3039 & -2.2814 & -2.1693 & -1.3269 & -1.7126 & -0.2710 & & 1.0289 & & 2.7995 & & 5.5802 & 0.0734 & 10.8486 & 1.7293 \\
\hline 0.3293 & -2.2596 & -2.1402 & -1.2896 & -1.7480 & -0.2404 & -1.5104 & 1.0539 & -1.2790 & 2.8276 & -0.9629 & 5.6316 & -0.3582 & 10.9682 & 1.1326 \\
\hline 0.3546 & -2.2325 & -2.1172 & -1.2529 & -1.8130 & -0.2185 & -1.6632 & 1.0662 & -1.5299 & 2.8382 & -1.3431 & 5.6590 & -0.9213 & 11.0674 & 0.3125 \\
\hline 0.3673 & -2.2139 & -2.1139 & -1.2353 & -1.8676 & -0.2112 & -1.7694 & 1.0679 & -1.6960 & 2.8367 & -1.5911 & 5.6620 & -1.2923 & 1068 & -0.2531 \\
\hline 0.3799 & -2.1978 & -2.1101 & -1.2222 & -1.9223 & -0.2101 & -1.8757 & 1.0612 & -1.8624 & 2.8237 & -1.8398 & 5.6476 & -1.6646 & 11.1158 & -0.8220 \\
\hline 0.3926 & -2.1735 & -2.1251 & -1.2106 & -2.0115 & -0.2101 & -2.0274 & 1.0539 & -2.0894 & 2.8078 & -2.1731 & 5.6257 & -2.1662 & 11.1136 & -1.6181 \\
\hline 0.4306 & -2.1093 & -2.2454 & -1.2192 & -2.3906 & -0.2569 & -2.6302 & 0.9718 & -2.9650 & 2.6711 & -3.4395 & 5.4121 & -4.0728 & 10.8350 & -4.7171 \\
\hline 0.4686 & -2.1082 & -2.9199 & -1.2657 & -3.5551 & -0.3312 & -4.2482 & 0.8355 & -5.1369 & 2.4205 & -6.4228 & 4.9564 & -8.4502 & 9.9928 & -11.9335 \\
\hline 0.4831 & -2.1434 & -3.6303 & -1.3017 & -4.6390 & -0.3938 & -5.6775 & 0.7280 & -6.9886 & 2.2409 & -8.8934 & 4.6390 & -11.9863 & 9.3717 & -17.6402 \\
\hline 0.4859 & -2.1416 & & -1.3007 & & -0.3985 & -6.2665 & 0.7149 & & 2.2149 & & 4.5878 & -13.3694 & 9.2581 & -19.8226 \\
\hline 0.4887 & -2.1482 & -4.3263 & -1.3127 & -5.6382 & -0.4202 & -6.9584 & 0.6805 & -8.6171 & 2.1615 & -11.0311 & 4.4993 & -14.9861 & 9.0846 & -22.3648 \\
\hline 0.4916 & -2.1470 & -4.9132 & -1.3194 & -6.4632 & -0.4362 & -8.0079 & 0.6535 & -9.9443 & 2.1182 & -12.7649 & 4.4252 & -17.4018 & 8.9294 & -26.1308 \\
\hline 0.4944 & -2.1659 & -5.7760 & -1.3566 & -7.6631 & -0.4901 & -9.5282 & 0.5809 & -11.8610 & 2.0181 & -15.2605 & 4.2748 & -20.8633 & 8.6577 & -31.4902 \\
\hline 0.4972 & -2.2192 & -7.5385 & -1.4467 & -10.0870 & -0.6073 & -12.5867 & 0.4349 & -15.7042 & 1.8303 & -20.2445 & 4.0097 & -27.7398 & 8.2136 & -42.0454 \\
\hline 0.5000 & -4.0297 & -19.1916 & -3.8189 & -25.8551 & -3.5078 & -32.3504 & -3.1173 & -40.4296 & -2.6727 & -52.1831 & -2.0784 & -71.6080 & -0.9253 & -108.8500 \\
\hline
\end{tabular}


Table 4.20: Normalized T-stress values for $t / W=4.0$

\begin{tabular}{|c|c|c|c|c|c|c|c|c|c|c|c|c|c|c|}
\hline \multirow{2}{*}{$z / t$} & \multicolumn{2}{|c|}{$\mathrm{a} / \mathrm{w}=\mathbf{0 . 1}$} & \multicolumn{2}{|c|}{$\mathrm{a} / \mathrm{w}=\mathbf{0 . 2}$} & \multicolumn{2}{|c|}{$\mathbf{a} / \mathbf{w}=\mathbf{0 . 3}$} & \multicolumn{2}{|c|}{$\mathrm{a} / \mathrm{w}=0.4$} & \multicolumn{2}{|c|}{$\mathrm{a} / \mathrm{w}=0.5$} & \multicolumn{2}{|c|}{$\mathrm{a} / \mathrm{w}=\mathbf{0 . 6}$} & \multicolumn{2}{|c|}{$\mathbf{a} / \mathbf{w}=\mathbf{0 . 7}$} \\
\hline & $T_{11}$ & $T_{33}$ & $T_{11}$ & $\boldsymbol{T}_{33}$ & $T_{11}$ & $\boldsymbol{T}_{33}$ & $T_{11}$ & $\boldsymbol{T}_{33}$ & $T_{11}$ & $T_{33}$ & $T_{11}$ & $\boldsymbol{T}_{33}$ & $T_{11}$ & $\boldsymbol{T}_{33}$ \\
\hline 0.0000 & -2.4771 & -2.8491 & -1.5822 & -2.0031 & -0.6156 & -1.1503 & 0.5825 & -0.2373 & 2.2292 & 0.8042 & 4.8477 & 2.1330 & 9.9282 & 4.1978 \\
\hline 0.0445 & -2.4752 & -2.8410 & -1.5810 & -1.9995 & -0.6148 & -1.1508 & 0.5831 & -0.2414 & 2.2297 & 0.7965 & 4.8483 & 2.1217 & 9.9289 & 4.1824 \\
\hline 0.1039 & -2.4668 & -2.8082 & -1.5752 & -1.9847 & -0.6106 & -1.1518 & 0.5869 & -0.2569 & 2.2337 & 0.7670 & 4.8525 & 2.0778 & 9.9333 & 4.1226 \\
\hline 0.1633 & -2.4485 & -2.7474 & -1.5612 & -1.9546 & -0.5980 & -1.1492 & 0.6009 & -0.2805 & 2.2498 & 0.7178 & 4.8699 & 2.0035 & 9.9504 & 4.0218 \\
\hline 0.2227 & -2.4148 & -2.6542 & -1.5319 & -1.9022 & -0.5663 & -1.1368 & 0.6406 & -0.3096 & 2.2980 & 0.6482 & 4.9223 & 1.8984 & 10.0004 & 3.8850 \\
\hline 0.2558 & -2.3871 & -2.5862 & -1.5057 & -1.8604 & -0.5346 & -1.1247 & 0.6822 & -0.3313 & 2.3491 & 0.5942 & 4.9788 & 1.8190 & 10.0543 & 3.7895 \\
\hline 0.2740 & -2.3682 & -2.5424 & -1.4866 & -1.8324 & -0.5102 & -1.1171 & 0.7149 & -0.3479 & 2.3897 & 0.5543 & 5.0244 & 1.7623 & 10.0986 & 3.7254 \\
\hline 0.2923 & -2.3468 & -2.4942 & -1.4638 & -1.8008 & -0.4799 & -1.1099 & 0.7554 & -0.3704 & 2.4401 & 0.5035 & 5.0822 & 1.6913 & 10.1560 & 3.6483 \\
\hline 0.3014 & -2.3349 & -2.4677 & -1.4502 & -1.7835 & -0.4611 & -1.1075 & 0.7805 & -0.3860 & 2.4715 & 0.4704 & 5.1192 & 1.6456 & 10.1942 & 3.5998 \\
\hline 0.3106 & -2.3226 & -2.4411 & -1.4365 & -1.7661 & -0.4429 & -1.1051 & 0.8045 & -0.4019 & 2.5011 & 0.4369 & 5.1537 & 1.5994 & 10.2298 & 3.5508 \\
\hline 0.3197 & -2.3092 & -2.4121 & -1.4202 & -1.7477 & -0.4202 & -1.1051 & 0.8341 & -0.4242 & 2.5383 & 0.3928 & 5.1988 & 1.5387 & 10.2789 & 3.4863 \\
\hline 0.3288 & -2.2955 & -2.3831 & -1.4040 & -1.7291 & -0.3984 & -1.1053 & 0.8623 & -0.4469 & 2.5729 & 0.3480 & 5.2405 & 1.4772 & 10.3240 & 3.4210 \\
\hline 0.3563 & -2.2491 & -2.2861 & -1.3421 & -1.6741 & -0.3153 & -1.1269 & 0.9654 & -0.5576 & 2.7007 & 0.1465 & 5.4040 & 1.1967 & 10.5222 & 3.1098 \\
\hline 0.3837 & -2.1958 & -2.1802 & -1.2652 & -1.6308 & -0.2207 & -1.1889 & 1.0735 & -0.7368 & 2.8323 & -0.1603 & 5.5831 & 0.7598 & 10.7709 & 2.5868 \\
\hline 0.4111 & -2.1297 & -2.0711 & -1.1655 & -1.6389 & -0.1205 & -1.3484 & 1.1743 & -1.0616 & 2.9517 & -0.6856 & 5.7632 & -0.0152 & 11.0919 & 1.5384 \\
\hline 0.4385 & -2.0461 & -1.9843 & -1.0669 & -1.7514 & -0.0512 & -1.6597 & 1.2266 & -1.5989 & 3.0006 & -1.5224 & 5.8433 & -1.2803 & 11.3168 & -0.3495 \\
\hline 0.4659 & -1.9229 & -2.1020 & -1.0130 & -2.2244 & -0.0367 & -2.4474 & 1.2051 & -2.7721 & 2.9225 & -3.2475 & 5.6894 & -3.9113 & 11.1316 & -4.6692 \\
\hline 0.4775 & -1.8922 & -2.3174 & -1.0190 & -2.6621 & -0.0563 & -3.0906 & 1.1560 & -3.6689 & 2.8171 & -4.5176 & 5.4874 & -5.8236 & 10.7624 & -7.8832 \\
\hline 0.4813 & -1.8921 & -2.4582 & -1.0223 & -2.9055 & -0.0644 & -3.4294 & 1.1345 & -4.1246 & 2.7699 & -5.1479 & 5.3952 & -6.7564 & 10.5775 & -9.4466 \\
\hline 0.4850 & -1.8993 & -2.6515 & -1.0297 & -3.2238 & -0.0792 & -3.8635 & 1.1029 & -4.7013 & 2.7068 & -5.9374 & 5.2774 & -7.9161 & 10.3411 & -11.3766 \\
\hline 0.4887 & -1.9060 & -2.9584 & -1.0321 & -3.7013 & -0.0914 & -4.4998 & 1.0722 & -5.5343 & 2.6412 & -7.0623 & 5.1518 & -9.5508 & 10.0756 & -14.0644 \\
\hline 0.4925 & -1.9204 & -3.4843 & -1.0472 & -4.4803 & -0.1241 & -5.5175 & 1.0130 & -6.8499 & 2.5369 & -8.8185 & 4.9674 & -12.0717 & 9.7009 & -18.1471 \\
\hline 0.4962 & -1.9199 & -4.6340 & -1.0580 & -6.1020 & -0.1575 & -7.5924 & 0.9527 & -9.4933 & 2.4364 & -12.3093 & 4.7842 & -17.0112 & 9.3029 & -26.0088 \\
\hline 0.5000 & -2.4813 & -14.1231 & -1.8038 & -19.0035 & -1.0732 & -23.8715 & -0.1646 & -30.0195 & 1.0137 & -39.1246 & 2.8275 & -54.3842 & 6.2262 & -84.0476 \\
\hline
\end{tabular}




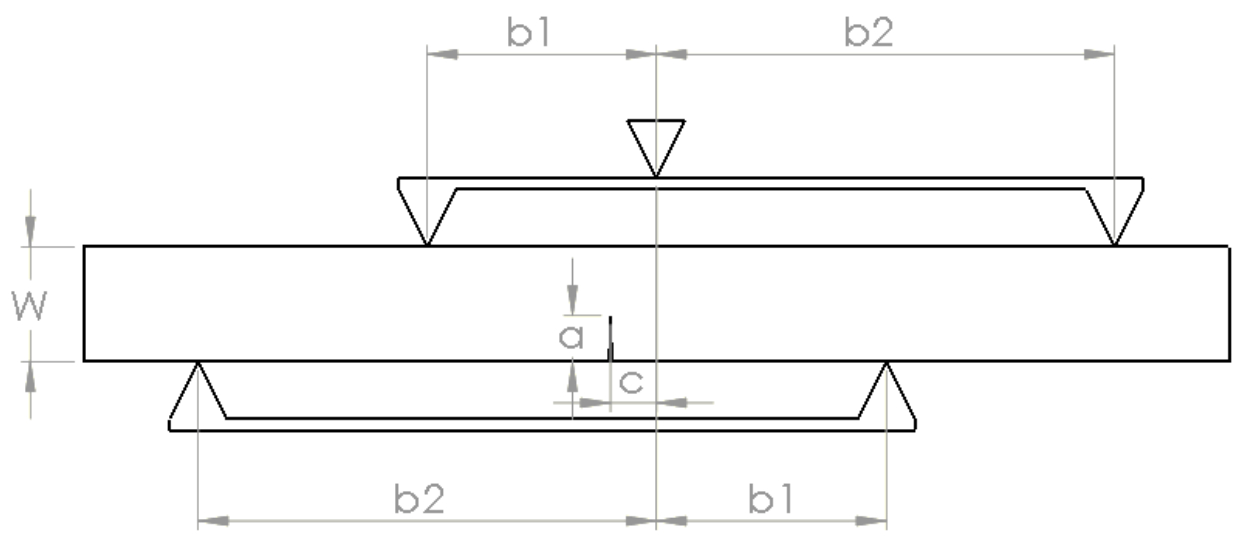

Figure 4.1: The asymmetric four-point shear specimen

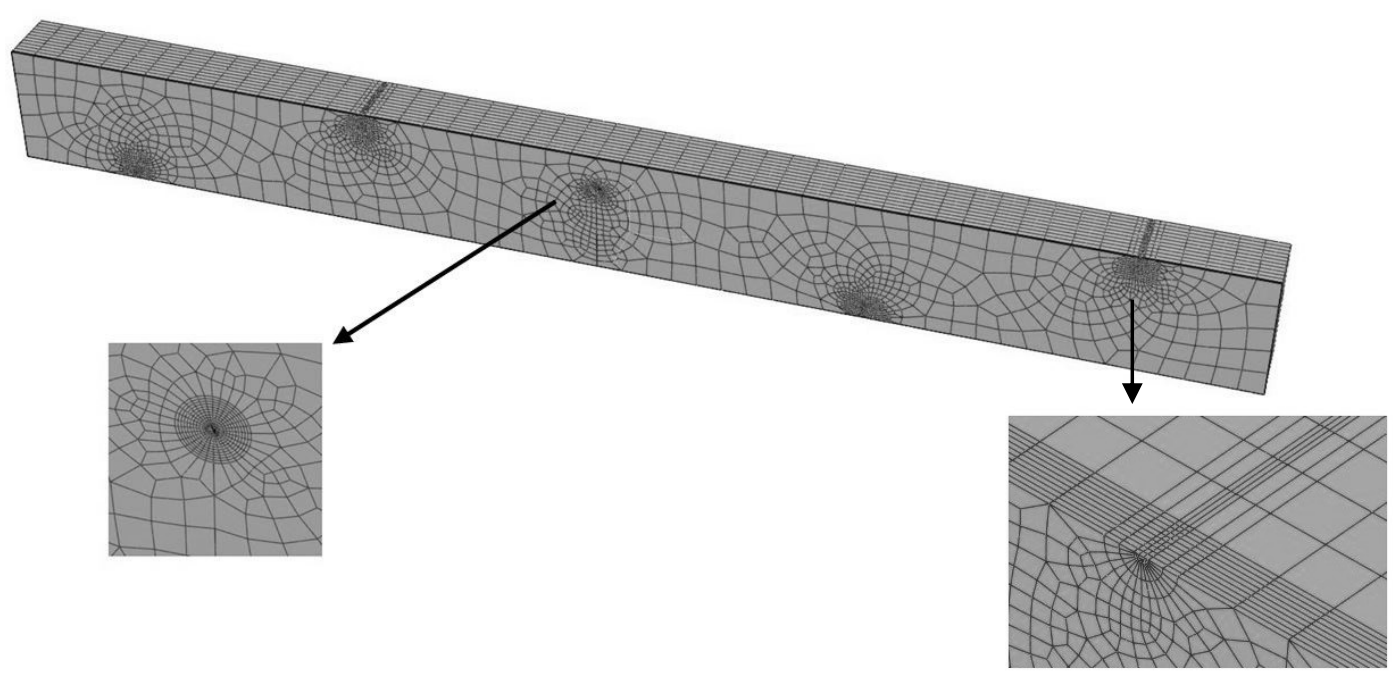

Figure 4.2: Typical mesh for AFPS specimen with $\mathrm{a} / \mathrm{W}=\mathbf{0 . 7}, \mathrm{c} / \mathrm{W}=\mathbf{0 . 1}$, and $\mathrm{t} / \mathrm{W}=1.0$ 


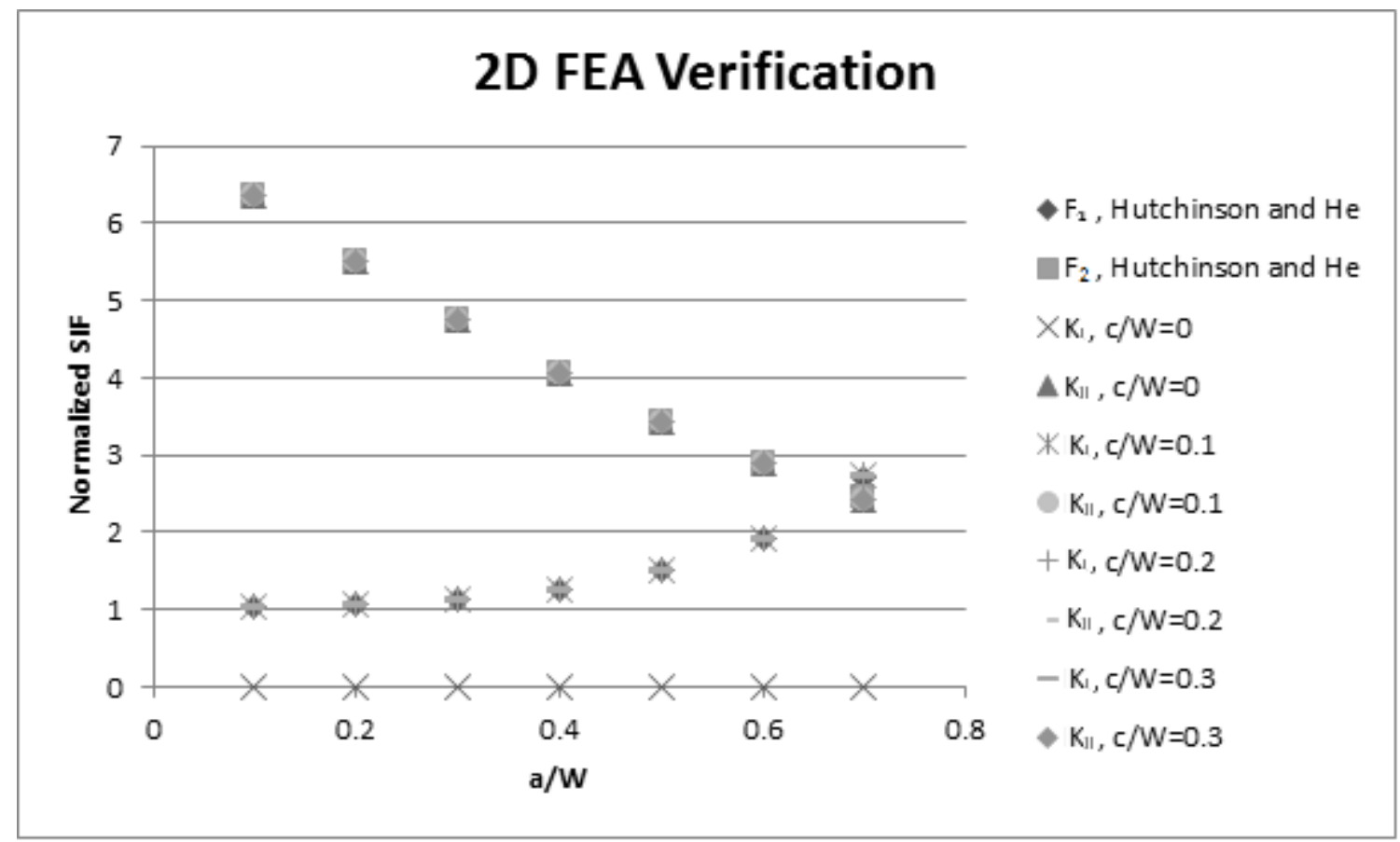

Figure 4.3: 2D FEA Comparison with Literature Results

\section{D FEA Midplane Results Comparison}

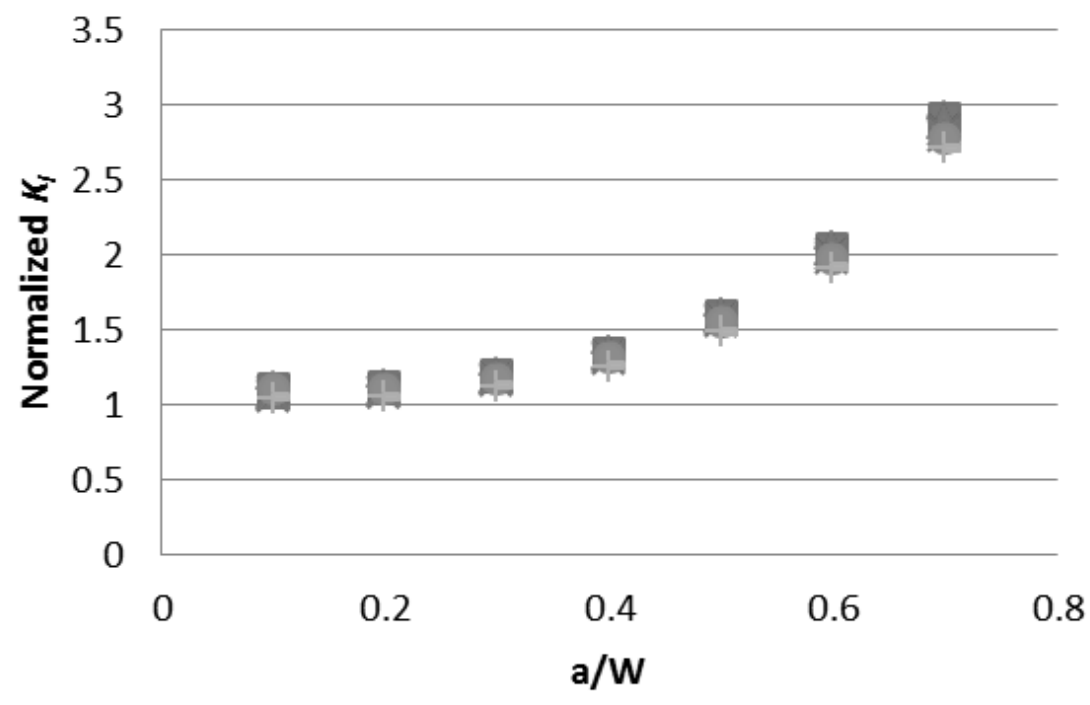

$\diamond \mathrm{t} / \mathrm{W}=0.1$

$\mathrm{t} / \mathrm{W}=0.2$

$\Delta \mathrm{t} / \mathrm{W}=0.5$

$\times \mathrm{t} / \mathrm{W}=1.0$

$* \mathrm{t} / \mathrm{W}=2.0$

$\mathrm{t} / \mathrm{W}=4.0$

$+\mathrm{t} / \mathrm{W}=4.0 \mathrm{v}=0$

- Reference

Figure 4.4: 3D FEA mid plane results comparison for $\mathrm{c} / \mathrm{W}=0.1$ 


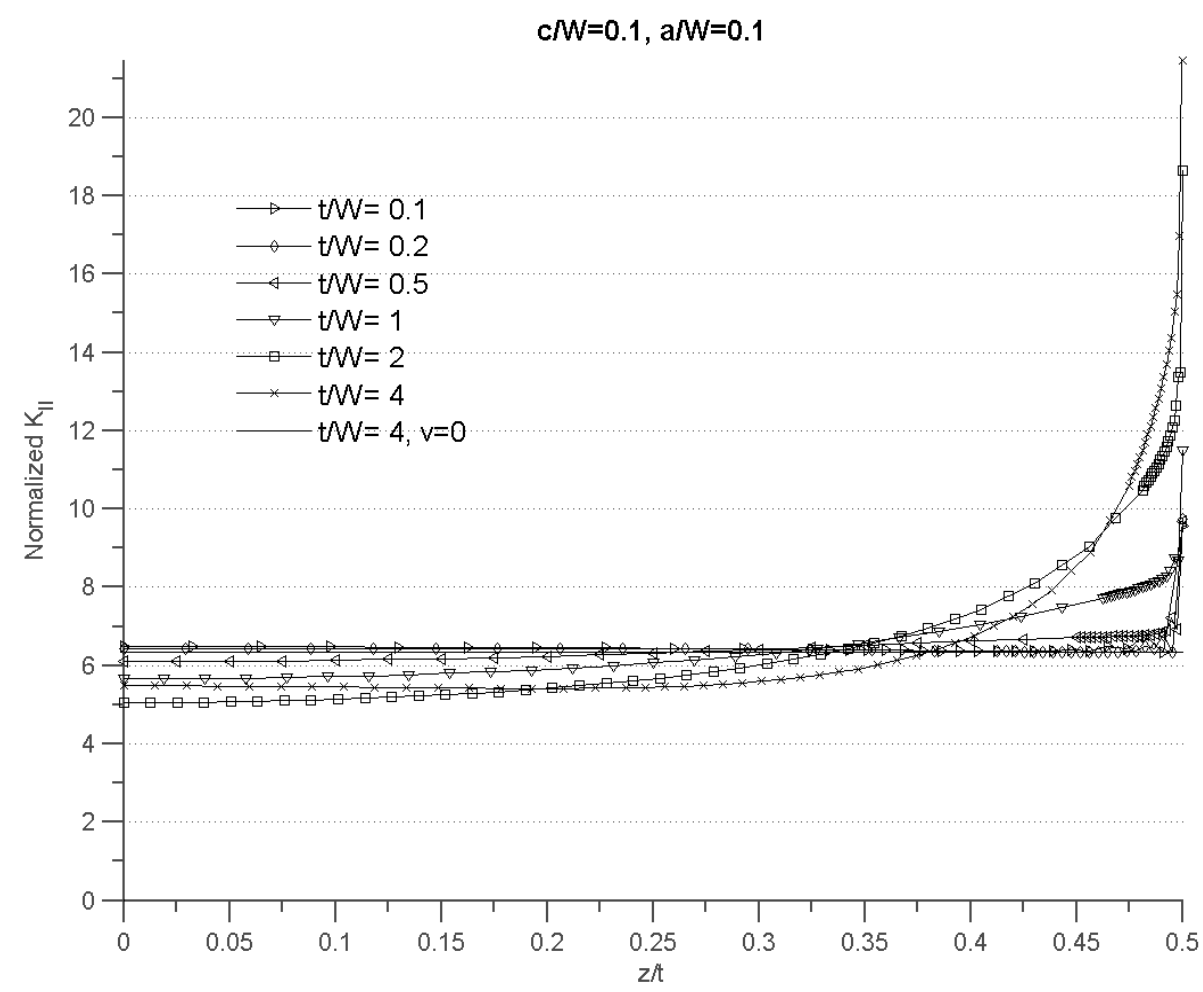

Figure 4.5: Normalized $K_{I I}$ for $\mathrm{a} / \mathrm{W}=0.1$

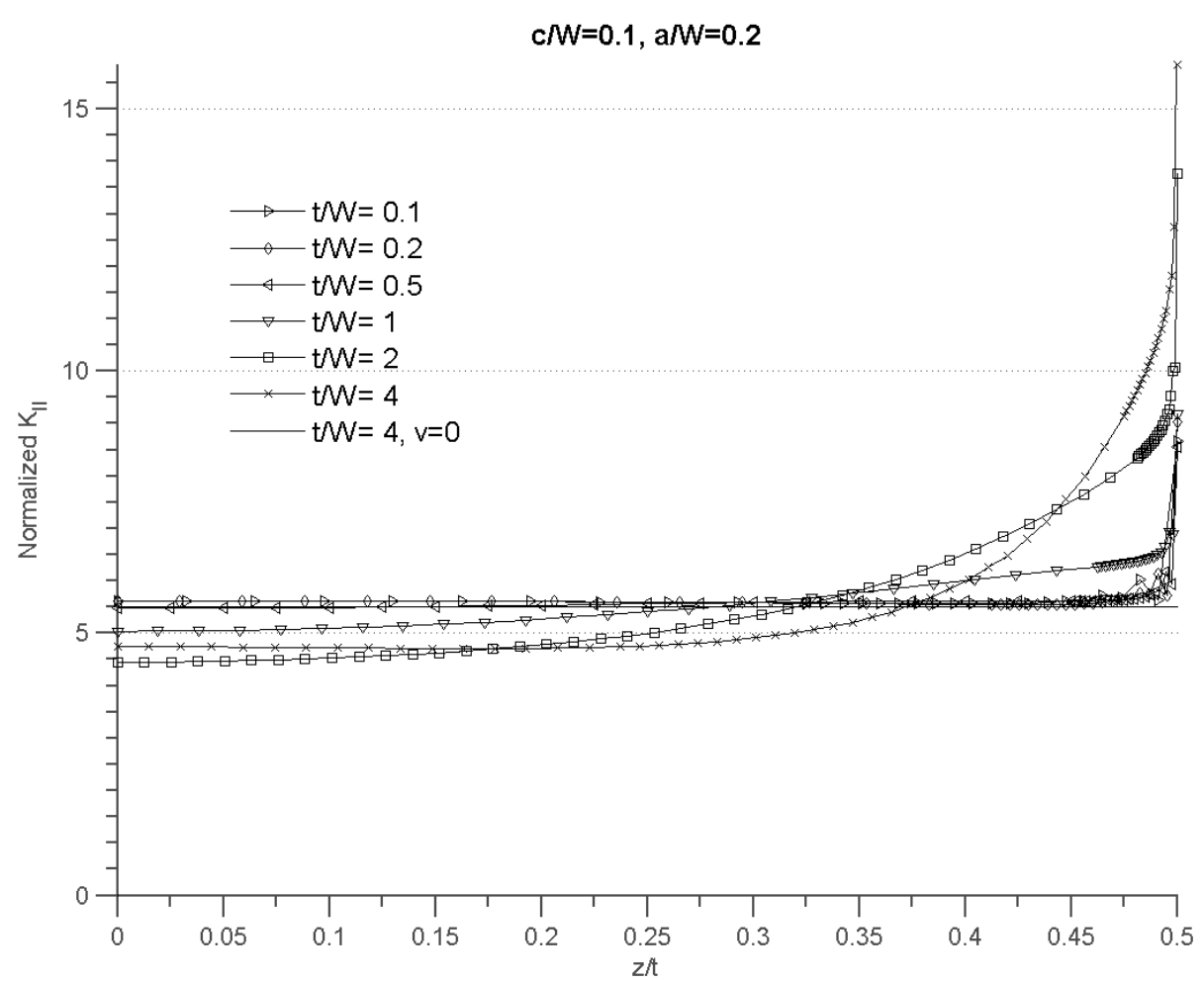

Figure 4.6: Normalized $K_{I I}$ for $\mathrm{a} / \mathrm{W}=\mathbf{0 . 2}$ 


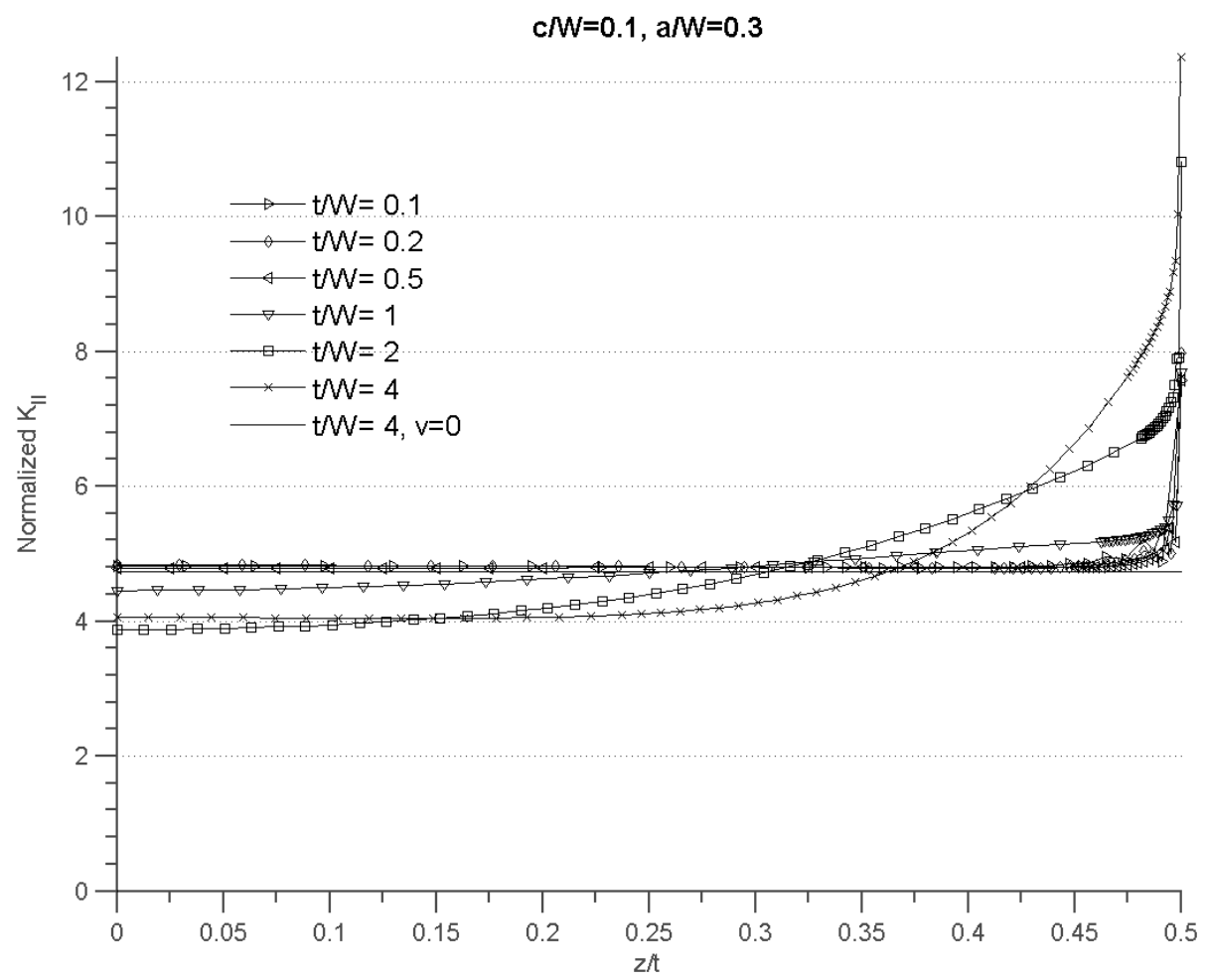

Figure 4.7: Normalized $K_{I I}$ for $\mathrm{a} / \mathrm{W}=\mathbf{0 . 3}$

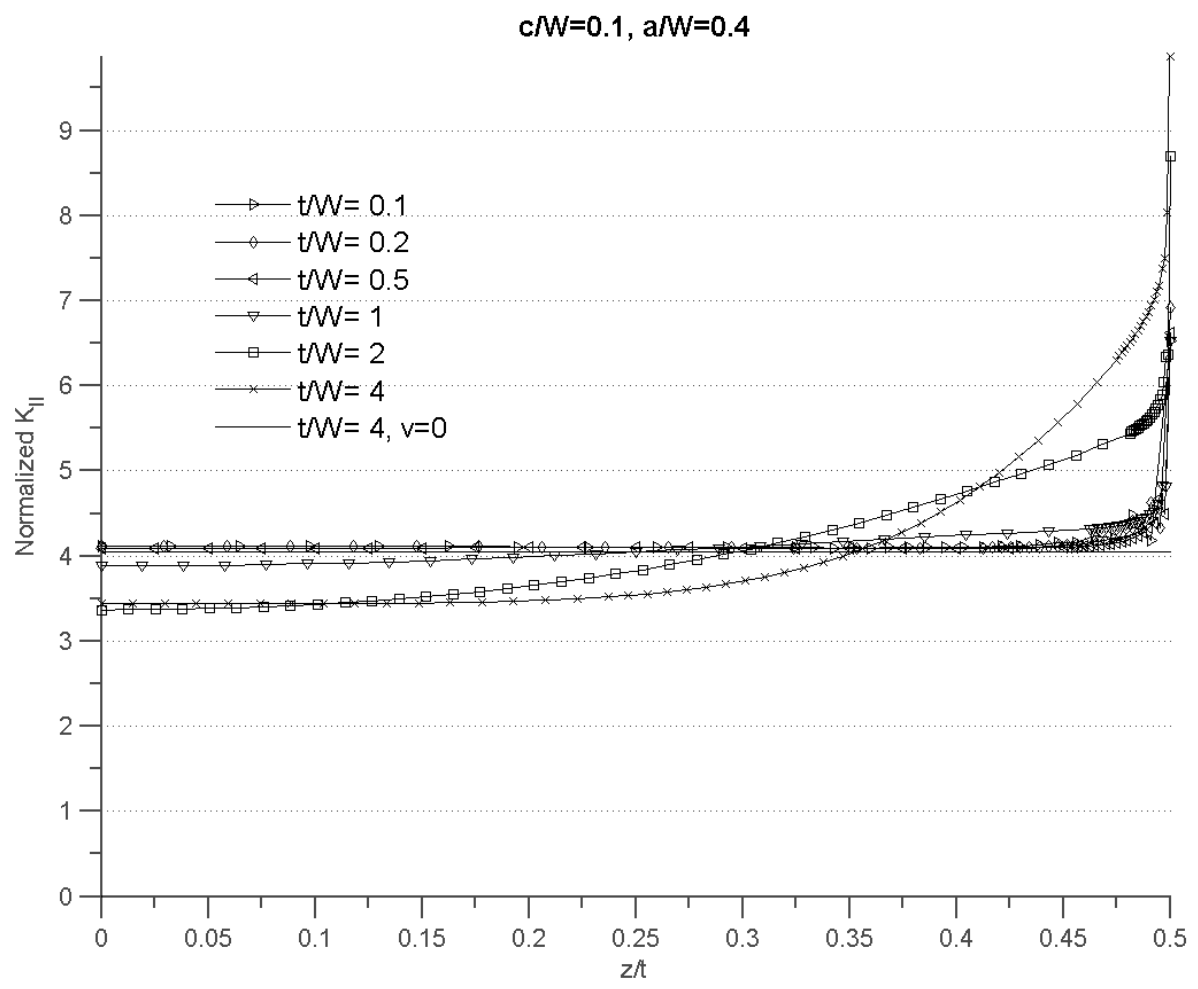

Figure 4.8: Normalized $K_{I I}$ for $\mathrm{a} / \mathrm{W}=\mathbf{0 . 4}$ 


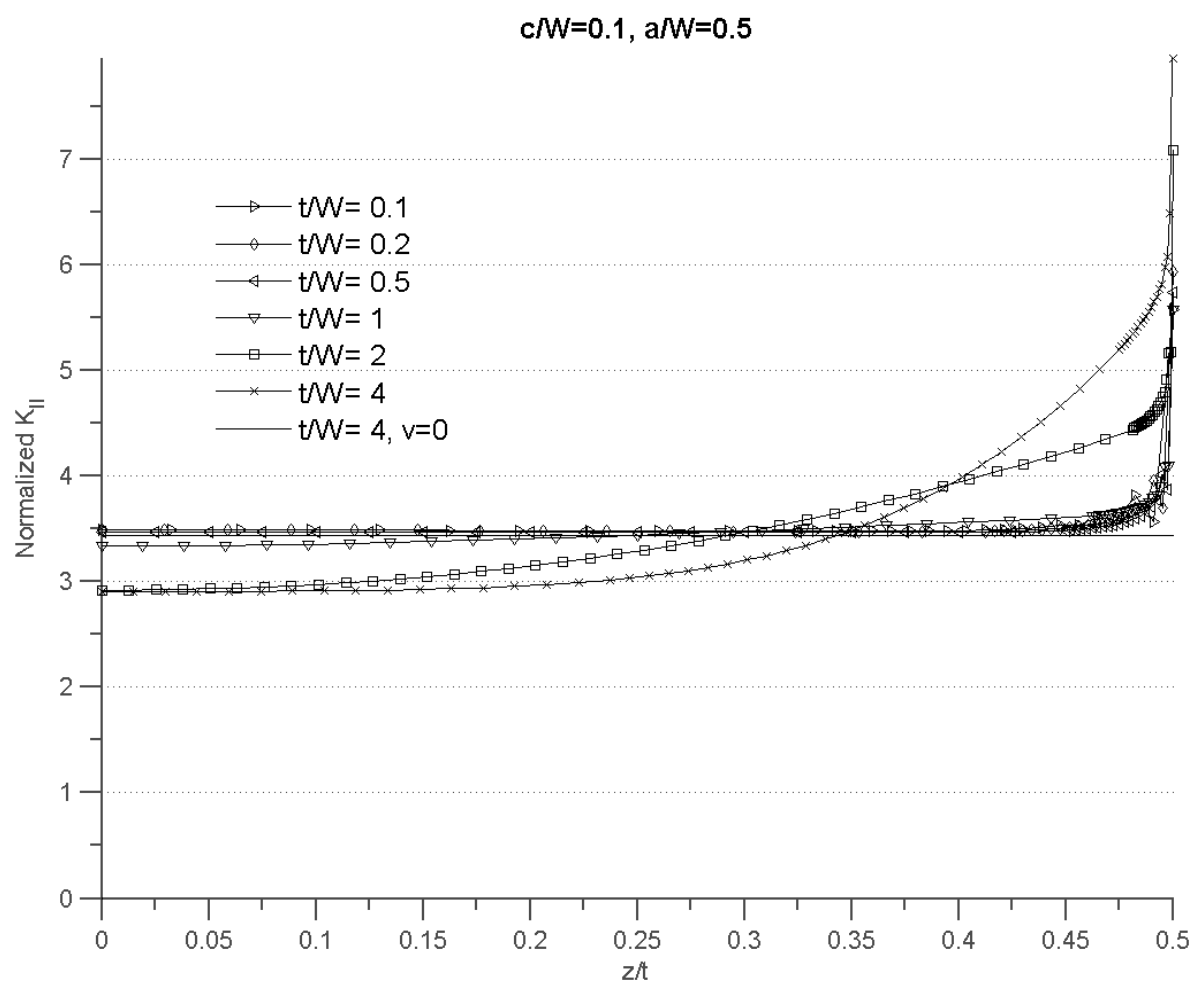

Figure 4.9: Normalized $K_{I I}$ for $\mathrm{a} / \mathrm{W}=0.5$

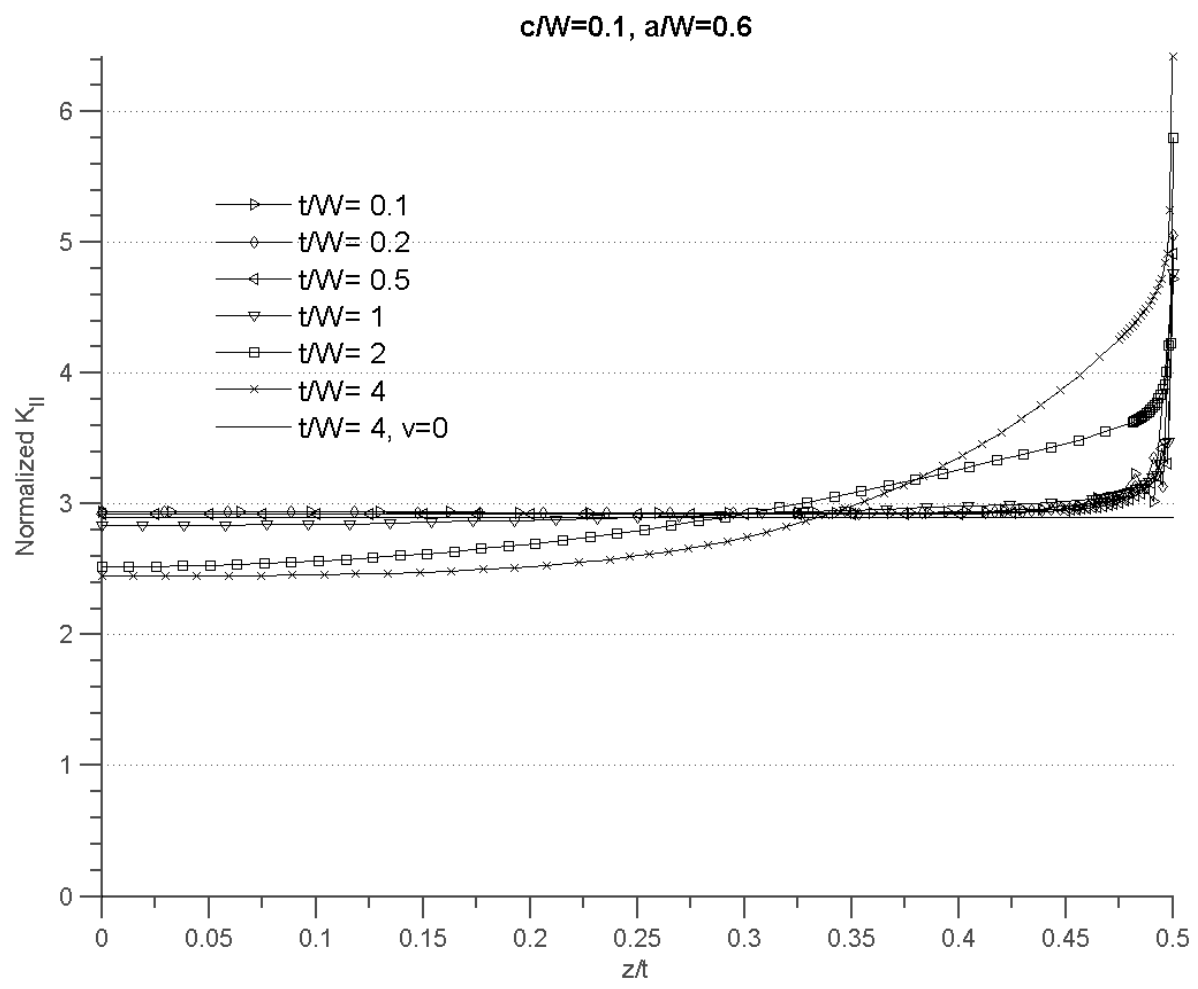

Figure 4.10: Normalized $K_{I I}$ for $\mathrm{a} / \mathrm{W}=\mathbf{0 . 6}$ 


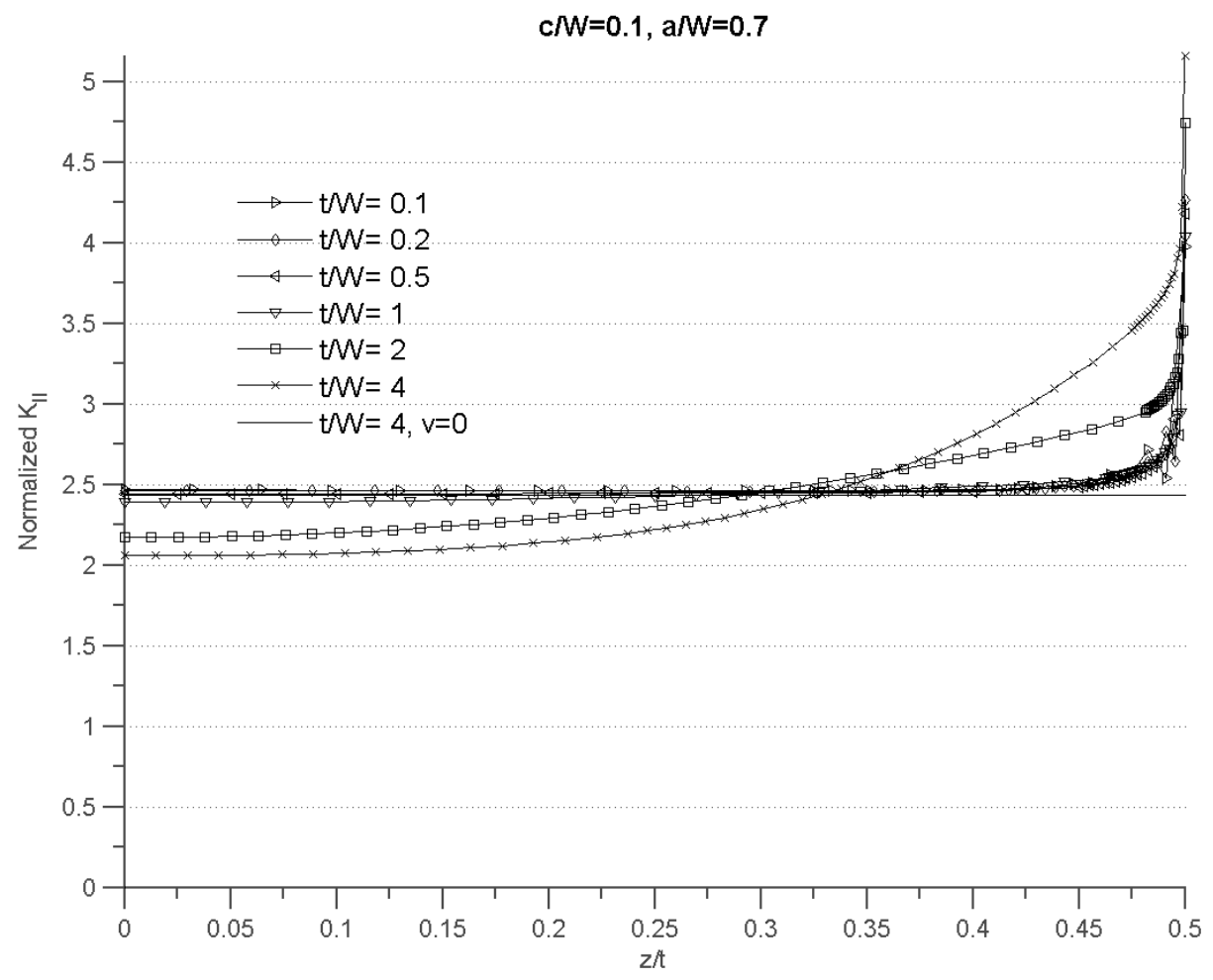

Figure 4.11: Normalized $K_{I I}$ for $\mathrm{a} / \mathrm{W}=0.7$ 


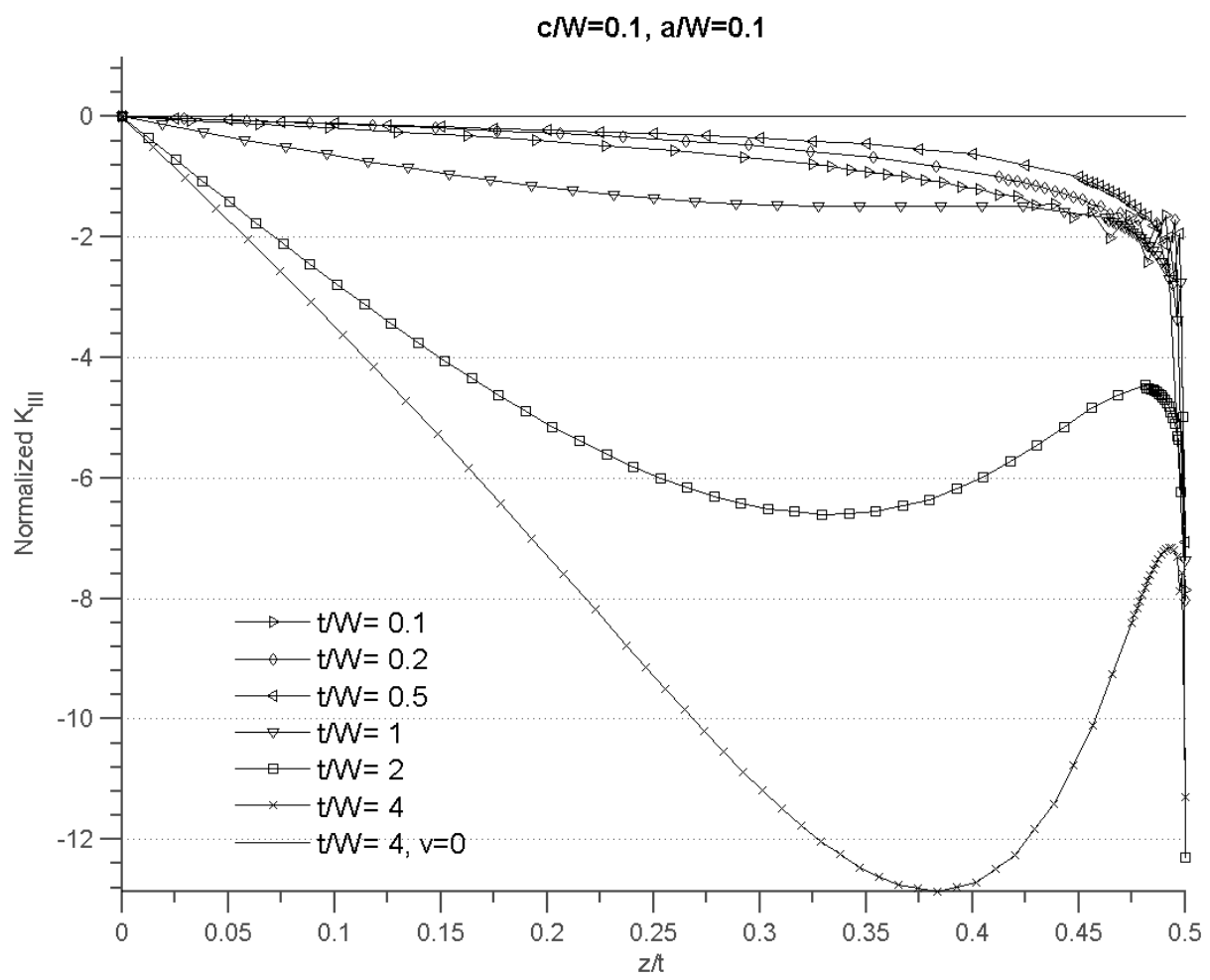

Figure 4.12: Normalized $K_{I I I}$ for a/W=0.1

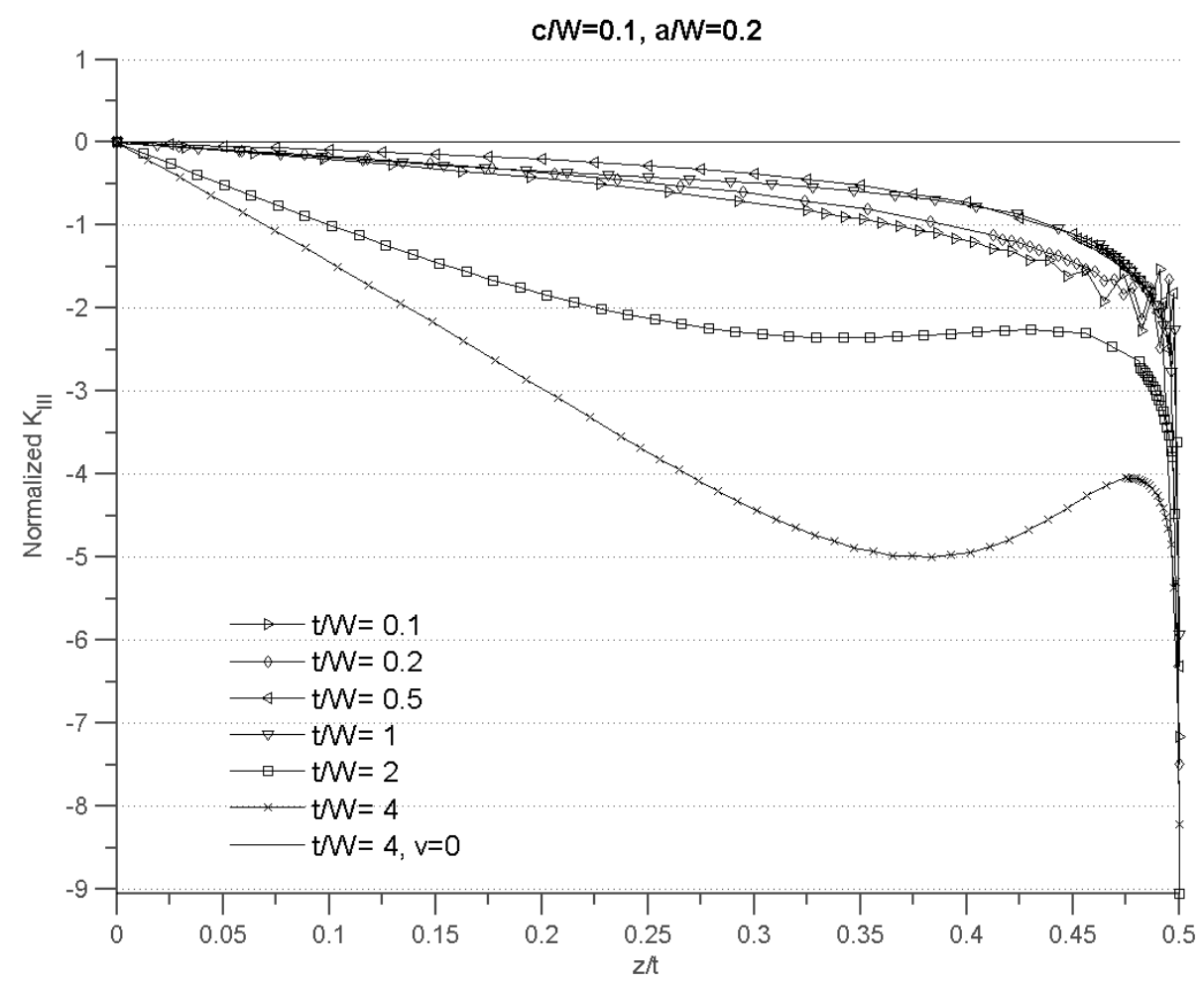

1Figure 4.13: Normalized $K_{I I}$ for $a / W=0.2$ 


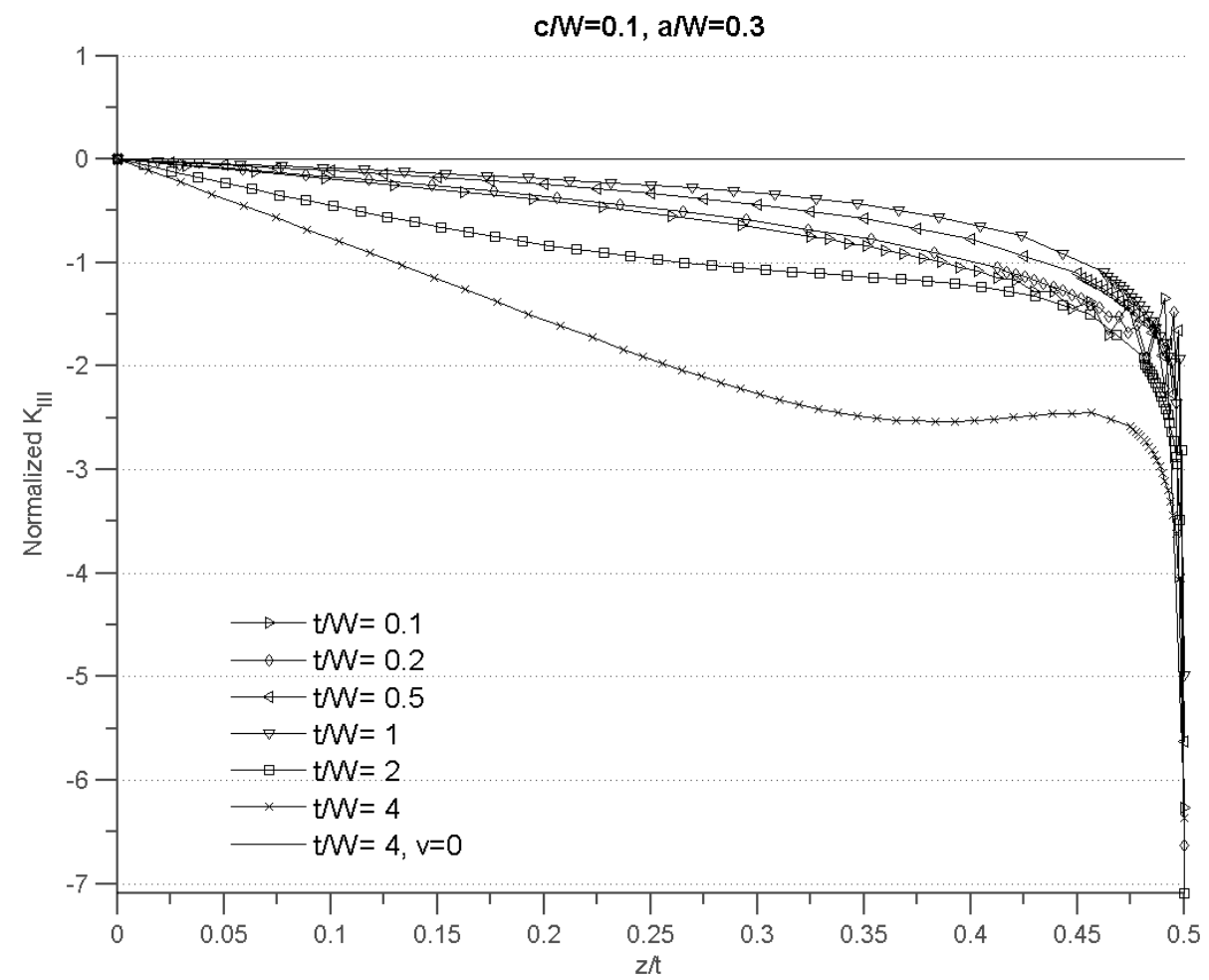

Figure 4.14: Normalized $K_{I I I}$ for a/W=0.3

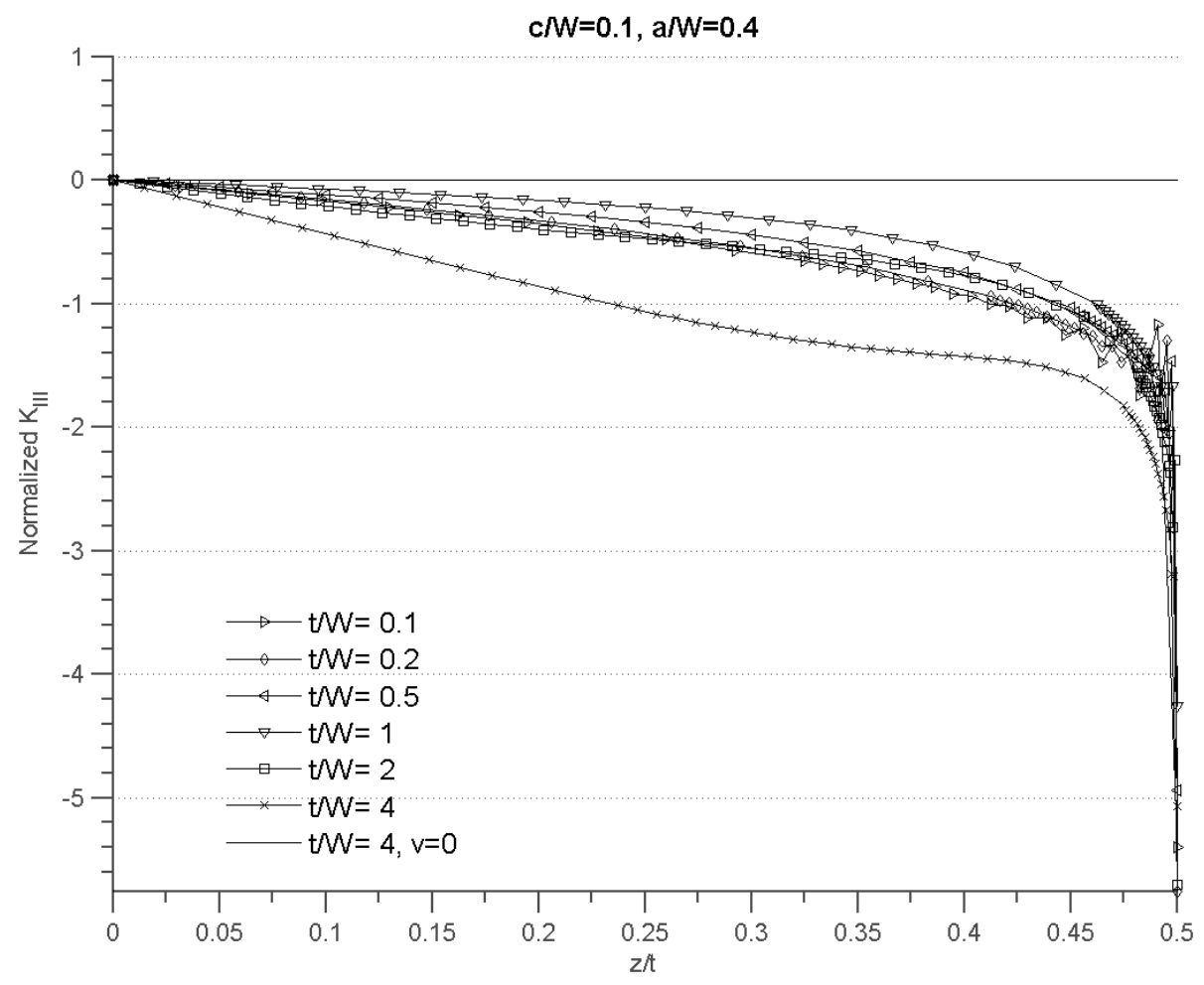

Figure 4.15: Normalized $K_{I I I}$ for $a / \mathrm{W}=0.4$ 


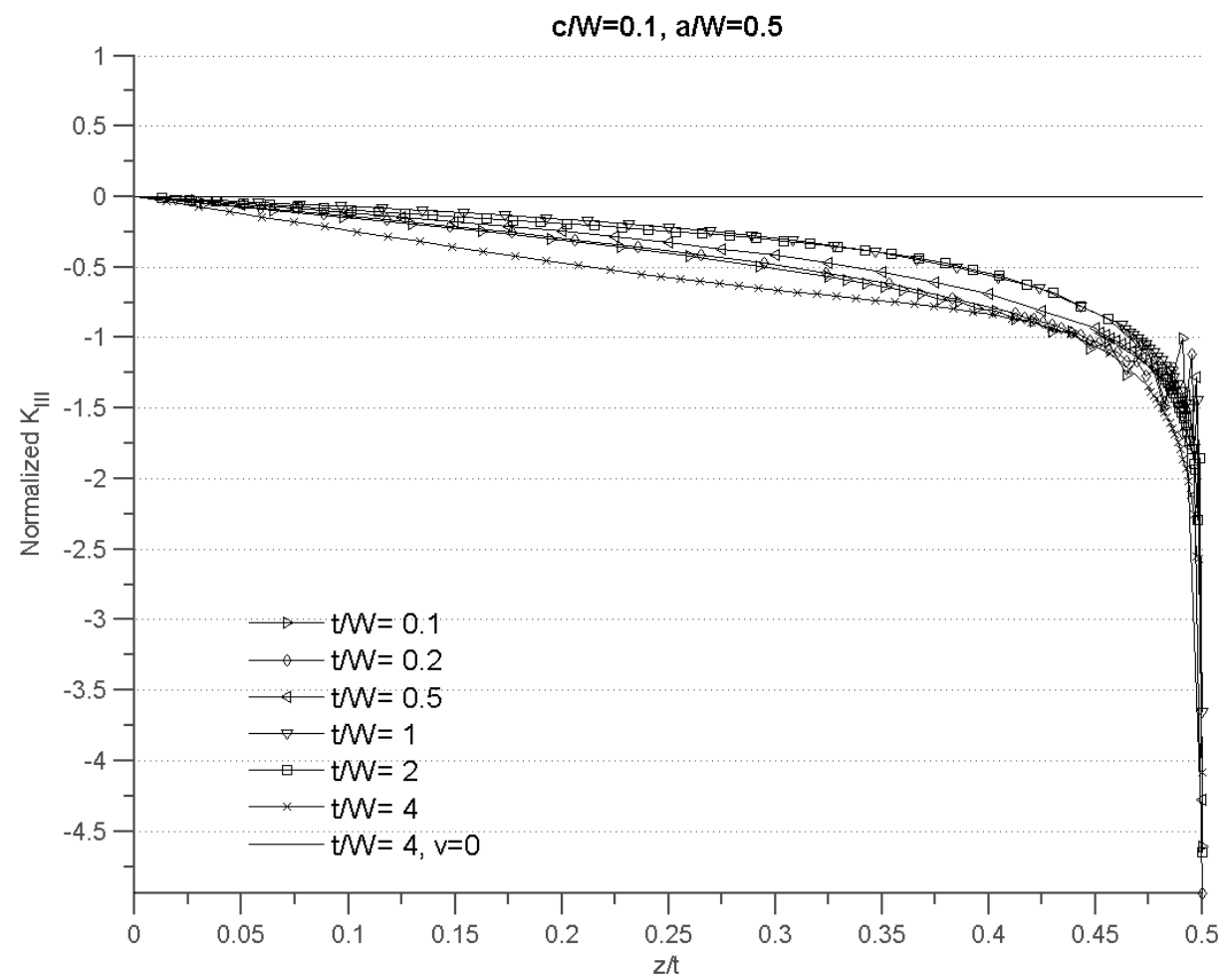

Figure 4.16: Normalized $K_{I I}$ for $\mathrm{a} / \mathrm{W}=0.5$

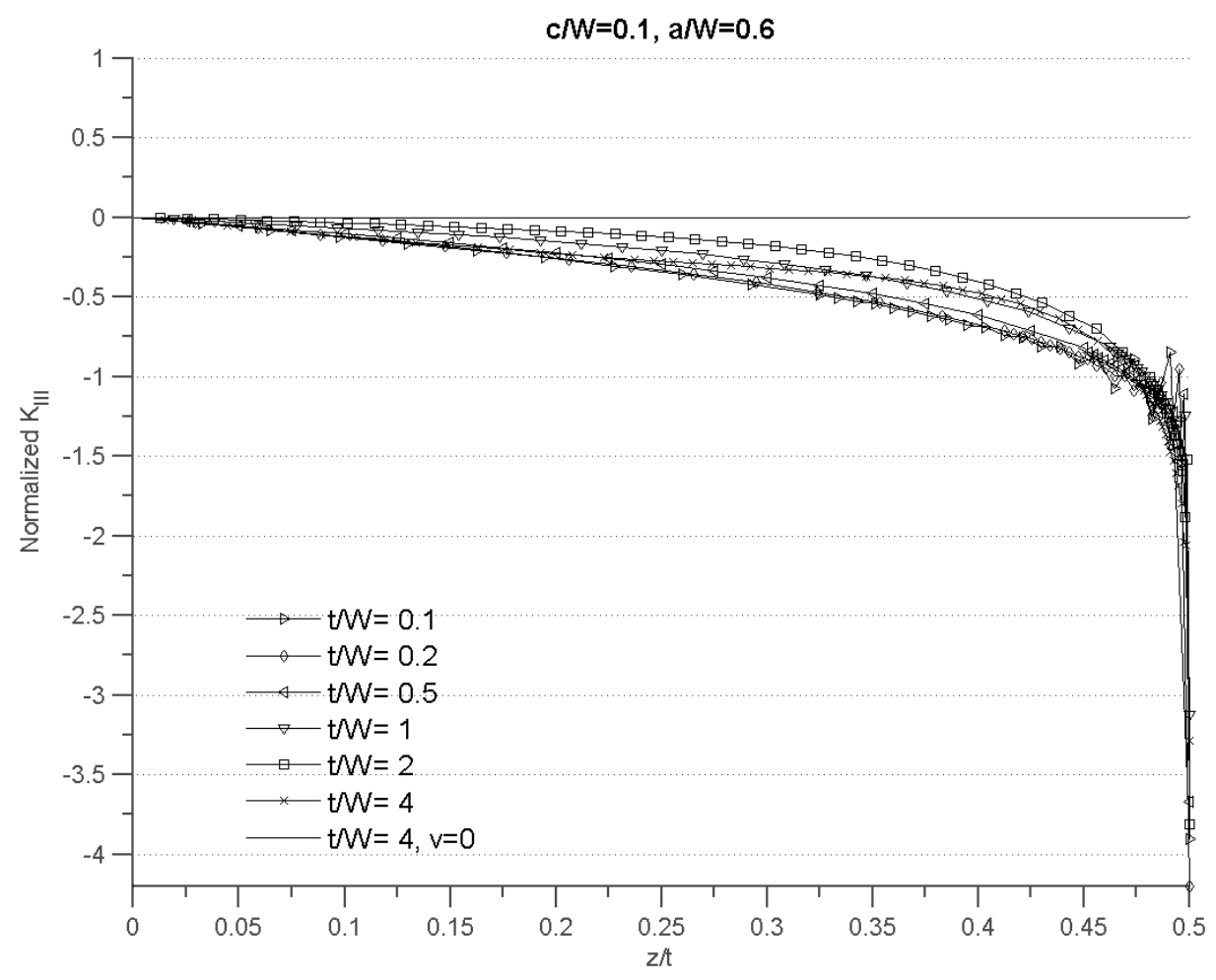

Figure 4.17: Normalized $K_{I I I}$ for a/W=0.6 


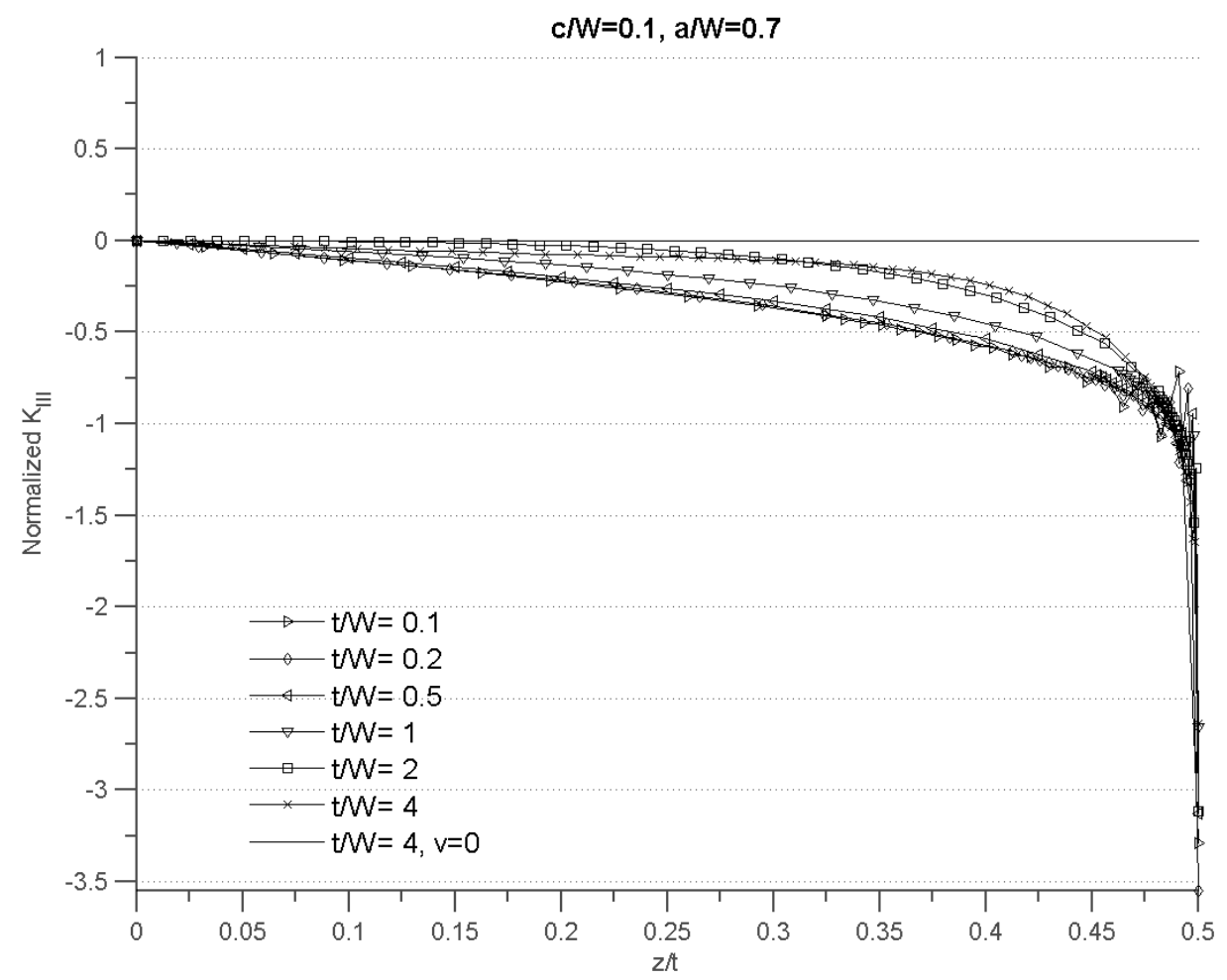

Figure 4.18: Normalized $K_{I I}$ for $\mathrm{a} / \mathrm{W}=0.7$ 


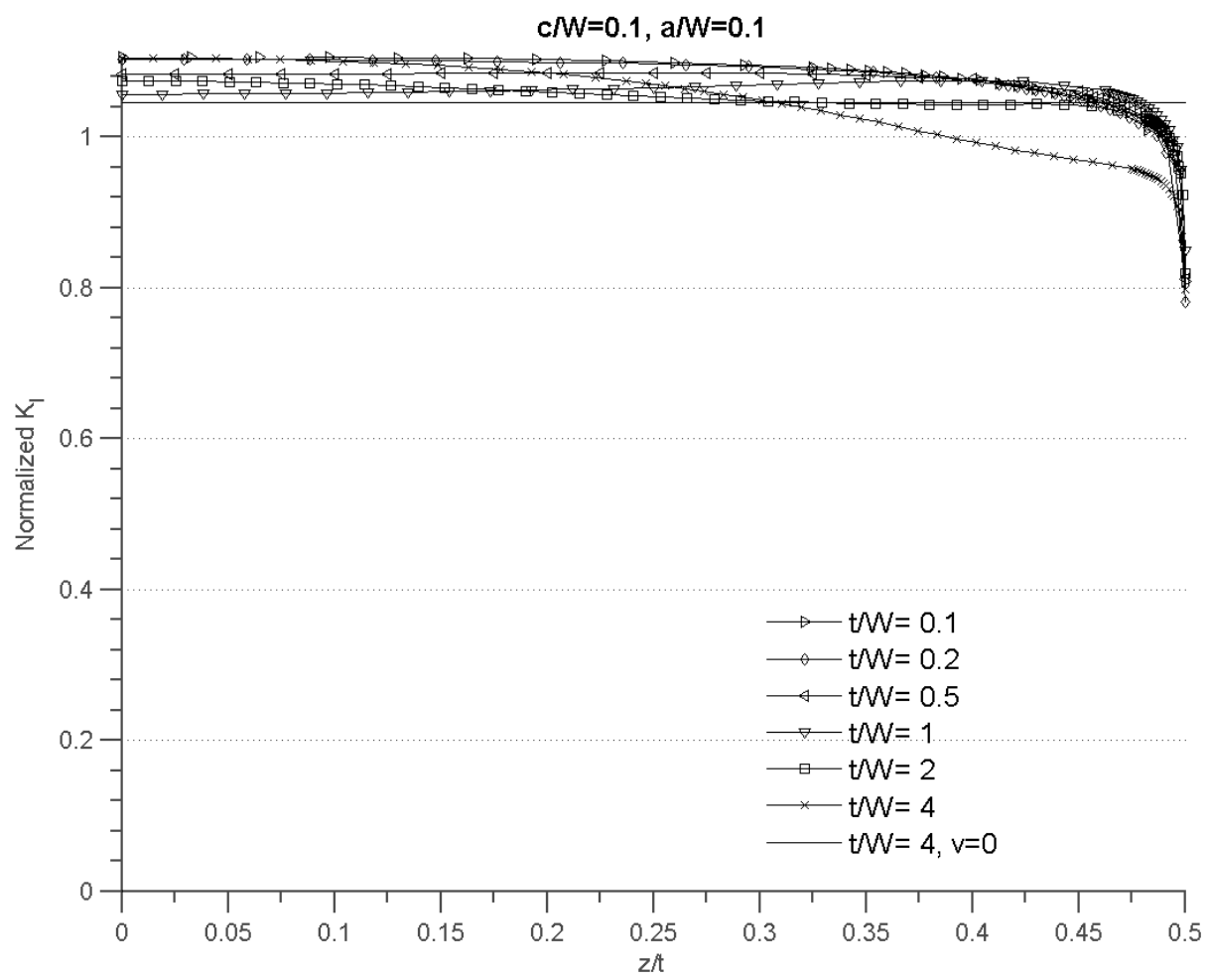

Figure 4.19: Normalized $K_{I}$ for a/W=0.1

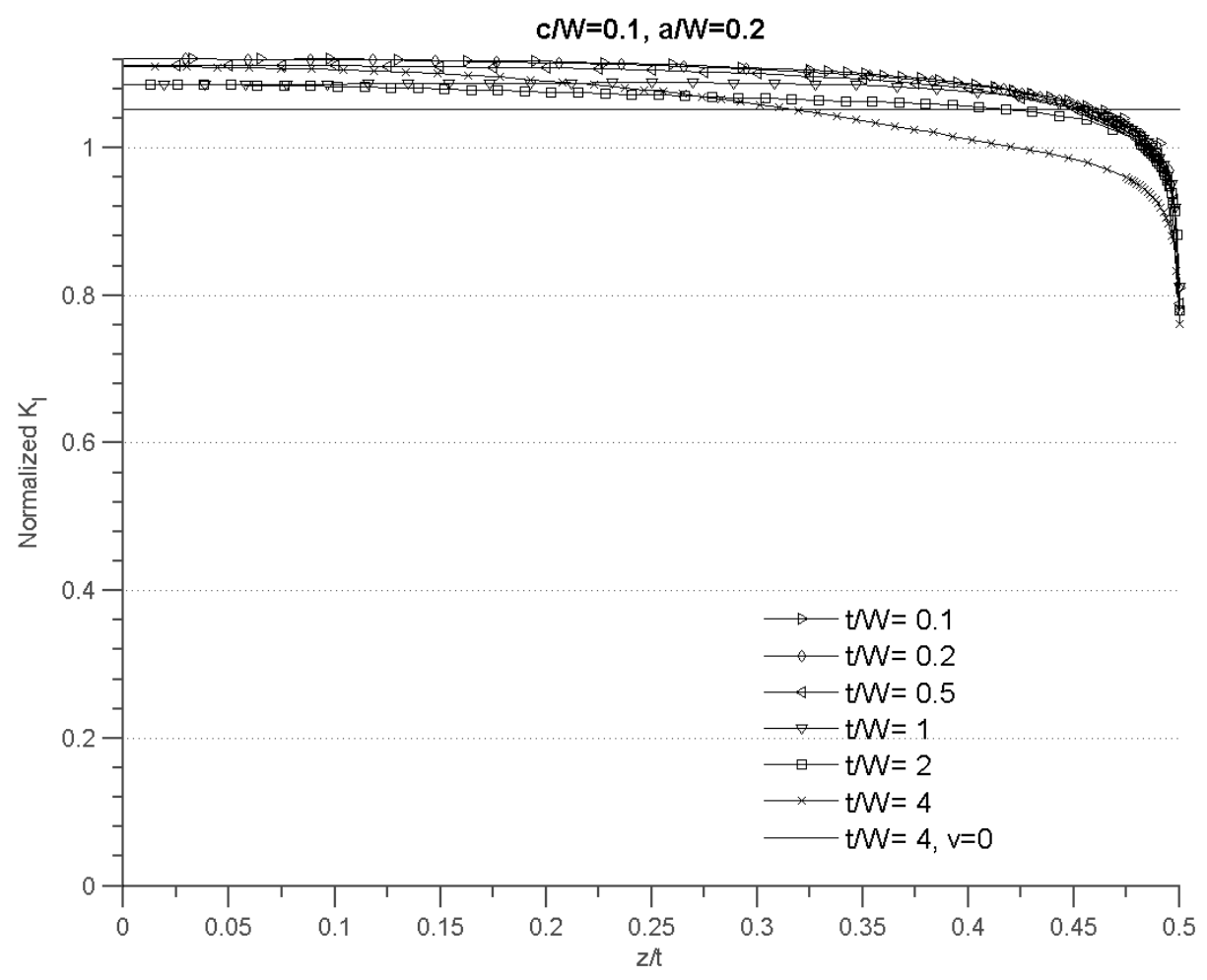

Figure 4.20: Normalized $K_{I}$ for $\mathrm{a} / \mathrm{W}=\mathbf{0 . 2}$ 


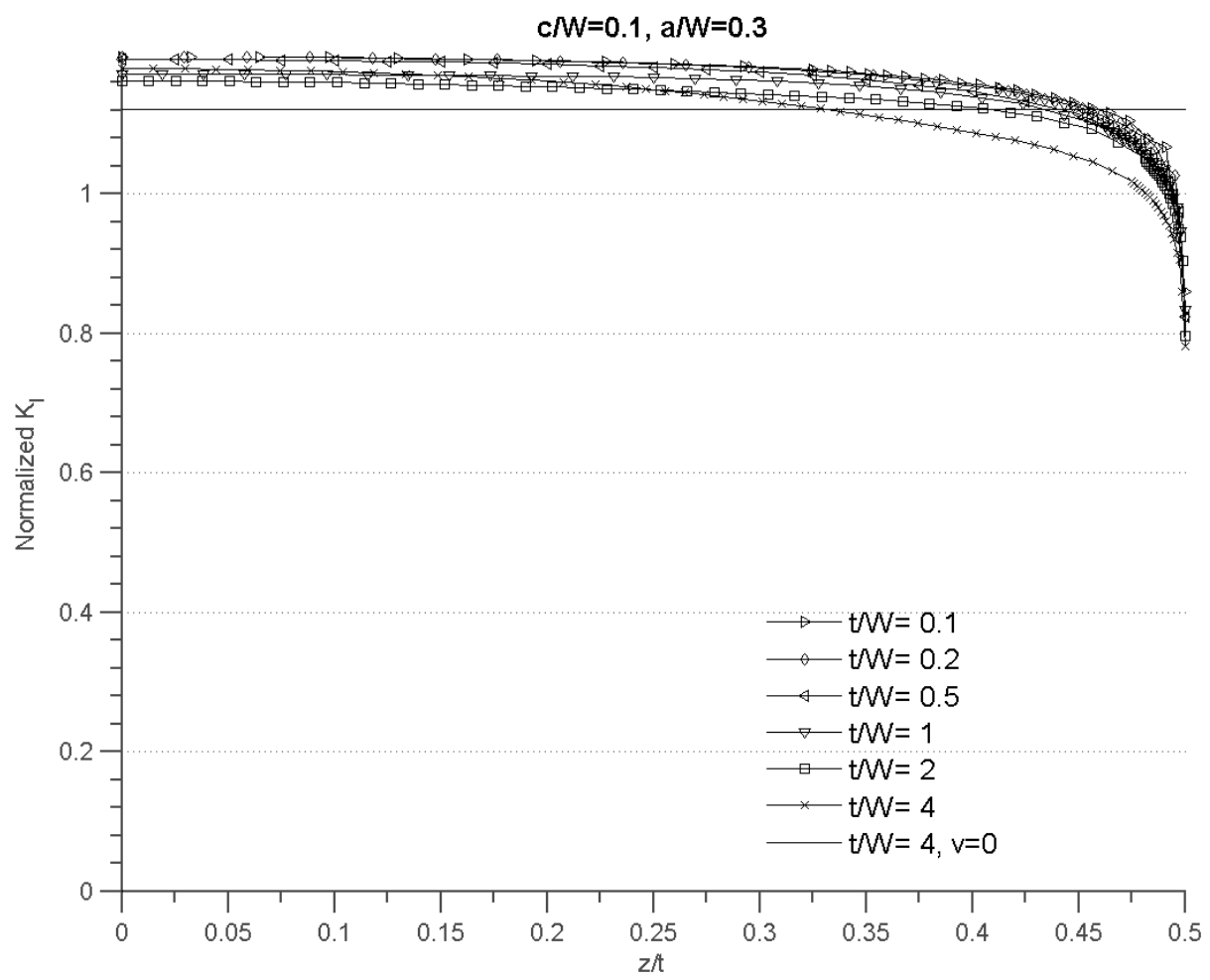

Figure 4.21: Normalized $K_{I}$ for $\mathrm{a} / \mathrm{W}=\mathbf{0 . 3}$

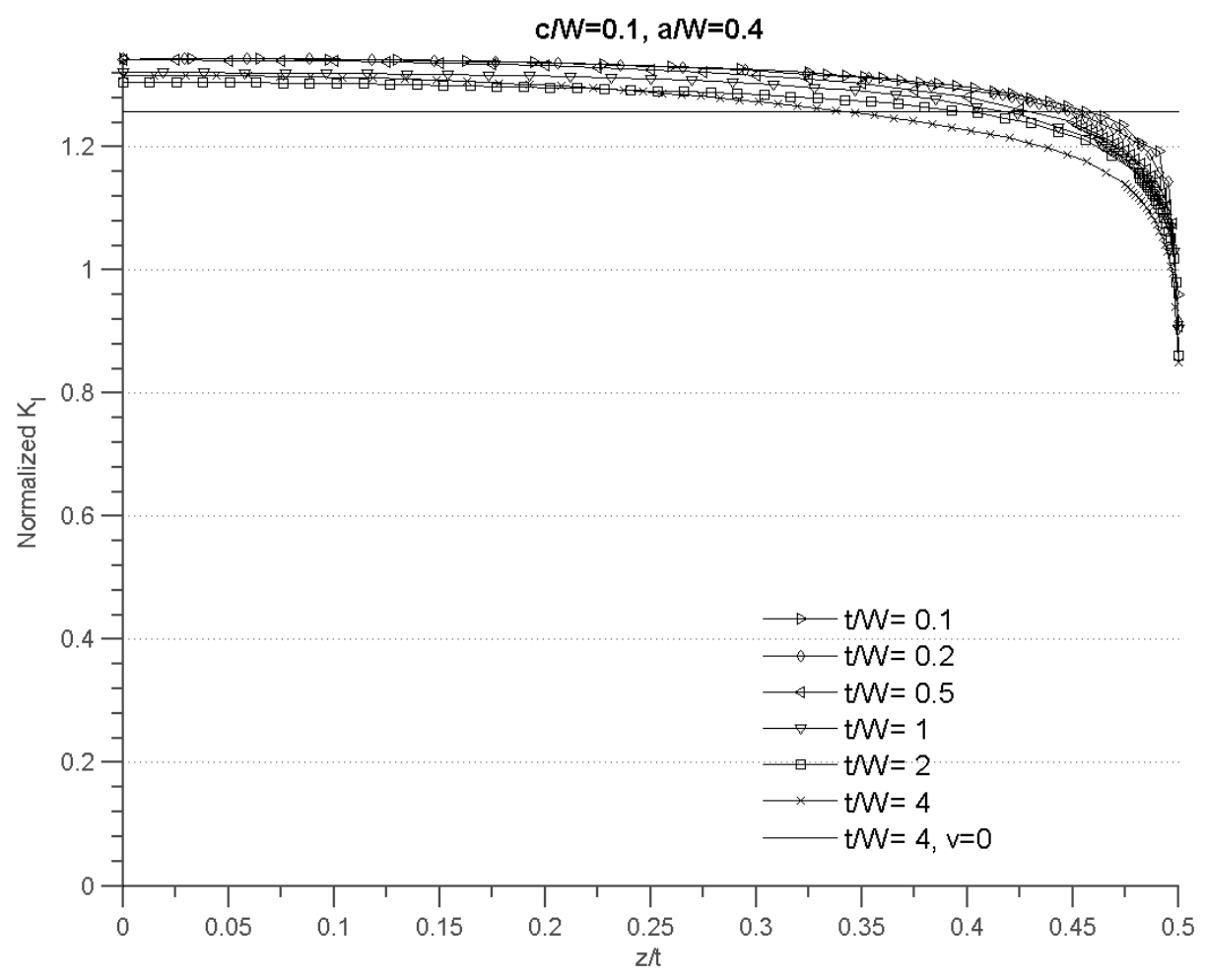

Figure 4.22: Normalized $K_{I}$ for $\mathrm{a} / \mathrm{W}=0.4$ 


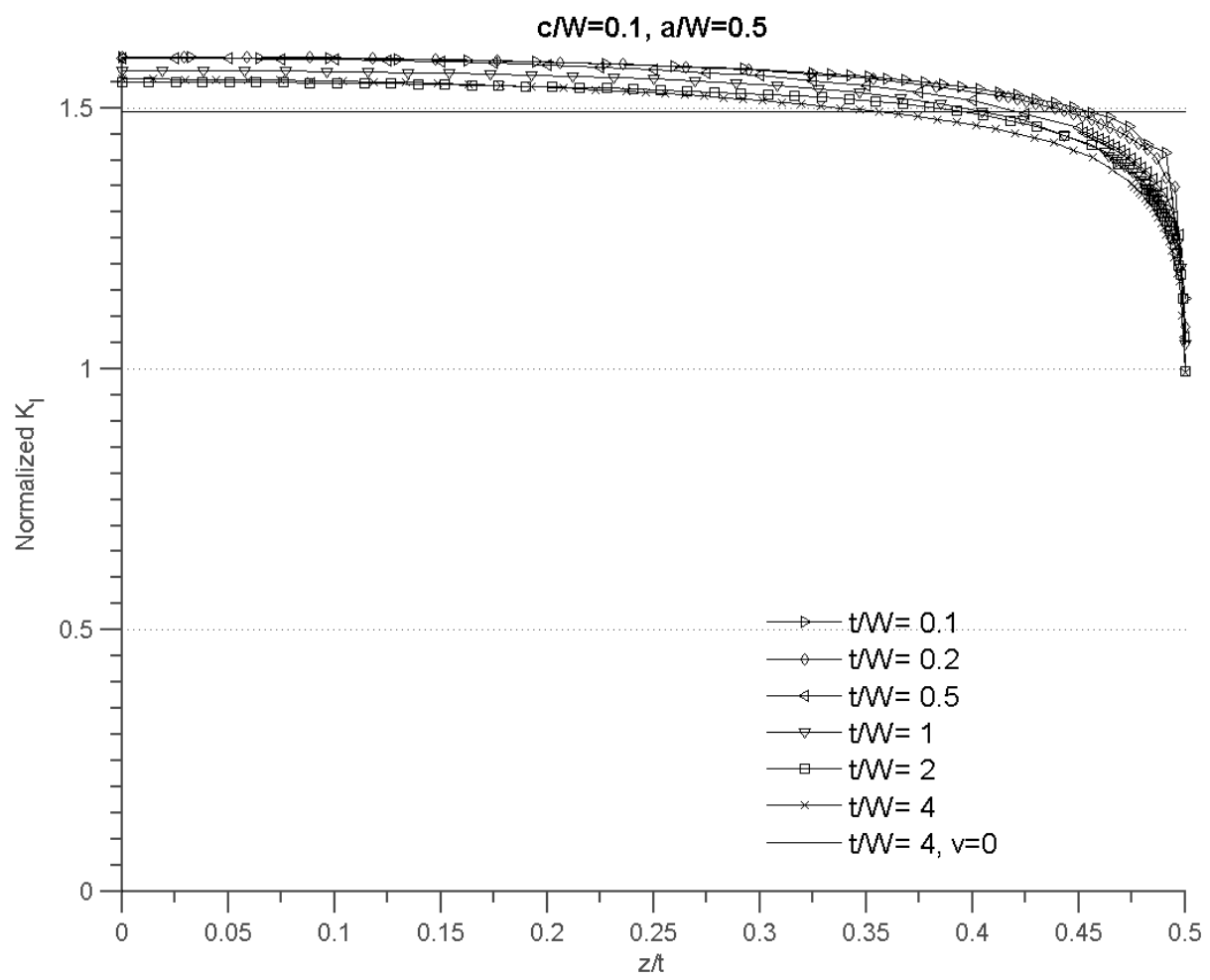

Figure 4.23: Normalized $K_{I}$ for $\mathrm{a} / \mathrm{W}=\mathbf{0 . 5}$

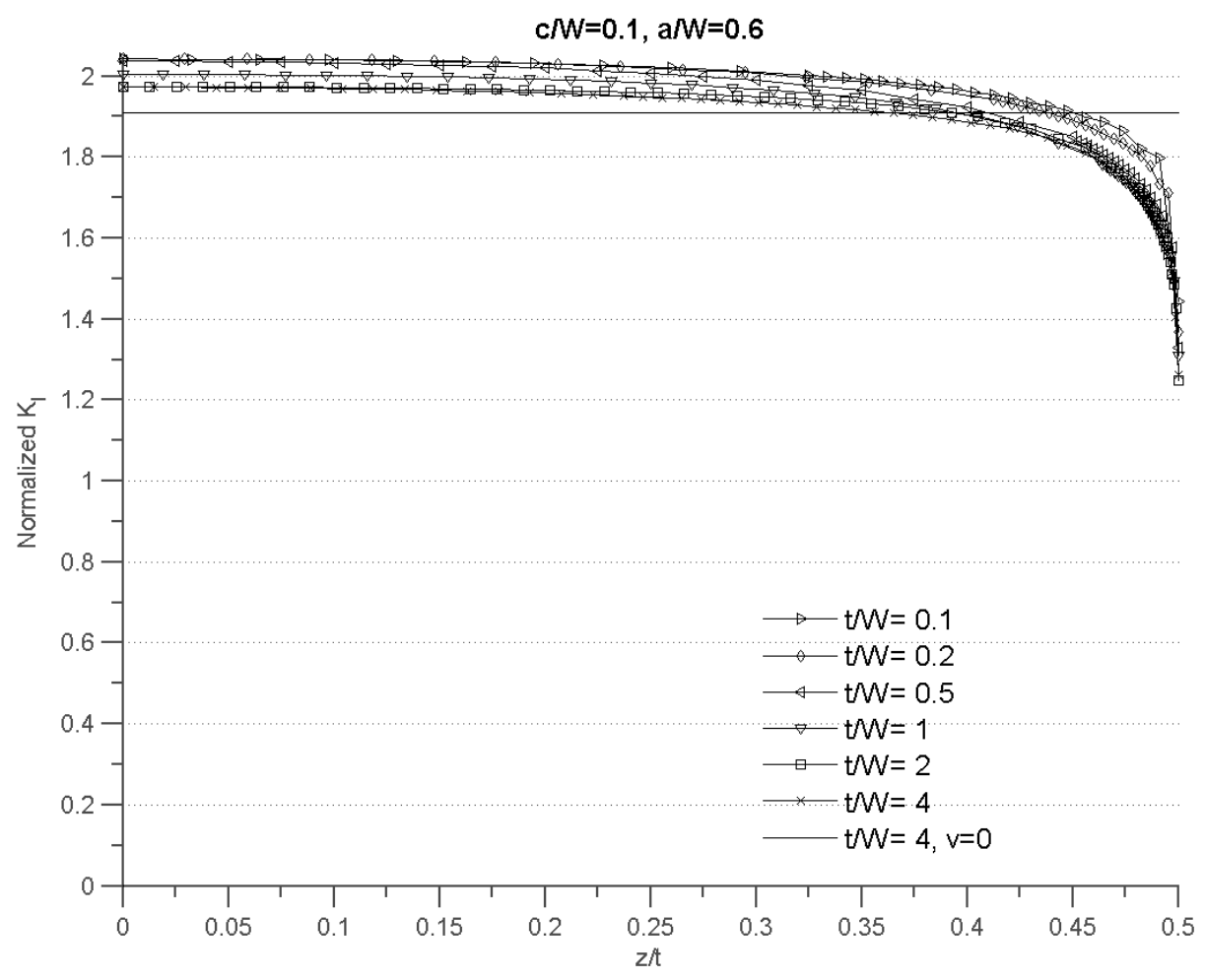

Figure 4.24: Normalized $K_{I}$ for $\mathrm{a} / \mathrm{W}=0.6$ 


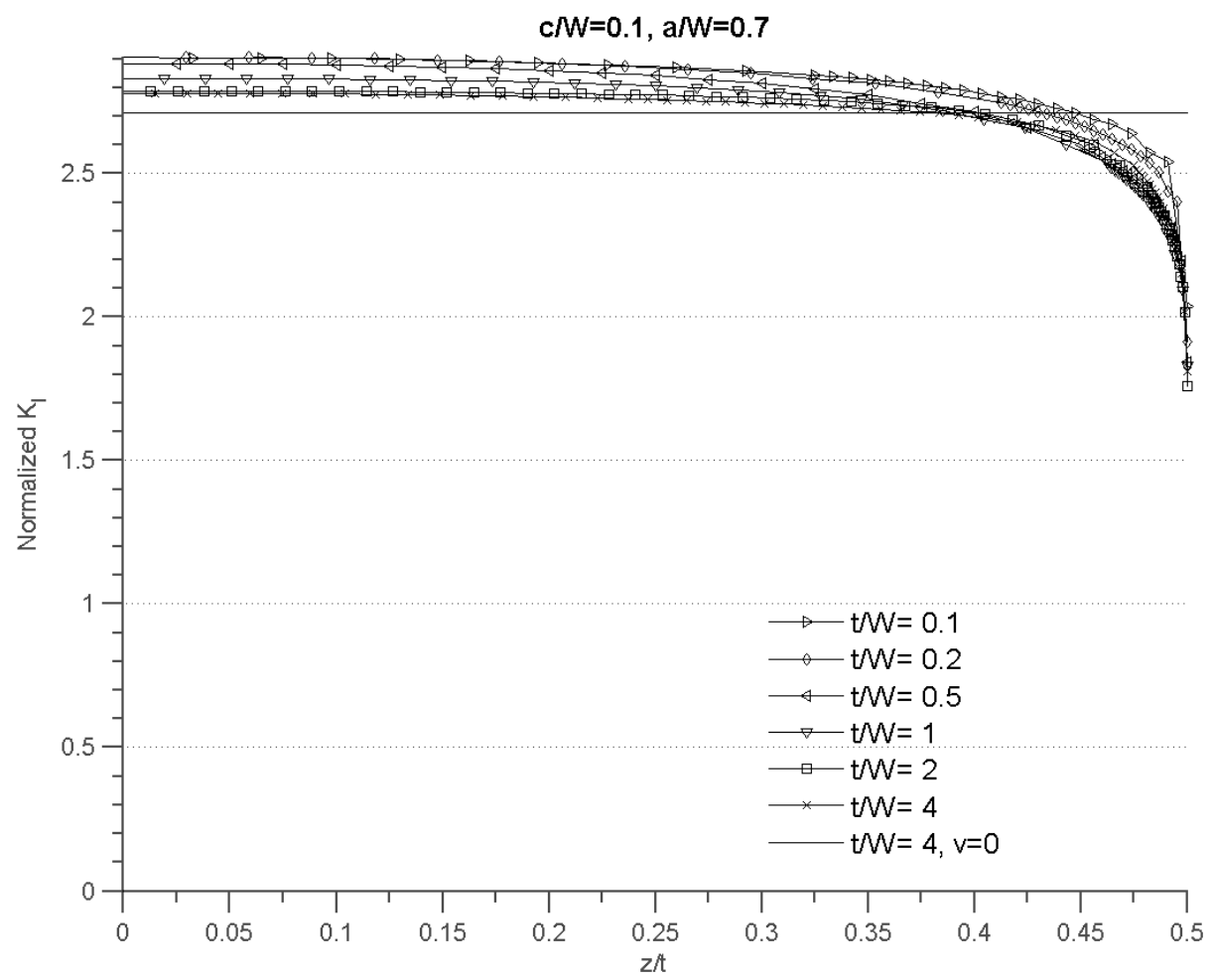

Figure 4.25: Normalized $K_{I}$ for $\mathrm{a} / \mathrm{W}=\mathbf{0 . 7}$ 


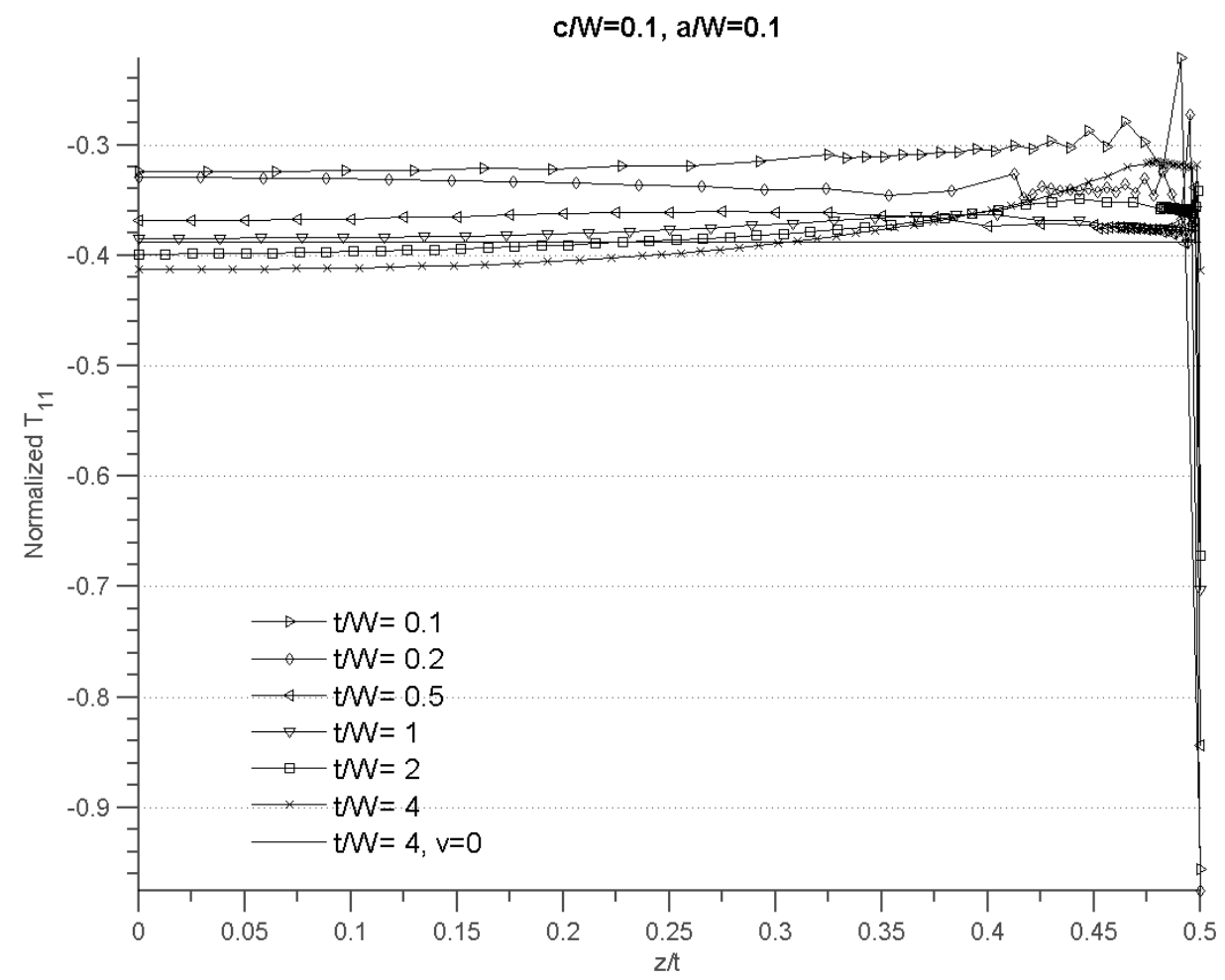

Figure 4.26: Normalized $T_{11}$ for $\mathrm{a} / \mathrm{W}=0.1$

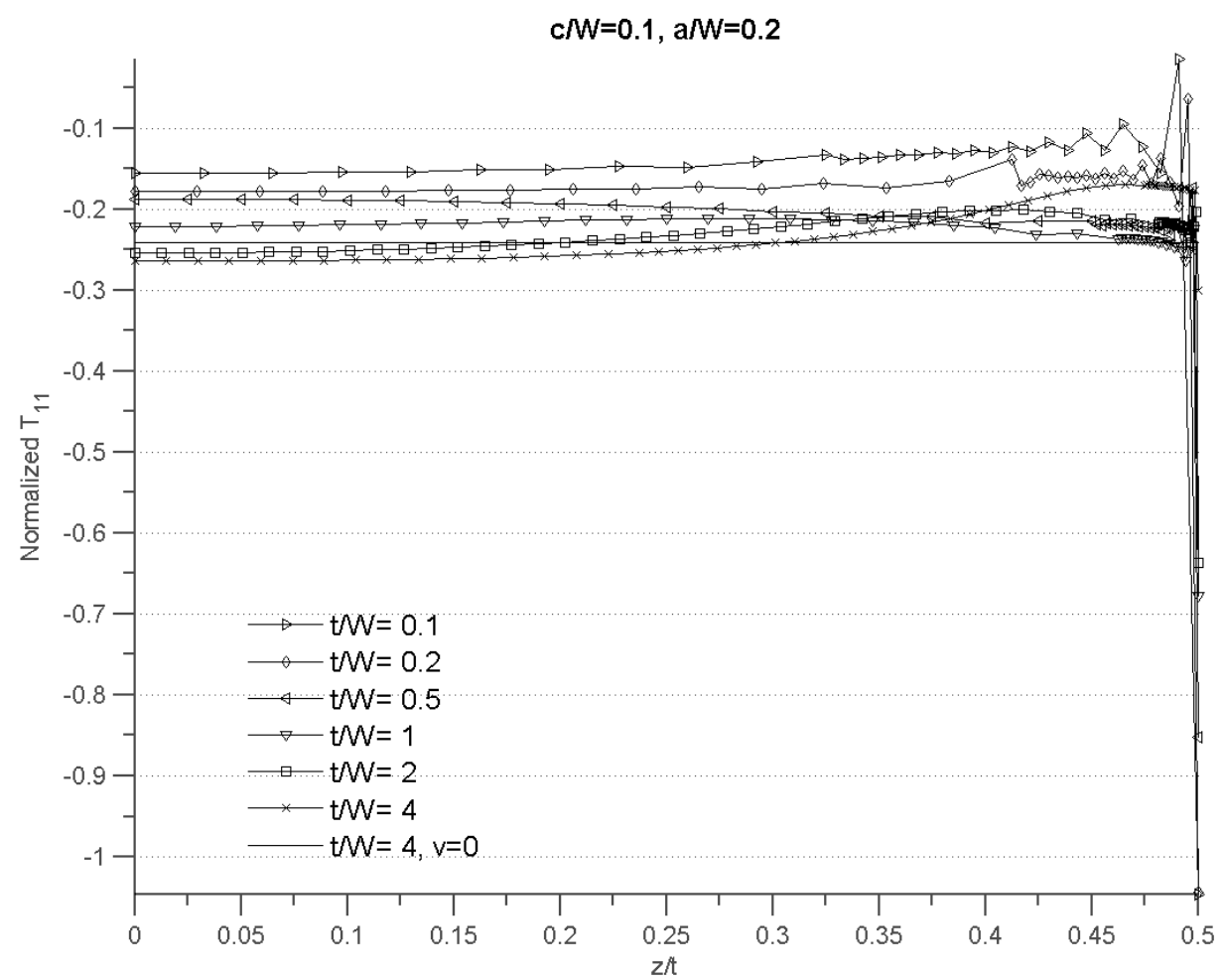

Figure 4.27: Normalized $T_{11}$ for $\mathrm{a} / \mathrm{W}=0.2$ 


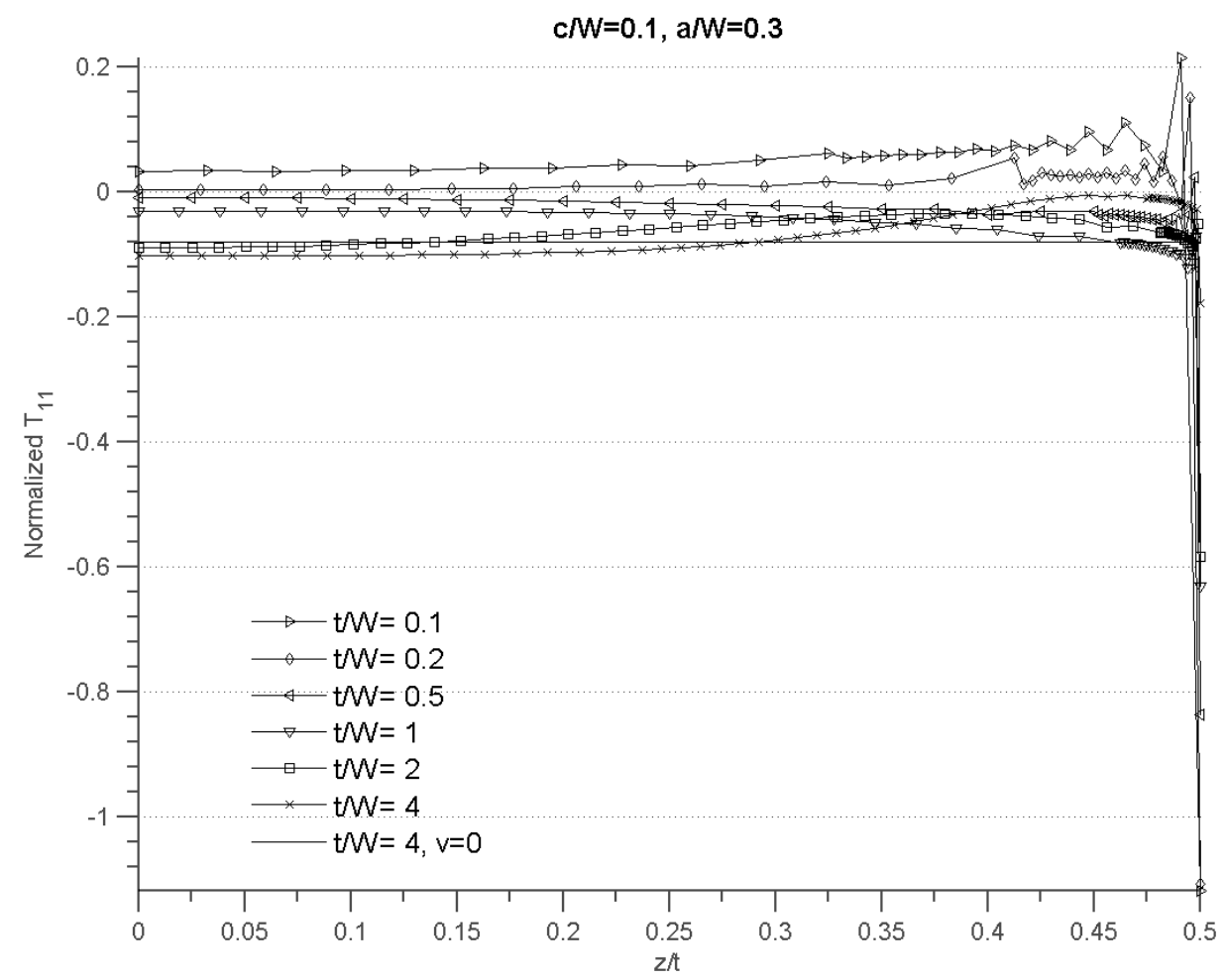

Figure 4.28: Normalized $T_{11}$ for $\mathrm{a} / \mathrm{W}=\mathbf{0 . 3}$

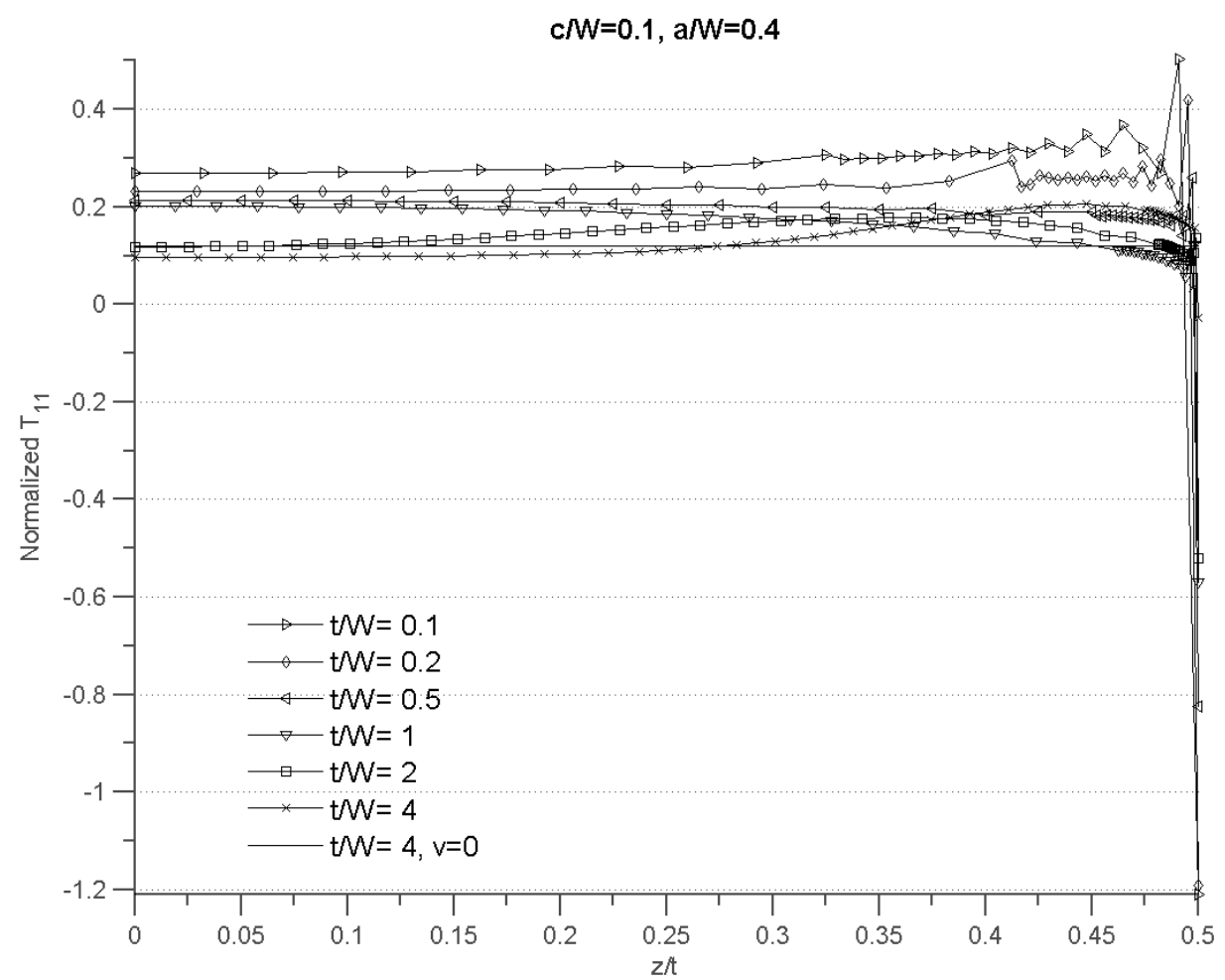

Figure 4.29: Normalized $T_{11}$ for $\mathrm{a} / \mathrm{W}=0.4$ 


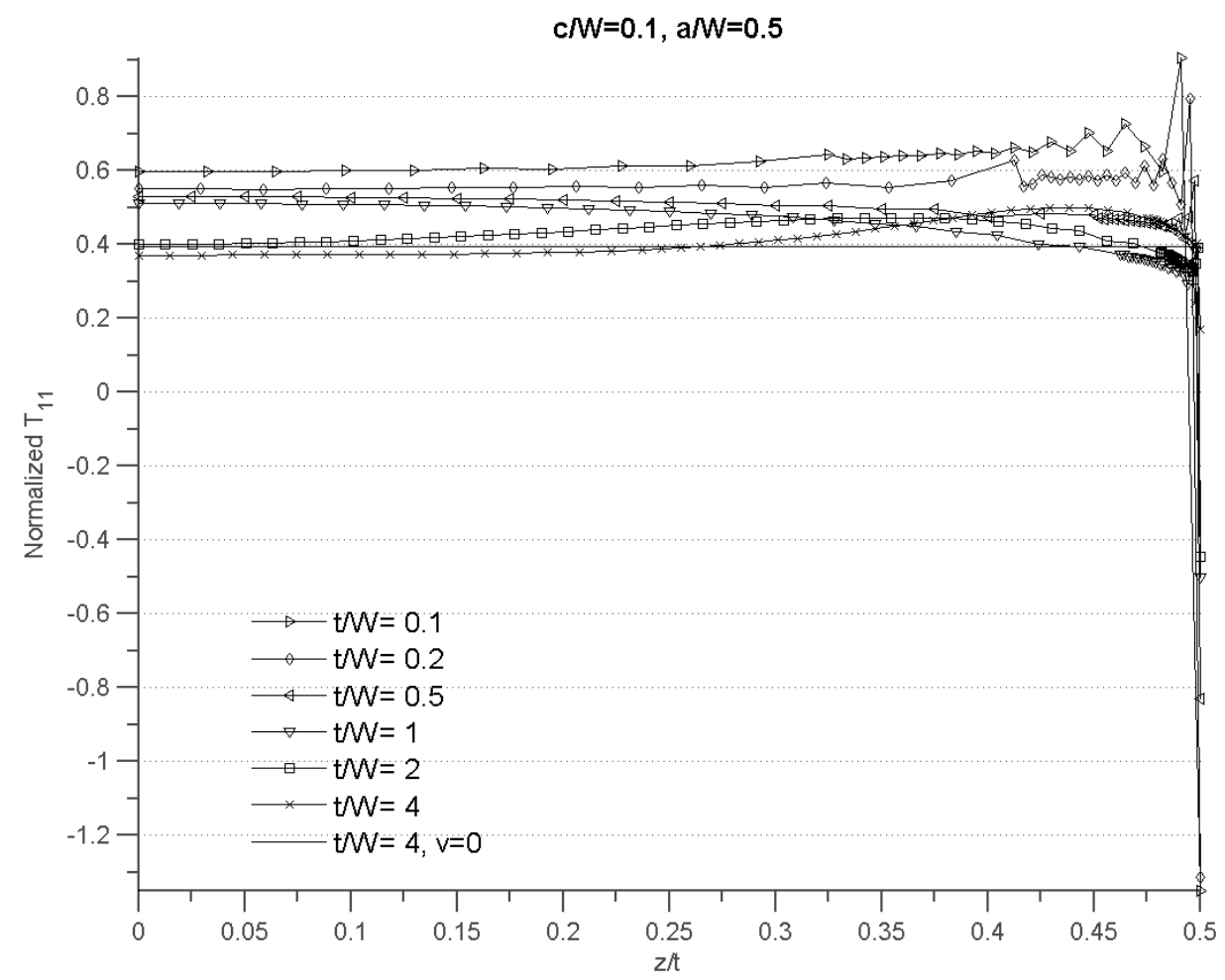

Figure 4.30: Normalized $T_{11}$ for $\mathrm{a} / \mathrm{W}=0.5$

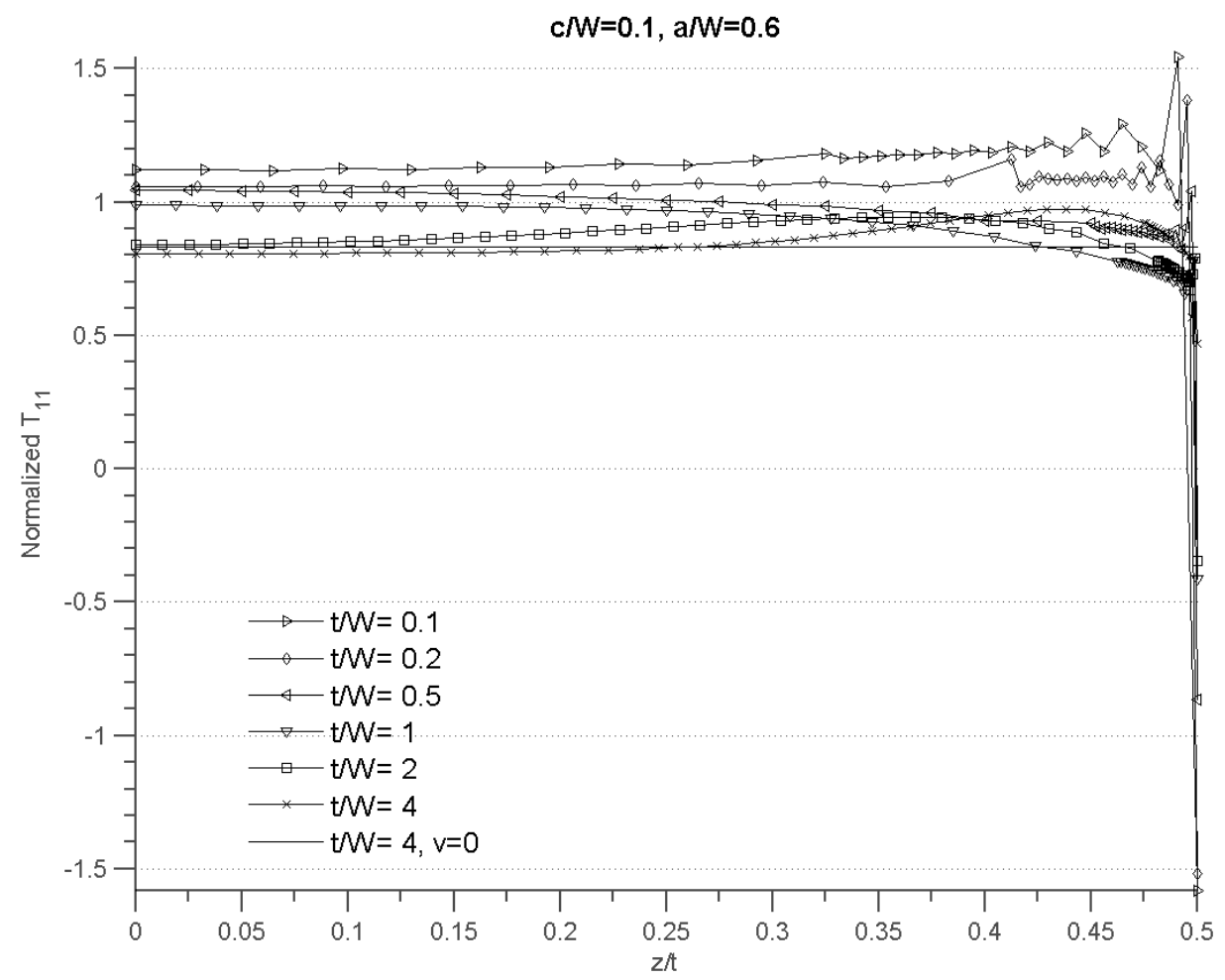

Figure 4.31: Normalized $T_{11}$ for $\mathrm{a} / \mathrm{W}=0.6$ 


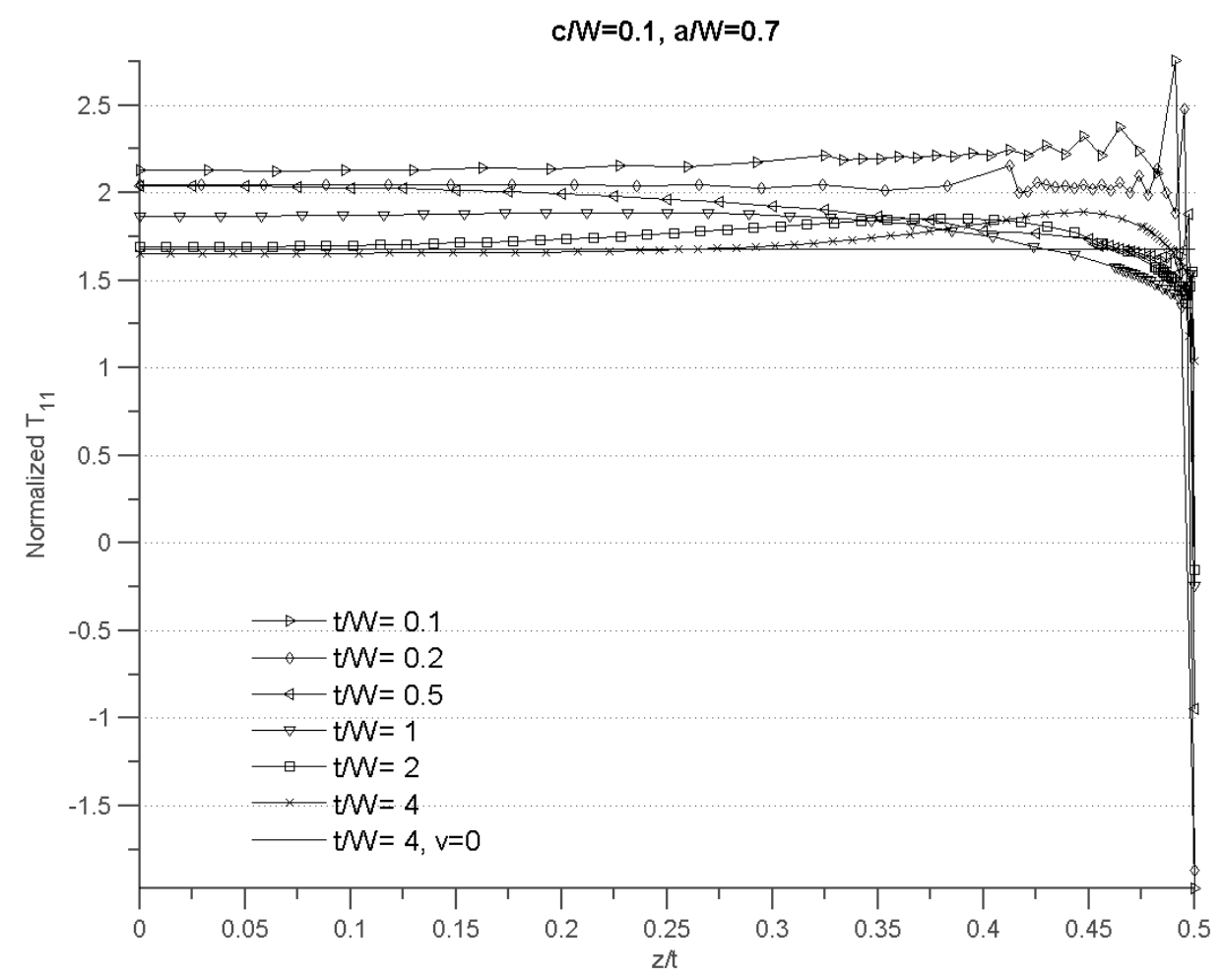

Figure 4.32: Normalized $T_{11}$ for $\mathrm{a} / \mathrm{W}=0.7$ 


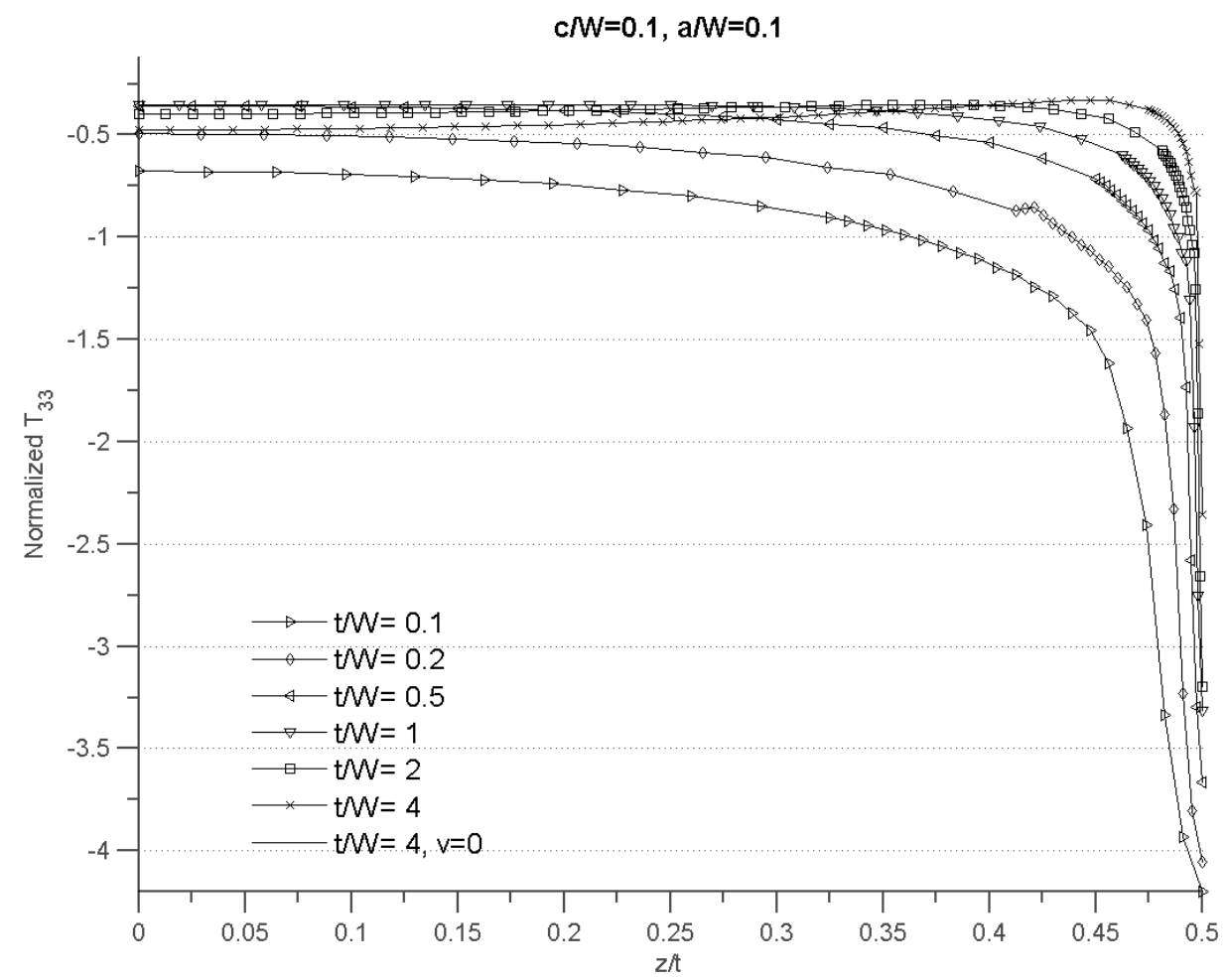

Figure 4.33: Normalized $T_{33}$ for $\mathrm{a} / \mathrm{W}=\mathbf{0 . 1}$

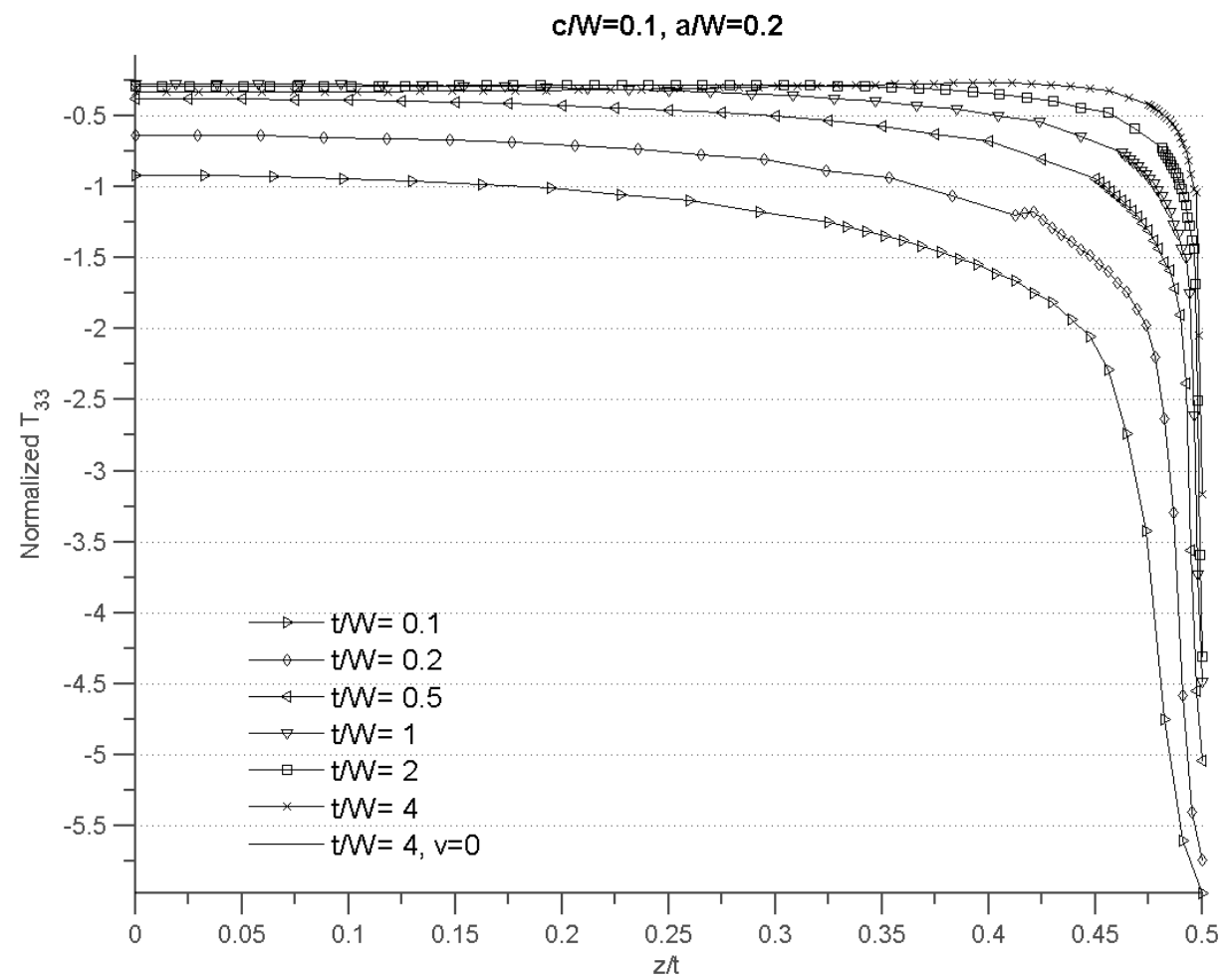

Figure 4.34: Normalized $T_{33}$ for $\mathrm{a} / \mathrm{W}=0.2$ 


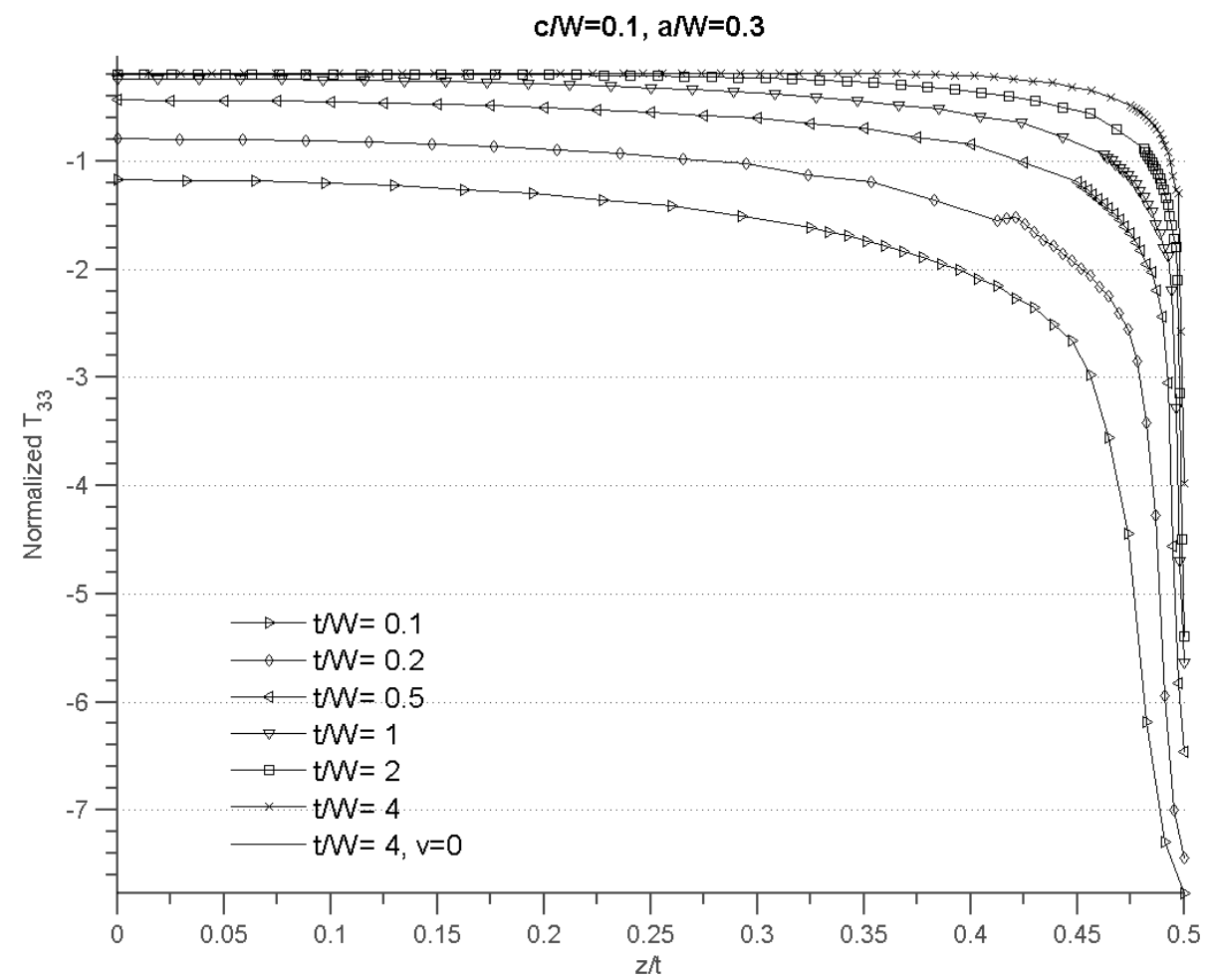

Figure 4.35: Normalized $T_{33}$ for $\mathrm{a} / \mathrm{W}=\mathbf{0 . 3}$

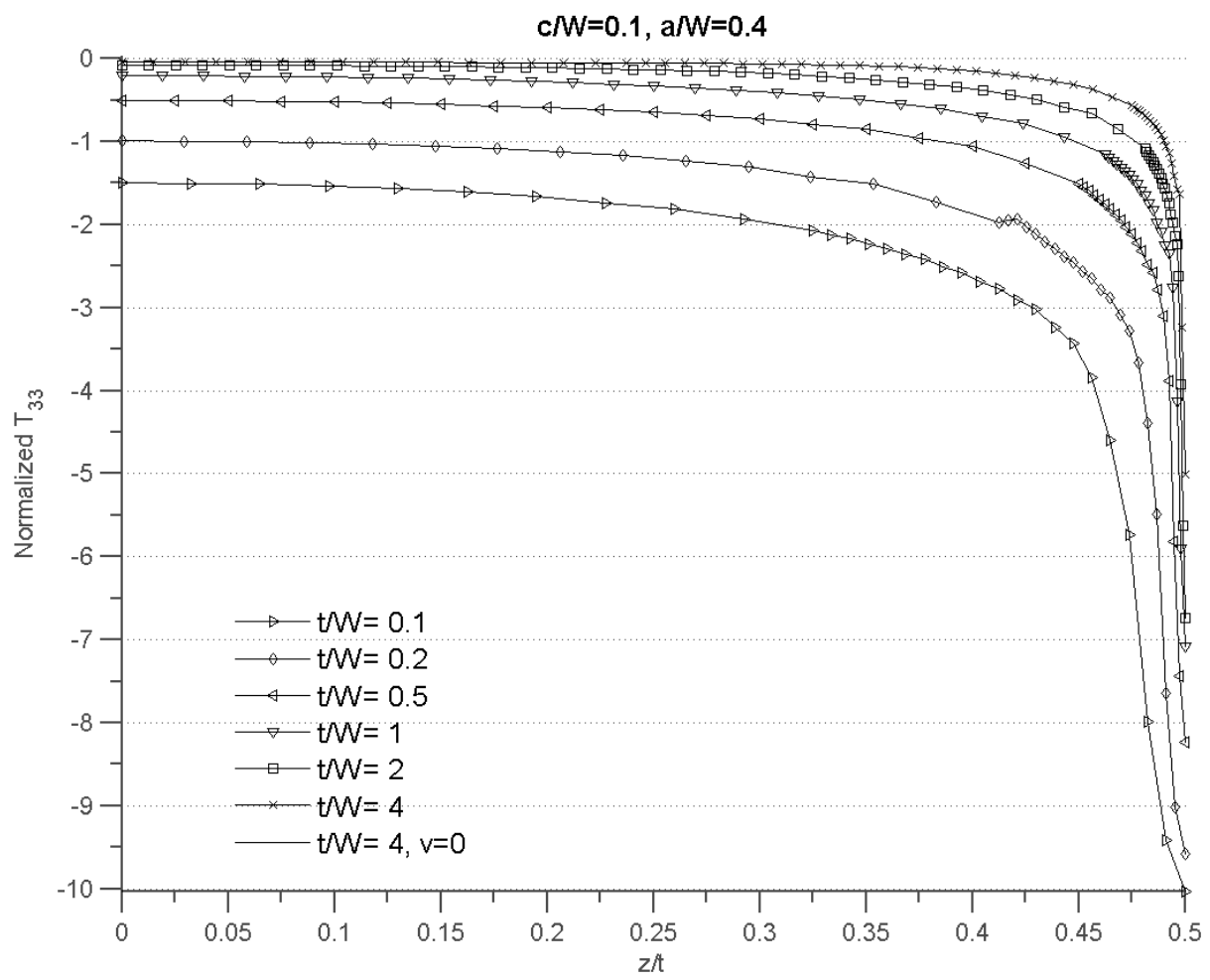

Figure 4.36: Normalized $T_{33}$ for $\mathrm{a} / \mathrm{W}=0.4$ 


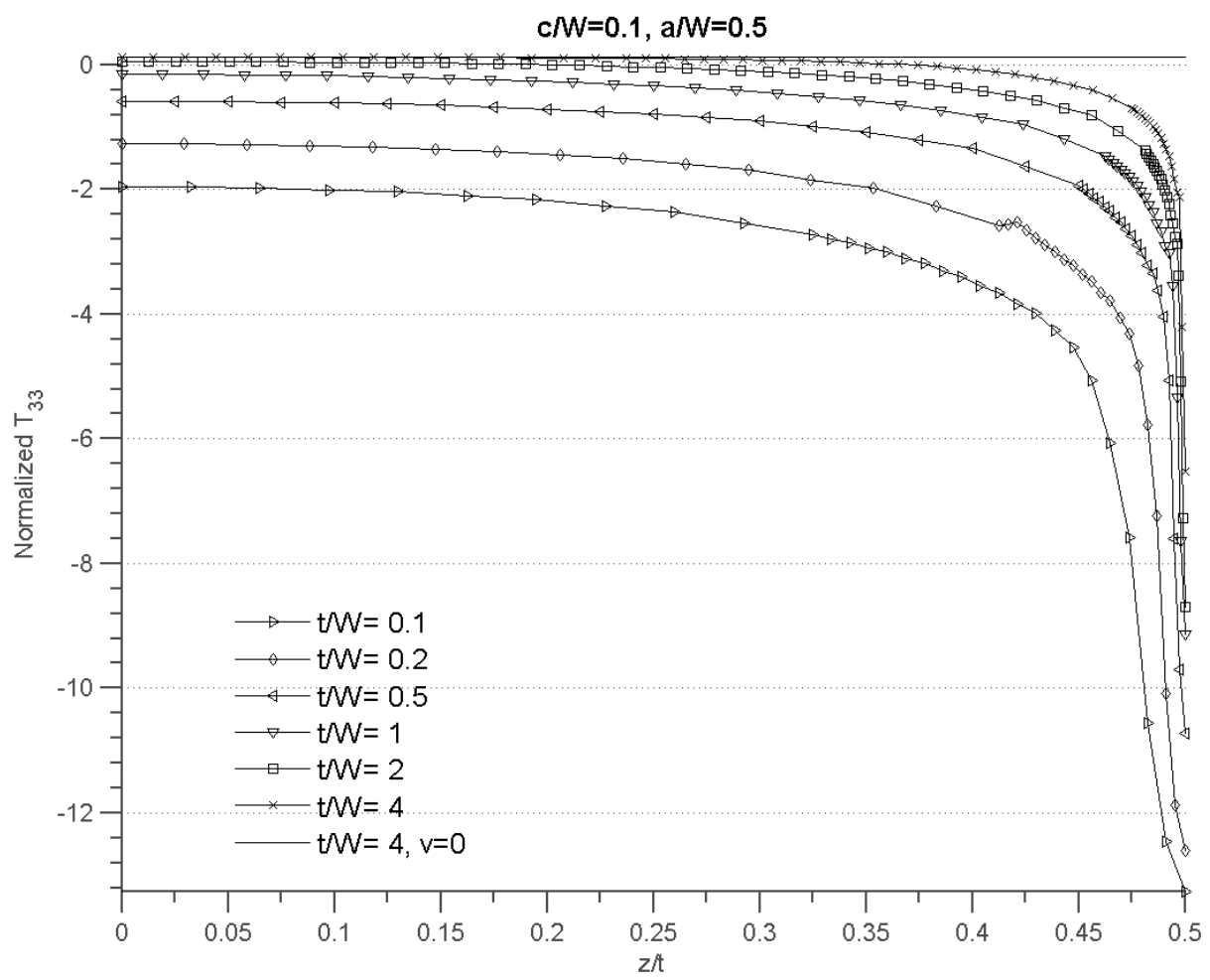

Figure 4.37: Normalized $T_{33}$ for $\mathrm{a} / \mathrm{W}=\mathbf{0 . 5}$

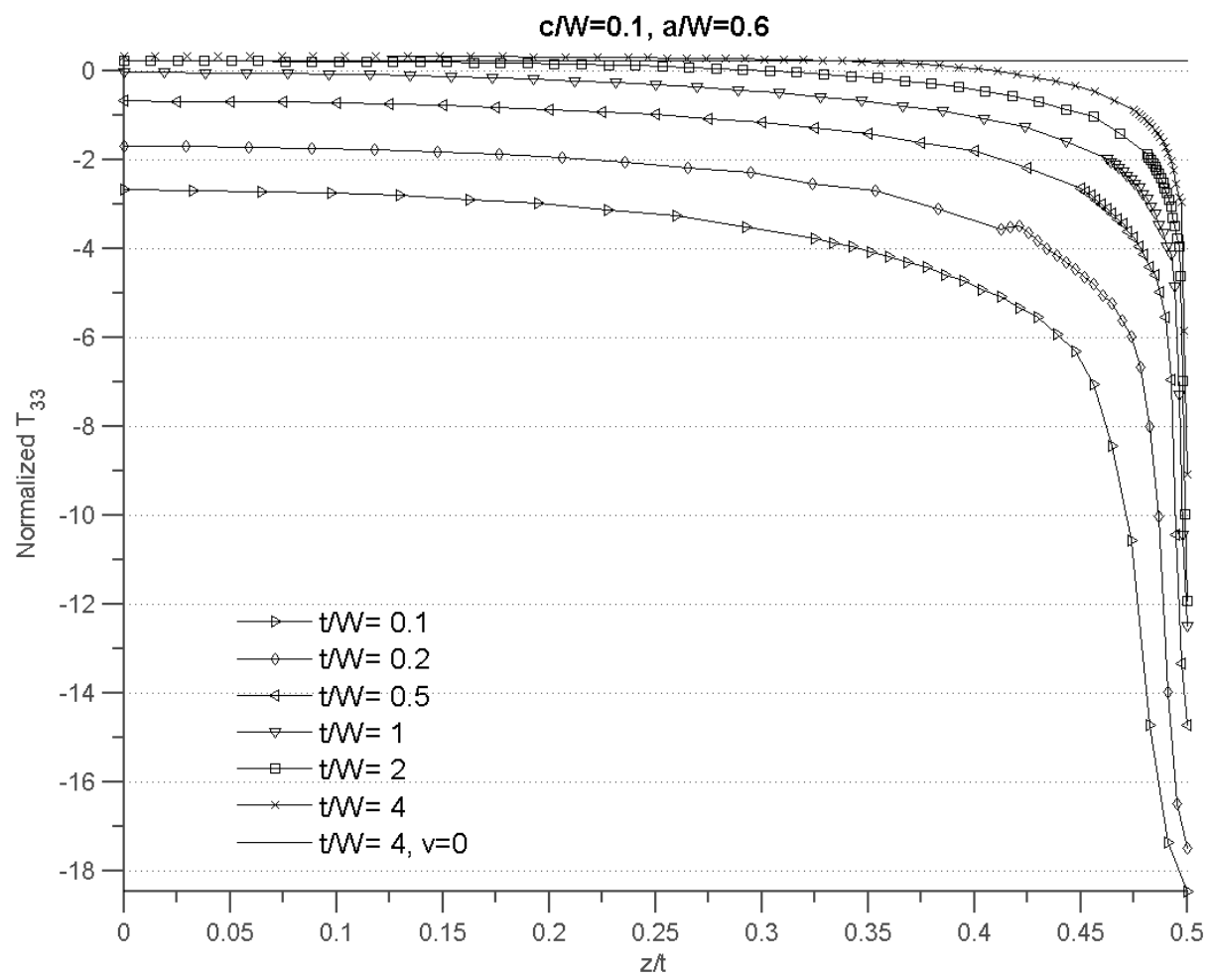

Figure 4.38: Normalized $T_{33}$ for $\mathrm{a} / \mathrm{W}=0.6$ 


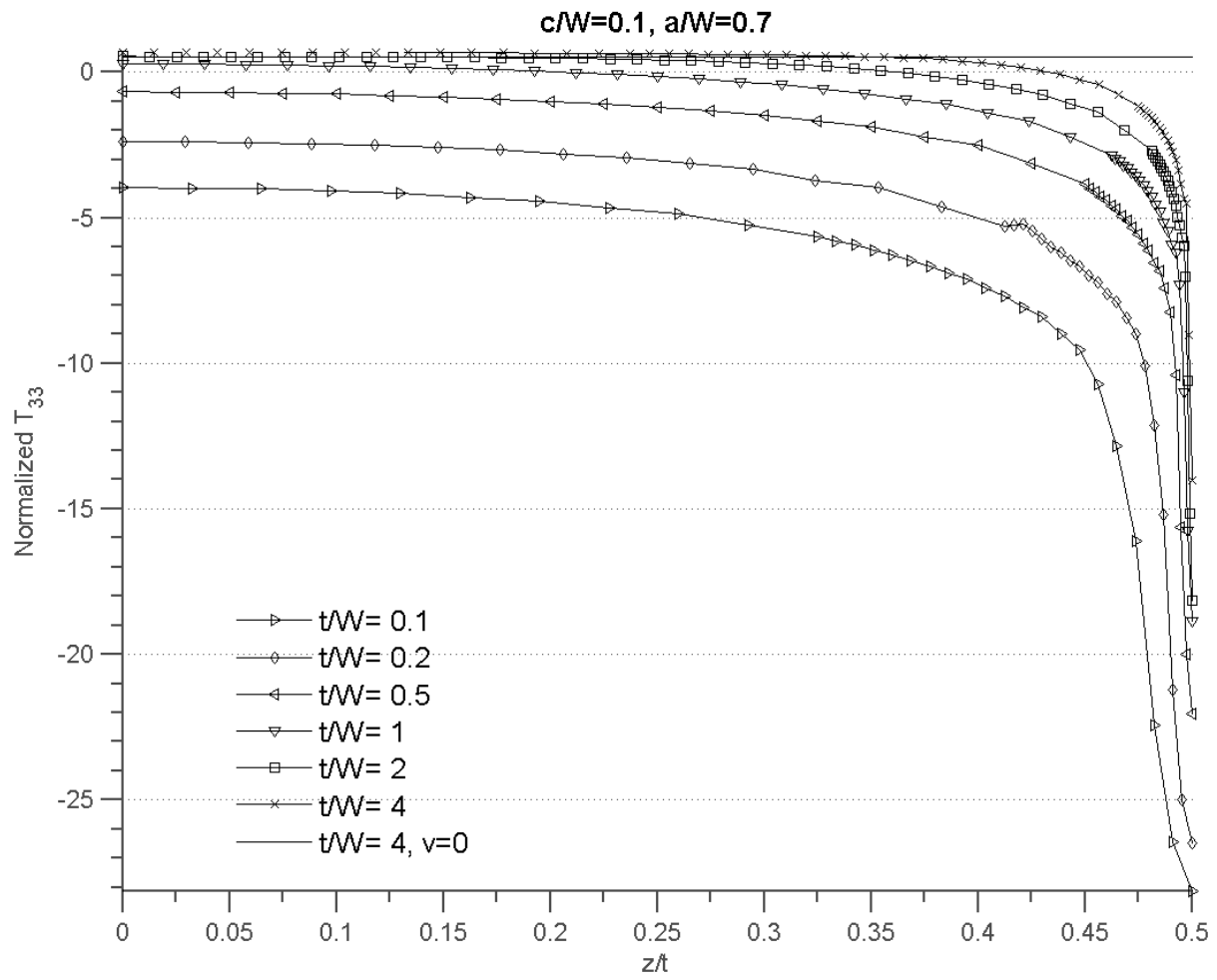

Figure 4.39: Normalized $T_{33}$ for $\mathrm{a} / \mathrm{W}=0.7$ 

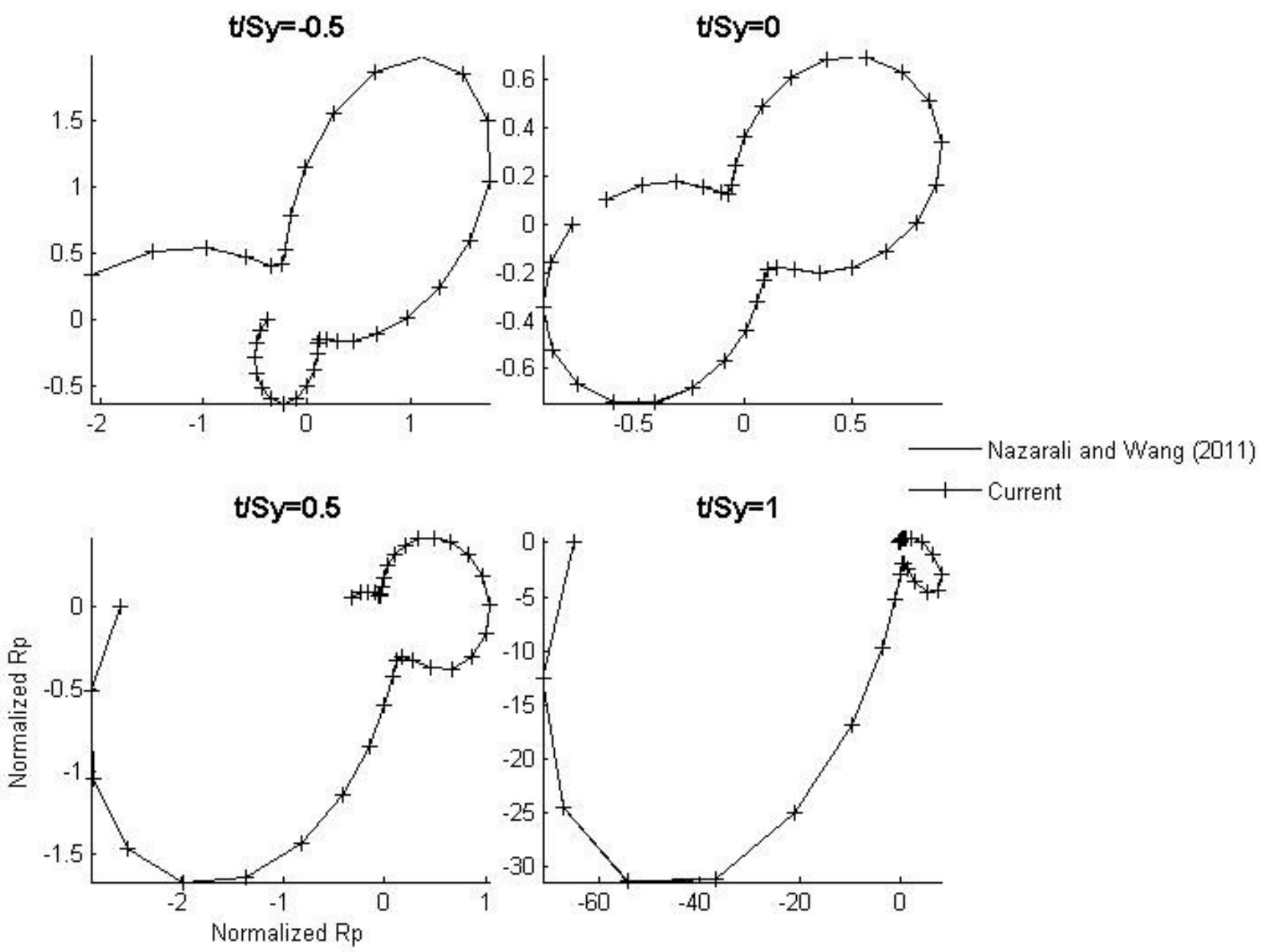

Figure 4.40: Plastic zone verification for $K_{I I} / K_{I}=1.0$ 


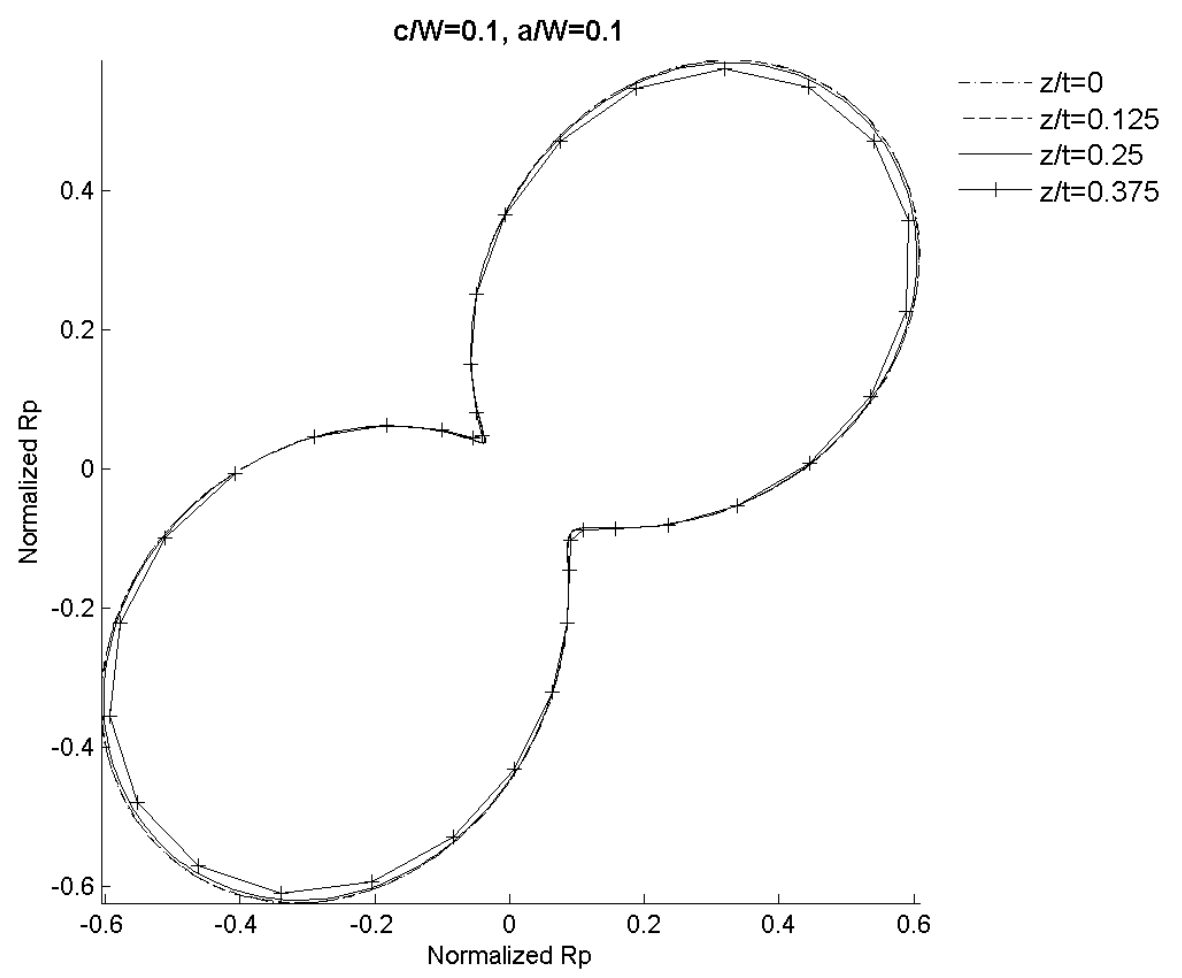

Figure 4.41: Plastic zone map for $t / W=0.1, a / W=0.1$

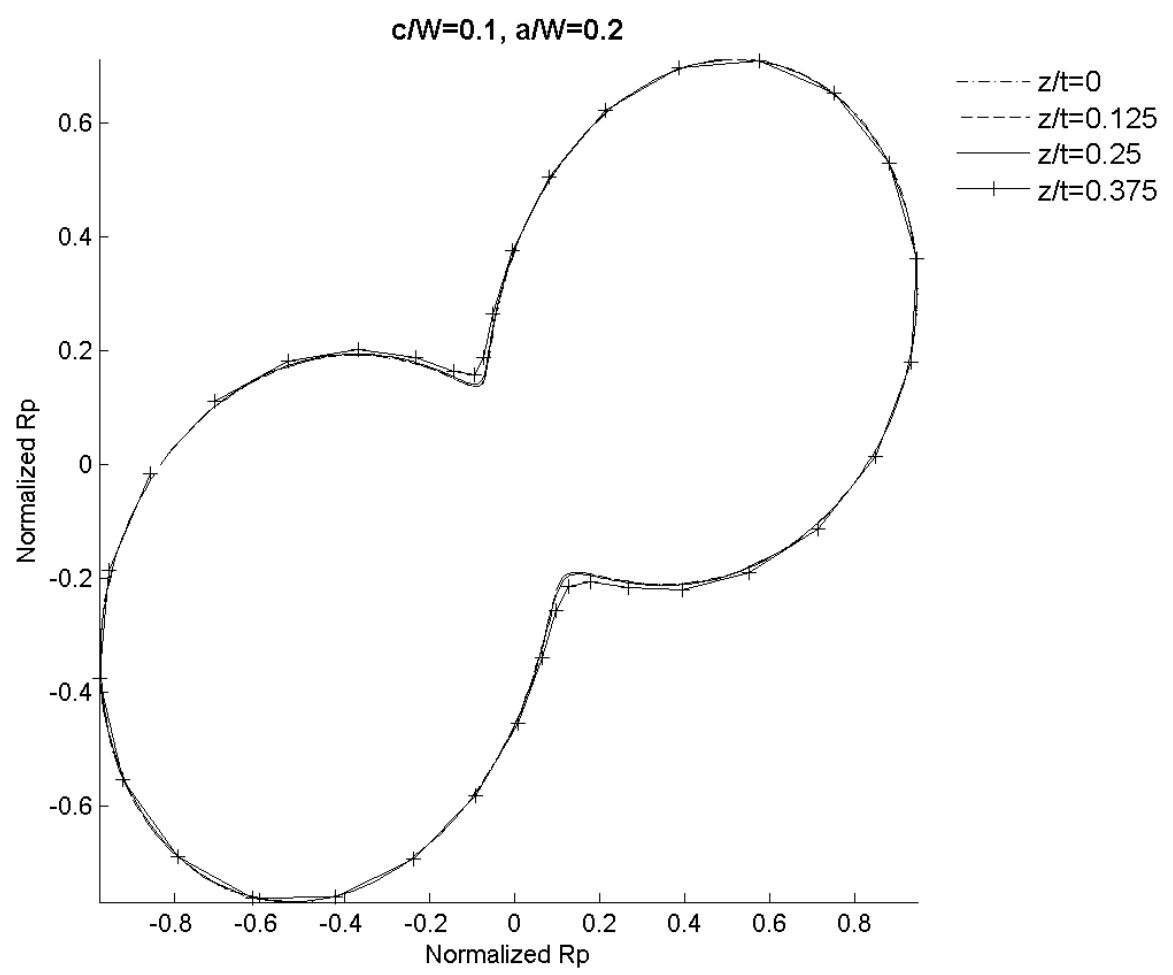

Figure 4.42: Plastic zone map for $t / W=0.1, a / W=0.2$ 


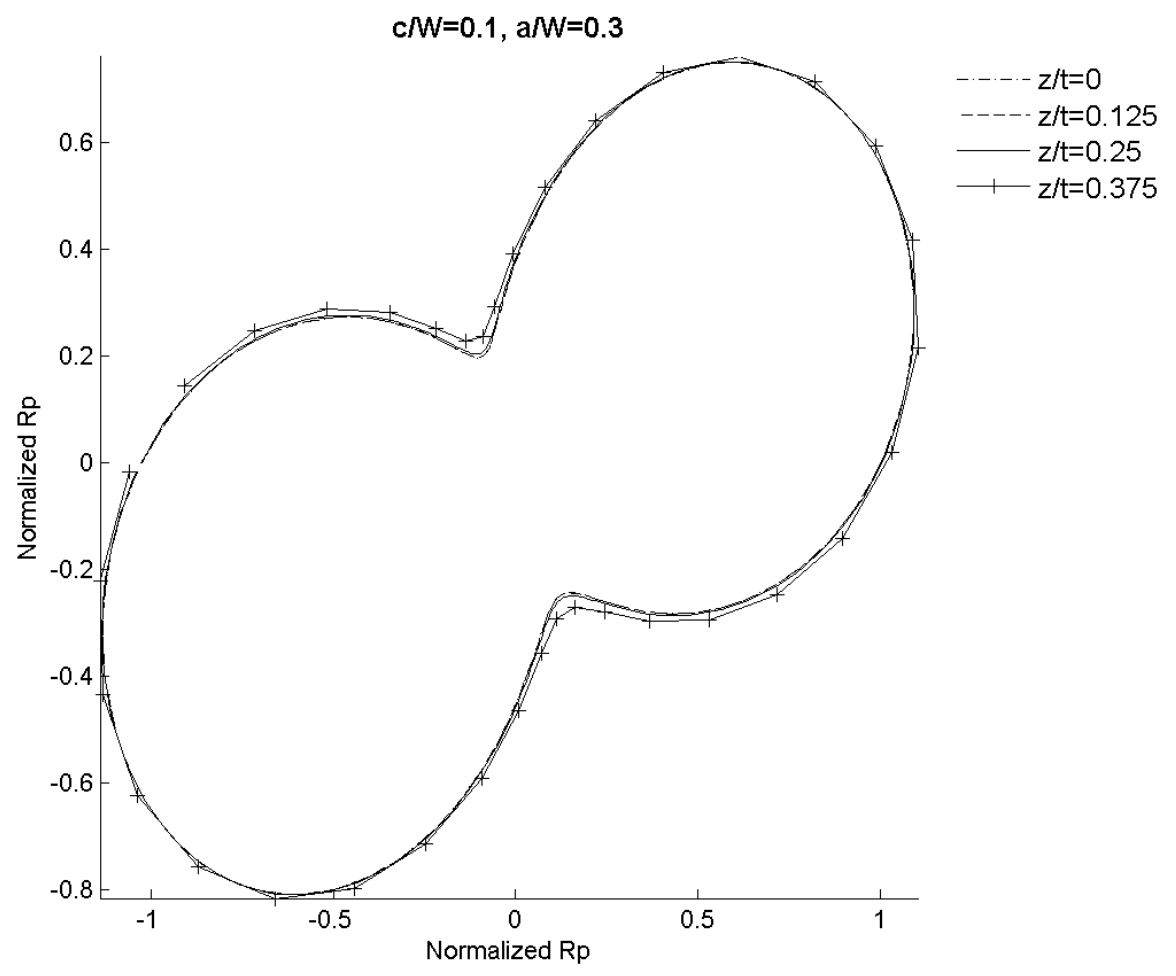

Figure 4.43: Plastic zone map for $t / W=0.1, a / W=0.3$

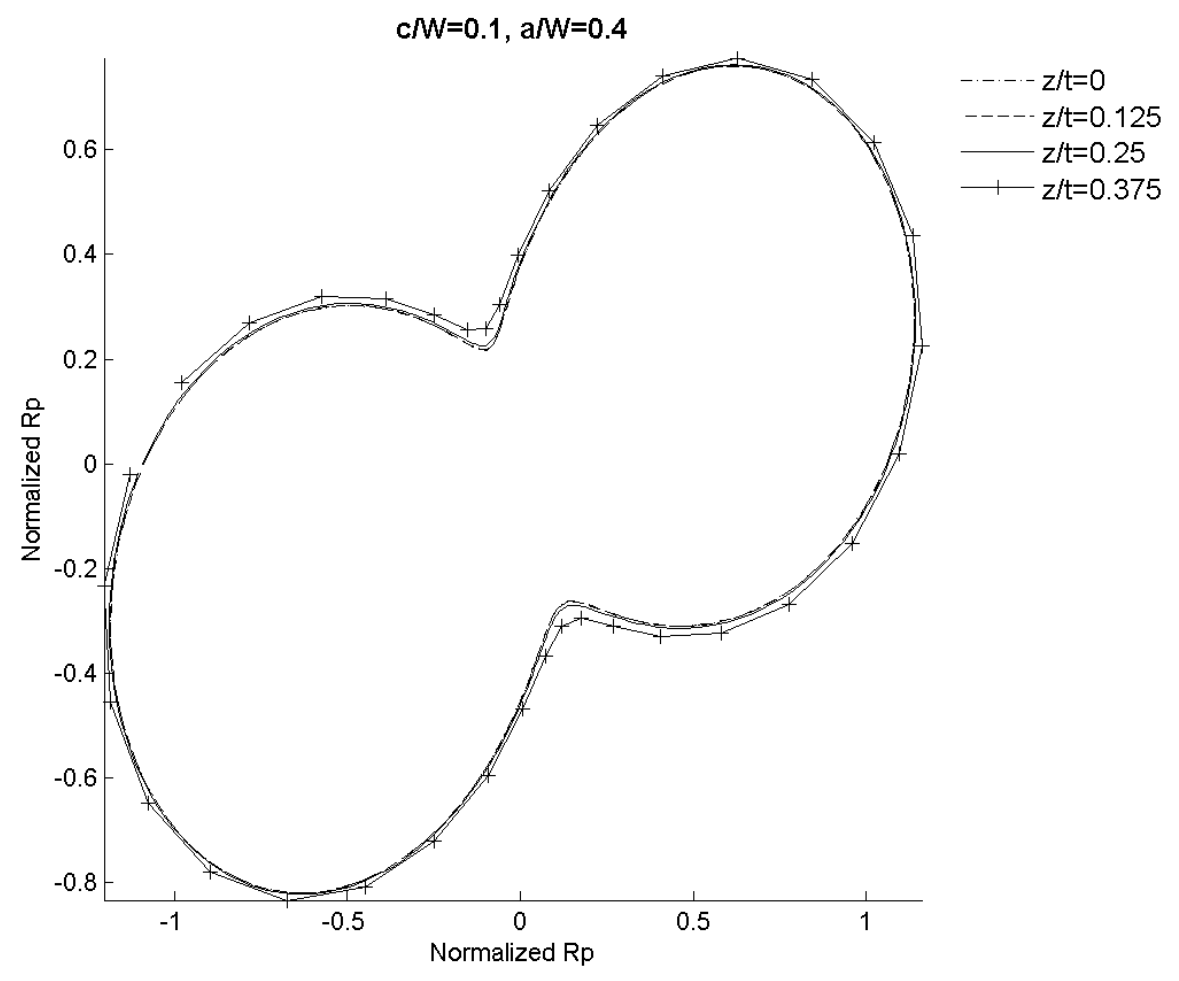

Figure 4.44: Plastic zone map for $\mathrm{t} / \mathrm{W}=\mathbf{0 . 1}, \mathrm{a} / \mathrm{W}=\mathbf{0 . 4}$ 


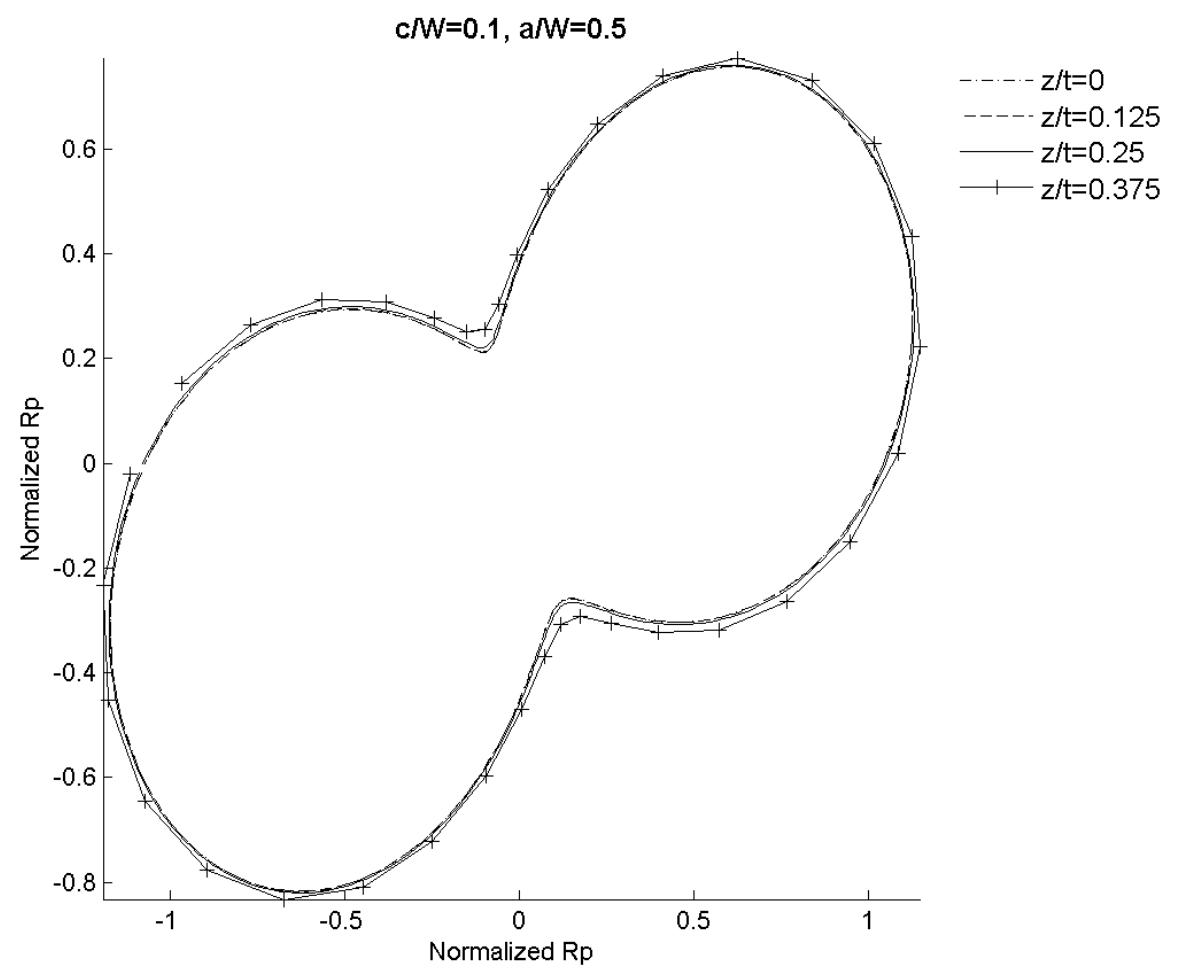

Figure 4.45: Plastic zone map for $t / W=0.1, a / W=0.5$

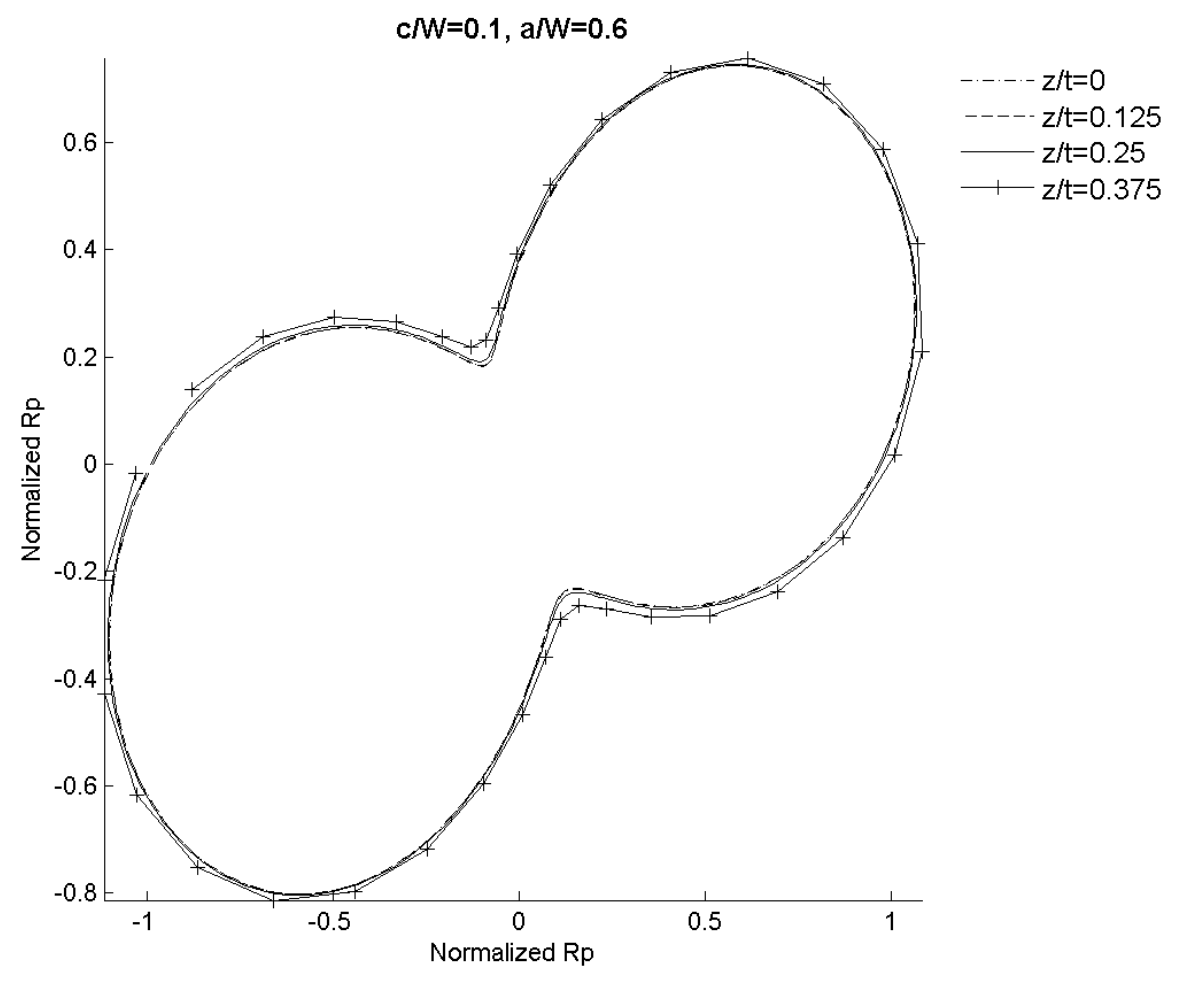

Figure 4.46: Plastic zone map for $t / W=0.1, a / W=0.6$ 


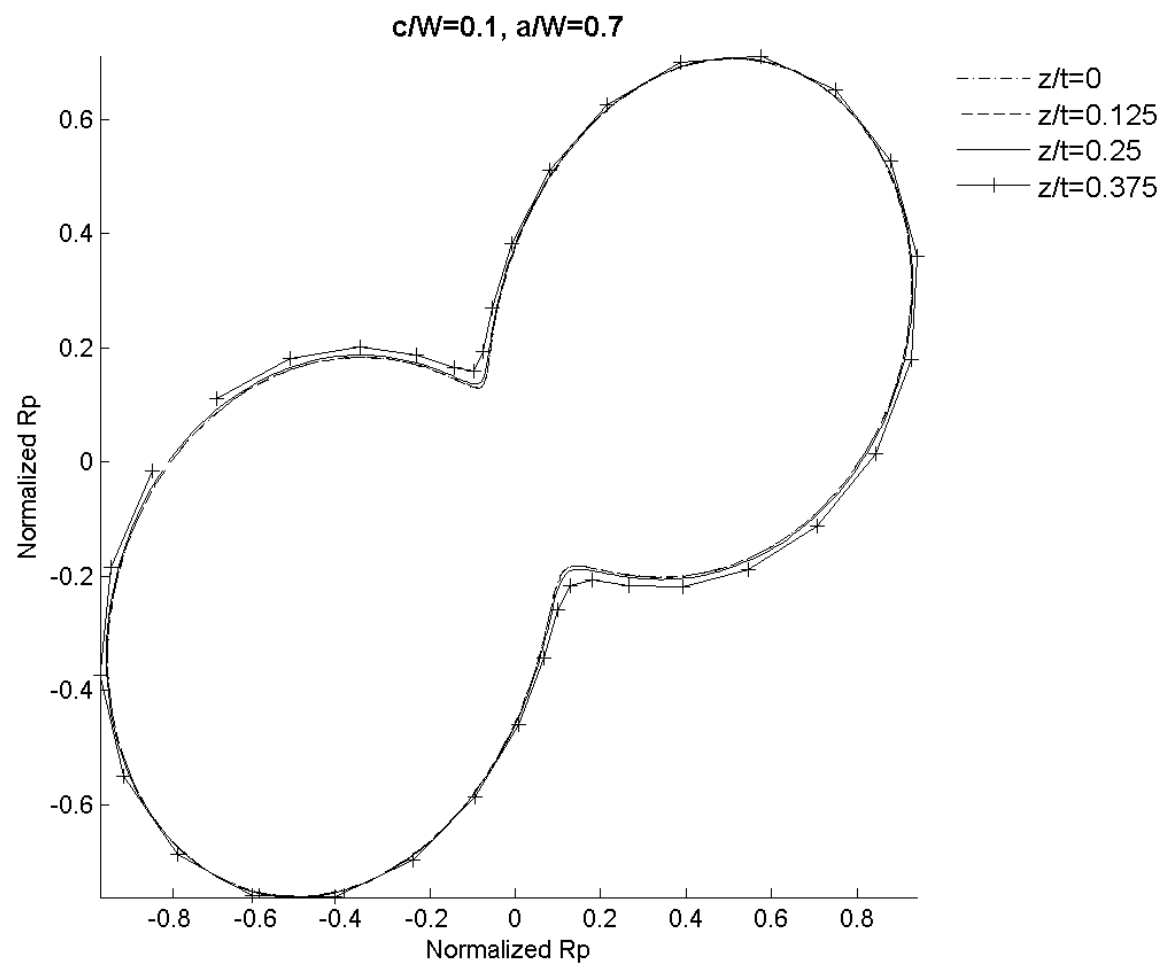

Figure 4.47: Plastic zone map for $\mathrm{t} / \mathrm{W}=\mathbf{0 . 1}, \mathrm{a} / \mathrm{W}=\mathbf{0 . 7}$ 


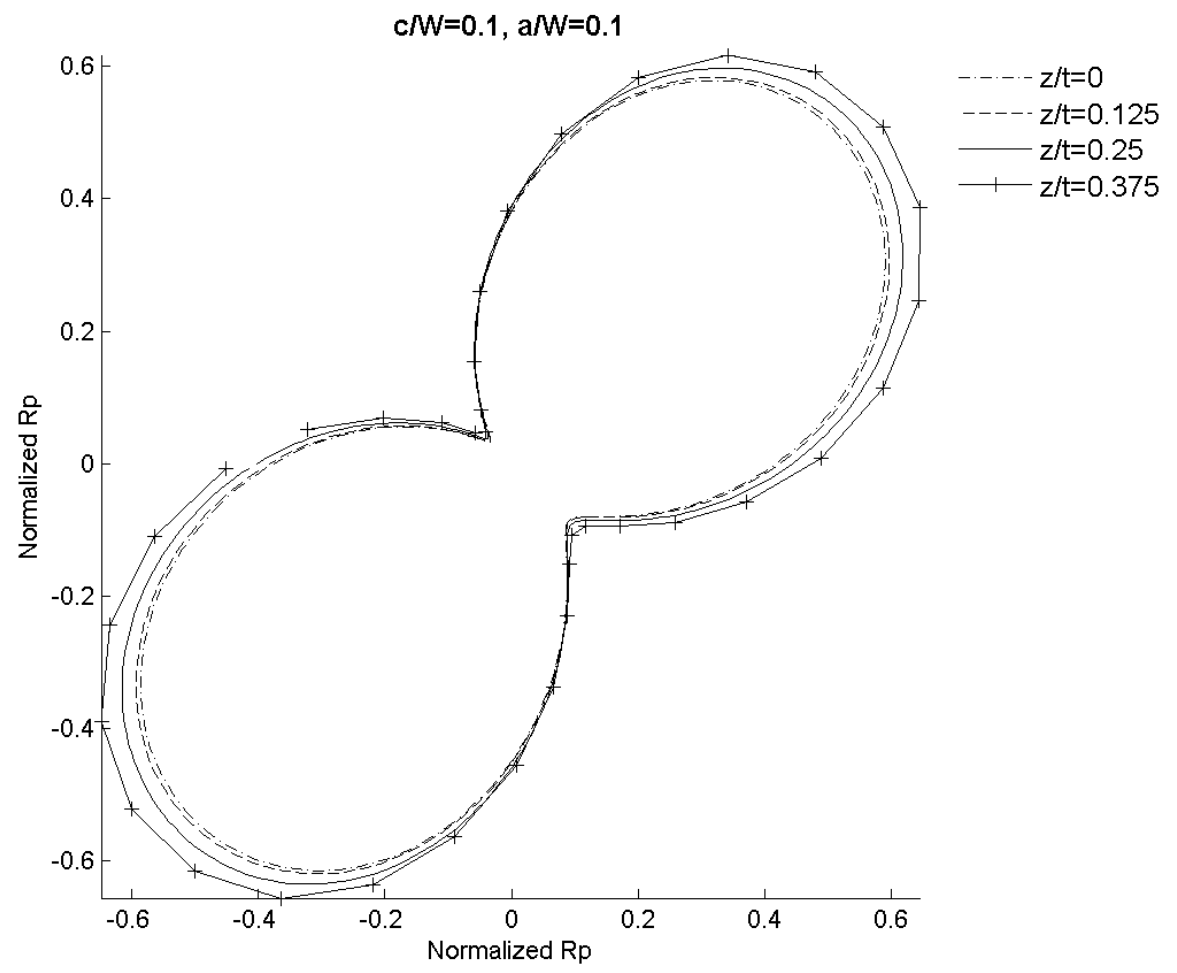

Figure 4.48: Plastic zone map for $\mathrm{t} / \mathrm{W}=0.5, \mathrm{a} / \mathrm{W}=\mathbf{0 . 1}$

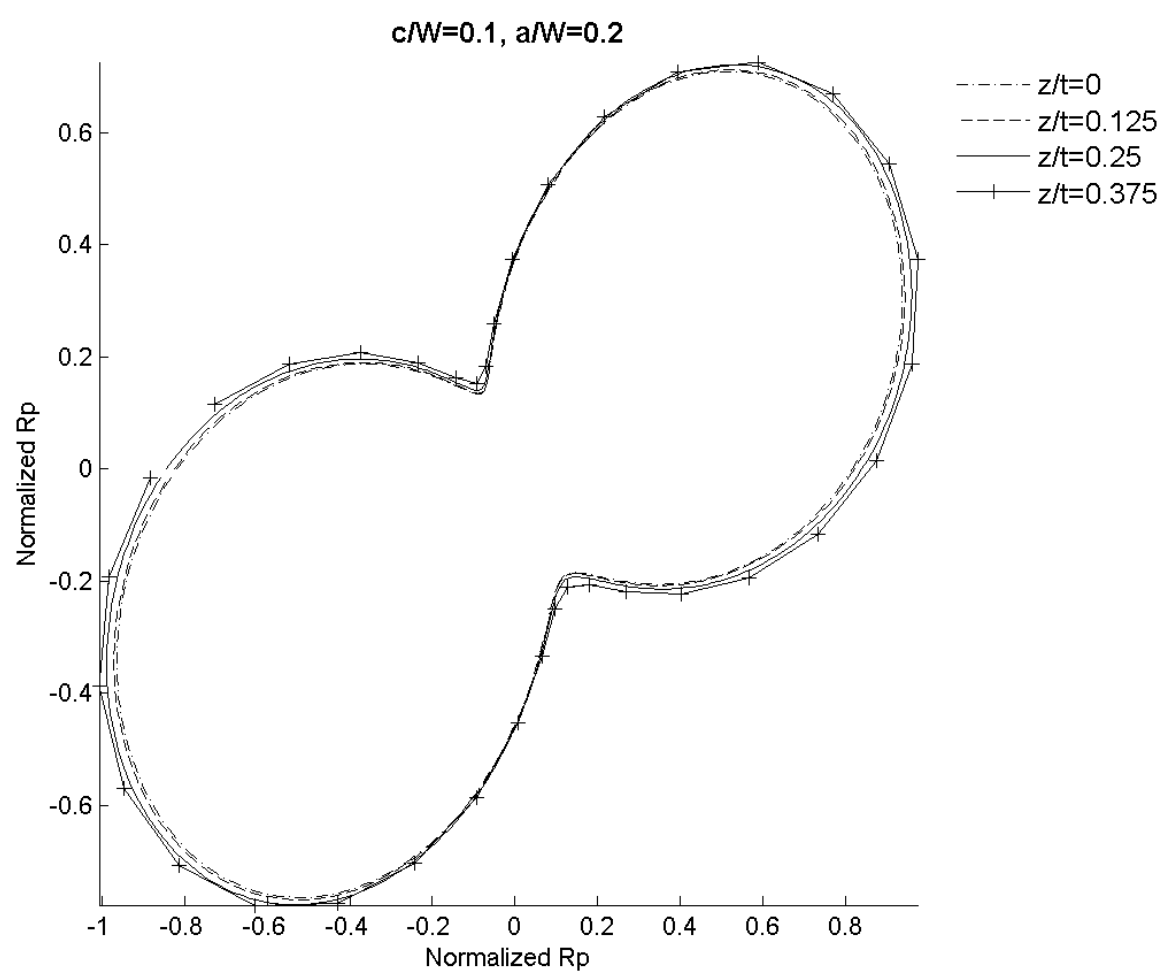

Figure 4.49: Plastic zone map for $t / W=0.5, a / W=0.2$ 


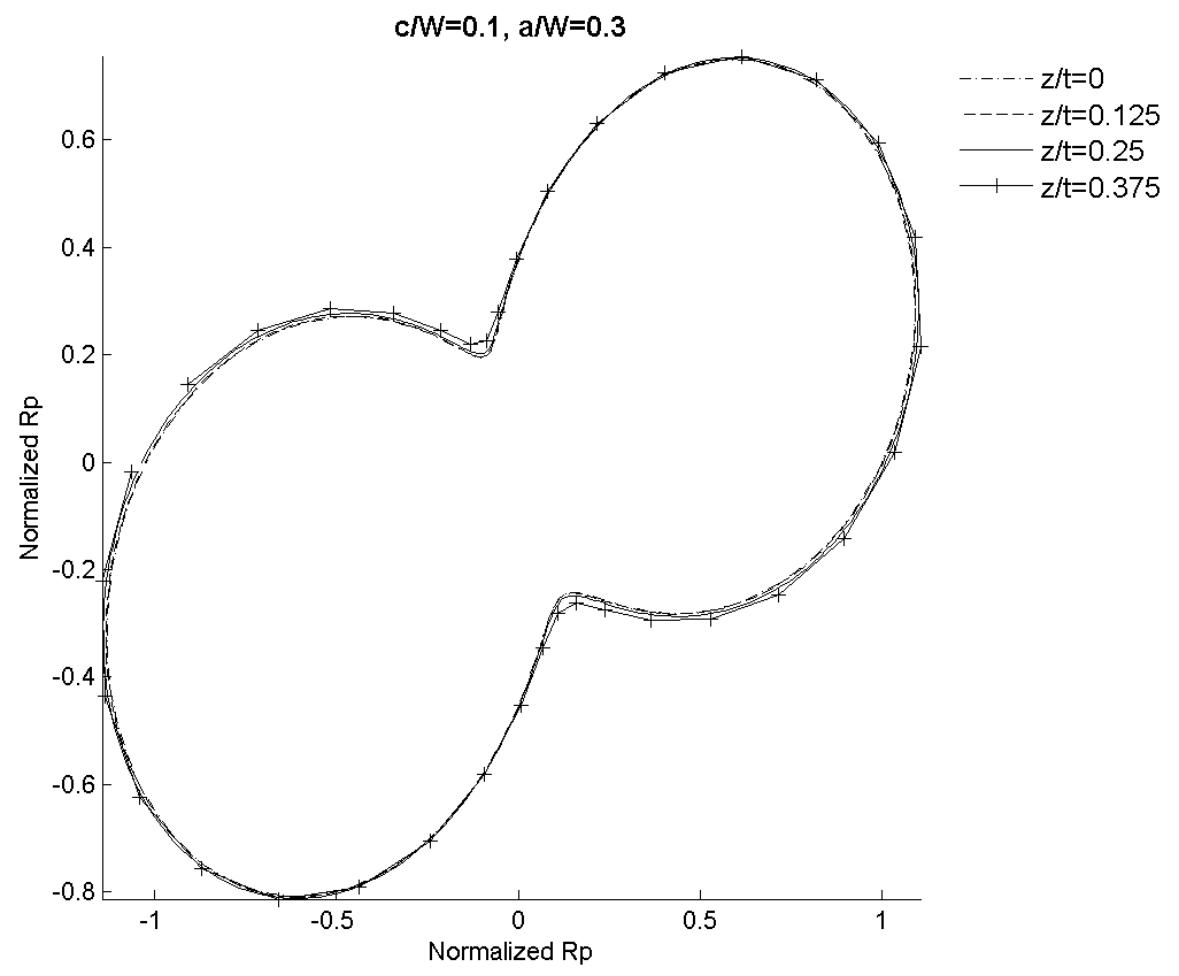

Figure 4.50: Plastic zone map for $t / W=0.5, a / W=0.3$

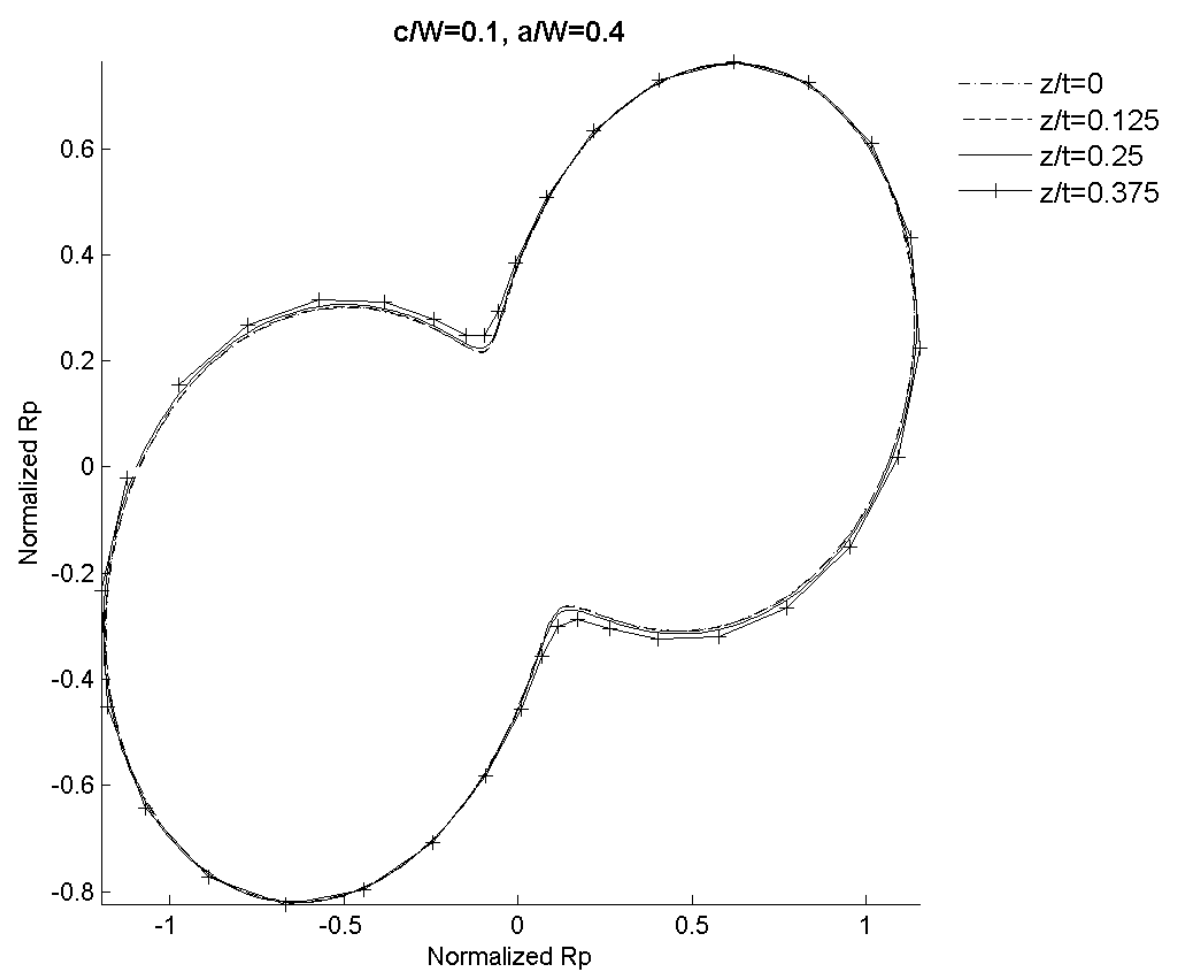

Figure 4.51: Plastic zone map for $t / W=0.5, a / W=0.4$ 


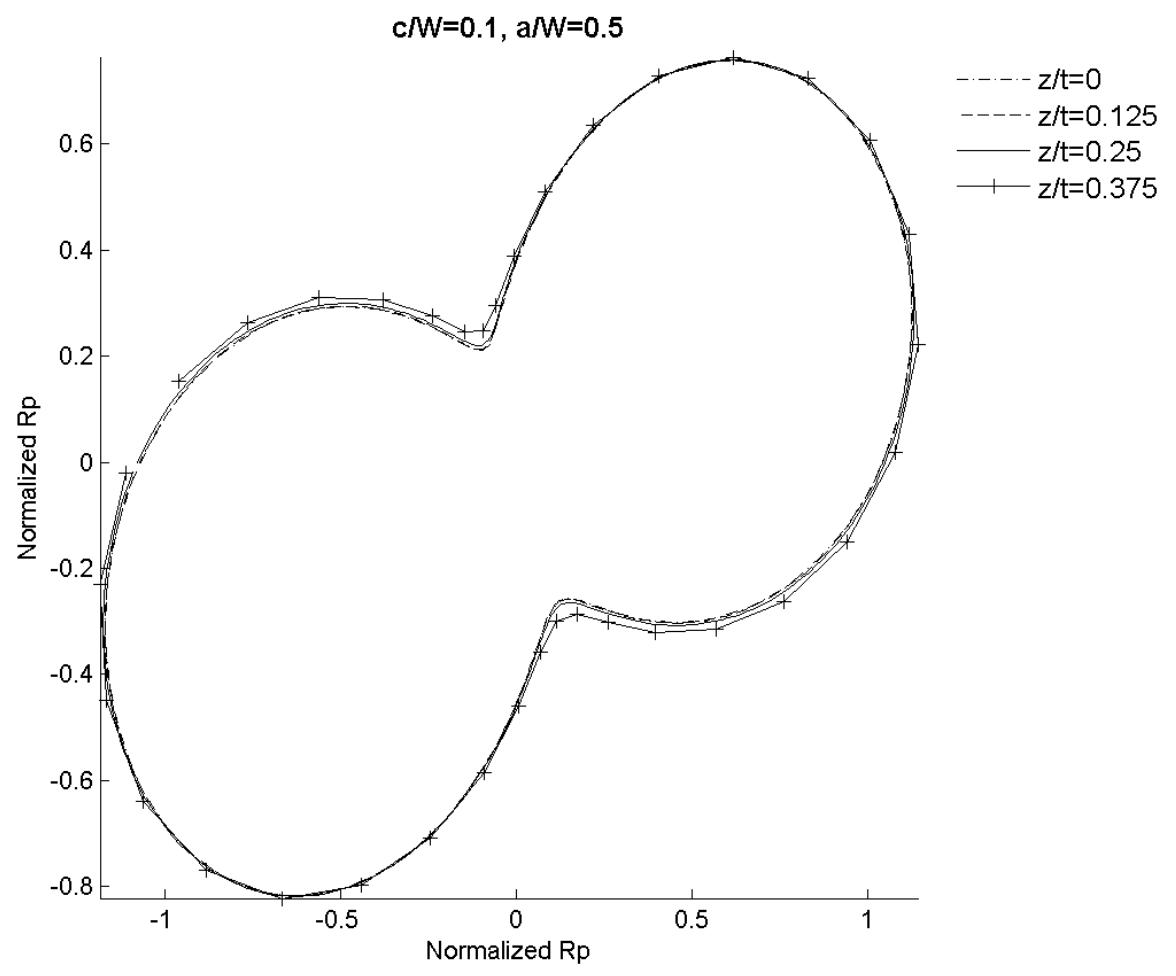

Figure 4.52: Plastic zone map for $t / W=0.5, a / W=0.5$

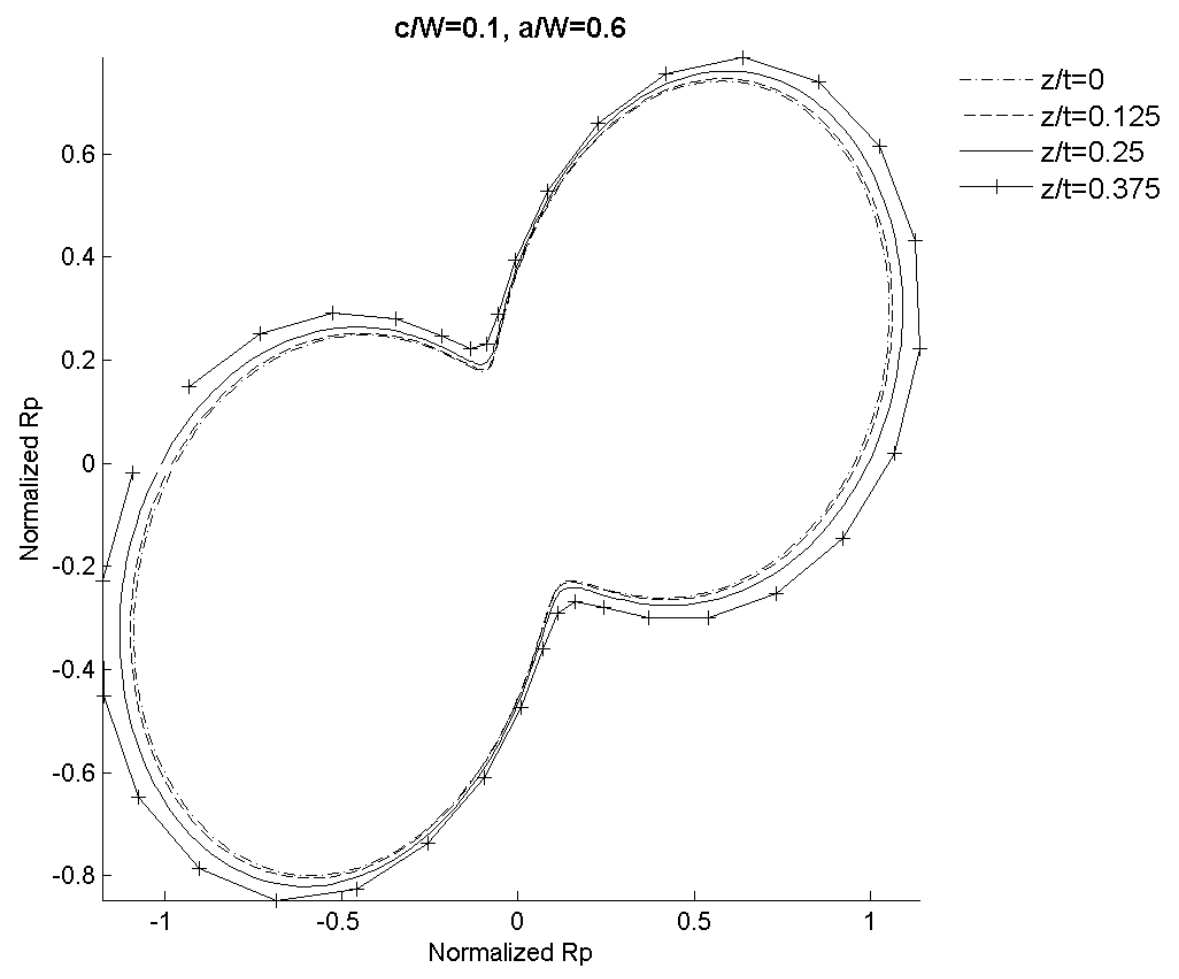

Figure 4.53: Plastic zone map for $\mathrm{t} / \mathrm{W}=\mathbf{0 . 5}, \mathrm{a} / \mathrm{W}=\mathbf{0 . 6}$ 


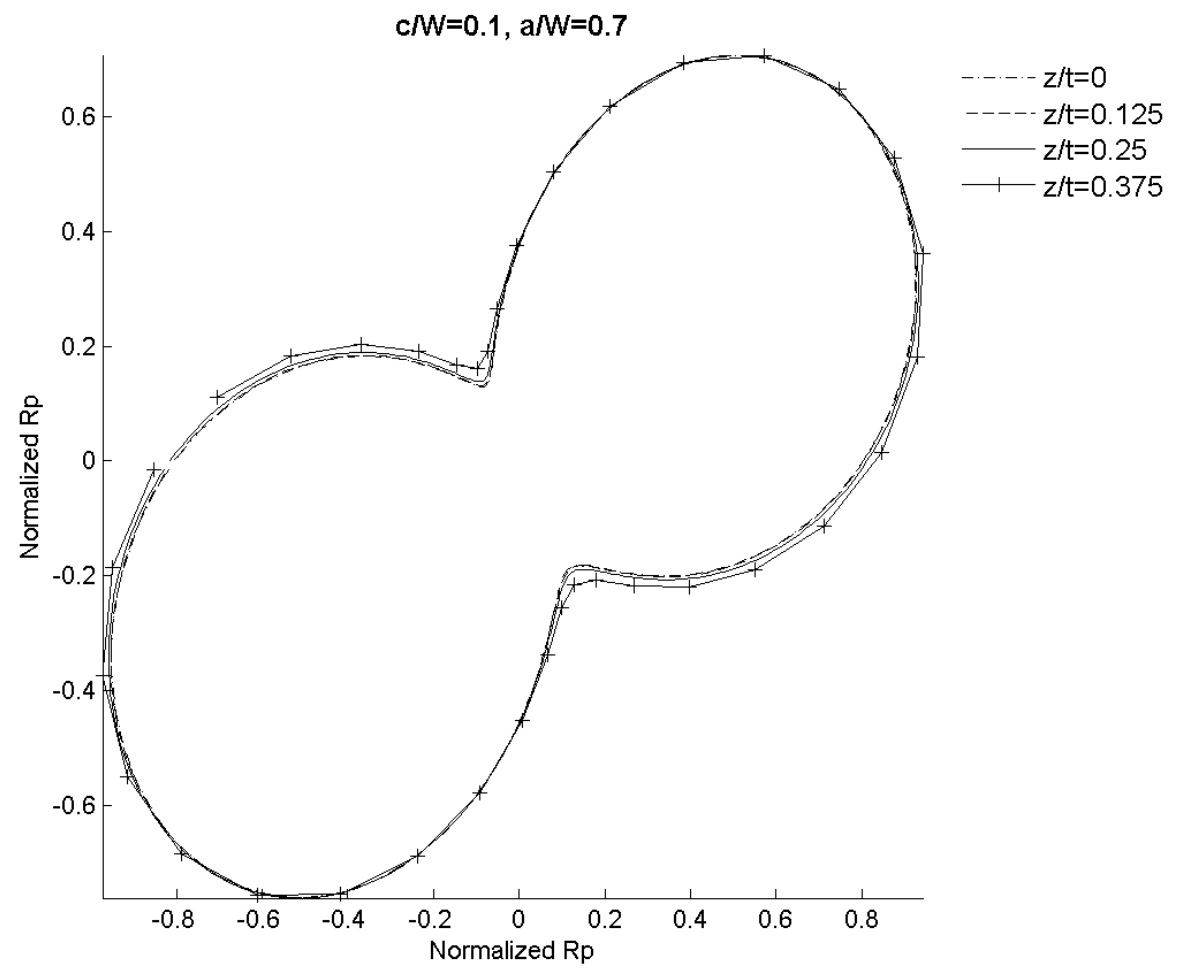

Figure 4.54: Plastic zone map for $\mathrm{t} / \mathrm{W}=\mathbf{0 . 5}, \mathrm{a} / \mathrm{W}=\mathbf{0 . 7}$ 


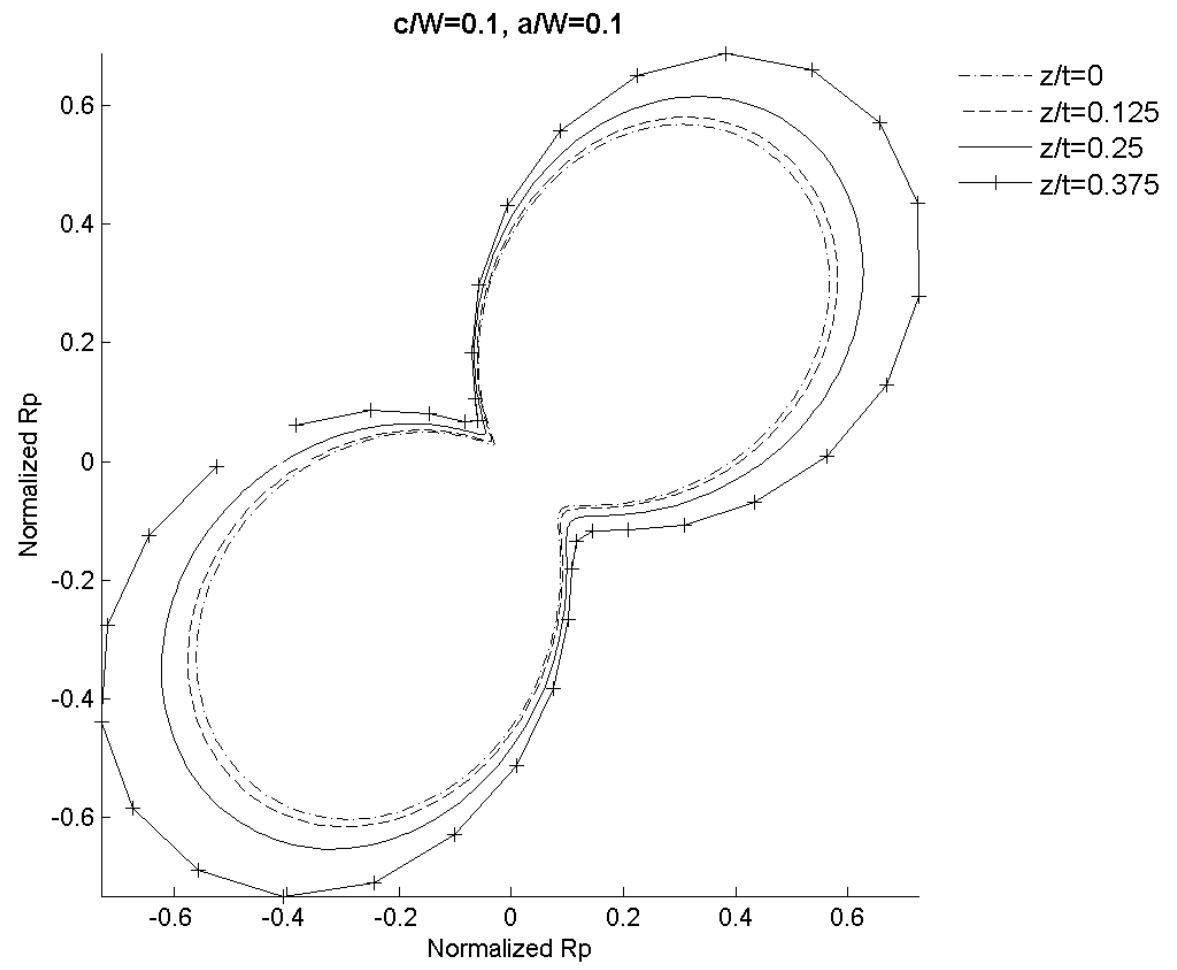

Figure 4.55: Plastic zone map for $t / W=1.0, a / W=0.1$

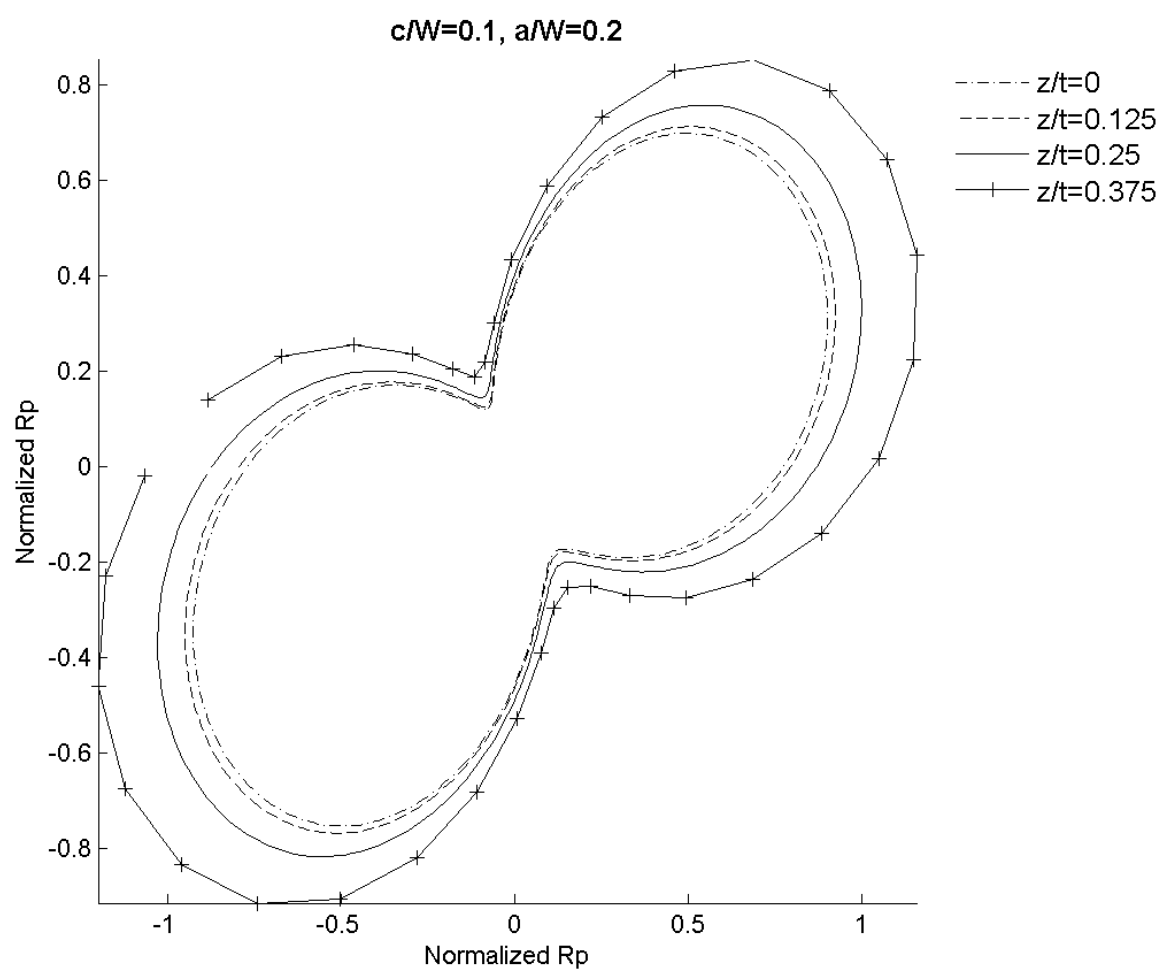

Figure 4.56: Plastic zone map for $t / W=1.0, a / W=0.2$ 


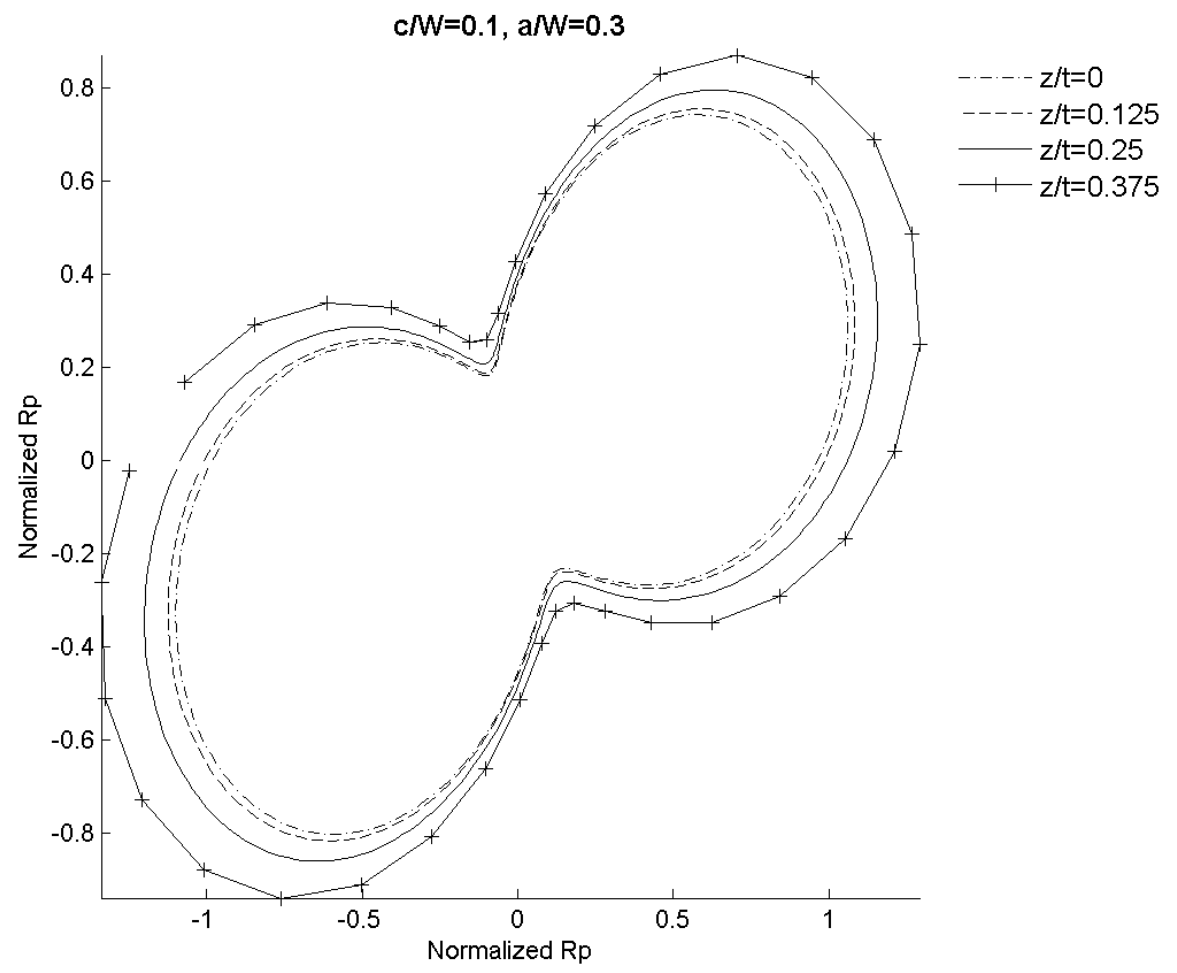

Figure 4.57: Plastic zone map for $t / W=1.0, a / W=0.3$

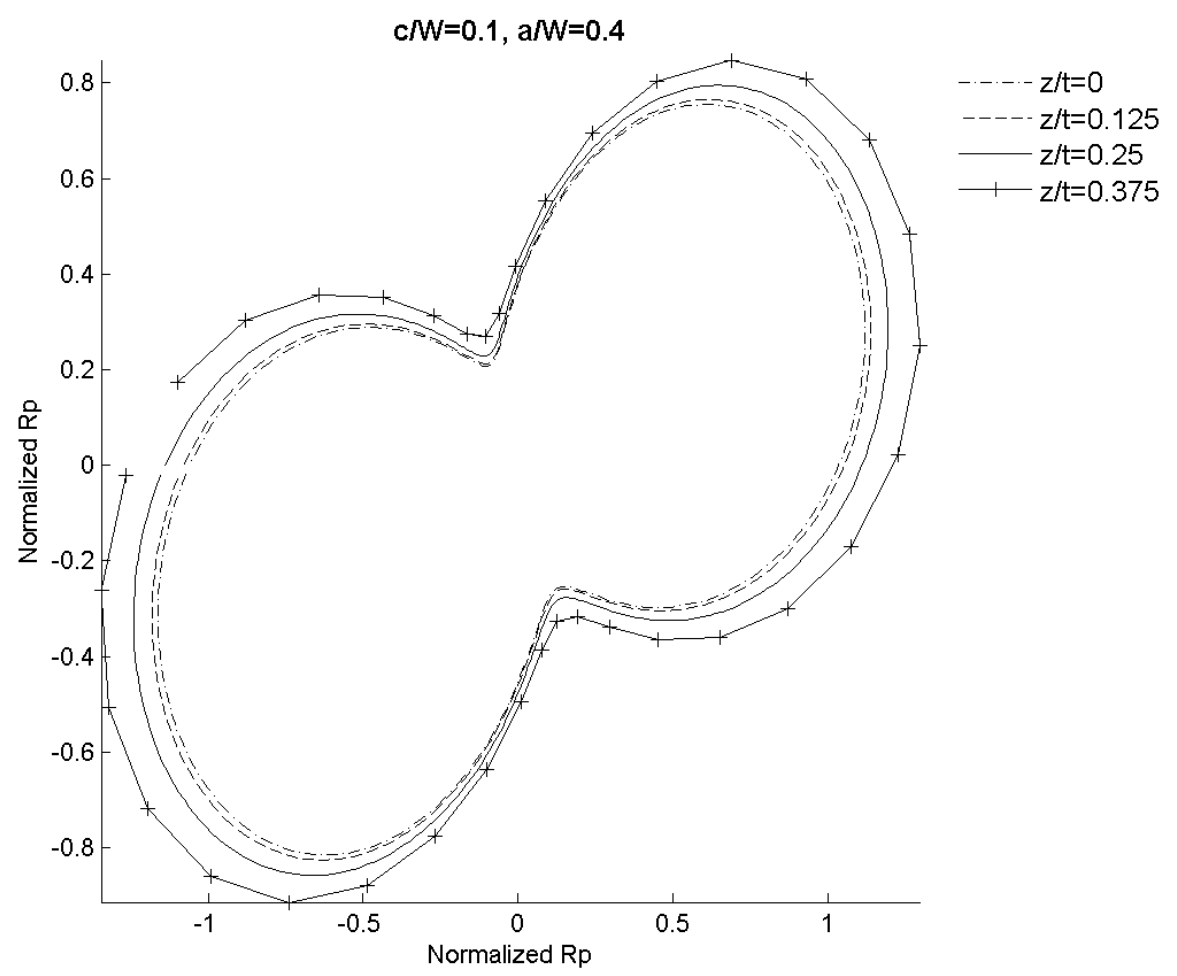

Figure 4.58: Plastic zone map for $\mathrm{t} / \mathrm{W}=1.0, \mathrm{a} / \mathrm{W}=\mathbf{0 . 4}$ 


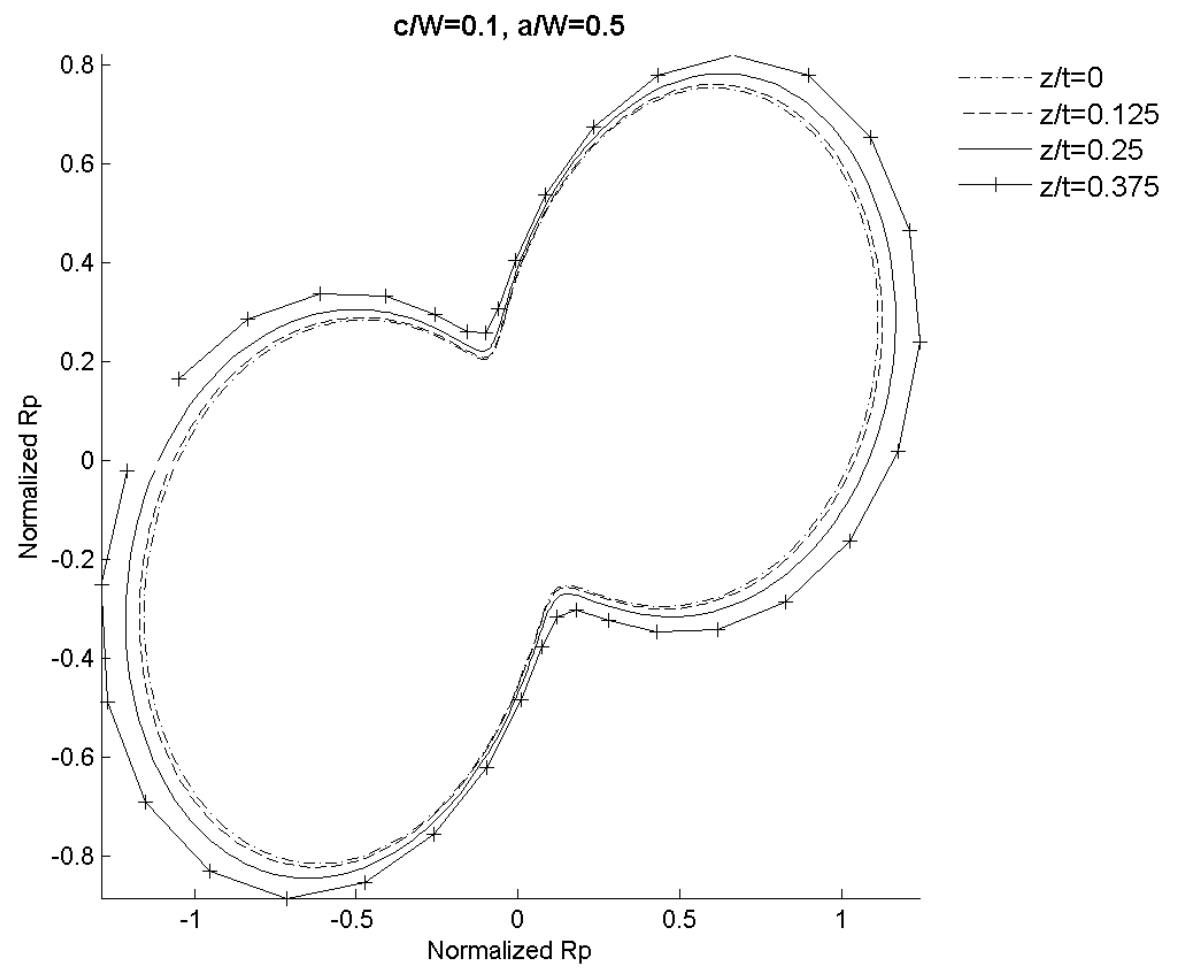

Figure 4.59: Plastic zone map for $t / W=1.0, a / W=0.5$

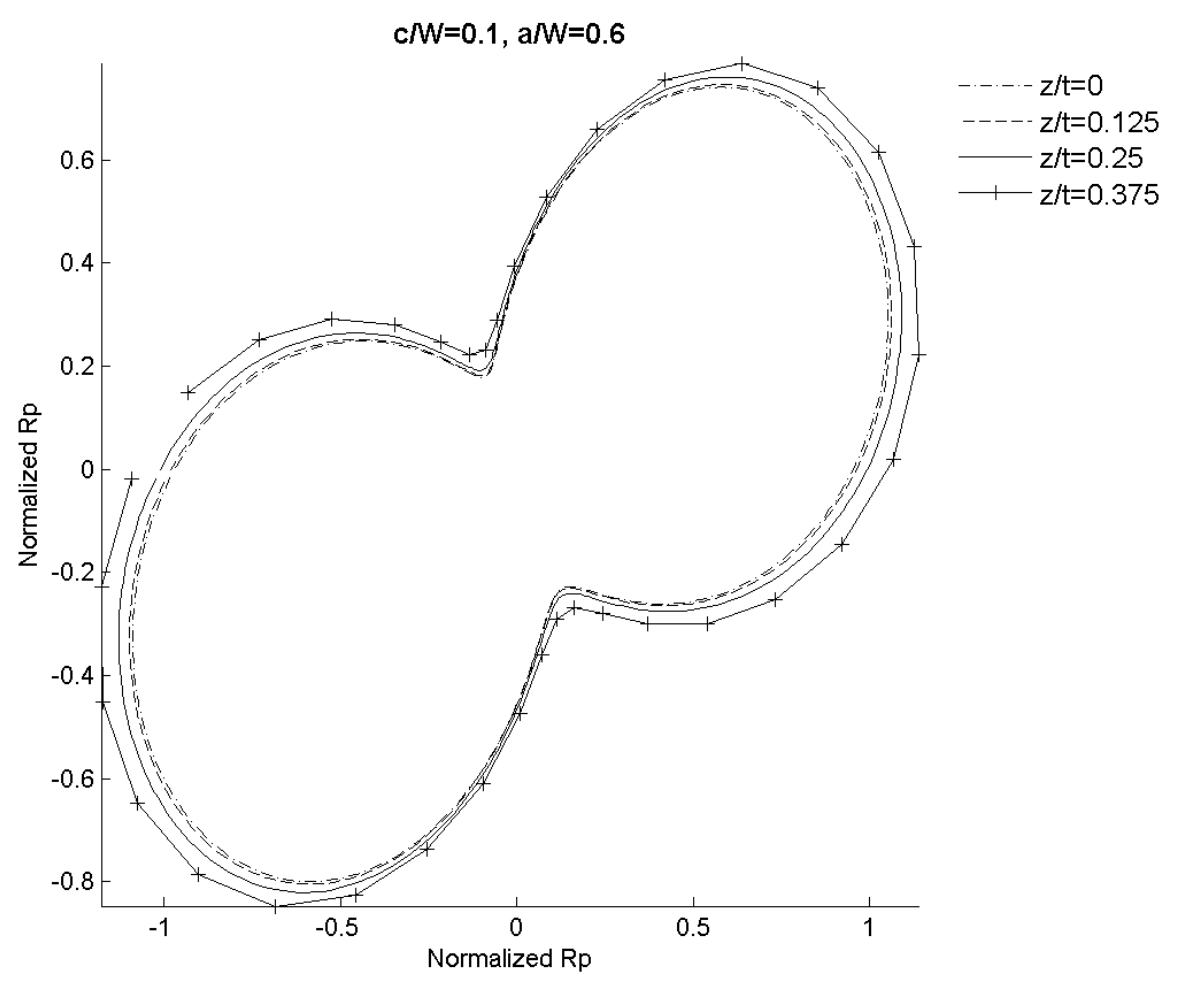

Figure 4.60: Plastic zone map for $t / W=1.0, a / W=0.6$ 


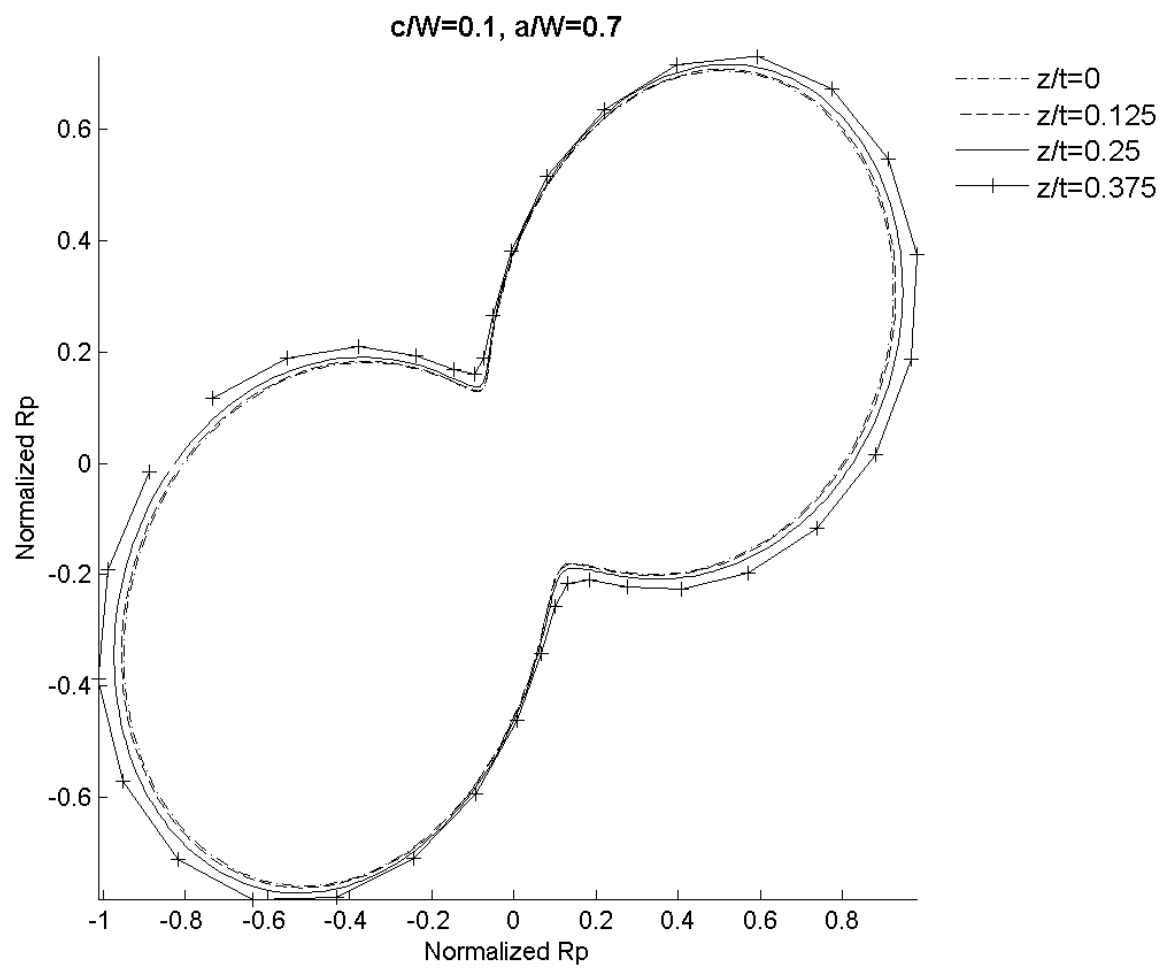

Figure 4.61: Plastic zone map for $t / W=1.0, a / W=0.7$ 


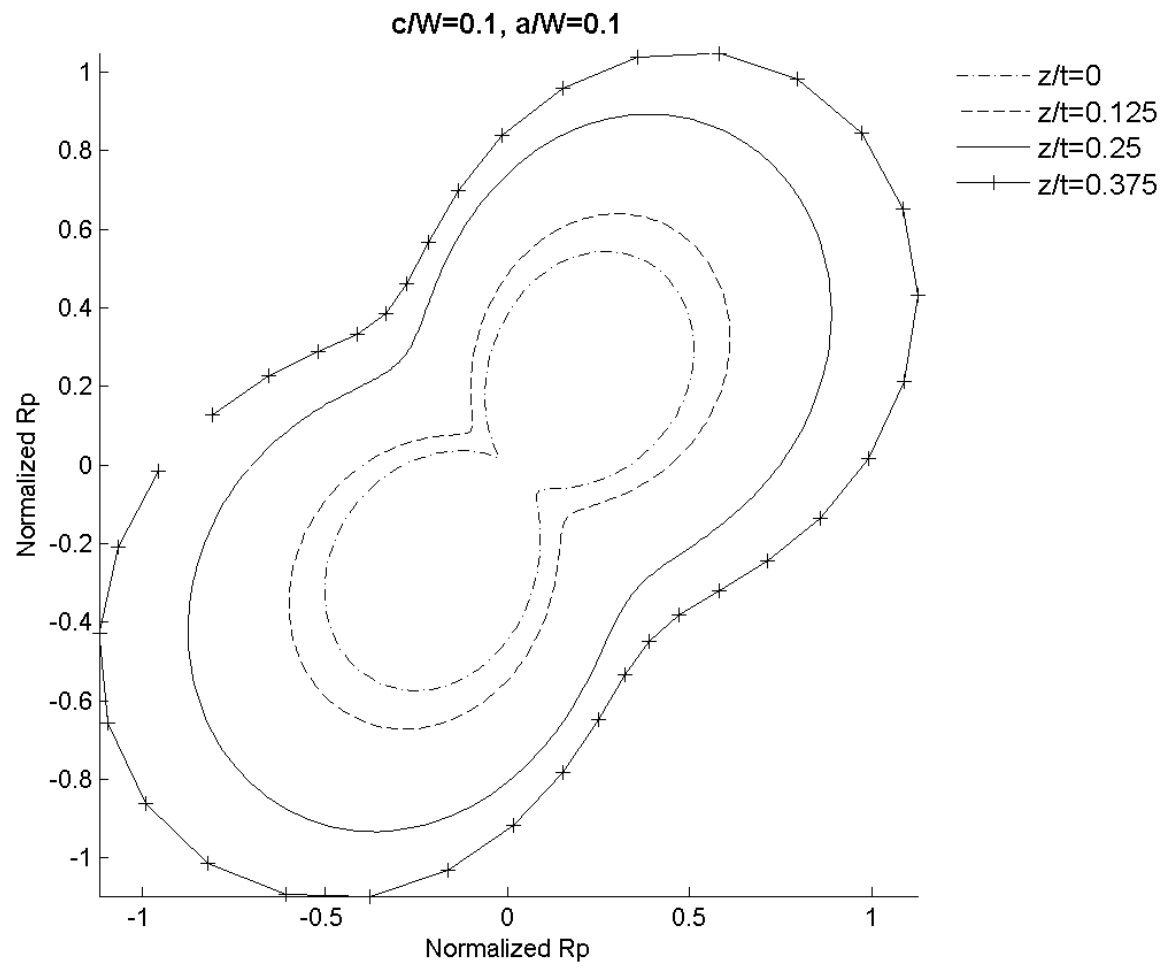

Figure 4.62: Plastic zone map for $\mathrm{t} / \mathrm{W}=\mathbf{2 . 0}, \mathrm{a} / \mathrm{W}=\mathbf{0 . 1}$

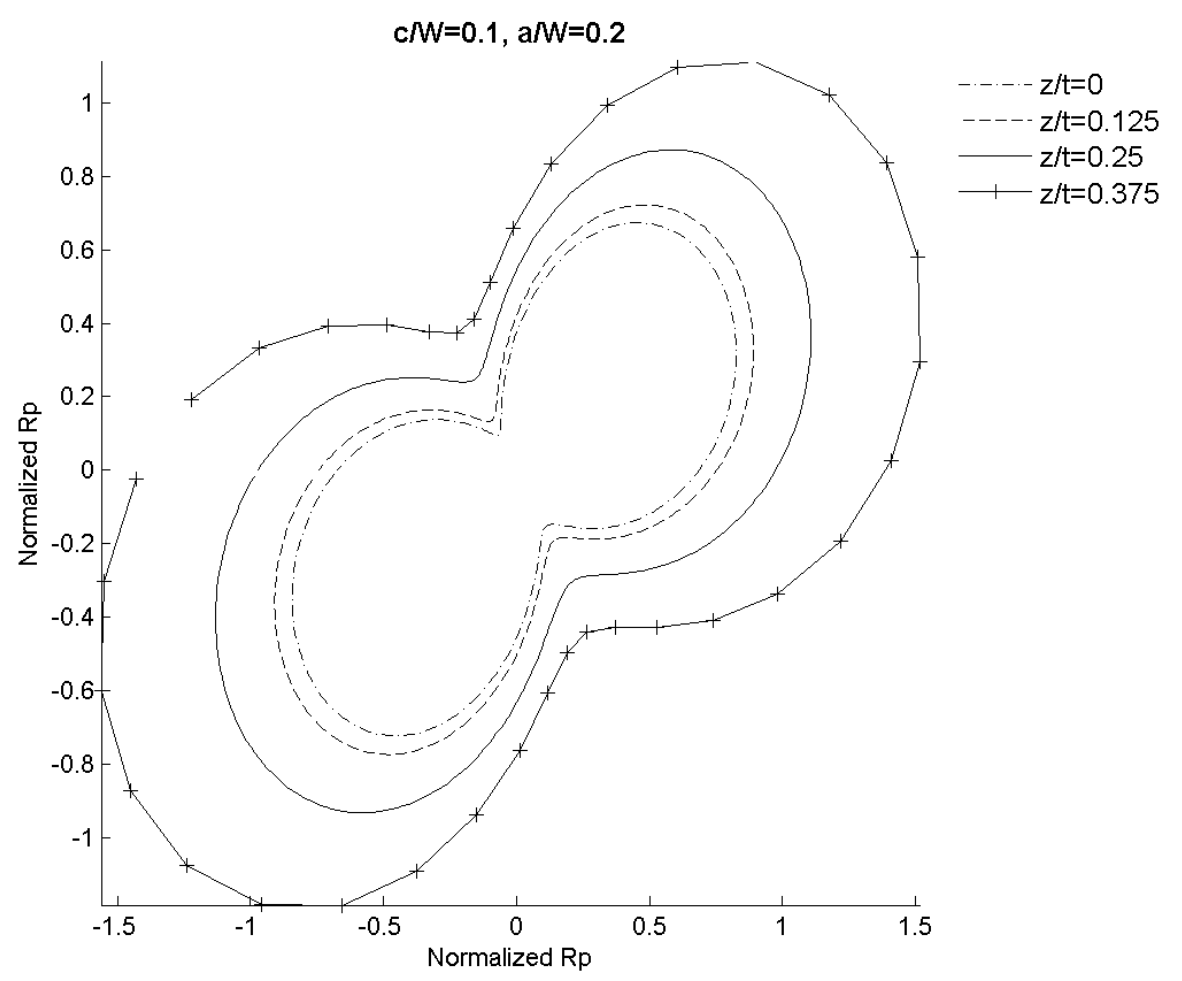

Figure 4.63: Plastic zone map for $t / W=2.0, a / W=0.2$ 


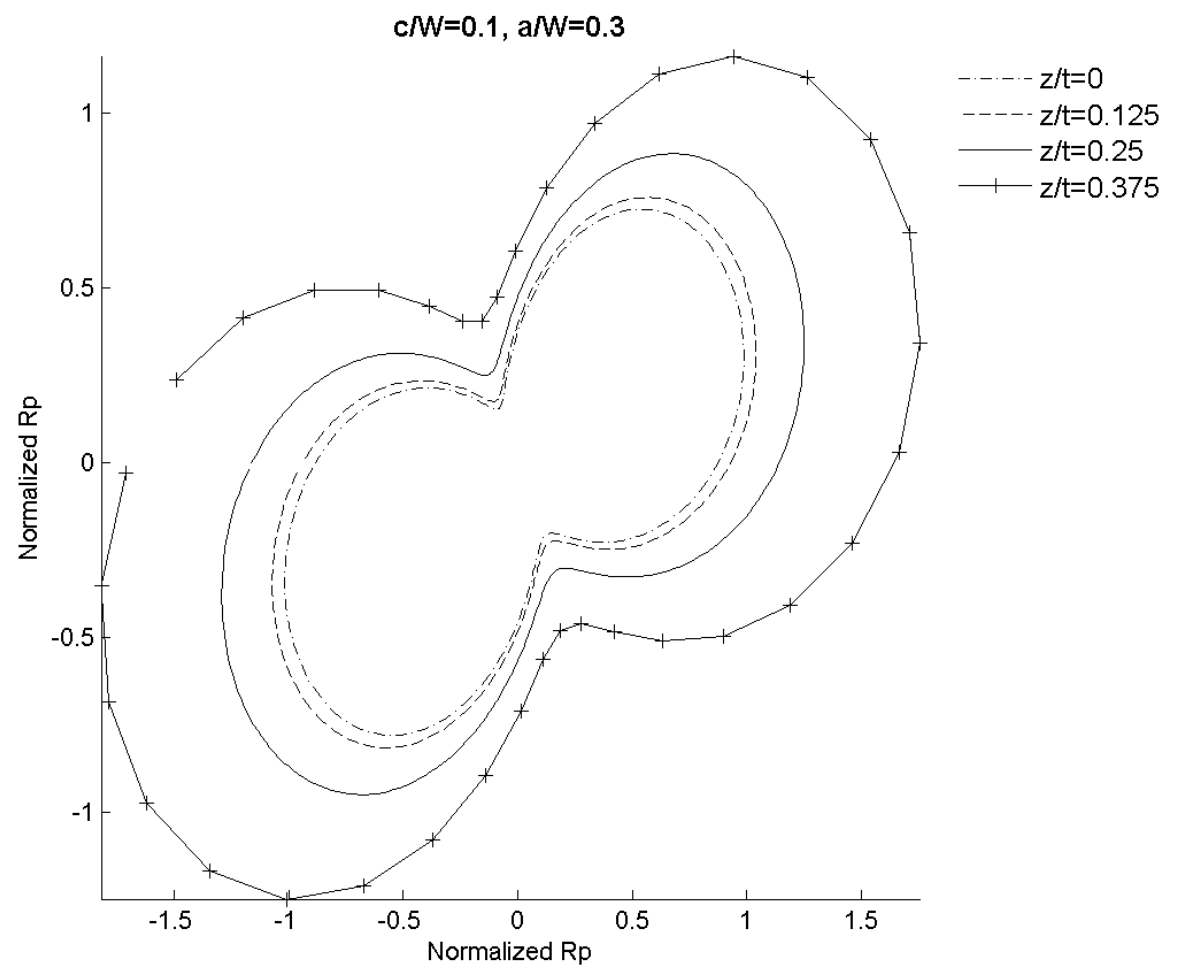

Figure 4.64: Plastic zone map for $t / W=2.0, a / W=0.3$

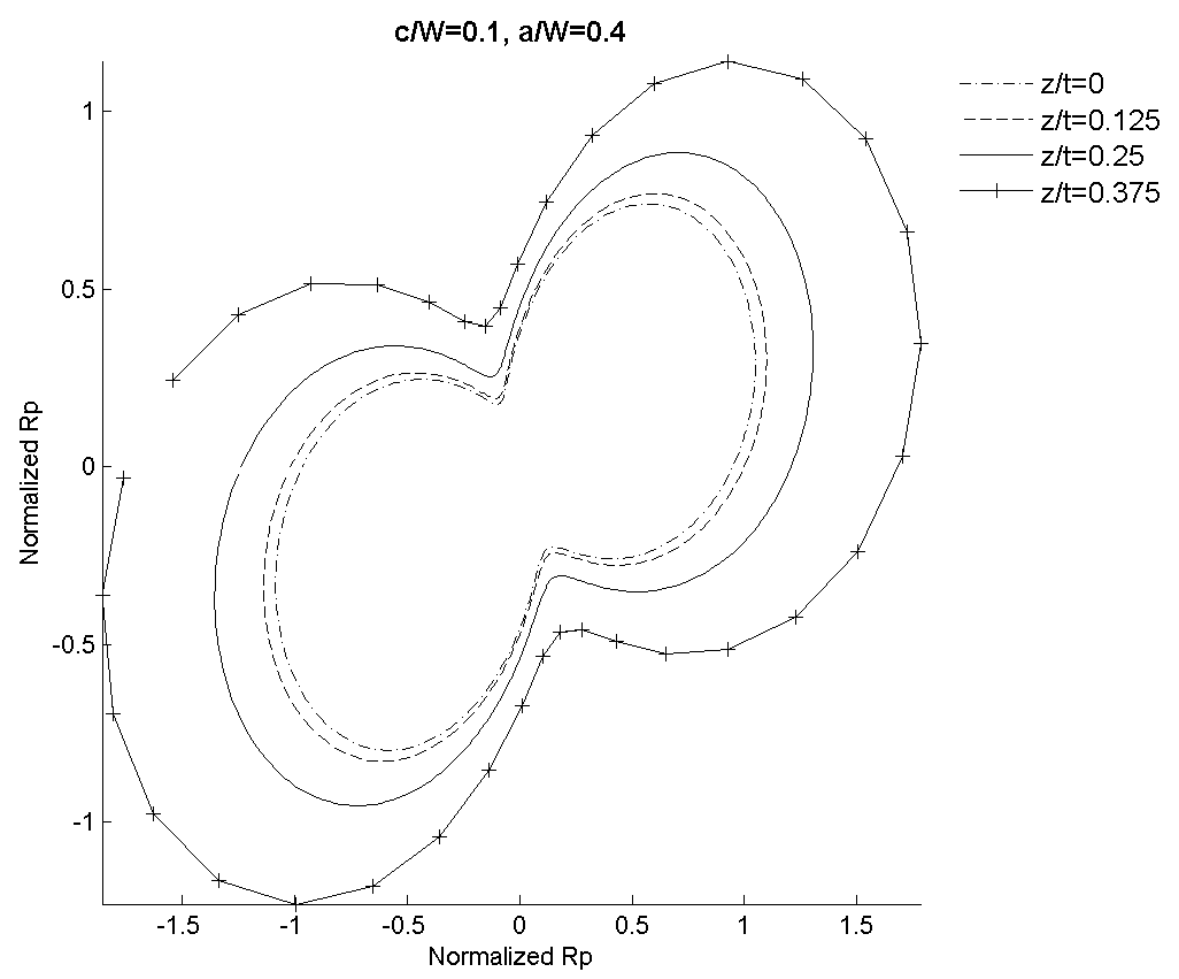

Figure 4.65: Plastic zone map for $t / W=2.0, a / W=0.4$ 


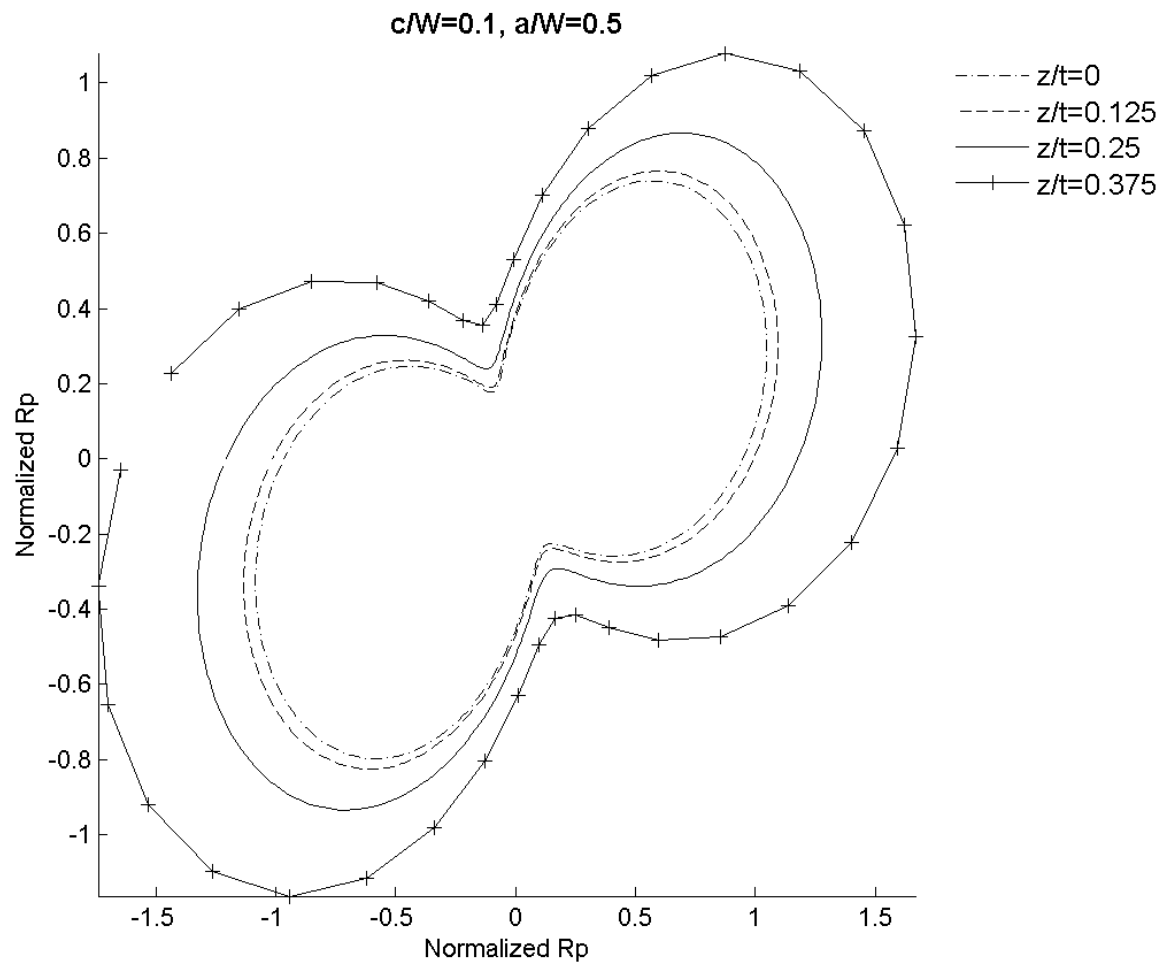

Figure 4.66: Plastic zone map for $t / W=2.0, a / W=0.5$

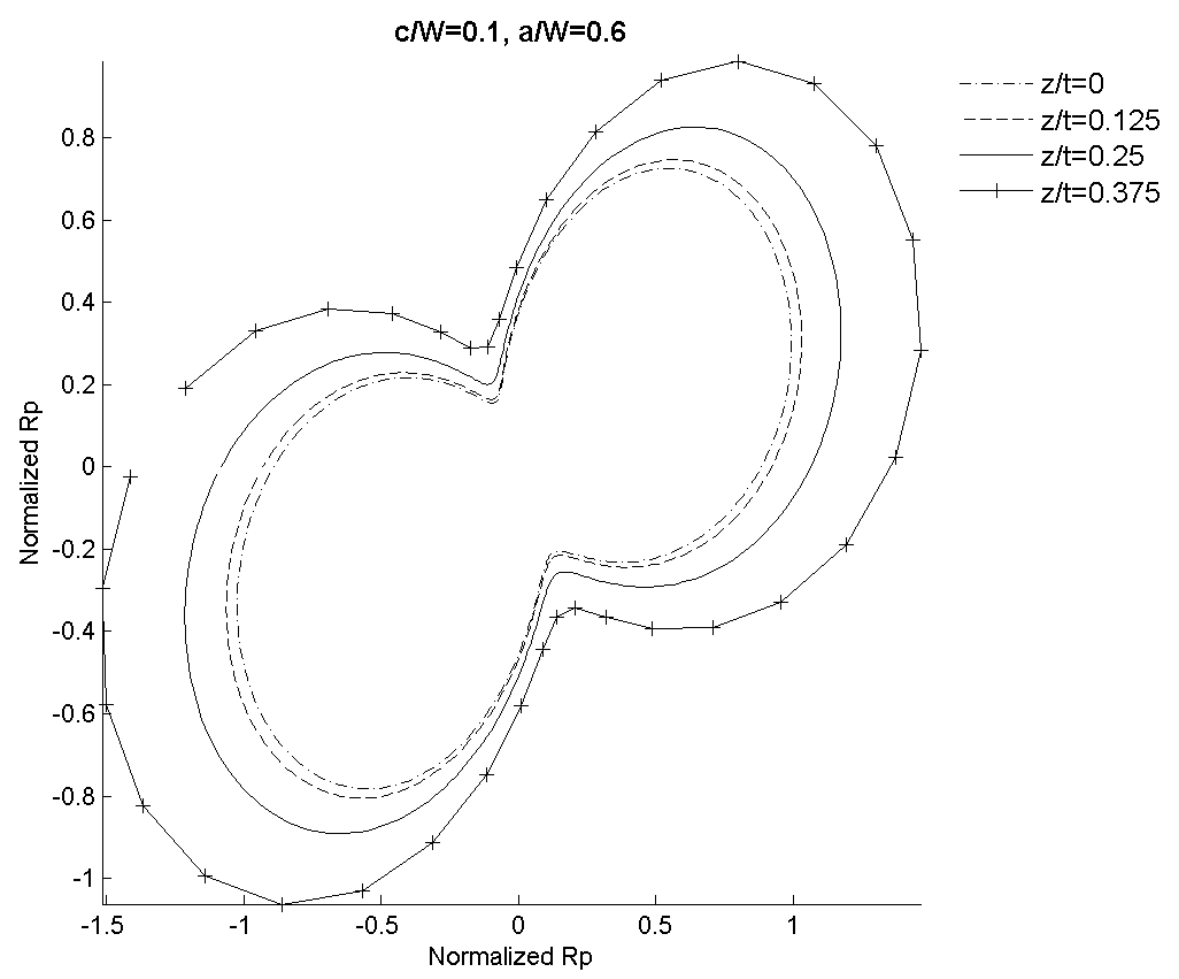

Figure 4.67: Plastic zone map for $t / W=2.0, a / W=0.6$ 


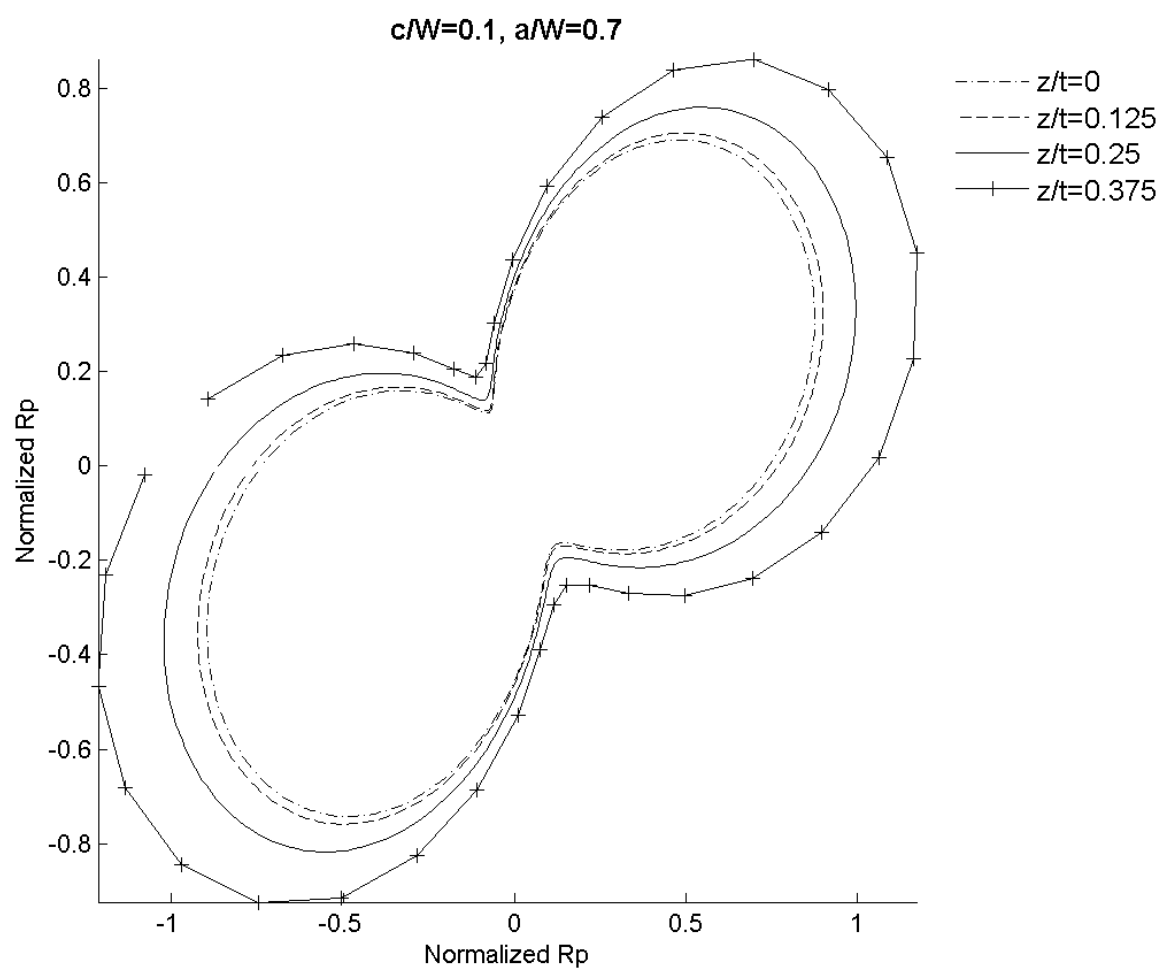

Figure 4.68: Plastic zone map for $\mathrm{t} / \mathrm{W}=\mathbf{2 . 0}, \mathrm{a} / \mathrm{W}=\mathbf{0 . 7}$ 


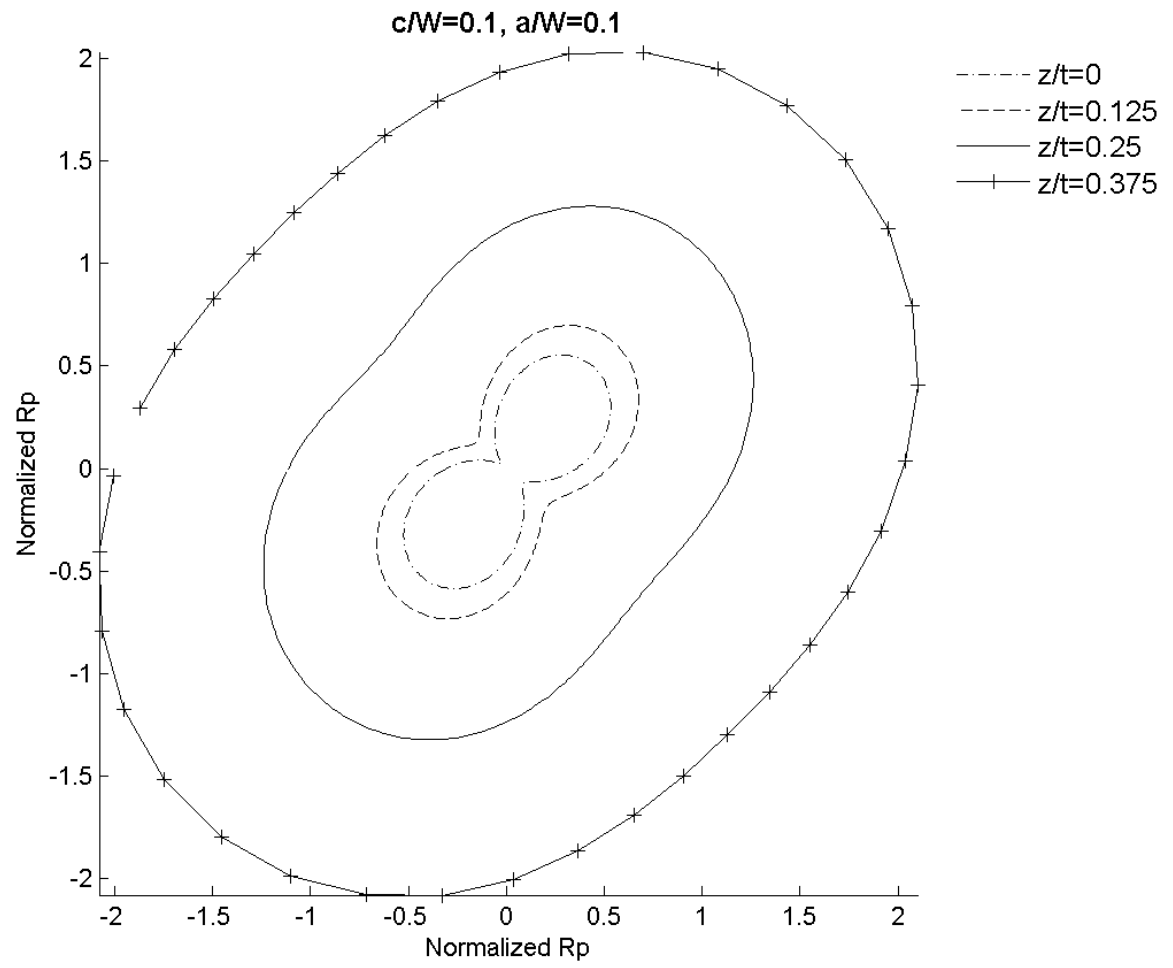

Figure 4.69: Plastic zone map for $t / W=4.0, a / W=0.1$

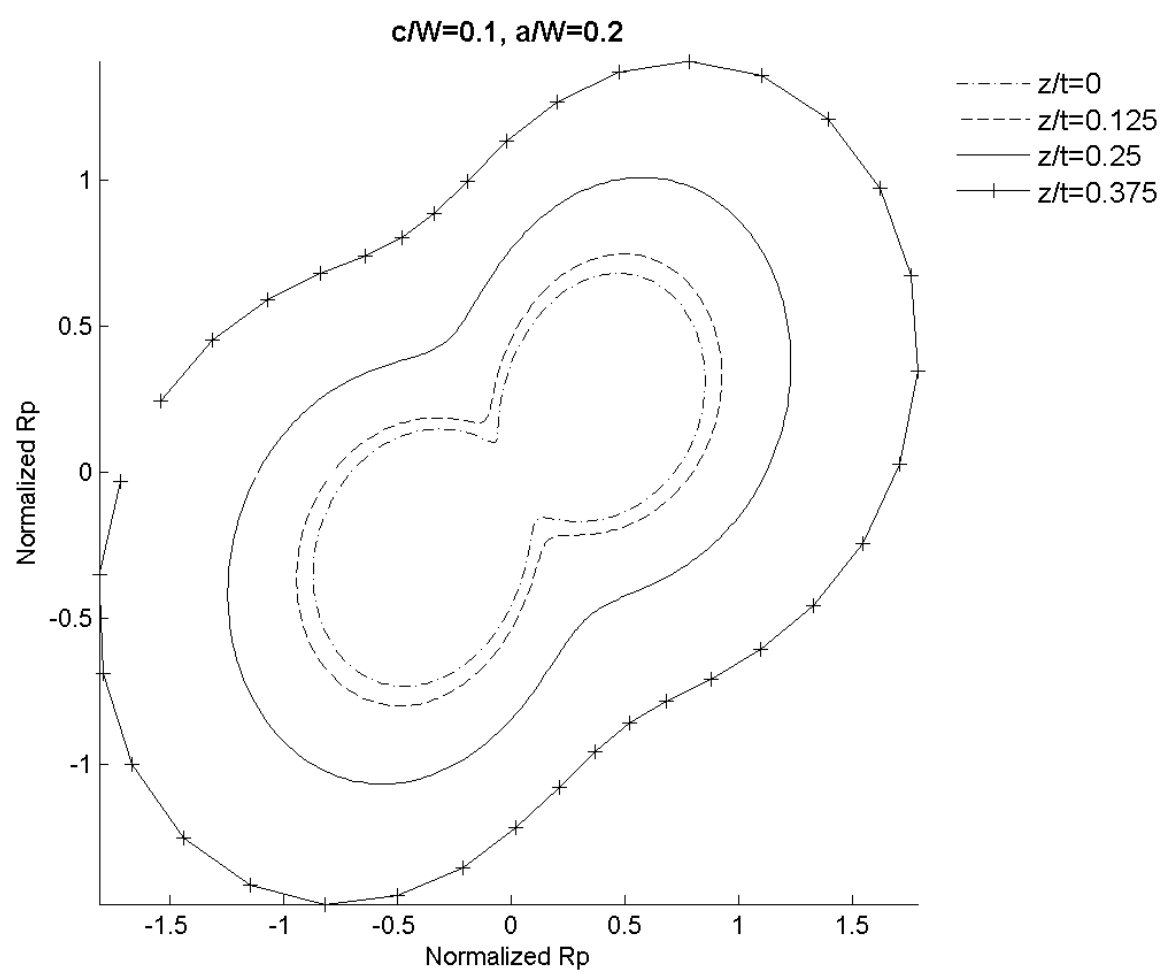

Figure 4.70: $P$ lastic zone map for $\mathrm{t} / \mathrm{W}=4.0, \mathrm{a} / \mathrm{W}=\mathbf{0 . 2}$ 


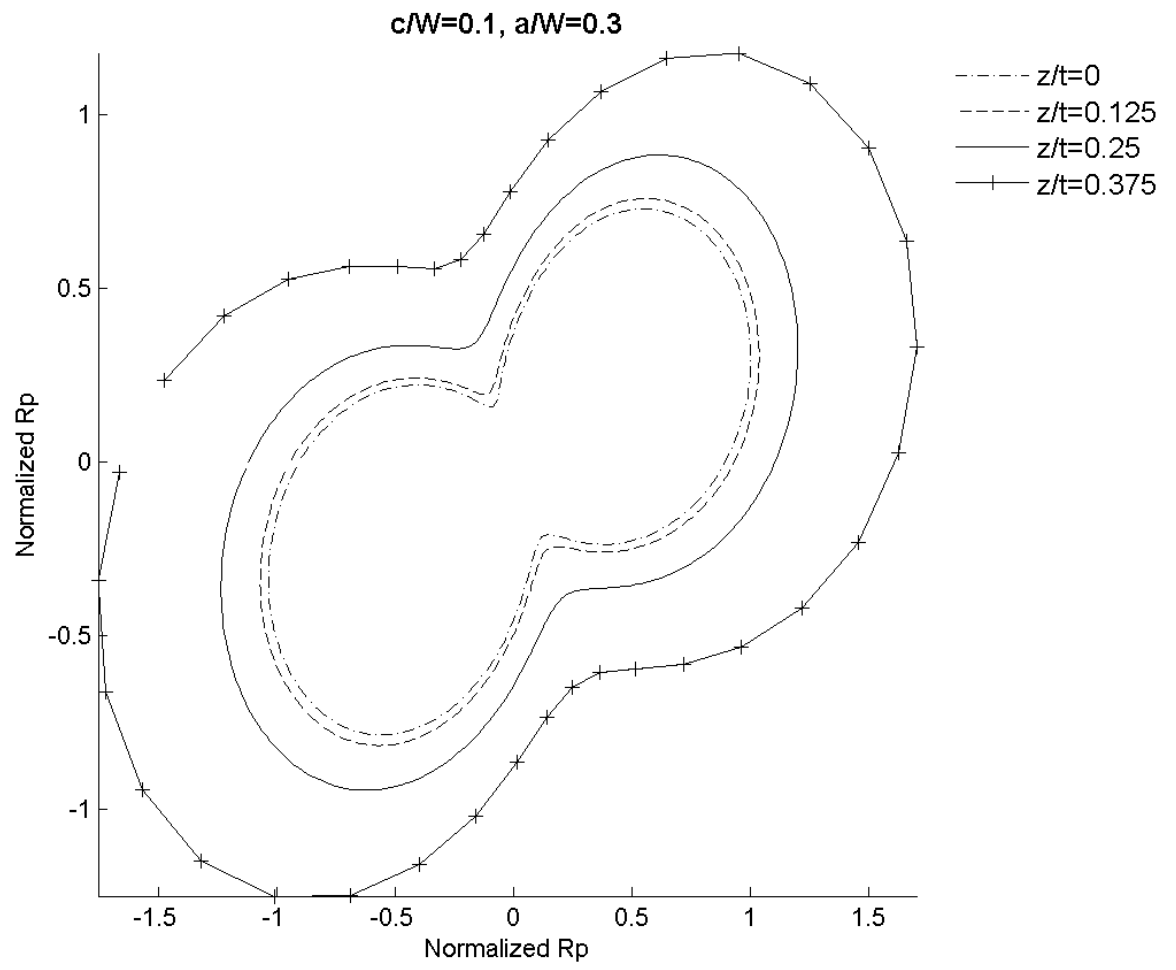

Figure 4.71: Plastic zone map for $t / W=4.0, a / W=0.3$

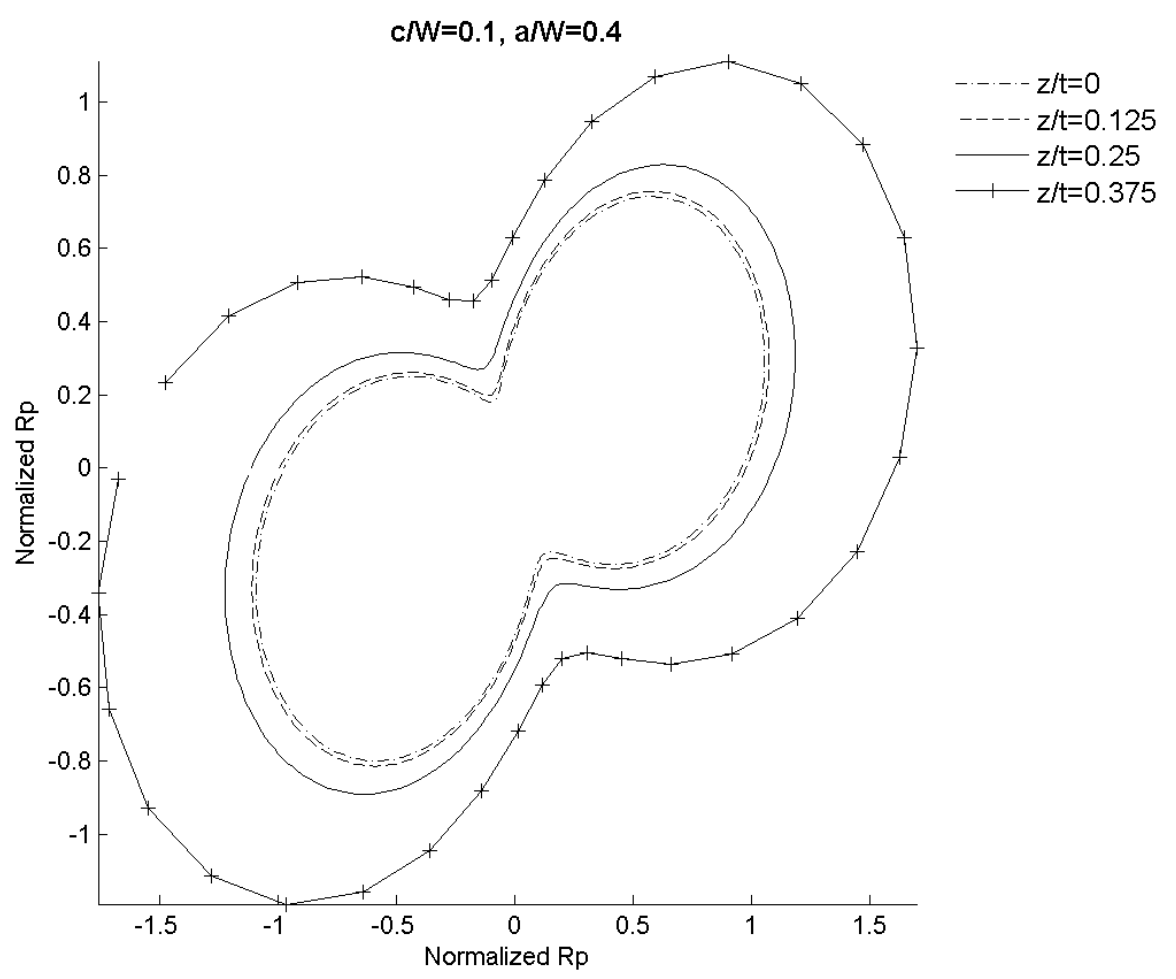

Figure 4.72: Plastic zone map for $t / W=4.0, a / W=0.4$ 


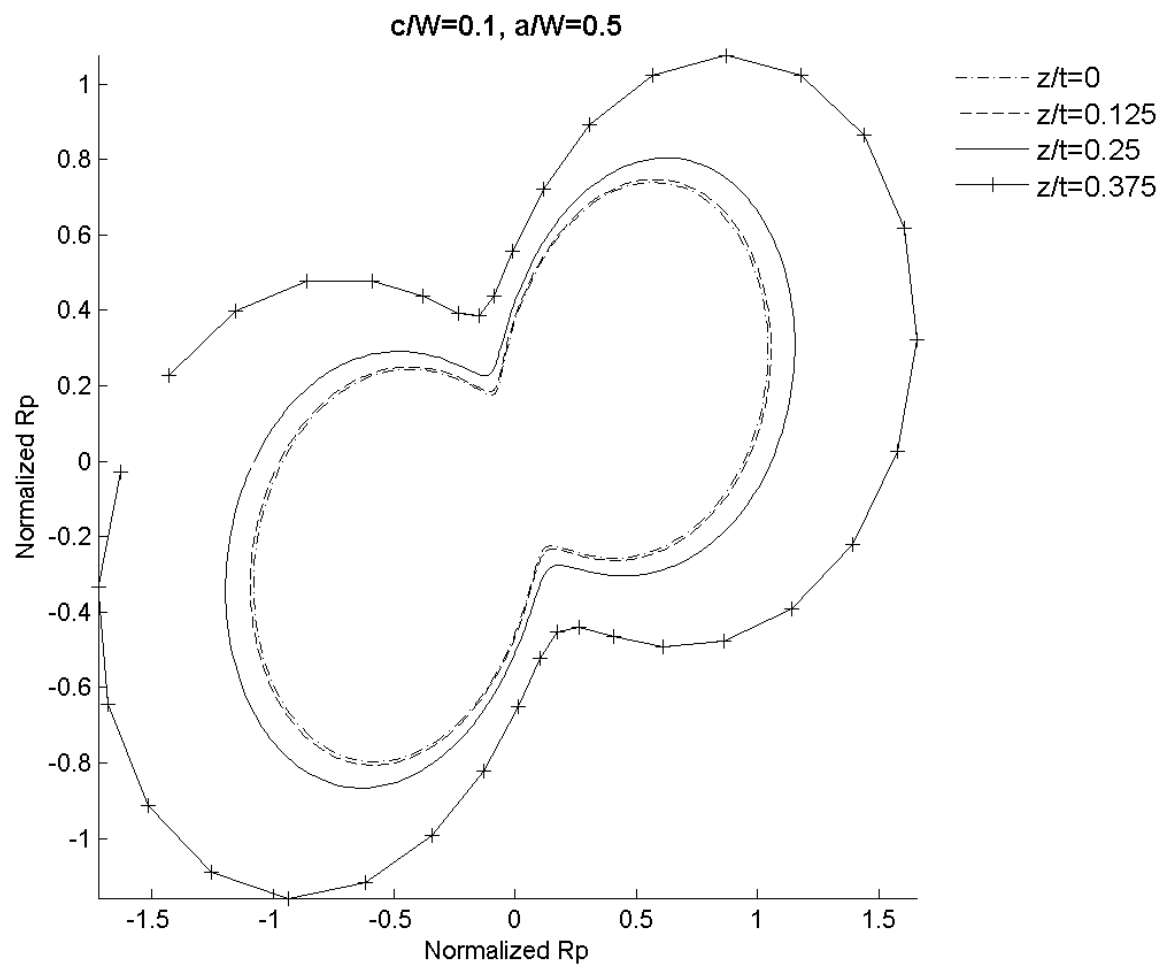

Figure 4.73: Plastic zone map for $t / W=4.0, a / W=0.5$

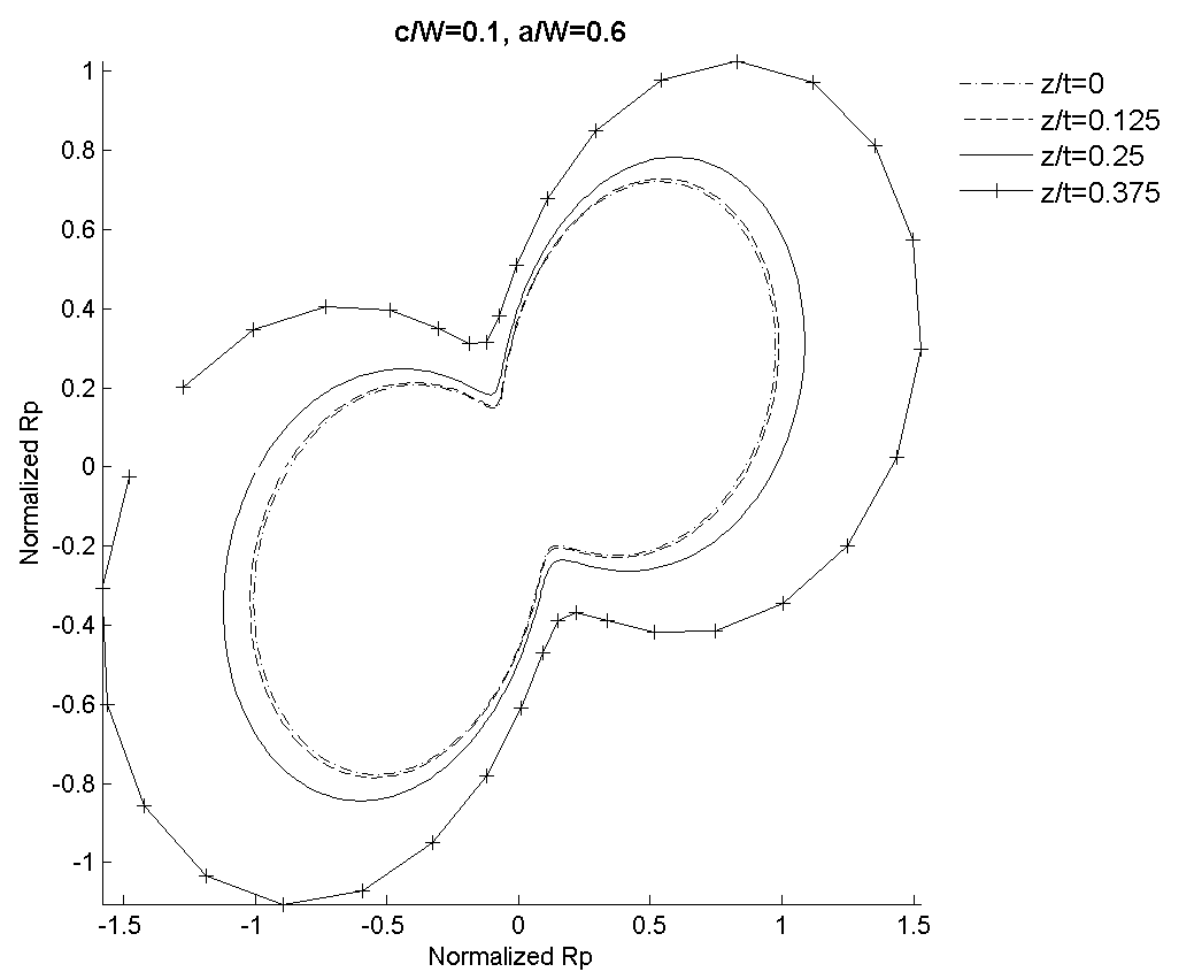

Figure 4.74: Plastic zone map for $t / W=4.0, a / W=0.6$ 


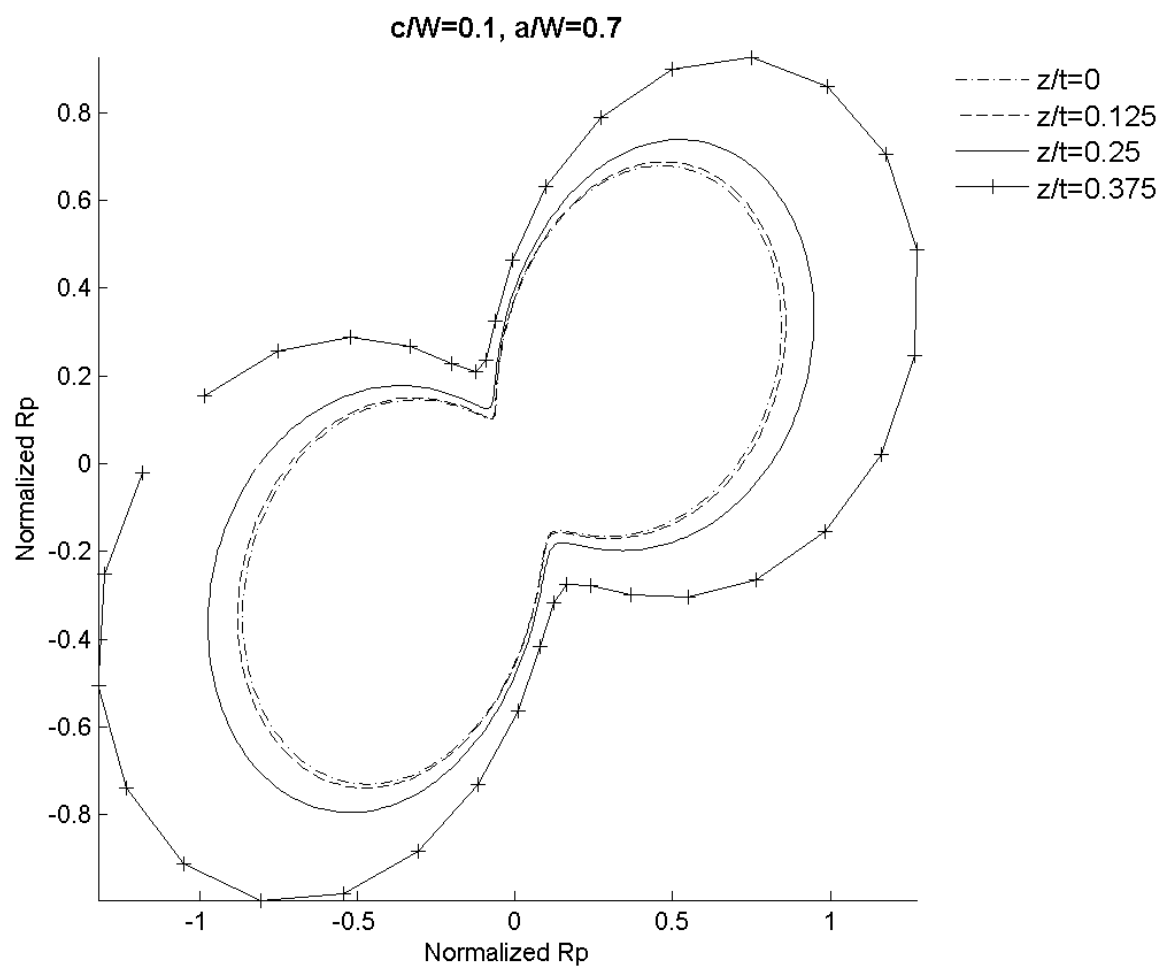

Figure 4.75: Plastic zone map for $t / W=4.0, a / W=0.7$ 


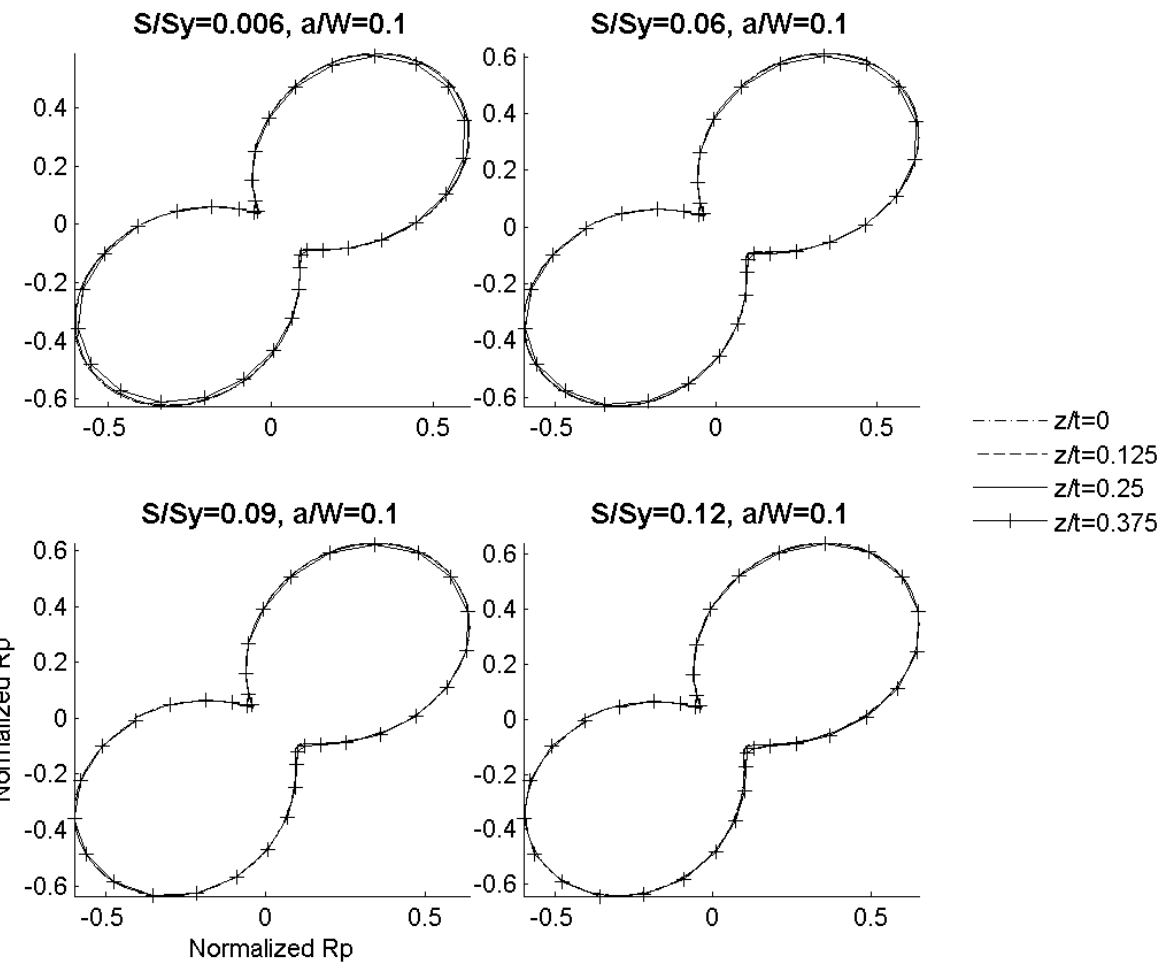

Figure 4.76: Plastic zone map including $T$-stress for $t / W=0.1, a / W=0.1$
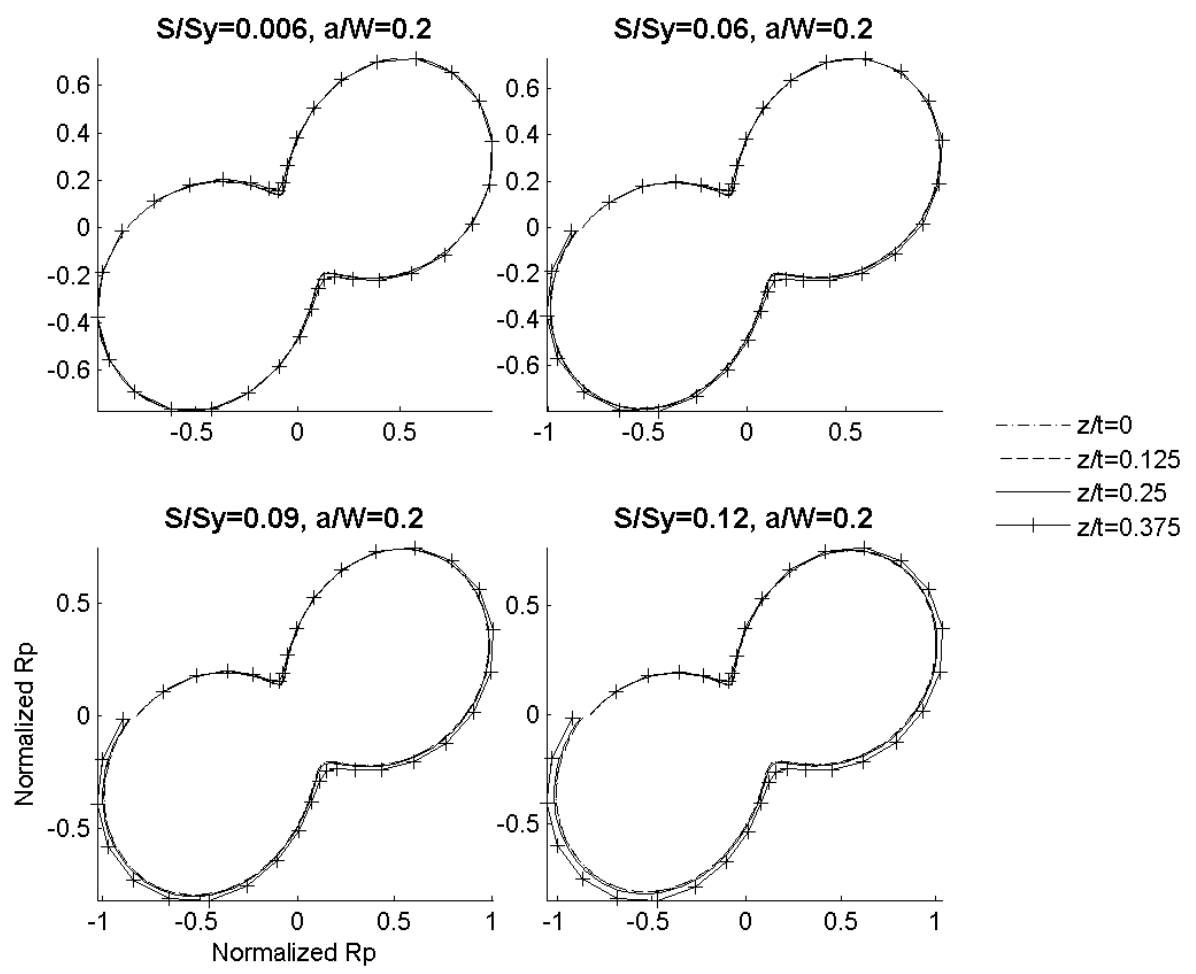

Figure 4.77: Plastic zone map including $T$-stress for $t / W=0.1, a / W=0.2$ 

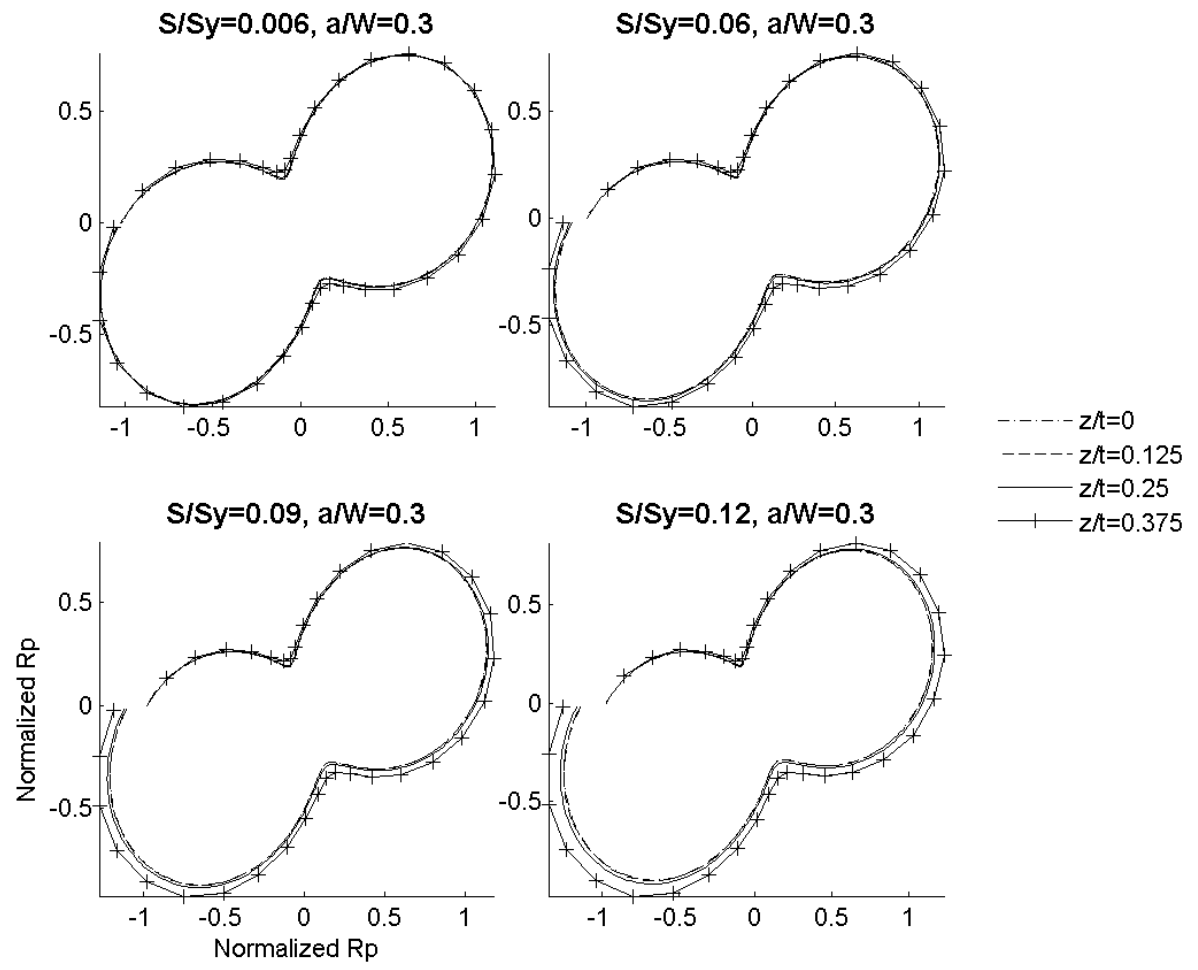

Figure 4.78: Plastic zone map including $T$-stress for $t / W=0.1, a / W=0.3$
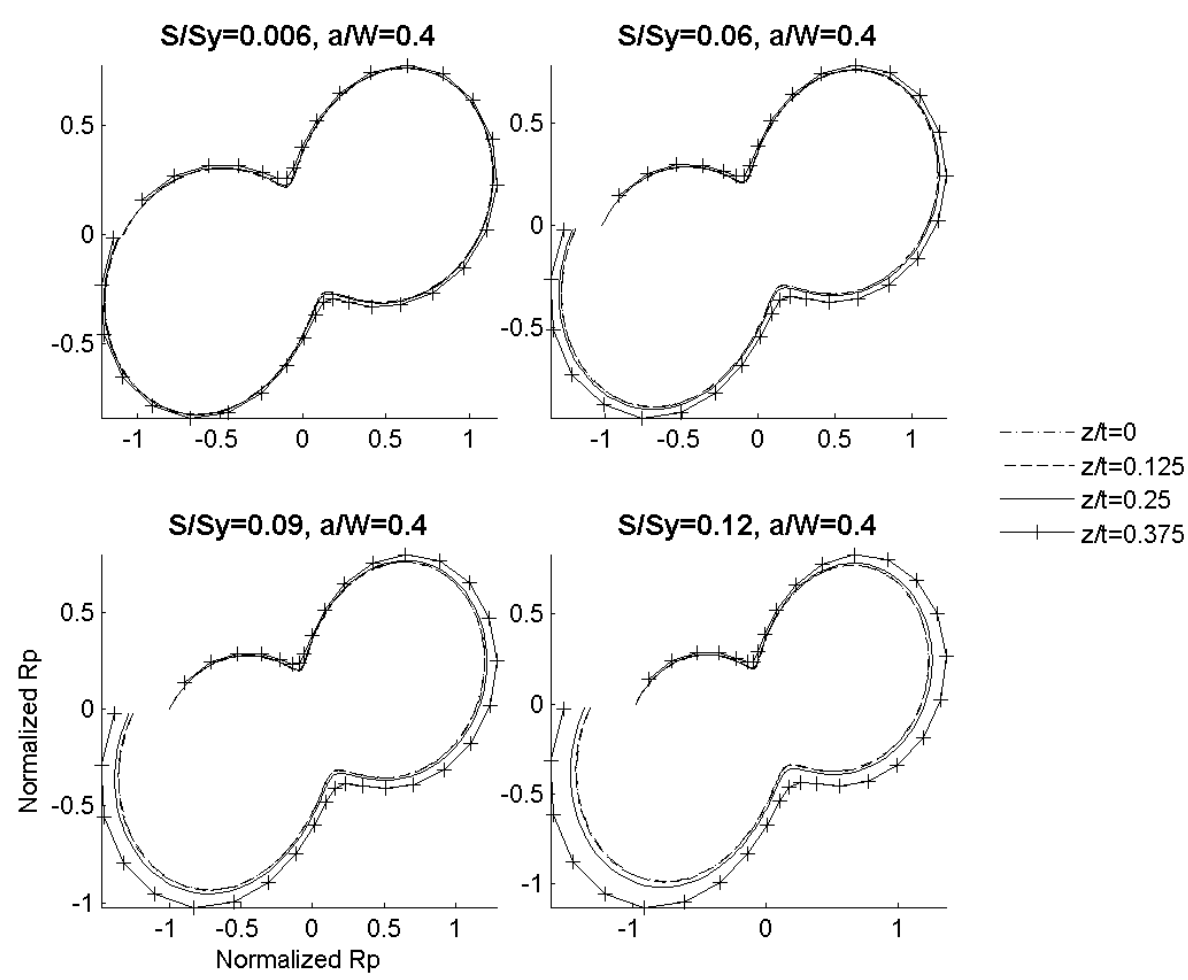

Figure 4.79: Plastic zone map including $T$-stress for $t / W=0.1, a / W=0.4$ 

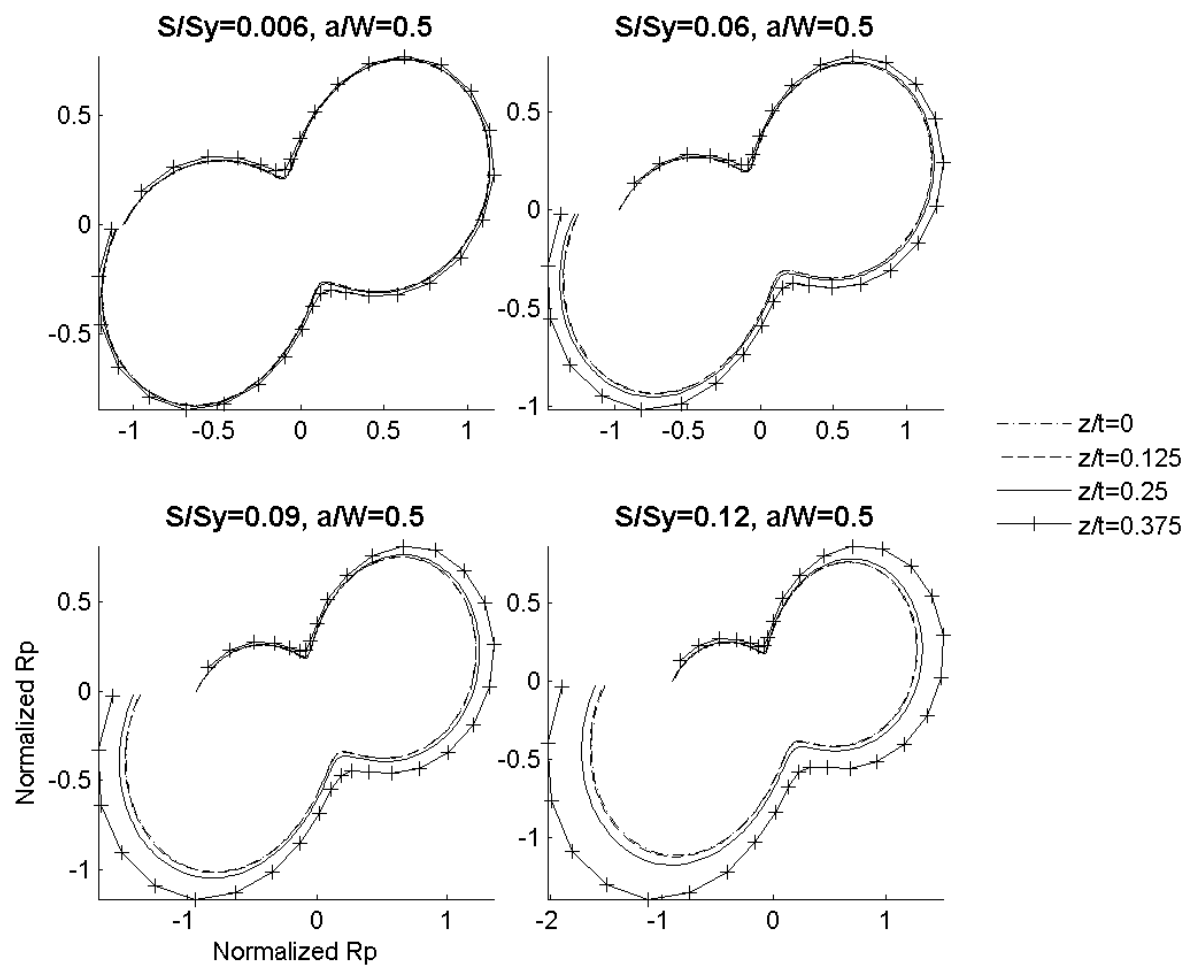

Figure 4.80: Plastic zone map including $T$-stress for $t / W=0.1, a / W=0.5$
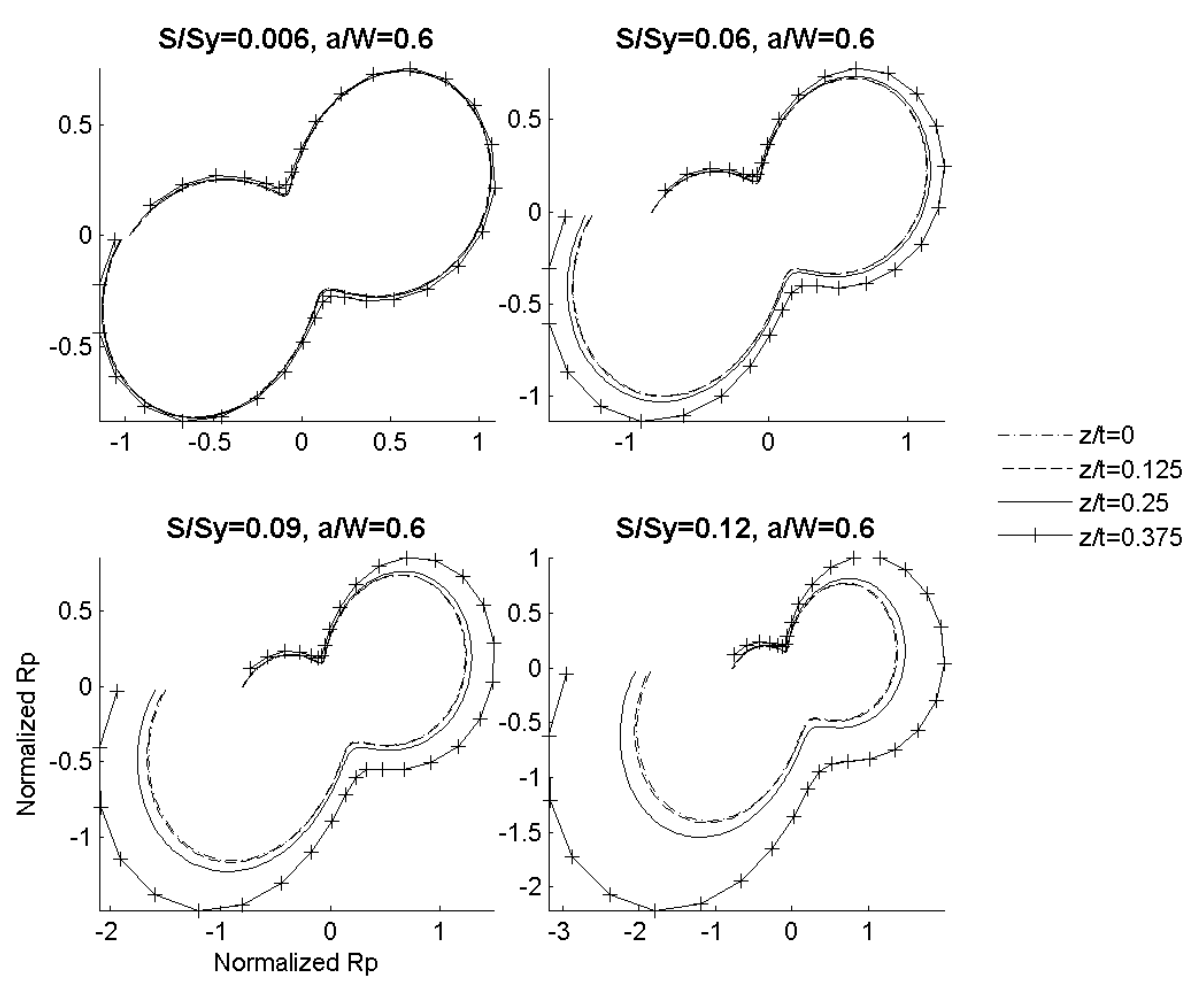

Figure 4.81: Plastic zone map including $T$-stress for $t / W=0.1, a / W=0.6$ 

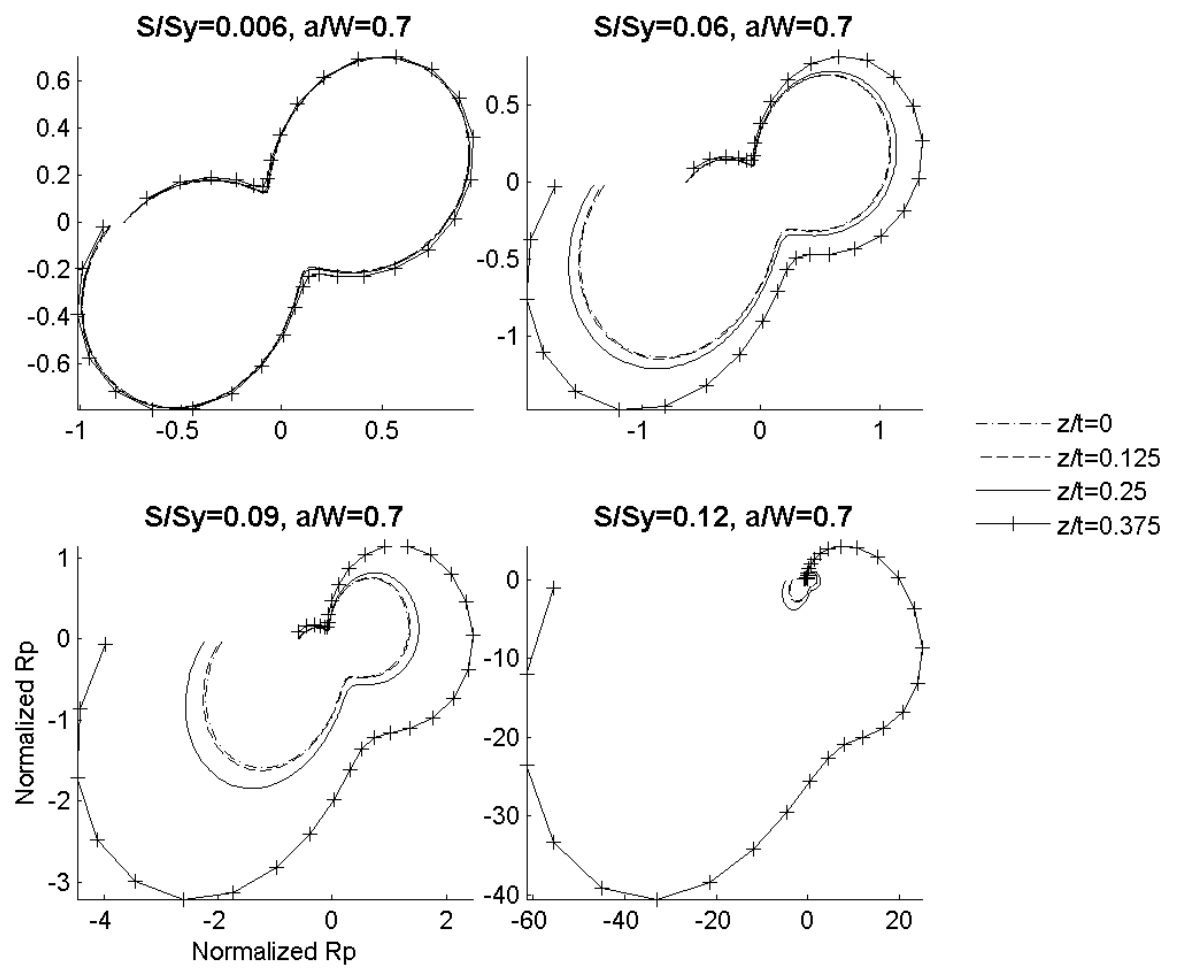

Figure 4.82: Plastic zone map including T-stress for $t / \mathrm{W}=0.1, \mathrm{a} / \mathrm{W}=\mathbf{0 . 7}$ 

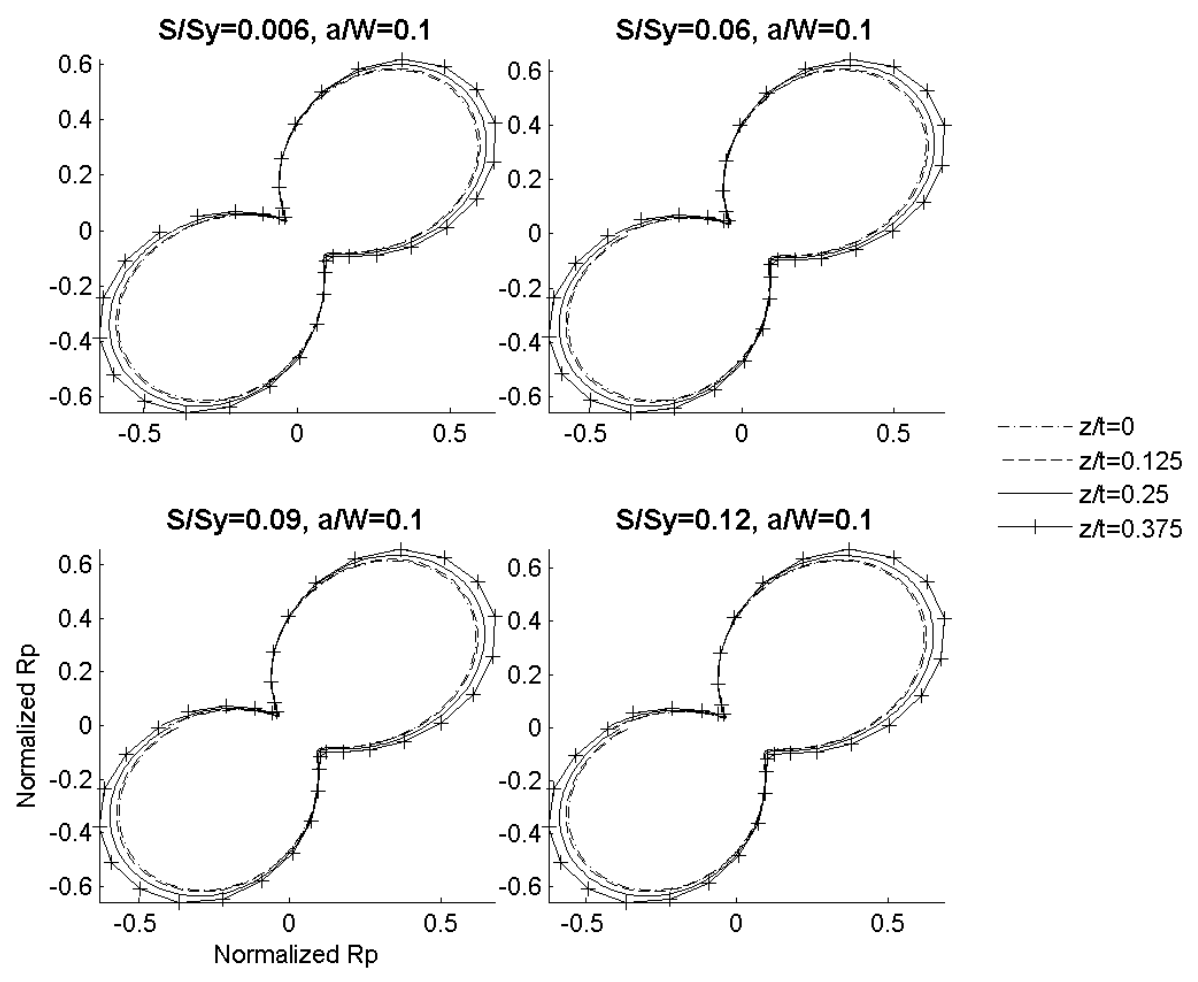

Figure 4.83: Plastic zone map including $T$-stress for $t / W=0.5, a / W=0.1$
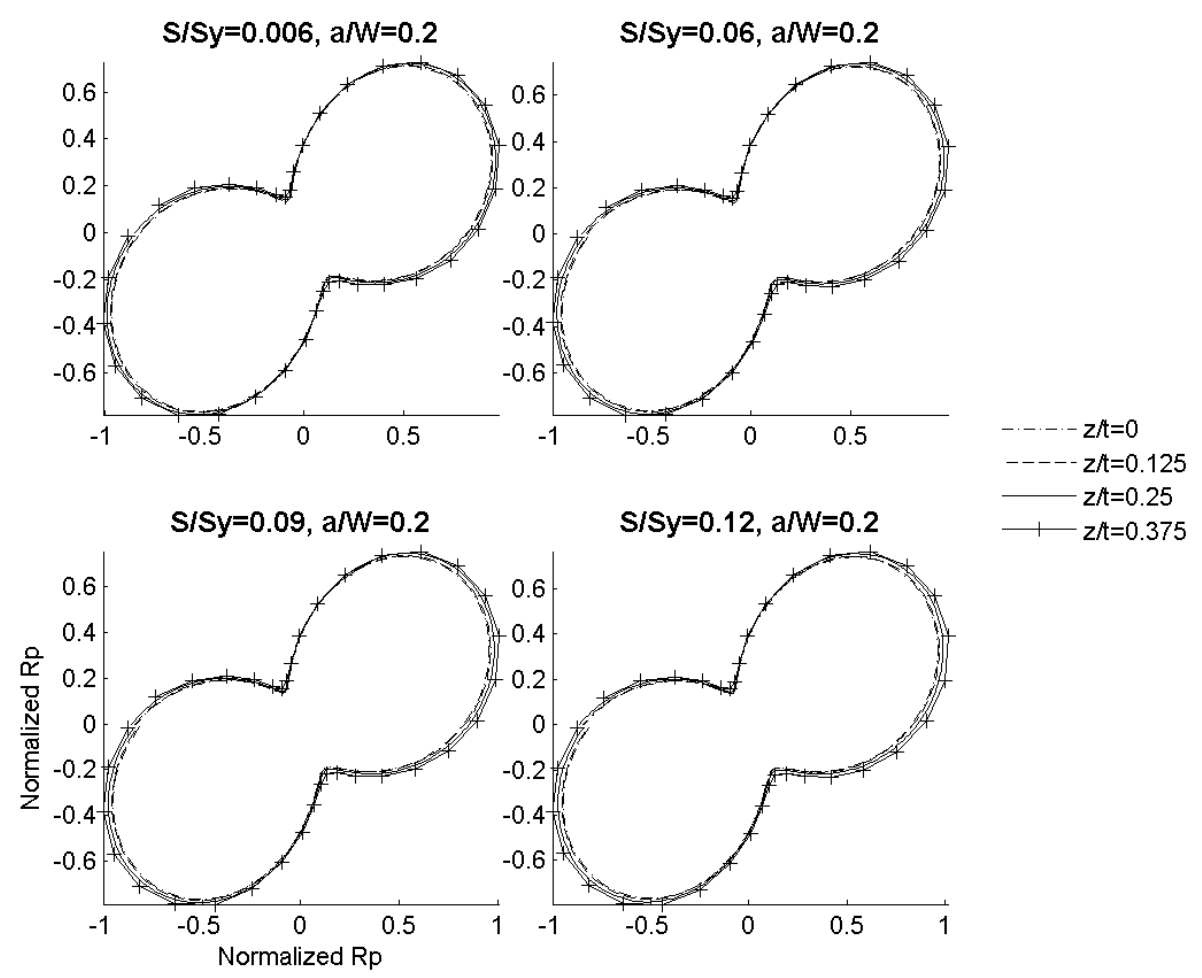

Figure 4.84: Plastic zone map including $T$-stress for $t / W=0.5, a / W=0.2$ 

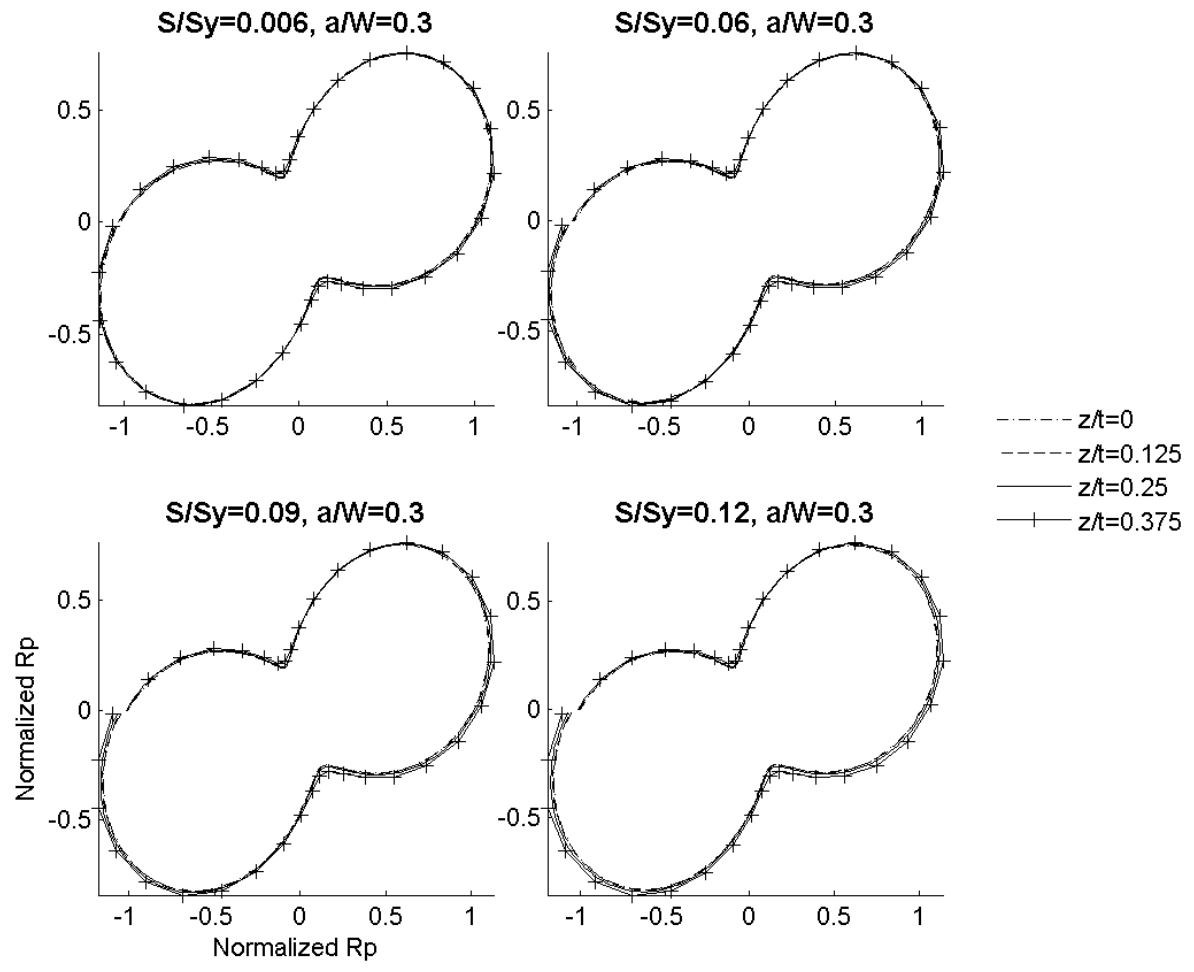

Figure 4.85: Plastic zone map including T-stress for $t / W=0.5, a / W=0.3$
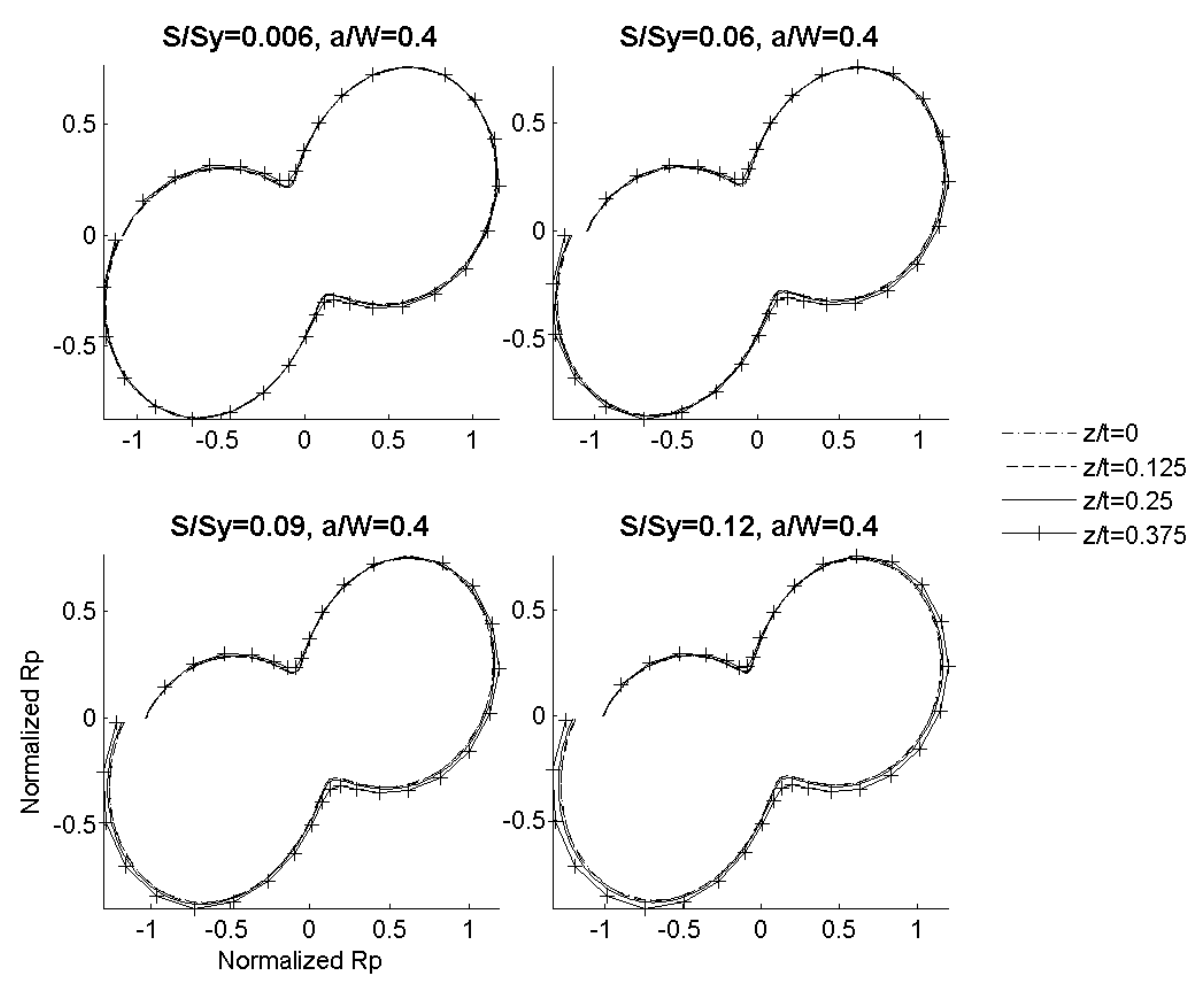

Figure 4.86: Plastic zone map including $T$-stress for $t / W=0.5, a / W=0.4$ 

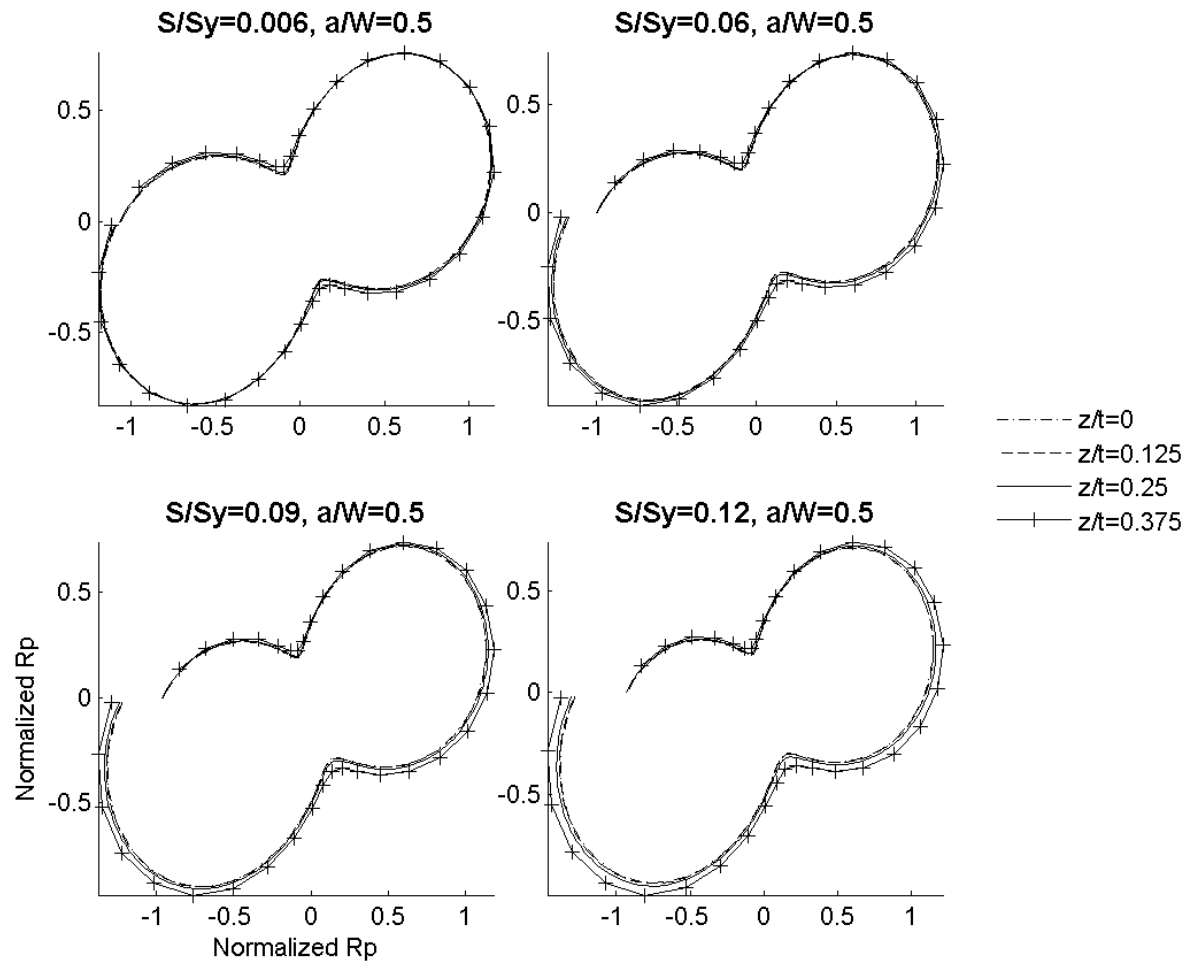

Figure 4.87: Plastic zone map including T-stress for $\mathrm{t} / \mathrm{W}=\mathbf{0 . 5}, \mathrm{a} / \mathrm{W}=\mathbf{0 . 5}$
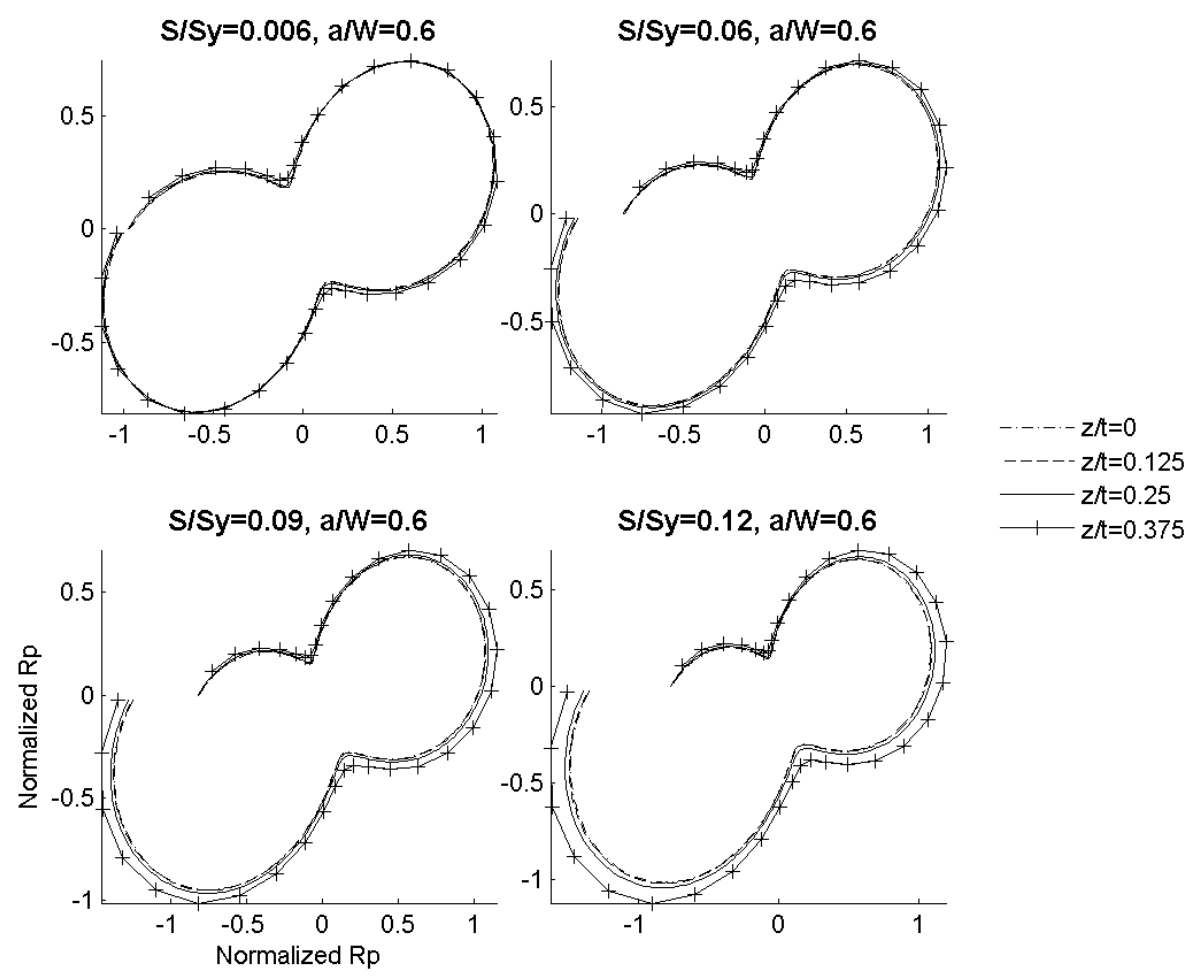

Figure 4.88: Plastic zone map including T-stress for $t / W=0.5, a / W=0.6$ 

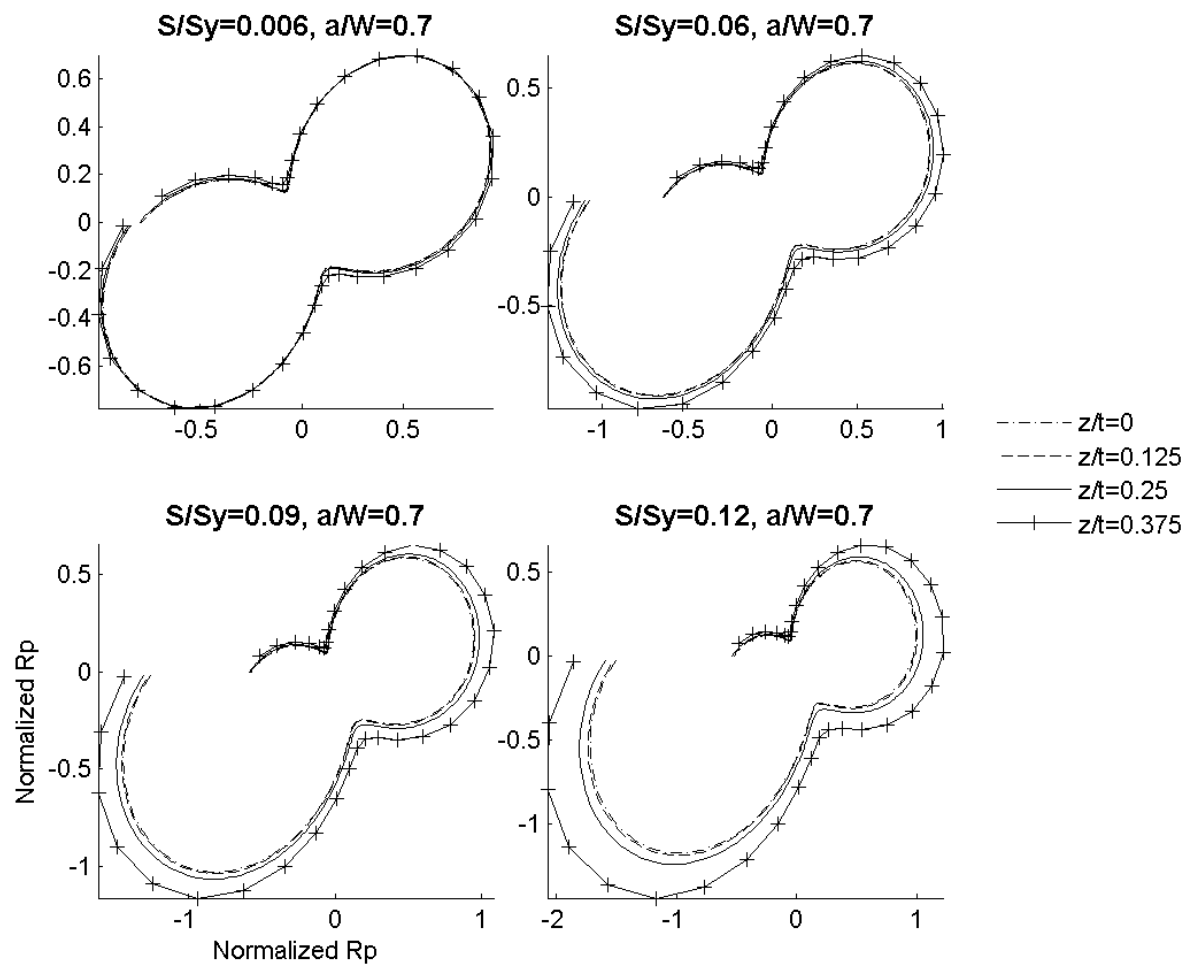

Figure 4.89: Plastic zone map including $T$-stress for $\mathrm{t} / \mathrm{W}=\mathbf{0 . 5}, \mathrm{a} / \mathrm{W}=\mathbf{0 . 7}$ 

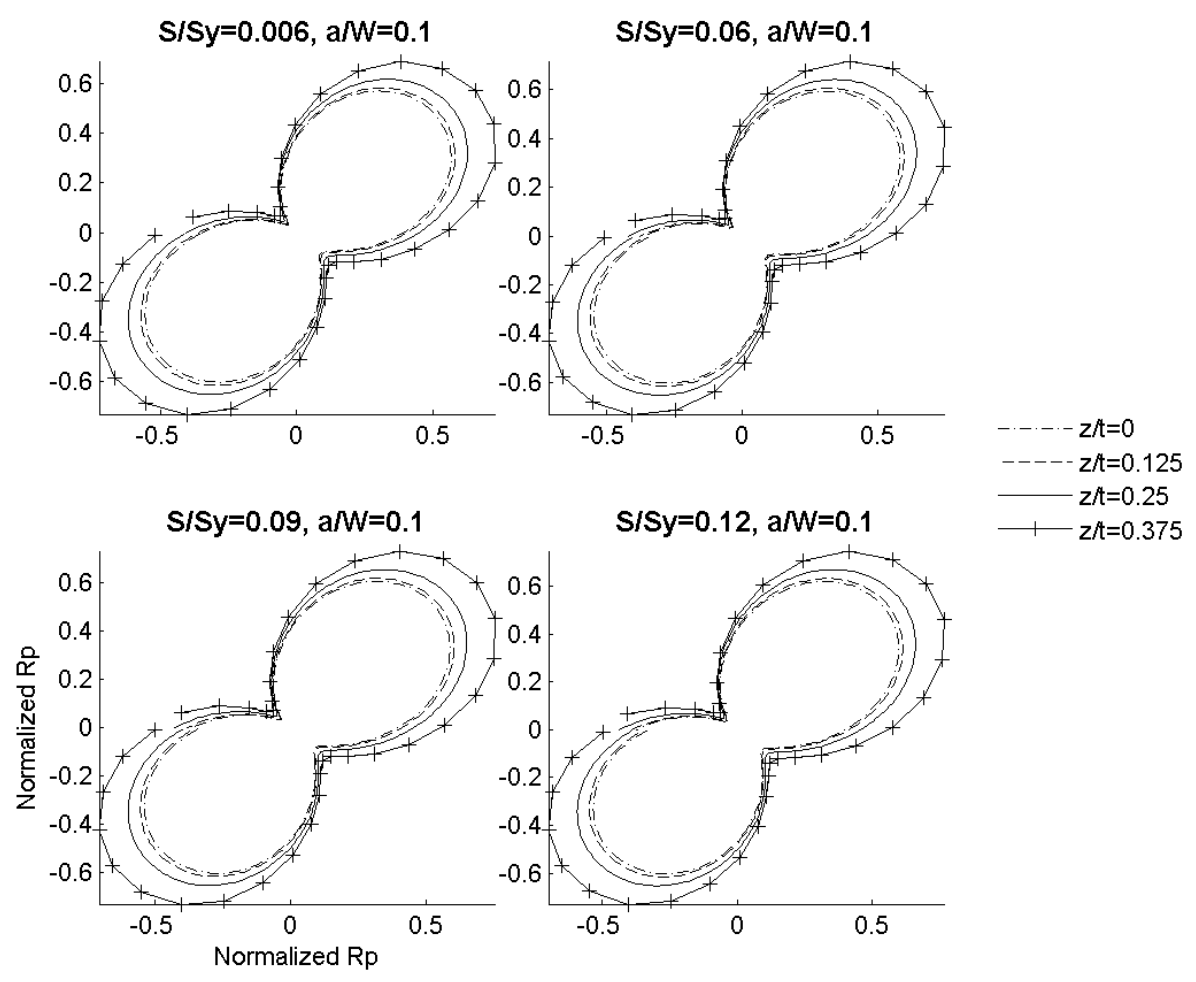

Figure 4.90: Plastic zone map including $T$-stress for $t / W=1.0, a / W=0.1$
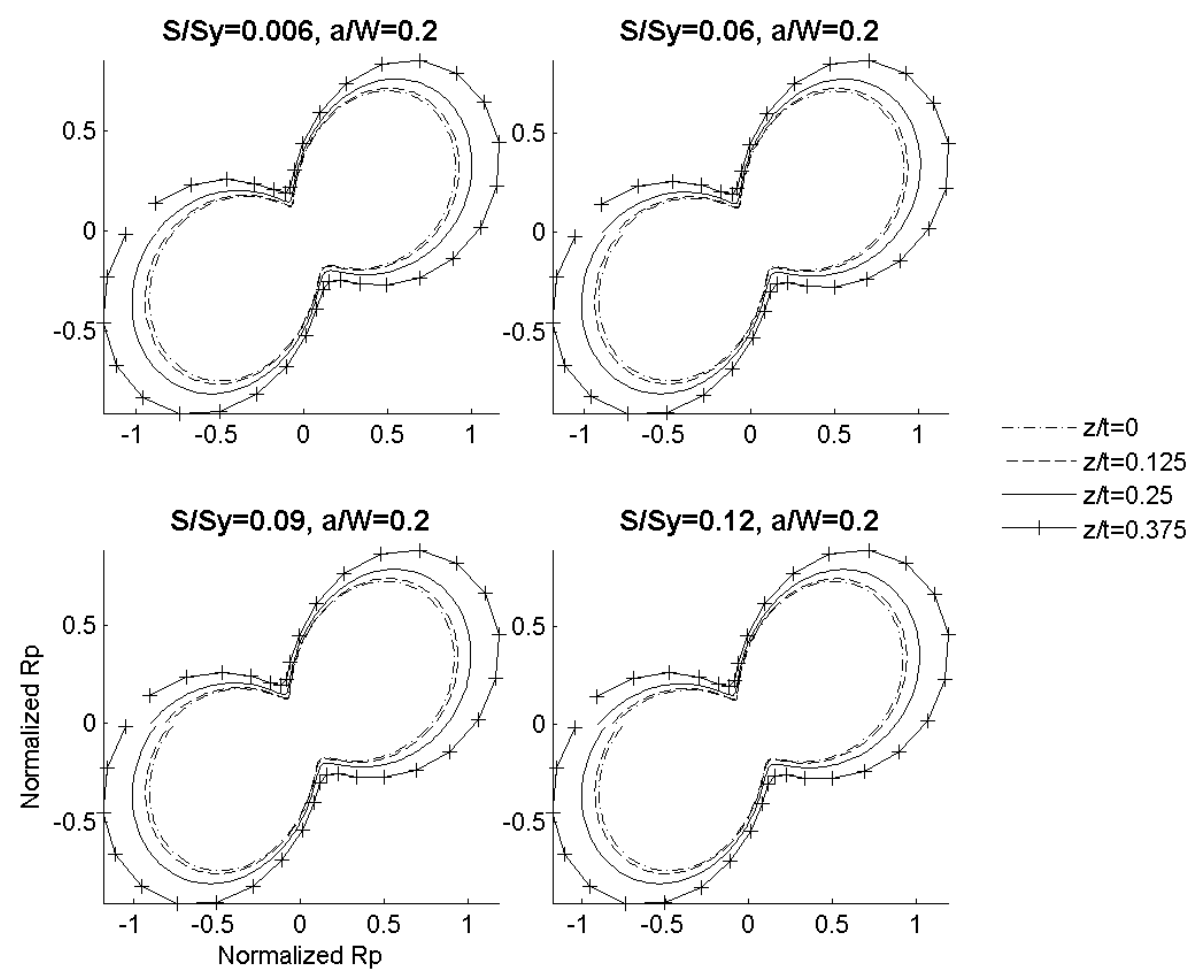

Figure 4.91: Plastic zone map including T-stress for $t / W=1.0, a / W=0.2$ 

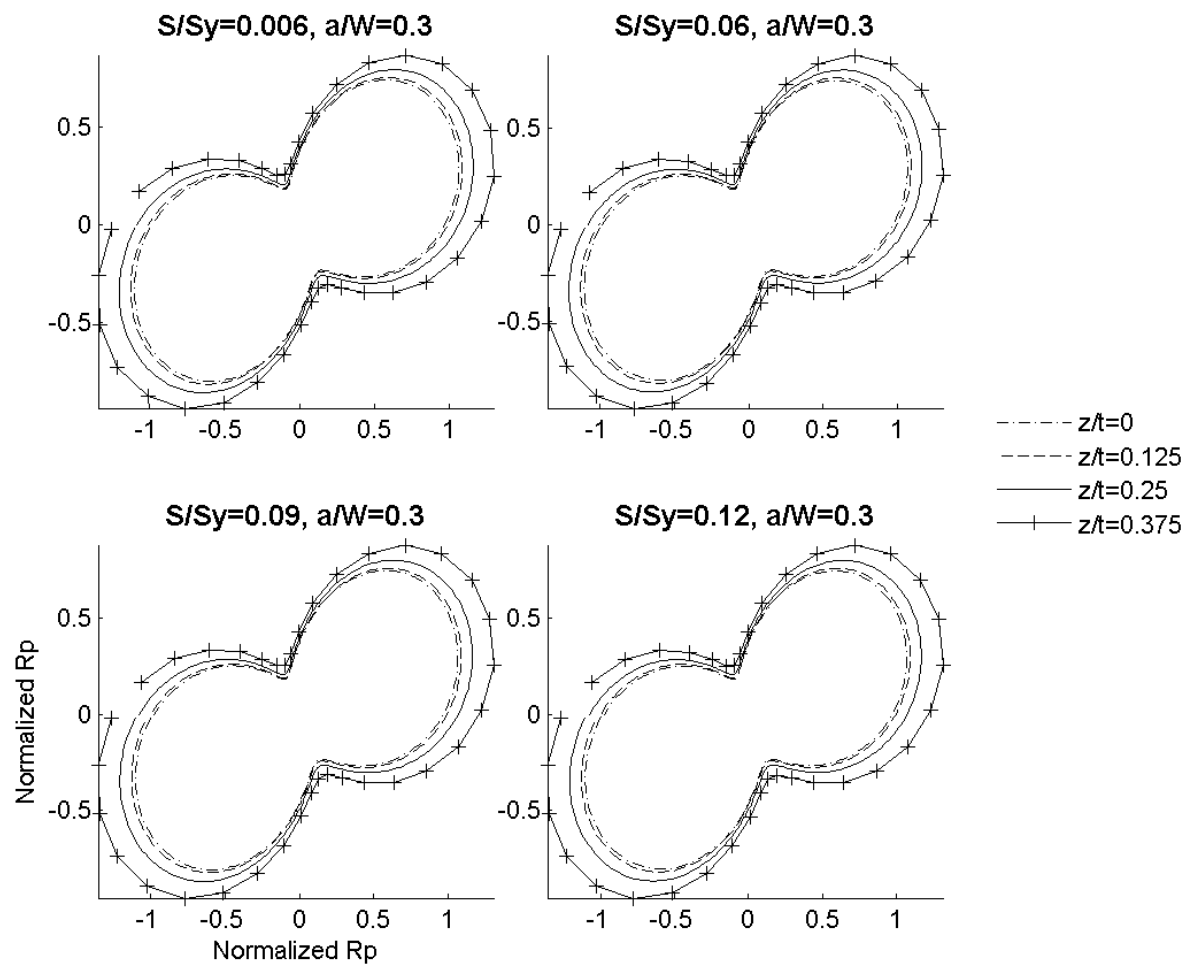

Figure 4.92: Plastic zone map including T-stress for $t / W=1.0, a / W=0.3$
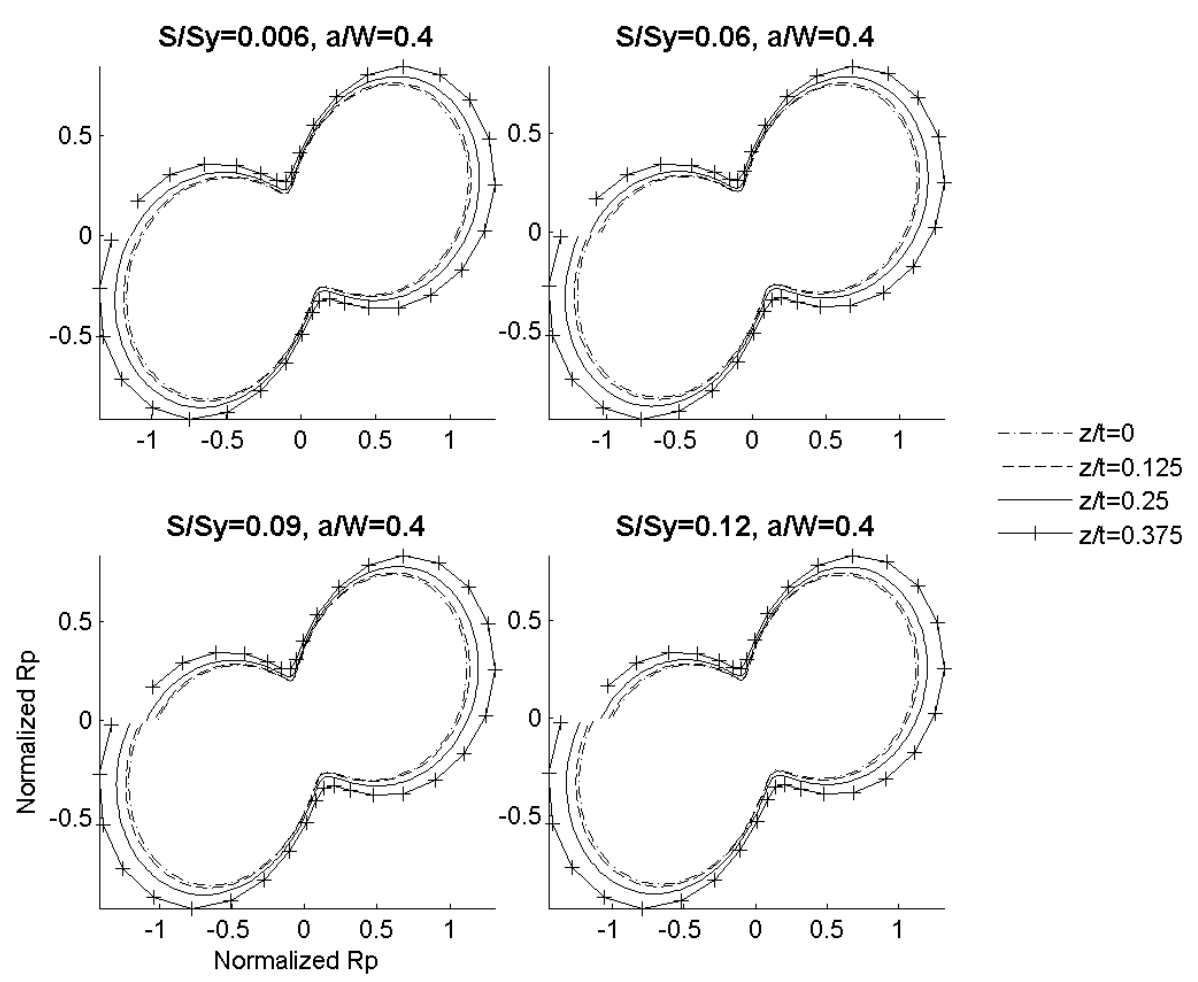

Figure 4.93: Plastic zone map including T-stress for $t / W=1.0, a / W=0.4$ 

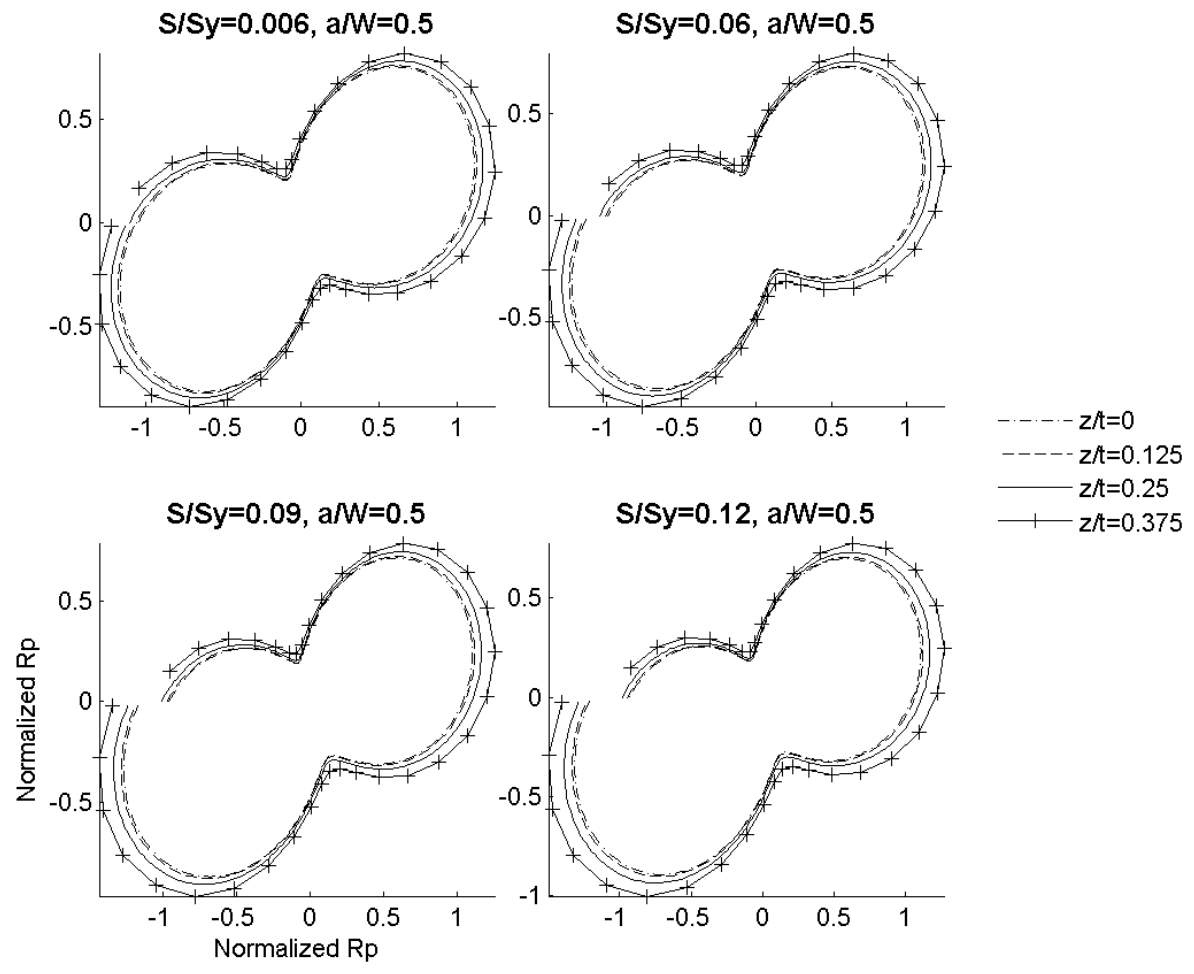

Figure 4.94: Plastic zone map including $T$-stress for $t / W=1.0, a / W=0.5$
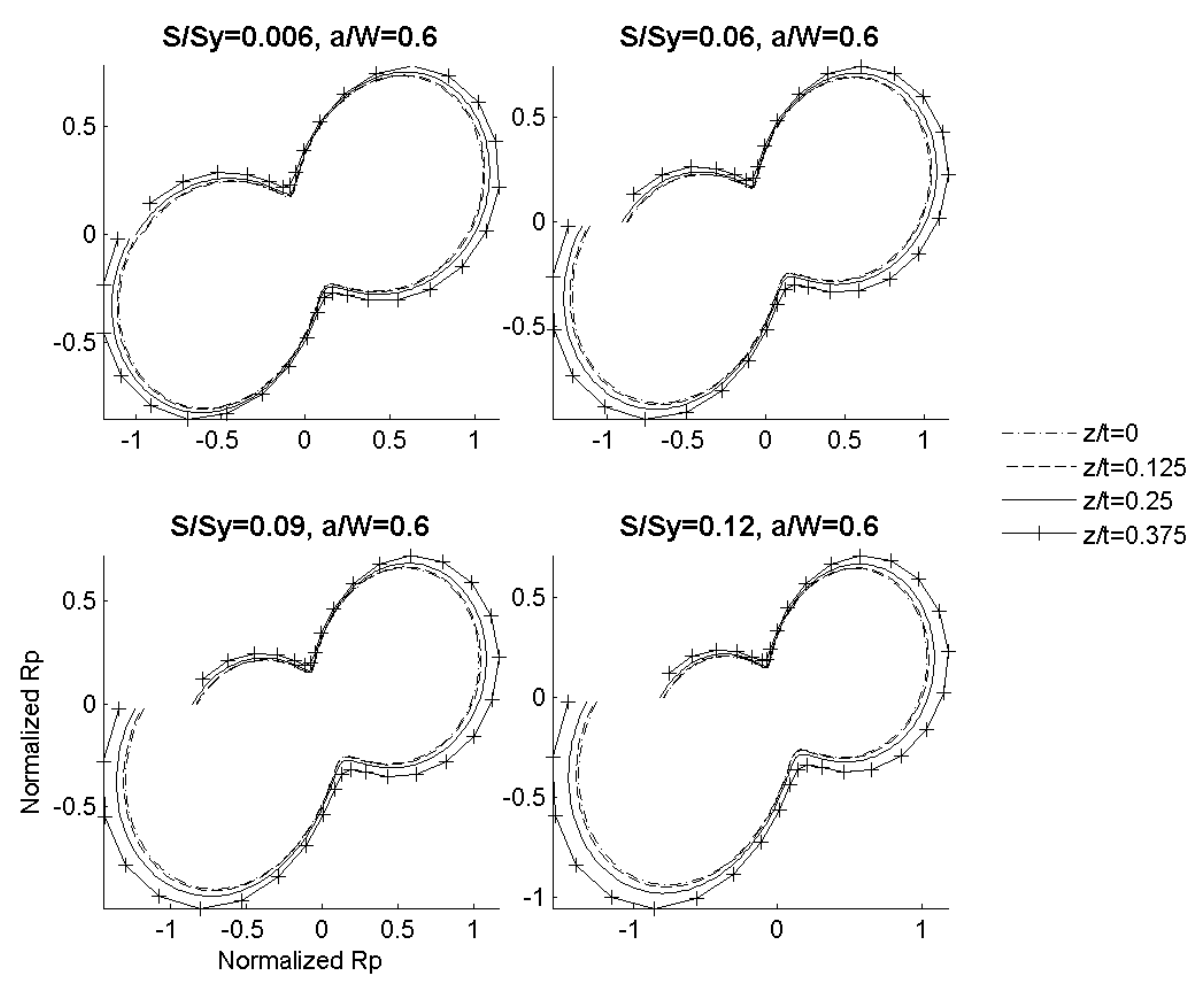

Figure 4.95: Plastic zone map including $T$-stress for $t / W=1.0, a / W=0.6$ 

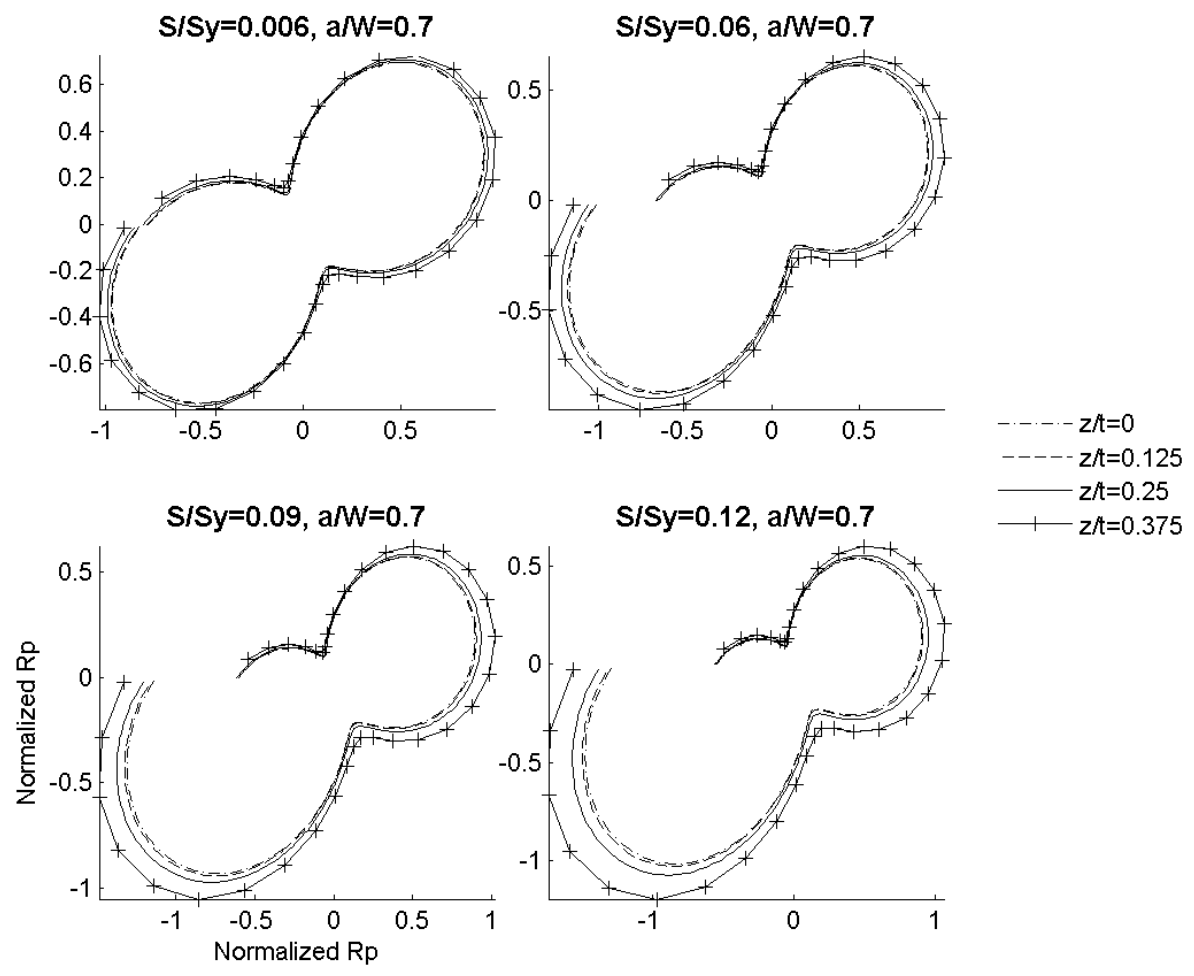

Figure 4.96: Plastic zone map including $T$-stress for $t / W=1.0, a / W=0.7$ 

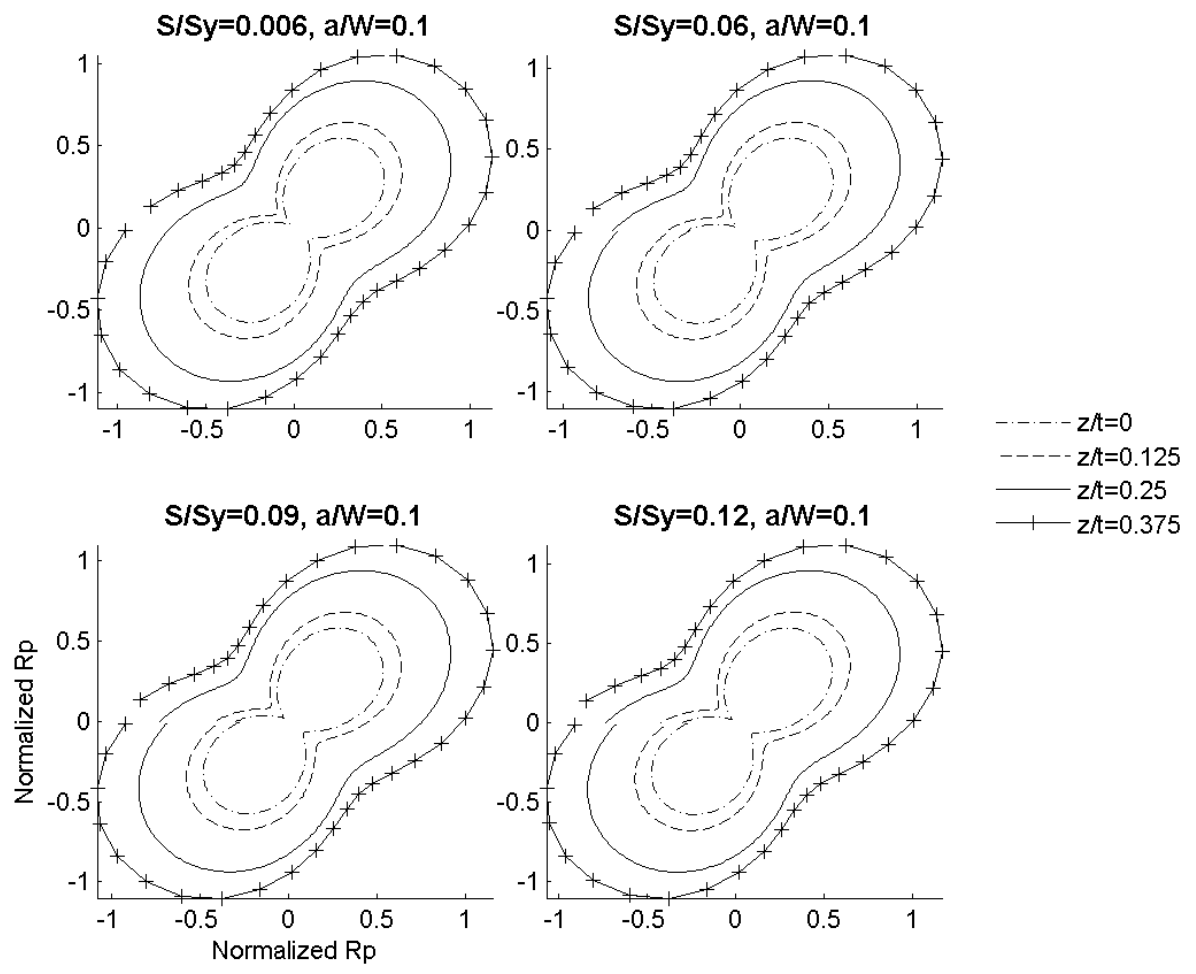

Figure 4.97: Plastic zone map including $T$-stress for $t / W=2.0, a / W=0.1$
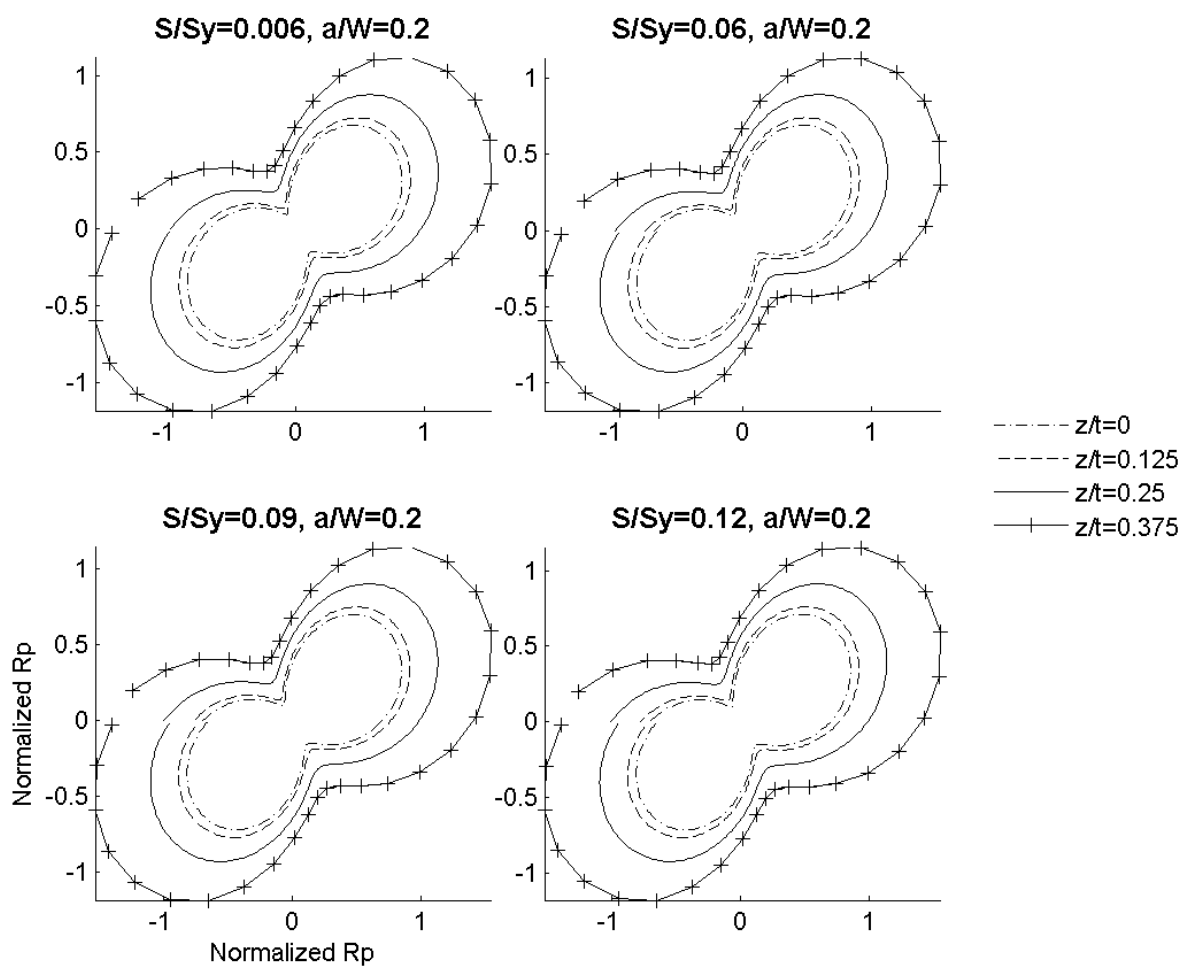

Figure 4.98: Plastic zone map including $T$-stress for $t / W=2.0, a / W=0.2$ 

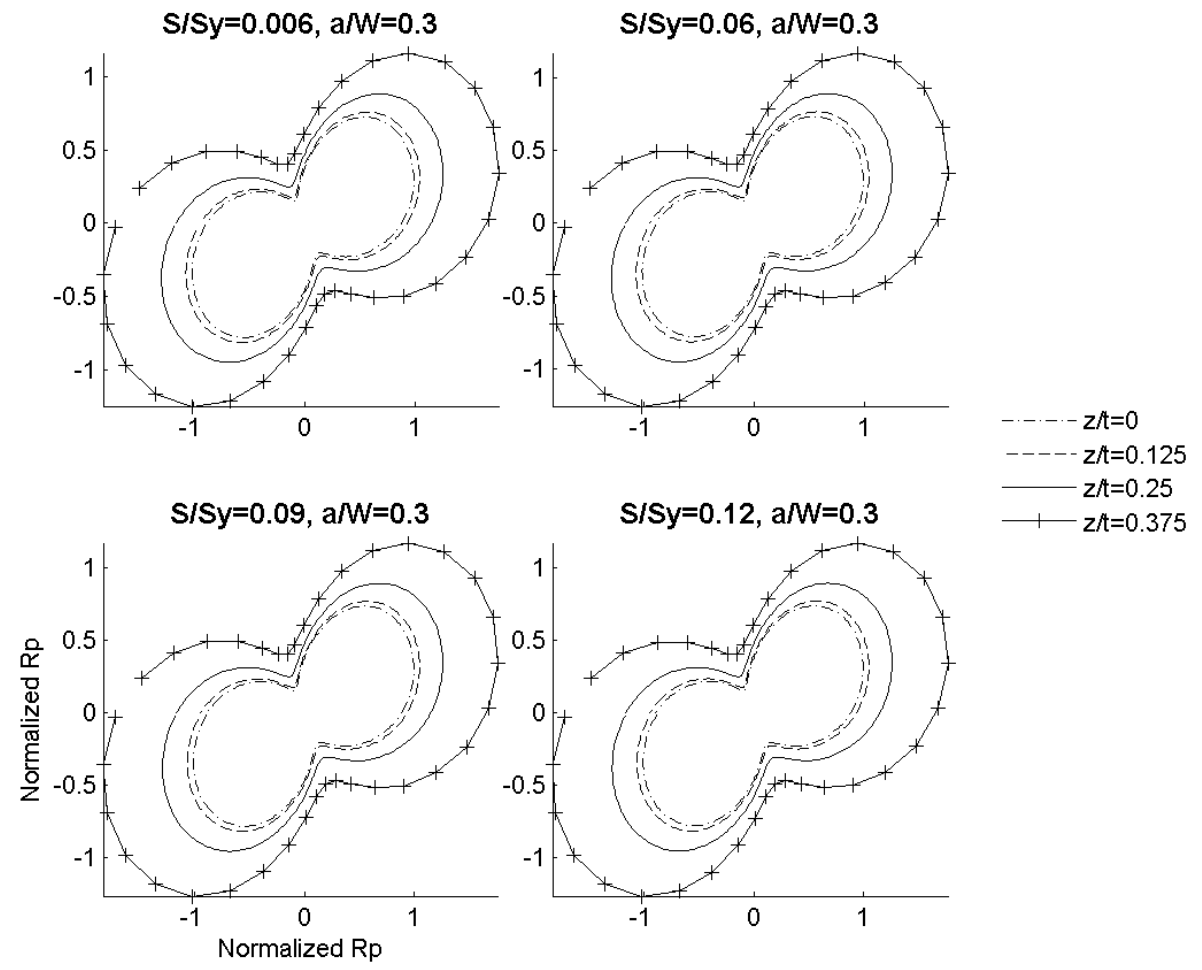

Figure 4.99: Plastic zone map including $T$-stress for $t / W=2.0, a / W=0.3$
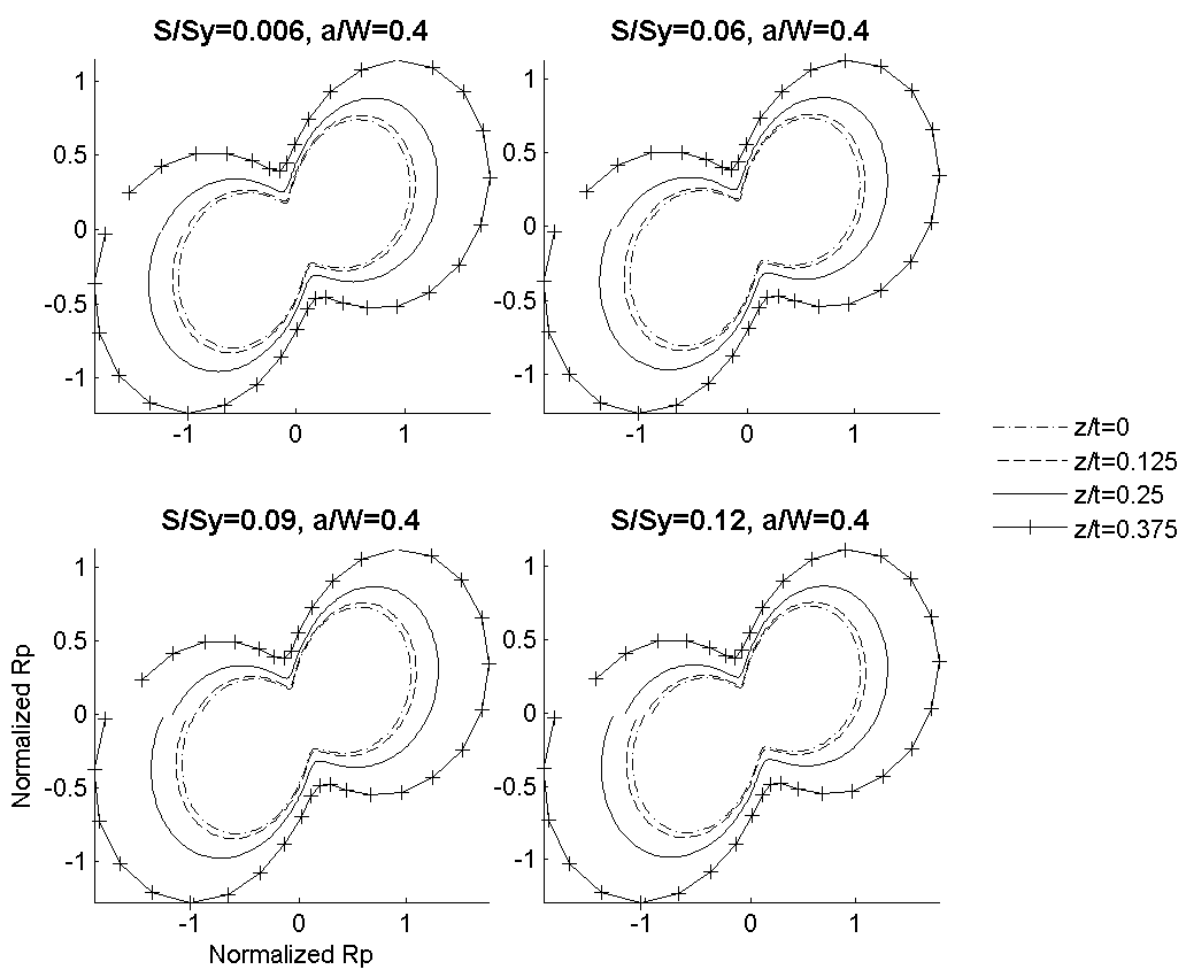

Figure 4.100: Plastic zone map including $T$-stress for $t / W=2.0, a / W=0.4$ 

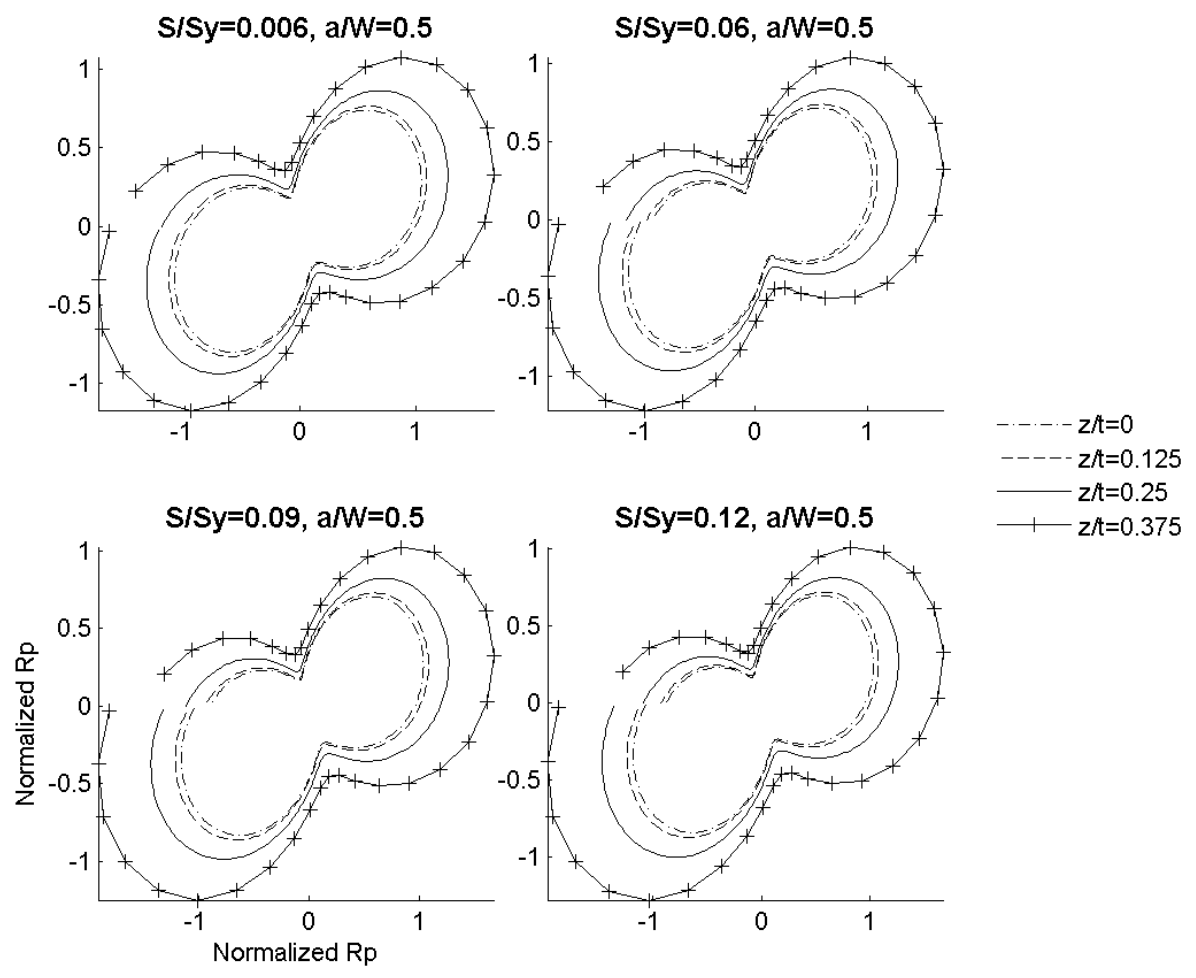

Figure 4.101: Plastic zone map including T-stress for $t / W=2.0, a / W=0.5$
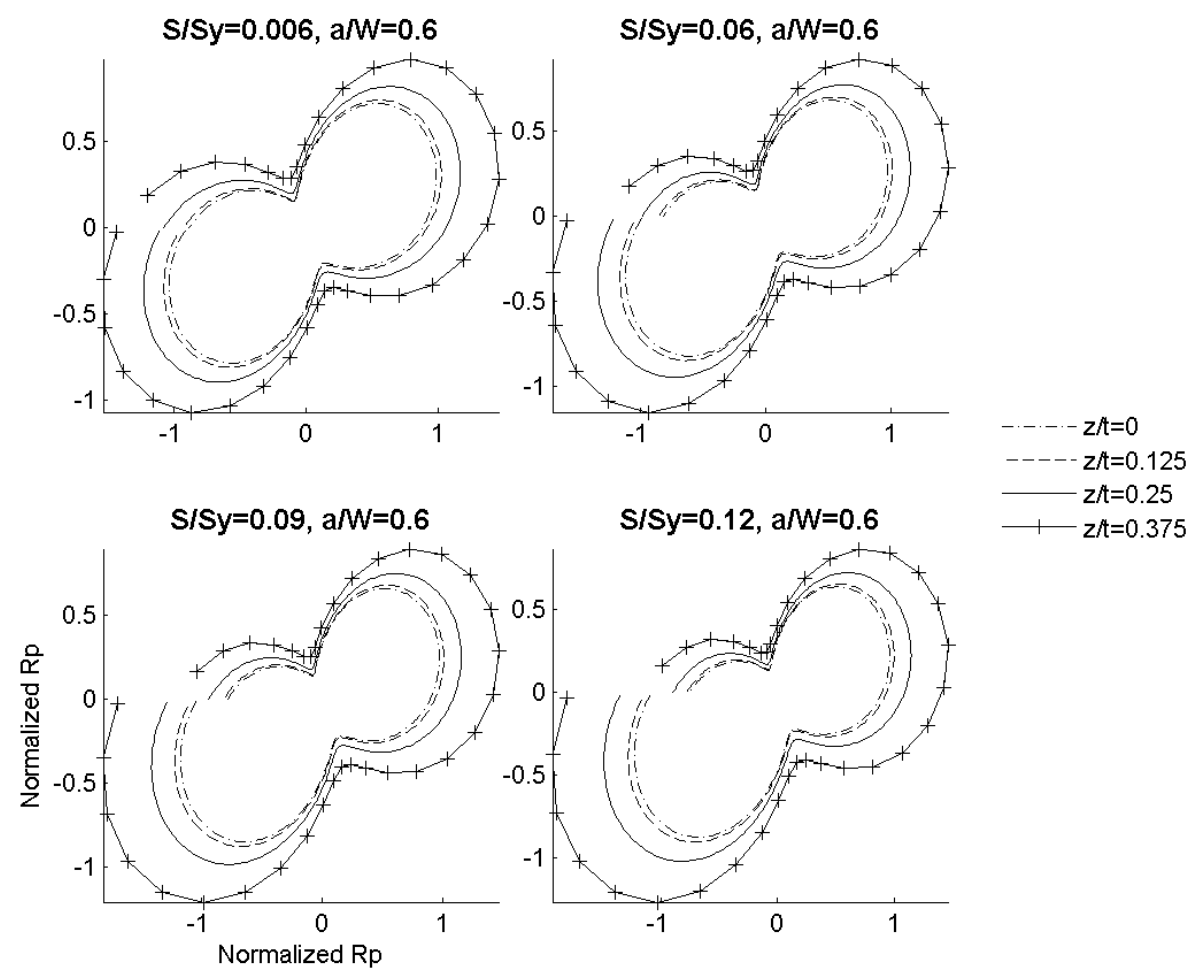

Figure 4.102: Plastic zone map including $T$-stress for $t / W=2.0, a / W=0.6$ 

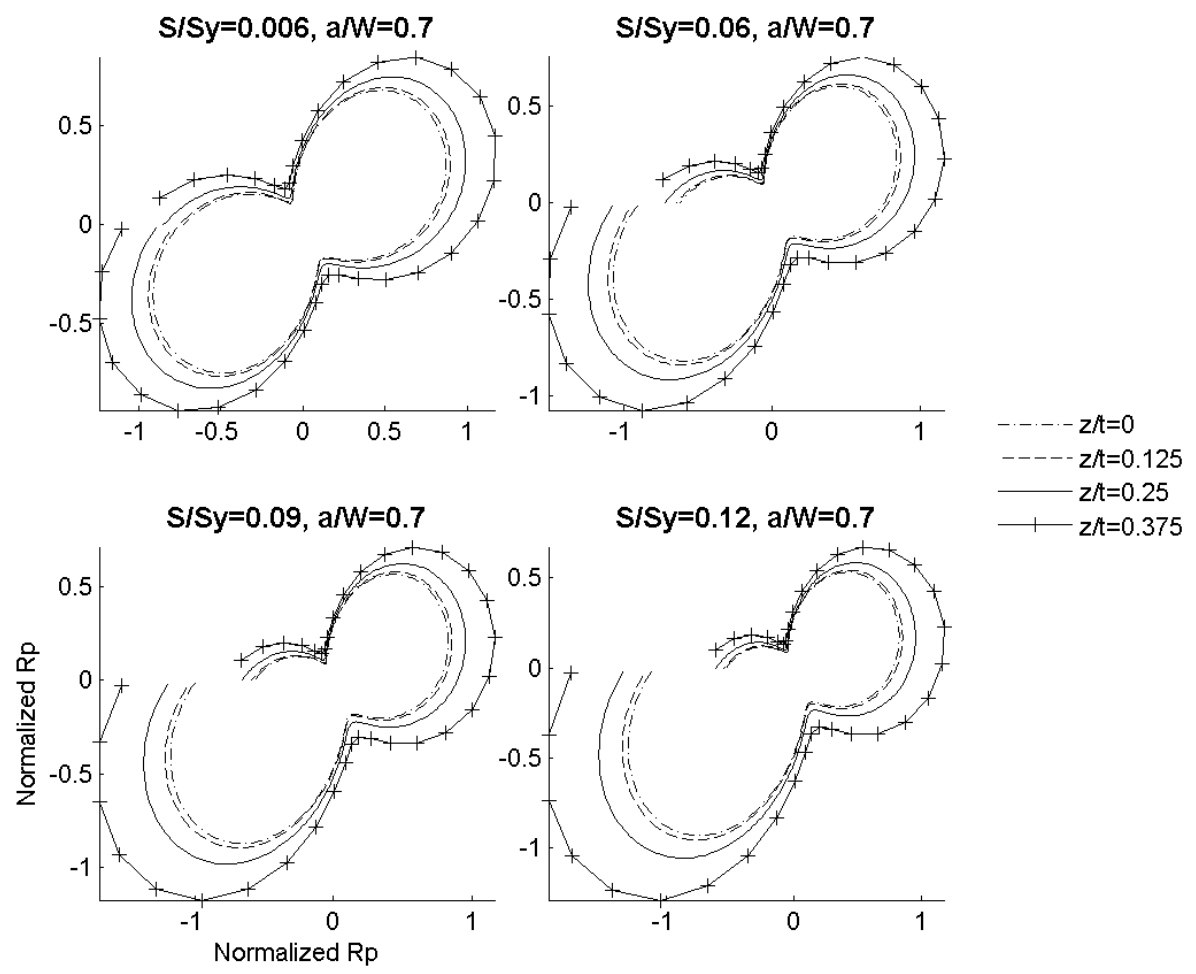

Figure 4.103: Plastic zone map including T-stress for $t / W=2.0, a / W=0.7$ 

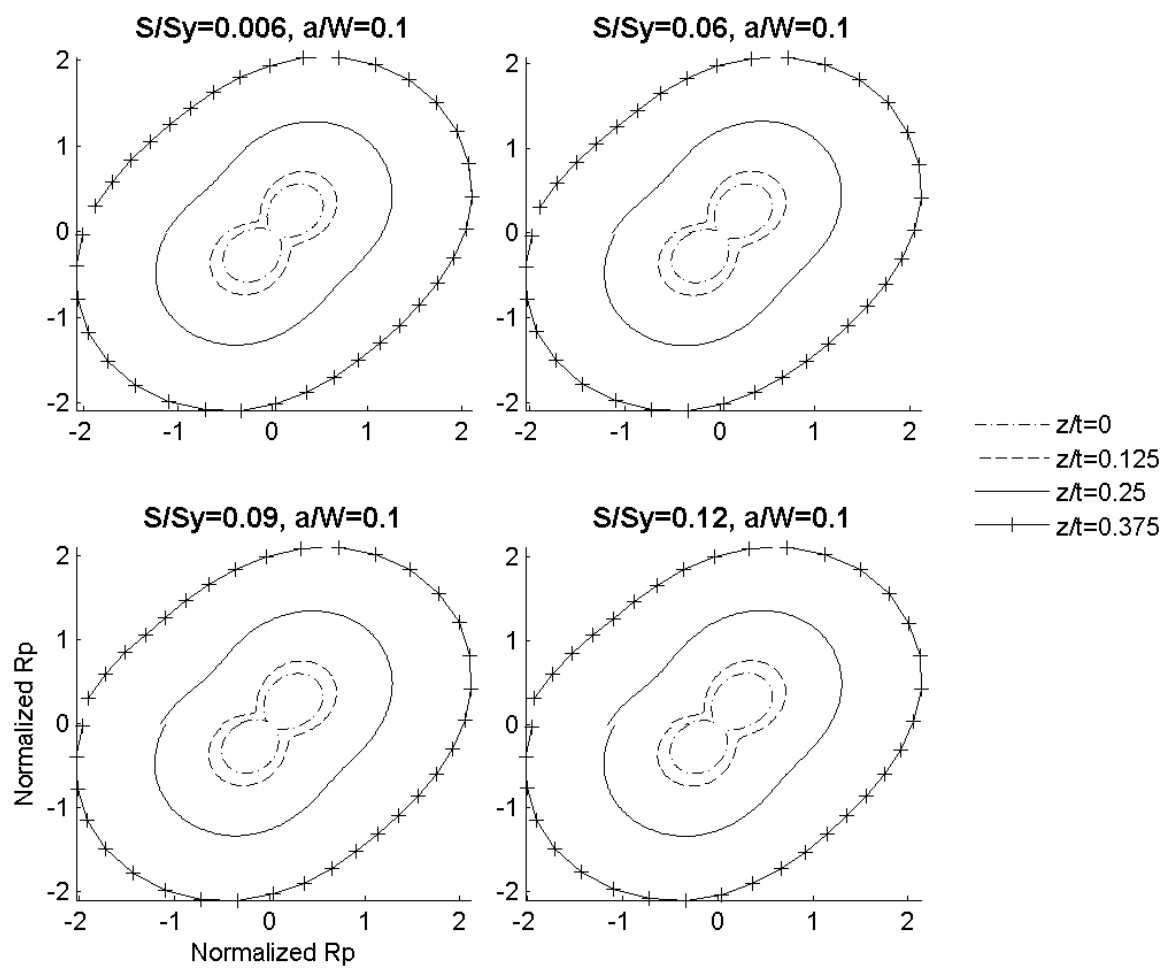

Figure 4.104: Plastic zone map including $T$-stress for $t / W=4.0, a / W=0.1$
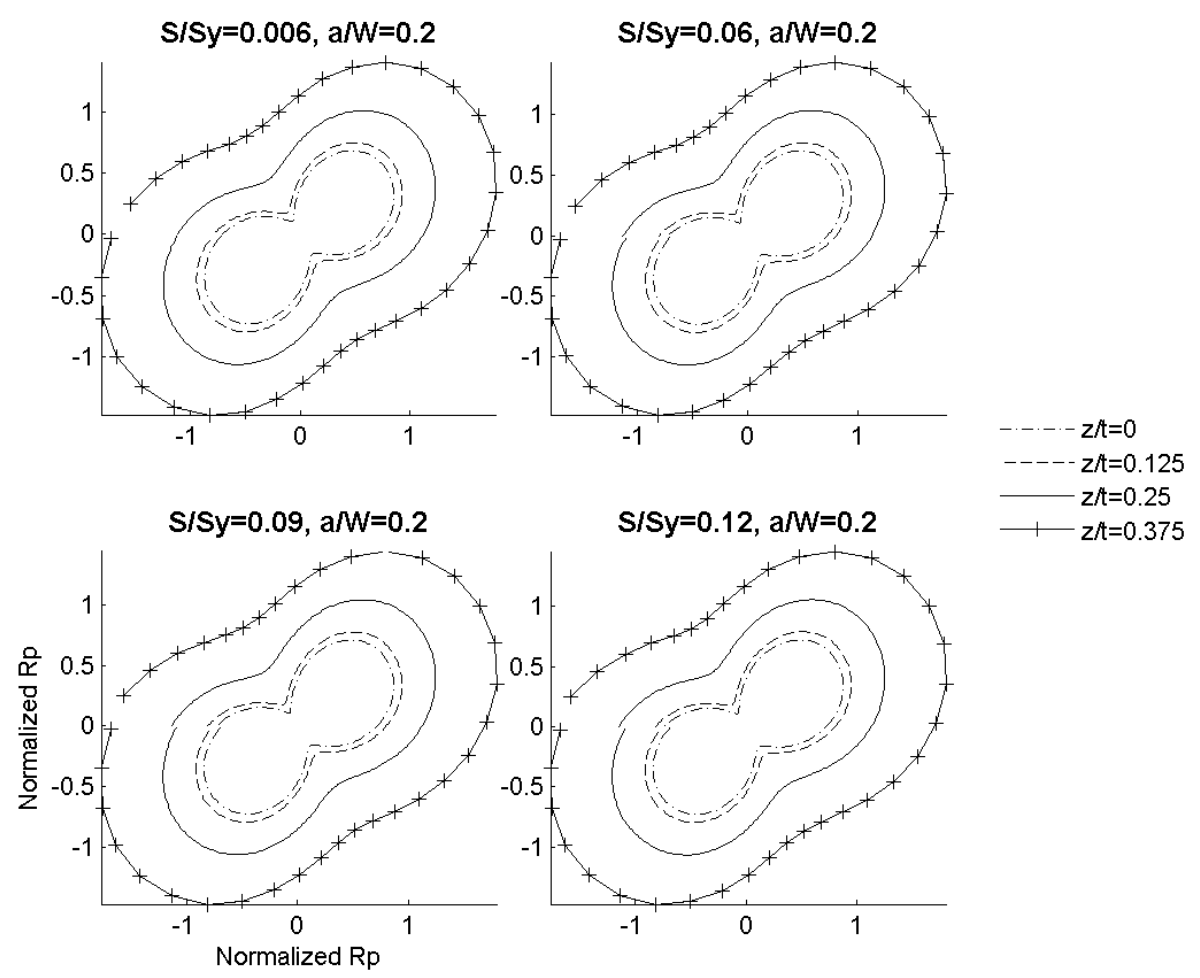

Figure 4.105: Plastic zone map including $T$-stress for $t / W=4.0, a / W=0.2$ 

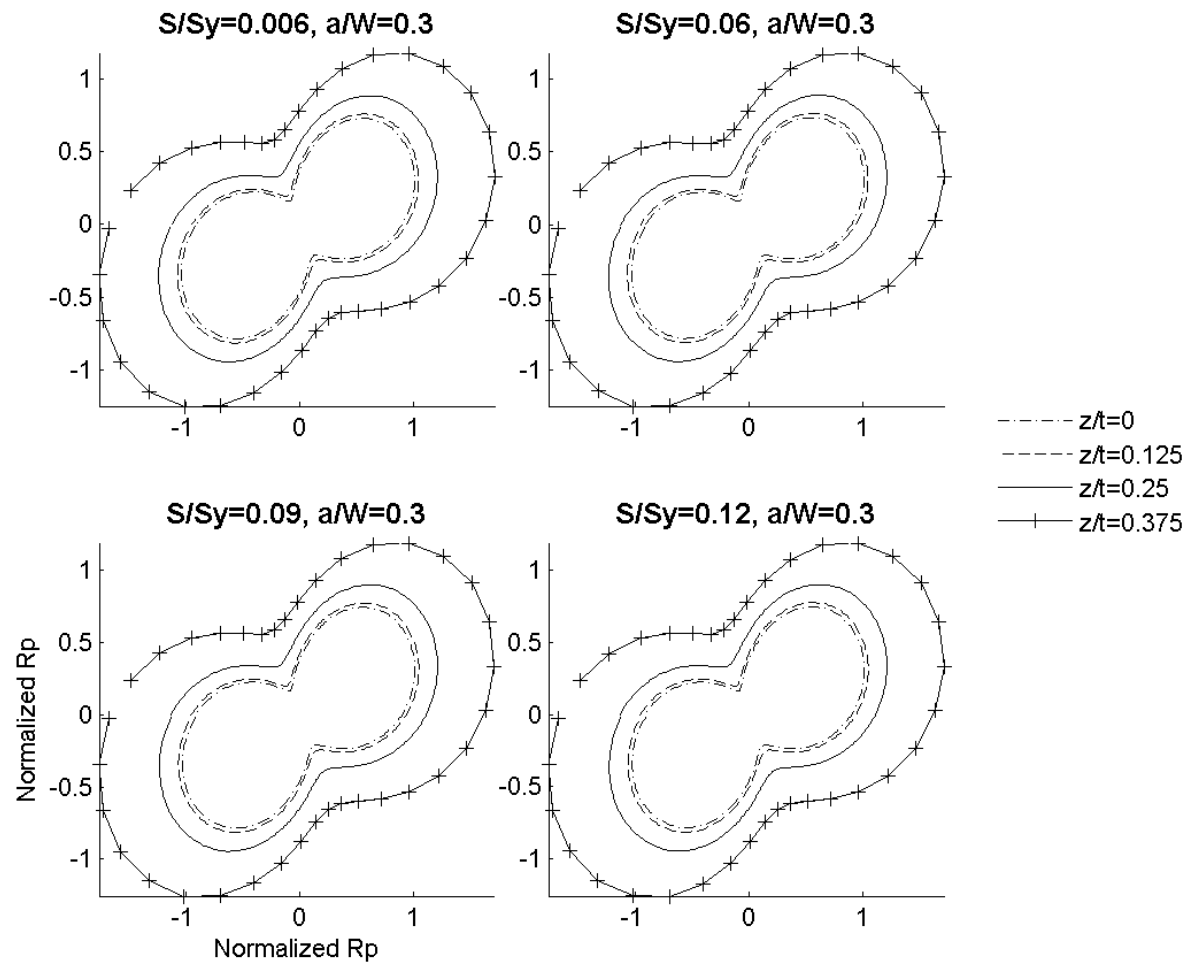

Figure 4.106: Plastic zone map including $T$-stress for $t / W=4.0, a / W=0.3$
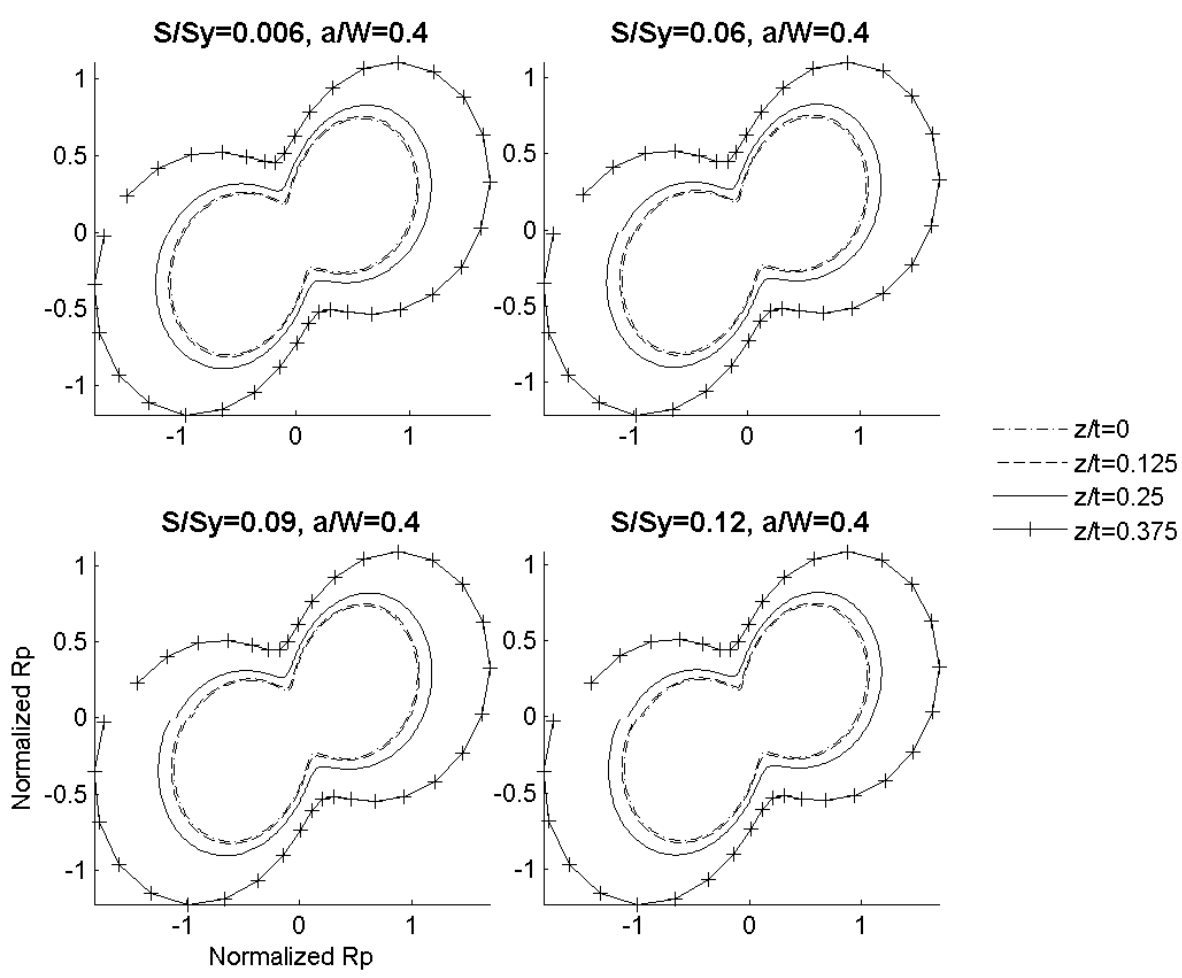

Figure 4.107: Plastic zone map including $T$-stress for $t / W=4.0, a / W=0.4$ 

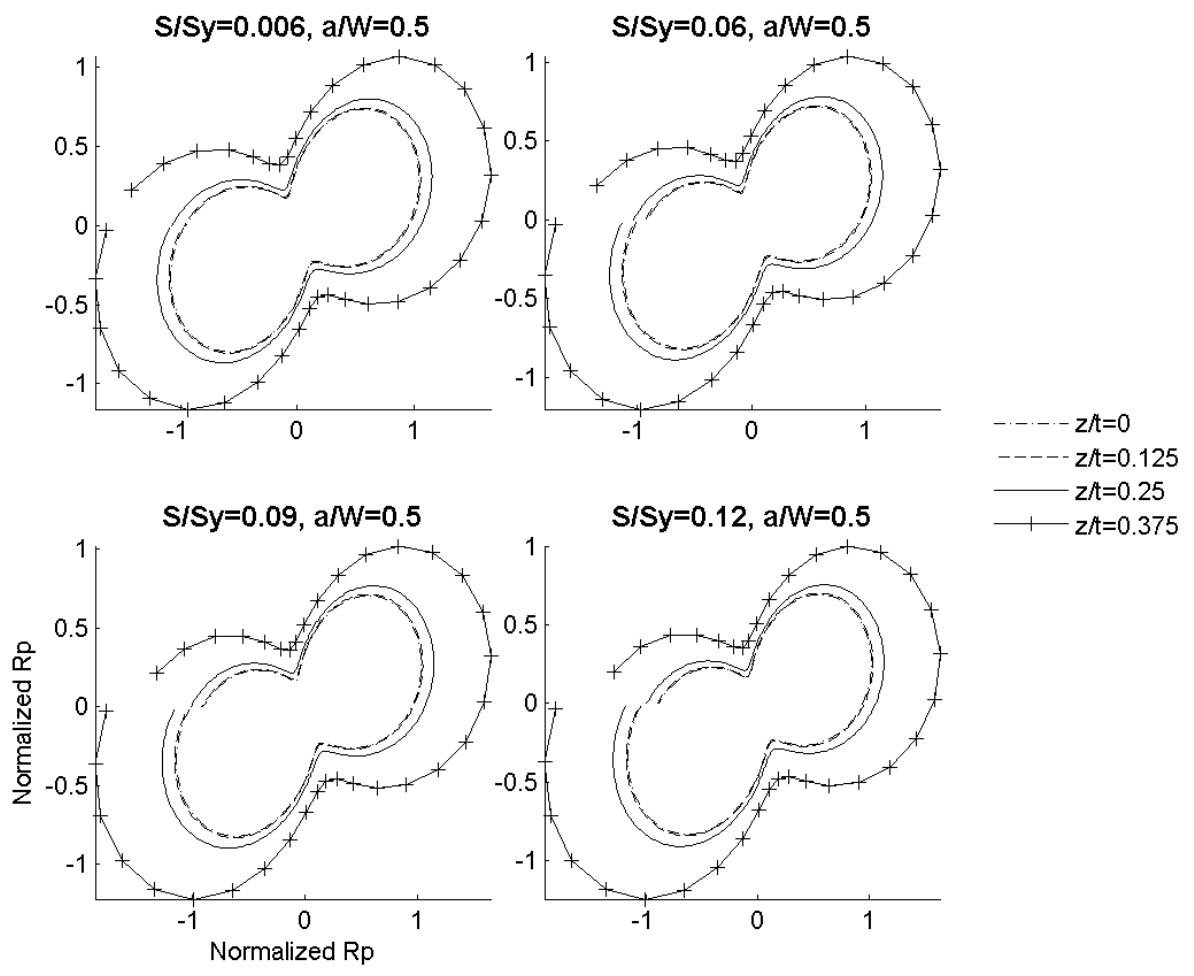

Figure 4.108: Plastic zone map including $T$-stress for $t / W=4.0, a / W=0.5$
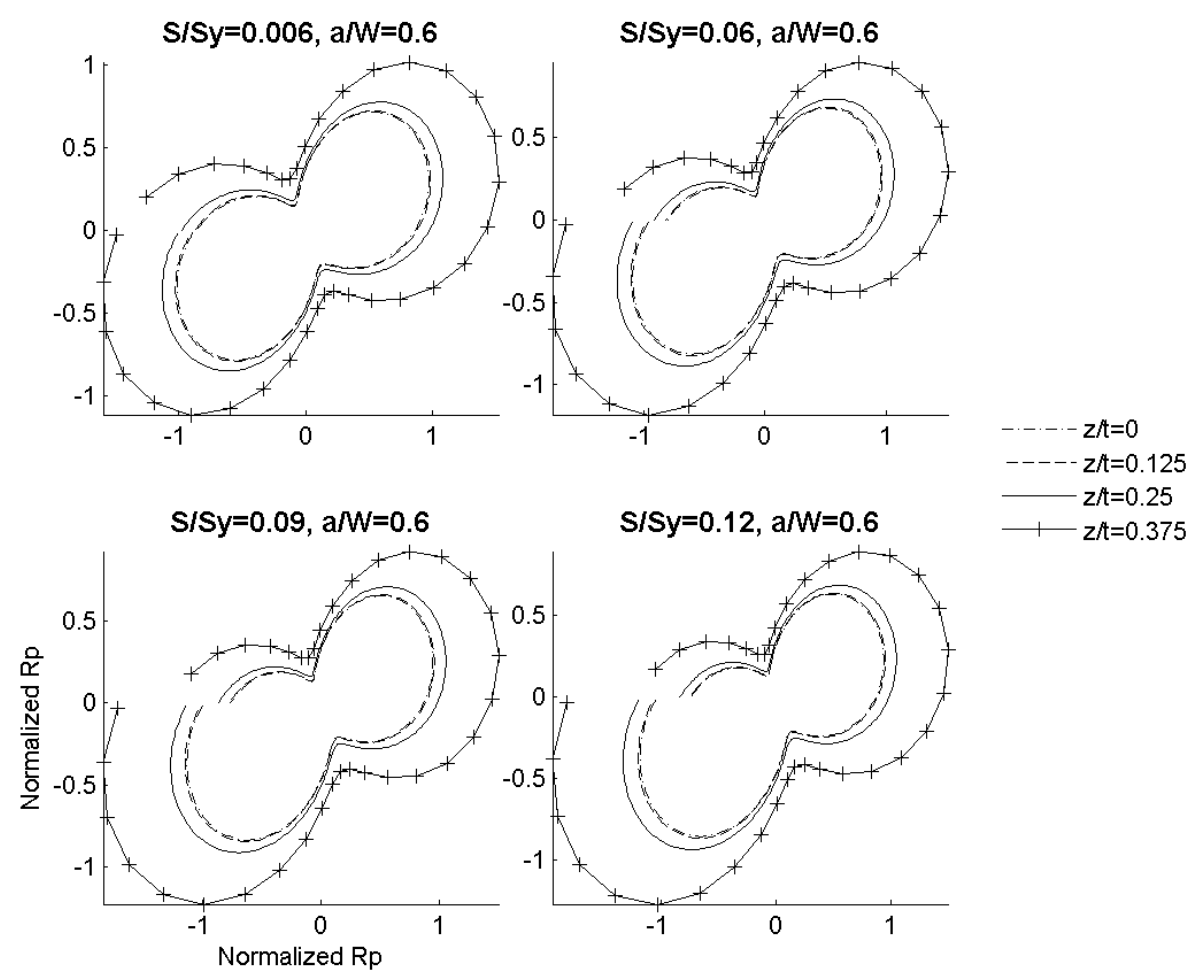

Figure 4.109: Plastic zone map including $T$-stress for $t / W=4.0, a / W=0.6$ 

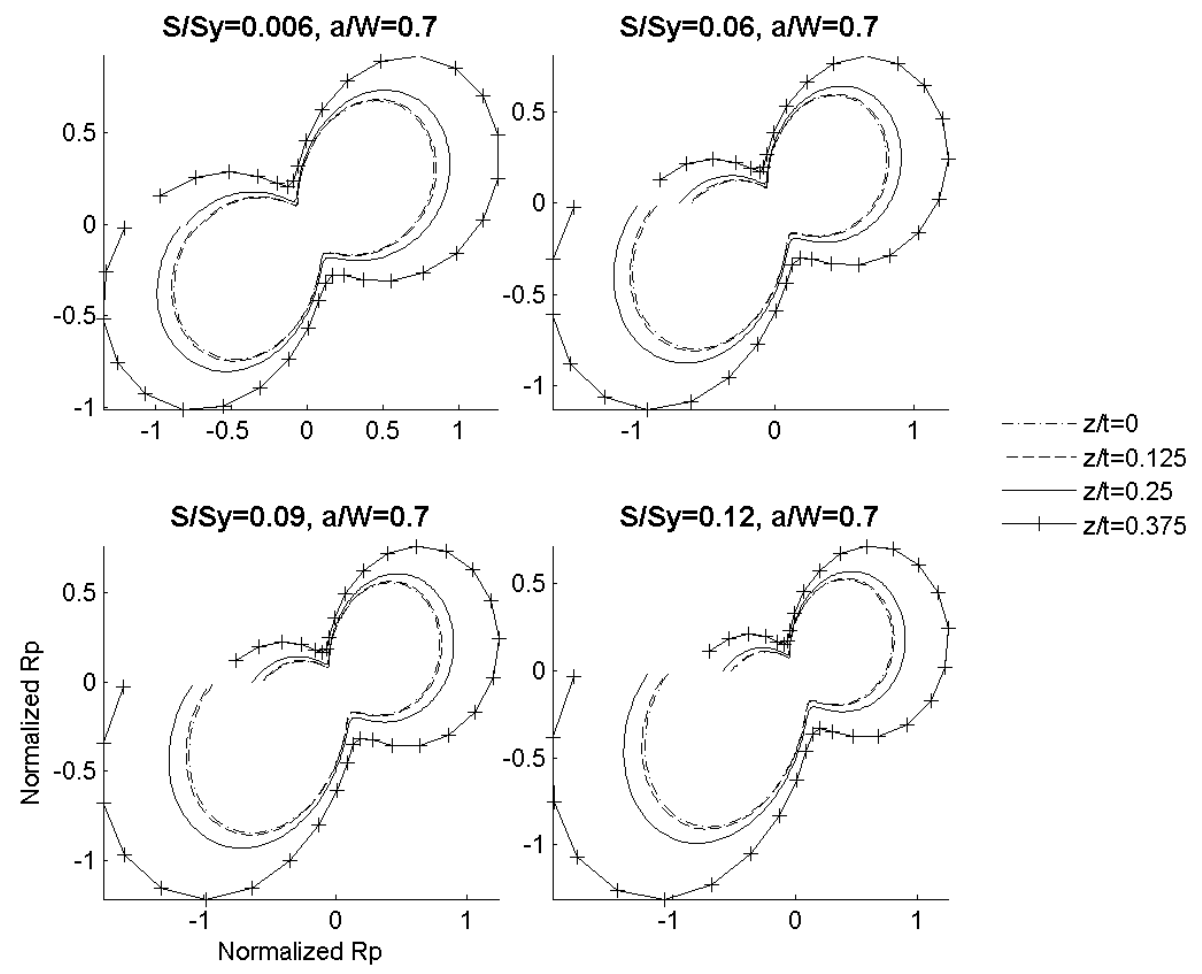

Figure 4.110: Plastic zone map including T-stress for $t / W=4.0, a / W=0.7$ 


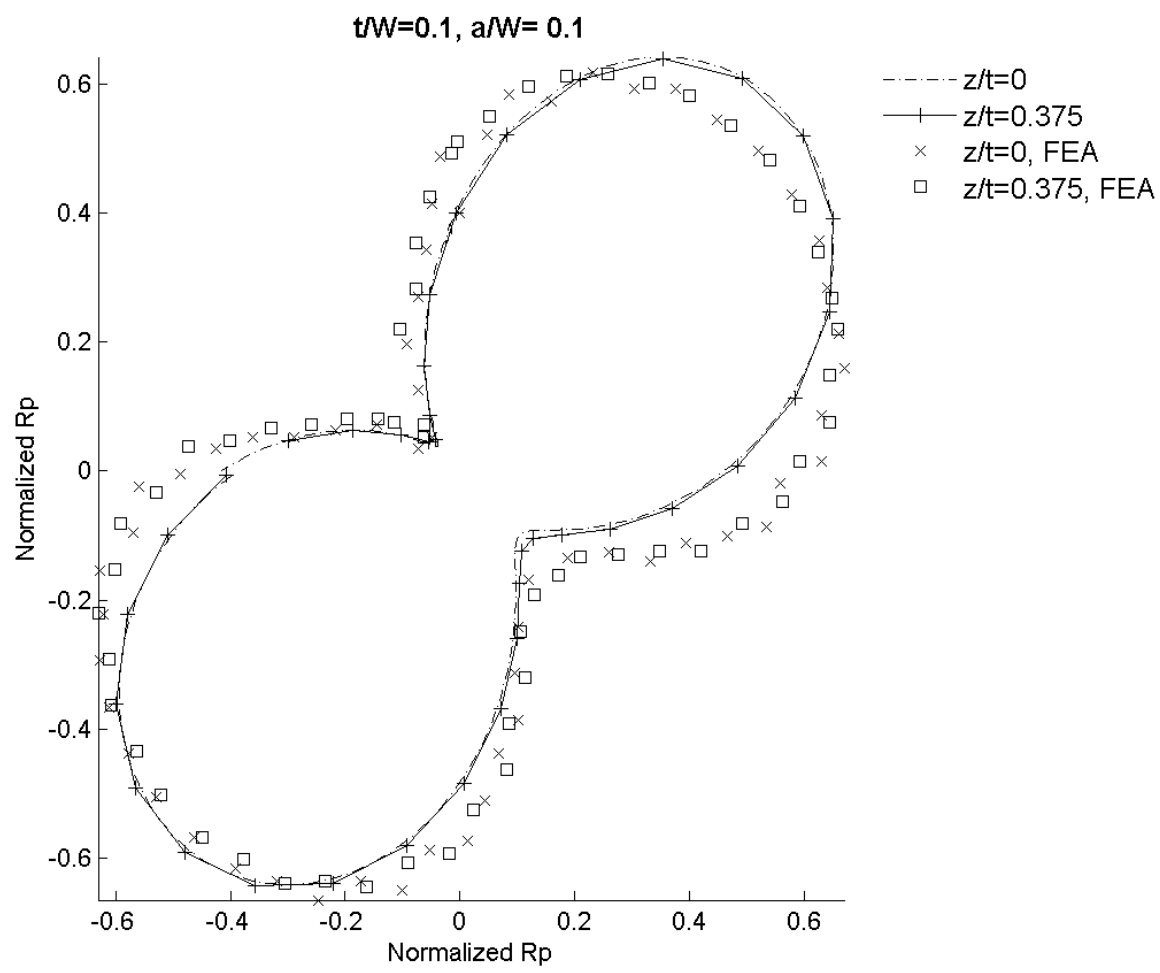

Figure 4.111: Plastic zone comparison for $\mathrm{t} / \mathrm{W}=\mathbf{0 . 1}, \mathrm{a} / \mathrm{W}=\mathbf{0 . 1}, \mathrm{S} / \mathrm{Sy}=\mathbf{0 . 1 2}$

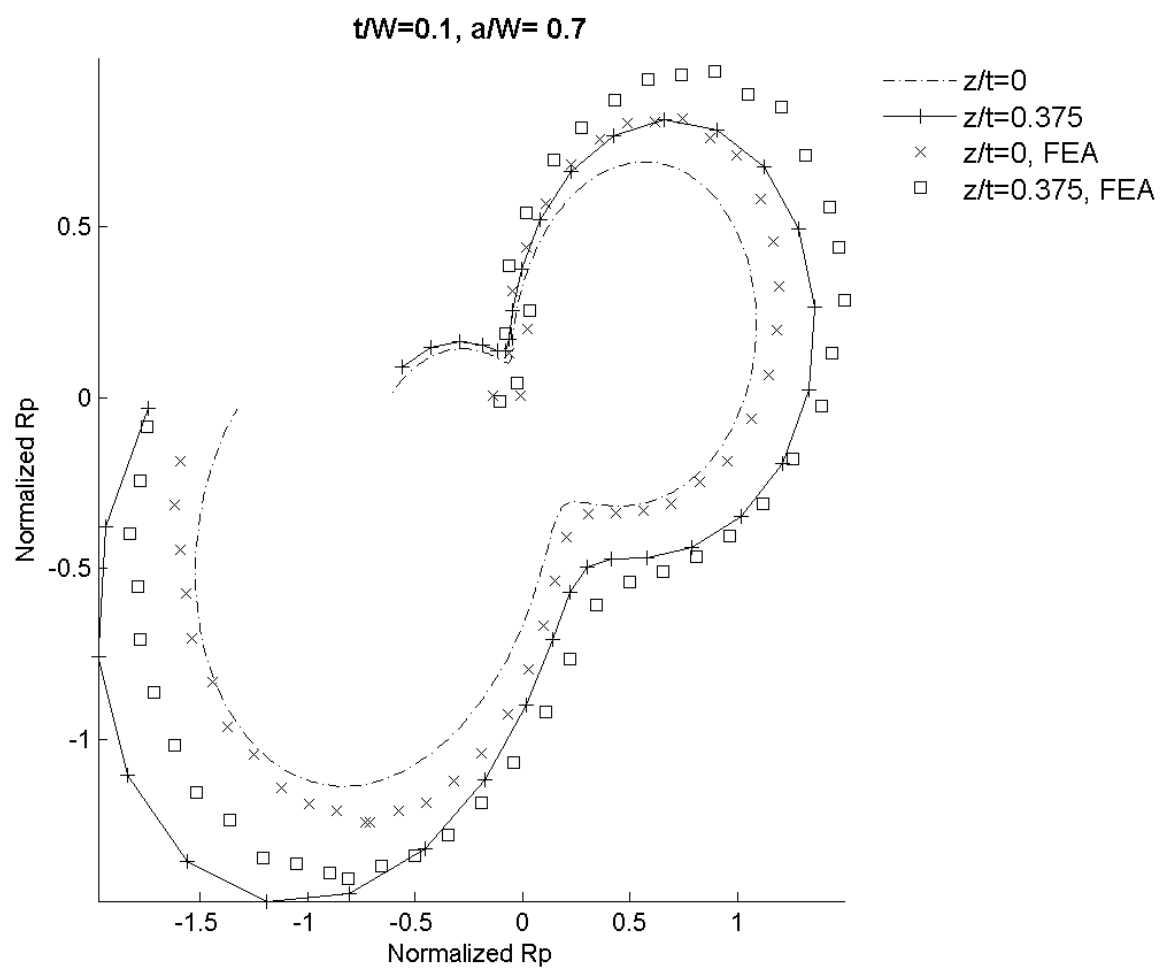

Figure 4.112: Plastic zone comparison for $\mathrm{t} / \mathrm{W}=\mathbf{0 . 1}, \mathrm{a} / \mathrm{W}=\mathbf{0 . 7}, \mathrm{S} / \mathrm{Sy}=\mathbf{0 . 0 6}$ 


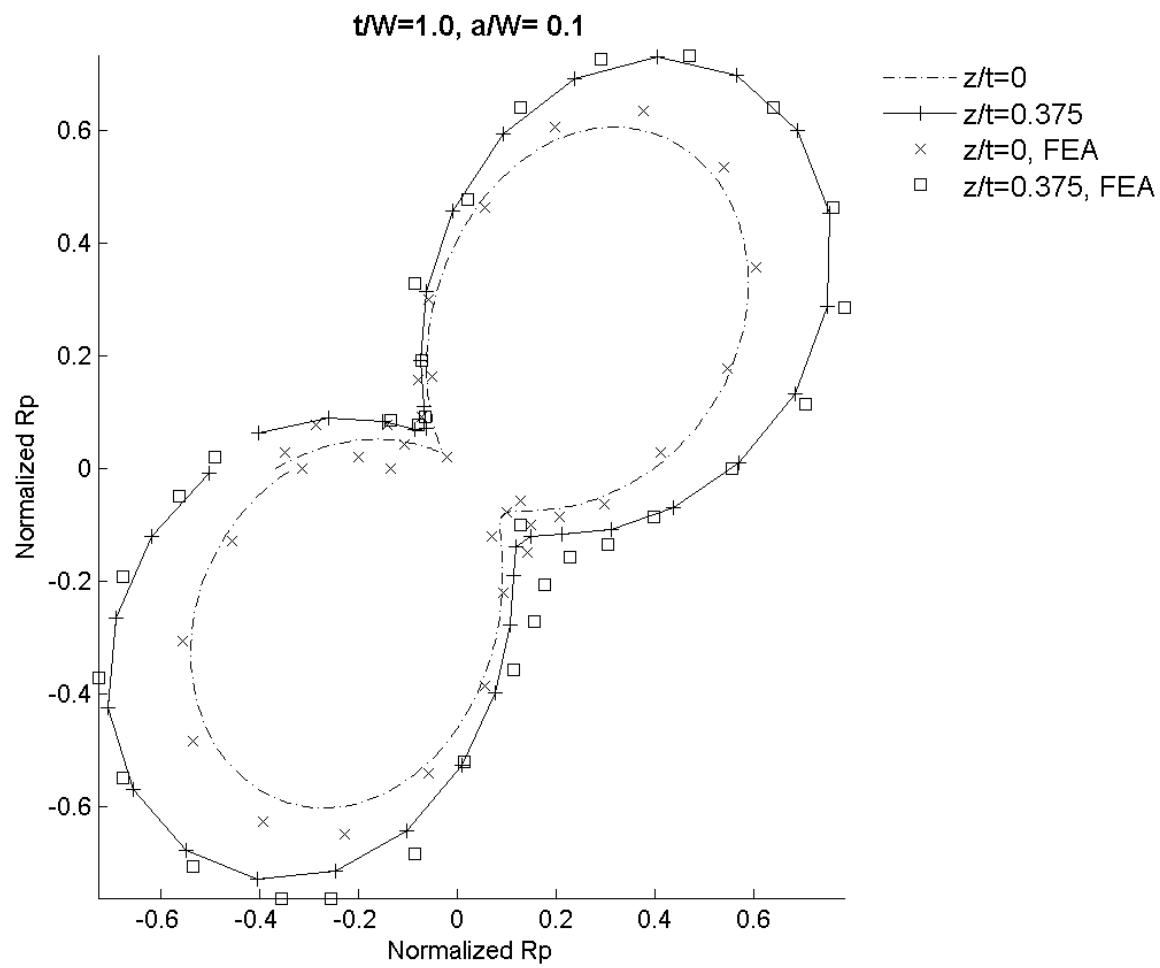

Figure 4.113: Plastic zone comparison for $\mathrm{t} / \mathrm{W}=\mathbf{0 . 1}, \mathrm{a} / \mathrm{W}=\mathbf{0 . 1}, \mathrm{S} / \mathrm{Sy}=\mathbf{0 . 0 9}$

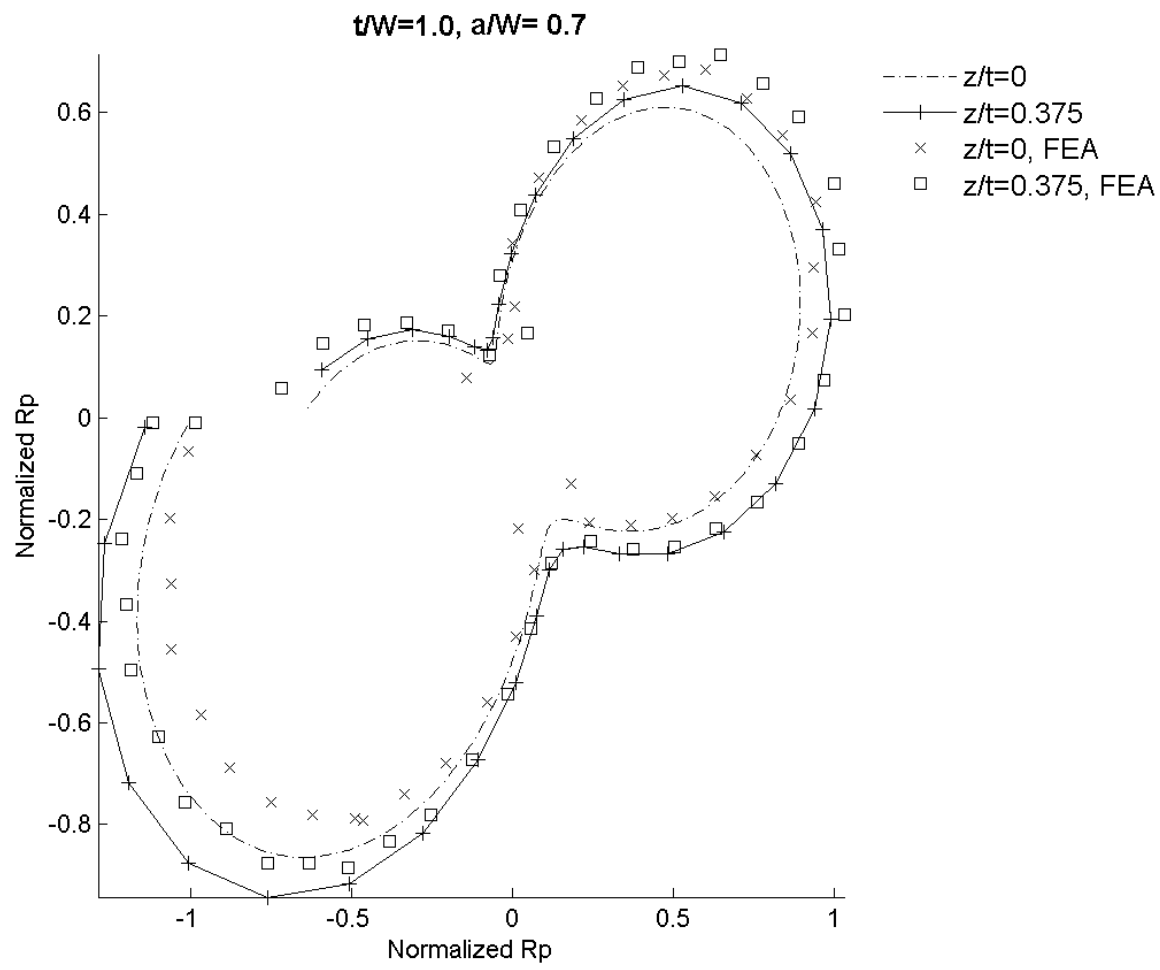

Figure 4.114: $P$ lastic zone comparison for $\mathrm{t} / \mathrm{W}=1.0, \mathrm{a} / \mathrm{W}=\mathbf{0 . 7}, \mathrm{S} / \mathrm{Sy}=\mathbf{0 . 0 6}$ 


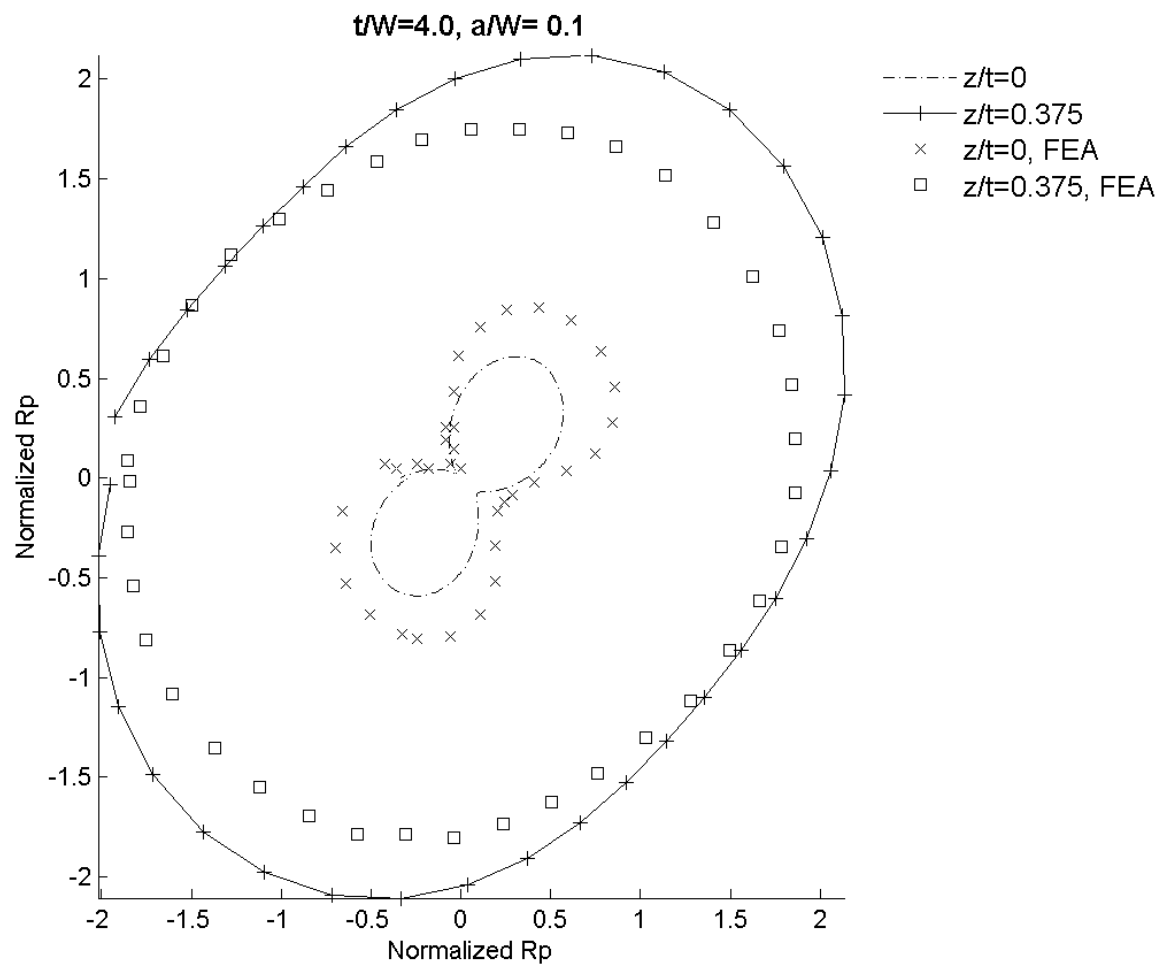

Figure 4.115: Plastic zone comparison for $t / W=4.0, a / W=0.1, S / S y=0.12$

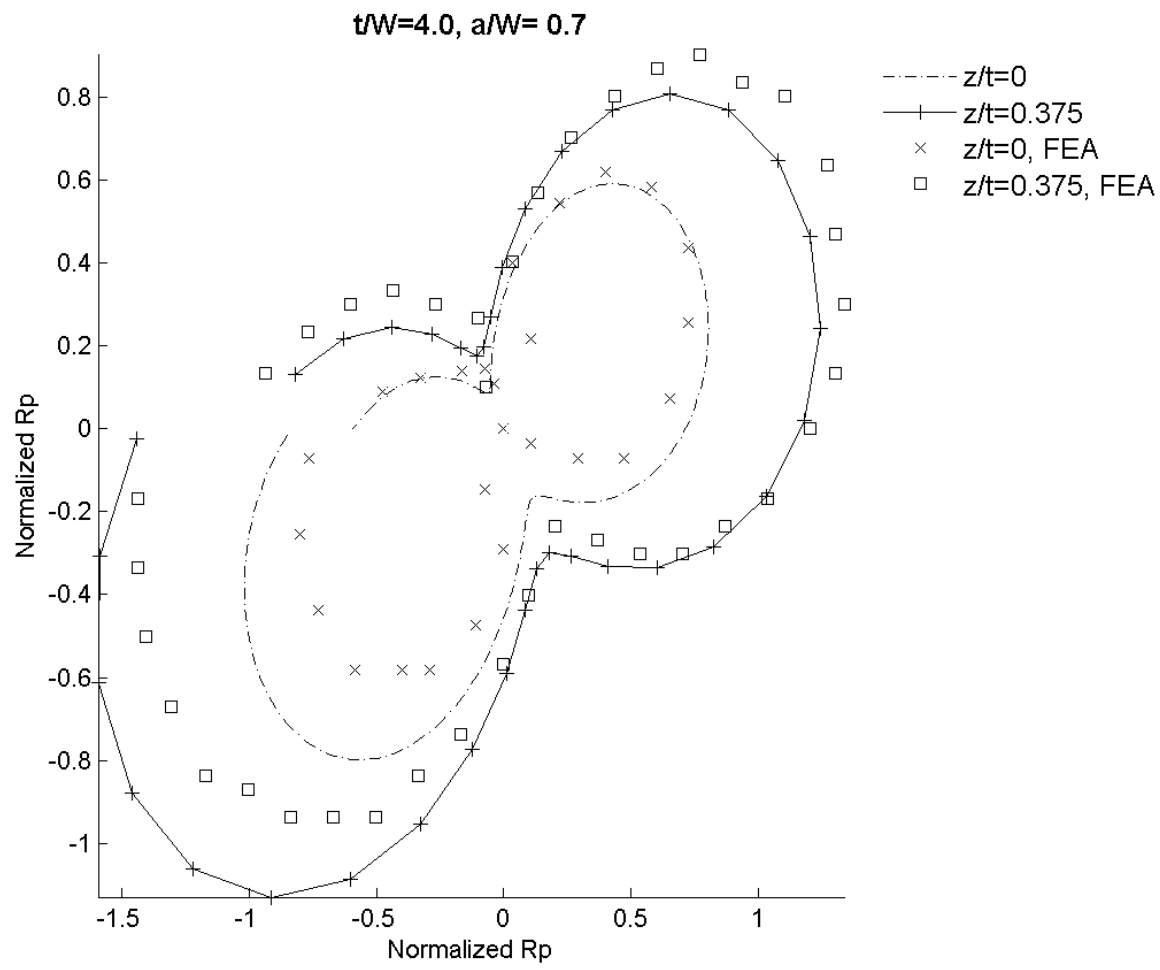

Figure 4.116: Plastic zone comparison for $\mathrm{t} / \mathrm{W}=4.0, \mathrm{a} / \mathrm{W}=\mathbf{0 . 7}, \mathrm{S} / \mathrm{Sy}=\mathbf{0 . 0 6}$ 


\section{Chapter 5: Discussion and Conclusion}

In the fracture testing of materials, determination of material properties is the primary motivation. In order to interpret the results of these tests, accurate expressions for SIF must be known, in terms of specimen and crack geometry. The current research provides numerical solutions for the fracture parameters required to analyze most cases of mixedmode I/II loading in the AFPS specimen.

In the current research, the LEFM parameters are calculated for the FPS and AFPS. In the four-point shear specimen, pure mode II shear loading results in zero values of all fracture parameters except mode II and III SIFs. The asymmetric four-point shear specimen represents a mixed-mode I/II loading case, with non-zero values of all SIF and T-stress parameters. Currently, the values of $K_{I}, K_{I I}, K_{I I I}, T_{11}$, and $T_{33}$ were calculated for a wide range of plate thicknesses, and crack depths. These results are provided at a number of points through-thickness, and plotted for each case considered.

For most cases considered, the size and shape of the plastic zone is predicted, and used to visualize the stress distribution surrounding the crack. For the asymmetric four-point shear specimen, the plastic zone is calculated at several load levels, in order to show the varying effect of T-stress at different load levels. Only a single load level case is shown for the pure mode II loading scenario, which is independent of relative loading when considering purely elastic material behaviour. For some select cases, the plastic zone predicted by equating the Williams (1957) expansion with the Von-Mises yield criterion is compared against the plastic zone extracted from the stress contours of elastic FEA, showing good agreement.

\subsection{D Fracture Analysis of FPS Specimen}

In Chapter 3, the apparent coupling of mode II and mode III SIFs - due to the effects of Poisson's ratio on a finite-thickness specimen - has been investigated thoroughly, for the

specific case of the four-point-shear specimen. The results from the current finite element analysis have shown that the thickness of specimen, as well as depth of crack, have a large effect on the stress surrounding the crack tip. 
The results can be split into two distinct groups; thick plate cases $(t / W>=1.0)$ and thin plate cases $(t / W<1.0)$. The thin plate results are very similar to the literature solution values for $K_{I I}$ and $K_{I I I}$, and the values of these SIFs do not vary significantly throughthickness.

The thick plate cases were observed to show more significant 3D effects than the thin cases for pure mode II loading. The values of $K_{I I}$ at the mid plane decreases inversely with thickness; this effect is more pronounced at shallow cracks. Through-thickness variation of $K_{I I}$ is significant for thick plates, with values increasing approaching the free edge. The calculated values of $K_{I I I}$ were seen to be extreme for shallow cracks in thick plates, with large variations through-thickness, and maximum magnitudes between $z / t=0.3$ to 0.4 .

The effect of including $K_{I I I}$ in the fracture analysis of pure mode II test specimens is most easily seen through plastic zone analysis. Using the size and shape of the plastic zone as an indicator of the stress surrounding the crack tip, the influence of $K_{I I I}$ can be visually seen.

In the thin plate cases, the values of $K_{I I I}$ are small, and $K_{I I}$ does not have much variation through-thickness. This combination of $K_{I I}$ and $K_{I I I}$ produces plastic zones that are all very similar, and are consistent through-thickness. The shape of the plastic zone is representative of pure mode II loading, and the $K_{I I I}$ influence is minimal.

The results for the thick plate cases are drastically different by inspection of the plastic zone. For thick cases, large $K_{I I I}$ values close to the free edge contribute to large variation in plastic zone through-thickness. The combination of large $K_{I I}$ and $K_{I I I}$ magnitudes at locations near the free edge result in a correspondingly large plastic zone, with a shape resembling pure mode III loading (circular). These effects are most noticeable at shallow cracks, and appear to increase with plate thickness.

Overall, it would appear that 3D fracture analysis for pure mode II test specimens would be most important for relatively thick plates and shallow cracks. From the current results, thin plates could be predicted reasonably by considering only $K_{I I}$ terms in the analysis. 
For thick plate cases, it would be necessary to include the effects of finite thickness by considering both $K_{I I}$ and $K_{I I I}$ in the fracture analysis.

\subsection{D Fracture Analysis of AFPS Specimen}

In Chapter 4, mixed-mode loading of the AFPS specimen was studied, and the values of all fracture parameters were obtained. In this specimen, mode I loading is introduced by means of asymmetry, resulting in bending stresses at the crack. This bending stress does not have any effect on the values of $K_{I I}$ and $K_{I I I}$, which are observed to have the same magnitude and through-thickness variation as those calculated for the pure mode II plate cases.

The values of $K_{I}, T_{11}$, and $T_{33}$ show trends consistent with the previous work of Jin and Wang (2014); $K_{I}$ decreasing towards the free edge, for all crack depths and plate thicknesses; $T_{11}$ larger for thinner plates, fairly constant value through-thickness; and $T_{33}$ also larger for thinner plates, with sharply decreasing values towards the free edge. These trends are what is expected, since in the calculation of the SIF and T-stress values, each mode of loading is mostly independent of one another. The exception is for the T-stress component $T_{33}$, which could see influence from mode II loading in its calculation, which includes the strain term $e_{33}$.

For mixed-mode loading, the plastic zone is calculated in two different ways: including only SIF terms, and including all SIF and T-stress terms. In this way, the effect of including T-stress in fracture analysis of mixed-mode loading can be seen.

When considering only SIF terms, the plastic zone shape is a combination of the shape of each of the pure modes: I, II, and III. Mixed-mode loading exhibits a plastic zone shape between pure mode I and II; with the addition of mode III SIF, the plastic zone is enlarged. For the cases which have large magnitudes of $K_{I I I}$, such as thick plates with shallow cracks, a similar enlargement in plastic zone size, as was seen for the pure mode II loading case, is again seen approaching the free edge. For small $K_{I I I}$ this increase can be small and unnoticeable, but for larger magnitudes, the plastic zone increase can be 
extreme. In the cases of $t / W>1.0$, the most shallow cracks had plastic zones of typical mixed-mode behaviour at the mid plane, and much larger, circular (mode III dominated), plastic zones approaching the free edge.

With the inclusion of T-stress values in the plastic zone analysis, the effect of relative loading becomes important. When considering only SIF values, the plastic zone shape calculated is constant, regardless of load level. Due to the definition of T-stress, it is unrealistic to have T-stress values higher than the value of the considered materials yield stress. The plastic zone cannot be described generally for all load levels and particular specimen geometry; the relative loading level must also be known.

For very low loads, the inclusion of T-stress terms is insignificant to the predicted plastic zone size, as predicted by earlier researchers such as Nazarali and Wang (2011). At higher loads the plastic zone becomes discontinuous at the crack tip, and the T-stress terms are seen to increase the size of the plastic zone; deep cracks and thin plates have the largest values of T-stress, and exhibit the most extreme effects.

From the specimens studied in this analysis, two extremes can be identified: thick plates with shallow cracks, and thin plates with deep cracks. In each of these cases, the extreme effect of including $K_{I I I}$ and T-stress in 3D fracture analysis is demonstrated.

In thick plates containing shallow cracks, the large $K_{I I I}$ values dominate both pure mode II and mixed-mode I/II loading cases. Large circular plastic zones are observed close to the free edge for both loading cases, and in mixed-mode loading, the T-stress effect is minimal for even the largest load levels.

Thin plates containing deep cracks represent the extreme effects of T-stress; even at relatively low load levels, large T-stress appears to be a dominant feature in the plastic zone approaching the free edge. While in pure mode II loading, there is no T-stress effect on the plastic zone, and the plastic zone is fairly constant through-thickness, in mixedmode loading, T-stress effects are highest approaching the free edge.

These two extreme cases serve to highlight the importance of analyzing fracture in 3D, and including all SIF and T-stress terms in the analysis. It is not possible to only consider 
2D plates, as neither state of plane stress or plane strain can adequately describe a finitethickness plate. For pure mode I, thick plate cases have been seen to have SIF values approach plane strain solutions, however, from the current results, there is no similar behaviour for pure mode II or mixed-mode I/II loading. The current analysis provides a comprehensive set of solutions for SIF and T-stress values for the FPS and AFPS specimens, which represent typical mixed-mode fracture specimens. By accounting for finite-thickness specimens, and including the full set of fracture parameters, mixed mode fracture test results can be correctly interpreted over a wide range of specimen and crack geometries.

Overall, it has been demonstrated that $K_{I I I}$ components cannot be ignored for both FPS and AFPS specimens. For the AFPS specimen, the contributions of $T_{11}$ and $T_{33}$ should also be considered in interpreting fracture toughness results. It was also observed that the plastic zone will have significant size and shape variations from the mid-plane to the free surface. The current results show that the thickness of the specimen has a large effect on the values of the fracture parameters, and must therefore be accounted for. The results of this thesis will be very useful for the interpretation of experimental test results of mixed mode fracture toughness. 


\section{Appendices}

\section{Appendix A : Plastic Zone Calculation Method (Nazarali and Wang 2011)}

Nazarali and Wang derived an equation to calculate the plastic zone size and shape for a mixed-mode I/II loading case. The following equations are used as a reference solution to verify the current method for calculating the plastic zone.

Considering only modes I and II, the plastic zone is found to be described by the following:

$$
r_{p}(\theta)=\frac{1}{\pi}\left[\frac{K}{\sigma_{Y S}}\right]^{2}\left[\frac{1}{c^{*}}\left\{-[b] t_{y}-\sqrt{[b]^{2} t_{y}^{2}-[a] c^{*}}\right\}\right]^{2}
$$

Net SIF $K=\sqrt{K_{I}^{2}+K_{I I}^{2}}$

$$
\text { Phase angle: } \emptyset=\tan ^{-1} \frac{K_{I I}}{K_{I}}
$$

$$
\text { Normalized T-stress: } t_{y}=\frac{T}{\sigma_{Y S}}
$$

$$
c^{*}=[c] t_{y}^{2}-2
$$

$$
\begin{aligned}
{[a]=\left[\cos ^{2} \emptyset\right.} & \left\{\frac{3}{2} \sin ^{2} \theta+V(\cos \theta+1)\right\} \\
& +\cos \emptyset \sin \emptyset\{2 \sin \theta(3 \cos \theta-V)\} \\
& \left.+\sin ^{2} \emptyset\left\{6-\frac{9}{2} \sin ^{2} \theta-V(\cos \theta-1)\right\}\right]
\end{aligned}
$$




$$
\begin{gathered}
{[b]=\left[\cos \emptyset\left\{\cos \frac{\theta}{2}\left(-3\left\{\cos ^{2} \frac{\theta}{2}-\cos ^{2} \theta\right\}+V\right)\right\}\right.} \\
\left.+\sin \emptyset\left\{\sin \frac{\theta}{2}\left(-3\left\{\cos ^{2} \frac{\theta}{2}+\cos ^{2} \theta\right\}-V\right)\right\}\right] \\
{[c]=\left[\frac{1}{2}\{3+V\}\right]} \\
V=(1-2 v)^{2}
\end{gathered}
$$

The radius of the plastic zone is given as:

$$
\frac{\mathrm{r}_{\mathrm{p}}(\theta)}{\mathrm{h}}=\frac{1}{2}\left[\frac{1}{\mathrm{c}^{*}}\left\{-[\mathrm{b}] \mathrm{t}_{\mathrm{y}}-\sqrt{[\mathrm{b}]^{2} \mathrm{t}_{\mathrm{y}}^{2}-[\mathrm{a}] \mathrm{c}^{*}}\right\}\right]
$$

where

$$
h=\frac{1}{\pi}\left(\frac{K}{\sigma_{Y S}}\right)^{2}
$$




\section{Bibliography}

Aliha, M. R M, and Saghafi, H. (2013). The effects of thickness and Poisson's ratio on 3D mixed-mode fracture. Engineering Fracture Mechanics 98 (1), 15-28.

Anderson, T.1. (2005). Fracture mechanics: fundamentals and applications. Third edition. Boca Raton: CRC Press.

Ayatollahi, M. R., and Aliha, M. R M. (2005). Cracked Brazilian disc specimen subjected to mode II deformation. Engineering Fracture Mechanics 72 (4), 493-503.

Ayatollahi, M. R., and Aliha, M. R M. (2007). Fracture toughness study for a brittle rock subjected to mixed mode I/II loading. International Journal of Rock Mechanics and Mining Sciences 44 (4), 617-624.

Ayatollahi, M. R., and Zakeri, M. (2007). T-stress effects on isochromatic fringe patterns in mode II. International Journal of Fracture 143 (2), 189-194.

Cotterell, B., and Rice, J. R. (1980). Slightly curved or kinked cracks. International Journal of Fracture 16 (2), 155-169.

Dassault Systèmes/ SIMULIA (2013). Modeling fracture and failure with Abaqus. Providence, RI.

Dhondt, G., Chergui, A., and Buchholz, F. G. (2001). Computational fracture analysis of different specimens regarding $3 \mathrm{~d}$ and mode coupling effects. Engineering Fracture Mechanics 68 (4), 383-401.

Fett, T. (2001). Stress intensity factors and T-stress for internally cracked circular disks under various boundary conditions. Engineering Fracture Mechanics 68 (9), 1119 1136.

Griffith, A.A. (1921). The phenomena of rupture and flow in solids VI . Philosophical Transactions of the Royal Society of London, Series A, 221, 163-198.

Hallback, N., and Nilsson F. (1994). Mixed-mode I / II fracture behaviour of an aluminium alloy. Journal of the Mechanics and Physics of Solids 42 (9), 1345-1374.

He, M.Y., Cao, H.C., and Evans, A.G. (1990). Mixed-mode fracture: the four-point shear specimen. Acta Metallurgica et Materialia 38 (5), 839-846.

He, M. Y., and Hutchinson, J. W. (2000). Asymmetric four-point crack specimen. Journal of Applied Mechanics 67 (1), 207-209.

Inglis, C. E. (1913). Stresses in a plate due to the presence of cracks and sharp corners. Trans. I.N.A. XLIV (15), 219-230. 
Irwin, G. (1957, September). Analysis of stresses and strains near the end of a crack traversing a plate. Journal of Applied Mechanics, 361-364.

Jin, Z., and Wang, X. (2015, June). Characteristics of crack front stress fields in threedimensional single edge cracked plate specimens under general loading conditions. Theoretical and Applied Fracture Mechanics 77, pp. 14-34.

Kačianauskas, R., Mroz, Z., Žarnovskij, V., and Stupak, E. (2005). Three-dimensional correction of the stress intensity factor for plate with a notch. International Journal of Fracture 136 (1-4), 75-98.

Kudari, S.K., and Kodancha, K.G. (2010). 3D finite element analysis on crack-tip plastic zone. International Journal of Engineering, Science and Technology 2 (6): 47-58.

Kwon, S.W., and Sun, C.T. (2000). Characteristics of three-dimensional stress fields in plates with a through-the-thickness crack. International Journal of Fracture 104 (3), 289-314.

Larsson, S.G., and Carlsson, A.J. (1973). Influence of non-singular stress terms and specimen geometry on small-scale yielding at crack tips in elastic-plastic materials. Journal of the Mechanics and Physics of Solids 21 (4), 263-277.

Maccagno, T.M., and Knott, J.F. (1985). Brittle fracture under mixed modes I and II loading. International Journal of Fracture 29 (4), R49-R57.

Maccagno, T.M., and Knott, J.F. (1991). The low temperature brittle fracture behaviour of steel in mixed modes I and II. Engineering Fracture Mechanics 38 (2-3), 111128.

MathWorks (2015). Matlab Documentation. Retrieved April 12, 2015, from MathWorks website: http://www.mathworks.com/help/symbolic/vpasolve.html

Matos, C.G. and Dodds, R. H., Jr. (1997). Nonlinear analysis of thin fracture specimens using solid, isoparametric finite elements. Civil Engineering Studies: Structural Research Series 621. Urbana, Illinois: University of Illinois.

Matvienko, Y.G., and Pochinkov, R.A. (2013). effect of nonsingular T-stress components on the plastic-deformation zones near the tip of a mode I crack. Russian Metallurgy (Metally) 2013 (4), 262-271.

McClung, R. C. (1991). Crack closure and plastic zone sizes in fatigue. Fatigue and Fracture of Engineering Materials and Structures 14 (4), 455-468.

Molla-Abbasi, K., and Schütte, H. (2008). On the full set of elastic T-stress terms of internal elliptical cracks under mixed-mode loading condition. Engineering Fracture Mechanics 75 (6), 1545-1568. 
Nakamura, T., and Parks, D.M. (1989). Antisymmetrical 3-D stress field near the crack front of a thin elastic plate. International Journal of Solids and Structures 25 (12), $1411-1426$.

Nakamura, T., and Parks, D.M. (1990). Three-dimensional crack front fields in a thin ductile plate. Journal of the Mechanics and Physics of Solids 38 (6), 787-812.

Nazarali, Q., and Wang, X. (2011). The effect of T-stress on crack-tip plastic zones under mixed-mode loading conditions. Fatigue and Fracture of Engineering Materials and Structures 34 (10), 792-803.

Qu, J., and Wang, X. (2006). Solutions of T-stresses for quarter-elliptical corner cracks in finite thickness plates subject to tension and bending. International Journal of Pressure Vessels and Piping 83 (8), 593-606.

Rice, J. R. (1974). Limitations to the small scale yielding approximation for crack tip plasticity. Journal of the Mechanics and Physics of Solids 22 (1), 17-26.

Roy, Y.A., and Narasimhan, R. (1998). J-dominance in mixed mode ductile fracture specimens. International Journal of Fracture 88 (3), 259-279.

Shahani, A.R., and Tabatabaei, S.A. (2008). Computation of mixed mode stress intensity factors in a four-point bend specimen. Applied Mathematical Modelling 32 (7), $1281-1288$.

Shih, C.F., and Asaro, R.J. (1989, January). Elastic-plastic analysis of a collinear array of cracks on a bimaterial interface. Materials Science and Engineering, A: Structural Materials: Properties, Microstructure and Processing 107, 145-157.

Sih, G.C. (1971). A review of the three-dimensional stress problem for a cracked plate. International Journal of Fracture Mechanics 7 (1), 39-61.

SIMULIA (2014). Abaqus Theory Manual. Retrieved May 15, 2015 from Abaqus website: http://abaqus.ethz.ch:2080/v6.11/pdf_books/THEORY.pdf

Tohgo, K., and Ishii, H. (1991, January). Elastic-plastic fracture toughness test under mixed mode loading. Key Engineering Materials: Fracture and Strength '90 (5152), 307-312.

Toshio, N., and Parks, D.M. (1992). Determination of elastic T-stress along threedimensional crack fronts using an interaction integral. International Journal of Solids and Structures 29 (13), 1597-1611.

United States Coast Guard (1957). Ship research and the ship structure committee. Proceedings of the Merchant Marine Council 14 (11), 175-177, 189. 
Wang, X., and Bell, R. (2004). Elastic T-stress solutions for semi-elliptical surface cracks in finite thickness plates subject to non-uniform stress distributions. Engineering Fracture Mechanics 71 (9-10), 1477-1496.

Williams, M.L. (1957). On the stress distribution at the base of a stationary crack. Journal of Applied Mechanics, 111-114.

Zehnder, A.T., and Viz, M.J. (2005). Fracture mechanics of thin plates and shells under combined membrane, bending, and twisting loads. Applied Mechanics Reviews 58 (1), 37-48. 